\author{
B\&R GB0606020
}

\title{
3He TARGET/BLANKET
}

\section{TOPICAL REPORT}

\section{March 1995}

\author{
DISCLAIMER
}

This report was prepared as an account of work sponsored by an agency of the United States Government. Neither the United States Government nor any agency thereof, nor any of their employees, makes any warranty, express or implied, or assumes any legal liability or responsibility for the accuracy, completeness, or usefulness of any information, apparatus, product, or process disclosed, or represents that its use would not infringe privately owned rights. Reference herein to any specific commercial product, process, or service by trade name, trademark, manufacturer, or otherwise does not necessarily constitute or imply its endorsement, recommendation, or favoring by the United States Government or any agency thereof. The views and opinions of authors expressed herein do not necessarily state or reflect those of the United States Government or any agency thereof.

Submitted to

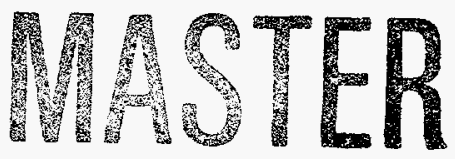

US Department of Energy

Office of Tritium Technology, DP 17

DISTAIDUTION OF THS DOCUMERT IS UNLMITED 



\section{DISCLAMMER}

Portions of this document may be illegible in electronic image products. Images are produced from the best available original document. 



\section{APT ${ }^{3}$ He TARGET/BLANKET TOPICAL REPORT}

\section{TABLE OF CONTENTS}

ACRONYM LIST

XIII

APT OVERVIEW

0.1

1.0 EXECUTIVE SUMMARY

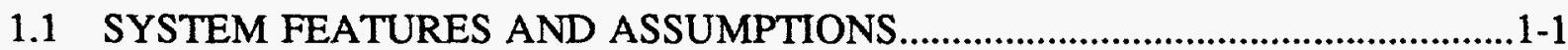

1.2 DESIGN CRITERIA AND ASSUMPTIONS ……...............................................1-1

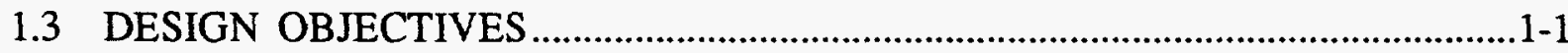

1.4 DESIGN OVERVIEW, 3/8-GOAL SYSTEM ……................................................

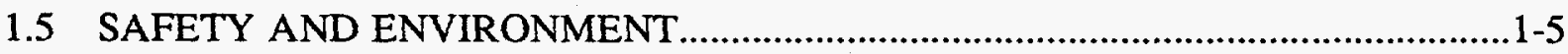

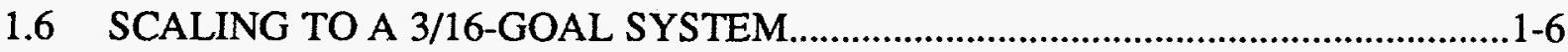

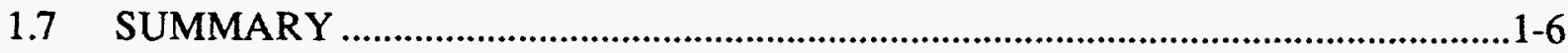

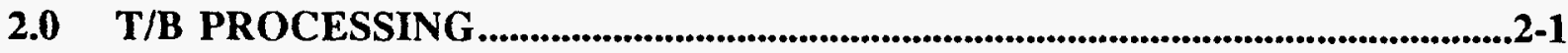

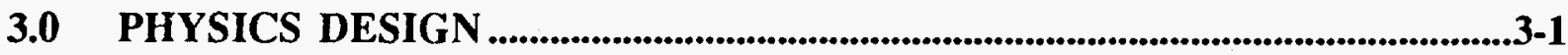

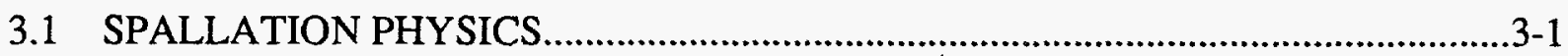

3.2 REFERENCE TARGET/BLANKET PHYSICS MODEL ..........................................3-2

3.3 CALCULATIONAL METHODOLOGY ...................................................................

3.3.1 Overview of the LAHET Code System .............................................................3-6

3.3.2 Overview of CINDER'90

3.3.3 Comparison between LCS Calculations and Experimental Data.........................3-9

3.3.3.1 Bare-Target Integral Neutron Leakage ...................................................3-10

3.3.3.2 LANSCE Neutron Spectra ...............................................................3-10

3.3.3.3 Los Alamos FERFICON Conversion Measurements .........................3-10

3.3.3.4 Russian Energy Deposition Measurements.............................................3-12

3.3.3.5 LANSCE Activation Measurement.....................................................3-13 


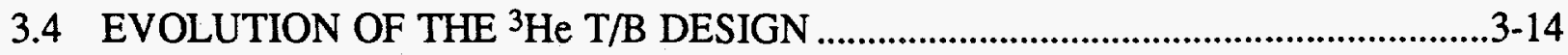

3.4.1 Fundamental Target Neutronics.......................................................................3-14

3.4.2 Physics Models ............................................................................................16

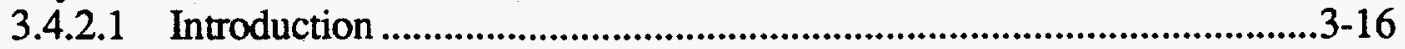

3.4.2.2 Design Evolution.................................................................................3-19

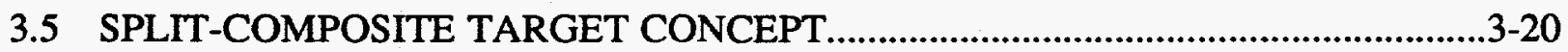

3.6 PHYSICS DESIGN OF THE NEUTRON SOURCE..............................................24-24

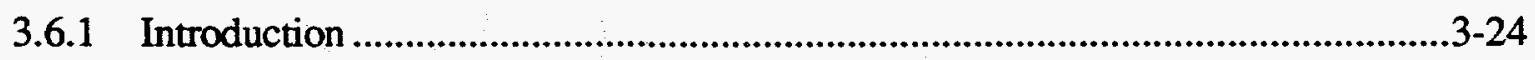

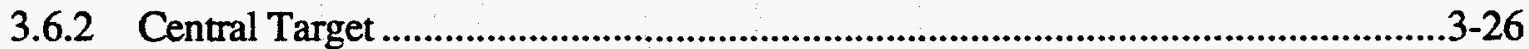

3.6.2.1 Rod Bundle Pattern Study ...............................................................2-26

3.6.2.2 Flux-Trap Gap Size Investigations .........................................................3-31

3.6.2.3 ${ }^{3} \mathrm{He}$ Target Chamber Pressure Studies.................................................3-32

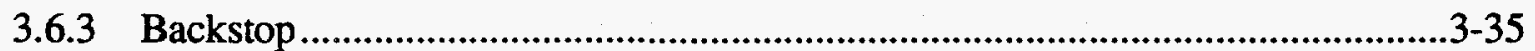

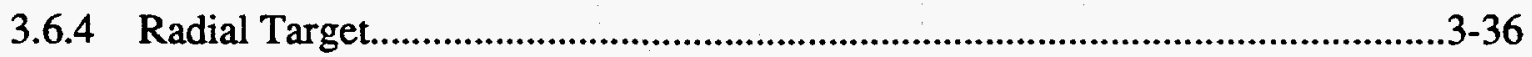

3.6.4.1 Optimization of Radial Target Length.....................................................3-36

3.6.4.2 Effects of Material Composition of the Radial Target ..........................3-37

3.6.4.3 Optimization of Radial Target Thickness...............................................3-40

3.6.5 Neutron Source Performance Optimization Studies...........................................3-42

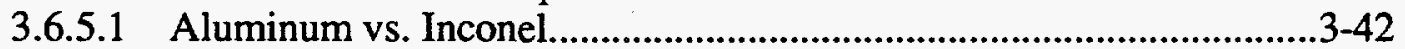

3.6.5.2 Target Chamber Wall Thickness ..........................................................

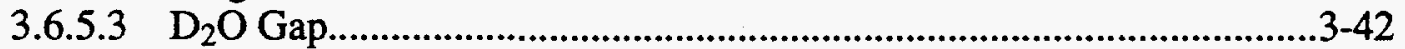

3.6.5.4 Proton Beam Tube.........................................................................44

3.7 PHYSICS DESIGN OF THE MODERATOR/REFLECTOR ...................................3-47

3.7.1 Moderator and Reflector Material ........................................................................

3.7.2 Tube Modeling and ${ }^{3} \mathrm{He}$ Pressure......................................................................48

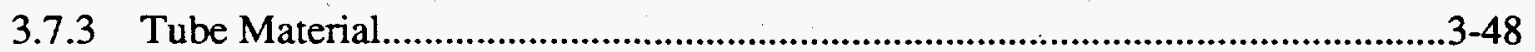

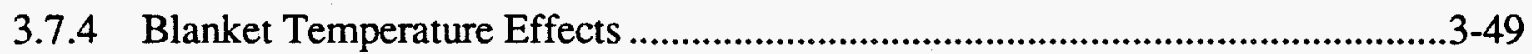

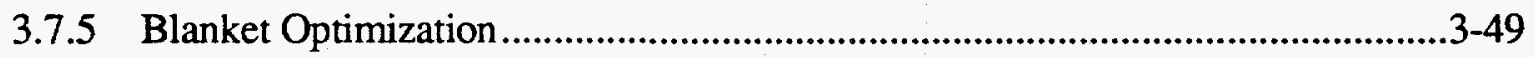

3.7.6 Tube Arrangement......................................................................................

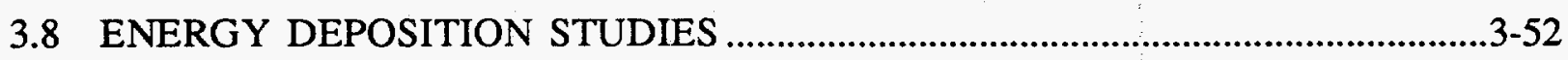

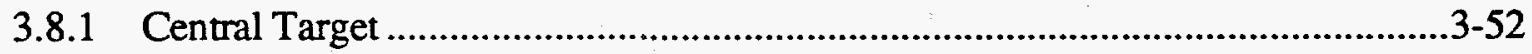

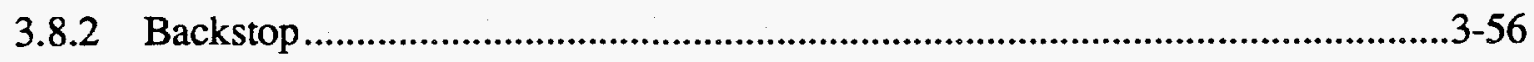

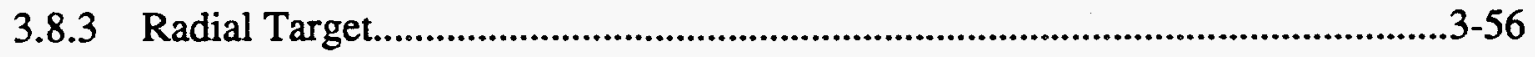

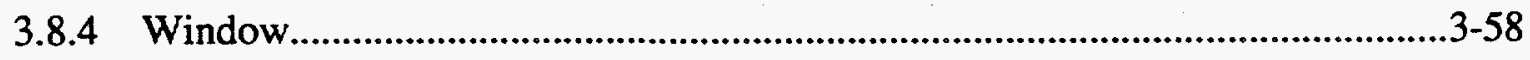


3.8.5 Rod Bundles. $.3-59$

3.9 RADIONUCLIDE PRODUCTION (REFERENCE DESIGN) ………........................61

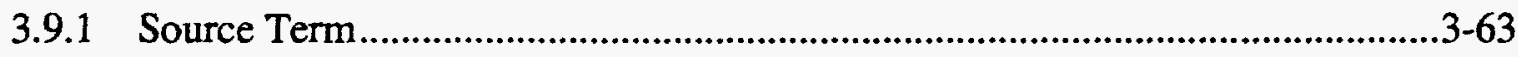

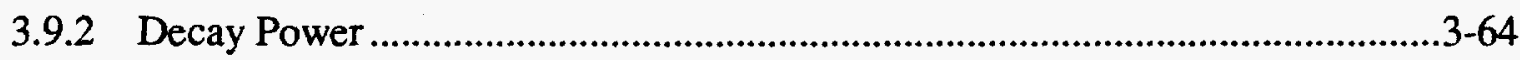

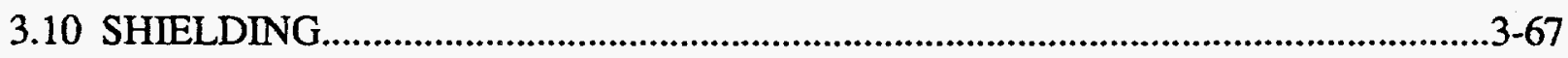

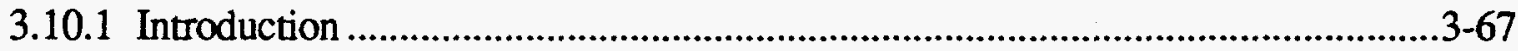

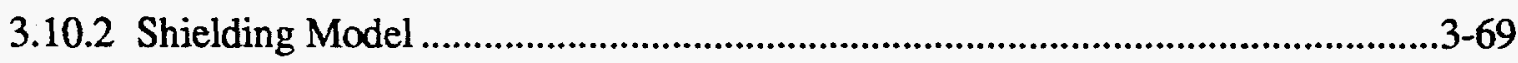

3.10.3 Comparisons between Shielding Model, Simulations, and Experiment..............3-70

3.10.4 Application of the Shielding Model to the ${ }^{3} \mathrm{He}$ T/B System ..............................3-71

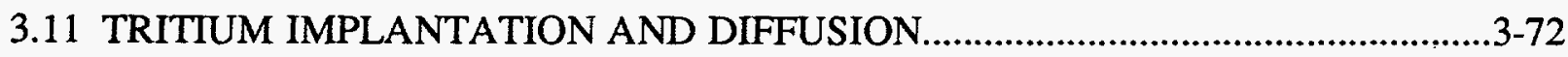

3.12 BEAMSTOP

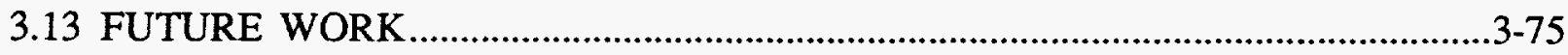

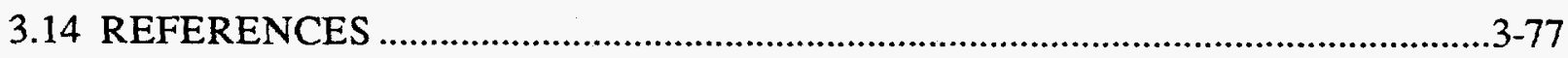

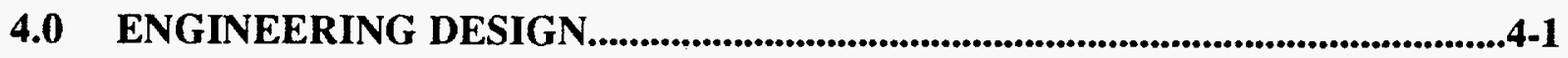

4.1 INTRODUCTION AND ENGINEERING CONSIDERATIONS .............................4-1

4.1.1 T/B Components ......................................................................................

4.1.2 T/B Heat Removal Systems ........................................................................4-3

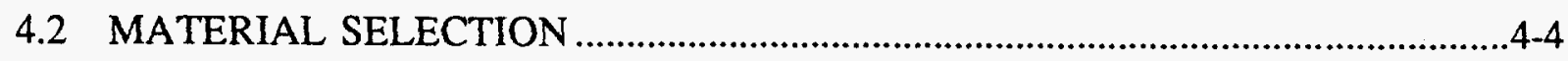

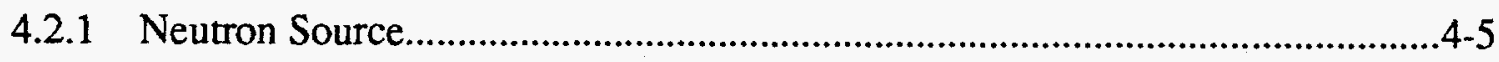

4.2.2 Beam Entrance Window, ${ }^{3} \mathrm{He}$ Chamber, and Rod Bundle Ducts......................4-6

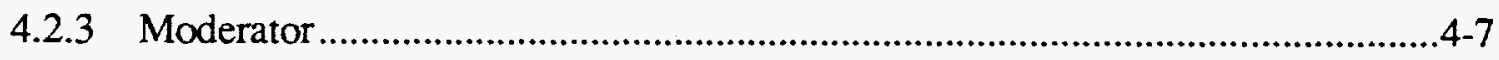

4.2.4 ${ }^{3} \mathrm{He}$ Heat Exchanger and Blanket Tubes ........................................................8

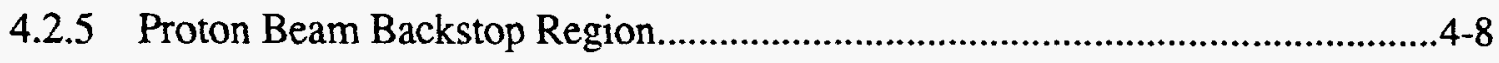

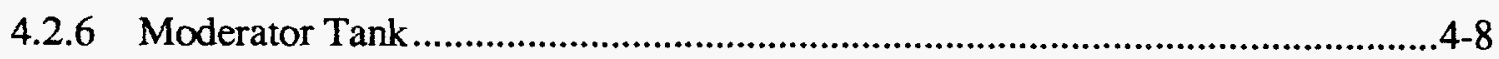

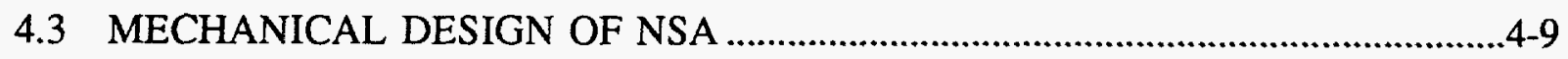

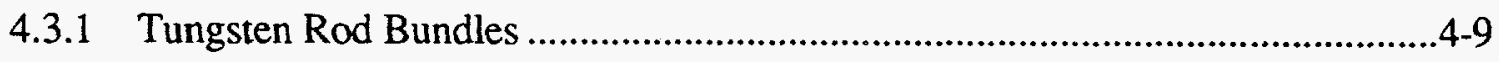

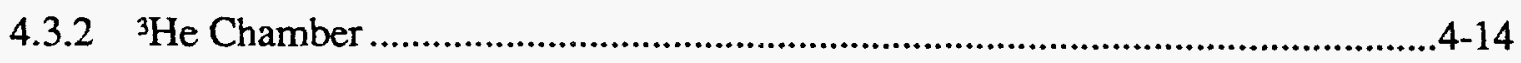

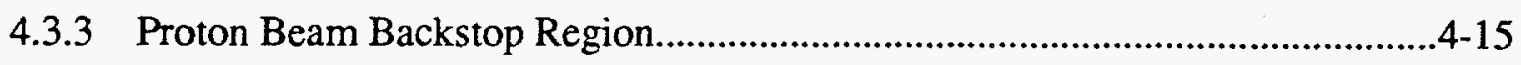

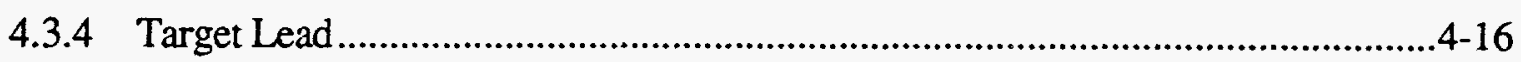

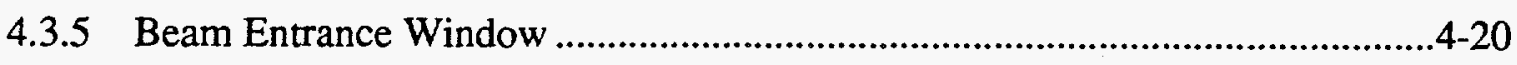




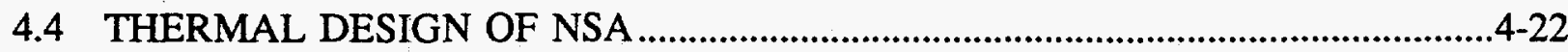

4.4.1 Tungsten Rod Bundles ...................................................................................4-22

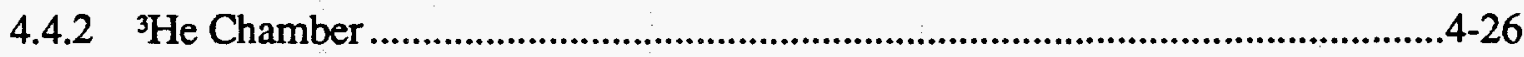

4.4.3 Proton Beam Backstop Region.......................................................................4-26

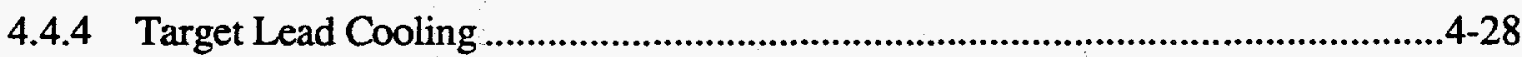

4.4.5 Beam Entrance Window .............................................................................33

4.5 MECHANICAL DESIGN OF TARGET MODULE.................................................4-34

4.6 TARGET/BLANKET HEAT TRANSPORT SYSTEMS ............................................4-50

4.6.1 Tungsten Heat Transport System (THTS)......................................................4-50

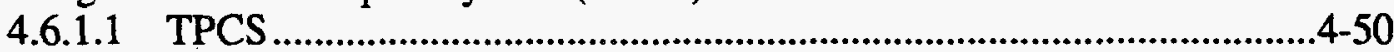

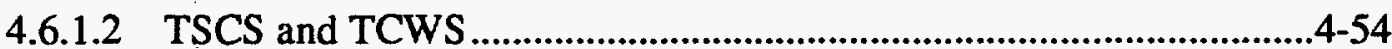

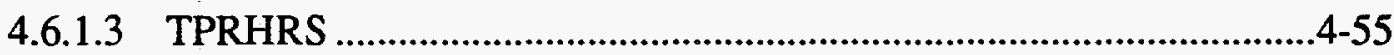

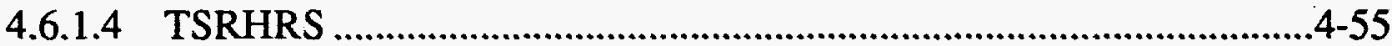

4.6.2 Moderator Heat Transport Systems (MTHTS) ............................................4-55

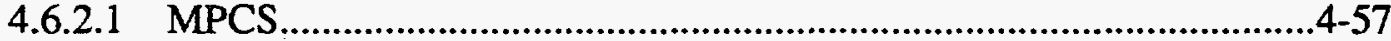

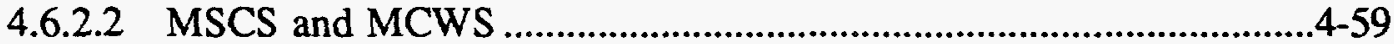

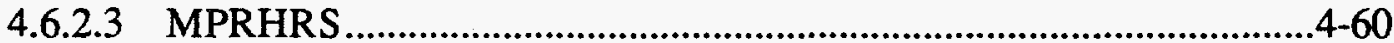

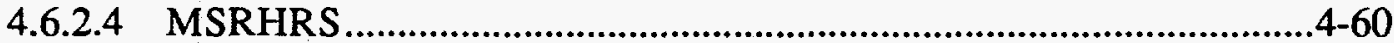

4.6.3 ${ }^{3}$ He Heat Transport Systems (HHTS) ..........................................................64

4.6.3.1 NSA Coolant System (NCS) .....................................................4-62

4.6.3.2 Blanket Coolant System (BCS) ....................................................4-65

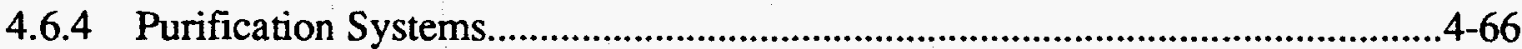

4.7 INSTRUMENTATION CONTROL AND PROTECTION SYSTEMS .......................4-67

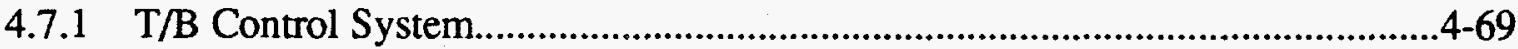

4.7.2 T/B Control Concept......................................................................................69

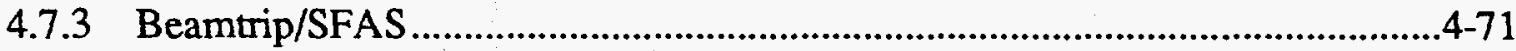

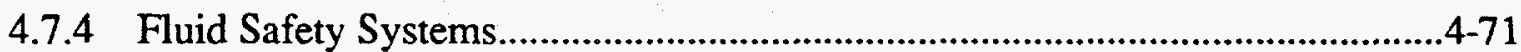

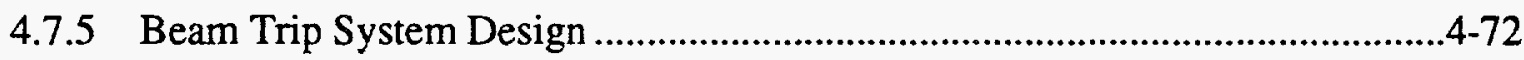

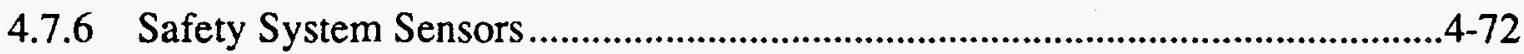

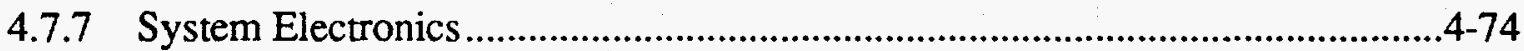

4.7.8 Accelerator Beamtrip Mechanism ..................................................................

4.8 REMOTE-HANDLING SYSTEMS AND OPERATION........................................4-76

4.8.1 Introduction and Summary...........................................................................

4.8.2 Target Module Refurbishment.........................................................................4-77

4.8.3 Target Module Retargeting Operation............................................................4-79

4.8.4 Target Module Disassembly..........................................................................4-83

4.8.5 NSA Target Disassembly Sequence.....................................................................4-84 


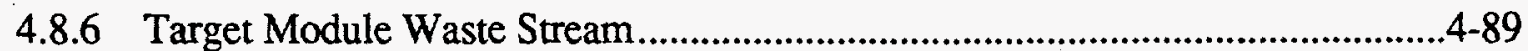

4.9 BEAMSTOP

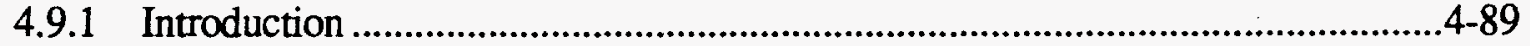

4.9.2 Beamstop Mechanical Design ....................................................................4-91

4.9.3 Beamstop Physics Analysis ..............................................................................4-92

4.9.4 Beamstop Thermal Analysis ..............................................................................4-94

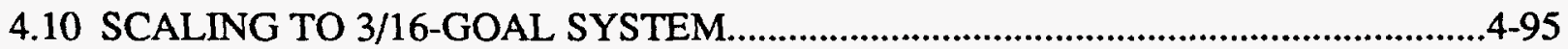

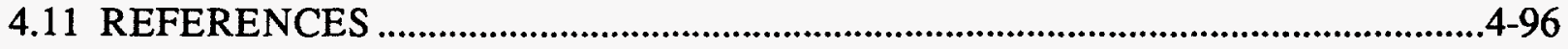

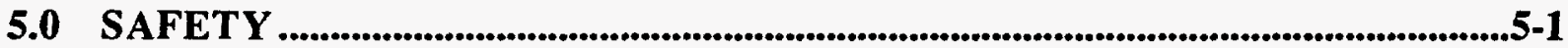

5.1 SAFETY APPROACH AND DESIGN CRITERIA …..............................................

5.2 SUMMARY OF PEIS ACCIDENT SCENARIOS ................................................

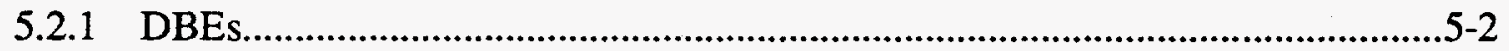

5.2.1.1 Single Helium Tube Break ……………................................................5-3

5.2.1.2 Beam Entrance Window Failure .....................................................5-3

5.2.1.3 Fire in Radwaste System................................................................5-4

5.2.1.4 LBLOCA with One Active Residual Heat Removal System ................5-4

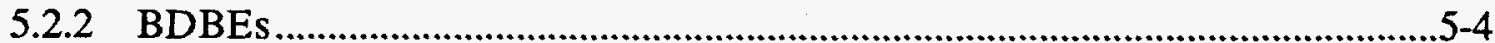

5.2.2.1 Single Assembly Flow Blockage with Delayed Beam Trip .................5-4

5.2.2.2 Multiple Helium Tube Break or Chamber Failure..................................5-5

5.2.2.3 LBLOCA with Total Failure of Active Residual-Emergency

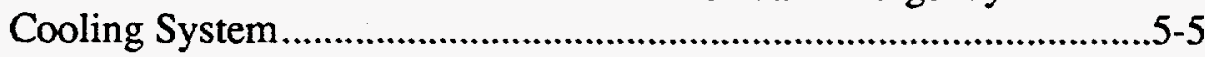

5.3 SUMMARY OF TRAC MODEL AND LBLOCA ANALYSES .............................5-6

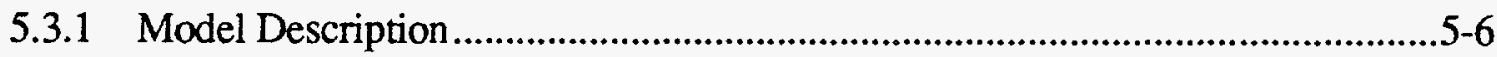

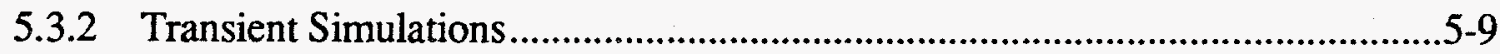

5.4 WINDOW COOLING BY RADIATION HEAT TRANSFER

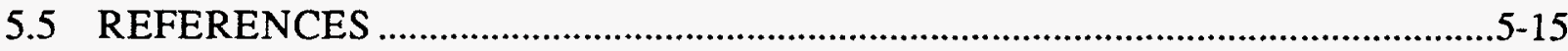

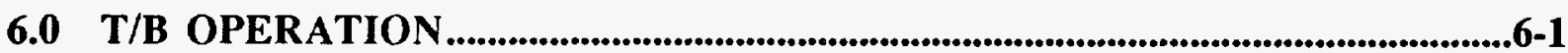

7.0 T/B INTERFACES WITH BOP .........................................................................

8.0 TRITIUM PROCESSING SYSTEMS ..........................................................................8-1

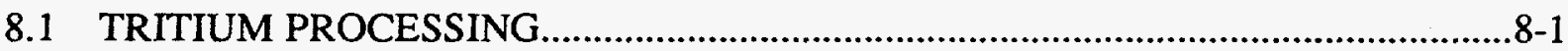




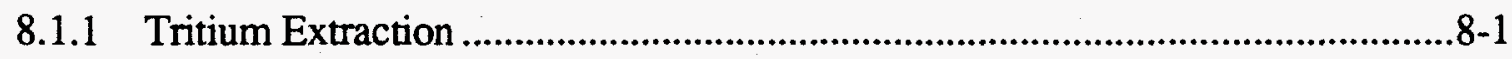

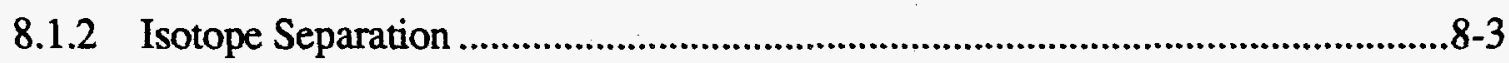

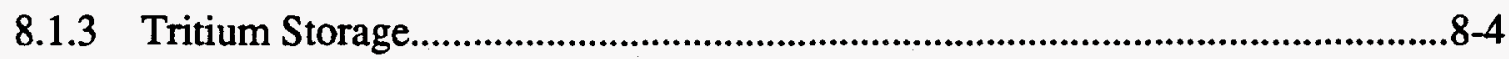

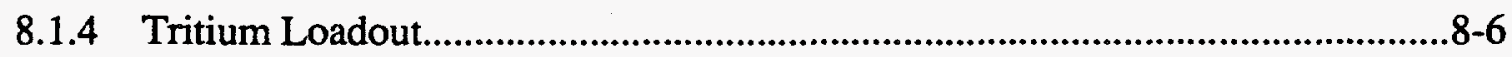

8.2 MODERATOR TRITIUM ACTIVATION

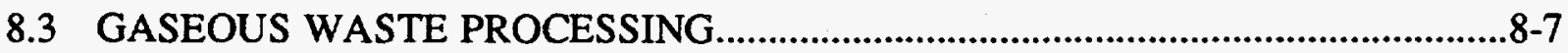

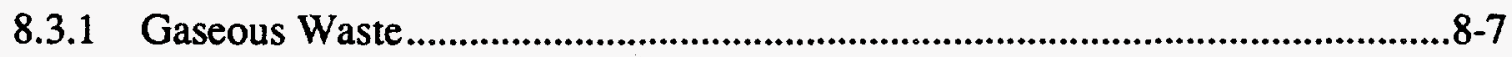

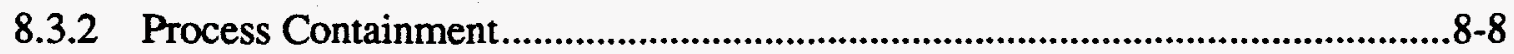

8.3.3 Emergency Cleanup ................................................................................

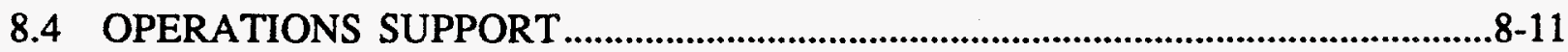

8.4.1 Monitoring and Control .................................................................................

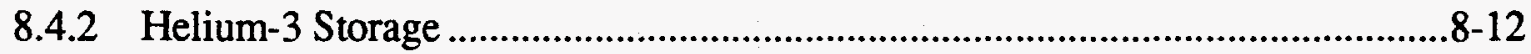

8.4.3 Analytical Laboratory …............................................................................

8.4.4 Tritium Monitoring........................................................................................8-14

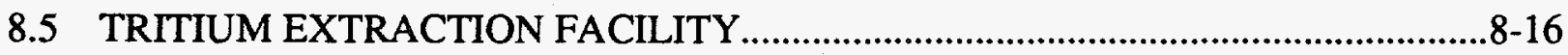

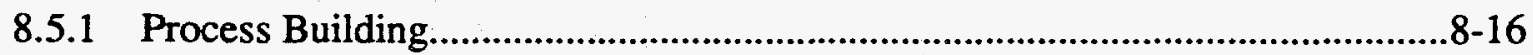

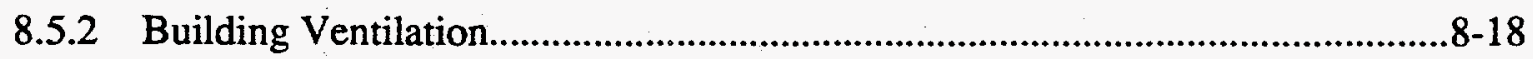

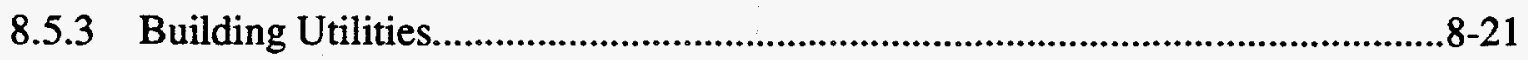

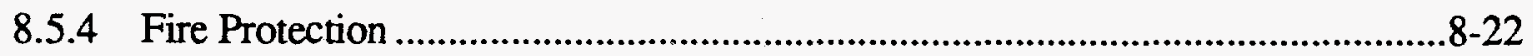

9.0 TECHNOLOGY BASE AND FUTURE DEVELOPMENT ...................................9-1

\section{LIST OF FIGURES}

Fig. 3-1. Illustration of spallation physics ……...........................................................

Fig. 3-2. Cross-sectional views of the APT physics reference model.................................3-3

Fig. 3-3. LCS codes and data files............................................................................

Fig. 3-4. Absolute neutron yields from a cylindrical lead target $20 \mathrm{~cm}$ diam x $60 \mathrm{~cm}$ long. Squares denote LCS results.

Fig. 3-5. Calculated and measured neutron energy spectra from the LANSCE highintensity $\mathrm{H}_{2} \mathrm{O}$ moderator. 
Fig. 3-6. LCS geometry for the Russian energy deposition calculations. 3-12

Fig. 3-7. Total low-energy neutron production and leakage for solid right-circular cylindrical targets bombarded on axis by $1-\mathrm{GeV}$ protons

Fig. 3-8. Tungsten cylindrical target performance relative to lead.

Fig. 3-9. Illustration of various target geometries.

Fig. 3-10. Neutronic performance of various target geometries with equal areas $\left(5775 \mathrm{~cm}^{2}\right)$.

Fig 3-11. Illustration of various target geometries, coolant flow directions, and target chamber-wall geometries.

Fig. 3-12. Basic target configurations

Fig. 3-13. Total neutron production, spallation plus $(\mathrm{n}, \mathrm{xn})$, and leakage for $50-\mathrm{cm}-$ diam targets.

Fig. 3-14. Relative proton beam power density and tritium production as a function of target diameter for cylindrical targets.

Fig. 3-15. Relative tritium production vs. the height of the reentrant head.

Fig. 3-16. Low-energy $(<20 \mathrm{MeV})$ neutron surface flux at a radius of $20 \mathrm{~cm}$ from a 30-cm-diam $\mathrm{W}-\mathrm{Pb}$ split-composite target.

Fig. 3-17. Schematic of the APT target/blanket system.

Fig. 3-18. Cross sectional view of the APT Physics-IV model used in the rod bundle pattern study.

Fig. 3-19. Cross-sectional view of a 91-rod-bundle unit.

Fig. 3-20. The 60-cm region just downstream of the proton beam window of the central target zone for the six cases studied.

Fig. 3-21. Cross-sectional view of the APT T/B showing the central target zone layout for (a) case 1, (b) case 3 and (c) case 4

Fig. 3-22. Tritium production (relative to production at $100 \mathrm{psia}$ ) and fraction of tritium produced within the target chamber as a function of ${ }^{3} \mathrm{He}$ target chamber pressure.

Fig. 3-23. Absorption in the tungsten target material and the radial lead target relative to absorption at $100 \mathrm{psia}$ as a function of ${ }^{3} \mathrm{He}$ target chamber pressure.

Fig. 3-24. Tritium production as a function of Zircaloy backstop length. 3-36

Fig. 3-25. Initial optimization of radial lead zone thickness with a $10.2 \mathrm{v} \%$ coolant fraction.

Fig. 3-26. Optimization of radial lead zone thickness with a $30 \%$ coolant fraction.

Fig. 3-27. Optimization of radial lead zone thickness with structure.

Fig. 3-28. Dependence of tritium production on target chamber wall thickness. 3-43 
Fig. 3-29. Variation of tritium production with $\mathrm{D}_{2} \mathrm{O}$ gap thickness. ...................................4-43

Fig. 3-30. Cross-sectional view of the beam tube area of the base case model BT1...........3-45

Fig. 3-31. Cross-sectional view of the beam tube area of model BT2 with a cylindrical beam tube.

Fig. 3-32. Neutron captures by ${ }^{3} \mathrm{He}$ vs. moderator thickness for $\mathrm{D}_{2} \mathrm{O}$, graphite, and Be moderators.

Fig. 3-33. Typical tube arrangement and dimensions used for optimizing the spacing, S.

Fig. 3-34. Central target zone rod bundle layout showing the bundle-numbering convention used to identify rod bundle positions.

Fig. 3-35. Power density variation with axial position for various radial regions in the radial lead zone.

Fig. 3-36. Radial variation of the "high-Z" power density, averaged over axial extent and azimuth, in the radial lead target.

Fig. 3-37. Bundle layout and numbering for case B3, where the target regions are modeled as discrete, homogeneous hexagons.

Fig. 3-38. Closeup of the layout and numbering of the detailed bundle modeled in case B4.

Fig. 3-39. Nuclide chart with APT reference design medium-energy reaction products.

Fig. 3-40. Distribution of APT mass by element $Z$.

Fig. 3-41. Time dependence of the decay power for the ${ }^{3} \mathrm{He}$ APT target/blanket and the relative core power for shutdown of the NPR Heavy Water Reactor.

Fig. 3-42. Comparison of neutron production spectra from fission and spallation.

Fig. 3-43. Energy-dependent neutron production cross-sections for 800-MeV protons incident on iron as a function of angle with respect to the proton beam.

Fig. 3-44. Case III geometry

Fig. 3-45. Average high-energy neutron dose and energy from the upstream, downstream, and radial surfaces of the APT T/B, as calculated by the Monte Carlo code LAHET.

Fig. 3-46. Dose rate at the shield outer surface vs. inner shield thickness. 3-73

Fig. 3-47. Schematic of the APT target region. .3-74

Fig. 4-1. Neutron Source Assembly (NSA). 4-10

Fig. 4-2. Tungsten Rod Bundle Layout Pattern (Rev. 1.5) $4-11$ 
Fig. 4-3. Tungsten Rod Bundle End Assembly 4-11

Fig. 4-4. Hexagonal Duct Deformation Due To Internal Pressure Loading. $4-13$

Fig. 4-5. Target Lead Configuration. 4-18

Fig. 4-6. Finite Element Model Of Beam Entrance Window 4-21

Fig. 4-7. Target Lead $\mathrm{D}_{2} \mathrm{O}$ Fraction. 4-29

Fig. 4-8. Moderator $\mathrm{D}_{2} \mathrm{O}$ Coolant Flow Arrangement. 4-30

Fig. 4-9. Target Lead Cooling. 4-31

Fig. 4-10. Target Lead Temperature....................................................................................4-33

Fig. 4-11. Target Cavity and Shielding .........................................................................36

Fig. 4-12. Target Module. …….....................................................................................4-38

Fig. 4-13. General Arrangement - Cross Section Elevation. ................................................4-39

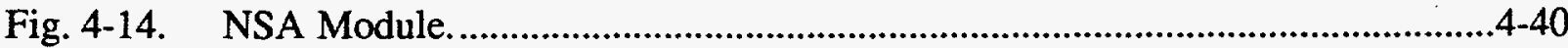

Fig. 4-15. Target Module Assembly...............................................................................4-41

Fig. 4-16. Target Module General Arrangement - Side Section Elevation.........................4-43

Fig. 4-17. Target Module Tungsten $\mathrm{D}_{2} \mathrm{O}$ Arrangement - Cross Section Elevation. ...........4-44

Fig. 4-18. Tungsten $\mathrm{D}_{2} \mathrm{O}$ Coolant Flow Arrangement .....................................................4-45

Fig. 4-19. NSA Helium Flow Arrangement...............................................................4-47

Fig. 4-20. Helium Blankets......................................................................................4-49

Fig. 4-21. T/B Heat Transport Systems (Rev. 1.5)...........................................................4-51

Fig. 4-22. Tungsten Primary Coolant System Flow Schematic......................................4-52

Fig. 4-23. Tungsten Primary Residual Heat Removal System Flow Schematic.................4-56

Fig. 4-24. Moderator Primary Coolant System Flow Schematic........................................4-58

Fig. 4-25. Moderator Primary Residual Heat Removal System Flow Schematic................4-61

Fig. 4-26. NSA Coolant System Flow Schematic.....................................................4-63

Fig. 4-27. Blanket Coolant System Flow Schematic...........................................................4-64

Fig. 4-28. Purification System Schematic...................................................................4-68

Fig. 4-29. LANL APT Control Concept. ....................................................................4-70

Fig. 4-30. Beamtrip/SFAS Overview. .........................................................................4-75

Fig. 4-31. Target Module Assembly......................................................................4-78

Fig. 4-32. Decay Heat vs. Time. ..............................................................................4-79

Fig. 4-33. Retargeting - Target Disassembly Sequence....................................................4-81

Fig. 4-34. Target Cavity and Shielding.....................................................................

Fig. 4-35. Target Rotation Device...............................................................................4-86

Fig. 4-36. Disassembly Sequence..................................................................................4-87 
Fig. 4-37. NSA Target Ready for Tungsten Removal......................................................4-88

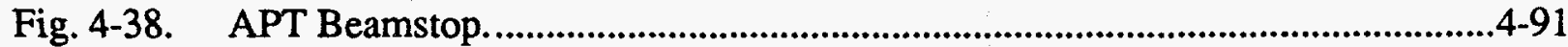

Fig. 4-39. Beamstop Neutron Leakage Flux From Cylindrical Surface................................4-93

Fig. 5-1. APT primary cooling loop schematic as modeled with TRAC. .........................5-7

Fig. 5-2. Pressures in the APT moderator tank during an LBLOCA of a tungsten rod bundle cooling system main coolant pipe, 0 to $10 \mathrm{~s}$.

Fig. 5-3. Maximum structure temperatures for $200 \%$ cold-leg break unprotected LOCA.

Fig. 6-1. APT Complex Control System.

Fig. 8-1. DOE generic components diagram required by tritium supply process..............8-2

Fig. 8-2. APT TE system.........................................................................................

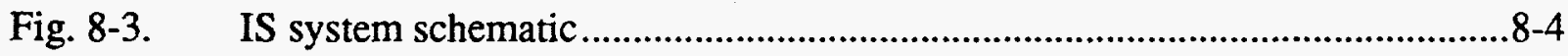

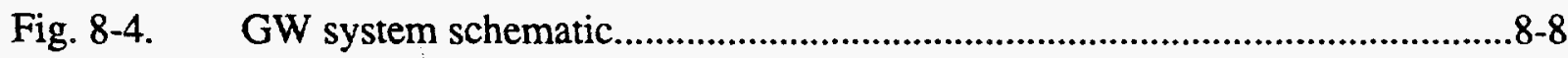

Fig. 8-5. Preconceptual layout-Tritium Extraction Facility .............................................8-17

Fig. 8-6. HVAC schematic_-Tritium Extraction Facility .................................................8-20 


\section{LIST OF TABLES}

Table 1-1. Comparison of Material Inventory …….........................................................1-4

Table 1-2. Summary of Masses for A Single Target/Blanket Module ...............................1-6

Table 3-1. Neutron Production by Material in the ${ }^{3} \mathrm{He}$ Physics Reference Model .............3-5

Table 3-2. Energy Balance Sheet for the ${ }^{3} \mathrm{He}$ Physics Reference Model .............................3-5

Table 3-3. Physical Characteristics of the FERFICON Targets.........................................3-10

Table 3-4. Comparison of Measured and Calculated Conversions for Thorium and Depleted Uranium Targets Bombarded by $800-\mathrm{MeV}$ Protons...........................3-12

Table 3-5. Total Energy Deposited over Axial Length for Radii of $5 \mathrm{~cm}$ and $10 \mathrm{~cm}$

Table 3-6. Decay Dose Values Calculated by LCS/CINDER'90 and Experimental

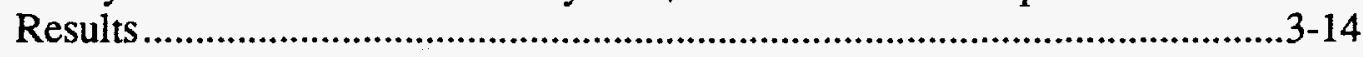

Table 3-7. Results of the Rod Bundle Pattern Study......................................................3-31

Table 3-8. Relative Tritium Production of Various ${ }^{3} \mathrm{He}$ APT Source and Blanket

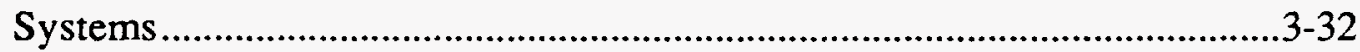

Table 3-9. Relative Tritium Production of ${ }^{3} \mathrm{He}$ APT Lower-Target and Flux-Trap Systems

Table 3-10. Comparison of the Neutronic Performance of Several Backstop Materials.

Table 3-11. Effects of Coolant Fraction in Radial Lead Zone on Tritium

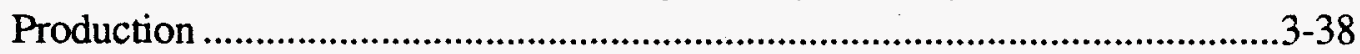

Table 3-12. Heavy Water Distribution in Radial Target Lead Zone......................................3-38

Table 3-13. Material Composition for the SILC Lead Cladding Design..............................3-39

Table 3-14. Effect of Aluminum-1100 Cladding on APT System Performance ................3-39

Table 3-15. Effects of Adding Structural Material to Target Lead Zone.............................3-39

Table 3-16. Tritium Production As A Function Of Beam Tube Configuration....................3-44

Table 3-17. Tritium Production as a Function of Blanket ${ }^{3} \mathrm{He}$ Pressure ...............................3-48

Table 3-18. Average Power Densities in the 88 Rod Bundles and Inconel Clad ..................3-54

Table 3-19. Power densities in the backstop.....................................................................

Table 3-20. Energy Deposited in Various APT Windows...................................................3-59

Table 3-21. Fluxes, Nuclide Production for Each Material in the Reference Physics Model .

Table 3-22. Summary of CINDER' 90 Calculated Time Dependence of the Macroscopic Neutron Absorption During Operation 
Table 3-23. Results of Least-Squares Fits of Eq. (3-3) to Monte Carlo Beamspill Simulations.

Table 3-24. Comparison of Moyer Model Results for Case III with Calculated and Experimental Results.

Table 4-1. Neutron Source Material Comparison ................................................................4-5

Table 4-2. Structural Material Comparisons.............................................................4-7

Table 4-3. Target Lead Plates Preconceptual Design ............................................................4-19

Table 4-4. Target Lead Parameters (Preconceptual Approximation) .................................4-19

Table 4-5. Tungsten Power Densities in Each Rod Bundle Row ........................................4-23

Table 4-6. Bundle Characteristics for Each Group .......................................................4-24

Table 4-7. High- and Mid-Range-Power Rod Bundles and Ducts ....................................4-25

Table 4-8. Backstop Power Density Summary ............................................................24

Table 4-9. Target Lead Cooling Preconceptual Design Parameters ..................................4-32

Table 4-10. Estimated Target Module Weight Breakdown ..................................................4-37

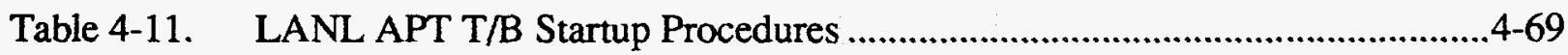

Table 4-12. LANL APT T/B Event vs. Trip Matrix ....................................................4-73

Table 4-13. LANL APT T/B Trip Function List.......................................................4-73

Table 4-14. Estimated Target Module Waste Stream Breakdown Per Target Module Replacement ..............................................................................................4-90

Table 4-15. LAHET-MCNP Energy Deposition Results...................................................4-92

Table 4-16. LAHET-MCNP Proton and Neutron Flux Summary.....................................4-93

Table 4-17. Plate Temperatures and Dimensions ..............................................................4-94

Table 4-18. Summary of Masses for a Single Target/Blanket Module...............................4-95

Table 5-1. APT Tungsten Rod Bundle Cooling System Design Values ...........................5-8

Table 5-2. APT Tungsten Rod Bundle Cooling System Design Parameters.....................5-8

Table 5-3. Comparison of Key Parameters for APT Tungsten Rod Bundle Cooling System LOCA Simulations.........................................................................5-10

Table 5-4. Break Flow, Integrated Break Flow, and Break Temperature for HotLeg LBLOCA, June 1993 Model

Table 8-1. $\quad$ APT Storage Requirements.....................................................................

Table 8-2. APT Tritium Monitors ……………….....................................................

Table 8-3. Tritium Extraction Facility Space Allocation....................................................8-18 


\section{ACRONYM LIST}

6ACM01 beam profile monitor (harp) at LAMPF target station A-6

6AHP01 beam profile monitor (harp) at LAMPF target station A-6

$A \& P \quad$ amplitude and phase

ABAQUS finite element code

acfm

ac frequency modulation

$\mathrm{ACI}$

American Concrete Institute

$\mathrm{A} / \mathrm{E}$

architect engineer

AISC

American Institute of Steel and Concrete

AL

analytical laboratory

Al-2.7Mg

aluminum with 2.7 by weight magnesium

ALARA

as low as reasonably achievable

ALCLAD

aluminum alloy clad with another aluminum alloy

AMESS

autonomous machine environmental surveillance system

AMTEL Advanced Materials Testing and Evaluation Laboratory

ANS

American Nuclear Society

ANS

advanced neutron source

ANSI

American National Standards Institute

AOO

anticipated operational occurrence

$\mathrm{AOP}$

annual operating plan

APDF

Accelerator Performance Demonstration Facility

APT

accelerator production of tritium

ARMS

Accelerator Remote Maintenance System

ASCE

American Society of Civil Engineers

ASHRAE American Society of Heating, Refrigerating, and Air Conditioning Engineers

ASME American Society of Mechanical Engineers

ASTM American Society for Testing Materials

ATR advanced test reactor

ATS Accelerator Test Stand

ATW accelerator transmutation of waste

B biocide

$\mathrm{B} \& \mathrm{~W}$ Babcock \& Wilcox

BCDTL bridge-coupled drift tube linac 
BDBA beyond design basis accident

BDBE beyond design basis event

BEAR beam experiment aboard a rocket

BL beta lambda

BL0 butt-line zero

BLM beamloss monitor

BNL

Brookhaven National Laboratory

BOP

balance of plant

BPM

beam-position monitor

BPSA

beam power spectrum analyzer

BU

building utility

BV

building ventilation

BWR

boiling water reactor

CAM

continuous air monitor

$\mathrm{CB}$

circulation blower

CBP

capacitive beam monitor

$\mathrm{CCL}$

coupled-cavity linac

CCLFISH

computer code for CCL design

CCTV

closed circuit television

CDC

cryogenic distillation columns

CDR

Conceptual Design Report

CERN

European Organization for Nuclear Research (Organization Européenne pour la Recherche Nucléaire)

CETS connector environmental test stand

CF

charcoal filters

CFC

constant-flow cooling

CFR

code of federal regulations

CFS

common file system

CGA

Compressed Gas Association

CHF critical heat flux

CI

corrosion inhibitor

CPM

critical path method

CR catalytic reactor

CRADA Cooperative Research and Development Agreement 


$\begin{array}{ll}\text { CRITS } & \text { Chalk River Injector Test Stand } \\ \text { CRNL } & \text { Chalk River Nuclear Laboratory } \\ \text { CS } & \text { Courant-Snyder } \\ \text { CT } & \text { cooling tower } \\ \text { Culham } & \text { Culham Laboratory (in England) } \\ \text { cw } & \text { continuous wave }\end{array}$

CWBCM continuous wave current monitor

CWBS contractor work breakdown structure

CWDD continuous-wave deuterium demonstrator

CWS circulating water system

$\mathrm{db} \quad$ dry bulb

DBA design basis accident

DBE design basis event

DBMS database management system

DBNP 2-2 Dibromo-3-Nitriloproprionamide

DBTT ductile-to-brittle transition temperature

dc direct current

DCCT de current transducer

DEMO demonstration fusion reactor device

DF duty factor

DoD Department of Defense

DOE Department of Energy

DOF degree of freedom

DOT Department of Transportation

DPA displacement per atom

DTL drift tube linac

DTLFISH A computer code

E neutron energy

EB electron beam weld

EBR-II engineering breeder reactor-II

ECCS emergency core-cooling system

ECN Netherlands Energy Research Foundation

ECR electron cyclotron resonance

EHV extremely high voltage 
EIA an electrical standard

EIS Environmental Impact Statement

EM electromagnet

EMF electromagnetic field

EMI electromagnetic interference

EMQ electromagnet quadrupole

EOL end of life

EPICS

experimental physics and industrial control system

ERAB Energy Research Advisory Board

ES\&H environmental, safety, and health

ESAAB Energy Systems Acquisition Advisory Board

FC Faraday cup

FD focus-defocus

FDC functional design criteria

FDDI fiber-distributed data interface

FDO focus defocus zero focus

FEA finite element analysis

FFTF fast flux test facility

FMEA failure mode and effects analysis

FMIT fusion materials irradiation test

FODO focus drift, defocus drift

FOFODODO focus drift, focus drift, defocus drift, defocus drift-focusing schemes

FPR functional performance requirements

FTA fault tree analysis

FYP Four-Year Plan

GBCA graphics-based control architecture

GBSC graphics-based supervisory control

GC gas chromatograph

GISC generic intelligent system control

GSF gross square feet

GRABASC graphics-based supervisory control

GSRD General Safety Requirements Document

GTA Ground Test Accelerator

GW gaseous waste 


$\begin{array}{ll}\text { HAZOP } & \text { hazards and operability } \\ \text { HE } & \text { high energy } \\ \text { 3He } & \text { helium-3 } \\ \text { HEBT } & \text { high-energy beam transport } \\ \text { HEPA } & \text { high-efficiency particulate adsorption } \\ \text { HERA } & \text { accelerator in Germany } \\ \text { 3HeT } & \text { 3He target } \\ \text { HETP } & \text { height equivalent to theoretical plate } \\ \text { HEX } & \text { heat exchanger } \\ \text { HFBR } & \text { high-flux beam reactor } \\ \text { HFIR } & \text { high-flux isotope reactor } \\ \text { HFR } & \text { high-flux reactor } \\ \text { HL } & \text { 3He loadin } \\ \text { HPN } & \text { high-pressure nitrogen } \\ \text { HS } & \text { 3He storage } \\ \text { HSE } & \text { health, safety, and environment } \\ \text { HTC } & \text { high tritium column } \\ \text { HTO } & \text { isotope of water-hydrogen-tritium-oxygen } \\ \text { HV } & \text { high voltage } \\ \text { HVAC } & \text { heating, ventilating, and air conditioning } \\ \text { HVAC } & \text { high-voltage alternating current } \\ \text { HVDC } & \text { high-voltage direct current } \\ \text { HVPS } & \text { high-voltage power system } \\ \text { HWR } & \text { heavy-water reactor } \\ \text { HX } & \text { heat exchanger } \\ \text { HXC } & \text { hydrogen extraction column } \\ \text { I/O } & \text { input/output } \\ \text { I\&C } & \text { instrumentation and controls } \\ \text { I\&Q } & \text { inphase and quadrature } \\ \text { IEEE } & \text { Institute of Electrical and Electronics Engineers } \\ \text { IEQ } & \text { isotopic equilibrator } \\ \text { IHX } & \text { input heat exchanger } \\ \text { IMS } & \text { intermediate matching section } \\ \text { INC } & \text { isotope and nuclear chemistry } \\ & \\ \text { HE } & \end{array}$


INEL

IOC

IOT

IP

IR

IS

ISI

ISIS

ISR

ITEP

ITER

JAERI

JOYO

JPL

KEK

KFA

LAHET

LAMPF

LAN

LANL

LANSCE

LASREF

LBLOCA

LCS

LTC

LE

LEBT

LEP

LES

LiAl

linac

LINDA

LLNL

LLRF
Idaho National Engineering Laboratory

input-out controllers

inductive output tube

Isotope Production (Facility)

infrared

isotope separation

in-service inspection

Rutherford Laboratory Neutron Facility

input storage receiver

Instituet for Theoretical and Experimental Physics (Moscow)

International Thermonuclear Experimental Reactor

Japan Atomic Energy Research Institute

Japanese testing reactor

Jet Propulsion Laboratory

National Laboratory for High-Energy Physics (Japan)

Kernforschunglage-Jülich (Nuclear Research Center)

Los Alamos High Energy Transport (code)

Los Alamos Meson Physics Facility

local area network

Los Alamos National Laboratory

Los Alamos Neutron Scattering Center

Los Alamos Spallation Radiation Effects Facility

large-break loss-of-coolant accident

LAHET code system

low tritium column

low energy

low-energy beam transport

large electron positron (collider at CERN)

low-energy system

lithium-aluminum

linear accelerator

accelerator beam diagnostics technique for measuring beam emmittence

Lawrence Livermore National Laboratory

low-level radio frequency 
$\mathrm{LN}_{2} \quad$ liquid nitrogen

LOCA loss-of-coolant accident

LOFA loss-of-flow accident

LOFT loss-of-fluid test

LPN low-pressure nitrogen

LPR low-pressure receiver

LTC low tritium column

LVDT linear variable differential transformer

LWR light water reactor

MANTIS a remote handling machine developed at CERN

MAWP maximum allowable working pressure

MBP microstrip beam probe

MBH million Btu's per hour

MC measurement and control

MCNP Monte Carlo neutron/photon transport

MCWS moderator circulating water system

MD moderator detritiation

MEBT medium-energy beam transport

MHTGR Modular High-Temperature, Gas-Cooled Reactor

MIC microbiologically induced corrosion

MIG metal inert gas

MIPS million instructions per second

MISS moderator isotope separation system

MMI man-machine interface

MPCS moderator primary coolant system

MPF Meson Physics Facility

MPRHRS moderator primary residual heat removal system

MRS monitored retrievable storage

MRTI Moscow RadioTechnical Institute

MS mass spectrometer

MSB molecular sieve bed

MSCS moderator secondary coolant system

MSRHRS moderator secondary residual heat removal system

MSRS molecular sieve regeneration system 
MST materials science and technology

MT moderator detritiation

MTBF meantime between failures

MTC main tritium column

MTHTS moderator tank heat transport system

MTTF mean time to failure

MTTR mean time to repair

NASA National Aeronautics and Space Administration

NBS National Bureau of Standards (now called NIST)

NC normal cleanup

NDE

nondestructive examination

NFS network file system

NEPA

National Environmental Policy Act

NFS net feet squared (net square feet)

NFS network file system

NIST National Institute of Standards and Technology

NNF nonreactor nuclear facilities

NP nuclear programs

NPB neutral particle beam

NPR New Production Reactor

NPSH net positive suction head

NSA neutron source assembly

NSF net square feet

OA/FA liquid-immersed, self-cooled/forced air-cooled transformer rating

OA/FA/FA liquid-immersed, self-cooled/forced air-cooled/forced air-cooled transformer rating

OA/FA/FOA liquid-immersed, self-cooled/forced air-cooled/forced liquid-cooled transformer rating

OBE operating basis earthquake

OFE oxygen-free electronic grade

OHX output heat exchanger

OM optical microscopy

ORNL Oak Ridge National Laboratory

P\&ID piping and instrument diagram

PARMILA an accelerator design code 


$\begin{array}{ll}\text { PBCM } & \text { pulsed beam current monitor } \\ \text { PC } & \text { process containment } \\ \text { PCS } & \text { primary coolant system } \\ \text { PEIS } & \text { Programmatic Environmental Impact Statement } \\ \text { PERM } & \text { palladium-silver alloy permeator } \\ \text { PKA } & \text { primary knock-on atom } \\ \text { PL } & \text { product loadout } \\ \text { PM } & \text { permanent magnet } \\ \text { PMQ } & \text { permanent magnet quadrupole } \\ \text { PNL } & \text { Pacific Northwest Laboratory } \\ \text { PRA } & \text { probabilistic risk assessment } \\ \text { PSI } & \text { Paul Scherrer Institute } \\ \text { PSM } & \text { phase spread monitor } \\ \text { PSR } & \text { proton storage ring } \\ \text { PSS } & \text { personal safety system } \\ \text { PVT } & \text { pressure/volume/temperature } \\ \text { PWR } & \text { pressurized water reactor } \\ \text { Q2 } & \text { hydrogen isotopes (H }, \text { HD, HT, } \mathrm{D}_{2}, \mathrm{DT}, \text { and/or } \mathrm{T}_{2} \text { ) } \\ \text { QA } & \text { quality assurance } \\ \text { QAPD } & \text { quality assurance program description } \\ \text { QD } & \text { quadrupole doublet } \\ \text { R\&D } & \text { research and development } \\ \text { RAM } & \text { radiation air monitor } \\ \text { RAMI } & \text { reliability, availability, maintainability, and inspectability } \\ \text { RD } & \text { Requirements Document } \\ \text { RD } & \text { room air detritiation } \\ \text { REC } & \text { rare earth cobalt } \\ \text { RF } & \text { radio frequency } \\ \text { RFP } & \text { request for proposal } \\ \text { RFQ } & \text { radio frequency quadrupole } \\ \text { RGDTL } & \text { ramped gradient drift tube linac } \\ \text { RH } & \text { remote handling } \\ \text { RHR } & \text { residual heat removal } \\ \text { RISO } & \text { a national laboratory in Denmark } \\ & \end{array}$

Revision 1.5 xxi 


$\begin{array}{ll}\text { RM } & \text { radiation monitor } \\ \text { RME } & \text { remote maintenance equipment } \\ \text { RMEX } & \text { exit radiation monitor } \\ \text { rms } & \text { root mean square } \\ \text { ROD } & \text { record of decision } \\ \text { ROV } & \text { remotely operated vehicle } \\ \text { RPT } & \text { reactor production of tritium } \\ \text { RTNS-II } & \text { rotating target neutron source-II } \\ \text { S\&S } & \text { safeguards and security } \\ \text { SAR } & \text { Safety Analysis Report } \\ \text { SC } & \text { secondary containment } \\ \text { SCBA } & \text { self-contained breathing apparatus } \\ \text { SCRF } & \text { superconducting radio frequency } \\ \text { SCS } & \text { secondary coolant system } \\ \text { SD } & \text { standard deviation } \\ \text { SDD } & \text { system design description } \\ \text { SDI } & \text { Strategic Defense Initiative } \\ \text { SEM } & \text { scanning electron microscopy } \\ \text { SF } & \text { safety factor } \\ \text { SFAS } & \text { safety features actuation system } \\ \text { SGHW } & \text { steam-generating heavy water (reactor) } \\ \text { SILC } & \text { spallation-induced lithium conversion } \\ \text { SiTR } & \text { silicon transformation reaction } \\ \text { SL/PGAD } & \text { site layout/plant general arrangement drawings } \\ \text { SLAC } & \text { Stanford Linear Accelerator } \\ \text { SNL } & \text { Sandia National Laboratories } \\ \text { SNOW } & \text { a computer code for injector design } \\ \text { SNQ } & \text { spallations-neutronenquelle (spallation neutron source) } \\ \text { SOP } & \text { standard operating procedure } \\ \text { SRL } & \text { Savannah River Laboratories } \\ \text { SRS } & \text { Savannah River Site } \\ \text { SS } & \text { stainless steel } \\ \text { SSC } & \text { Superconducting Super Collider } \\ \text { SSC } & \text { systems, structures, and components } \\ & \end{array}$


SSE Safe shutdown earthquake

STP spent target pool

SUPERFISH a computer code

SWM slow wire monitor

TA technical area

T/B target/blanket

TBS target/blanket system

TCWS tungsten circulating water system

TDH total dynamic head

TDS total dissolved solid

TE target extraction

TE tritum extraction

$\mathrm{TE}_{010}$ transverse electric (waveguide mode designation)

TEM transmission electron microscopy

TFTR Tokamak Fusion Test Reactor

Thompson CSF a French company

THTS

tungsten heat transport system

TIG

tungsten inert gas

TL

tritium loadout

TM

turbomolecular

TM

tritium monitoring

TOF time of flight

TOPAZ computer-based temperature analysis code

TOSCA 3D computer code for magnets

TPCS tungsten primary coolant system

TPL Tritium Processing Laboratory (Japan)

TPRHRS tungsten primary residual heat removal system

TPS tritium processing system

TRAC Transient Reactor Analysis Code

TS tritium storage

TSCS tungsten secondary coolant system

TSFF Tritium Science and Fabrication Facility

TSRHRS tungsten secondary residual heat removal system

TSS tritium separation system 
APT ${ }^{3}$ He TARGET/BLANKET TOPICAL REPORT

LA-UR-95-2071

TSTA tritium systems test assembly

TSTF tritium systems test facility

UPS uninterrupted power source

USNRC US Nuclear Regulatory Commission

UI University of Illinois

UT ultrasonic test

UTCI universal tool coupling interface

UTIC universal tool interface coupling

Vac volts alternating current

VCO voltage-controlled oscillator

VCR a high-vacuum fitting

VAc volts direct current

VFC variable-flow cooling

VHN Wickers hardness number

VPCE vapor phase catalytic exchange

VPM video profile monitor

VSWR voltage-standing wave ratio

WBS work breakdown structure

WCWS window circulating water system

WHTS window heat transport system

WNR weapons neutron research

WPCS window primary coolant system

WR waveguide-sizing unit

WSCS window secondary coolant system

$\mathrm{ZT}^{2} \quad$ cavity shunt impedance

Revision 1.5

xxiv

March 1995 


\section{APT OVERVIEW}

The Accelerator Production of Tritium (APT) project, sponsored by the Department of Energy/Defense Programs (DOE-DP), involves the preconceptual design of an accelerator system to produce tritium for the nation's stockpile of nuclear weapons. Tritium is an isotope of hydrogen used in nuclear weapons, and must be replenished because of radioactive decay (its half-life is approximately 12 years). Because the annual production requirement for tritium has greatly decreased since the end of the Cold War, an alternative approach to reactors for tritium production, based on a linear accelerator, is now being seriously considered.

The annual tritium requirement at the time this study was undertaken (1992-1993) was $3 / 8$ that of the 1988 goal, usually stated as $3 / 8$-Goal. Continued reduction in the number of weapons in the stockpile has led to a revised (lower) production requirement today (March, 1995). The production requirement needed to maintain the reduced stockpile, as stated in the recent Nuclear Posture Review (summer 1994) is approximately 3/16-Goal, half the previous level. The Nuclear Posture Review also requires that the production plant be designed to accomodate a production increase (surge) to $3 / 8$-Goal capability within five years, to allow recovery from a possible extended outage of the tritium plant. APT was reviewed by the DOE's Energy Research Advisory Board (ERAB) in late 1989 and then by the JASONs in early 1992. Both reviews were positive about the technology, but pointed out the need for a research and development program. As a result of these reviews, DOE decided to sponsor the preconceptual 3/8-Goal APT design activity.

A multi-laboratory team (Los Alamos, Sandia, and Brookhaven National Laboratories), collaborating with several industrial partners (Bechtel, Babcock \& Wilcox, Grumman, General Atomics, Maxwell Balboa, and Merrick), has developed a preconceptual APT design for the 3/8-Goal, operating at 75\% capacity. This report provides the results of the preconceptual design of the baseline 3He target/blanket system. An earlier version of this report (Revision 1.0) was issued in June, 1994. It contained the design results for the 3/8Goal system. This Revision 1.5 report corrects the errors in the previous revision, and adds some discussion on possible design changes that would be incorporated for the $3 / 16$-Goal system.

The APT tritium-supply option consists of a powerful linear accelerator that bombards a spallation target with high-energy protons. The final beam energy in the accelerator is 1000 $\mathrm{MeV}$ and the proton current is $200 \mathrm{~mA}$, which provides the $200 \mathrm{MW}$ of beam power needed for 3/8-Goal tritium production level. Beams from two $100-\mathrm{mA}$ low-energy linacs are combined to produce a $200-\mathrm{mA}$ current which is accelerated to the final energy in the highenergy linac. A beam transport system conveys the $1000-\mathrm{MeV}$ beam to one of two identical target/blanket systems where the tritium is produced. A schematic diagram of the 3/8-Goal APT system is shown in Fig. O-1.

Neutrons are produced in the spallation target and are absorbed in a blanket material to produce tritium. Two spallation targets are currently under investigation: (1) a tungsten neutron-source target, and (2) a lead neutron-source target. The tungsten target is the primary system and the lead target is a backup system. In the tungsten target concept, the 
neutrons are captured by gaseous $3 \mathrm{He}$, an isotope of helium, which is continuously circulated through the tungsten target region and the heavy-water blanket region to produce tritium via neutron capture. Because tritium beta decays to ${ }^{3} \mathrm{He}$, a sufficient inventory of ${ }^{3} \mathrm{He}$ is readily available for conversion. In the lead target concept, the lead neutron source is surrounded by a lithium-aluminum blanket and the tritium is produced by neutron capture in the lithium isotope, 6 $\mathrm{Li}$. This target is referred to as the Spallation-Induced Lithium-Conversion (SILC) target. This report describes in detail the preconceptual design of the ${ }^{3} \mathrm{He}$ Target/Blanket system. The backup SILC target concept is described in a separate report.

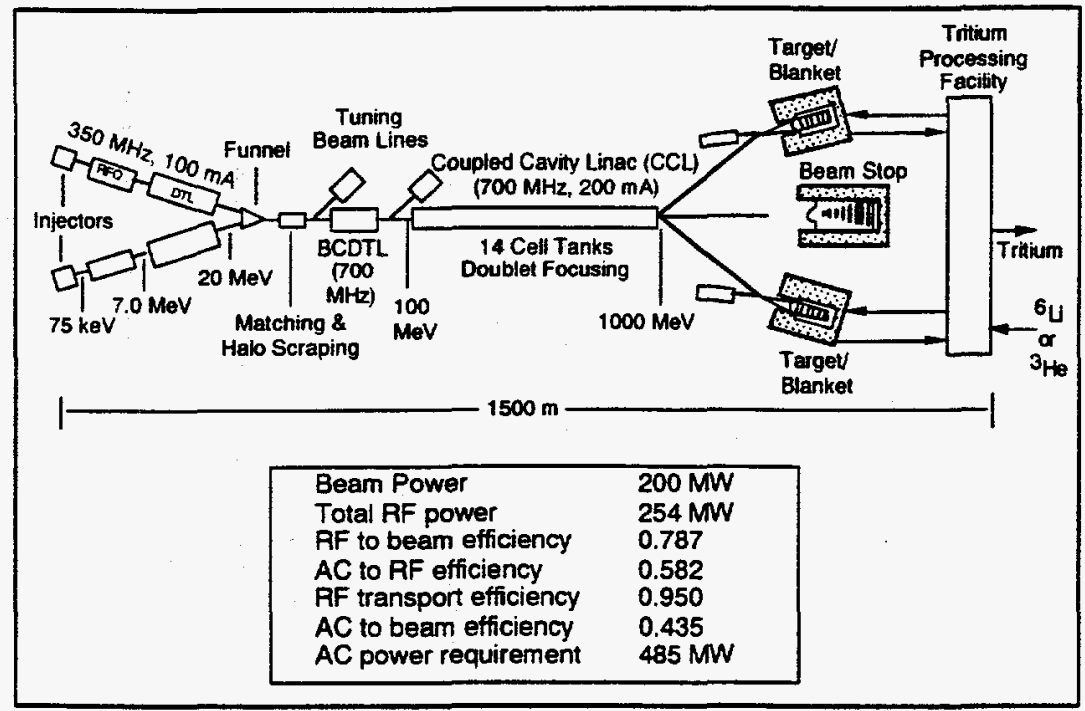

Fig. O-1. Schematic of the APT System (3/8-Goal)

For the $3 \mathrm{He}$ target/blanket, the tritium inventory is small because tritium is continuously extracted during operation. Also, the continuous on-line extraction process eliminates the need for fabrication and reprocessing of the lithium-aluminum blanket; therefore, the overall waste stream and attendant environmental impact are minimized. In both target concepts, tritium is produced without the use of fissionable materials; therefore, no high-level spent fuel waste is produced, and the overall safety impacts are significantly reduced compared with reactor systems.

Given the new 3/16-Goal tritium requirement expressed in the Nuclear Posture Review, a revised accelerator concept has been derived from the $3 / 8$-Goal system point design. In this 3/16-Goal (nominal) design, the beam current is reduced to $100 \mathrm{~mA}$, while the beam energy remains at $1000 \mathrm{MeV}$, providing a $100 \mathrm{MW}$ beam. Only a single low-energy linac is needed for this system (no beam combining). In the 3/16-Goal design, accelerating-structure frequencies are the same as in the 3/8-Goal system, and the beam-focusing magnet lattice is unchanged. The beam dynamics are identical to that of the $3 / 8$-goal linac because the number of protons per bunch remains the same. RF power and $A C$ power requirements are significantly smaller, however, because of the factor-of-two reduction in beam power. The high-energy beam transport is identical to that in the $3 / 8$-Goal system design. The accelerator tunnel and above-ground support buildings are constructed at the full size and 
scale needed for a $3 / 8$-Goal system, as are the buildings housing the target/blanket assemblies.

To provide the surge production capability implied in the Nuclear Posture Review, the 3/16-Goal linac could be upgraded to 3/8-Goal production by increasing the beam current to $200 \mathrm{~mA}$ (Fig. O-2). This would be accomplished by 1) adding a second 100-mA low-energy linac, 2) implementing the beam combiner, 3) adding RF power stations in the high-energy linac to supply the increased beam power, 4) replacing the target/blanket with a larger system, and 5) adding capacity in balance-of-plant systems (power distribution, cooling, etc.). The upgrade could be accomplished in two years.

Scaling of a 3/16-Goal accelerator concept from the 3/8-goal APT point design provides a reasonable starting point for a reduced capacity system, bounding values for ES\&H impacts, and a basis for construction and operating cost estimates. However, such an approach may not generate an optimum design in terms of costs, performance, technical risk, and other factors. Trade studies are planned during the APT conceptual design phase to determine the optimum accelerator configuration to satisfy the Nuclear Posture Review requirements.

The target/blanket design for the 3/16-Goal system is reduced in cross section from that of a 3/8-Goal target/blanket design, maintaining approximately the same power density in the neutron source materials. A comparison of the materials required for a single target/blanket module for both the 3/16 and 3/8-Goal systems is given in Table 0-1 below.

Table O-1. Summary Of Masses For A Single Target/Blanket Module

\begin{tabular}{lcc}
\hline Material & 3/16 Goal & 3/8 Goal \\
\hline Aluminum & 4419 & 5497 \\
Heavy Water & 28175 & 34583 \\
Helium-3 & 7.6 & 14.2 \\
Inconel & 3976 & 4972 \\
Lead & 53586 & 70801 \\
Stainless Steel & 14158 & 16618 \\
Tungsten & 778 & 1476 \\
Zircaloy-4 & 2320 & 4328 \\
\hline
\end{tabular}

The multi-laboratory team has presented APT as a promising alternative to the reactor concepts proposed for Complex-21. The validity of the design has been confirmed by several reviews that have taken place. since the conclusion of the 1992-1993 3/8-Goal design study, including a very thorough and detailed assessment by a "red team" from Lawrence Livermore National Laboratory and a second review by the JASONs. Given the requirements of a reduced weapons stockpile, APT offers both significant safety, environmental, and production-flexibility advantages in comparison with reactor systems, and the prospect of successful development in time to meet the US defense requirements of the 21st Century. 


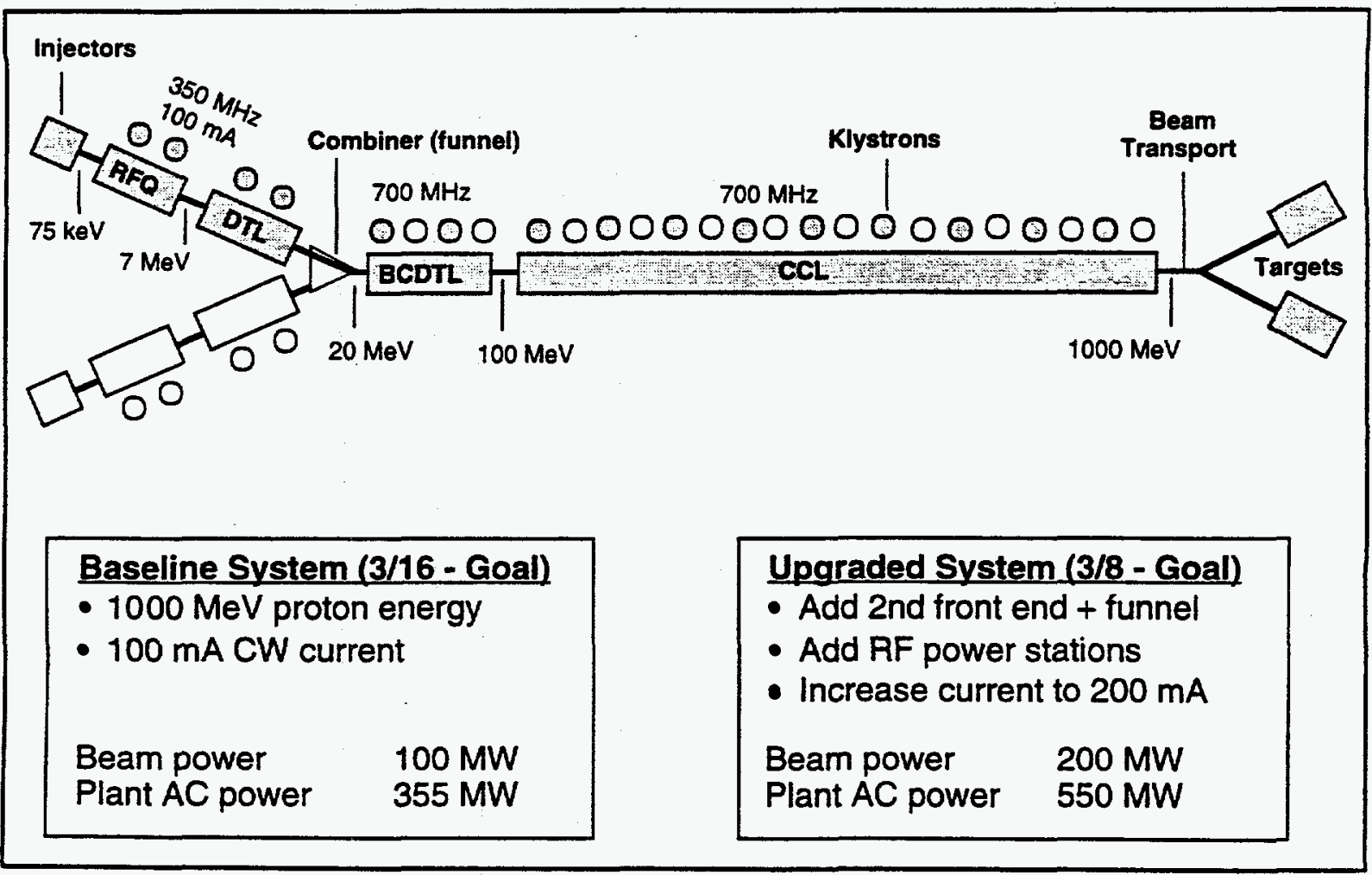

Fig. 0-2. 3/16-Goal APT Accelerator with Upgrade to 3/8-Goal 


\section{EXECUTIVE SUMMARY}

CHAPTER 1

Page

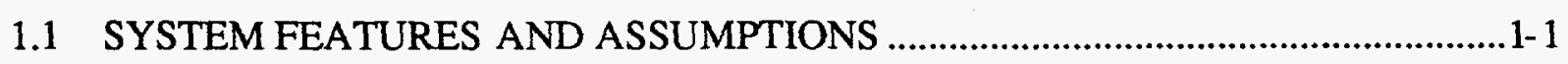

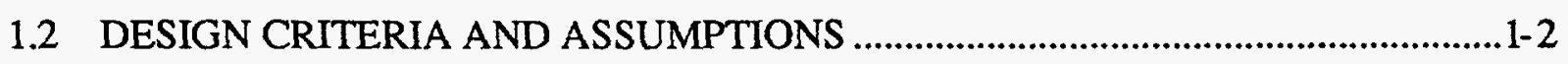

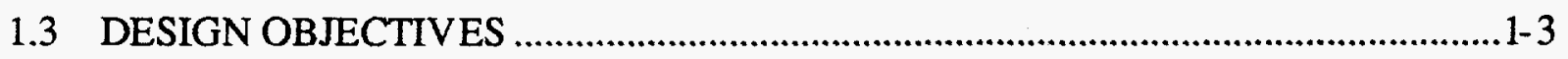

1.4 DESIGN OVERVIEW, 3/8-GOAL SYSTEM ……...................................................

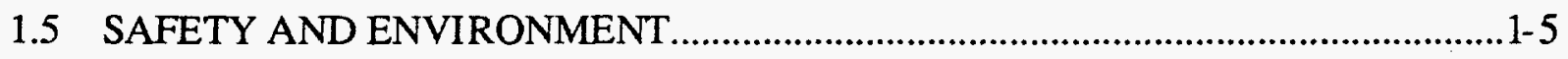

1.6 SCALING TO A 3/16-GOAL SYSTEM

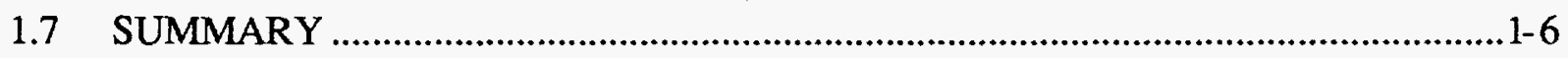

\section{LIST OF TABLES}

Table 1-1. Comparison of Material Inventory ……..................................................

Table 1-2. Summary of Masses for A Single Target/Blanket Module ...............................1-6 



\subsection{EXECUTIVE SUMMARY}

The 3 He target/blanket (T/B) preconceptual design for the $3 / 8$-Goal facility is based on a $1000-\mathrm{MeV}, 200-\mathrm{mA}$ accelerator to produce a high-intensity proton beam that is expanded and then strikes one of two T/B modules. Each module consists of a centralized neutron source made of tungsten and lead, a proton beam backstop region made of zirconium and lead, and a moderator made of $\mathrm{D}_{2} \mathrm{O}$. Helium-3 gas is circulated through the neutron source region and the blanket to create tritium through neutron capture. The gas is continually processed to extract the tritium with an online separation process.

\section{$1.1 \quad$ SYSTEM FEATURES AND ASSUMPTIONS}

System features and assumptions that strongly influenced the design were:

- use of a 1000-MeV, 200-mA accelerator (200 MW of beam power),

- overall system availability of $75 \%$,

- tritium production of $3 / 8$ of the 1988 requirement (3/8-Goal) produced per annum,

- centralized neutron source with 1-year or longer lifetime,

- use of a $\mathrm{D}_{2} \mathrm{O}$ moderator with ${ }^{3} \mathrm{He}$ feedstock material to produce tritium through the $(n, p)$ reaction,

- "defense in depth" safety philosophy which includes active plus passive cooling systems; diverse redundant backup systems, and multiple radionuclide barriers, and

- continuous extraction and processing of the tritium produced in the system.

The target/blanket system, the accelerator system and the tritium handling components make up an Accelerator Production of Tritium system that has the following distinct beneficial features:

- Tritium is produced without the use of any fissile material.

- There will be no need to handle, store, and dispose of spent nuclear fuel.

- Nuclear criticality will not be a consideration in design or operation.

- The system will be easier to site and license than systems using fissile material.

- The accelerator can be shut off within milliseconds, greatly facilitating prevention or mitigation of the consequences of accidents.

- The materials used in the target/blanket system produce very low residual heat, again facilitating the prevention or mitigation of the consequences of accidents. 
- The system produces the $3 / 8$-Goal quantity of tritium at a low life-cycle cost.

\subsection{DESIGN CRITERIA AND ASSUMPTIONS}

Many design criteria were set by the requirement that the system operate continuously with $200 \mathrm{MW}$ of proton beam. For example, the "beamspot" size was required to be expanded to a size of $42 \times 91 \mathrm{~cm}$ (16.5 $\times 35.8 \mathrm{in}$.) to reduce the power density in the window and in the neutron source region to acceptable levels.

To meet the system availability requirement of $75 \%$ or greater, the design criteria evolved to the use of two T/B modules. At any given time, one module will be in production and one will be in retargeting or standby, thereby increasing the potential availability. The design of the target module provides for replacement which occurs after beam shutdown. More complex operations such as assembly, disassembly, and target lead recovery and re-use can be performed offline. The design locates the heavy water and helium connectors outside the primary shielding to avoid seal material irradiation damage and the associated potential for leakage. The design also allows for recovery and re-use of most of the target lead for mixed waste minimization.

To meet the tritium goal requirement, it was necessary for the $T / B$ design to achieve high conversion efficiency of $3 \mathrm{He}$ into tritium. Such a conversion efficiency required an innovative, highly re-entrant neutron source design, which minimized neutron loss through the large beam entrance surface. This goal was accomplished in the physics design by effectively using neutron-source geometry and $3 \mathrm{He}$ as a neutron decoupler to minimize parasitic capture in tungsten. In addition, the entrance beam profile was kept as small as practical, thereby minimizing the amount of tungsten.

Choosing a $\mathrm{D}_{2} \mathrm{O}$ moderator was dictated by efficiency. Heavy water had a clear advantage over other possible materials, based on a study that compared the tritium production efficiency of $3 \mathrm{He}$ contained in graphite, beryllium, or $\mathrm{D}_{2} \mathrm{O}$ moderators. The choice of ${ }^{3} \mathrm{He}$ as a target material was made because of the large neutron-absorption cross section for tritium production and because of the capability for a simple, continuous extraction of tritium. Because $3 \mathrm{He}$ is the byproduct of tritium decay, a sufficient inventory is available in the Defense Complex for use.

Maximizing the safety and environmental characteristics of the system led to further design choices. The high beam power required using a high-temperature neutron source material. Parameter studies confirmed that tungsten was the best candidate overall for the region struck by the direct proton beam, with lead placed in lower power regions to enhance neutron production.

Because of existing accelerator experience, Inconel 718 was chosen as the baseline structural material in the neutron source and as the beam entrance window material. At the APT proton flux levels, the Inconel lifetime is expected to be 1 year or longer. Stainless steel offers a potential neutronic and fabrication advantage. A planned materials irradiation program will investigate these and other materials.

Continuous extraction and processing of tritium minimizes the possibility that radioactive material that would be released in an off-normal event and reduces the amount of 
material required for radioactive material disposal. In addition, tritium separation from the ${ }^{3} \mathrm{He}$ and $\mathrm{H}$ can be accomplished in an essentially waste-free process using cryogenic distillation technology. The design chosen in this study was based on the technology developed by the Tritium System Test Assembly (TSTA) at Los Alamos with 10 years of operational experience that has demonstrated extremely low tritium release levels and minimum worker exposure.

\subsection{DESIGN OBJECTIVES}

Considering the above design criteria and assumptions, the following design objectives were adopted for the target/blanket systems:

- Produce the goal amount of tritium with adequate margin. Assume $75 \%$ overall capacity ( $365 \times 0.75=273.75$ full power days per year).

- Where possible, use existing technology.

- Incorporate "safety by design" to minimize worker hazards and hazards to the public.

\subsection{DESIGN OVERVIEW, 3/8-GOAL SYSTEM}

In summary, the design that has evolved for the $3 / 8$-Goal system consists of the incident proton beam, which is expanded to a beamspot size of $42 \times 91 \mathrm{~cm}(16.5 \times 35.8 \mathrm{in}$.). The beam passes through a double-wall vacuum interface window that is constructed of Inconel 718 and cooled with $\mathrm{D}_{2} \mathrm{O}$. The protons then enter a $118 \mathrm{~cm}(46.5 \mathrm{in}$.) diameter by $4.5 \mathrm{~m}$ (177 in.) long vessel (also made of Inconel) that contains ${ }^{3} \mathrm{He}$ at a moderate pressure of 2.07 $\mathrm{MPa}$ (300 psia) and 88 tungsten rod bundles that are distributed along the length of the vessel. The protons strike the tungsten and produce high-energy neutrons through the process of spallation. Behind the Inconel vessel is a proton beam backstop region made of zirconium and lead that provides an additional source of neutrons and fully stops the proton beam and any secondary particles.

The Inconel vessel is surrounded by an annulus of lead that is cooled with $\mathrm{D}_{2} \mathrm{O}$. The preconceptual design of the lead region has focused on an unclad, stacked-plate concept. Neutron multiplication occurs in the lead through additional spallation and (n,xn) reactions. Surrounding the lead is a thin annulus of $3 \mathrm{He}$ contained in aluminum tubing, a $\mathrm{D}_{2} \mathrm{O}$ moderator, another annulus of $3 \mathrm{He}$, a second $\mathrm{D}_{2} \mathrm{O}$ moderator, and a third annulus of $3 \mathrm{He}$. Neutrons are moderated to near thermal energies in the $\mathrm{D}_{2} \mathrm{O}$. Tritium is produced through neutron capture in $3 \mathrm{He}$ that is circulated and processed continually. Thermal neutrons that are scattered back into the neutron source are preferentially captured in the $3 \mathrm{He}$, thereby reducing parasitic capture in the tungsten. The entire assembly of the neutron source vessel and surrounding blanket of lead, ${ }^{3} \mathrm{He}$, and $\mathrm{D}_{2} \mathrm{O}$ are contained in a stainless steel moderator tank. The moderator tank and primary coolant piping outside of the moderator tank form a highly reliable radionuclide retention boundary.

The mechanical design of the components in a T/B module has undergone significant change from that presented in Revision 0 to simplify the design and improve reliability. The 
details of these changes are presented in Section 4.5. The following table shows the significant reduction in mass of the Revision 1.5 components inside one moderator tank including the tank wall compared to the Revision 0 design. Note, the heavy water is that for one moderator tank and does not include the heavy water in the primary cooling systems. All masses are in kilograms.

Table 1-1. Comparison of Material Inventory

\begin{tabular}{lccc}
\hline Material & Rev. 0 Mass & Rev. 1.5 Mass & Rev. 1.5/Rev. 0 \\
\hline Aluminum & 8904 & 5497 & 0.62 \\
Heavy Water & 340141 & 34583 & 0.10 \\
Inconel & 6907 & 4972 & 0.72 \\
Lead & 94064 & 70801 & 0.75 \\
Steel & 398291 & 16618 & 0.04 \\
Tungsten & 1674 & 1476 & 0.88 \\
Zircaloy-4 & 2601 & 4328 & 1.66 \\
Helium-3 & 23.2 & 14.2 & 0.61 \\
Total Mass & 852605 & 138289 & 0.16 \\
\hline \hline
\end{tabular}

The incident proton beam for the $3 / 8$-Goal design contains $200 \mathrm{MW}$ of power. Of this total, approximately $77 \mathrm{MW}$ is deposited in the tungsten rod bundles. Sixteen rows of rod bundles (placed in alternating rows of five bundles in one row and six bundles in the next row, for a total of 88 bundles) serve as the "targets" for the high-energy proton beam from the accelerator. The remaining power is distributed to the beam entrance window, proton beam backstop region, target lead, $\mathrm{D}_{2} \mathrm{O}$ moderator, moderator vessel, helium chamber, blanket structures, shield, and $3 \mathrm{He}$. The tungsten rod bundles are cooled with $\mathrm{D}_{2} \mathrm{O}$ at moderate pressure $1.44 \mathrm{MPa}$ (209 psia). Each of the rod bundles is composed of 91 tungsten rods wrapped with tungsten wire that makes four complete turns in the $96 \mathrm{~cm}$ (37.8 in.) length of the rod. Bundle dimensions are based on a nominal rod diameter of $0.3175 \mathrm{~cm}$ (0.125 in.) and a nominal wire diameter of $0.1016 \mathrm{~cm}(0.040 \mathrm{in}$.$) . Rod and wire sizes are$ varied by position in the rows with smaller rods used in the higher power density front end rows where the beam strikes first and larger rods used in the lower power density back end rows. The rod bundles are held in hexagonal Inconel ducts and are supported to minimize thermal and mechanical stress. Full-scale hydraulic tests show that the pressure drop is within design specifications. With the small-diameter rods, an adequate heat transfer area maintains low temperatures $\left(180^{\circ} \mathrm{C}\right)$ and small radial gradients $\left(7.7^{\circ} \mathrm{C}\right.$ over $\left.0.14 \mathrm{~cm}\right)$ in the tungsten. The peak rod-heat flux of $1.82 \mathrm{MW} / \mathrm{m}^{2}$ is similar to that of existing light water power reactors. Full-length heated rod tests show that this heat flux is obtainable with a safety factor of 2.0 .

The tungsten rod bundle cooling system is composed of two independent cooling loops. Each loop circulates the $\mathrm{D}_{2} \mathrm{O}$ out of the moderator tank to a shielded equipment room that contains the primary coolant pump and heat exchanger. The heat is transferred to an intermediate loop containing light water, and then through a heat exchanger to a third loop that dumps the heat to the atmosphere with a cooling tower. The intermediate loop reduces the potential for release of the $\mathrm{D}_{2} \mathrm{O}$ coolant and its activation products to the environment. 
The moderator tank cooling system also operates at low pressure $0.3 \mathrm{MPa}$ ( 45 psia) and consists of multiple cooling loops. This system removes the heat from the beam entrance window, the radial lead, the backstop, the ${ }^{3} \mathrm{He}$, and the remainder of the moderator tank components.

\subsection{SAFETY AND ENVIR ONMENT}

An APT safety approach is being developed for the T/B systems using inherent safety features and active and passive engineered safety systems to meet or exceed the safety requirements. The safety approach will ensure that a beamtrip and adequate cooling are provided if T/B system parameters deviate from the design operating range.

The beamtrip system will be designed to IEEE-603 Standards with two-out-of-four trip logic and will be designed to have both a primary and secondary trip function for each transient. These protection system features should put initiating events with failure to trip beam in the residual risk category. Residual risk events have a probability of less than $10^{-7}$ per year. The engineered safety systems include both active and passive cooling systems to remove the decay heat. By having two $100 \%$-active residual heat removal systems, the effects of a single failure can be accommodated. By having both active and passive residual heat removal systems, the effects of multiple failures can also be accommodated. For the tungsten rod bundles, detailed system analyses show that during an unprotected large-break loss-of-coolant accident (LBLOCA), the rod bundles are cooled by natural circulation and remain below $160^{\circ} \mathrm{C}\left(320^{\circ} \mathrm{F}\right)$. Water that is spilled from the break is below the saturation temperature so that the pressure response on the confinement is minimal.

There are many inherent features in the APT system that provide environmental and safety advantages. The decay heat during the first seconds after shutdown in the tungsten is only $0.9 \%$ of total power (compared with $6 \%$ for a reactor) and is reduced to $0.28 \%$ in one day. The use of tungsten offers the added safety advantage because of its high temperature capability and $3410^{\circ} \mathrm{C}\left(6170^{\circ} \mathrm{F}\right)$ melting point. The accelerator can be shut off very quickly-less than $1 \mathrm{~ms}$ after a trip signal. The APT does not use fissile or fertile material, making reactivity-induced transients impossible. Waste disposal is simplified enormously because the irradiated tungsten and Inconel structures (replaced every 1 year or longer) can be disposed of with on-site burial.

Continuous processing is a unique feature in the $3 \mathrm{He}$-APT system that cannot be safely accomplished with reactors because of the large reactivity worth of the target nuclei. As $3 \mathrm{He}$ gas is circulated through the neutron source assembly and blanket, tritium is created through a $(n, p)$ reaction. Tritium and other hydrogen isotopes are continually removed through a palladium leak to maintain the tritium content in the ${ }^{3} \mathrm{He}$ gas at a low level. The required separations of hydrogen isotopes are made in a single cryogenic fractional distillation column at an on-site Tritium Purification Facility. The feed is pretreated with membrane separation and molecular sieve sorption to remove impurities, $3 \mathrm{He}$, and any condensable materials. The impurities are sent to the gas waste system, and the $3 \mathrm{He}$ is returned to the T/B. A catalytic equilibrator is included in the feed stream to assure complete equilibration. This converts $\mathrm{H}_{2}$ and $\mathrm{T}_{2}$ to HT. This stream is fed to a cryogenic distillation column. A protium-enriched stream is produced from the top of the column. The $\mathrm{T}_{2}$-enriched reboiler product is 99 mol\% tritium. As part of a fusion research program, the TSTA at Los Alamos developed and 
demonstrated this cryogenic distillation technology at a larger scale than that needed for APT.

The continuous processing offers significant environmental, safety, and cost advantages. Because the tritium is continuously extracted from the system, the tritium inventory that could potentially be released is very small. In addition, tritium separation from the $3 \mathrm{He}$ is an essentially waste-free process (i.e. no fabrication or reprocessing of target rods). Also, by continuously removing the tritium from the ${ }^{3} \mathrm{He}$, the need for an expensive tritium extraction facility is eliminated.

\subsection{SCALING TO A 3/16-GOAL SYSTEM}

Because of further potential stockpile reductions, the tritium production requirement may be reduced to 3/16-Goal. A 3/16-Goal design concept has been derived from the detailed 3/8-Goal system design. In the 3/16-Goal design, the beam current has been reduced from $200 \mathrm{~mA}$ to $100 \mathrm{~mA}$ while keeping the energy at $1000 \mathrm{MeV}$.

The target/blanket design for the 3/16-Goal system is reduced in cross section from that of a 3/8-Goal target/blanket design, maintaining approximately the same power density in the neutron source materials. A comparison of the materials required for a single target/blanket module for both the $3 / 16$ and $3 / 8$-Goal systems is given in the following table.

Table 1-2. Summary of Masses for A Single Target/Blanket Module

\begin{tabular}{lcc}
\hline \multicolumn{1}{c}{ Material } & 3/16 Goal & 3/8 Goal \\
\hline Aluminum & 4419 & 5497 \\
Heavy Water & 28175 & 34583 \\
Helium-3 & 7.6 & 14.2 \\
Inconel & 3976 & 4972 \\
Lead & 53586 & 70801 \\
Stainless Steel & 14158 & 16618 \\
Tungsten & 778 & 1476 \\
Zircaloy-4 & 2320 & 4328 \\
\hline \hline
\end{tabular}

\subsection{SUMMARY}

In the preconceptual design presented, all of the design criteria and objectives have been achieved. The proposed system is safe and efficient, and meets the goal amount of tritium production using conventional, proven technology in essentially all areas of design. All design reviews have been positive; comments and suggestions have been incorporated where possible. The Lab-industry design team is well prepared to proceed with the conceptual design. 


\section{T/B PROCESSING}

CHAPTER 2

This chapter has been deleted in Revision 1.5; the material found in Revision 0 has been incorporated in the Executive Summary, Chapter 3, and Chapter 4 in Revision 1.5. 



\section{PHYSICS DESIGN}

CHAPTER 3

Page

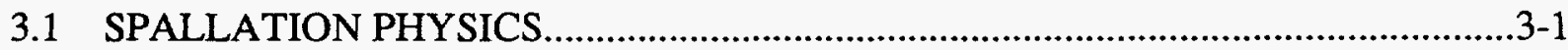

3.2 REFERENCE TARGET/BLANKET PHYSICS MODEL ……................................3-2

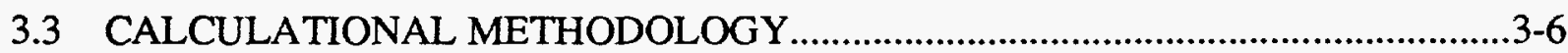

3.3.1 Overview of the LAHET Code System ...............................................................3-6

3.3.2 Overview of CINDER'90

3.3.3 Comparison between LCS Calculations and Experimental Data..........................3-9

3.3.3.1 Bare-Target Integral Neutron Leakage.....................................................3-10

3.3.3.2 LANSCE Neutron Spectra ...............................................................10

3.3.3.3 Los Alamos FERFICON Conversion Measurements ...........................3-10

3.3.3.4 Russian Energy Deposition Measurements............................................3-12

3.3.3.5 LANSCE Activation Measurement...................................................3-13

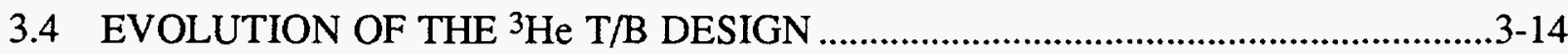

3.4.1 Fundamental Target Neutronics.......................................................................

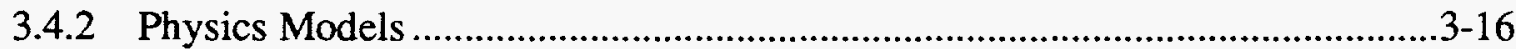

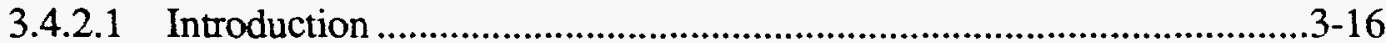

3.4.2.2 Design Evolution......................................................................19

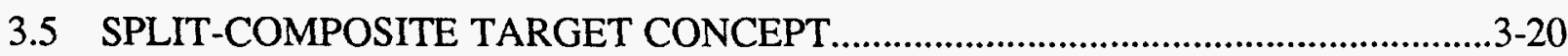

3.6 PHYSICS DESIGN OF THE NEUTRON SOURCE ...............................................2-24

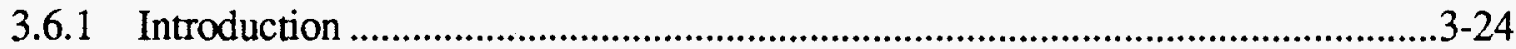

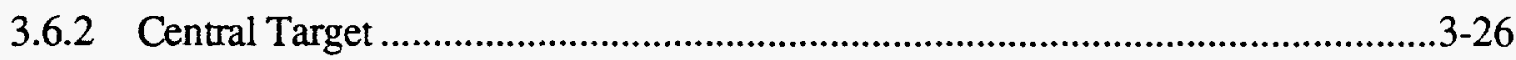

3.6.2.1 Rod Bundle Pattern Study ..............................................................3-26

3.6.2.2 Flux-Trap Gap Size Investigations ................................................3-31

3.6.2.3 ${ }^{3} \mathrm{He}$ Target Chamber Pressure Studies...............................................3-32

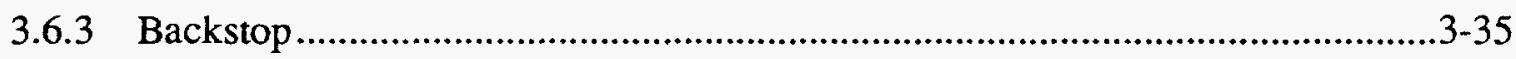

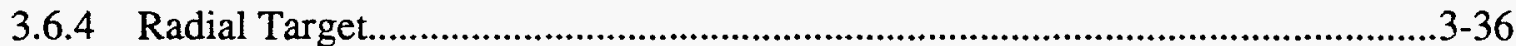

3.6.4.1 Optimization of Radial Target Length....................................................3-36

3.6.4.2 Effects of Material Composition of the Radial Target .........................3-37

3.6.4.3 Optimization of Radial Target Thickness.................................................3-40

3.6.5 Neutron Source Performance Optimization Studies .............................................3-42

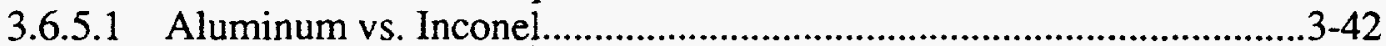

3.6.5.2 Target Chamber Wall Thickness .................................................... $3-42$

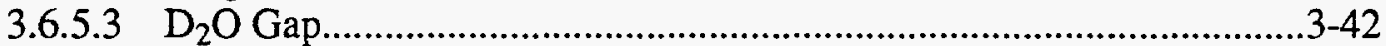

3.6.5.4 Proton Beam Tube....................................................................3-44 
3.7 PHYSICS DESIGN OF THE MODERATOR/REFLECTOR ...................................3-47

3.7.1 Moderator and Reflector Material ....................................................................47

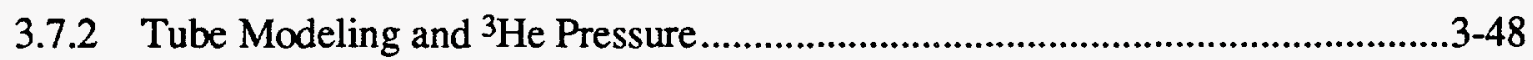

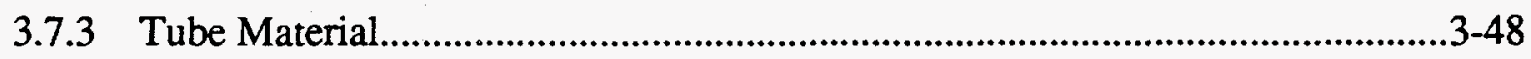

3.7.4 Blanket Temperature Effects .........................................................................49

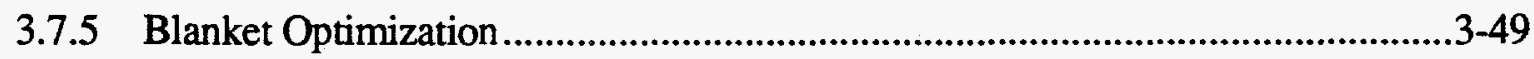

3.7.6 Tube Arrangement.............................................................................................3-50

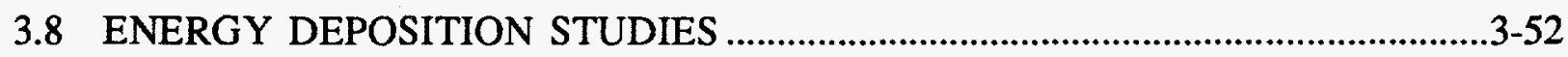

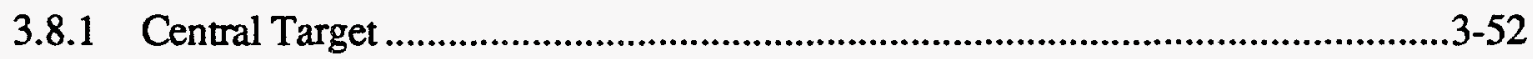

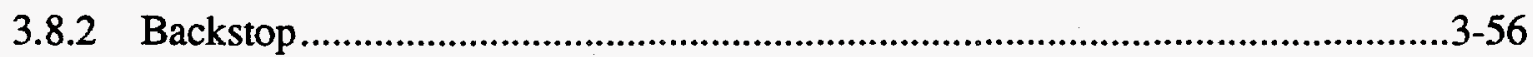

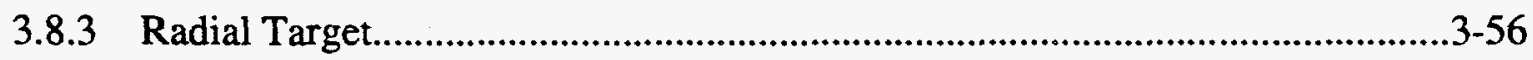

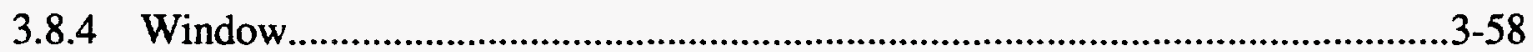

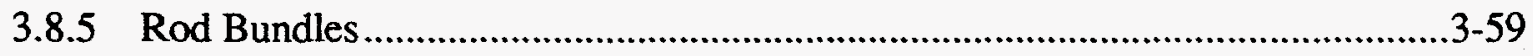

3.9 RADIONUCLIDE PRODUCTION (REFERENCE DESIGN) ..................................

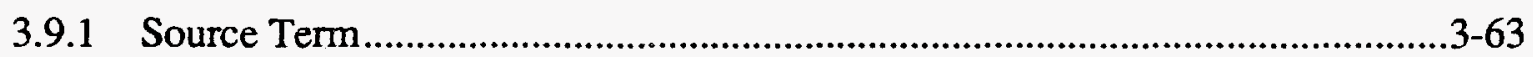

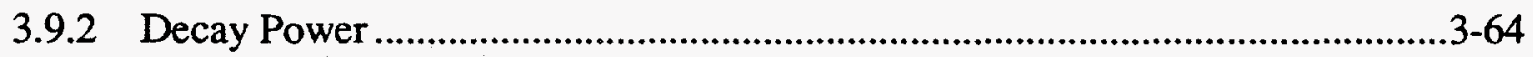

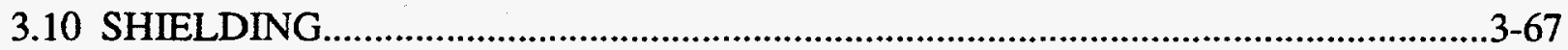

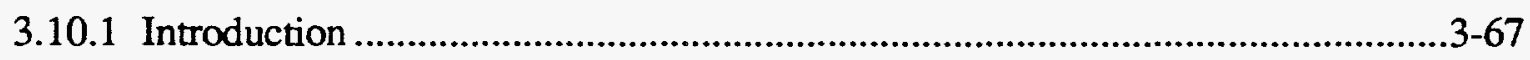

3.10.2 Shielding Model ..........................................................................................3-69

3.10.3 Comparisons between Shielding Model, Simulations, and Experiment..............3-70

3.10.4 Application of the Shielding Model to the ${ }^{3} \mathrm{He}$ T/B System ................................3-71

3.11 TRITIUM IMPLANTATION AND DIFFUSION...................................................3-72

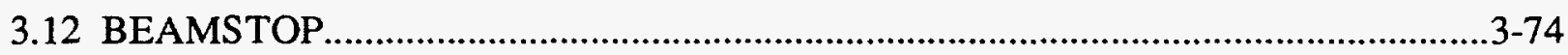

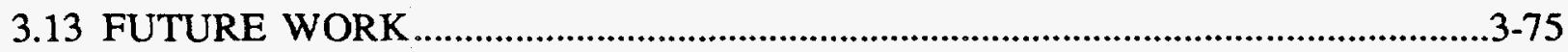

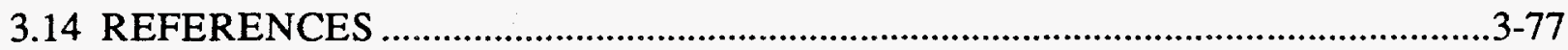




\section{LIST OF FIGURES}

Page

Fig. 3-1. Illustration of spallation physics 3-1

Fig. 3-2. Cross-sectional views of the APT physics reference model............................3-3

Fig. 3-3. LCS codes and data files.........................................................................

Fig. 3-4. Absolute neutron yields from a cylindrical lead target $20 \mathrm{~cm}$ diam x $60 \mathrm{~cm}$ long. Squares denote LCS results

Fig. 3-5. Calculated and measured neutron energy spectra from the LANSCE highintensity $\mathrm{H}_{2} \mathrm{O}$ moderator.

Fig. 3-6. LCS geometry for the Russian energy deposition calculations.

Fig. 3-7. Total low-energy neutron production and leakage for solid right-circular cylindrical targets bombarded on axis by $1-\mathrm{GeV}$ protons.

Fig. 3-8. Tungsten cylindrical target performance relative to lead

Fig. 3-9. Illustration of various target geometries

Fig. 3-10. Neutronic performance of various target geometries with equal areas $\left(5775 \mathrm{~cm}^{2}\right)$

Fig 3-11. Illustration of various target geometries, coolant flow directions, and target chamber-wall geometries.

Fig. 3-12. Basic target configurations.

Fig. 3-13. Total neutron production, spallation plus $(n, x n)$, and leakage for $50-\mathrm{cm}-$ diam targets.

Fig. 3-14. Relative proton beam power density and tritium production as a function of target diameter for cylindrical targets.

Fig. 3-15. Relative tritium production vs. the height of the reentrant head.

Fig. 3-16. Low-energy ( $<20 \mathrm{MeV})$ neutron surface flux at a radius of $20 \mathrm{~cm}$ from a 30-cm-diam W-Pb split-composite target.

Fig. 3-17. Schematic of the APT target/blanket system.

Fig. 3-18. Cross-sectional view of the APT Physics-IV model used in the rod bundle pattern study.

Fig. 3-19. Cross-sectional view of a 91-rod-bundle unit.

Fig. 3-20. The 60-cm region just downstream of the proton beam window of the central target zone for the six cases studied.

Fig. 3-21. Cross-sectional view of the APT T/B showing the central target zone layout for (a) case 1, (b) case 3 and (c) case 4 . 
Fig. 3-22. Tritium production (relative to production at $100 \mathrm{psia}$ ) and fraction of tritium produced within the target chamber as a function of ${ }^{3} \mathrm{He}$ target chamber pressure.

Fig. 3-23. Absorption in the tungsten target material and the radial lead target relative to absorption at $100 \mathrm{psia}$ as a function of ${ }^{3} \mathrm{He}$ target chamber pressure.

Fig. 3-24. Tritium production as a function of Zircaloy backstop length.

Fig. 3-25. Initial optimization of radial lead zone thickness with a $10.2 \mathrm{v} \%$ coolant fraction.

Fig. 3-26. Optimization of radial lead zone thickness with a $30 \%$ coolant fraction.

Fig. 3-27. Optimization of radial lead zone thickness with structure.

Fig. 3-28. Dependence of tritium production on target chamber wall thickness...................3-43

Fig. 3-29. Variation of tritium production with $\mathrm{D}_{2} \mathrm{O}$ gap thickness. .................................4-43

Fig. 3-30. Cross-sectional view of the beam tube area of the base case model BT1...........3-45

Fig. 3-31. Cross-sectional view of the beam tube area of model BT2 with a cylindrical beam tube.

Fig. 3-32. Neutron captures by ${ }^{3} \mathrm{He}$ vs. moderator thickness for $\mathrm{D}_{2} \mathrm{O}$, graphite, and Be moderators.

Fig. 3-33. Typical tube arrangement and dimensions used for optimizing the spacing, S.

Fig. 3-34. Central target zone rod bundle layout showing the bundle-numbering convention used to identify rod bundle positions.

Fig. 3-35. Power density variation with axial position for various radial regions in the radial lead zone.

Fig. 3-36. Radial variation of the "high-Z" power density, averaged over axial extent and azimuth, in the radial lead target.

Fig. 3-37. Bundle layout and numbering for case B3, where the target regions are modeled as discrete, homogeneous hexagons.

Fig. 3-38. Closeup of the layout and numbering of the detailed bundle modeled in case B4.

Fig. 3-39. Nuclide chart with APT reference design medium-energy reaction products.

Fig. 3-40. Distribution of APT mass by element $Z$. .3-65

Fig. 3-41. Time dependence of the decay power for the ${ }^{3} \mathrm{He}$ APT target/blanket and the relative core power for shutdown of the NPR Heavy Water Reactor.

Fig. 3-42. Comparison of neutron production spectra from fission and spallation. 3-67

Fig. 3-43. Energy-dependent neutron production cross-sections for $800-\mathrm{MeV}$ protons incident on iron as a function of angle with respect to the proton beam. 
Fig. 3-44. Case III geometry. 3-71

Fig. 3-45. Average high-energy neutron dose and energy from the upstream, downstream, and radial surfaces of the APT T/B, as calculated by the Monte Carlo code LAHET.

Fig. 3-46. Dose rate at the shield outer surface vs. inner shield thickness.............................3-73

Fig. 3-47. Schematic of the APT target region.......................................................................3-74

\section{LIST OF TABLES}

Page

Table 3-1. Neutron Production by Material in the ${ }^{3} \mathrm{He}$ Physics Reference Model .................3-5

Table 3-2. Energy Balance Sheet for the ${ }^{3} \mathrm{He}$ Physics Reference Model .............................3-5

Table 3-3. Physical Characteristics of the FERFICON Targets..............................................3-10

Table 3-4. Comparison of Measured and Calculated Conversions for Thorium and Depleted Uranium Targets Bombarded by 800-MeV Protons............................3-12

Table 3-5. Total Energy Deposited over Axial Length for Radii of $5 \mathrm{~cm}$ and $10 \mathrm{~cm}$..........3-13

Table 3-6. Decay Dose Values Calculated by LCS/CINDER' 90 and Experimental

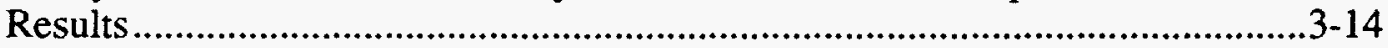

Table 3-7. Results of the Rod Bundle Pattern Study........................................................3-31

Table 3-8. Relative Tritium Production of Various ${ }^{3} \mathrm{He}$ APT Source and Blanket Systems.

Table 3-9. Relative Tritium Production of ${ }^{3} \mathrm{He}$ APT Lower-Target and Flux-Trap Systems

Table 3-10. Comparison of the Neutronic Performance of Several Backstop Materials.........3-35

Table 3-11. Effects of Coolant Fraction in Radial Lead Zone on Tritium Production ...........3-38

Table 3-12. Heavy Water Distribution in Radial Target Lead Zone.......................................3-38

Table 3-13. Material Composition for the SILC Lead Cladding Design..................................3-39

Table 3-14. Effect of Aluminum-1100 Cladding on APT System Performance ...................3-39

Table 3-15. Effects of Adding Structural Material to Target Lead Zone.................................3-39

Table 3-16. Tritium Production as a Function Of Beam Tube Configuration...........................3-44

Table 3-17. Tritium Production as a Function of Blanket ${ }^{3} \mathrm{He}$ Pressure ..................................3-48

Table 3-18. Average Power Densities in the 88 Rod Bundles and Inconel Clad ....................3-54

Table 3-19. Power densities in the backstop....................................................................3-56

Table 3-20. Energy Deposited in Various APT Windows................................................3-59

Table 3-21. Fluxes, Nuclide Production for Each Material in the Reference Physics Model 
Table 3-22. Summary of CINDER' 90 Calculated Time Dependence of the Macroscopic Neutron Absorption During Operation $.3-64$

Table 3-23. Results of Least-Squares Fits of Eq. (3-3) to Monte Carlo Beamspill Simulations..

Table 3-24. Comparison of Moyer Model Results for Case III with Calculated and Experimental Results. 


\subsection{PHYSICS DESIGN}

\subsection{SPALLATION PHYSICS}

Spallation-a reaction in which a high-energy primary particle (e.g., a 1-GeV proton) interacts with a target nucleus-is thought to take place in two stages. First, in the intranuclear cascade phase, the incident proton creates a high-energy particle cascade inside the nucleus. In this phase, some high-energy ( $>20 \mathrm{MeV})$ secondary particles and low-energy $(<20 \mathrm{MeV})$ cascade particles escape the nucleus, leaving the nucleus in a highly excited state. This spallation model holds when the deBrogile wavelength of the incident particle is short enough to interact with individual nucleons inside the nucleus. Second, in the evaporation phase, the excited nucleus relaxes, primarily by emitting low-energy $(<20 \mathrm{MeV})$ evaporation neutrons. Low-energy spallation neutrons are the low-energy cascade-evaporation neutrons. Figure 3-1 shows these basic processes. Figure 3-1 also shows that for thick targets, high-energy secondary particles (plus their progeny) can undergo further spallation reactions. Also, for some target materials, low-energy spallation neutrons can enhance neutron production through low-energy " $(\mathrm{n}, \mathrm{xn})$ " reactions. Total low-energy neutron production from a target is the lowenergy spallation neutron production plus the net production from low-energy "( $\mathrm{n}, \mathrm{xn})$ " reactions.

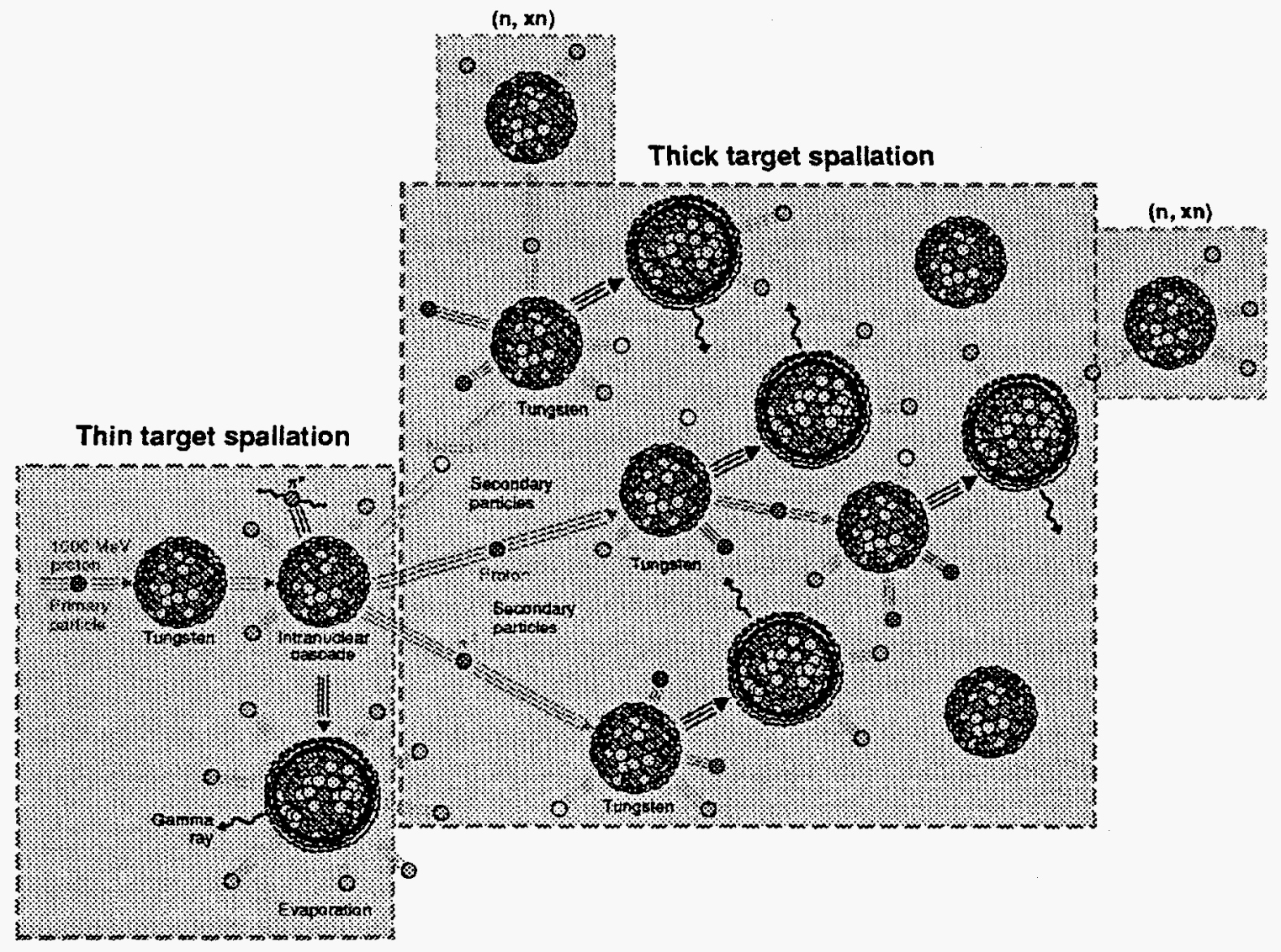

Fig. 3-1. Illustration of spallation physics 


\subsection{REFERENCE TARGET/BLANKET PHYSICS MODEL}

The reference physics model of the APT ${ }^{3} \mathrm{He}$ target/blanket has four major components: the central tungsten target region; the Zircaloy-lead backstop zone; the radial lead target region; and the heavy water ${ }^{3} \mathrm{He}$ blanket zone. The design parameters of the first three are highly interdependent. The design parameters of the blanket region are not strongly dependent on the details of the first three components.

The physics model includes many of the details of the engineering design. Structural material is included in the model to the degree that it is known. Where practicable, cells are introduced that explicitly represent structure, such as annuli of Inconel around the tungsten rod bundles that simulate the hex cans which hold the rod bundles. Where engineering design lacks specific detail, or where such detail is impractical to model, structural material is homogenized with other material; for example, $3 \mathrm{v} \%$ of Zircaloy -4 and $10 \mathrm{v} \%$ of $\mathrm{D}_{2} \mathrm{O}$ is mixed with $\mathrm{Pb}$ in the radial lead target region to represent support structure, coolant, and target. If done correctly, homogenization has a negligible effect on the calculated results.

Horizontal and vertical slices of the reference physics model are shown in Figs. 3-2a and $3-2 b$, respectively; the slices are taken through the proton beam centerline. The proton beam first strikes the proton beam window, which is modeled as two $0.125-\mathrm{cm}$-thick $(0.0492 \mathrm{in}$.) hemi-cylindrical Inconel-718 plates, between which is a $0.25-\mathrm{cm}\left(0.0984 \mathrm{in}\right.$.) gap of $\mathrm{D}_{2} \mathrm{O}$. The beam then enters the central target zone, which is modeled as 88 Inconel-718-clad tungsten rod bundles arranged in alternating rows of five and six bundles per row, for a total of 16 rows. In the model, the ninety-one $0.3175-\mathrm{cm}$-diam ( $0.125 \mathrm{in}$.) wire-wrapped tungsten rods comprising a rod bundle are homogenized with the surrounding $\mathrm{D}_{2} \mathrm{O}$ coolant to produce a 4.389 -cm-diam $\times 96-\mathrm{cm}$-long (1.728 in. $\times 37.80$ in.) rod bundle. Each consecutive five-bundle and six-bundle row forms a "group" of bundles. There are eight groups in all, each with eleven rod bundles. The tungsten volume fraction varies with the rod-bundle group, representing a variation in rod and wire-wrap size between groups. This variation reduces the need for orificing flow to the bundles, as described in Section 4.4.1. The Inconel-718 hexagonal can containing the rod bundle is modeled as an annulus with an equivalent volume, having an outside diameter of $4.548 \mathrm{~cm}$ (1.791 in.). A row of six bundles has a width of $47 \mathrm{~cm}(18.50 \mathrm{in}$.). The central target is located inside a $118-\mathrm{cm}$-diam $\times 450-\mathrm{cm}$-long (46.46 in. $\times 177.2$ in.) Inconel-718 target chamber, which is pressurized to 300 psia with ${ }^{3} \mathrm{He}$ (mass density of $1.874 \times 10^{-3} \mathrm{~g} / \mathrm{cm}^{3}$, or $\left.6.772 \times 10^{-5} \mathrm{lb}_{\mathrm{m}} / \mathrm{in} .{ }^{3}\right)$. The dimensions are inside dimensions and end cap to end cap. The ${ }^{3} \mathrm{He}$ within the target chamber and heat exchanger is modeled in the Monte Carlo transport code as having a temperature of $400 \mathrm{~K}\left(261^{\circ} \mathrm{F}\right)$. Fifteen stiffening rings, which are required for structural integrity of the target chamber, are modeled explicitly. Elliptical end caps are modeled on each end of the chamber. Downstream of the target chamber is the backstop; it is modeled as four 15 -cm-thick (5.9055 in.) Zircaloy-4 disks each separated by a 5-cm-thick (1.9685 in.) region of ${ }^{3} \mathrm{He}$, followed by a 30 -cm-thick (11.811 in.) disk of lead. The backstop diameter is the same as that of the target chamber.

Surrounding the cylindrical surfaces of the target chamber and backstop is a 28 -cm-thick (11.2036 in.) radial lead target, with a $\mathrm{D}_{2} \mathrm{O}$ coolant volume fraction of $10 \%$ and a Zircaloy-4 structural volume fraction of $3 \%$. The engineering design uses Inconel-718 as its structural 


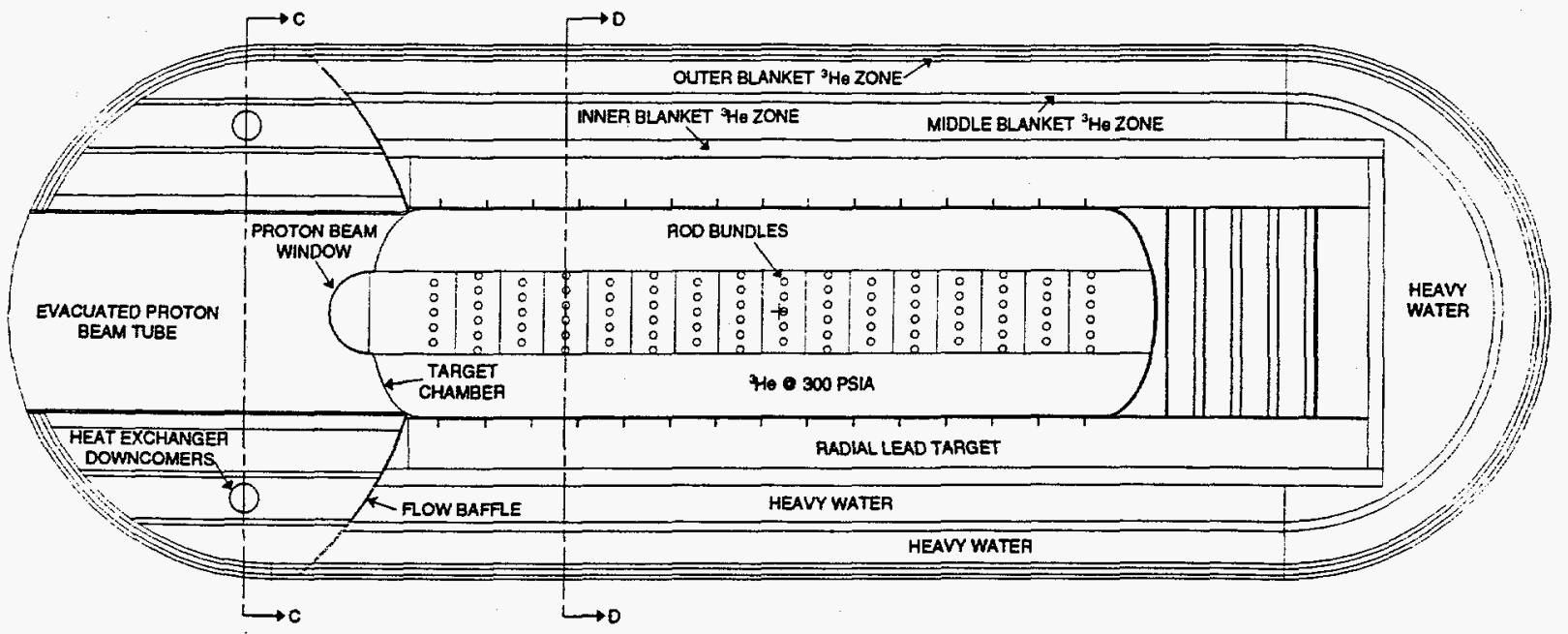

(a)

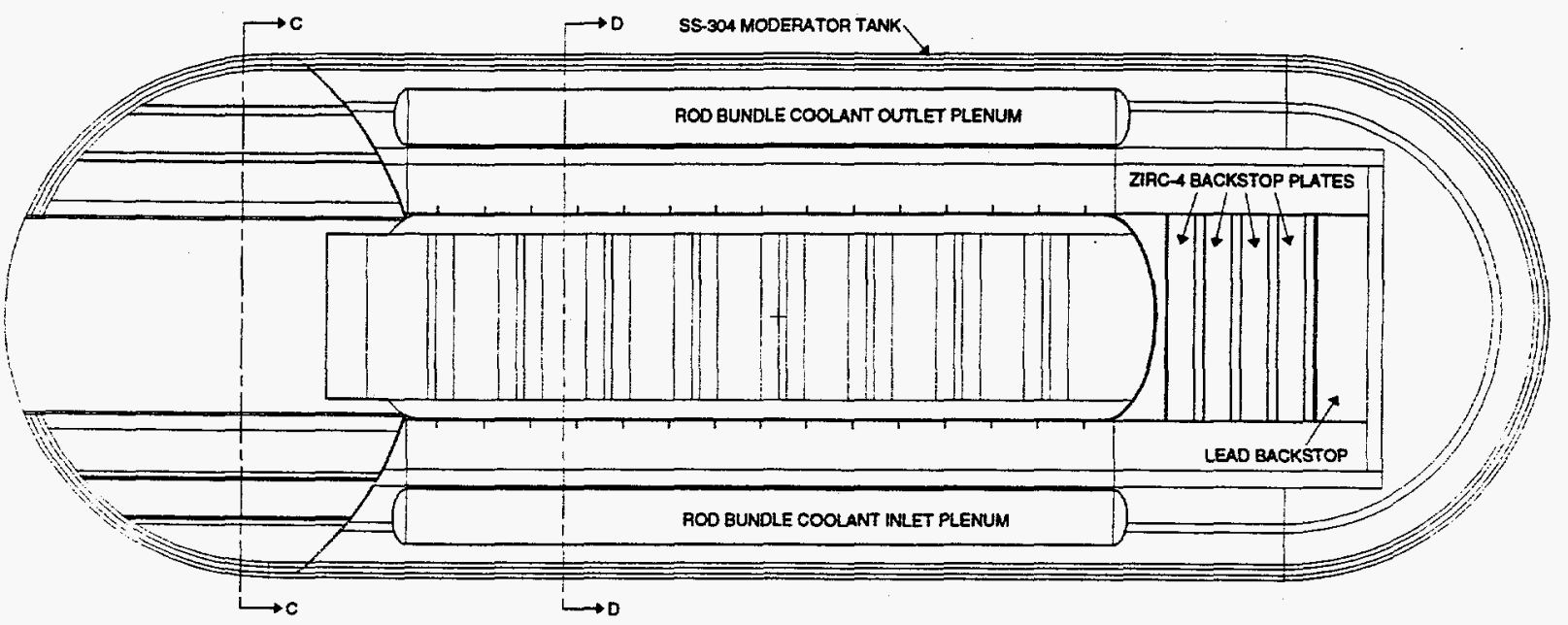

(b)

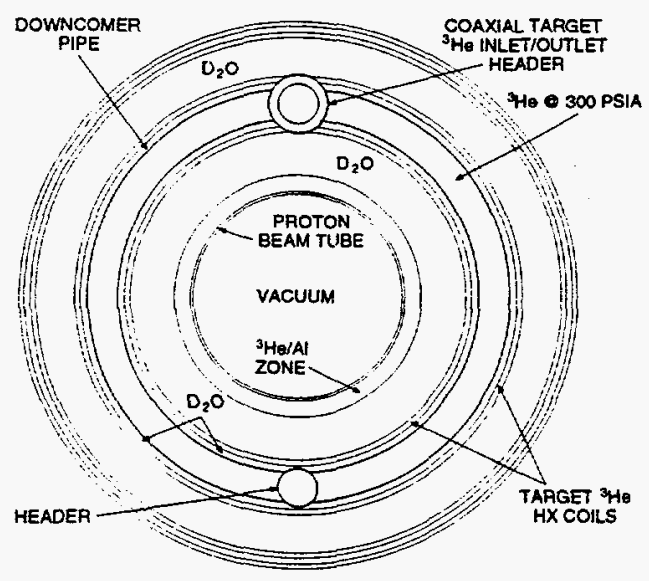

(c)

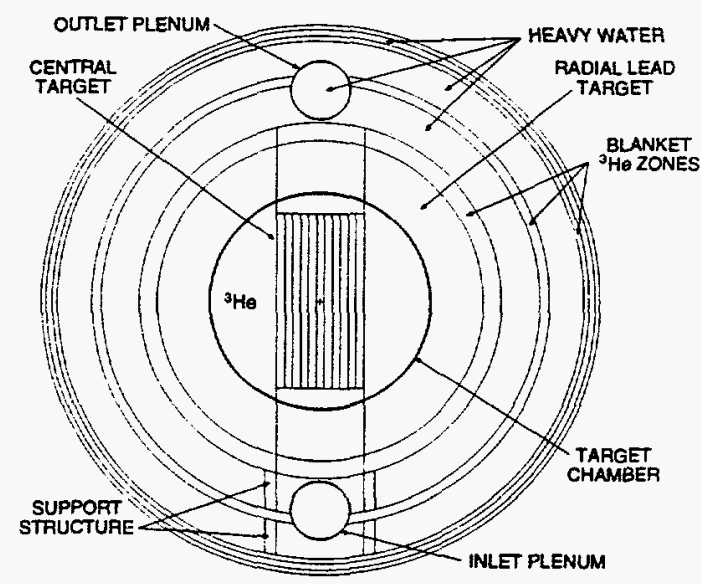

(d)

Fig. 3-2. Cross-sectional views of the APT physics reference model (a)

Horizontal cross section (plan view). (b) Vertical cross section (elevation view). (c) End view, Section C-C. (d) End view, Section D-D. 
material in this region; this inconsistency between the physics model and engineering design remains to be resolved. Above and below the central target zone, the rod bundle coolant pipes penetrate the radial lead target. In the physics model, we homogenize an appropriate amount of Inconel-718 and $\mathrm{D}_{2} \mathrm{O}$ material within this portion of the radial lead target to take account of these pipes; the same procedure is used to model these pipes within the target chamber, as well. A $0.5-\mathrm{cm}$-thick (0.1969 in.) annular $\mathrm{D}_{2} \mathrm{O}$ zone is sandwiched between the target chamber and radial lead target. This water gap provides cooling to the target chamber and clearance between the radial lead target and the target chamber. The outer surface of the radial lead target defines the border between the target and the blanket.

Just outside the radial lead target is the first array of ${ }^{3} \mathrm{He}$-filled aluminum tubes, which is modeled as a homogeneous region of $54.72 \mathrm{v} \%{ }^{3} \mathrm{He}, 20.23 \mathrm{v} \% \mathrm{Al}-1100$, and $25.05 \mathrm{v} \% \mathrm{D}_{2} \mathrm{O}$. This 9.713-cm-thick (3.824 in.) annular ${ }^{3} \mathrm{He}$ region, called the inner blanket ${ }^{3} \mathrm{He}$ zone, extends from the flow baffle to the end of the backstop. This is followed by a $20.68-\mathrm{cm}$-thick $(8.140$ in.) annular region of $\mathrm{D}_{2} \mathrm{O}$, which serves to moderate the fast neutrons escaping from the target region. Surrounding this heavy water zone is a $5.101-\mathrm{cm}$-thick (2.008 in.) annular region, called the middle blanket ${ }^{3} \mathrm{He}$ zone, whose composition is $52.90 \mathrm{v} \%{ }^{3} \mathrm{He}, 18.24 \mathrm{v} \% \mathrm{Al}-1100$, and $28.86 \mathrm{v} \% \mathrm{D}_{2} \mathrm{O}$. This is followed by a $18.58-\mathrm{cm}$-thick $(7.317 \mathrm{in}$.) annular reflector region of heavy water, which is enclosed by the outer blanket ${ }^{3} \mathrm{He}$ zone of thickness $2.737 \mathrm{~cm}(1.078$ in.), composed of $39.47 \mathrm{v} \%{ }^{3} \mathrm{He}, 23.14 \mathrm{v} \%$ Al-1100, and $37.39 \mathrm{v} \% \mathrm{D}_{2} \mathrm{O}$. The thickness and composition for each of the three blanket ${ }^{3} \mathrm{He}$ zones models the tube design described in Section 4.5. The rod-bundle coolant plena, which reside in the blanket, are modeled explicitly (see Figs. 3-2b and 3-2d); their composition is Inconel-718. Below the radial lead target are cells, containing Al-1100, that represent support structure. Throughout the model, heavy water is assumed to have a $0.25 \mathrm{a} \%$ contamination of $\mathrm{H}_{2} \mathrm{O}$.

A spherically-shaped 0.9525 -cm-thick ( 0.375 in.) Inconel-718 flow baffle, which extends from the downstream end of the proton beam tube to the moderator tank, divides the blanket into two regions. The region upstream of the flow baffle contains the heat exchanger for the target chamber ${ }^{3} \mathrm{He}$ (see Fig. 3-2c). The heat exchanger has six components modeled: annularshaped inner and outer banks of heat exchanger coils, a 32.39-cm-OD (12.75 in.) coaxial header at the top, a $21.91-\mathrm{cm}$-OD (8.625 in.) header on the bottom, and two $16.83-\mathrm{cm}-\mathrm{OD}$ (6.625 in.) toroidally-shaped downcomer pipes that connect the two headers. The coils are homogenized Al-1 $100(24.3 \mathrm{v} \%)$ and $300-p s i a{ }^{3} \mathrm{He}(75.7 \mathrm{v} \%)$. The Al-1100 pipes composing the headers and downcomers are modeled explicitly, and are filled with $300-$ psia ${ }^{3} \mathrm{He}$. Surrounding the proton beam tube is a ${ }^{3} \mathrm{He}$ zone whose composition and thickness are the same as the inner ${ }^{3} \mathrm{He}$ zone in the blanket. Since this zone is not part of the heat exchanger, its ${ }^{3} \mathrm{He}$ pressure is $100 \mathrm{psia}$. One other ${ }^{3} \mathrm{He}$ zone exists in this region-it is a spherically-shaped zone that wraps within the front end of the moderator tank and is simply an extension of the outer blanket ${ }^{3} \mathrm{He}$ zone. The remainder of the volume in this region is filled with heavy water.

Table 3-1 shows the relative neutron production as a function of material in the system. The central target zone is responsible for nearly half of the total neutron production, and the radial lead target accounts for most of the other half. About $93 \%$ of the neutron production comes from spallation and other reactions above $20 \mathrm{MeV}$; the remainder is a result of $(n, x n)$ reactions below $20 \mathrm{MeV}$. The present reference design meets the tritium production goal. 
Table 3-1. Neutron Production by Material in the ${ }^{3} \mathrm{He}$ Physics Reference Model

$\begin{array}{ccc}\begin{array}{c}\text { Relative production by } \\ \text { spallation and other }\end{array} & \begin{array}{c}\text { Relative production } \\ \text { by }(\mathrm{n}, \mathrm{xn}) \text { below }\end{array} & \begin{array}{c}\text { Total relative } \\ \text { production }\end{array}\end{array}$

Material reactions above $20 \mathrm{MeV}(\%)$

$20 \mathrm{MeV}(\%)$

(\%)

\begin{tabular}{lrrr} 
Tungsten target material & 43.1 & 1.6 & 44.7 \\
Radial Pb target & 38.7 & 4.7 & 43.3 \\
Zircaloy-4 / Pb backstop & 5.6 & 0.4 & 6.0 \\
Inconel-718 structure & 2.5 & 0.1 & 2.6 \\
Heavy water & 1.7 & 0.4 & 2.1 \\
All others & 1.2 & 0.1 & 1.3 \\
\hline
\end{tabular}

Table 3-2 gives an energy balance for the target/blanket system. Of the total beam power incident on the target/blanket, $74.4 \%$ is deposited as heat. Another $3.1 \%$ "leaks" from the system; that is, it is carried off by particles that escape from the target/blanket. The remaining $22.5 \%$ is converted to mass via nuclear reactions.

Table 3-2. Energy Balance Sheet for the ${ }^{3} \mathrm{He}$ Physics Reference Model

\begin{tabular}{|c|c|c|c|}
\hline & $\begin{array}{c}\text { Energy } \\
\text { (MeV/proton) }\end{array}$ & $\begin{array}{c}\text { Power }^{*} \\
\text { (MW) }\end{array}$ & $\begin{array}{c}\text { Relative } \\
\text { Error }\end{array}$ \\
\hline \multicolumn{4}{|l|}{ High-Energy ( $>20 \mathrm{MeV}$ ) Leakage } \\
\hline upstream surface & 0.78 & 0.16 & 0.034 \\
\hline downstream surface & 1.30 & 0.26 & 0.040 \\
\hline cylindrical surface & 14.12 & 2.82 & 0.011 \\
\hline neutrinos (all surfaces) & 13.38 & 2.68 & 0.110 \\
\hline \multicolumn{4}{|l|}{ Low-Energy $(<20 \mathrm{MeV})$ Leakage: } \\
\hline upstream surface & 0.50 & 0.10 & 0.020 \\
\hline downstream surface & 0.04 & 0.01 & 0.081 \\
\hline cylindrical surface & 0.96 & 0.19 & 0.016 \\
\hline Total Leakage Energy & 31.08 & 6.22 & 0.048 \\
\hline \multicolumn{4}{|l|}{ Energy Deposition by Material: } \\
\hline Inconel structure & 54.43 & 10.89 & 0.004 \\
\hline heavy water & 29.75 & 5.95 & 0.004 \\
\hline SS-304 moderator tank & 1.79 & 0.36 & 0.017 \\
\hline aluminum structure & 4.41 & 0.88 & 0.009 \\
\hline radial lead target & 185.86 & 37.17 & 0.003 \\
\hline Zircaloy backstop & 77.65 & 15.53 & 0.005 \\
\hline lead backstop & 1.32 & 0.26 & 0.031 \\
\hline target chamber ${ }^{3} \mathrm{He}$ & 12.71 & 2.54 & 0.003 \\
\hline heat exchanger ${ }^{3} \mathrm{He}$ material & 0.43 & 0.09 & 0.013 \\
\hline inner blanket ${ }^{3} \mathrm{He}$ material & 12.23 & 2.45 & 0.005 \\
\hline middle blanket ${ }^{3} \mathrm{He}$ material & 2.94 & 0.59 & 0.007 \\
\hline outer blanket ${ }^{3} \mathrm{He}$ material & 0.68 & 0.14 & 0.016 \\
\hline backstop ${ }^{3} \mathrm{He}$ material & 1.71 & 0.34 & 0.020 \\
\hline tungsten target material & 358.13 & 71.63 & 0.002 \\
\hline Total Deposited Energy & 744.04 & 148.81 & 0.001 \\
\hline \multicolumn{4}{|l|}{ Energy Balance: } \\
\hline total leakage energy & 31.08 & 6.22 & 0.048 \\
\hline total deposited energy & 744.04 & 148.81 & 0.001 \\
\hline conversion to mass & 224.88 & 44.97 & \\
\hline Total & 1000.00 & 200.00 & \\
\hline
\end{tabular}

*Assumes a proton beam current of $200 \mathrm{~mA}$. 


\subsection{CALCULATIONAL METHODOLOGY}

\subsubsection{Overview of the LAHET Code System}

Los Alamos National Laboratory has world-class Monte Carlo computational capability applicable to spallation-neutron source design with the requisite computer hardware and experienced people to set up and run the codes and interpret the results. One part of the computational tool is based on the LANL version of the HETC Monte Carlo code for the transport of nucleons, pions, and muons, which was originally developed at ORNL [3-1]. Because of major modifications and additions made to the HETC code at LANL, the version of HETC has been renamed LAHET, and the system of codes based on LAHET (useful in spallation neutron source design) is designated as the LAHET Code System (LCS) [3-2]. A brief description of the breadth of capabilities that the LCS puts at the users' disposal follows below.

The LCS is a sophisticated code system based on several subcomponents, among which LAHET and MCNP are the major players. LAHET itself is used for the transport and interaction of nucleons, pions, and muons at energies less than $4 \mathrm{GeV}$. LAHET uses the Bertini or the ISABEL model to describe the physics of the intranuclear cascade and uses the Dresner evaporation model for the last phase of the nuclear interaction. The Fermi breakup model replaces the Dresner model for describing the evaporation process for light nuclei. A pre-equilibrium model was recently added as an intermediate stage between the intranuclear phase and the evaporation phase. Two fission models (Rutherford-Appleton or ORNL) complement the set of physics models LAHET uses.

The MCNP code is a design-production code for low-energy neutron/photon/electron Monte Carlo transport. The code is distributed and used internationally by nuclear-systems designers. The MCNP code is geared toward the transport of neutrons, photons, and electrons in matter and uses very detailed cross sections for several hundred isotopes to describe the interaction of neutrons and photons with matter down to sub-thermal energies. The version of the code used in the present study, MCNP4XE, makes use of ENDF/B-V cross sections for neutron and photon reactions. Thermal neutron reactions use the free gas model or, when available, detailed scattering kernels, known as the $S(\alpha, \beta)$ treatment. For photons, the code takes coherent and incoherent scattering, fluorescent emission after photoelectric absorption, and various other physical processes into account. Contrary to multigroup codes, MCNP is a general-purpose, continuous-energy, generalized-geometry, and time-dependent Monte Carlo transport code. It is capable of handling arbitrarily complex 3D geometries. The output MCNP produces ranges from neutron and gamma-ray fluxes and currents to energy deposition, from energy fluxes to gas production, and from radiation doses to criticality eigenvalues.

Particle transport in both LAHET and MCNP is based on Monte Carlo techniques. The philosophy used in the LAHET code is to treat all interactions by protons, pions, and muons within LAHET but to treat neutron interactions only above a cutoff energy, typically $20 \mathrm{MeV}$. Any low-energy $(<20 \mathrm{MeV})$ neutron emerging from a reaction has its kinematic parameters recorded on a neutron file (NEUTP) for subsequent transport. For LAHET, a version of MCNP (called HMCNP) has been modified to accept the NEUTP file as an input source to 
complete the low-energy neutron transport using continuous-energy, ENDF/B-based, neutron cross-section libraries. Low-energy, transported neutrons can participate in nuclear reactions and produce additional particles.

During a LAHET calculation, a large quantity of information is recorded on a separate file, which another piece of code, PHT - a photon source generating code - can subsequently analyze to produce a source for HMCNP. The HMCNP phase of the calculation is then executed as a coupled neutron/photon transport problem. The photons originate either from the decay of neutral pions produced in the intranuclear cascade phase or by the deexcitation of residual nuclei after the evaporation phase. In a coupled neutron/photon problem, the neutron file NEUTP and the gamma-ray file GAMTP are merged (by the MRGNTP code in the LCS) to produce a combined neutron/photon file COMTP that describes the low-energy neutron source and high-energy-produced gamma-ray source for the entire system. HMCNP is then used to transport these neutrons and photons plus gamma rays produced from neutron-induced reactions below $20 \mathrm{MeV}$.

In addition, both LAHET and HMCNP can write history files, called HISTP and HISTX, respectively, that contain a (nearly) complete description of events occurring during the computations. The code HTAPE (another code in the LCS suite of codes) is used to postprocess the HISTP and HISTX files to extract a variety of information. The edit options available with HTAPE include surface current and flux; cell-average neutron flux particle production spectra; residual mass production; mean excitation energy; mass-energy balance, gas production, and energy deposition by cell or material; pulse shape analysis of surface current; and global emission spectrum in polar and azimuthal bins. The relationships of the various codes in the LCS and the files that carry information from one to another are shown in Fig. 3-3.

In summary, for the APT ${ }^{3} \mathrm{He} \mathrm{T} / \mathrm{B}$ system, LAHET is used mainly to (1) study the generation of neutrons and photons resulting from the interaction of the proton beam with the target system; (2) calculate energy deposition and power densities from the high-energy particles streaming into the various parts of the T/B system; and (3) predict spallation product production and neutron fluxes for subsequent radionuclide production and decay calculations with the CINDER' 90 code, the description of which follows below.

\subsubsection{Overview of CINDER'90}

The temporal concentrations of nuclides depleted and produced in materials subject to irradiation are described by a large set of coupled differential equations. Each nuclide's concentration is determined by the history of gains from neutron absorption reactions (spallation, fission, $(n, \gamma),(n, 2 n)$, etc.) and radioactive decay of parent nuclides, as well as losses from its own decay and particle absorption. The solution for these nuclide concentrations was simplified with the CINDER code, which resolved the complicated nuclide couplings into linear chains, each chain representing a unique path from nuclide to nuclide, resulting in small independent sets of differential equations describing the rate of change of partial concentrations of nuclides in each chain. This reduces the solution of a large set of coupled differential equations to the solution of a number of small sets of differential equations 


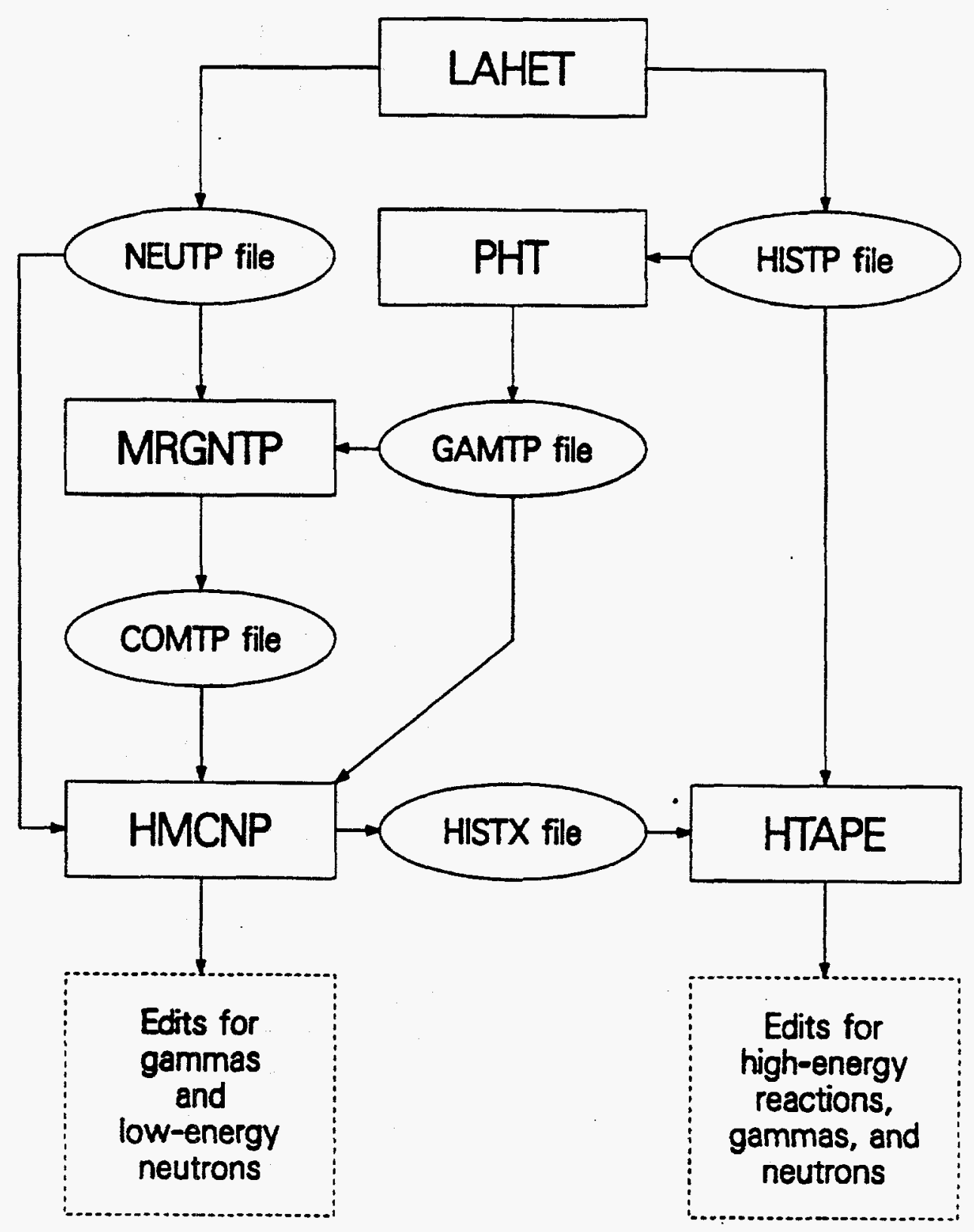

Fig. 3-3. LCS codes and data files

that are each characterized by a single generalized form. Because of the linear nature of the chain (a result of the Markov process), the generalized equations are solved sequentially for the partial concentration of each nuclide in the chain. The total nuclide concentrations are obtained by summing partial concentrations.

Calculations of radionuclide inventories in high-current, medium- and high-energy accelerator targets have required the development of a new inventory code (CINDER'90), evolved from earlier versions of CINDER [3-3 through 3-8] and REAC, [3-9 through 3-12] 
and continuing development of cross-section and decay data. CINDER'90 uses these data with problem-specific spallation production and neutron-flux data calculated using the LCS.

The CINDER'90 code differs from earlier CINDER versions in that earlier versions required the development of a library of transmutation chains before a calculation. Users selected chains of such libraries to follow transmutation paths that the user considered necessary and sufficient for the problem, and chains developed for one problem were not necessarily applicable to others. The CINDER' 90 code uses a library of basic nuclear data to trace all possible transmutation paths, determining the partial concentration and associated activity of each nuclide as well as the integrated transmutation of each nuclide during a time increment. A nuclide's integrated transmutation, called the passerby, indicates the sum of subsequent partial concentrations in chains continuing from the nuclide. CINDER'90 examines each nuclide's partial concentration, activity, and passerby to determine whether a chain should be terminated relative to input significance criteria.

CINDER'90 accumulates nuclide concentrations and activities from nuclide properties as they are calculated. It then combines the post-processing data with decay and neutron absorption data to obtain density (atoms/barn-cm and $\mathrm{kg}$ ); activity $\left(\mathrm{Ci} / \mathrm{cm}^{3}\right.$ and $\mathrm{Ci}$ ); decay power (W/ $/ \mathrm{cm}^{3}$ and watts); macroscopic neutron absorption $\left(\mathrm{cm}^{-1}\right)$; and decay spectra properties listed by nuclide, element $\mathrm{Z}$, and mass $\mathrm{A}$. The code also tabulates major contributors $(\geq 0.1 \%$ ) to mass, activity, decay power, and macroscopic absorption.

The individual nuclide and aggregate results-activity inventory, decay power, macroscopic neutron absorption, etc.-have many practical applications. Some applications require the transport of the decay source to obtain a desired response, such as dose or doseequivalent rates. Transmutation calculation results are limited in accuracy by appropriate problem definition and by the validity of the nuclear data used in the calculation (i.e., neutronabsorption cross sections, decay constants for each nuclide transmutation path, and associated branching fractions to ground and isomeric states produced). Additional data describing the energy spectra and toxicity associated with the decay of radionuclides are required. The collection, calculation, and evaluation of the data are ongoing efforts currently involving dozens of scientists internationally. A detailed description of current CINDER'90 development activities is given in Refs. 3-13 and 3-14.

\subsubsection{Comparison between LCS Calculations and Experimental Data}

In the analysis of the APT system, the LCS is used to predict neutron production, neutron reaction rates, energy deposition, and nuclide production and destruction rates to assess the $3 \mathrm{He}$ $T / B$ neutronic performance, as input for engineering decisions and for coupling to CINDER'90. Additionally, CINDER'90 is used to determine the time-dependent isotopic concentrations based on input from LCS calculations and to predict the resulting gamma-decay spectra. The fidelity with which the calculations reproduce experimental data is one indicator of confidence in the validity of the APT model. To determine the accuracy of these calculational techniques with regard to APT analysis, a number of benchmark calculations were performed to compare with existing experimental data. 


\subsubsection{Bare-Target Integral Neutron Leakage}

One test for the LAHET Code System consists of estimating neutron yields from simple targets. This particular test, although straightforward, is very important because it relates directly to the ability of LCS to reliably predict absolute neutron yields in the energy range of interest for APT. Other tests, even more stringent in nature, are described in subsequent sections.

Recent measurements by Vassilkov et al. [3-15] in Dubna define an excellent test problem for LCS. They measured absoluted neutron yields from a thick cylindrical lead target at various proton beam energies. More precisely, the target was a natural lead cylinder, $20 \mathrm{~cm}$ in diameter and $60 \mathrm{~cm}$ long. The JINR synchrocyclotron in Dubna was used to produce a focused proton beam at various energies ranging from 990 to $3650 \mathrm{MeV}$. In the experiment, researchers measured absolute neutron yields with threshold fission detectors.

The physics parameters (Bertini model, pre-equilibrium model, etc.) used in these LCS calculations are the same as those used in the APT calculations. Figure 3-4 shows the results produced by LCS, as well as the experimental data of Vassilkov et al. The agreement between the LCS results and the experimental data over the entire energy range is excellent.

\subsubsection{LANSCE Neutron Spectra}

The neutron flux from the high-intensity $\mathrm{H}_{2} \mathrm{O}$ moderator at the Manuel J. Lujan Neutron Scattering Center (LANSCE) has been measured from $0.025 \mathrm{eV}$ to $100 \mathrm{keV} \mathrm{[3-16]} \mathrm{and} \mathrm{the}$ measured values compared with calculated predictions. The results are shown in Fig. 3-5 from $0.025 \mathrm{eV}$ to $10 \mathrm{keV}$. The agreement between calculations and measurements is at the $20 \%$ level. This is a very stringent test of the LCS because of the complex geometry and composition of the LANSCE target-moderator-reflector-shield system.

\subsubsection{Los Alamos FERFICON Conversion Measurements}

As part of the fertile-to-fissile conversion (FERFICON) program [3-17] at LANL, the axial distributions of fissions and of fertile-to-fissile conversions in thick, depleted uranium and thorium targets bombarded by $800-\mathrm{MeV}$ protons were measured. Table 3-3 gives the physical characteristics of the targets.

\begin{tabular}{ccccc}
\hline \hline Table 3-3. & Physical Characteristics of the FERFICON Targets & \\
\hline Material & $\begin{array}{c}\text { Number of } \\
\text { Rods }\end{array}$ & $\begin{array}{c}\text { Density } \\
\left(\mathbf{g} / \mathbf{c m}^{2}\right)\end{array}$ & $\begin{array}{c}\text { Diameter } \\
(\mathbf{c m})\end{array}$ & $\begin{array}{c}\text { Length } \\
\text { (cm) }\end{array}$ \\
\hline Depleted Uranium* & 37 & 19.04 & $19.70^{* *}$ & 30.46 \\
Thorium & 19 & 11.38 & $18.28^{* * *}$ & 36.31 \\
\hline \hline
\end{tabular}

*0.251 w\% $235 \mathrm{U}$.

**Effective diameter of the clustered target $(D=d \sqrt{n})$ with an individual rod diameter of $3.239 \mathrm{~cm}$.

***Effective diameter of the clustered target with an individual rod diameter of $4.194 \mathrm{~cm}$. 


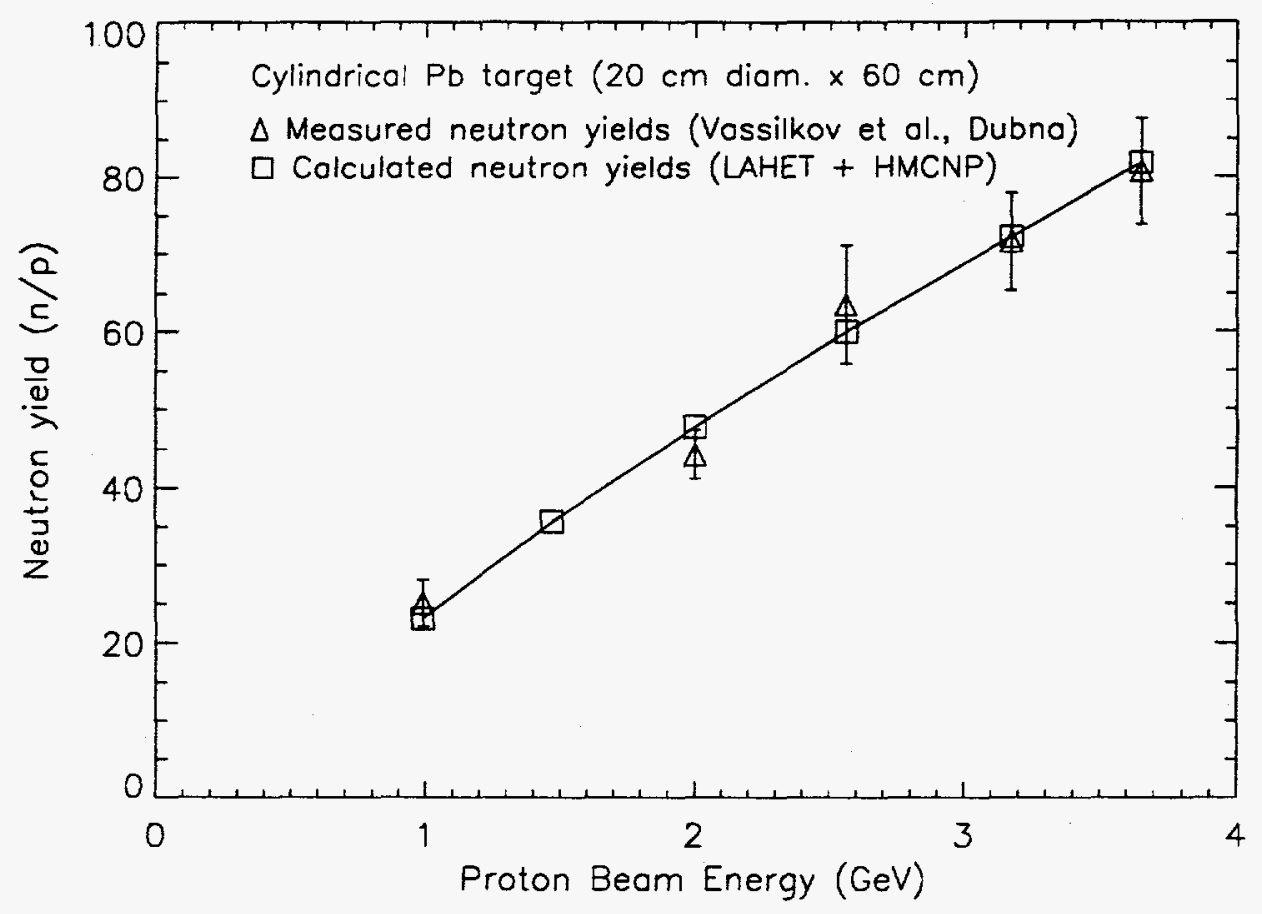

Fig. 3-4. Absolute neutron yields from a cylindrical lead target $20 \mathrm{~cm}$ diam $\times 60 \mathrm{~cm}$ long. Squares denote LCS results; triangles denote experimental results by Vassilkov et al. The solid line is placed to guide the eye.

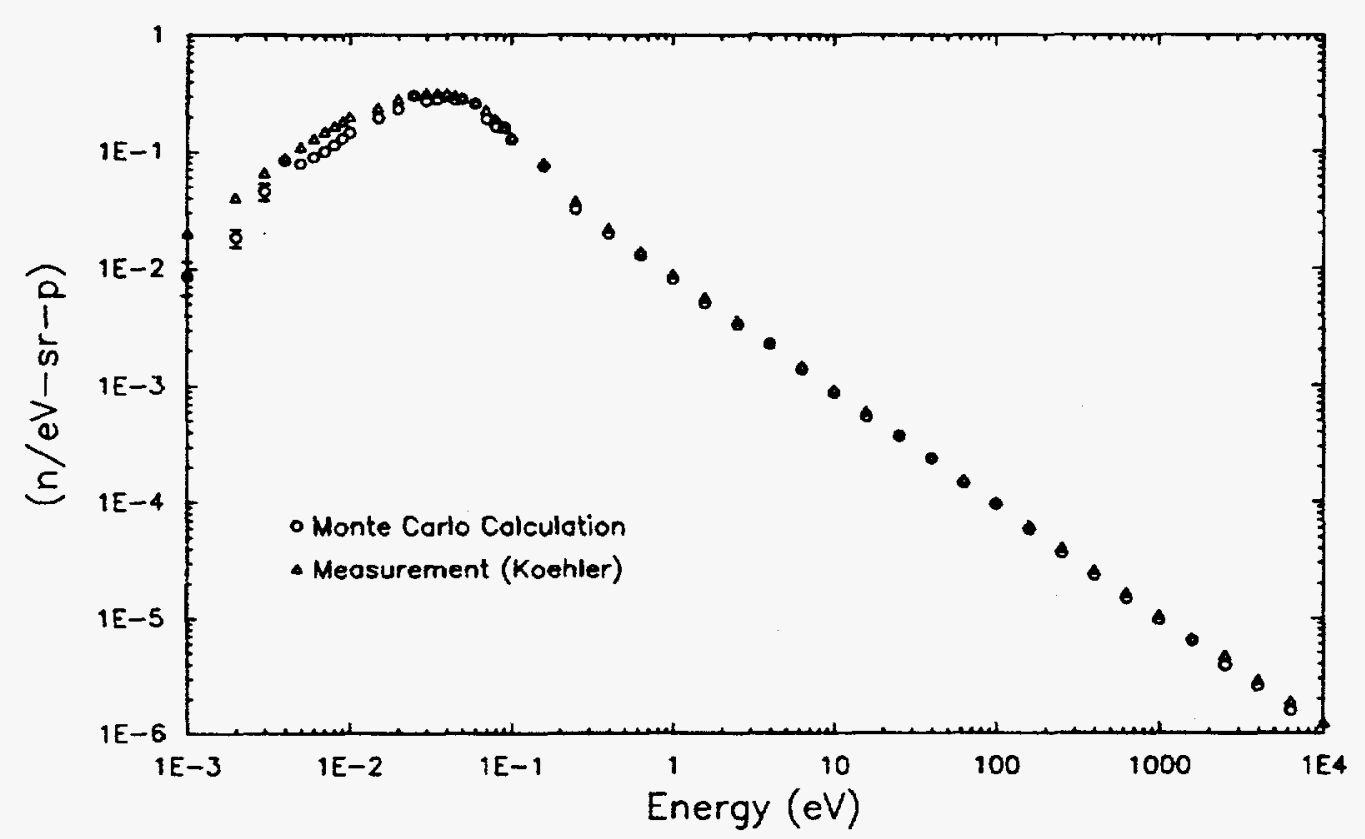

Fig. 3-5. Calculated and measured neutron energy spectra from the LANSCE high-intensity $\mathrm{H}_{2} \mathrm{O}$ moderator. 
Table 3-4 shows measured conversion results compared to calculated predictions. The results indicate that the "source term" for low-energy $(<20 \mathrm{MeV})$ neutron production is being handled well by LAHET.

Table 3-4. Comparison of Measured and Calculated Conversions for Thorium and Depleted Uranium Targets Bombarded by $800-\mathrm{MeV}$ Protons

\begin{tabular}{ccc}
\hline Target & $\begin{array}{c}\text { Measured Conversion } \\
\text { (atoms/protons) }\end{array}$ & $\begin{array}{c}\text { Calculated Conversion } \\
\text { (atoms/protons) }\end{array}$ \\
\hline Thorium & $1.25 \pm 0.01$ & $1.27 \pm 0.01$ \\
Depleted Uranium & $3.81 \pm 0.01$ & $3.88 \pm 0.03$ \\
\hline \hline
\end{tabular}

\subsubsection{Russian Energy Deposition Measurements}

Because the thermal-hydraulic design of the $3 \mathrm{He} \mathrm{T} / \mathrm{B}$ system is based on calculated energy deposition, it is one of the most important pieces of information transferred to the engineering task. To estimate the accuracy of the LCS with regard to energy deposition, LCS predictions were compared with the experimental results of Belyakov-Bodin et al. [3-18 through 3-20]. These comparisons were made for protons with energies of 800,1000 , and $1200 \mathrm{MeV}$ on lead, bismuth, beryllium, carbon, aluminum, and uranium. A comparison was not performed on energy deposition in ungsten because experimental data are not available.

The experimental apparatus used by Belyakov-Bodin et al., shown in Fig. 3-6, consisted of 24 blocks, each $2.5 \mathrm{~cm}$ thick, $20 \mathrm{~cm}$ in diameter, and fabricated with internal thermocouples for temperature measurements. The LAHET/HMCNP geometry set up to model the experimental configurations of Belyakov-Bodin consisted of a cylinder divided into a total of 48 cells, each $2.5 \mathrm{~cm}$ thick, divided into two radial regions $(r \leq 5 \mathrm{~cm}$ and $5 \mathrm{~cm}<r \leq 10 \mathrm{~cm})$. Table 3-5 compares the calculated results with experimental data. No specific trends in discrepancies between the calculated results and the experiment can be identified that encompass all of the elements. The results for lead, bismuth, and uranium agreed reasonably

BEAM DISTRIBUTION

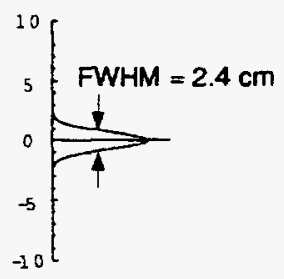

SIDE VIEW
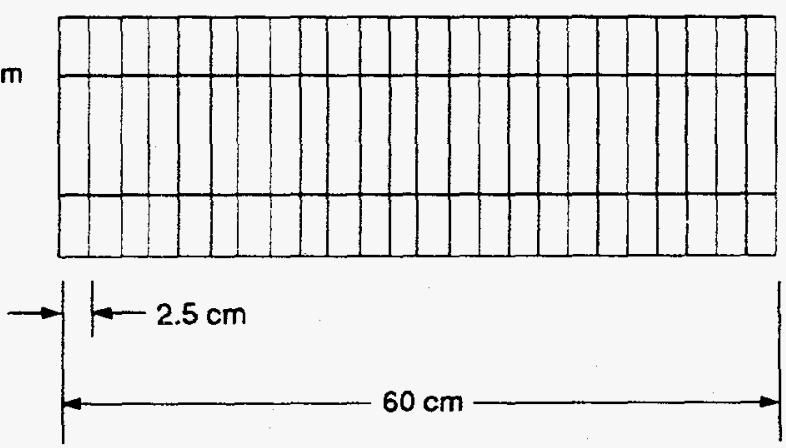

END VIEW

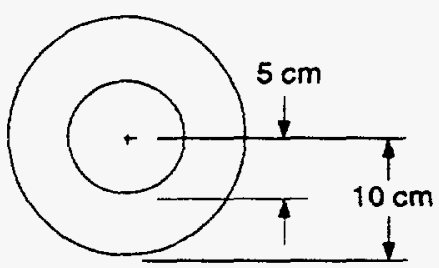

Fig. 3-6. LCS geometry for the Russian energy deposition calculations. 
well with the experimental results. For the lighter materials, however, the LCS inconsistently matched the experimental data. Nevertheless, for heavy materials, the LCS accurately predicts energy deposition values for both total deposition and deposition at specific locations within the targets, with the total energy deposition being correct within approximately $20 \%$. Energy deposition values at specific locations are even more accurate. Therefore, the LCS is valid for use in predicting energy deposition for tungsten targets because of tungsten's high atomic mass. For lighter elements, the uncertainty in the predictions is greater. This is partly because the Bertini intranuclear cascade and Dresner evaporation models are statistical in nature and work better for heavier nuclei. Also, the nuclear level density is not modeled as well for light nuclei.

\begin{tabular}{lcccccc}
\hline Table 3-5. & \multicolumn{6}{c}{ Total Energy Deposited over Axial Length for Radii of $5 \mathrm{~cm}$ and $10 \mathrm{~cm}$} \\
\hline Material & $\begin{array}{c}\text { Proton } \\
\text { Energy } \\
\text { (MeV) }\end{array}$ & $\begin{array}{c}\text { Reasured } \\
\text { Radius } \\
\text { (cm) }\end{array}$ & $\begin{array}{c}\text { Deposited } \\
\text { Energy } \\
\text { (MeV) }\end{array}$ & $\begin{array}{c}\text { Quoted } \\
\text { Experimental } \\
\text { Error (\%) }\end{array}$ & $\begin{array}{c}\text { Calculated } \\
\text { Deposited } \\
\text { Energy } \\
\text { (MeV) }\end{array}$ & $\begin{array}{c}\text { Deviation } \\
\text { between } \\
\text { Calc. and } \\
\text { Exp. (\%) }\end{array}$ \\
\hline Lead & 800 & 5 & 380 & 7.0 & 475 & 25.0 \\
Lead & 800 & 10 & 460 & 10.0 & 523 & 13.7 \\
Lead & 1000 & 5 & 450 & 7.0 & 540 & 20.0 \\
Lead & 1000 & 10 & 520 & 10.0 & 608 & 16.9 \\
Lead & 1200 & 5 & 530 & 8.0 & 611 & 15.3 \\
Lead & 1200 & 10 & 600 & 11.0 & 687 & 14.5 \\
Bismuth & 800 & 5 & 430 & 4.0 & 469 & 9.1 \\
Bismuth & 800 & 10 & 520 & 6.0 & 525 & 1.0 \\
Bismuth & 1000 & 5 & 470 & 4.0 & 526 & 11.9 \\
Bismuth & 1000 & 10 & 570 & 6.0 & 600 & 5.3 \\
Bismuth & 1200 & 5 & 470 & 8.0 & 589 & 25.3 \\
Bismuth & 1200 & 10 & 570 & 10.0 & 672 & 17.9 \\
Uranium & 800 & 5 & 1090 & 4.4 & 1205 & 10.6 \\
Uranium & 800 & 10 & 1570 & 5.8 & 1622 & 3.3 \\
Uranium & 1000 & 5 & 1460 & 6.7 & 1497 & 2.5 \\
Uranium & 1000 & 10 & 2170 & 7.2 & 2083 & -4.0 \\
Uranium & 1200 & 5 & 1700 & 3.5 & 1784 & 4.9 \\
Uranium & 1200 & 10 & 2530 & 4.1 & 2540 & 0.4 \\
\hline \hline
\end{tabular}

*Error defined as (Calc. Value - Exp. Value)/(Exp. Value).

\subsubsection{LANSCE Activation Measurement}

A comparison of predicted and observed residual dose rate measurements of the LANSCE tungsten target after removal for storage was done to test the accuracy of the LCS coupled with CINDER' 90 with regard to predicting residual nuclide activity due to long-term proton bombardment.

From November 1985 through October 1990, the LANSCE spallation target received a time-integrated beam current of about $0.25 \mathrm{~A} \cdot \mathrm{h}$ of $800-\mathrm{MeV}$ protons with a documented operational history. The target was removed for disposal on April 7, 1991. The gamma dose rates from decay of activation products in the target were measured along the outside surface of the target and in the $14-\mathrm{cm}$ gap between the upper and lower targets. A coupled LCS/CINDER'90 calculation was done to predict these dose rates. 
A comparison of the measured values and the calculated results is given in Table 3-6. The greatest difference between the LCS results and the experimental values occurred at the downstream end of the lower target assembly, where the LCS prediction was 2.4 times greater than the experimental measurement. This was also the only value predicted by the LCS that was greater than the measured value. The coupled LCS/CINDER' 90 calculation predicted the resulting dose levels surrounding the target to within a factor of about 2 , which is remarkably good agreement for this type of computation. Furthermore, the gamma-ray dose had significant activation contributions from both spallation (predominantly in the tungsten) and parasitic neutron absorption (primarily in the steel), and the successful prediction of that dose illustrates the ability of LCS/CINDER'90 to accurately account for both mechanisms of radionuclide production. Finally, the material composition of the LANSCE target makes this measurement a strong validation for the use of LCS/CINDER'90 for APT activation calculations. Combining the LCS with CINDER' 90 results in a very useful package for the analysis of APT radionuclide waste streams and subsequent dose levels generated by the activated material.

Table 3-6. Decay Dose Values Calculated by LCS/CINDER'90 and Experimental Results

\begin{tabular}{lccc}
\hline \multicolumn{1}{c}{ Location } & $\begin{array}{c}\text { LCS } \\
(\mathbf{k R / h})\end{array}$ & $\begin{array}{c}\text { Measurement } \\
(\mathbf{k R} / \mathbf{h})\end{array}$ & Ratio \\
\hline Top of Upper Target Assembly & 0.07 & 0.11 & 0.64 \\
Middle of Upper Target Assembly & 0.25 & 0.39 & 0.64 \\
Bottom of Upper Target Assembly & 0.93 & 1.30 & 0.72 \\
Center of Flux Trap & 2.21 & 3.40 & 0.65 \\
Top of Lower Target Assembly & 0.74 & 1.55 & 0.48 \\
Middle of Lower Target Assembly & 0.65 & 0.65 & 1.00 \\
Bottom of Lower Target Assembly & 0.46 & 0.19 & 2.42 \\
\hline \hline
\end{tabular}

\subsection{EVOLUTION OF THE ${ }^{3} \mathrm{He}$ T/B DESIGN}

The application of the Los Alamos split-composite target concept to APT (the 3He T/B design) has extended knowledge of the neutronics of spallation-neutron sources. To gain insight into this unique $\mathrm{T} / \mathrm{B}$ system, it is necessary to provide a background of basic target neutronics before describing the evolution of the $3 \mathrm{He} \mathrm{T} / \mathrm{B}$ design.

\subsubsection{Fundamental Target Neutronics}

Maximizing total low-energy neutron production, which depends on target material and geometry, as well as incident particle type and energy, is an important aspect of spallation neutron source target design-however, it is only part of the story. Once low-energy neutrons are produced inside the target, they must escape (leak) from the target before they can be moderated (slowed down by scattering) to energies that are useful in a variety of applications. Leakage maximization is therefore a crucial aspect of spallation-neutron source target design. The geometry and parasitic absorption of the target primarily control the leakage of low-energy neutrons for a given target material. Figure 3-7 illustrates these effects by comparing neutron 


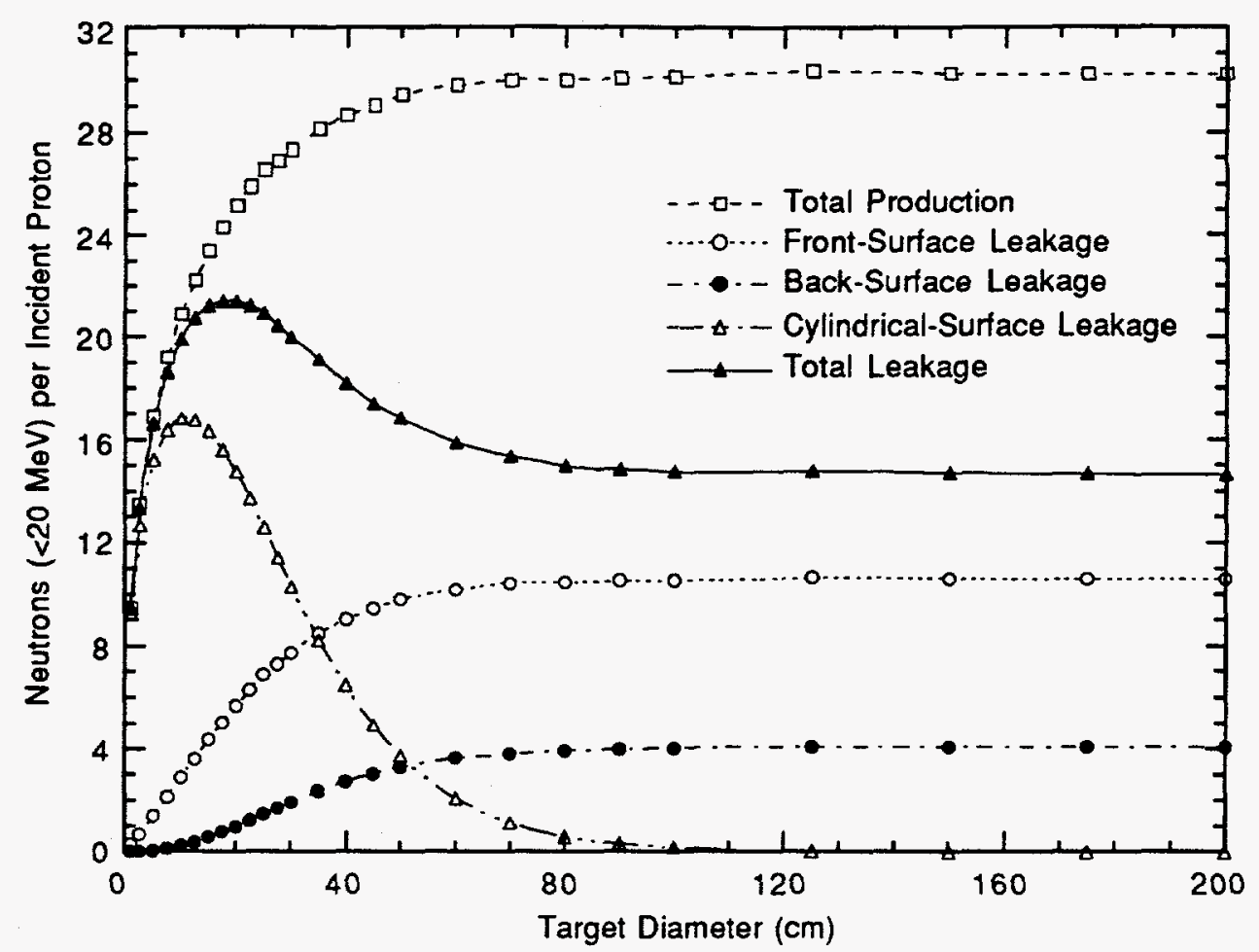

(a)

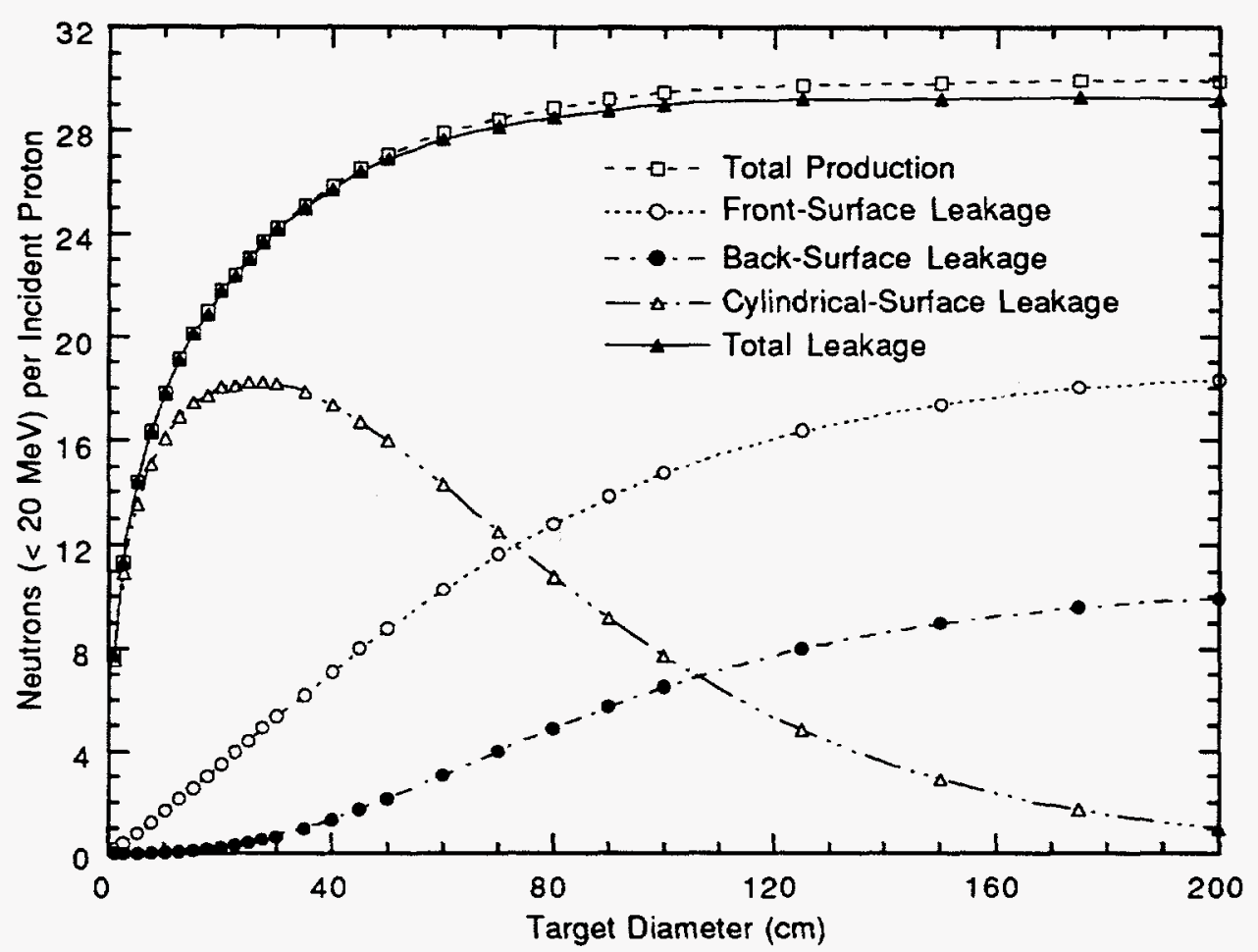

(b)

Fig. 3-7. Total low-energy neutron production and leakage for solid rightcircular cylindrical targets bombarded on axis by 1.GeV protons. (a) A 30-cm-long natural tungsten target; (b) a 55-cm-long natural lead target. 
production and leakage for two typical spallation-neutron source target materials, lead and tungsten. The increased parasitic absorption in tungsten is significant at large target diameters, yet tungsten produces more low-energy neutrons than lead. As can be seen, leakage from the front surface of the targets dominates the total leakage at large target diameters.

Figure 3-7 also illustrates the flexibility and complexity of designing spallation targets. For example, if target compactness and neutron leakage from the cylindrical surface are important, then one would choose a tungsten target with a diameter of approximately $10 \mathrm{~cm}$. Indeed, this is the case for the LANSCE-pulsed spallation neutron source application for materials science research [3-21]. On the other hand, if total neutron leakage alone dominates the target design criteria, one would choose a solid lead target with a diameter of about $100 \mathrm{~cm}$. However, the engineering realities concerning target structural support and target cooling must be addressed; these criteria have a tremendous impact on spallation target design.

Figure 3-8 shows ratios of total low-energy neutron production and leakage of stoppinglength targets of tungsten and lead. Note in Fig. 3-8 that the low-energy neutron production of tungsten equals or exceeds that of lead at all target diameters.

Figure 3-9 illustrates cylindrical, square, and rectangular (slab) targets where the proton beam would be incident (perpendicularly) into the plane of the paper. In the view shown, rectangular targets with large aspect ratios (long side/short side) are called slab targets. Shown in Fig. 3-10 are the ratios of total low-energy neutron production, leakage, and absorption of stopping-length tungsten and tungsten with $50 \%$ heavy water coolant for square and rectangular targets relative to circular targets. Stopping-length targets are long enough to rangeout the primary $(1000-\mathrm{MeV})$ protons. In the calculations, the proton beamspot was centered and assumed to have the shape of the target, with an area of $5000 \mathrm{~cm}^{2}$. Figures $3-10 \mathrm{a}$ and 3$10 \mathrm{~b}$ show that neutron leakage increases with the target aspect ratio. A slab-target geometry is employed in the ${ }^{3} \mathrm{He}$ target design. Figures $3-9 \mathrm{a}$ and $3-9 \mathrm{~b}$ also show that it is more important to have a slab target when coolant material is present.

\subsubsection{Physics Models}

\subsubsection{Introduction}

In the $\mathrm{T} / \mathrm{B}$ design, ${ }^{3} \mathrm{He}$ is used inside and outside the neutron-producing zones. Heavy water is used to cool the targets, as well as for a moderator and a reflector outside the target zones. A moderator is necessary to slow down source neutrons to thermal velocities, where the tritium production cross section for ${ }^{3} \mathrm{He}$ is large. A reflector minimizes neutron leakage from the entire system and increases the tritium production efficiency. This aggregate of the $\mathrm{D}_{2} \mathrm{O}$ moderator and reflector and the ${ }^{3} \mathrm{He}$ outside the target zones is called the "blanket." The concept of a split-composite target (see Section 3.5) is used in the ${ }^{3} \mathrm{He}$ T/B design. To simulate the varied concepts considered in the evolution of the ${ }^{3} \mathrm{He} \mathrm{T} / \mathrm{B}$ design, a number of physics models were created to calculate the neutronic performance of the various concepts with the LCS code package. The concepts considered and the physics models used in the calculations are described below. 


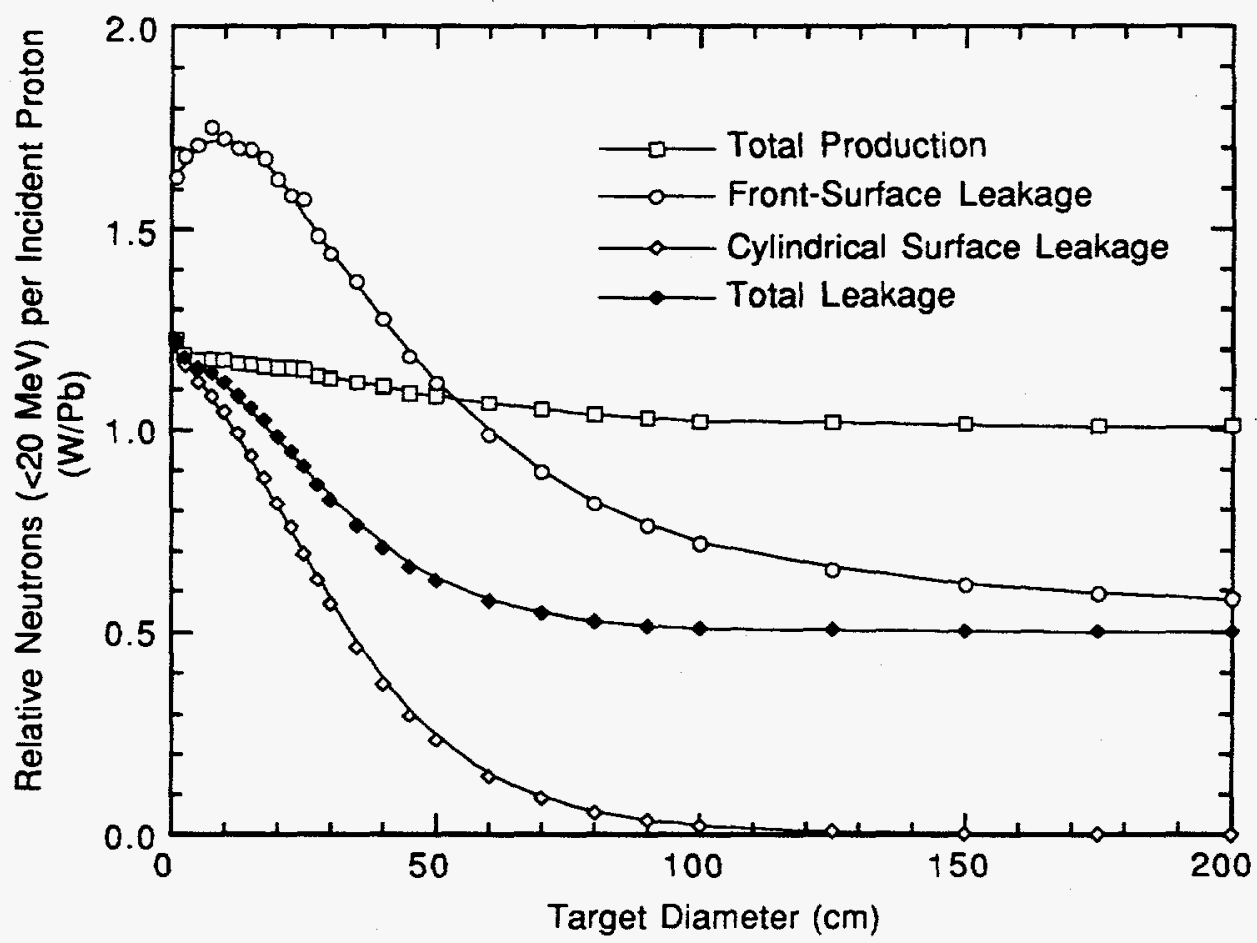

Fig. 3-8. Tungsten cylindrical target performance relative to lead.

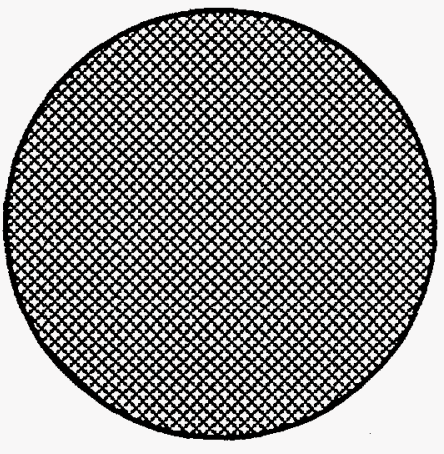

(a)

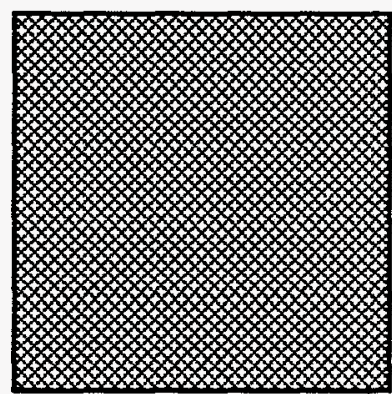

(b)

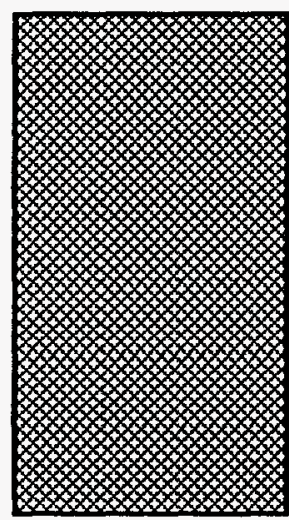

(c)

Fig. 3-9. Illustration of various target geometries: a) circular; b) square (aspect ratio of 1 ); and c) rectangular (slab, with an aspect ratio of approximately 2). The proton beam would be incident into the plane of the paper. 

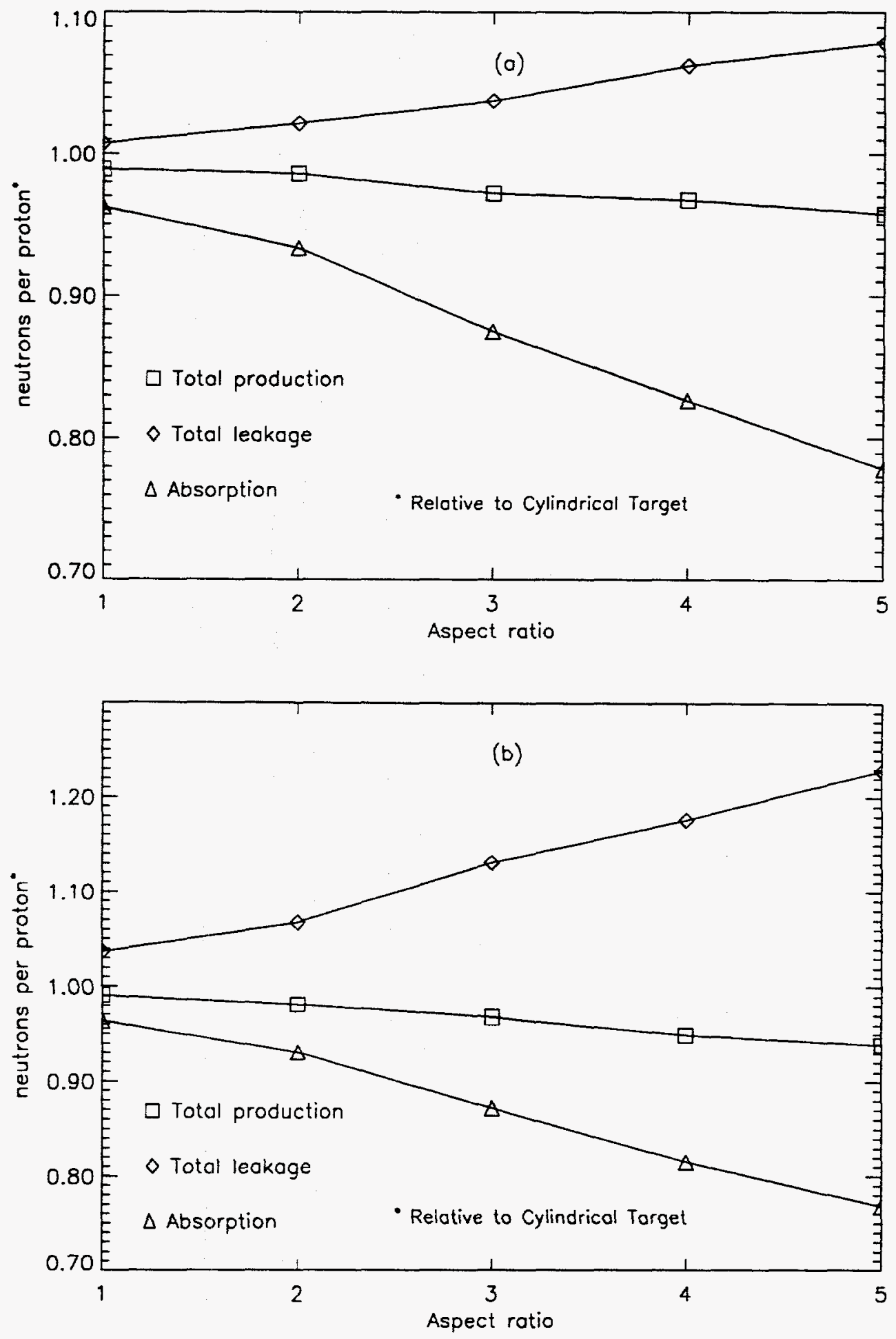

Fig. 3-10. Neutronic performance of various target geometries with equal areas $\left(5775 \mathrm{~cm}^{2}\right)$. (a) The pure tungsten targets are $30 \mathrm{~cm}$ long and (b) the tungsten $/ \mathrm{D}_{2} \mathrm{O}(50 \mathrm{v} \%)$ targets are $60 \mathrm{~cm}$ long. 


\subsubsection{Design Evolution}

A coaxially-cooled $3 \mathrm{He}$ target design was first presented at the APT design review held by the JASONS [3-22]. In a coaxially-cooled target, the $\mathrm{D}_{2} \mathrm{O}$ coolant flow is parallel to the proton beam. The Physics-I model is an application of a coaxially-cooled target with a cylindrical target chamber to the $3 \mathrm{He} \mathrm{T} / \mathrm{B}$ design. The central tungsten target zone of the design presented at the JASON review was $35 \mathrm{~cm} \times 35 \mathrm{~cm}$. One concern raised by the JASONS during their review was that the peak power density in the tungsten $(\sim 4 \mathrm{MW} / \ell)$ was too high. A solution to this potential problem was presented, which evoked a special property of the split-composite target (the so-called neutronically reentrant feature described later). Figure 3-11a shows a coaxially-cooled target inside a circular target chamber.

As the ${ }^{3} \mathrm{He} \mathrm{T} / \mathrm{B}$ design continued to evolve through close interactions between the LANL physics and engineering design teams, the coaxial cooling employed in the target presented to the JASONS proved to be a design problem. Also, the presence of the coaxial inlet/outlet cooling channels between the central and radial target zones degraded tritium production by $\sim 5 \%$. The coaxially-cooled target design was dropped in favor of a cross-flow-cooled target, where the coolant flow is at right angles to the proton beam. Figs. 3-11b and 3-11c illustrate cross-flow-cooled square and slab targets with a rectangular target chamber.

The next phase in the evolution of the $3 \mathrm{He} \mathrm{T} / \mathrm{B}$ design employed a cross-flow-cooled slab target. The Physics-II model is an application of the cross-flow-cooled slab target with a rectangular target chamber to the ${ }^{3} \mathrm{He} \mathrm{T} / \mathrm{B}$ design. The neutronic performance of the slab-target concept with a rectangular target chamber was found to be satisfactory. However, there was a potential flaw in the design from a mechanical viewpoint because the stresses in the flat target chamber wall became excessive, requiring a wall that was too thick from neutronic and thermal-hydraulic viewpoints. System engineering, including cooling of the target chamber, became complicated. Therefore, the idea of a rectangular target chamber was dropped in favor of a cylindrical target chamber but the slab-target design was retained (Fig. 3-11d).

The Physics-III and -IV models both apply the concept of a cross-flow-cooled slab target with a cylindrical target chamber to the ${ }^{3} \mathrm{He} \mathrm{T} / \mathrm{B}$ design. The cylindrical target chamber is much more satisfactory from a mechanical/stress viewpoint. The Physics-III model did not have a proton beam backstop zone to catch all the scattered proton beam from the central target region; even so, the neutronic performance was adequate. The Physics-IV model incorporated a backstop and demonstrated the backstop's role as an integral part of the target system neutron production, as well as the need for a backstop to enhance the overall tritium production efficiency of the ${ }^{3} \mathrm{He}$ T/B design. The Physics-IV model was used in a variety of parametric studies to enhance the neutronic performance of the ${ }^{3} \mathrm{He} \mathrm{T} / \mathrm{B}$ system. The blanket for the Physics-IV model has three ${ }^{3} \mathrm{He}$ decoupler/absorption zones, a moderator region, and a reflector zone. 


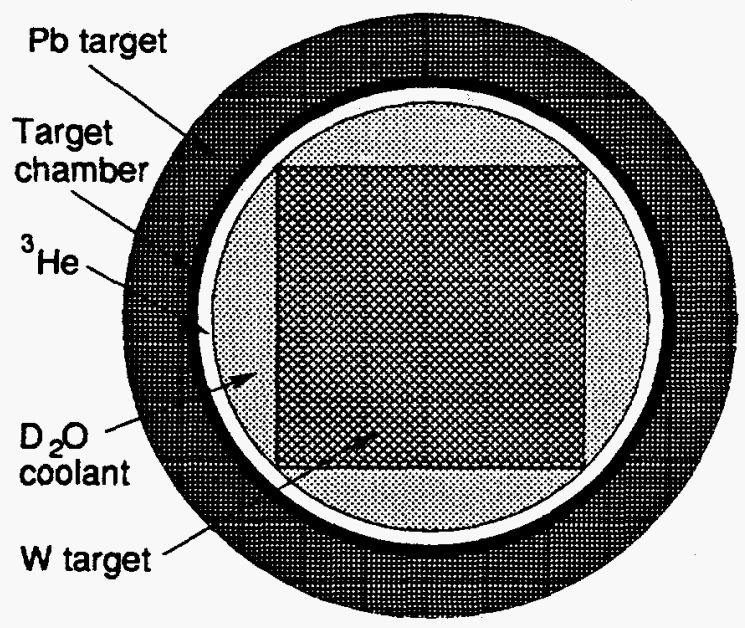

(a)

$\mathrm{Pb}$ target $/ \mathrm{D}_{2} \mathrm{O}$ coolant transition region -

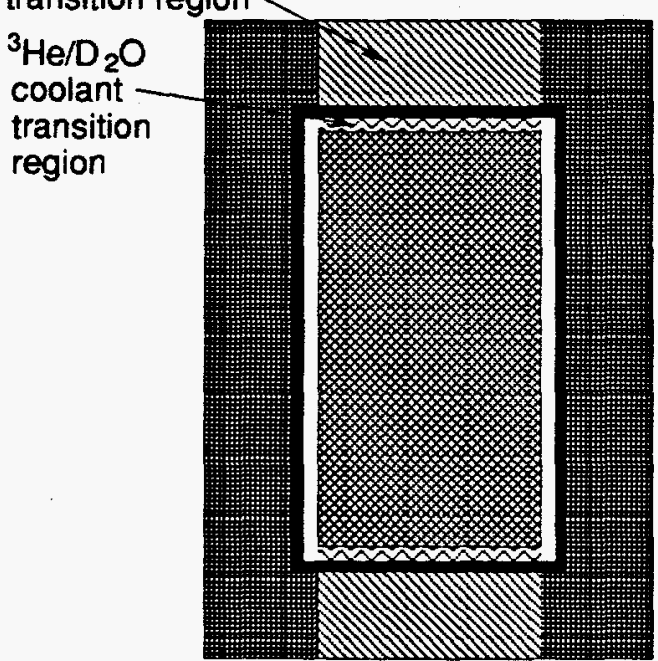

(c)

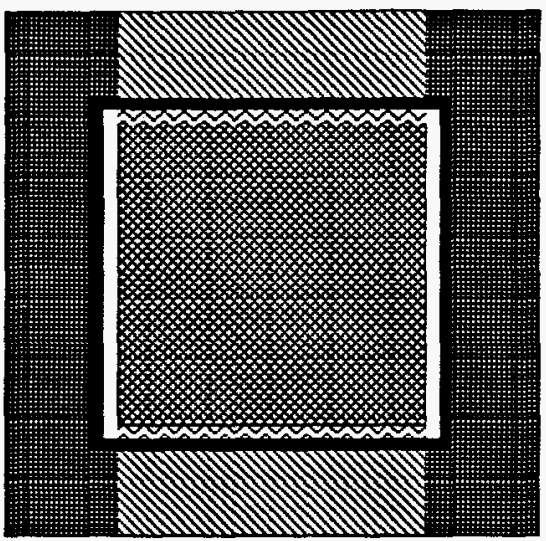

(b)

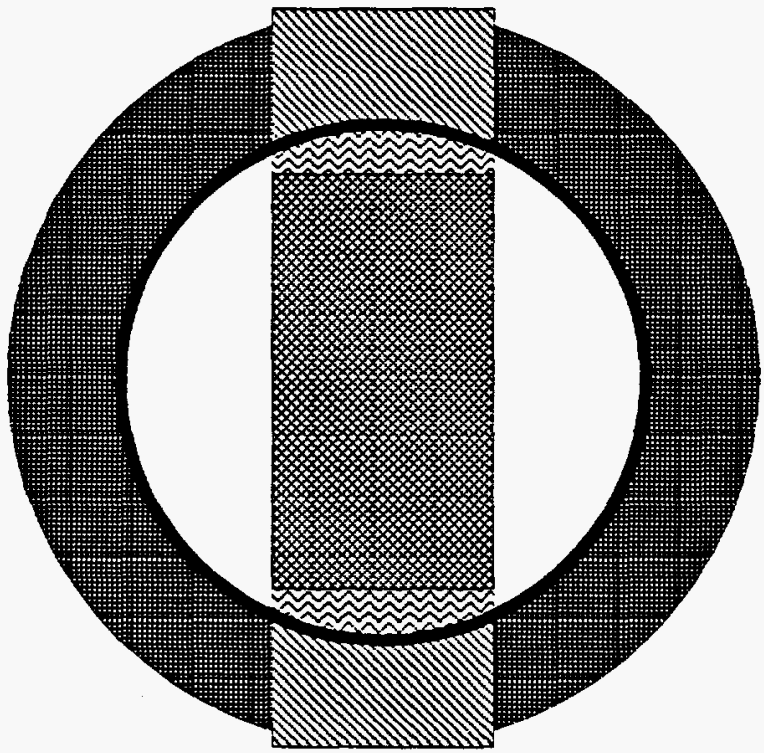

(d)

Fig 3-11. Illustration of various target geometries, coolant flow directions, and target chamberwall geometries: (a) square target, coaxially cooling, and cylindrical target chamber; (b) square target, cross-flow cooling, and square target chamber; (c) rectangular (slab) target, cross-flow cooling, and rectangular target chamber; and (d) rectangular (slab) target, cross-flow cooling, and cylindrical target chamber. The ${ }^{3} \mathrm{He}$ APT target/blanket reference design is based on (d).

\subsection{SPLIT-COMPOSITE TARGET CONCEPT}

For tungsten (and any other neutron absorber), the detrimental parasitic absorption effect is improved by going from a solid target (Fig. 3-12a) to a split target (Fig. 3-12b), which also helps mitigate excessive neutron leakage from the front surface of the target. 
The term flux trap refers to the spaces between the target segments of a split target. Splitting a target increases low-energy neutron leakage and reduces parasitic neutron absorption. However, neutron production is also degraded because secondary high-energy particles escape the central target zone. This effect is mitigated by a radially composite target consisting of a central region and a radial zone (Fig. 3-12c). Depending on the application of the split-composite target concept, it may also be desirable to have a target both axially and radially composite, as well as split (Fig. 3-12d).

a)

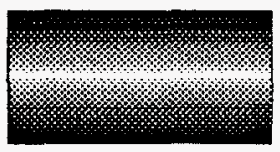

b)

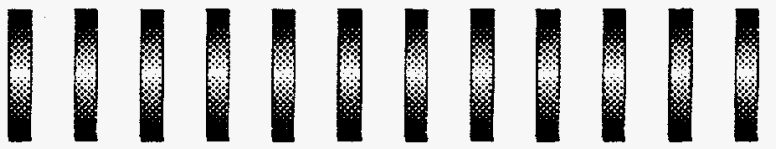

c)

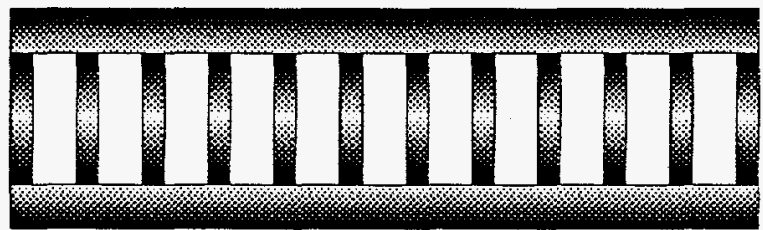

d)

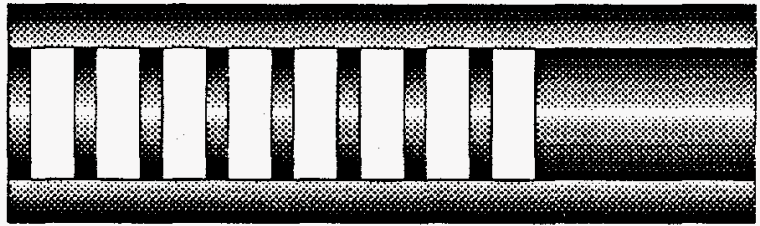

Fig. 3-12. Basic target configurations: a) solid cylindrical target; b) split target; c) split, radially composite target; d) split-composite target-with the addition of a backstop of material different from the central target material, this target is both radially and axially composite.

Figure 3-13 quantitatively shows the effects of splitting a target and making the target both split and radially composite. Note that splitting the target increases neutron leakage for fluxtrap gaps that are $5 \mathrm{~cm}$, decreases front-surface neutron leakage, and decreases neutron production (as the flux-trap gaps increase). Making the target both split and radially composite enhances both the total low-energy neutron leakage and production.

The split-composite target concept has four unique features. First, the concept reduces parasitic neutron absorption in the central target region and enhances radial (or lateral, for square and slab central targets) leakage of both low-energy neutrons and high-energy secondary particles. Enhanced leakage allows the use of high-neutron-absorption materials, such as tungsten, in the central target zone; lead can be used in the radial or lateral target zone to enhance neutron production and utilization (Fig. 3-13). Second, the split-composite target is neutronically reentrant: the central target zone is split (by flux traps) axially, and the neutron 


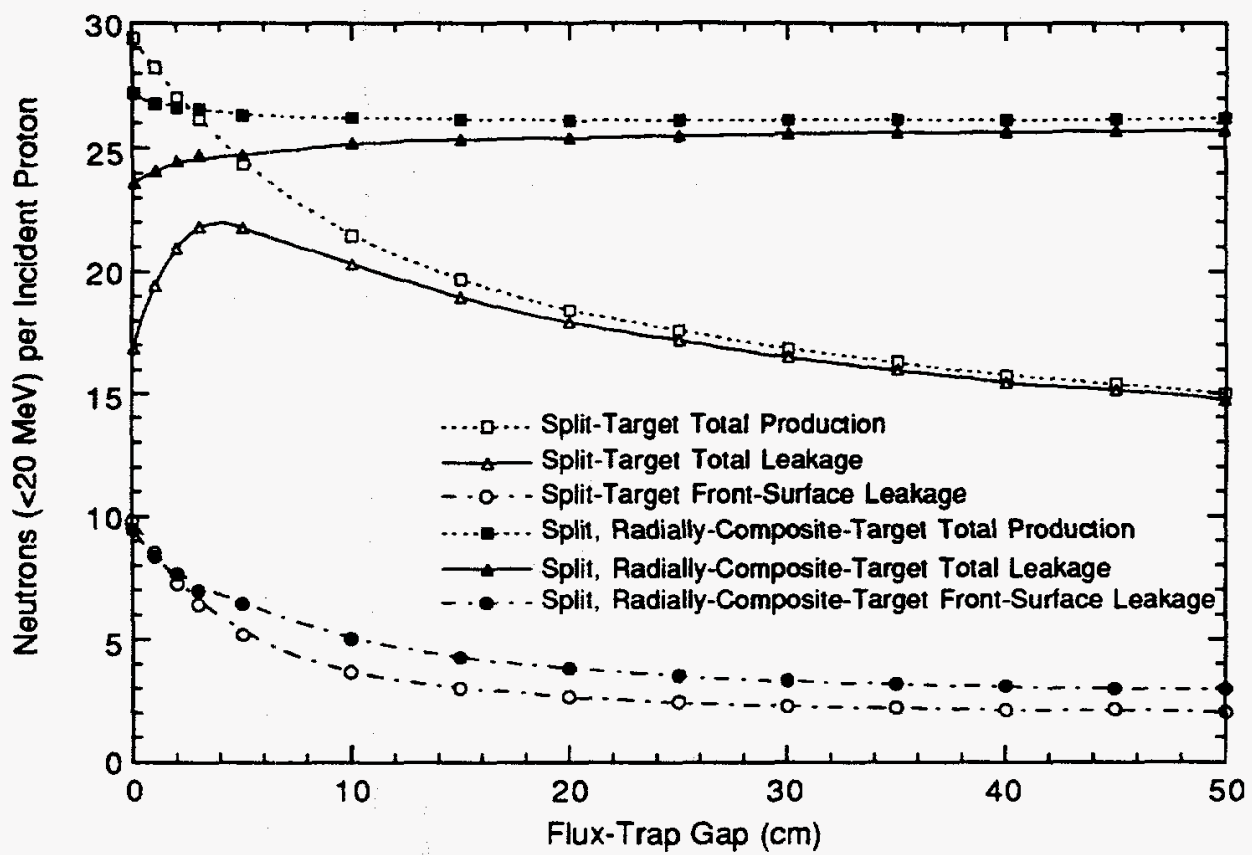

Fig. 3-13. Total neutron production, spallation plus $(\mathrm{n}, \mathrm{xn})$, and leakage for $50-\mathrm{cm}-\mathrm{diam}$ targets. Neutron production is enhanced by the addition of the radial lead zone. Neutron leakage from the top surface is decreased by splitting the central target tungsten zone.

production is spatially distributed deeper along the target axis (Fig. 3-13). Neutron leakage is reduced from the front target surface viewpoint, allowing the use of large target diameters and reduced power densities without large front-surface leakage losses. Figure 3-14 illustrates the latter effects for some early calculations done for $800-\mathrm{MeV}$ protons incident on a split, radially composite target of $\mathrm{W} / \mathrm{Pb}$. Figure 3-14 shows that going from a target diameter of $40 \mathrm{~cm}$ to $115 \mathrm{~cm}$, the tritium production decreases by $\sim 12 \%$, while the proton beam power density decreases by about a factor of 10 . Some of this loss in tritium production efficiency can be mitigated by increasing the height of the reentrant head (Fig. 3-15); the reentrant head refers to the distance from the proton beam window to the front, upstream surface of the APT blanket. Third, the central target zone of a split-composite target allows for geometric decoupling; the axial splitting of the central tungsten target zone accomplishes this geometric effect (Fig. 3-12b). Geometric decoupling also enhances leakage of high-energy secondary particles into the radial target zone to cause spallation reactions; it also increases low-energy neutron leakage from the central target zone. Production of low-energy spallation neutrons in the radial target zone increases their chance to escape (leak) from the split-composite target. Fourth, a split-composite target allows for the possibility of using neutronic decoupling-employing neutron absorbing material in and between target regions to reduce parasitic neutron absorption in the target itself. The following section discusses the use of ${ }^{3} \mathrm{He}$ for neutronic decoupling.

Another benefit of the split-composite target is that the axial distribution of the neutron flux is relatively flat over $\sim 1.5 \mathrm{~m}$, depending on the size of the flux-trap gap. Figure 3-16 illustrates this effect for an early set of calculations done with $800-\mathrm{MeV}$ protons. Splitting the target spreads out radiation damage over larger areas and should extend the lifetime of individual components. Axially splitting the central target zone helps reduce power densities in 


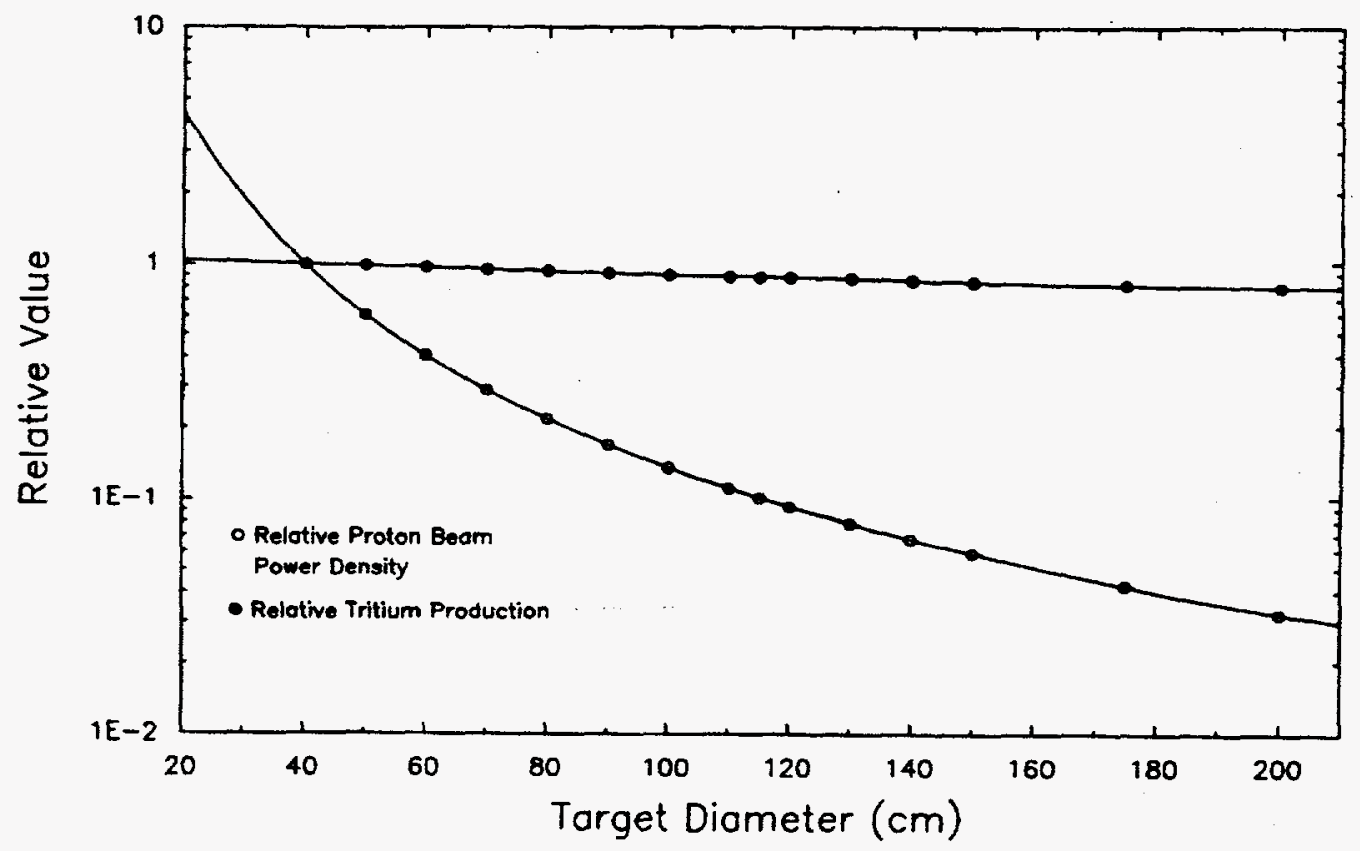

Fig. 3-14. Relative proton beam power density and tritium production as a function of target diameter for cylindrical targets. The reference target diameter is $40 \mathrm{~cm}$. The circular proton beam diameter varies with target diameter and is equal to the target diameter minus $5 \mathrm{~cm}$. The overall $T / B$ axial extent is $350 \mathrm{~cm}$, the reentrant head $100 \mathrm{~cm}$, and the proton energy $800 \mathrm{MeV}$. The target is a W-Pb split-composite target.

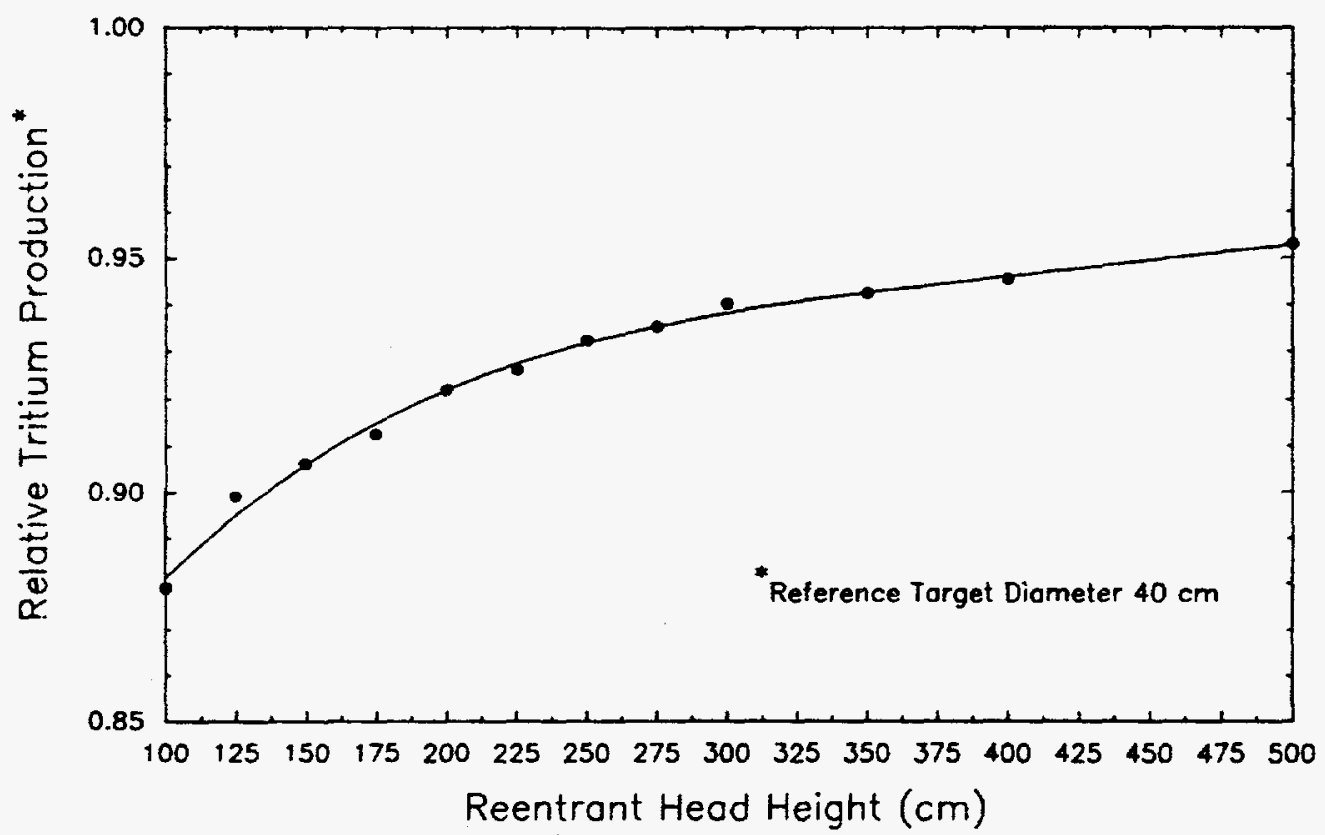

Fig. 3-15. Relative tritium production vs. the height of the reentrant head. The reference target diameter is $40 \mathrm{~cm}$. The target diameter is $115 \mathrm{~cm}$, and the proton energy $800 \mathrm{MeV}$. The target is a $\mathbf{W}$-Pb split-composite target. 
the radial target region. The axial flattening of the neutron flux provides high neutron fluxes over relatively large volumes. The application of the split-composite target to the APT is described in Ref. 3-23.

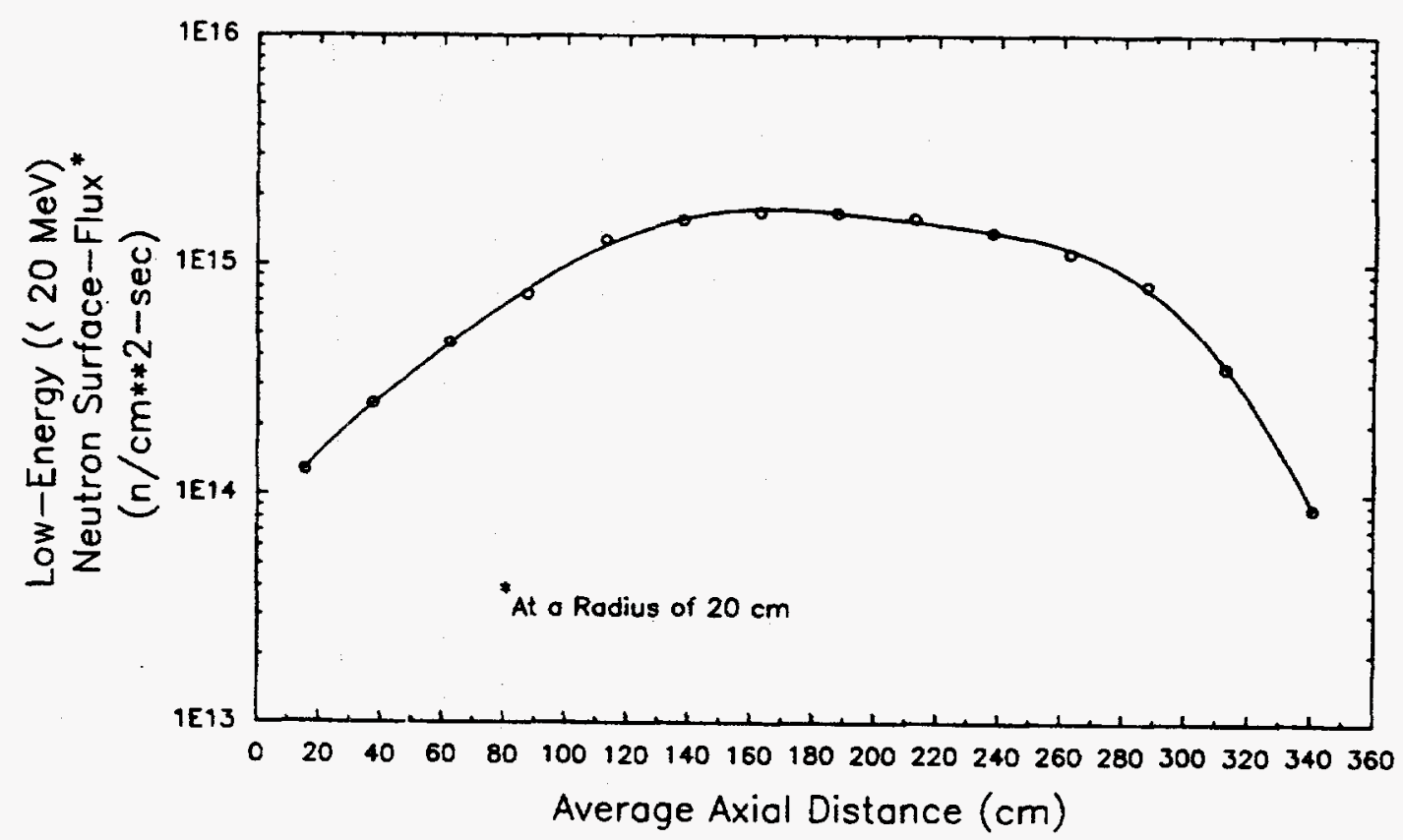

Fig. 3-16. Low-energy ( $<20 \mathrm{MeV}$ ) neutron surface flux at a radius of $20 \mathrm{~cm}$ from a $30-\mathrm{cm}$. diam W-Pb split-composite target. The W-Pb target is surrounded by a $\mathrm{D}_{2} \mathrm{O}$ moderator/reflector with ${ }^{3} \mathrm{He}$ decoupler/absorber regions.

\subsection{PHYSICS DESIGN OF THE NEUTRON SOURCE}

\subsubsection{Introduction}

The Los Alamos APT concept is an ideal application of the split-composite target concept. It takes advantage of the large thermal cross section ( $\sim 5300$ barns $)$ for the reaction

$$
3 \mathrm{He}+n \rightarrow p+\mathrm{T}
$$

to convert ${ }^{3} \mathrm{He}$ to tritium. Here, $n, p$, and $T$ stand for neutron, proton, and tritium, respectively.

Figure 3-17 illustrates the various components of the Los Alamos APT design. The proton beam strikes tungsten target elements in the central target region, producing neutrons through the spallation process. The backstop region ranges out the primary protons and catches any small-angle scattered protons from the central target region producing neutrons through the spallation process. The backstop also helps reduce the heat load in the moderator tank and shield in the downstream direction from the proton beam. The radial target zone is an integral part of the ${ }^{3} \mathrm{He}$ target system; protons and neutrons escaping the central target zone can strike the radial target and produce neutrons. The ${ }^{3} \mathrm{He}$ inside the target chamber is used to neutronically decouple the target elements in the central target region from each other and from 
the radial-target and backstop zones. The first ${ }^{3} \mathrm{He}$ layer in the blanket region has two functions: to neutronically decouple the radial target from the moderator and to produce tritium. The moderator slows down low-energy spallation neutrons so they can produce tritium more efficiently. The ${ }^{3} \mathrm{He}$ region between the moderator and reflector is there to produce tritium. The reflector enhances tritium production and minimizes neutron-leakage losses. The outer ${ }^{3} \mathrm{He}$ regions of the blanket region improve tritium-production efficiency. The importance of these ${ }^{3} \mathrm{He}$ zones needs further evaluation as more constraints, such as ${ }^{3} \mathrm{He}$ and $\mathrm{D}_{2} \mathrm{O}$ inventory, are imposed on the design.

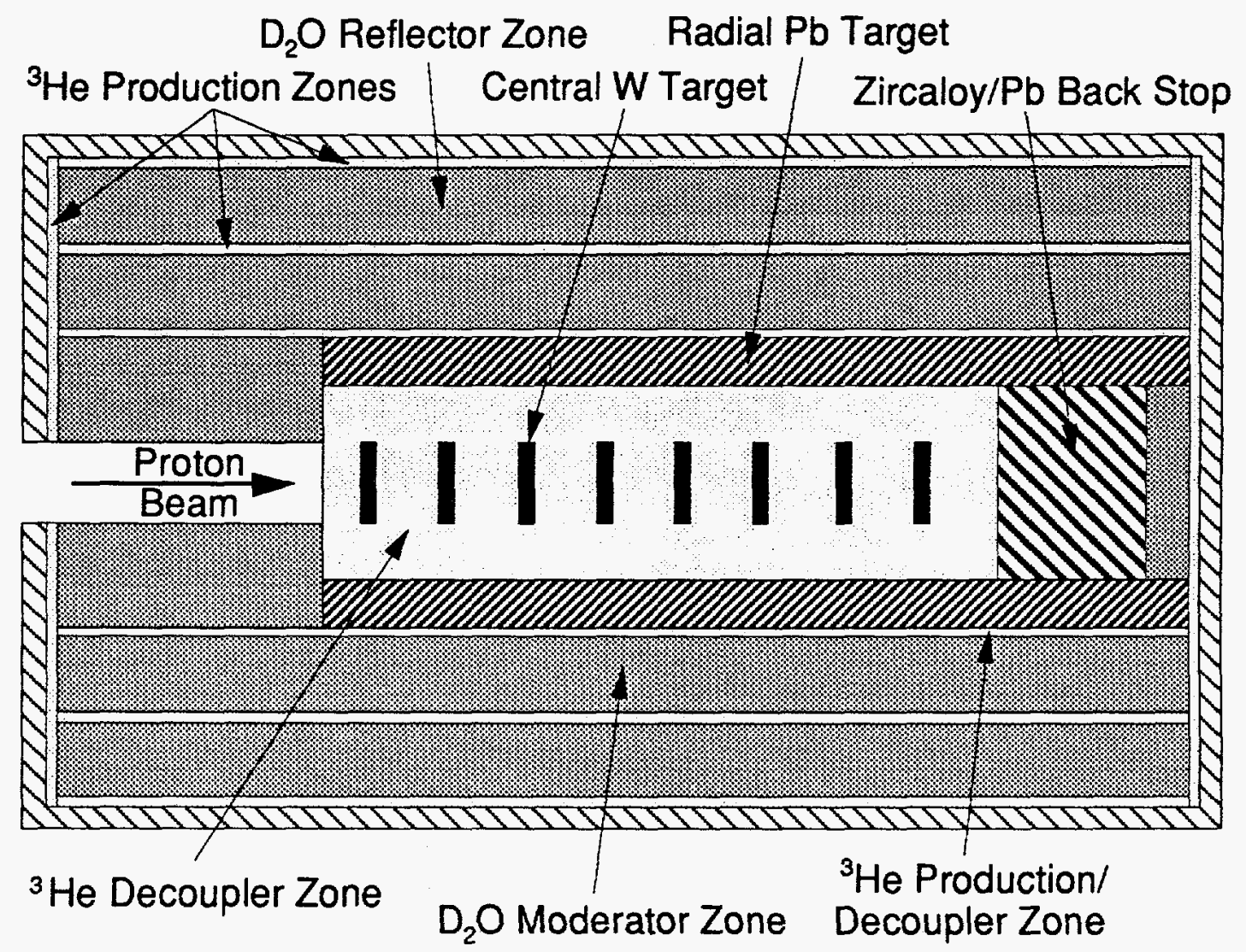

Fig. 3-17. Schematic of the APT target/blanket system.

Tungsten is used in the central target zone followed by a Zircaloy-4/lead backstop zone (Fig. 3-17). The central target and backstop zones are surrounded with a radial target region of lead, making the target both axially and radially composite. In addition, using the splitcomposite target design increases leakage and decreases parasitic absorption enough that tungsten can be used in the central target region. Because tungsten is a high-temperature material it can handle high proton beam powers; the target diameter may be adjusted to achieve the desired power densities.

For the ${ }^{3} \mathrm{He}$ T/B design, the $1-\mathrm{GeV}, 42 \mathrm{~cm} \times 91 \mathrm{~cm}(16.5 \mathrm{in} \times 35.8 \mathrm{in}),. 200-\mathrm{MW}$ proton beam strikes the central tungsten target region. Heavy water is prevalent throughout the target regions: in the rod bundles $\left(33\right.$ to $\left.54 \mathrm{v} \% \mathrm{D}_{2} \mathrm{O}\right)$; in the radial lead target $\left(10 \mathrm{v} \% \mathrm{D}_{2} \mathrm{O}\right)$; and in 
the backstop plates ( 3 to $15 \mathrm{v} \% \mathrm{D}_{2} \mathrm{O}$ ). The central tungsten target zones account for $45 \%$ of the total low-energy neutron production; the Zircaloy-4/Pb backstop for about $6 \%$; and the radial lead target region for $43 \%$. About $93 \%$ of the low-energy neutron production results from spallation reactions; the rest result from low-energy $(n, x n)$ reactions. As mentioned above, placing ${ }^{3} \mathrm{He}$ between the central tungsten regions neutronically decouples the tungsten target zones from each other and from the radial lead target zone, thus offering an additional benefit-capturing neutrons using ${ }^{3} \mathrm{He}$ in the central-target zone to form tritium which reduces parasitic neutron absorption in the tungsten and helps minimize the radioactive waste stream. The radial lead target zone is neutronically decoupled from the $\mathrm{D}_{2} \mathrm{O}$ moderator by a ${ }^{3} \mathrm{He}$ region.

To avoid thermal and resonance neutron absorption in the $\mathrm{W} / \mathrm{D}_{2} \mathrm{O}$ rod bundles, it is necessary to minimize the amount of $\mathrm{D}_{2} \mathrm{O}$ in the central target region and increase the probability of absorption in ${ }^{3} \mathrm{He}$. These goals are achievable by increasing the flux-trap gaps (Sec. 3.6.2.2), by increasing the ${ }^{3} \mathrm{He}$ pressure (Sec. 3.6.2.3), by reducing the thickness of the rod bundle unit, and by reducing the rod-bundle $\mathrm{D}_{2} \mathrm{O}$ coolant fraction.

The APT design produces large numbers of low-energy neutrons with minimal frontsurface leakage and allows for use of large target sizes and a high-neutron-absorption material (tungsten) in the central target zone. The excellent neutronic decoupling property of ${ }^{3} \mathrm{He}$ assures good neutron efficiency. The main components involved in neutron production are described below, together with a variety of studies done to enhance the neutronic performance of the ${ }^{3} \mathrm{He} / \mathrm{B}$ system.

\subsubsection{Central Target}

\subsubsection{Rod Bundle Pattern Study}

The three major components of a split-composite target system are (1) a central target region composed of rod bundles, which the proton beam strikes; (2) a backstop zone to catch scattered protons, to range out the primary protons, and to reduce energy deposition in the downstream shield; and (3) a radial-target region to enhance neutron production. A study was performed to investigate the sensitivity of tritium production to the placement of rod bundles in the central target region. The motivation for the study involved an engineering consideration that favored a rod-bundle layout that offered adequate view factors for radiant heat transfer in the extremely unlikely event of a coolant dryout. The spacing of the rod bundles determine such view factors. The object of the study was to predict what benefit or penalty would result from changing the pattern of the rod bundles.

The Physics-IV model was used in these computations. In the base case, shown in Fig. 3-18, the central target zone consisted of eight $W / \mathrm{D}_{2} \mathrm{O}$ plates; in the rest of the cases the central target was made of 104 rod bundles (the current design employs 88 rod bundles). In the actual rod-bundle design, a single bundle consists of 91 individual, wire-wrapped rods in a hexagonal can (Fig. 3-19). The detail shown in Fig. 3-19 was not mocked up in the study. Instead circular rod-bundle units with a circular target canister were used with a central circular zone that was an homogenization of the 91 rods, wire wrap, and $\mathrm{D}_{2} \mathrm{O}$ coolant of an actual hexagonal rod-bundle unit. The areal densities of materials in each region in the direction of the proton beam were preserved. 


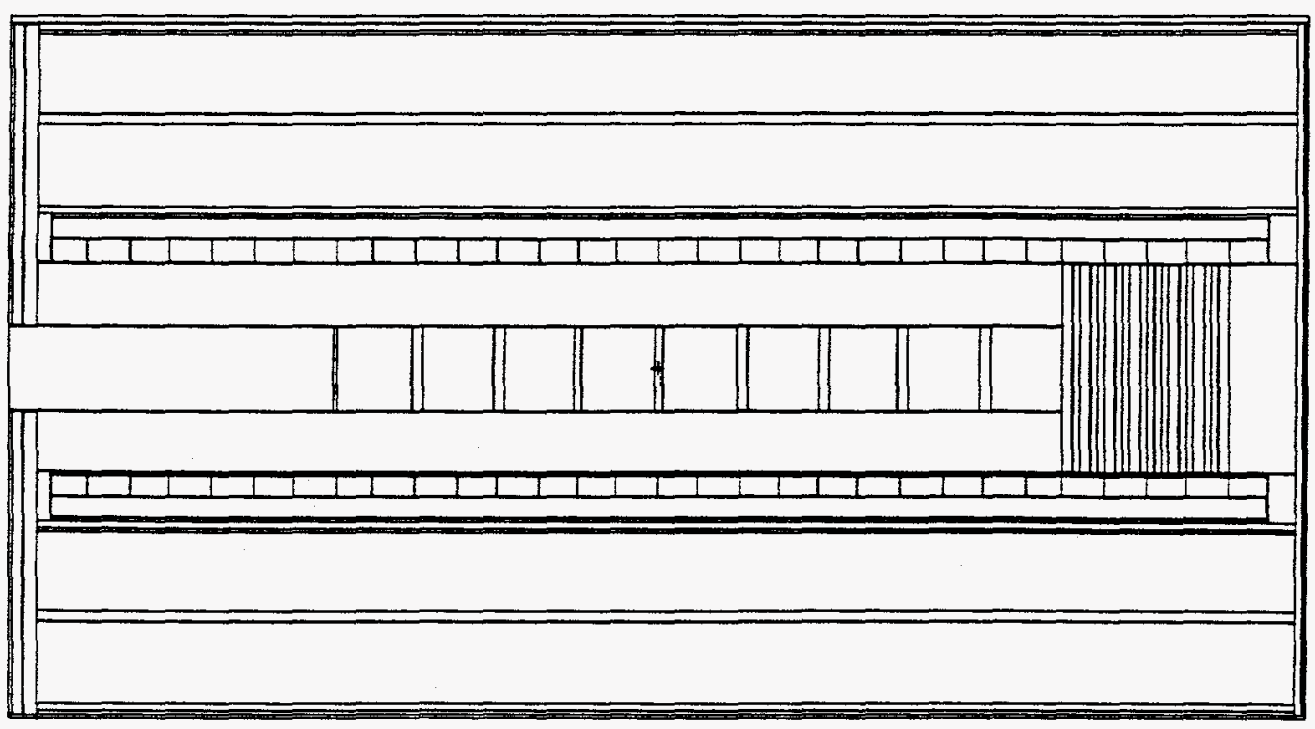

Fig. 3-18. Cross sectional view of the APT Physics-IV model used in the rod bundle pattern study. The radial lead target extends $7.5 \mathrm{~m}$ in the direction of the proton beam axis. The Zircaloy backstop is inside of the target chamber. The base case is shown here, with eight plates in the central target zone.

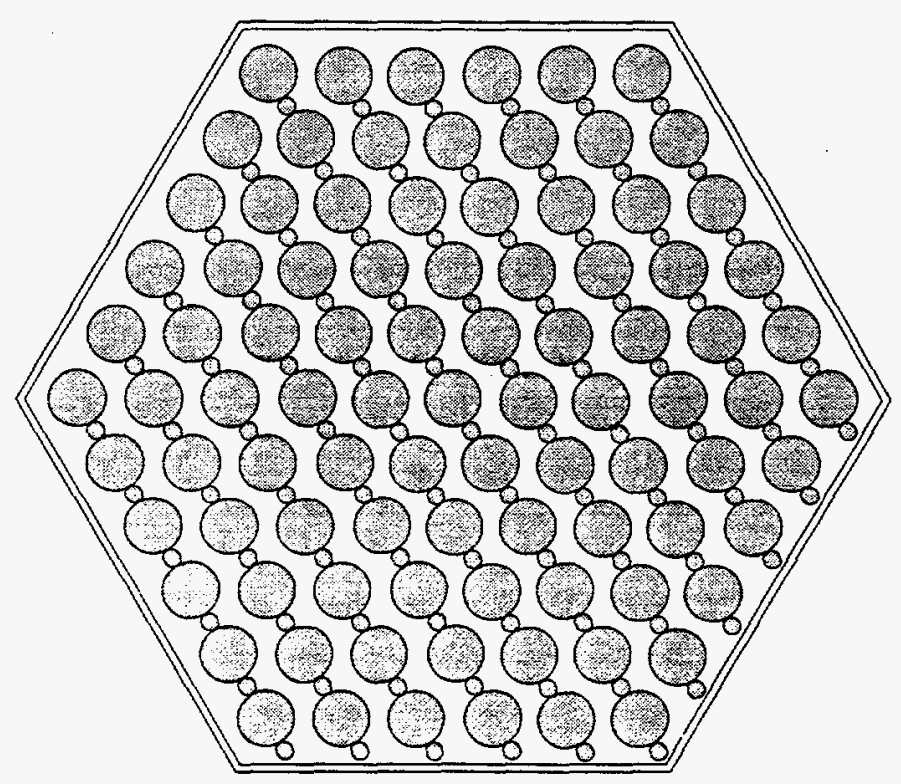

Fig. 3-19. Cross-sectional view of a 91-rod-bundle unit. 
The details of the parametric studies are given in Refs. 3-24 through 3-28. Flux traps are the spaces between rows of rod bundles. Several cases were studied:

- Base Case. Thirteen rod bundle units (i.e., two rows of bundles) are collapsed into a plate with the proper thicknesses and homogenized atom densities; eight such plates make up the central target zone (Fig. 3-20a), with constant flux-trap gaps;

- Case 1. A rod-bundle arrangement simulates the base case consisting of a row of seven rod bundles, followed by a row of six rod bundles where the pairs of rows are tangent, with constant flux-trap gaps (Fig. 3-20b);

- Case 2. A rod-bundle configuration where each pair of seven-bundle and sixbundle rows have the individual rod bundles form an equilateral triangle, with constant flux-trap gaps (Fig. 3-20c);

- Case 3. Each row of rod bundles (whether it comprises seven or six rod-bundle units) is equidistant from each other, with constant flux-trap gaps (Fig. 3-20d);

- Case 4. A barber-pole, rod-bundle layout forming equally separated diagonal rows-of-thirteen rod-bundle units making a $30^{\circ}$ angle with the proton beam axis, with constant flux-trap gaps (Fig. 3-20e);

- Case 5. A modified barber pole rod-bundle layout forming equally separated diagonal rows-of-thirteen rod-bundle units that have a $5-\mathrm{cm}$ larger separation (compared to Case 4) for the top half of the rod-bundle rows and a 5-cm smaller separation (compared to case 4) for the bottom half of the rod-bundle rows, with variable flux-trap gaps (Fig. 3-20f). 


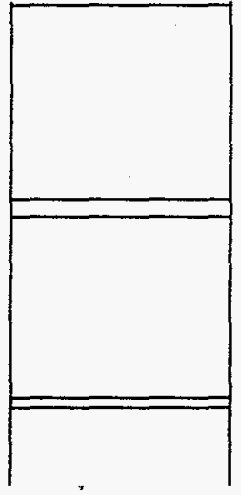

(a)

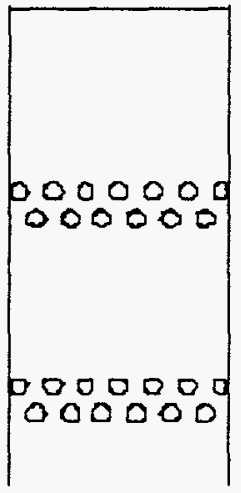

(c)

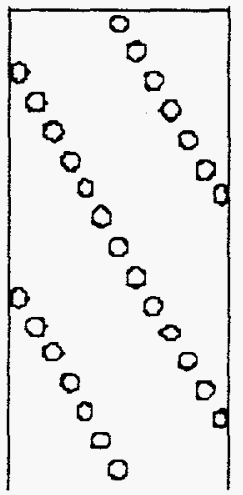

(e)

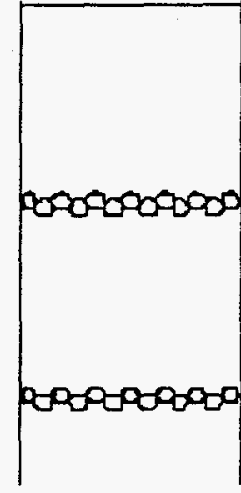

(b)

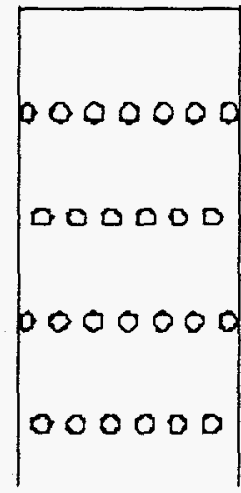

(d)

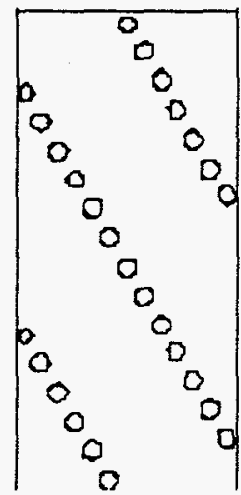

(f)

Fig. 3-20. The $60-\mathrm{cm}$ region just downstream of the proton beam window of the central target zone for the six cases studied. (a) The 13-rod-equivalent plate base case. (b) Case 1: close-packed rod bundles. (c) Case 2: equilaterally spaced rod bundles. (d) Case 3: constant flux-trap gaps. (e) Case 4: rod bundles arranged in a "barber pole" pattern. (f) Case 5: the modified "barber pole" pattern, where the first four flux-trap gaps were 10 cm larger than the last four flux-trap gaps. 
Figure 3-21 shows the placement of the rod-bundle patterns for Cases 1, 3, and 4 in the Physics-IV model of the $3 \mathrm{He}$ T/B system.

(a)

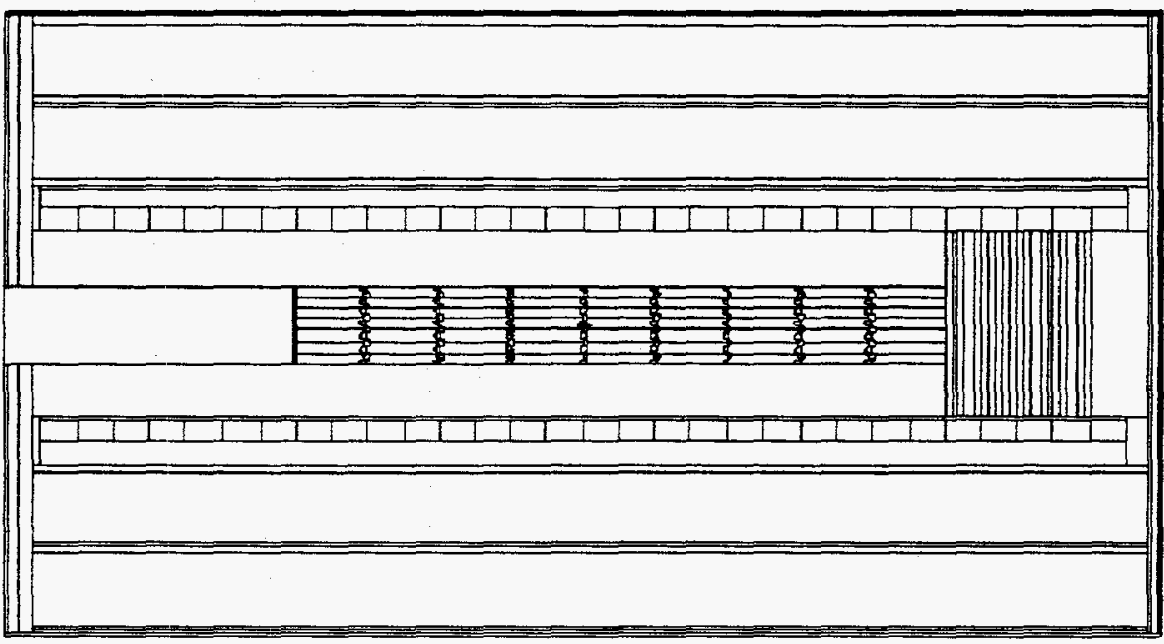

(b)

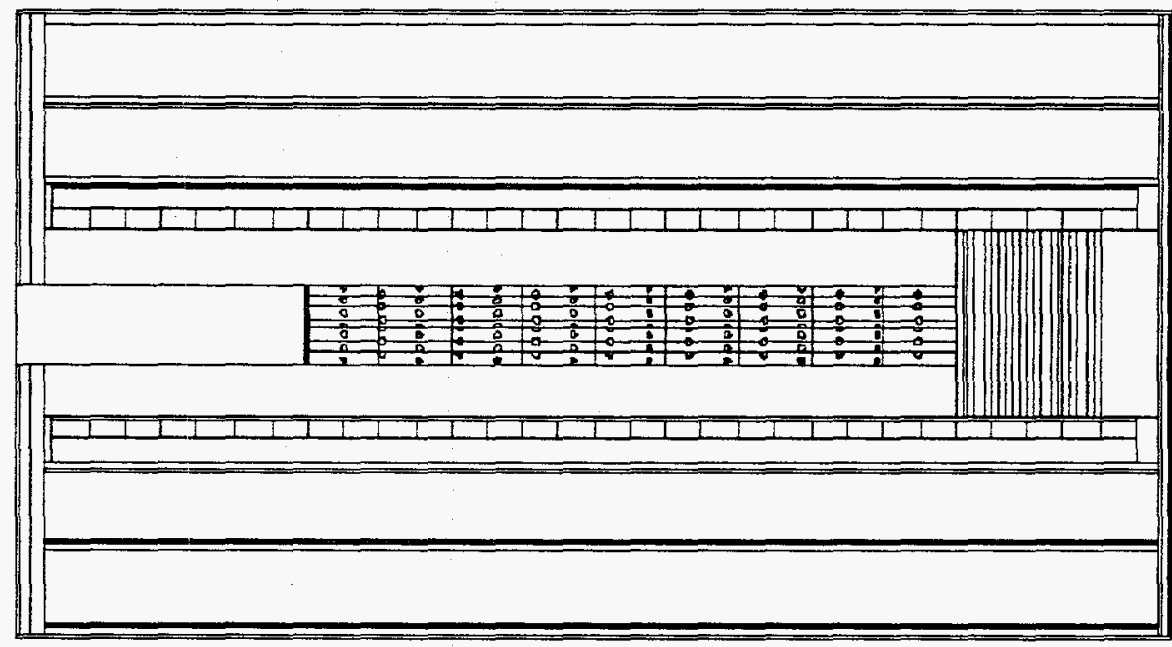

(c)

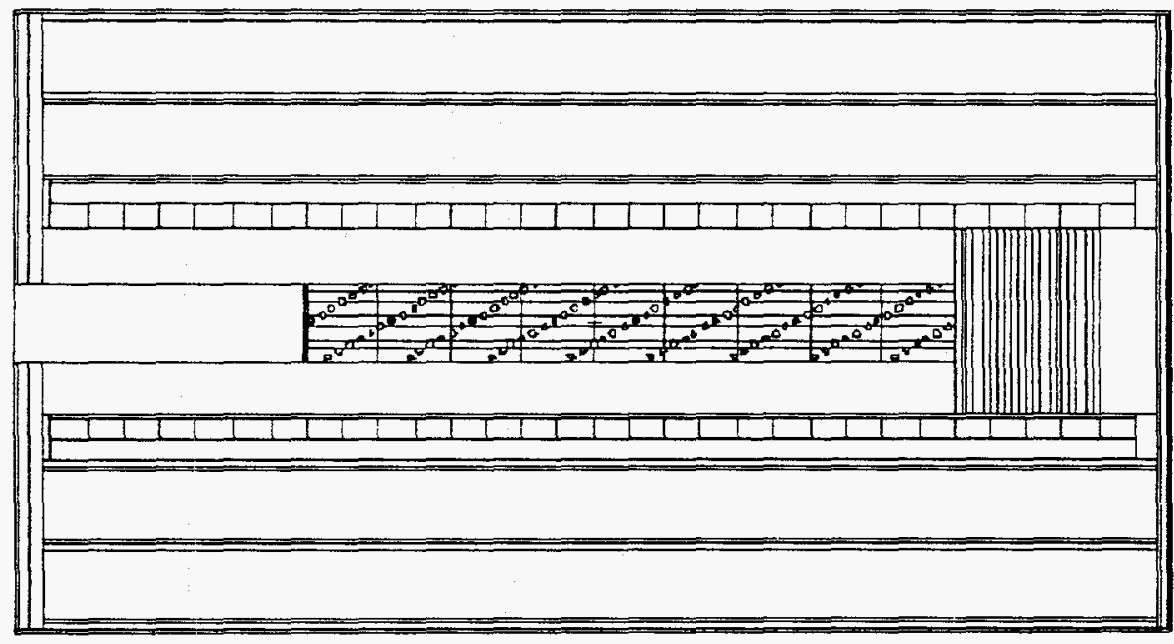

Fig. 3-21. Cross-sectional view of the APT T/B showing the central target zone layout for (a) case 1, (b) case 3 and (c) case 4 . 
The results of the calculations are shown in Table 3-7. Within the statistical errors, Cases 1,2 , and 4 have the same tritium production as the base case, showing that from a neutronics viewpoint, homogenized plate mockups of rod-bundle units give the same result as discrete mockups of individual rod-bundle units. The result for Case 3 shows a $-0.9 \%$ increase in tritium production, which is almost four standard deviations. The result for Case 5 is $\sim 0.4 \%$ below the base case in tritium production. The important conclusion is that, within $\sim 1 \%$, tritium production is independent of the rod-bundle pattern. The choice of the pattern for the rod bundles need not be dictated by neutronic performance considerations but instead, may be driven by mechanical design and thermal-hydraulics considerations.

\begin{tabular}{clcc}
\hline \hline Table 3-7. & Results of the Rod Bundle Pattern Study & \\
\hline Case & Target Pattern & $\begin{array}{c}\text { Relative Tritium } \\
\text { Production }\end{array}$ & Relative Error \\
\hline base & 13-rod-equivalent plates & 1.0000 & 0.0025 \\
1 & Close-packed rods & 1.0021 & 0.0025 \\
2 & Equilaterally spaced rods & 1.0021 & 0.0025 \\
3 & Constant flux-trap gaps & 1.0087 & 0.0024 \\
4 & "Barber pole" & 0.9980 & 0.0025 \\
5 & Modified "barber pole" & 0.9959 & 0.0025 \\
\hline
\end{tabular}

\subsubsection{Flux-Trap Gap Size Investigations}

An early study with LCS considered variants of the flux-trap thickness and radial target thickness and composition. Those calculations were done with the Physics-III APT model characterized by a rectangular central target zone of tungsten followed by lead with a lateral lead target. Variations of the lateral target dimensions and composition and the flux-trap gap were studied. In particular, the following parameter ranges were investigated:

- flux-trap thicknesses uniformly varied from 20 to $60 \mathrm{~cm}$;

- lateral target thickness varied from 15 to $30 \mathrm{~cm}$; and

- lateral target volume compositions limited to $3 / 4 \mathrm{~Pb}, 1 / 4 \mathrm{D}_{2} \mathrm{O} ; 3 / 8 \mathrm{~Pb}, 3 / 8 \mathrm{Be}, 1 / 4$ $\mathrm{D}_{2} \mathrm{O}$; and $3 / 4 \mathrm{Be}, 1 / 4 \mathrm{D}_{2} \mathrm{O}$.

The results of this study, tabulated in terms of relative tritium production, are given in Table 3-8.

These results indicate the following:

- no benefit with the use of $\mathrm{Be}$ in the lateral target;

- a lateral $\mathrm{Pb} / \mathrm{D}_{2} \mathrm{O}$ target thickness near $20 \mathrm{~cm}$ (7.87 in.) is best; and

- an increase in tritium production with increasing flux-trap gap size. 


\begin{tabular}{|c|c|c|c|c|c|c|}
\hline \multirow{2}{*}{$\begin{array}{l}\text { Flux Trap Dimension } \\
\text { (cm) }\end{array}$} & \multicolumn{6}{|c|}{ Lateral Target Dimension $(\mathrm{cm})$} \\
\hline & 15 & 17.5 & 20 & 22.5 & 25 & 30 \\
\hline 20 & & & & & & \\
\hline $303 / 4 \mathrm{~Pb}, 1 / 4 \mathrm{D}_{2} \mathrm{O}$ & 1.000 & & 1.015 & & 1.014 & 0.994 \\
\hline $\begin{array}{l}3 / 4 \mathrm{~Pb}, 1 / 4 \mathrm{D}_{2} \mathrm{O} \\
3 / 8 \mathrm{~Pb}, 3 / 8 \mathrm{Be}, 1 / 4 \mathrm{D}_{2} \mathrm{O} \\
40\end{array}$ & 1.030 & 1.046 & $\begin{array}{l}1.058 \\
0.0982\end{array}$ & 1.044 & 1.041 & 1.036 \\
\hline $\begin{array}{l}3 / 4 \mathrm{~Pb}, 1 / 4 \mathrm{D}_{2} \mathrm{O} \\
3 / 8 \mathrm{~Pb}, 3 / 8 \mathrm{Be}, 1 / 4 \mathrm{D}_{2} \mathrm{O} \\
3 / 4 \mathrm{Be}, 1 / 4 \mathrm{D}_{2} \mathrm{O}\end{array}$ & 1.049 & & $\begin{array}{l}1.065 \\
0.998 \\
0.849\end{array}$ & & $\begin{array}{l}1.061 \\
0.980\end{array}$ & 1.043 \\
\hline $\begin{array}{l}50 \\
3 / 4 \mathrm{~Pb}, 1 / 4 \mathrm{D}_{2} \mathrm{O} \\
3 / 8 \mathrm{~Pb}, 3 / 8 \mathrm{Be}, 1 / 4 \mathrm{D}_{2} \mathrm{O} \\
3 / 4 \mathrm{Be}, 1 / 4 \mathrm{D}_{2} \mathrm{O}\end{array}$ & 1.038 & & $\begin{array}{l}1.078 \\
0.993 \\
0.838\end{array}$ & & $\begin{array}{l}1.076 \\
0.984\end{array}$ & 1.053 \\
\hline $3 / 4 \mathrm{~Pb}, 1 / 4 \mathrm{D}_{2} \mathrm{O}$ & 0.987 & 1.046 & & 1.081 & & \\
\hline
\end{tabular}

A limited number of studies were done investigating changes in the composition and dimensions of the backstop and modifications to the flux traps. The results of these studies, tabulated in terms of relative tritium production, are given in Table 3-9. The reference calculation against which the other cases listed in Table 3-9 are compared has a backstop length of $37 \mathrm{~cm}$ and $20-\mathrm{cm}$ flux-trap gaps.

\begin{tabular}{lc}
\hline Table 3-9. & $\begin{array}{l}\text { Relative Tritium Production of 3He APT Lower-Target and Flux-Trap } \\
\text { Systems }\end{array}$ \\
\hline \multicolumn{1}{c}{ Description } & Relative 3He Production \\
\hline Lead Backstop & 1.000 \\
Reference Calculation & 0.995 \\
Backstop Shortened by $15 \mathrm{~cm}$ & 0.997 \\
Flux Traps Decreasing from 12.5 to $27.5 \mathrm{~cm}$ with & \\
$\quad$ Distance from Target Window & 0.936 \\
Inconel-718 Backstop & 0.938 \\
Replaced Lead Backstop with Inconel & 0.907 \\
Backstop Shortened by 20 cm & \\
Lower Two 6-cm Flux Traps Removed & 0.961 \\
Zircaloy-4 Backstop & \\
Replaced Lead Backstop with Zircaloy-4
\end{tabular}

\subsubsection{3He Target Chamber Pressure Studies}

As described in Section 3.6.1, ${ }^{3} \mathrm{He}$ in the target chamber serves as a neutronic decoupler. The ${ }^{3} \mathrm{He}$ readily absorbs the thermal neutrons reflected back into the target chamber from the $\mathrm{D}_{2} \mathrm{O}$ reflector instead of allowing them to be parasitically captured by the tungsten and 
structural material in the target. A more difficult problem than thermal absorption, however, is avoiding resonance absorption of epithermal neutrons by tungsten, Inconel, and lead. Evaporation neutrons born in the rod bundles downscatter into the resonance region of tungsten (approximately $5 \mathrm{eV}$ to $50 \mathrm{keV}$ ) after an average of about five collisions with deuterium. Heavy water is prevalent throughout the target region-in the rod bundles (33 to $\left.54 \mathrm{v} \% \mathrm{D}_{2} \mathrm{O}\right)$, in the radial lead target $\left(10 \mathrm{v} \% \mathrm{D}_{2} \mathrm{O}\right)$, and in the backstop plates ( 3 to $15 \mathrm{v} \%$ $\mathrm{D}_{2} \mathrm{O}$ ). To avoid resonance absorption (and thermal neutron absorption from moderation inside the rod bundles themselves), it is important to minimize the amount of $\mathrm{D}_{2} \mathrm{O}$ in the target region and increase the probability of absorption in ${ }^{3} \mathrm{He}$, which is achievable by increasing the fluxtrap gaps (Sec. 3.6.2.2) or by increasing the ${ }^{3} \mathrm{He}$ pressure.

The Physics-IV APT model was used to investigate the effect of ${ }^{3} \mathrm{He}$ target chamber pressure on tritium production and the results were found to be significant. The tritium production increased $4.2 \%$ when the pressure was doubled from 100 to 200 psia (Fig. 3-22). Also shown in Fig. 3-22 is the shift of production from the blanket to the target chamber with increasing target chamber pressure. The fraction of tritium produced in the target chamber increased from $48 \%$ at 225 psia to $58 \%$ at 900 psia.

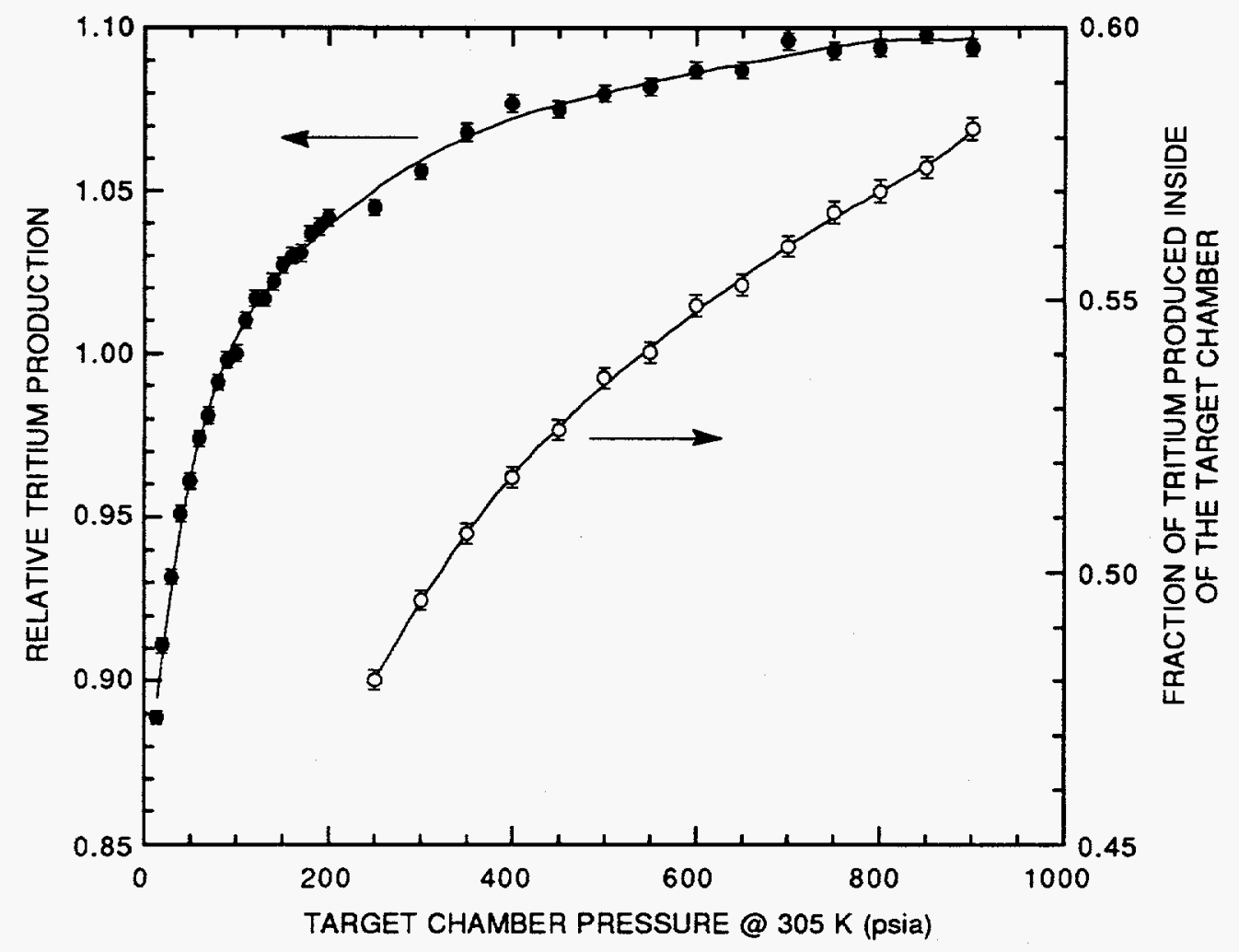

Fig. 3-22. Tritium production (relative to production at 100 psia) and fraction of tritium produced within the target chamber as a function of ${ }^{3} \mathrm{He}$ target chamber pressure.

As the target chamber pressure was increased, parasitic neutron capture in the tungsten and lead targets decreased (Fig. 3-23). Parasitic capture in tungsten is a factor of two lower at a 
pressure of 400 psia as compared to 100 psia and a factor of three lower at 800 psia as compared to $100 \mathrm{psia}$. The decrease in absorption by the radial lead target was not as dramatic because its absorption cross section is much smaller than that of tungsten and because the ${ }^{3} \mathrm{He}$ in the target chamber geometrically decouples the tungsten to a greater degree than it does the lead. About two-thirds of the increased absorption in $3 \mathrm{He}$ in going from 100 to $400 \mathrm{psia}$ is directly attributable to decreased capture in tungsten; reduced parasitic capture in the Inconel target chamber and hex cans and in the radial lead target account for the majority of the remainder. Over $90 \%$ of the increase in tritium production resulting from increasing the target chamber pressure from 400 to 800 psia comes from reduced parasitic capture in tungsten.

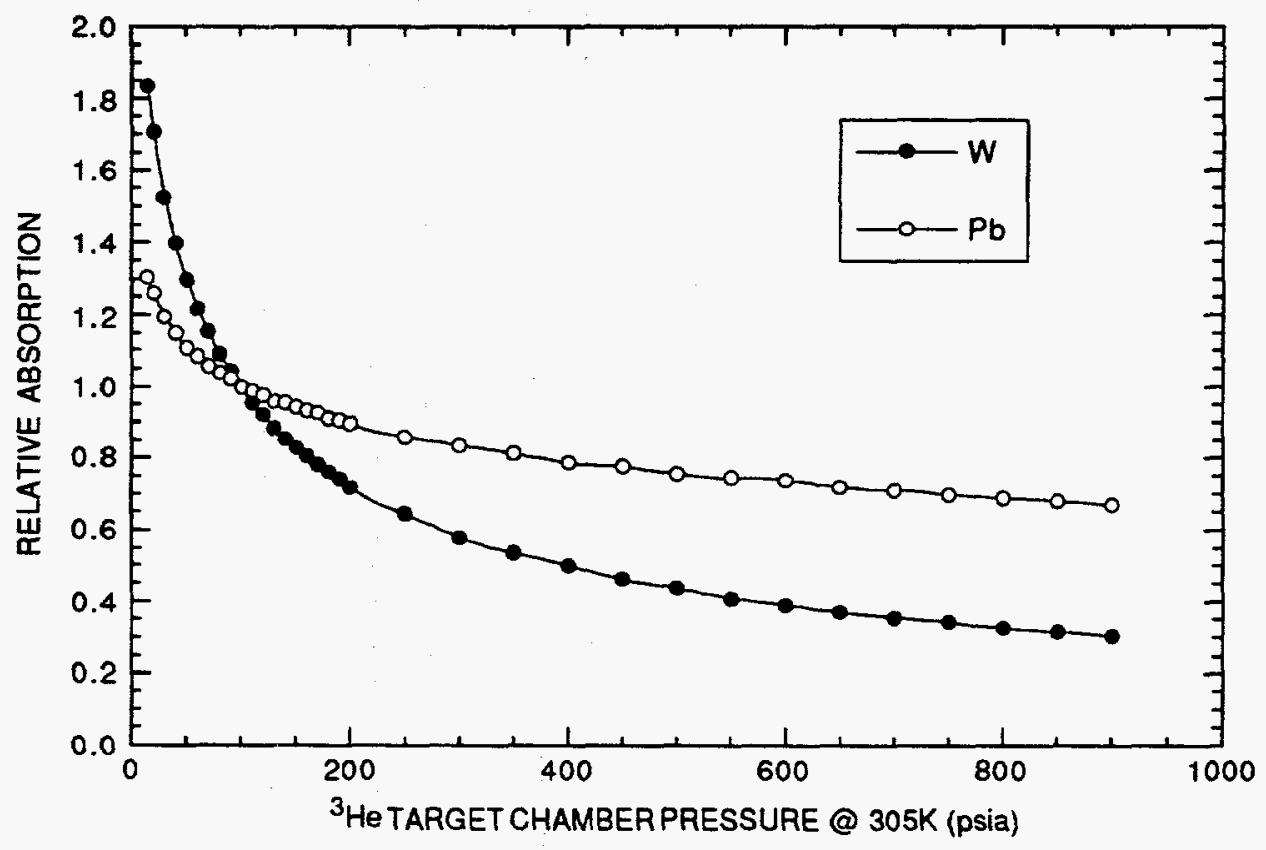

Fig. 3-23. Absorption in the tungsten target material and the radial lead target relative to absorption at 100 psia as a function of ${ }^{3} \mathrm{He}$ target chamber pressure.

The increased neutronic efficiency resulting from higher ${ }^{3} \mathrm{He}$ target chamber pressure has an additional benefit beyond increasing tritium production. By capturing in ${ }^{3} \mathrm{He}$ instead of tungsten, Inconel, and lead, target activation is reduced. This effect may prolong the life of target components and lead to a reduced waste stream.

It should be noted that additional structural material (in the form of thicker target chamber walls and/or more stiffening rings, thicker hex can walls, and thicker proton beam window) may be required for an appreciable increase in target chamber pressure. This dependence has not been taken into account in the present study, so that at high ${ }^{3} \mathrm{He}$ mass densities $\left(>2.5 \times 10^{-3}\right.$ $\mathrm{g} / \mathrm{cm}^{3}$ ), the gain in tritium production could be less than indicated here; however, additional structural material is not expected to change these results significantly. 
As designed, the target chamber (with a wall thickness of $0.3175 \mathrm{~cm}$ ) can withstand a maximum internal pressure of 300 psia [3-29]. Thus, 300 psia was selected because a net increase in tritium production of 5\% was observed without any change in chamber wall or window thickness. The average ${ }^{3} \mathrm{He}$ temperature within the target chamber is anticipated to be $400 \mathrm{~K}$. At 300 psia, this temperature leads to a ${ }^{3} \mathrm{He}$ mass density of $1.8744 \times 10^{-3} \mathrm{~g} / \mathrm{cm}^{3}$. This is the mass density used within the target chamber in the reference case.

\subsubsection{Backstop}

The backstop serves several functions: it stops the proton beam after the beam has slowed to an energy where it is no longer useful for the production of neutrons in tungsten; in stopping the proton beam (and other high-energy particles), it produces neutrons; finally, it reduces the deposited power density in the downstream moderator tank wall and subsequent shielding material. An optimized backstop design is a balance of these functions and other figures of merit, such as neutron efficiency and practicality of design.

A variety of materials were evaluated as backstop candidates: lead, tungsten, Inconel, and Zircaloy. In comparing these materials, a standard backstop design was used. This backstop was composed of 105 -cm-thick regions of the particular backstop material, each followed by a 5 -cm-thick ${ }^{3} \mathrm{He}$ zone, for a total backstop length of $1 \mathrm{~m}$. These studies were performed using the Physics-IV model. The results are listed in Table 3-10. If neutron production were the sole design criterion, pure lead would make the best backstop. Unfortunately, the average power density at the upstream face of the backstop is sufficiently high $(\sim 95 \mathrm{~kW} / \ell$ for pure lead) to preclude the use of lead at this position. While tungsten is the best neutron producer, it is also the greatest neutron absorber of the materials tested. Inconel is a fair neutron producer but a strong absorber. Zircaloy, a high-temperature material, is also a fair neutron producer and has a low capture cross section. Next to lead, Zircaloy is the best backstop material candidate of those analyzed.

\begin{tabular}{|c|c|c|c|c|c|}
\hline $\begin{array}{l}\text { Backstop } \\
\text { Region } \\
\text { Material }\end{array}$ & $\begin{array}{l}\text { Total } \\
\text { Neutron } \\
\text { Source* }\end{array}$ & $\begin{array}{c}\text { Neutron } \\
\text { Production in } \\
\text { Backstop** }\end{array}$ & $\begin{array}{c}\text { Neutron } \\
\text { Absorption in } \\
\text { Backstop** }\end{array}$ & $\begin{array}{l}\text { Net Gain of } \\
\text { Neutrons due } \\
\text { to Backstop** }\end{array}$ & $\underset{\text { Production }}{\text { Tritium }}$ \\
\hline $\begin{array}{c}\text { Inconel/ } / \mathrm{D}_{2} \mathrm{O} \\
(75 \vee \% / 25 \vee \%)\end{array}$ & 0.9760 & $4.14 \%$ & $3.00 \%$ & $1.14 \%$ & 0.9541 \\
\hline $\begin{array}{c}\text { Zircaloy/ } \mathrm{D}_{2} \mathrm{O} \\
(75 \vee \% / 25 \vee \%)\end{array}$ & 1.0000 & $5.48 \%$ & $0.99 \%$ & $4.49 \%$ & 1.0000 \\
\hline $\begin{array}{l}\text { Tungsten/ } / \mathrm{D}_{2} \mathrm{O} \\
(53 \mathrm{v} \% / 47 \mathrm{v} \%)\end{array}$ & 1.0536 & $11.46 \%$ & $10.52 \%$ & $0.94 \%$ & 0.9465 \\
\hline $\begin{array}{c}\mathrm{Lead} / \mathrm{D}_{2} \mathrm{O} \\
(59 \mathrm{v} \% / 41 \vee \%)\end{array}$ & 1.0406 & $9.56 \%$ & $0.14 \%$ & $9.42 \%$ & 1.0547 \\
\hline
\end{tabular}

*relative to that for a Zircaloy backstop

** relative to total neutron source

In an attempt to simplify the engineering design, the neutronic effect of reducing the number of backstop regions from 10 to 4 was investigated; the performance was found to be the same. In addition, the backstop length was optimized; results are shown in Fig. 3-24. For 
a backstop of $75 \mathrm{v} \%$ Zircaloy -4 and $25 \mathrm{v} \% \mathrm{D}_{2} \mathrm{O}$, an optimum occurs at a total length of about $60 \mathrm{~cm}$. Thus the reference design has four $15-\mathrm{cm}$-thick Zircaloy regions.

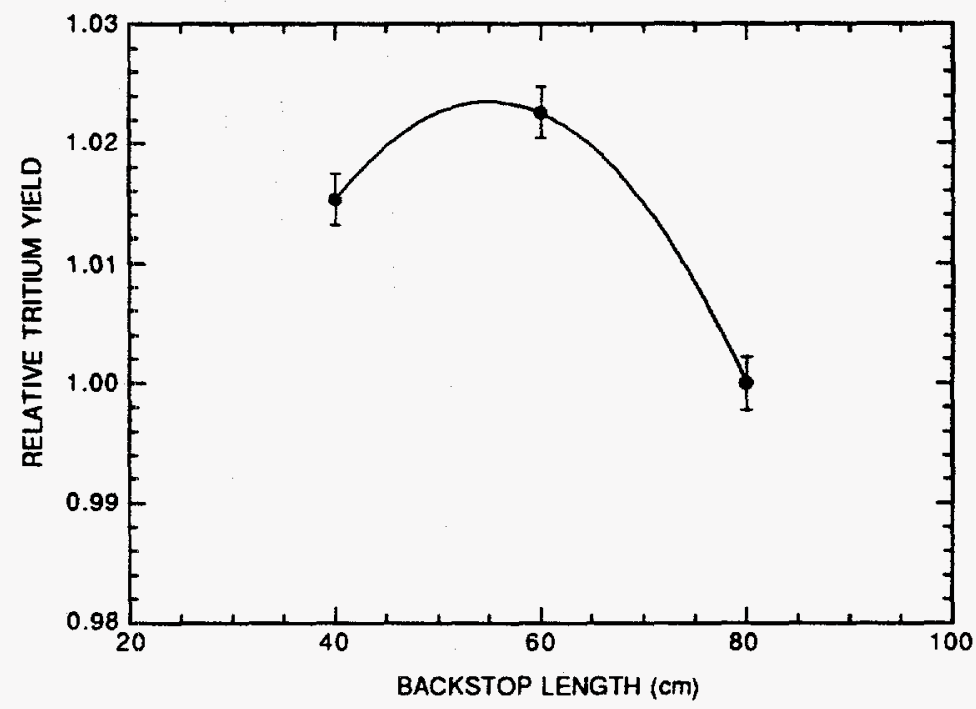

Fig. 3-24. Tritium production as a function of Zircaloy backstop length.

\subsubsection{Radial Target}

The radial target region is an important component of the ${ }^{3} \mathrm{He}$ target system because about $43 \%$ of the total low-energy neutron production comes from this target zone. Optimization of the neutronics of the radial target zone involves investigating the effects of the thickness of the zone, the axial length of the zone, and the zone's material composition. The main factors altered by changes in the radial target zone, which ultimately affect tritium production, are total spallation-neutron production and parasitic absorption in the radial target zone. The parametric calculations for the radial target zone optimization were done using the Physics-IV model with an Inconel-718 backstop.

\subsubsection{Optimization of Radial Target Length}

The calculations to optimize the length of the radial target zone were done in two stages. First, the length of the radial target zone near the entrance of the beam was altered; and second, the length of the radial target zone at the back end of the target was varied. Heavy water was the only material replaced or substituted for the radial target materials as a result of the geometry changes.

Changes in tritium production, neutron production, and lead absorption (absorption in the $\mathrm{Pb} / \mathrm{D}_{2} \mathrm{O}$ region) that resulted from changes in the length of the lead zone were computed. Although the lead absorption showed a much larger percentage change than the neutron production, it is much less important because the loss of neutrons to absorption in the lead region is only a small contribution to the overall neutron balance. The neutron production is 
roughly constant as long as the radial target zone extends upstream of the proton beam window, but the production begins to drop off if reduced beyond this point. Tritium production increases until the upstream end of the lead is even with the window because the reduction in the amount of lead in the system leads to a decrease in parasitic absorption. If the radial target zone starts downstream of the window, tritium production decreases due to a decrease in neutron production. Therefore, the optimal case occurs when the upstream end of the radial lead corresponds to the window location.

The length of the lead at the downstream end of the target near the backstop was varied. It was found that any reduction in the lead length at the back end in the target results in a reduction in tritium production. Conversely, increasing the length in the lead results in a production increase. Although the increase is questionable because its magnitude is roughly equal to only a single standard deviation in the Monte Carlo error, this effect is believed to be real; thus the optimal dimension selected was an increase in the length of lead at the downstream end of the backstop region of $16 \mathrm{~cm}(6.30 \mathrm{in}$.) from the base case.

A final case was computed using the optimal dimensions from the two studies described above. This scenario corresponds to decreasing the length of the radial target zone near the beam entrance by $175 \mathrm{~cm}$ and increasing the radial target zone length at the end of the target by $16 \mathrm{~cm}$. This case resulted in a $1.239 \pm 0.26 \%$ increase in tritium production, a $0.075 \pm 0.22 \%$ increase in neutron production, and a $16.564 \pm 0.32 \%$ decrease in the lead region absorption with respect to the base case. The overall volume of the lead region was reduced by $21.2 \%$.

\subsubsection{Effects of Material Composition of the Radial Target}

The radial target lead region is an important part of the APT T/B system because of its role in neutron production, as well as its ability to transmit neutrons generated in the tungsten rod bundles to the blanket ${ }^{3} \mathrm{He}$ regions. Small variations in the material composition of this region can generate large effects in overall system performance. However, the fact that a large amount of heat is deposited in the radial lead region, coupled with the mechanical properties of lead, compels target designers to incorporate a specific coolant fraction, as well as a certain amount of support structure. In an effort to quantify the effects of various proposed options, a number of material variations in this region were tested.

The effect of coolant fraction was one of these variations. The base case consisted of a $30-\mathrm{cm}$-thick lead annulus with a coolant volume fraction of $10.2 \%$. To determine the effects of coolant volume fraction on tritium production, the lead thickness was held constant, and the volume fraction of heavy water was varied from 15 to $40 \%$ (Table 3-11).

As seen in Table 3-11, any increase in coolant volume fraction in the lead zone causes a degradation in system performance due to both a decrease in spallation neutron production and an increase in absorption in the lead zone. Although an average coolant fraction of only $10.2 \%$ is required in the lead zone, the peaking of the power density distribution near the inner surface forces a higher coolant fraction in this region, with the coolant fraction dropping well below $10.2 \%$ near the outer lead surface. To avoid the penalties associated with having a higher coolant fraction throughout the entire lead zone, a coolant fraction gradient was incorporated in the design of the radial lead zone [3-31] (see Section 4.4.4). 


\begin{tabular}{cccc}
\hline \hline Table 3-11. & Effects of Coolant Fraction in Radial Lead Zone on Tritium Production \\
\hline $\begin{array}{c}\text { Coolant Volume } \\
\text { Fraction (\%) }\end{array}$ & $\begin{array}{c}\text { Change in Neutron } \\
\text { Production (\%) }\end{array}$ & $\begin{array}{c}\text { Change in Lead Region } \\
\text { Absorption (\%) }\end{array}$ & $\begin{array}{c}\text { Change in Total Tritium } \\
\text { Production (\%) }\end{array}$ \\
\hline 10.2 & Base Case & Base Case & Base Case \\
15.0 & $-1.32 \pm 0.22$ & $+26.0 \pm 0.48$ & $-2.24 \pm 0.25$ \\
25.0 & $-3.35 \pm 0.21$ & $+76.7 \pm 0.62$ & $-6.26 \pm 0.24$ \\
30.0 & $-4.51 \pm 0.21$ & $+94.4 \pm 0.66$ & $-8.14 \pm 0.24$ \\
35.0 & $-5.31 \pm 0.21$ & $+108.4 \pm 0.71$ & $-9.54 \pm 0.24$ \\
40.0 & $-6.74 \pm 0.21$ & $+114.9 \pm 0.71$ & $-11.6 \pm 0.24$ \\
\hline \hline
\end{tabular}

To test the effect this design would have on system performance, the heavy water distribution as specified in Section 4.4.4 was explicitly incorporated in the APT physics model. This was done by dividing the radial lead zone into 10 subdivisions using the thickness and heavy water fractions given in Table 3-12. This resulted in a net change in tritium production of $+0.09 \% \pm 0.26 \%$ from the reference case, which is a statistically insignificant increase. Thus, the gradient coolant fraction design is equivalent, with regard to physics analysis, to assuming an average coolant fraction of $10.2 \%$ throughout the entire radial lead zone.

\begin{tabular}{ccc}
\hline \hline Table 3-12. & Heavy Water Distribution in Radial Target Lead Zone \\
\hline Section & Radial Thickness $(\mathbf{c m})$ & Volume Percent $\mathbf{D}_{\mathbf{2}} \mathbf{O}$ \\
\hline 1 & 1.15 & 20.92 \\
2 & 1.40 & 17.18 \\
3 & 1.70 & 14.15 \\
4 & 2.00 & 12.03 \\
5 & 2.50 & 9.62 \\
6 & 3.20 & 7.52 \\
7 & 4.50 & 5.35 \\
8 & 5.75 & 4.18 \\
9 & 6.25 & 3.85 \\
10 & 6.65 & 3.62 \\
\hline \hline
\end{tabular}

The effect of cladding and structural materials was another variation tested. The addition of cladding and structural support material in the radial lead target zone can also have large effects on APT system performance. To evaluate the cladding effects, two cases were examined. Both cases maintained the $10.2 \mathrm{v} \%$ of heavy water coolant, with the remainder of the material being $84.8 \mathrm{v} \%$ lead and $5 \mathrm{v} \%$ aluminum- 1100 for the first case, and $79.8 \mathrm{v} \%$ lead and $10 \mathrm{v} \%$ aluminum- 1100 for the second case. Also, as a worst-case scenario, the SILC lead cladding configuration [3-32], including coolant fraction, was substituted into the radial lead zone. It should be noted that because the lead in the ${ }^{3} \mathrm{He}$ system is exposed to much lower power densities, the lead surface area (and consequently amount of cladding material) and coolant fraction of the SILC design are much too high (Table 3-13). The results for all three calculations are given in Table 3-14. Table 3-14 shows that additional cladding material in the lead zone both reduces neutron production and increases parasitic absorption. This behavior is similar to the behavior observed when the coolant volume fraction is increased (Table 3-11). In fact, by comparing Table 3-11 and Table 3-14, one can see that adding aluminum-1100 to 
Table 3-13. Material Composition for the SILC Lead Cladding Design

\begin{tabular}{lc}
\hline \multicolumn{1}{c}{ Material } & Volume Fraction \\
\hline $\mathrm{Pb}$ & 0.53798 \\
$\mathrm{Al}-1100$ & 0.17645 \\
$\mathrm{D}_{2} \mathrm{O}\left(0.25 \mathrm{a} \% \mathrm{H}_{2} \mathrm{O}\right)$ & 0.28557 \\
\hline
\end{tabular}

the lead zone is roughly equivalent to adding heavy water, with each 5\% addition causing a reduction in tritium production of between 1 and $2 \%$. It should be noted that the current reference design assumes that the lead is not clad.

\begin{tabular}{lccc}
\hline \hline Table 3-14. & Effect of Aluminum-1100 Cladding on APT System Performance \\
\hline $\begin{array}{c}\text { Lead Zone } \\
\text { Composition }\end{array}$ & $\begin{array}{c}\text { Change in Neutron } \\
\text { Production (\%) }\end{array}$ & $\begin{array}{c}\text { Change in Lead Region } \\
\text { Absorption (\%) }\end{array}$ & $\begin{array}{c}\text { Change in Total Tritium } \\
\text { Production (\%) }\end{array}$ \\
\hline $\begin{array}{l}10.2 \% \mathrm{D}_{2} \mathrm{O}, 84.8 \% \mathrm{~Pb}, \\
5 \% \mathrm{Al}-1100\end{array}$ & $-0.99 \pm 0.22$ & $+3.84 \pm 0.23$ & $-1.40 \pm 0.26$ \\
$10.2 \% \mathrm{D}_{2} \mathrm{O}, 79.8 \% \mathrm{~Pb}$, & $-1.89 \pm 0.22$ & $+15.60 \pm 0.44$ & $-2.54 \pm 0.25$ \\
$10 \% \mathrm{Al}-1100$ & $-8.7 \pm 0.20$ & $+99.50 \pm 0.70$ & $-13.5 \pm 0.20$ \\
$28.6 \% \mathrm{D}_{2} \mathrm{O}, 53.8 \% \mathrm{~Pb}$, & & & \\
$17.6 \% \mathrm{Al}-1100$ &
\end{tabular}

The effects of adding structural support material was determined by adding 3\% structure to the lead zone and testing different compositions of lead, aluminum-1100, and heavy water. Two materials were examined as possible structural materials: Zircaloy-4 and Inconel-718 (Table 3-15). The results show that while Zircaloy-4 and Inconel-718 both cause a similar degradation in neutron production (although Inconel-718 is worse), Inconel-718 is a much worse parasitic absorber. Hence, an increase in coolant fraction causes greater penalties if Inconel-718 is used than if Zircaloy-4 is present in the radial target lead region. To see if the added absorption for the worst Inconel-718 case could be alleviated by the addition of a decoupling zone, a zone of $1-\mathrm{cm}$ aluminum- $1100,5-\mathrm{cm}^{3} \mathrm{He}$, and $1-\mathrm{cm}$ aluminum-1100 was inserted in the middle of the lead target region. The addition of this decoupling zone resulted in a degradation in neutron production of $-5.67 \%$ from the reference case, which is statistically

\begin{tabular}{|c|c|c|c|c|}
\hline $\begin{array}{c}\text { Composition } \\
\text { (Pb, Al, } \mathrm{D}_{2} \mathrm{O} \text {, } \\
\text { Structure) }\end{array}$ & Structure & $\begin{array}{c}\text { Change in } \\
\text { Neutron } \\
\text { Production }(\%)\end{array}$ & $\begin{array}{l}\text { Change in Lead } \\
\text { Region } \\
\text { Absorption (\%) }\end{array}$ & $\begin{array}{c}\text { Change in Tritium } \\
\text { Production (\%) }\end{array}$ \\
\hline $\begin{array}{l}67 \%, 10 \%, 20 \%, 3 \% \\
77 \%, 5 \%, 15 \%, 3 \% \\
82 \%, 5 \%, 10 \%, 3 \% \\
67 \%, 10 \%, 20 \%, 3 \% \\
77 \%, 5 \%, 15 \%, 3 \% \\
82 \%, 5 \%, 10 \%, 3 \%\end{array}$ & $\begin{array}{c}\text { Zircaloy-4 } \\
\text { Zircaloy-4 } \\
\text { Zircaloy-4 } \\
\text { Inconel-718 } \\
\text { Inconel-718 } \\
\text { Inconel-718 }\end{array}$ & $\begin{array}{l}-5.08 \pm 0.21 \\
-2.87 \pm 0.21 \\
-1.47 \pm 0.22 \\
-5.81 \pm 0.21 \\
-3.28 \pm 0.21 \\
-1.49 \pm 0.22\end{array}$ & $\begin{array}{r}+81.88 \pm 0.62 \\
+55.35 \pm 0.53 \\
+32.40 \pm 0.45 \\
+214.30 \pm 0.01 \\
+175.15 \pm 0.88 \\
+128.10 \pm 0.73\end{array}$ & $\begin{array}{r}-8.73 \pm 0.24 \\
-5.34 \pm 0.25 \\
-3.34 \pm 0.25 \\
-15.86 \pm 0.23 \\
-11.37 \pm 0.23 \\
-7.89 \pm 0.24\end{array}$ \\
\hline
\end{tabular}


the same as without the decoupler. However, the absorption in the lead region was only $60.61 \%$ greater than the reference case, which is a substantial improvement over the $214.30 \%$ increase observed without the decoupler. The results of this study show that the majority of additional parasitic absorption can be eliminated by the addition of a decoupler region. Because the degradation in neutron production cannot be reduced, Zircaloy- 4 is the preferred structural material for the radial target zone.

\subsubsection{Optimization of Radial Target Thickness}

The initial optimization of the thickness of the radial lead zone (Fig. 3-25) was completed using the Physics-III model. This model had a lead coolant fraction of $10.2 \mathrm{v} \%$ and no cladding or support structure. To understand the effects of the coolant fraction on the optimal lead zone thickness, a $30 \%$ coolant volume fraction was arbitrarily selected and the optimal thickness determined (Fig. 3-26). For these and all subsequent calculations, the Physics-IV model was used. By comparing Fig. 3-25 with Fig 3-26, the optimal thickness is shown to decrease as the coolant volume fraction is increased, indicating that the additional absorption generated in the lead zone is greater than the additional neutron production achieved by maintaining a thicker region.

The data shown in Figs. 3-25 and 3-26 are normalized to different reference values (with the data shown in Fig. 3-25 normalized to the previously described base case). Hence, no comparison should be made with regard to the magnitudes of the yields. A final optimization on the radial lead zone thickness was performed for a zone composition of $82 \%$ lead, $5 \%$ aluminum-1100, 10\% heavy water, and 3\% Zircaloy- 4 by volume (Fig. 3-27). As with an

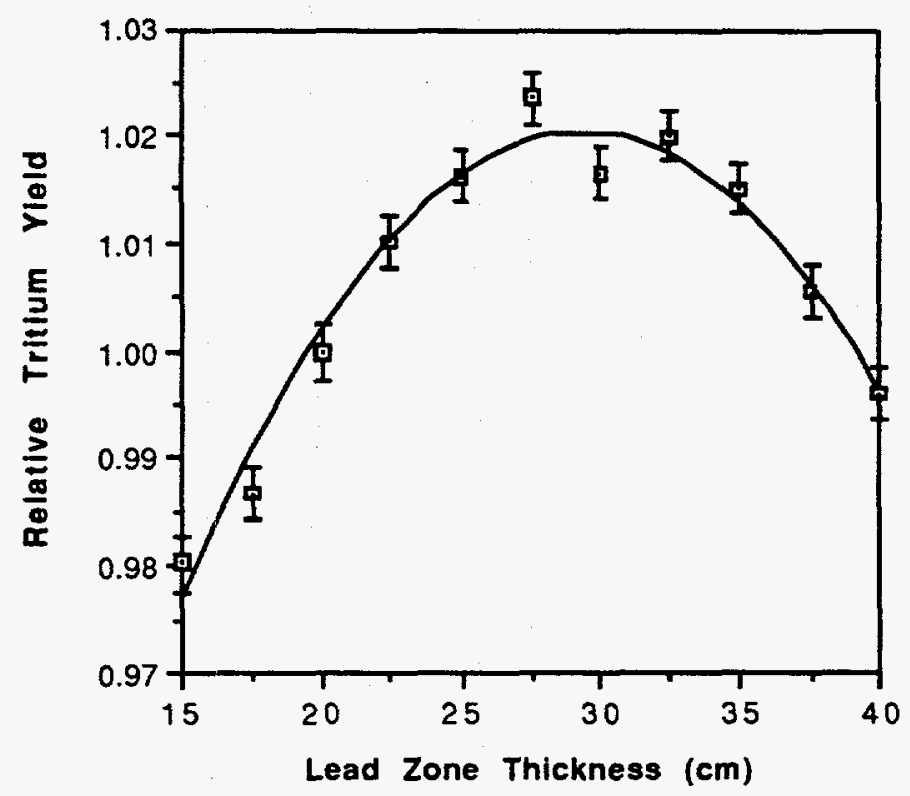

Fig. 3-25. Initial optimization of radial lead zone thickness with a 10.2 v\% coolant fraction. 


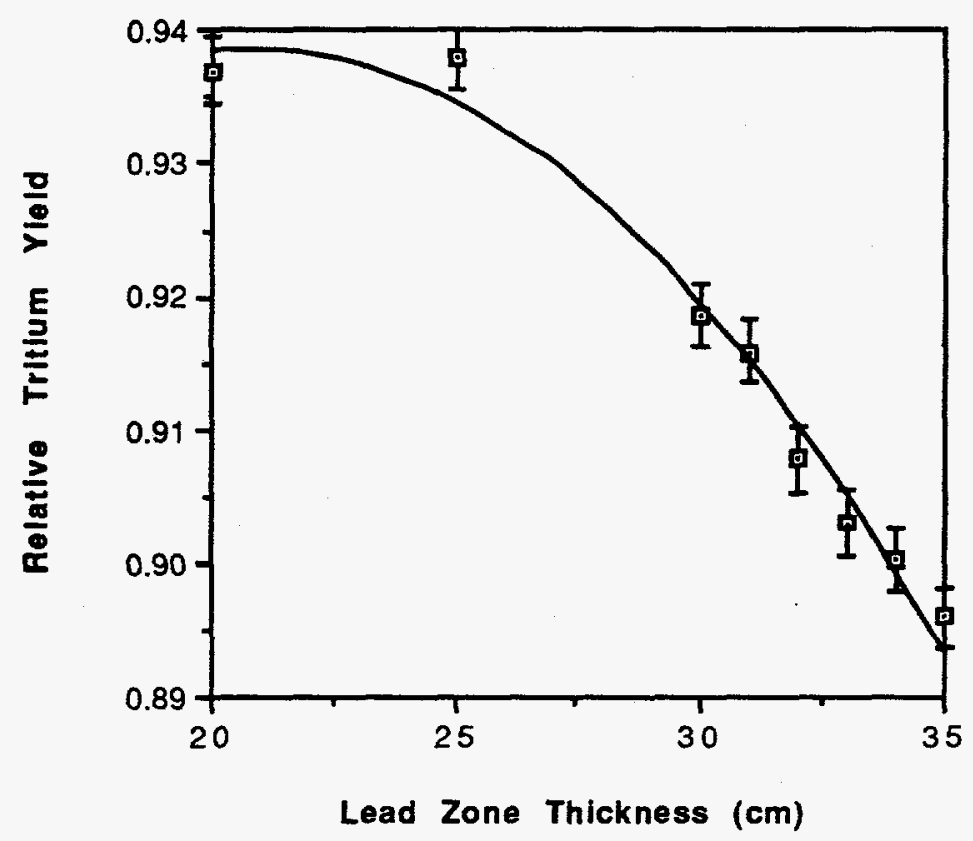

Fig. 3-26. Optimization of radial lead zone thickness with a $30 \%$ coolant fraction.

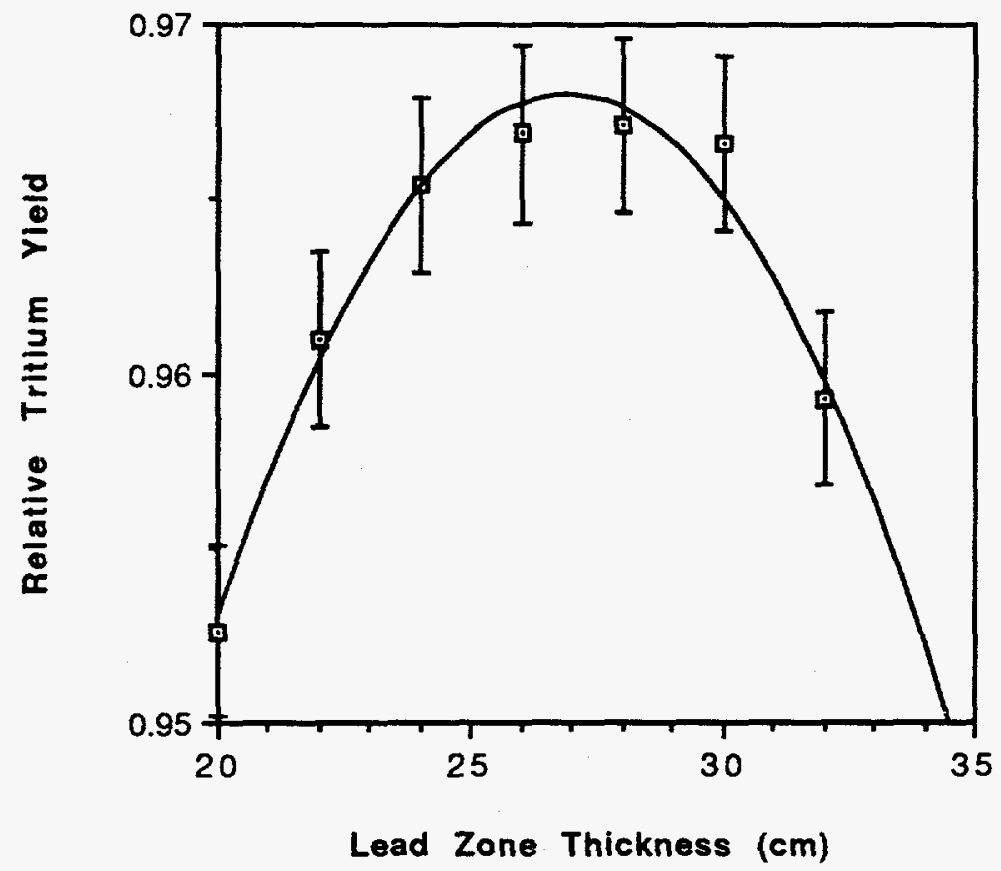

Fig. 3-27. Optimization of radial lead zone thickness with structure. 
increase in coolant, adding structure decreases the optimal zone thickness and results in a broad maximum in tritium yield, with the optimal thickness being about $27 \mathrm{~cm}$.

\subsubsection{Neutron Source Performance Optimization Studies}

\subsubsection{Aluminum vs. Inconel}

The Physics-V model was used to assess the penalty in substituting Al-2024 for Inconel718 for the rod-bundle canisters, target chamber, window, and rod-bundle cooling plena. No attempt was made to increase the thickness of aluminum over that of Inconel, which would be necessary because of differences in the structural properties of the two materials. A penalty in tritium production of about $5 \%$ is made for the use of Inconel-718. However, this allows us to use a high-temperature material (Inconel-718) in the central target region; furthermore, the use of Inconel rod-bundle canisters and target chamber simplifies the assembly of components.

\subsubsection{Target Chamber Wall Thickness}

Under normal operating conditions, the internal target chamber pressure exceeds the external pressure. If, during off-normal operation, internal pressure were lost, the external pressure could exceed the internal pressure by $20 \mathrm{psi}$. A structural analysis of the target chamber revealed that the initial value of $2.5 \mathrm{~mm}(0.0984 \mathrm{in}$.) for the target-chamber wall thickness was too thin to withstand an external pressure of 20 psi greater than the internal pressure. Thus, there was a desire to increase the wall thickness. A study was performed to determine the dependence of the target chamber wall thickness on tritium production; the results are shown in Fig. 3-28. At $2.5 \mathrm{~mm}(0.0984 \mathrm{in}$.), the slope of a curve through the data is about $-0.4 \% / \mathrm{mm}$; over the range studied ( 2.5 to $7 \mathrm{~mm}$, or 0.0984 to $0.276 \mathrm{in}$.) the average slope is about $-0.7 \% / \mathrm{mm}$, a rather large penalty. As a result of this study, the thickness of the target chamber wall was set at $0.3175 \mathrm{~cm}(0.125 \mathrm{in}$.), and stiffening rings were added to the target chamber wall. These rings provide the required structural integrity to withstand a total loss of internal target chamber pressure. By virtue of the much smaller volume of Inconel added, the neutronic impact of the stiffening rings is thought to be much less than that of increased wall thickness. To account for the neutronic penalty, stiffening rings have been included in the reference physics model.

\subsubsection{3 $\quad \mathrm{D}_{2} \mathrm{O}$ Gap}

A heavy-water gap is included between the Inconel target chamber and the radial lead target in all physics models. This gap serves two engineering functions: first, it provides clearance between the target chamber and radial lead target to allow for construction tolerances, and second, it is the coolant path for the target chamber wall. In the reference physics model, this gap is $5 \mathrm{~mm}$. A parametric study of the dependence of tritium production on this gap thickness was performed to assess whether the engineering tolerances and flow requirements might be relaxed. Figure 3-29 shows the results of this study-the magnitude of the vertical scale indicates the insensitivity of tritium production on gap thickness. The production is relatively constant over a gap of 2 to $7 \mathrm{~mm}(0.0787$ to $0.276 \mathrm{in}$.). Production then drops by 


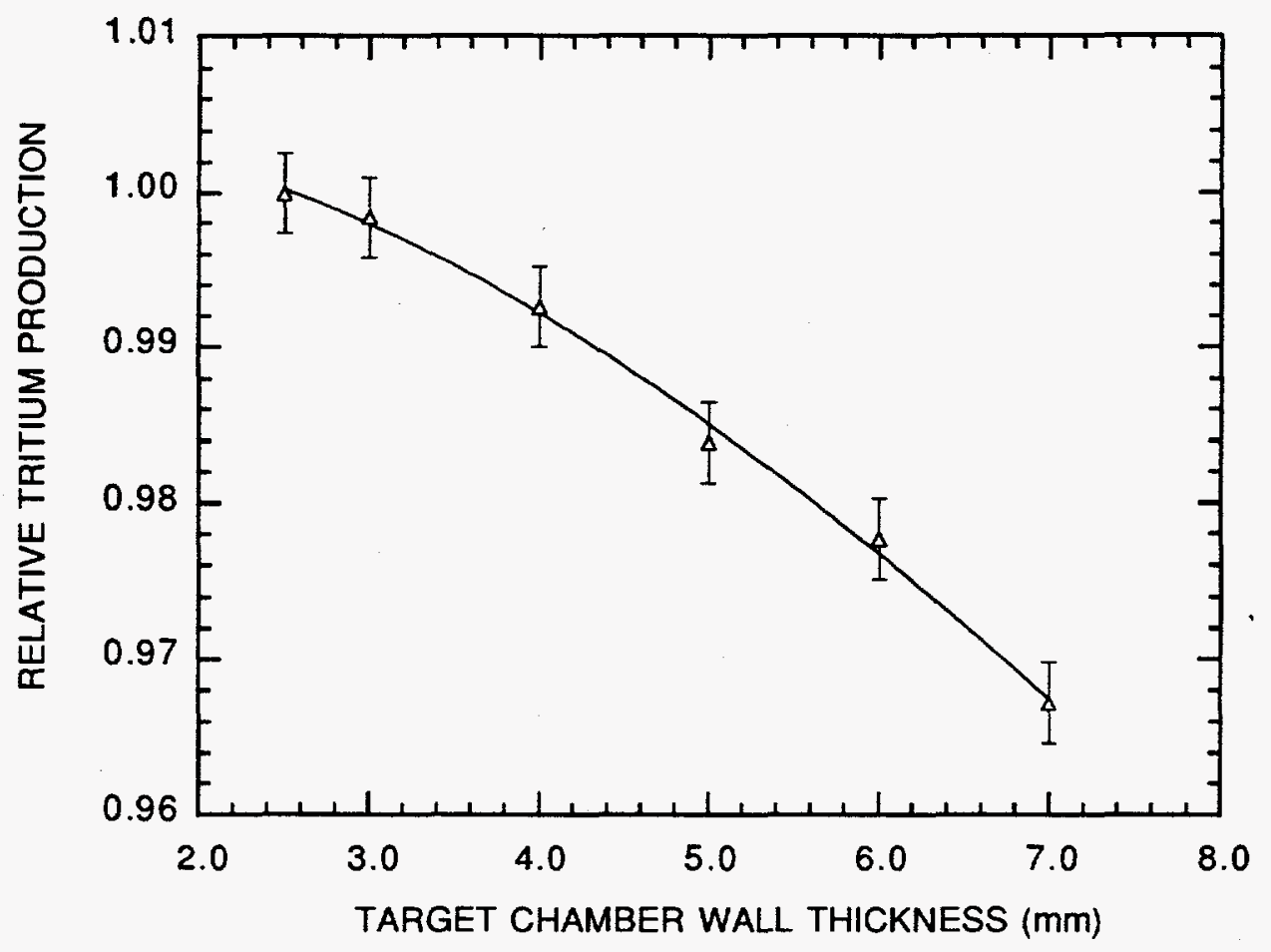

Fig. 3-28. Dependence of tritium production on target chamber wall thickness.

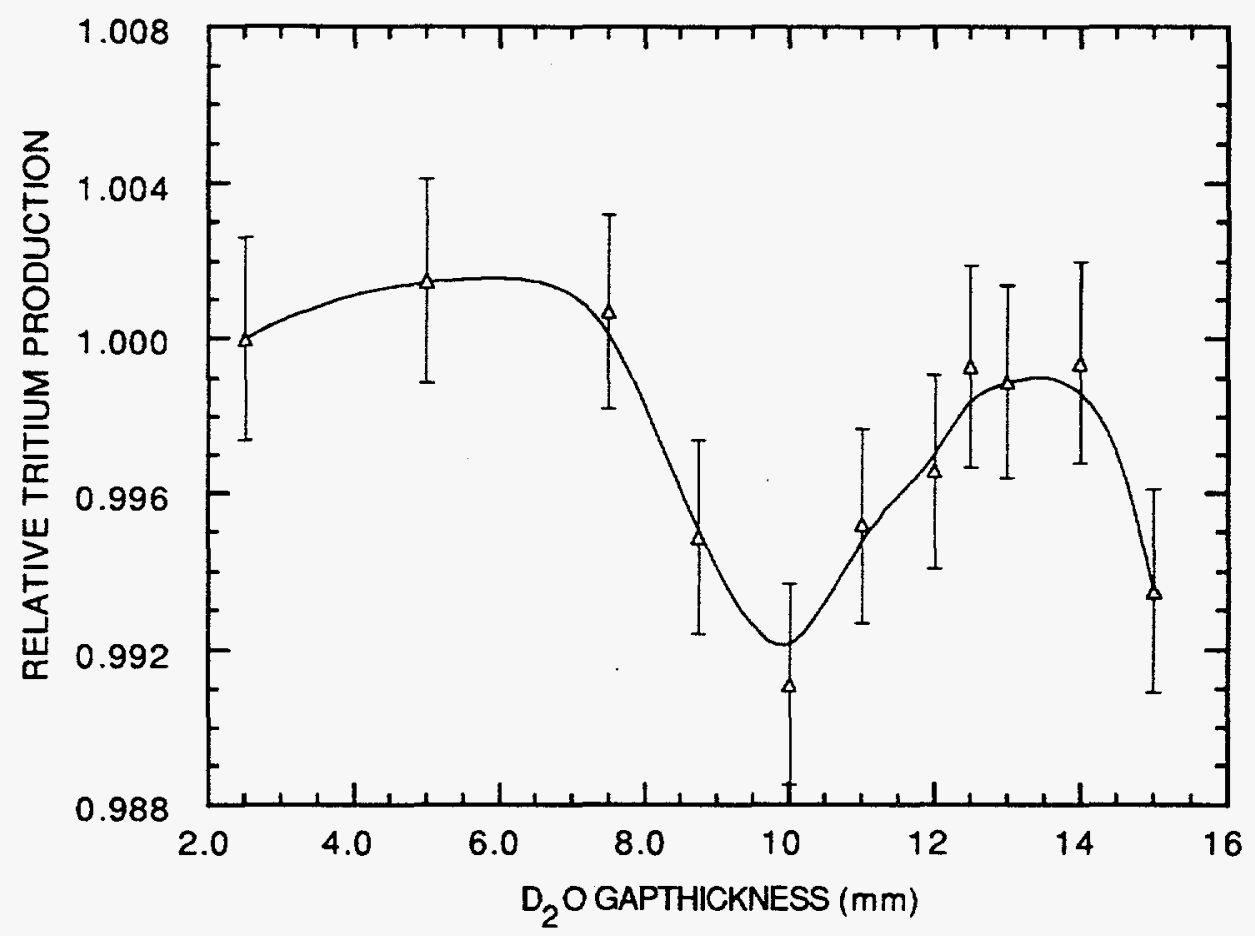

Fig. 3-29. Variation of tritium production with $\mathrm{D}_{2} \mathrm{O}$ gap thickness. 
about $1 \%$ at a gap of $10 \mathrm{~mm}(0.0394 \mathrm{in}$.) but returns to almost its initial value between 12 and $14 \mathrm{~mm}(0.472$ and $0.551 \mathrm{in}$.) of gap thickness. Beyond this thickness, production decreases once again. Given the complex dependence displayed in Fig. 3-29, it would be prudent to operate in a region with minimal sensitivity to the exact gap thickness. Thus, the $\mathrm{D}_{2} \mathrm{O}$ gap was fixed at $5 \mathrm{~mm}(0.197 \mathrm{in}$.).

\subsubsection{Proton Beam Tube}

The proton beam tube provides a vacuum path for the $42 \mathrm{~cm} \times 91 \mathrm{~cm}(16.5 \mathrm{in} \times 35.8 \mathrm{in}$.) proton beam just before the beam impinges on the $47 \mathrm{~cm} \times 96 \mathrm{~cm}(18.5 \mathrm{in} \times 37.8 \mathrm{in}$.) APT window. The base-case beam tube was rectangular in shape and fits $2.5 \mathrm{~cm}$ outside the window such that its inside dimensions are $52 \mathrm{~cm} \times 101 \mathrm{~cm}(20.5 \mathrm{in} \times 39.8 \mathrm{in}$.). Engineering considerations appear to require a cylindrical beam tube. Compared to the rectangular beam tube, our calculations show that the new cylindrical beam tube decreases total tritium production by only about $0.18 \%$.

In an effort to incorporate the new beam tube and to show what the tritium production penalties are and where they occur, tritium production was computed for all tritium producing cells in several beam tube models. The results are shown in Table 3-16 where tritium production is tabulated for all of the ${ }^{3} \mathrm{He}$ components of each beam tube model. The various beam tube models and comparison results are discussed below.

Table 3-16. Tritium Production as a Function Of Beam Tube Configuration (Normalized to Base Case Total Production $=100 \%$ )

\begin{tabular}{clcrrr}
\hline $\begin{array}{c}\text { LCS Cell } \\
\#\end{array}$ & \multicolumn{1}{c}{ Cell Description } & $\begin{array}{c}\text { BT1 } \\
\text { Base Case } \\
\text { Rectangle }\end{array}$ & $\begin{array}{c}\text { BT2 } \\
\text { Cylinder }\end{array}$ & $\begin{array}{c}\text { BT3 } \\
\text { Long } \\
\text { Cylinder }\end{array}$ & $\begin{array}{c}\text { BT4 } \\
\text { Short } \\
\text { Cylinder }\end{array}$ \\
\hline 3 & Beam Tube ${ }^{3} \mathrm{He}$ Wrap & 3.18 & 2.98 & 3.29 & 2.58 \\
952 & Hemispherical shell of ${ }^{3} \mathrm{He}$ & 0.04 & 0.06 & 0.04 & 0.11 \\
1500 & 1st cylindrical shell of ${ }^{3} \mathrm{He}$ & 30.76 & 31.41 & 31.38 & 31.11 \\
1900 & 2nd cylindrical shell of ${ }^{3} \mathrm{He}$ & 14.61 & 14.78 & 14.80 & 14.64 \\
2300 & 3rd cylindrical shell of ${ }^{3} \mathrm{He}$ & 1.13 & 1.10 & 1.13 & 1.10 \\
5 & Window ${ }^{3} \mathrm{He}$ & 0.39 & 0.38 & 0.39 & 0.38 \\
300 & 1st flux trap ${ }^{3} \mathrm{He}$ & 1.27 & 1.20 & 1.19 & 1.17 \\
301 & 2nd flux trap ${ }^{3} \mathrm{He}$ & 0.80 & 0.78 & 0.78 & 0.77 \\
302 & 3rd flux trap ${ }^{3} \mathrm{He}$ & 0.89 & 0.90 & 0.90 & 0.88 \\
$902+910$ & Target Chamber Header ${ }^{3} \mathrm{He}$ & 5.51 & 5.49 & 5.50 & 5.44 \\
$900+901$ & Target Chamber Side ${ }^{3} \mathrm{He}$ & 21.42 & 20.68 & 20.55 & 20.55 \\
& Balance of T/B & 20.01 & 20.05 & 19.99 & 19.93 \\
& Total Tritium Production & 100.00 & 99.82 & 99.94 & 98.67 \\
\hline \hline
\end{tabular}

BT1 - Base Case Rectangle - This is the base case beam tube model. It is a rectangular Inconel tube with inside dimensions of $52 \mathrm{~cm} \times 101 \mathrm{~cm}(20.5 \mathrm{in} \times 39.8 \mathrm{in}$.) and a wall thickness of $1.42875 \mathrm{~cm}(9 / 16 \mathrm{inch})$. The beam tube length is approximately $2 \mathrm{~m}(6.56 \mathrm{ft})$. The beam tube is wrapped with a 5.37-cm-thick (2.114 in.), rectangular, homogenized layer composed of $6.9 \mathrm{v} \% \mathrm{Al}-6061$ and $93.1 \mathrm{v} \%{ }^{3} \mathrm{He}$ at $100 \mathrm{psi}$ ("3 $\mathrm{He}-\mathrm{mix}$ "). A $0.5-\mathrm{cm}$-thick 
Inconel wall (window flange) was introduced to connect the perimeter on the window to the target tank. Figure 3-30 shows a horizontal cross-sectional view of the base case beam tube area. The tritium production results are shown in Table 3-16 where each ${ }^{3} \mathrm{He}$ component's percentage contribution to the total tritium production is tabulated. The results for all other models are also presented as percentages of the total tritium production of this base case.

BT2 - Cylinder - This case is like BT1 except the beam tube is a cylinder of inside radius $56 \mathrm{~cm}$ (22.0 in.) and wall thickness $1.42875 \mathrm{~cm}(9 / 16 \mathrm{in}$.). The inside radius was chosen to provide $2.5 \mathrm{~cm}$ ( $1 \mathrm{in}$.) clearance beyond the corners of the window to allow room to attach the beam tube to the target tank. This cylindrical beam tube was wrapped with a cylindrical layer of the same ${ }^{3} \mathrm{He}$-mix described in BT1. The window flange is also included. Figure 3-31 shows a horizontal cross-sectional view of the BT2 beam tube area. Compared to the base case (BT1), this beam tube reduces tritium production by $0.18 \% \pm 0.32 \%$, a statistically insignificant amount.

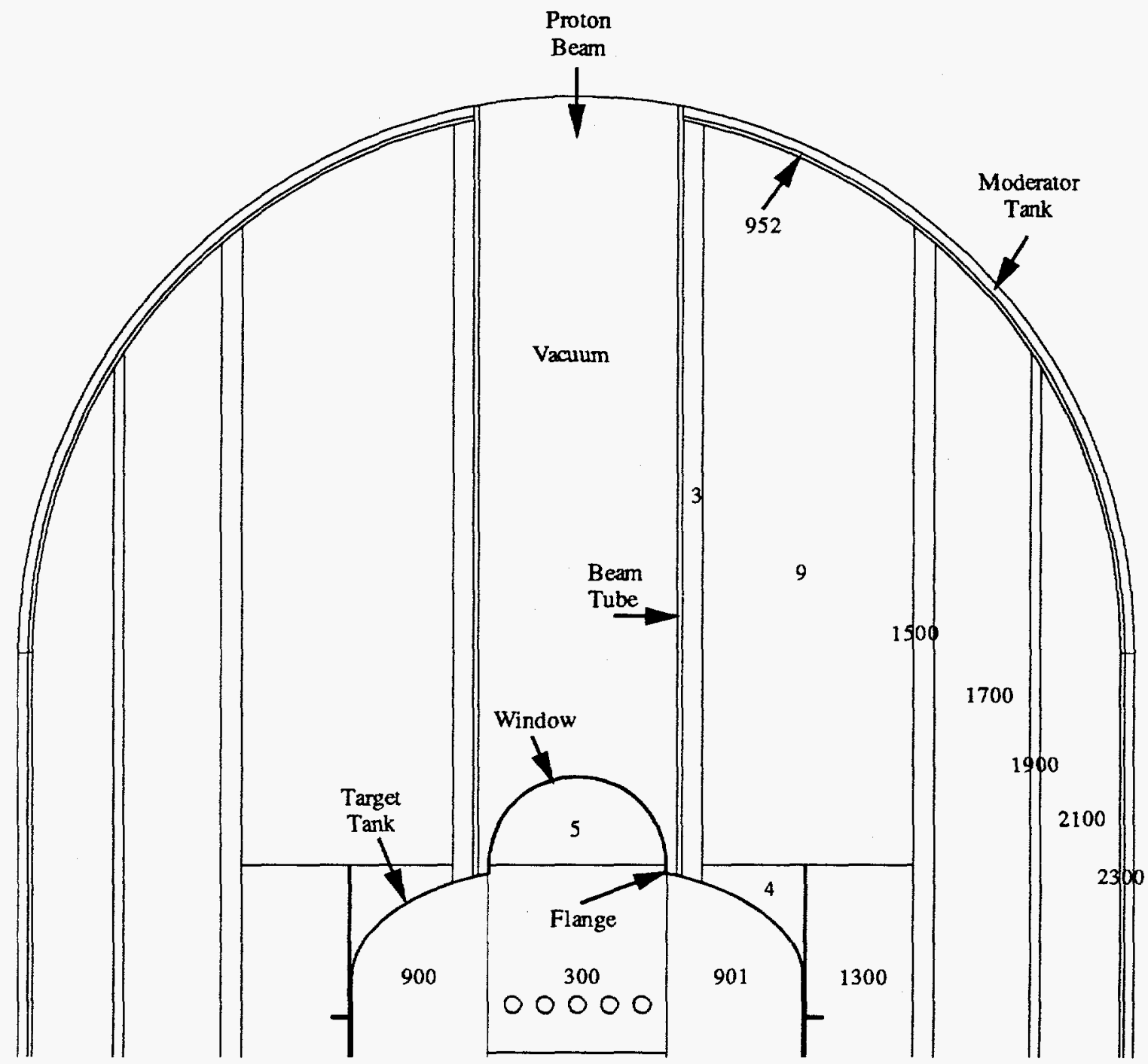

Fig. 3-30. Cross-sectional view of the beam tube area of the base case model BT1 which features a rectangular beam tube around the perimeter of the window.

The numbers correspond to LCS cell numbers identified in Table 3-16. 


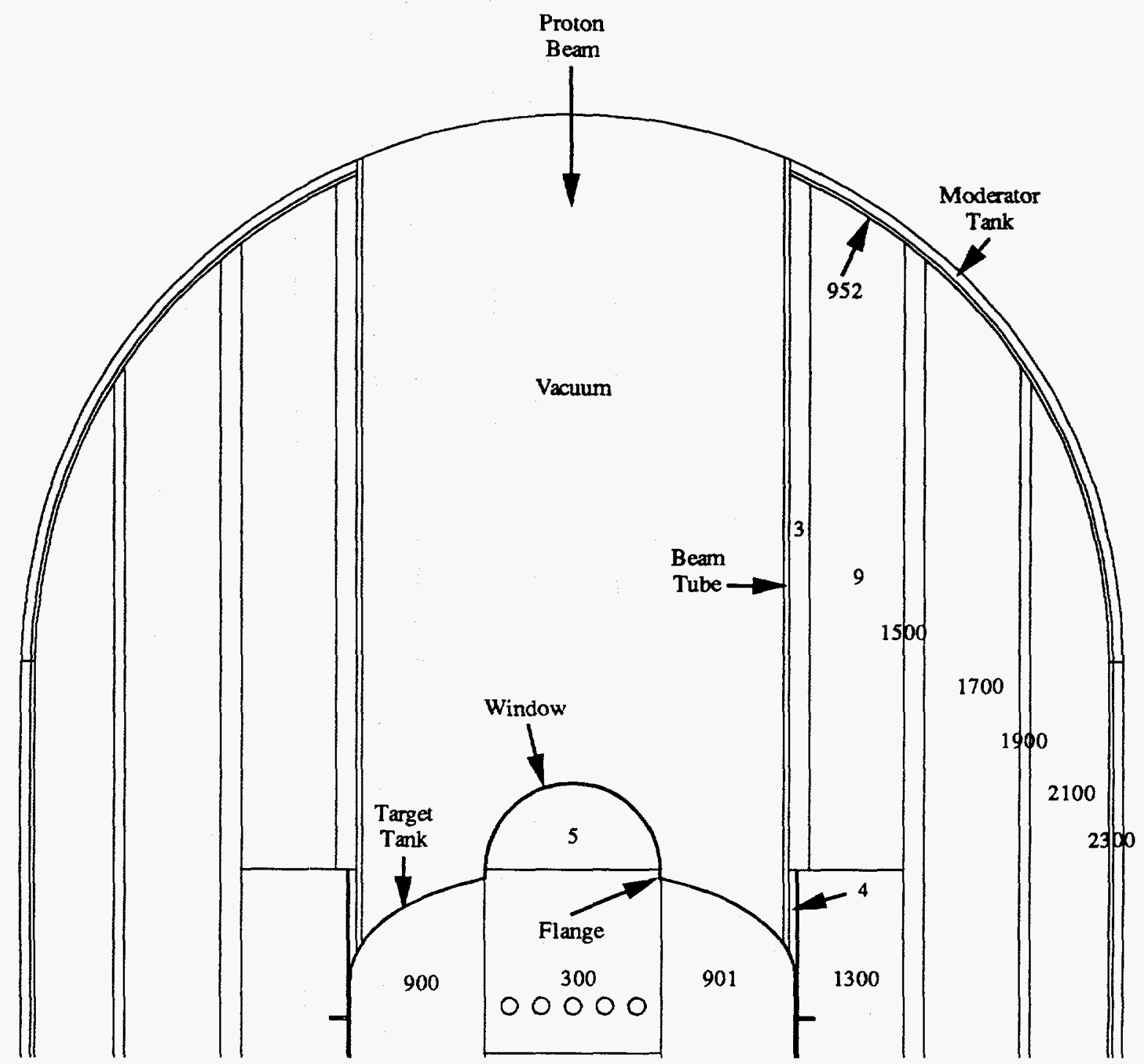

Fig. 3-31. Cross-sectional view of the beam tube area of model BT2 with a cylindrical beam tube. The numbers correspond to LCS cell numbers identified in Table 3-16.

BT3 - Long Cylinder - This case is like BT2 except the hemispherical front end of the moderator tank has been extended an additional $50 \mathrm{~cm}$ (19.7 in.) to try and reduce neutron leakage. Compared to the regular-length front end (BT2), this long front end increases the tritium production by only $0.12 \% \pm 0.32 \%$.

BT4 - Short Cylinder - This case is like BT2 except the hemispherical front end of the moderator tank has been reduced by $50 \mathrm{~cm}$ (19.7 in.). Compared to the regular length front end (BT2), this short front end reduces the tritium production by $1.15 \% \pm 0.32 \%$. This is caused by the increased neutron leakage up the beam tube vacuum and out the front end.

In conclusion, extension of the cylindrical beam tube beyond its $2-\mathrm{m}$ length of case BT2 does not improve the system performance. In addition, conversion from a rectangular to a 
circular beam tube cross section does not have a significant impact on tritium production. Hence the reference design uses a 2-m length, cylindrical beam tube, as in case BT2.

\subsection{PHYSICS DESIGN OF THE MODERATOR/REFLECTOR}

In the ${ }^{3} \mathrm{He}$ APT system, roughly half of the tritium production occurs in the moderator/reflector (blanket) region. The physics design objective for the moderator/ reflector was to efficiently maintain total tritium production while accommodating engineering, material inventory, safety, and neutron-source constraints that evolve with time.

\subsubsection{Moderator and Reflector Material}

The moderator and reflector must slow down and reflect source neutrons, without capturing them, to thermal velocities where the tritium production cross section of $3 \mathrm{He}$ is very large. Survey calculations were performed to investigate and compare the optimized efficiencies of $\mathrm{D}_{2} \mathrm{O}$, beryllium, and graphite as moderator/reflector materials [3-34]. Because of its low capture cross section, $\mathrm{D}_{2} \mathrm{O}$ was selected as the most efficient moderator. However, the smaller dimensions of a beryllium moderator may be useful if physical dimensions become limiting. Graphite has the lowest efficiency, which could become even lower if all realistic impurities were to be included (Fig. 3-32).

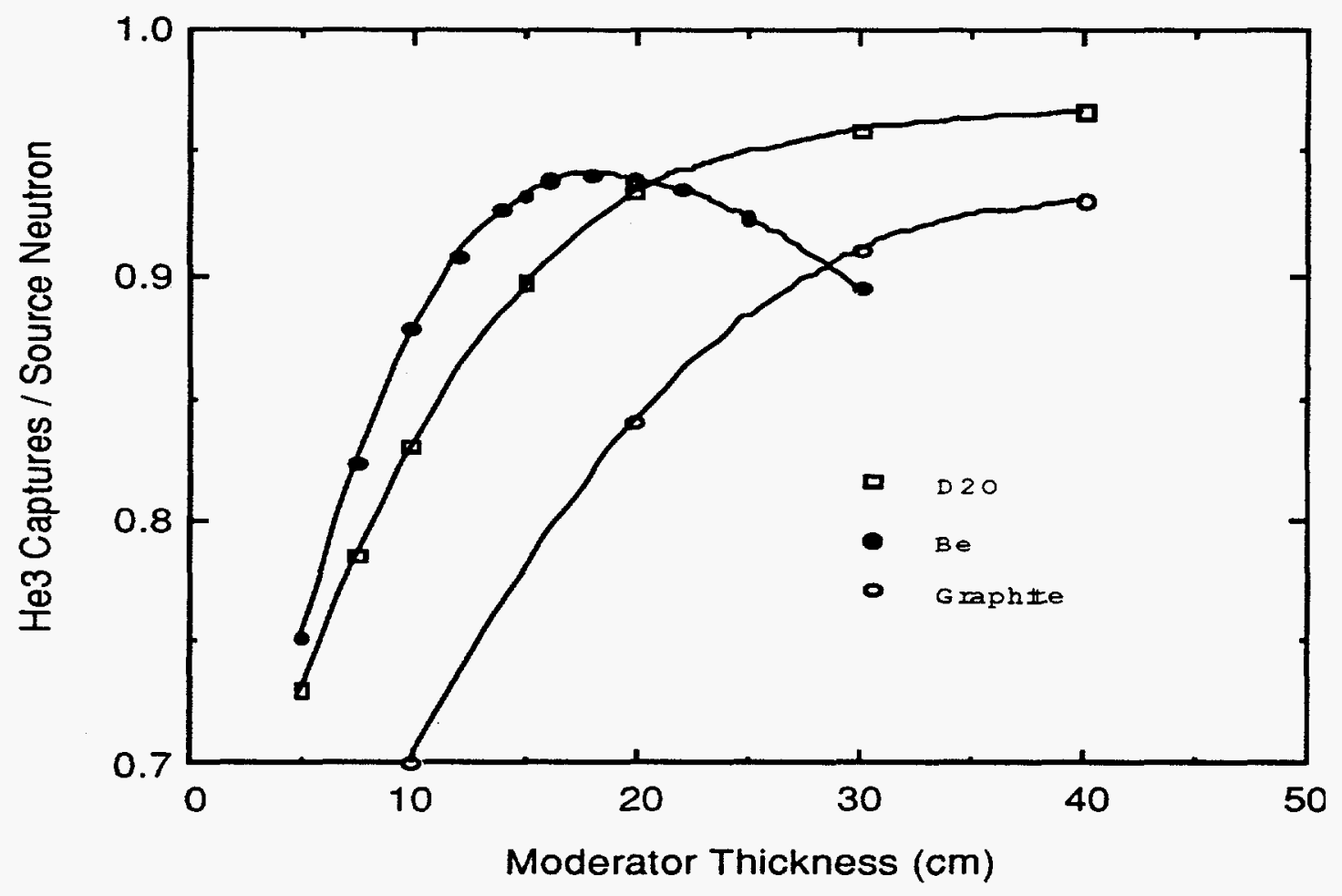

Fig. 3-32. Neutron captures by ${ }^{3} \mathrm{He}$ vs. moderator thickness for $\mathrm{D}_{2} \mathrm{O}$, graphite, and Be moderators. 


\subsubsection{Tube Modeling and ${ }^{3}$ He Pressure}

Designers currently visualize rows of tubes as the means to contain and transport ${ }^{3} \mathrm{He}$ throughout the blanket. This presents a modeling problem because it is not practical to model each of the tubes. A limited number of models were created to investigate the effect of modeling blanket ${ }^{3} \mathrm{He}$ tubing as cylindrical annuli, as annuli of two rows of tubes, and as annuli of three rows of tubes [3-35]. The results showed that for a constant ${ }^{3} \mathrm{He}$ inventory, the three models give essentially the same tritium production. This is the justification for using cylindrical shells to model the blanket ${ }^{3} \mathrm{He}$.

The same models mentioned above were used to investigate the effect of varying the pressure of $3 \mathrm{He}$ in the tubes [3-36]. The results showed that pressures above 100 psia did not increase tritium production. Only when the pressure was reduced to 40 psia did a statistically significant decrease in tritium production occur (Table 3-17). This is the justification for selecting 100 psia as an appropriate, base-case, blanket-tube pressure.

\begin{tabular}{lcccc}
\hline \hline Table 3-17. & Tritium Production as a Function of Blanket ${ }^{3 H e}$ Pressure & \\
\hline Region & $\mathbf{4 0 ~ P s i}$ & 70 Psi & 100 Psi & 111 Psi \\
\hline 1st annulus of ${ }^{3} \mathrm{He}$ & 50.79 & 53.72 & 55.36 & 55.80 \\
2nd annulus of ${ }^{3} \mathrm{He}$ & 18.14 & 17.55 & 17.16 & 17.03 \\
3rd annulus of ${ }^{3} \mathrm{He}$ & 0.72 & 0.70 & 0.70 & 0.70 \\
All other & $\underline{28.57}$ & $\underline{27.42}$ & $\underline{26.78}$ & $\underline{26.52}$ \\
Total & 98.22 & 99.39 & 100.00 & 100.05 \\
\hline \hline
\end{tabular}

*Percentages normalized to 100 total for 100 psia; relative error on totals is 0.0014 .

\subsubsection{Tube Material}

The selection of material for the tubes of the APT ${ }^{3} \mathrm{He}$ distribution system depends on many factors, including structural strength, amount of ${ }^{3} \mathrm{He}$, chemistry, corrosion, cost, tritium absorption, availability, and neutronic effects. A one-dimensional, cylindrical, TWODANT model was created to determine the first-order neutronic effects of candidate tube materials. Six different materials were tested to use as tube material: Al2024, Al6061, Croloy, Croloyal (Croloy with a $0.0127-\mathrm{cm}$ (0.005 in.) interior coating of Al6061), Zirc4 (Ziraloy-4), and Zirc4Cu (Zirc4 with a 0.0254-cm (0.01 in.) interior coating of copper). Al-2024, Al-6061, and Zircaloy -4 were shown to be essentially neutronically equivalent tube materials because they have little effect on tritium production. Croloy tubes, croloy linings, or copper linings significantly reduce the tritium production.

Specifically, the calculations showed that Al-2024, Al-6061, and Zircaloy-4 are essentially neutronically equivalent tube materials because the neutron absorption in the ${ }^{3} \mathrm{He}$ changes by less that $0.5 \%$. Croloy appears to absorb neutrons 5 to 13 times more readily than Zircaloy-4, $\mathrm{Al}-2024$, or Al-6061. On an equal-volume-tube-material basis, this absorption leads to a significant $4 \%$ (using Croloyal) to $5 \%$ (using Croloy) reduction in the neutrons absorbed by the ${ }^{3} \mathrm{He}$. Croloy would have to have very significant other benefits before this neutronic 
penalty should be accepted. Only $0.0254 \mathrm{~cm}(0.01 \mathrm{in}$.) of copper interior coating on Zircaloy-4 reduces the $3 \mathrm{He}$ neutron absorption by almost $2 \%$. This also seems to be a high neutronic penalty. Much thinner or much less absorbing coatings must be used. The tradeoff is between neutron absorption in the tube material and neutron absorption in the ${ }^{3} \mathrm{He}$. That is, whatever neutrons are absorbed in the tube material are subtracted from the neutron absorption in the ${ }^{3} \mathrm{He}$.

When Al-6061 is the tube wall material, $94.35 \%$ of the neutrons are captured by the ${ }^{3} \mathrm{He}$. This differs from an earlier study referred to in Sec. 3.7 .1 in which ${ }^{3} \mathrm{He}$ captured $96.8 \%$ of the neutrons. The difference was traced to the fact that the present study used a little water in the lead target region, whereas the earlier study used pure lead. This sensitivity to the water content of the lead target region is investigated more thoroughly in Sec. 3.6.4.2. Therefore, from a neutronics viewpoint, Al-2024, Al-6061, or Zircaloy-4 are suitable tubing materials. Subsequent neutronic analyses showed a marginal benefit in $t / p$ is derived if $\mathrm{Al}-1100$ is selected as the tubing material, as is the case in our reference design.

\subsubsection{Blanket Temperature Effects}

Thus far, most of the APT physics calculations have been performed with $300 \mathrm{~K}$ cross sections. The reasoning behind this expediency is that the APT temperatures are relatively moderate, there are no wildly temperature sensitive isotopes in the APT, temperaturedependent MCNP cross sections are difficult to obtain, and calculations must continue with what is available. Nevertheless, one may ask-would more realistic temperature-dependent cross sections significantly affect the APT? The investigation shows that $300 \mathrm{~K}$ cross sections are appropriate for the blanket.

In an attempt to answer the above question in part, blanket-temperature effects were tested with a 1D TWODANT model that has access to temperature dependent cross sections through the multigroup cross-section preparation code TRANSX [3-37]. In these calculations, temperature-induced dimensional and density changes were not included.

The calculations showed that temperature-induced microscopic cross-section changes have negligible effect in the blanket. Thus, the continued use of $300 \mathrm{~K} \mathrm{MCNP}$ cross sections in the blanket appears to be a justified, conservative assumption. Temperature-induced dimensional and density changes will have larger effects, but such changes can be incorporated explicitly into the physics modeling and their neutronic impact determined.

\subsubsection{Blanket Optimization}

Varying the thicknesses of the ${ }^{3} \mathrm{He}$ and $\mathrm{D}_{2} \mathrm{O}$ annuli can maximize tritium production while minimizing blanket volume. An initial optimization has preserved the blanket tritium production while reducing the ${ }^{3} \mathrm{He}$ and $\mathrm{D}_{2} \mathrm{O}$ blanket volumes by factors of 2.44 and 2.78 , respectively - a very significant finding.

A 1D, cylindrical, TWODANT model was generated that represented the old modeling of the blanket. That model had a $30-\mathrm{cm}$-thick annular central zone of lead, followed by three 5 -cm-thick annuli of ${ }^{3} \mathrm{He}$ separated by two 50 -cm-thick annuli of $\mathrm{D}_{2} \mathrm{O}$. 
In this study, the thicknesses of various annuli were varied in an attempt to maximize the ratio ( ${ }^{3} \mathrm{He}$ neutron absorptions)/( ${ }^{3} \mathrm{He}$ volume) with the constraints that the ${ }^{3} \mathrm{He}$ neutron absorptions remain $\geq 94.352 \%$ (which is the highest ${ }^{3} \mathrm{He}$ neutron absorption obtained in the above mentioned model using $\mathrm{Al}-6061$ as the tube material) and that no more than three ${ }^{3} \mathrm{He}$ annuli would be used. One possible improved arrangement consisted of the lead region, followed by $5 \mathrm{~cm}$ of ${ }^{3} \mathrm{He}, 25 \mathrm{~cm}$ of $\mathrm{D}_{2} \mathrm{O}, 2.5 \mathrm{~cm}$ of ${ }^{3} \mathrm{He}, 20 \mathrm{~cm}$ of $\mathrm{D}_{2} \mathrm{O}$, and $1 \mathrm{~cm}$ of ${ }^{3} \mathrm{He}$.

The 1D optimization results were compared to a detailed 3D MCNP model of the APT, and it was found that the total tritium production is conserved, and the regional shifts in tritium production adequately duplicated. The $3 \mathrm{D}$ results confirmed the $1 \mathrm{D}$ blanket optimization.

This initial attempt at blanket optimization indicates that it is possible to obtain the current amount of blanket tritium production with vastly smaller inventories of ${ }^{3} \mathrm{He}$ and $\mathrm{D}_{2} \mathrm{O}$. The smaller, but just as effective, blanket is incorporated in the reference physics model.

\subsubsection{Tube Arrangement}

A relationship between ${ }^{3} \mathrm{He}$ tube dimensions/arrangement and the annuli used in the physics modeling of these tubes was developed. By setting the spacing between ${ }^{3} \mathrm{He}$ cylinders to be $\sim 1.4$ times the radius of the ${ }^{3} \mathrm{He}$ cylinder, while preserving the annulus volume, the thickness variation can be minimized through the use of a double row of tubes.

The optimum ${ }^{3} \mathrm{He}$ tube dimensions and arrangement used to model the uniform annuli in physics simulations will ultimately have to be determined by detailed MCNP calculations. This present study is an effort to discover and justify some reasonably simple relationship that can be used in the interim. The results may also be useful to guide the ultimate optimization with MCNP.

Figure 3-33 is a possible way to approximate an annulus of $3 \mathrm{He}$ with a double row of discrete tubes. For tubes with an inside radius of $\mathrm{R}$ and a spacing $\mathrm{S}$ between the ${ }^{3} \mathrm{He}$ zones of two adjacent tubes, the average thickness $T_{\text {ave }}$ of ${ }^{3} \mathrm{He}$ is given by

$$
\mathrm{T}_{\mathrm{ave}}=\frac{\pi \mathrm{R}^{2}}{\mathrm{R}+\frac{\mathrm{S}}{2}}
$$

Because there is an infinite number of combinations of $R$ and $S$ that will give any particular average thickness, a study was conducted to determine how to select $R$ and $S$. A reasonable selection criterion would be to choose $\mathrm{R}$ and $\mathrm{S}$ to minimize the difference between the maximum ${ }^{3} \mathrm{He}$ thickness and the minimum ${ }^{3} \mathrm{He}$ thickness. Neglecting details of derivation, this scenario occurs when the peak in the sum of $3 \mathrm{He}$ thicknesses of overlapping tubes is equal to $2 \mathrm{R}$. This, in turn, occurs when

$$
S=(\sqrt{3}-1) 2 R=1.464 R
$$

and gives a maximum-to-minimum difference of

$$
\operatorname{Diff}=(1-\sqrt{2 \sqrt{3}-3}) 2 \mathrm{R}=0.638 \mathrm{R} .
$$




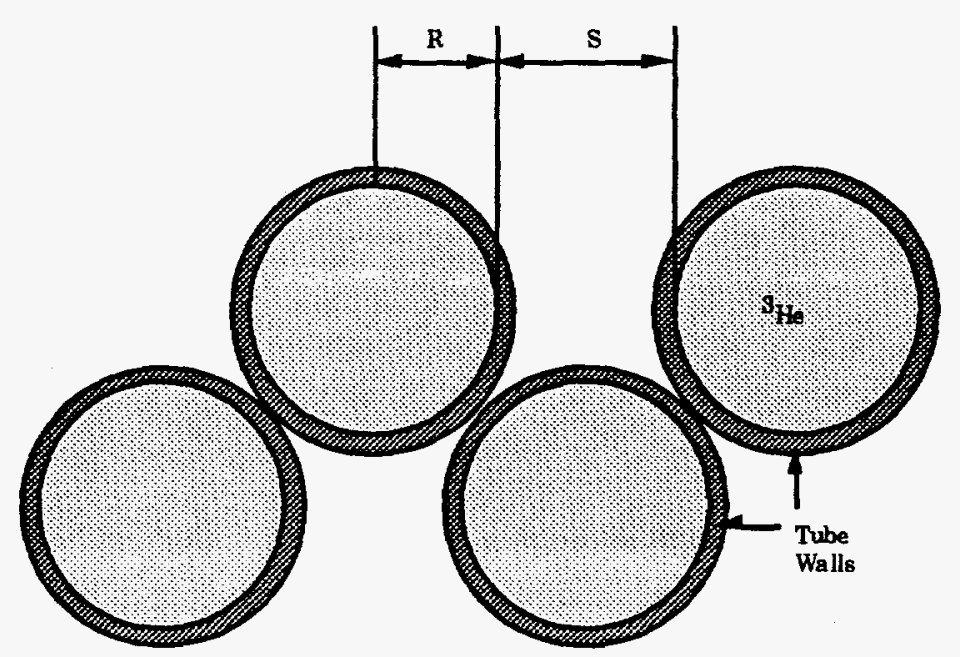

Fig. 3-33. Typical tube arrangement and dimensions used for optimizing the spacing, $S$. $R$ is the tube inner radius, and $S$ is the distance between the inner walls of adjacent tubes.

Thus, ${ }^{3} \mathrm{He}$ tubes, with an inside radius of $2.76 \mathrm{~cm}$ and spaced $4.04 \mathrm{~cm}$ apart, give an average ${ }^{3} \mathrm{He}$ thickness of $5 \mathrm{~cm}$, with a minimized difference of $1.76 \mathrm{~cm}$ between the maximum and minimum ${ }^{3} \mathrm{He}$ thicknesses.

Another reasonable selection criterion would be to minimize the variance of the ${ }^{3} \mathrm{He}$ thickness. This problem does not have an analytic solution. The problem must rely on numerical solutions. Again, neglecting details of derivation, the result is that for any given $R$, the ${ }^{3} \mathrm{He}$ thickness variance is minimized when

$$
S=1.38 \mathrm{R},
$$

and the standard deviation of the ${ }^{3} \mathrm{He}$ thickness is given by

$$
\sigma=0.17 \mathrm{R} \text {. }
$$

When comparing Eqs. (3-1) and (3-2), the two different criteria give nearly the same choice for $S$.

Under the constraint that only a double row of discrete tubes be used to simulate a uniform annulus, this study advances two different, logical criteria that can be used to relate the thickness of a uniform annulus to the dimensions/arrangement of the discrete tubes. Both criteria give nearly the same results and call for a spacing between tubes that leaves ample room for tube walls and support structure. 


\subsection{ENERGY DEPOSITION STUDIES}

\subsubsection{Central Target}

Average power densities in the rod bundles of the APT target have been calculated using the reference physics model. In this model, the central target zone has 88 individual rod bundles of circular cross section, arranged in the pattern shown in Fig. 3-34. Also shown is the bundle numbering convention used to identify rod bundle positions, and the dimensions of a cylindrically-equivalent rod-bundle cross section. The tungsten rods, tungsten wire wrap, and $\mathrm{D}_{2} \mathrm{O}$ coolant within a hex can are homogenized into a single $\mathrm{W} / \mathrm{D}_{2} \mathrm{O}$ zone. The areal densities of the $\mathrm{W} / \mathrm{D}_{2} \mathrm{O}$ zone and the can are equivalent to those of a 91-pin hexagonal rod bundle unit. The average power densities for both zones of each bundle are given in Table 3-18 for a rod-bundle length of $96 \mathrm{~cm}$ (37.8 in.). Also listed in this table are the W volume fractions in each rod bundle, which increases as a function of depth in the target (see Section 4.4.1 for an explanation of the selection of these volume fractions). Total power deposited in all $\mathrm{W} / \mathrm{D}_{2} \mathrm{O}$ zones is $71.8 \mathrm{MW}$ for a proton beam power of $200 \mathrm{MW}$. Total power in the Inconel clad is $4.84 \mathrm{MW}$.

The rod lengths are $96 \mathrm{~cm}(37.8 \mathrm{in}$.$) , and the beam size in the same dimension is 91 \mathrm{~cm}$ (35.8 in.). As the proton beam interacts with the target material, it grows in size due to Coulomb scattering and spallation, causing the beam to lose its well-defined "edge" as it passes through the target. Upon impinging on the first row of rod bundles (having passed through the proton beam window), most of the beam is within its original $91-\mathrm{cm}(35.8 \mathrm{in}$.) extent. Hence, in estimating the peak power density in a rod bundle in the first row, one should assume that the total power over the $96-\mathrm{cm}(37.8 \mathrm{in}$.) length is actually deposited over a $91-\mathrm{cm}$ (35.8 in.) length. This conservative assumption is applied not only to the bundles in the first row, but to all of the bundles in the target. The equivalent high- $Z$ power density in the $W$ is calculated by (conservatively) assuming that all power deposited in the $W / \mathrm{D}_{2} \mathrm{O}$ zone is deposited solely in the tungsten. Thus, the equivalent high- $Z$ peak power density is calculated by multiplying the values given in third column of Table $3-18$ by $(96 \div 91) \approx 1.05495$ and dividing by the appropriate $\mathrm{W}$ volume fraction. For the rod bundle with the highest power density (bundle \#8), this gives an equivalent high- $Z$ peak power density of $2.56 \mathrm{MW} / \ell$. Equivalent high- $Z$ peak power densities are shown in the fourth column of Table 3-18.

It should be mentioned that the average power densities listed in Table 3-18 are calculated by averaging over an entire bundle volume, and that spatial variations in the beam power density downstream of the first row of bundles may give rise to a spatial peaking within the rod bundles, which has not been accounted for here.

On a 6-bundle row, the original beam width ( $42 \mathrm{~cm}$, or 16.5 in.) is smaller than the row width $(47 \mathrm{~cm}$, or $18.5 \mathrm{in}$.). Since the proton beam strikes only roughly half of an edge bundle in these rows (e.g., bundles \#6 and \#11 in the second row), the average power density in these bundles is about half the average power density of the other bundles in these rows. However, the peak power density within these bundles is roughly equivalent to the peak power density seen in the other bundles on the same row.

Looking at the bundles near the center of each row, one sees that the power density in the second row is higher by about $10 \%$ than the power density in the first row; further, the power 

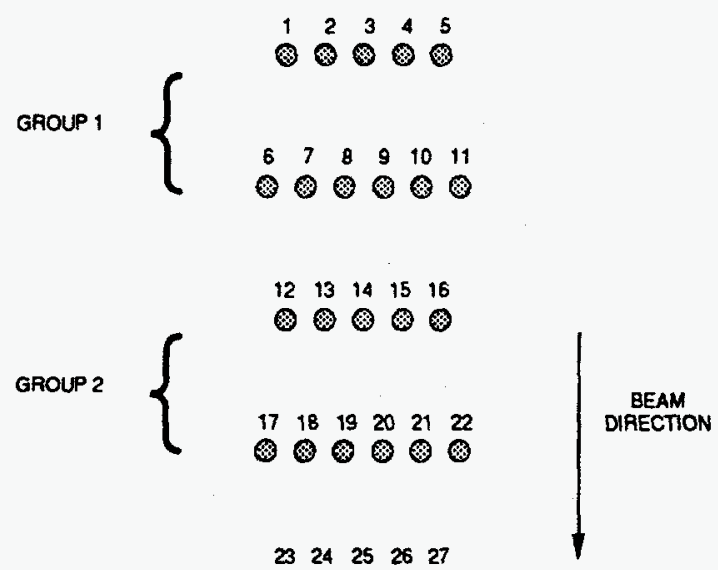

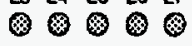

GROUP $3\left\{\begin{array}{l}2829313233 \\ 2830 * 0\end{array}\right.$

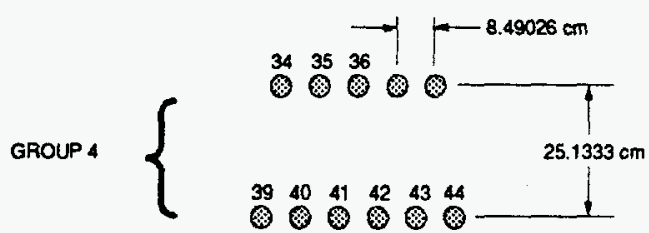

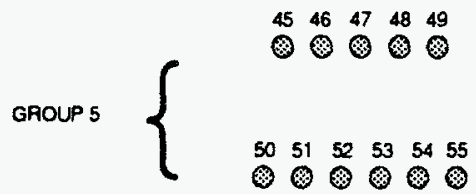

cylindrically equivalent 91-rod bundle geometry:

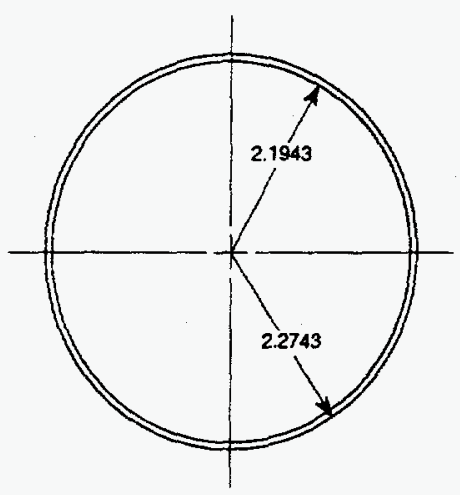

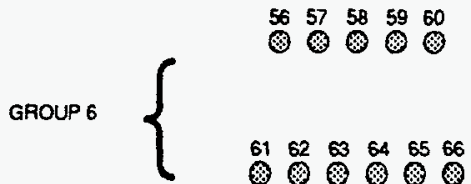

$\begin{array}{lllll}67 & 68 & 69 & 70 & 71\end{array}$

GROUP 7 $\left\{\begin{array}{l}7273757677 \\ 7000 \%\end{array}\right.$

$\begin{array}{lllll}78 & 79 & 80 & 81 & 82\end{array}$

0000

GROUP 8

B3 $84 \quad 85 \quad 86 \quad 87 \quad 88$

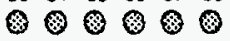

Fig. 3-34. Central target zone rod bundle layout showing the bundlenumbering convention used to identify rod bundle positions. At right are the dimensions of an equivalent rod bundle cross section. 


\begin{tabular}{|c|c|c|c|c|c|c|}
\hline $\begin{array}{c}\text { Rod } \\
\text { Bundle } \\
\text { Number }\end{array}$ & $\begin{array}{c}\mathbf{W} \\
\text { Volume } \\
\text { Fraction }\end{array}$ & 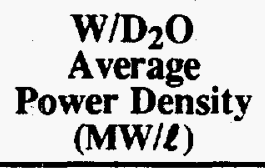 & $\begin{array}{l}\text { W Peak } \\
\text { Power } \\
\text { Density } \\
(\mathrm{MW} / \ell)\end{array}$ & $\begin{array}{c}\text { Relative } \\
\text { Error }\end{array}$ & $\begin{array}{c}\text { Inconel Clad } \\
\text { Average } \\
\text { Power Density } \\
\text { (MW/ })\end{array}$ & $\begin{array}{c}\text { Relative } \\
\text { Error }\end{array}$ \\
\hline 1 & 0.4634 & 0.989 & 2.251 & 0.018 & 1.000 & 0.046 \\
\hline 2 & 0.4634 & 1.004 & 2.286 & 0.017 & 1.008 & 0.044 \\
\hline 3 & 0.4634 & 1.007 & 2.292 & 0.018 & 1.061 & 0.054 \\
\hline 4 & 0.4634 & 0.988 & 2.249 & 0.017 & 1.024 & 0.047 \\
\hline 5 & 0.4634 & 0.996 & 2.268 & 0.018 & 1.003 & 0.044 \\
\hline 6 & 0.4634 & 0.489 & 1.114 & 0.024 & 0.554 & 0.059 \\
\hline 7 & 0.4634 & 1.081 & 2.460 & 0.017 & 1.077 & 0.044 \\
\hline 8 & 0.4634 & 1.126 & 2.564 & 0.016 & 1.110 & 0.037 \\
\hline 9 & 0.4634 & 1.106 & 2.518 & 0.016 & 1.149 & 0.043 \\
\hline 10 & 0.4634 & 1.086 & 2.472 & 0.016 & 1.134 & 0.056 \\
\hline 11 & 0.4634 & 0.509 & 1.159 & 0.024 & 0.529 & 0.065 \\
\hline 12 & 0.5021 & 0.830 & 1.743 & 0.018 & 0.900 & 0.062 \\
\hline 13 & 0.5021 & 0.864 & 1.816 & 0.018 & 0.924 & 0.043 \\
\hline 14 & 0.5021 & 0.888 & 1.865 & 0.018 & 0.940 & 0.056 \\
\hline 15 & 0.5021 & 0.845 & 1.775 & 0.018 & 0.894 & 0.047 \\
\hline 16 & 0.5021 & 0.820 & 1.724 & 0.018 & 0.902 & 0.058 \\
\hline 17 & 0.5021 & 0.517 & 1.087 & 0.023 & 0.496 & 0.053 \\
\hline 18 & 0.5021 & 1.018 & 2.138 & 0.016 & 0.965 & 0.046 \\
\hline 19 & 0.5021 & 1.088 & 2.286 & 0.016 & 1.047 & 0.051 \\
\hline 20 & 0.5021 & 1.043 & 2.192 & 0.016 & 0.987 & 0.042 \\
\hline 21 & 0.5021 & 1.026 & 2.155 & 0.017 & 0.940 & 0.043 \\
\hline 22 & 0.5021 & 0.511 & 1.073 & 0.023 & 0.488 & 0.048 \\
\hline 23 & 0.5498 & 0.738 & 1.415 & 0.019 & 0.696 & 0.050 \\
\hline 24 & 0.5498 & 0.842 & 1.615 & 0.018 & 0.812 & 0.055 \\
\hline 25 & 0.5498 & 0.820 & 1.573 & 0.018 & 0.791 & 0.041 \\
\hline 26 & 0.5498 & 0.796 & 1.527 & 0.017 & 0.783 & 0.051 \\
\hline 27 & 0.5498 & 0.743 & 1.426 & 0.019 & 0.720 & 0.042 \\
\hline 28 & 0.5498 & 0.446 & 0.856 & 0.023 & 0.400 & 0.067 \\
\hline 29 & 0.5498 & 0.848 & 1.627 & 0.018 & 0.755 & 0.039 \\
\hline 30 & 0.5498 & 0.878 & 1.685 & 0.017 & 0.790 & 0.040 \\
\hline 31 & 0.5498 & 0.836 & 1.603 & 0.018 & 0.742 & 0.053 \\
\hline 32 & 0.5498 & 0.820 & 1.574 & 0.018 & 0.715 & 0.051 \\
\hline 33 & 0.5498 & 0.435 & 0.835 & 0.023 & 0.415 & 0.078 \\
\hline 34 & 0.5973 & 0.617 & 1.089 & 0.020 & 0.525 & 0.055 \\
\hline 35 & 0.5973 & 0.767 & 1.355 & 0.019 & 0.650 & 0.048 \\
\hline 36 & 0.5973 & 0.788 & 1.392 & 0.018 & 0.673 & 0.051 \\
\hline 37 & 0.5973 & 0.767 & 1.354 & 0.019 & 0.654 & 0.057 \\
\hline 38 & 0.5973 & 0.628 & 1.109 & 0.021 & 0.527 & 0.062 \\
\hline 39 & 0.5973 & 0.380 & 0.671 & 0.026 & 0.313 & 0.071 \\
\hline 40 & 0.5973 & 0.630 & 1.112 & 0.020 & 0.528 & 0.049 \\
\hline 41 & 0.5973 & 0.709 & 1.253 & 0.018 & 0.595 & 0.047 \\
\hline 42 & 0.5973 & 0.682 & 1.205 & 0.019 & 0.576 & 0.067 \\
\hline 43 & 0.5973 & 0.641 & 1.132 & 0.020 & 0.531 & 0.058 \\
\hline 44 & 0.5973 & 0.367 & 0.648 & 0.027 & 0.312 & 0.072 \\
\hline 45 & 0.6498 & 0.484 & 0.786 & 0.022 & 0.393 & 0.072 \\
\hline 46 & 0.6498 & 0.639 & 1.037 & 0.021 & 0.506 & 0.055 \\
\hline 47 & 0.6498 & 0.643 & 1.045 & 0.019 & 0.523 & 0.052 \\
\hline 48 & 0.6498 & 0.621 & 1.009 & 0.019 & 0.487 & 0.059 \\
\hline 49 & 0.6498 & 0.480 & 0.778 & 0.022 & 0.367 & 0.055 \\
\hline 50 & 0.6498 & 0.301 & 0.488 & 0.028 & 0.237 & 0.081 \\
\hline 51 & 0.6498 & 0.471 & 0.765 & 0.021 & 0.378 & 0.048 \\
\hline 52 & 0.6498 & 0.557 & 0.904 & 0.021 & 0.443 & 0.057 \\
\hline
\end{tabular}




\begin{tabular}{|c|c|c|c|c|c|c|}
\hline $\begin{array}{c}\text { Rod } \\
\text { Bundle } \\
\text { Number }\end{array}$ & $\begin{array}{c}\mathbf{W} \\
\text { Volume } \\
\text { Fraction }\end{array}$ & 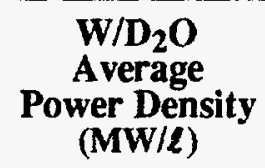 & $\begin{array}{l}\text { W Peak } \\
\text { Power } \\
\text { Density } \\
(\mathrm{MW} / \ell)\end{array}$ & $\begin{array}{c}\text { Relative } \\
\text { Error }\end{array}$ & $\begin{array}{c}\text { Inconel Clad } \\
\text { Average } \\
\text { Power Density } \\
(\mathrm{MW} / \ell)\end{array}$ & $\begin{array}{c}\text { Relative } \\
\text { Error }\end{array}$ \\
\hline 53 & 0.6498 & 0.566 & 0.918 & 0.021 & 0.433 & 0.060 \\
\hline 54 & 0.6498 & 0.478 & 0.777 & 0.022 & 0.399 & 0.062 \\
\hline 55 & 0.6498 & 0.295 & 0.479 & 0.028 & 0.230 & 0.073 \\
\hline 56 & 0.6745 & 0.349 & 0.545 & 0.026 & 0.273 & 0.085 \\
\hline 57 & 0.6745 & 0.466 & 0.728 & 0.023 & 0.347 & 0.058 \\
\hline 58 & 0.6745 & 0.500 & 0.782 & 0.021 & 0.394 & 0.062 \\
\hline 59 & 0.6745 & 0.452 & 0.707 & 0.022 & 0.353 & 0.082 \\
\hline 60 & 0.6745 & 0.351 & 0.549 & 0.025 & 0.270 & 0.066 \\
\hline 61 & 0.6745 & 0.235 & 0.368 & 0.029 & 0.176 & 0.095 \\
\hline 62 & 0.6745 & 0.350 & 0.547 & 0.025 & 0.256 & 0.076 \\
\hline 63 & 0.6745 & 0.418 & 0.654 & 0.023 & 0.304 & 0.049 \\
\hline 64 & 0.6745 & 0.421 & 0.658 & 0.022 & 0.335 & 0.056 \\
\hline 65 & 0.6745 & 0.338 & 0.529 & 0.025 & 0.256 & 0.068 \\
\hline 66 & 0.6745 & 0.236 & 0.369 & 0.030 & 0.174 & 0.071 \\
\hline 67 & 0.6745 & 0.250 & 0.391 & 0.029 & 0.194 & 0.050 \\
\hline 68 & 0.6745 & 0.312 & 0.487 & 0.025 & 0.243 & 0.079 \\
\hline 69 & 0.6745 & 0.347 & 0.542 & 0.024 & 0.273 & 0.036 \\
\hline 70 & 0.6745 & 0.323 & 0.505 & 0.026 & 0.257 & 0.046 \\
\hline 71 & 0.6745 & 0.255 & 0.400 & 0.028 & 0.194 & 0.042 \\
\hline 72 & 0.6745 & 0.163 & 0.255 & 0.033 & 0.122 & 0.095 \\
\hline 73 & 0.6745 & 0.241 & 0.377 & 0.029 & 0.196 & 0.052 \\
\hline 74 & 0.6745 & 0.276 & 0.432 & 0.026 & 0.225 & 0.041 \\
\hline 75 & 0.6745 & 0.283 & 0.443 & 0.027 & 0.206 & 0.075 \\
\hline 76 & 0.6745 & 0.234 & 0.366 & 0.028 & 0.166 & 0.050 \\
\hline 77 & 0.6745 & 0.161 & 0.251 & 0.034 & 0.127 & 0.048 \\
\hline 78 & 0.6745 & 0.177 & 0.277 & 0.034 & 0.133 & 0.073 \\
\hline 79 & 0.6745 & 0.223 & 0.349 & 0.031 & 0.182 & 0.065 \\
\hline 80 & 0.6745 & 0.238 & 0.372 & 0.028 & 0.188 & 0.063 \\
\hline 81 & 0.6745 & 0.223 & 0.348 & 0.030 & 0.173 & 0.053 \\
\hline 82 & 0.6745 & 0.172 & 0.269 & 0.032 & 0.132 & 0.072 \\
\hline 83 & 0.6745 & 0.120 & 0.187 & 0.037 & 0.079 & 0.047 \\
\hline 84 & 0.6745 & 0.162 & 0.254 & 0.033 & 0.127 & 0.053 \\
\hline 85 & 0.6745 & 0.187 & 0.292 & 0.030 & 0.145 & 0.058 \\
\hline 86 & 0.6745 & 0.182 & 0.285 & 0.031 & 0.145 & 0.043 \\
\hline 87 & 0.6745 & 0.166 & 0.259 & 0.032 & 0.136 & 0.065 \\
\hline 88 & 0.6745 & 0.121 & 0.190 & 0.038 & 0.094 & 0.063 \\
\hline
\end{tabular}

density in the fourth row is higher by about $20 \%$ than the power density in the third row. This can be explained by the impact that spallation reactions and Coulombic interactions have on the beam power density profile. As the protons pass through the rod bundles in the first row, Coulomb scattering and spallation impart an angular divergence of the order of a few tens of milliradians to that portion of the beam that interacts with the bundles, which leads to a spreading of this portion of the beam such that, by the time the beam reaches the second row, about $10 \%$ of the beam that interacted with the first row also strikes the second row. Of course, the beam between the bundles passes on to the second row undisturbed. Thus, the beam power to which the bundles in the second row are subjected is about $10 \%$ higher than that which the first-row bundles see. This difference is amplified for the third- and fourth-row bundles through the same processes. 


\subsubsection{Backstop}

As described in Section 3.6.3, lead would make the best backstop material were it not for its relatively poor mechanical properties. Because the deposited power declines with depth into the backstop, the optimum backstop design would use a high-temperature material at the front of the backstop and switch over to lead at the point where the deposited power density in the lead becomes tolerable from a thermal-hydraulics standpoint. Thus the axial variation of the power density in the backstop is an important parameter in the optimum design of the target system. Using the reference physics model, the power densities in the five high- $\mathrm{Z}$ regions of the backstop were calculated. The results are reported in Table 3-19. In this study, all five regions have a heavy water coolant volume fraction of $25 \%$. In the reference physics model, these fractions have been reduced to $15 \%, 13 \%, 8 \%$, and $5 \%$ in the four $15-\mathrm{cm}$-thick (5.91 in.) Zircaloy- 4 regions, and to $3 \%$ in the $30-\mathrm{cm}$-thick (11.8 in.) lead region. There exists the potential to enhance the net neutron production in the backstop over the present design by reducing the amount of Zircaloy in the backstop with a concomitant increase in the coolant fraction in the lead.

\begin{tabular}{lcc}
\hline \hline Table 3-19. $\quad$ Power densities in the backstop. & \\
\hline \multicolumn{1}{c}{ Backstop Region } & $\begin{array}{c}\text { Volume-Averaged Power } \\
\text { Density (kW/ })\end{array}$ & Relative Error \\
& 45.30 & 0.0069 \\
1st Zircaloy-4 zone & 29.67 & 0.0085 \\
1st Zircaloy-4 zone & 12.19 & 0.0127 \\
1st Zircaloy-4 zone & 4.15 & 0.0206 \\
1st Zircaloy-4 zone & 1.31 & 0.0245 \\
Lead zone & & \\
\hline \hline
\end{tabular}

\subsubsection{Radial Target}

A series of runs was executed using the Physics-IV model with a Zircaloy-4 backstop in which the radial lead target was broken into two radial sections, with the inner section varying in thickness from 0.5 to $22.5 \mathrm{~cm}$. In addition, the 7.5 -m-long lead target was divided into 30 axial segments (subsequent optimization studies shortened this length). In this model, the lead zone has an inner radius of $66.32 \mathrm{~cm}$ and an outer radius of $96.32 \mathrm{~cm}$. Results of these runs are presented in Fig. 3-35, where a proton beam current of $200 \mathrm{~mA}$ has been assumed. The high- $Z$ power density is calculated by assuming that all of the power deposited in a cell is deposited solely in the lead. Thus, the high- $Z$ power density is obtained by dividing the power density by the lead volume fraction of 0.898 . The peak high- $Z$ power density of $0.048 \mathrm{MW} / \ell$ occurs at the inner surface of the lead zone, about $2 \mathrm{~m}$ downstream of the beam entrance window. Because the power densities are obtained by averaging over azimuth, the actual peak power density may be greater than this value because the targets, being rectangular in cross section, are not azimuthally symmetric. The total power deposited in the radial lead target in this particular model is $34.7 \mathrm{MW}$.

The average high- $\mathrm{Z}$ power density as a function of radius is plotted in Fig. 3-36. It shows the power density falling dramatically with the radial position, from $25 \mathrm{~kW} / \ell$ at the inner surface to less than $1 \mathrm{~kW} / \ell$ in the outer $7.5-\mathrm{cm}$ region. 


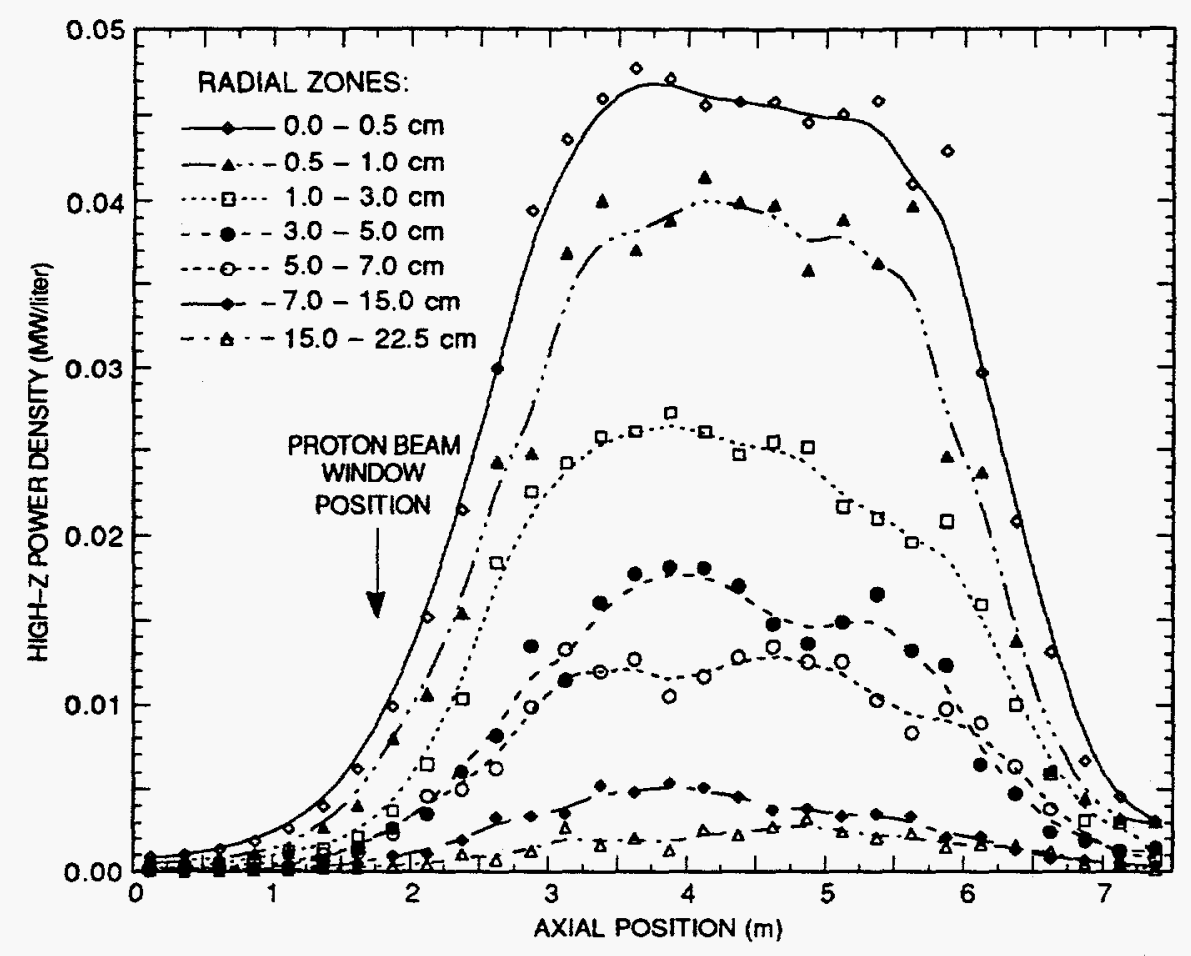

Fig. 3-35. Power density variation with axial position for various radial regions in the radial lead zone.

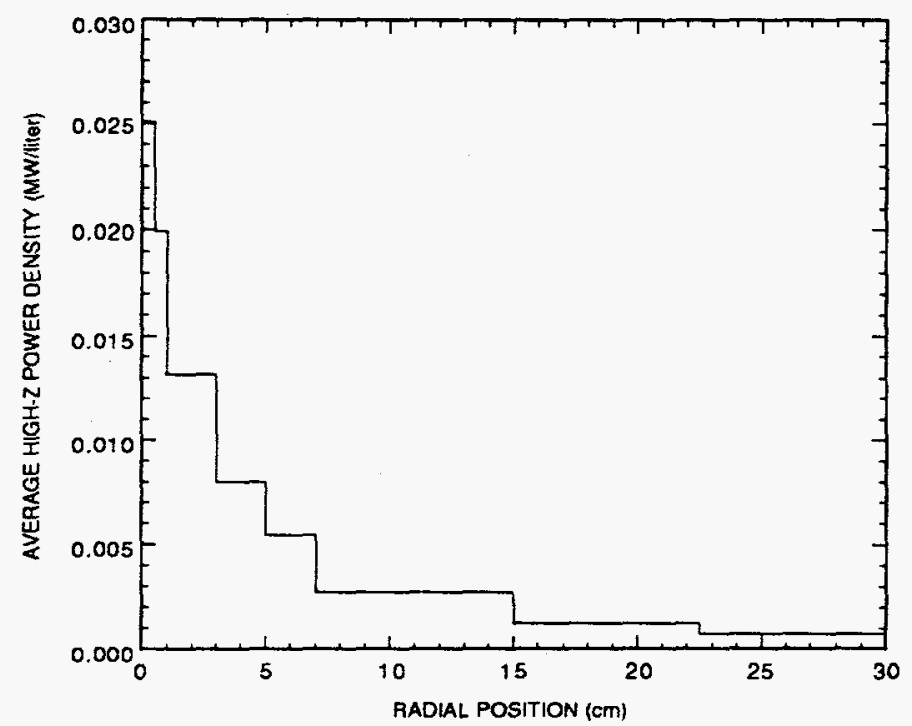

Fig. 3-36. Radial variation of the "high-Z" power density, averaged over axial extent and azimuth, in the radial lead target. 


\subsubsection{Window}

A variety of APT window sizes and shapes were modeled to determine the effects of structure requirements, safety requirements, and homogenization on the energy deposited in a window. The basic results include the following:

- Any requirement that increases the window mass in the beam will increase both the energy deposited in the window and the window cooling requirements, and

- Homogenized windows are adequate models for predicting the energy deposited in the window.

For this study, a 55-cm $\times 105-\mathrm{cm}$ window (with limited geometric models that included enough of the APT system to adequately compute the window interaction) was used, with a $50-\mathrm{cm} \times 100-\mathrm{cm}$ incident proton beam at $1 \mathrm{GeV}$. The window was followed by $49.5 \mathrm{~cm}$ of ${ }^{3} \mathrm{He}$, followed by $2 \mathrm{~cm}$ of homogenized target bundles, followed by $30 \mathrm{~cm}$ of ${ }^{3} \mathrm{He}$, followed by another $2 \mathrm{~cm}$ of target material. The studies included the following window models:

- W1 Model. This window is a homogenized plate $0.5 \mathrm{~cm}$ thick and composed of $50 \mathrm{v} \%$ Inconel-718 and $50 \mathrm{v} \%$ water ( $99.75 \mathrm{a} \% \mathrm{D}_{2} \mathrm{O}$ and $0.25 \mathrm{a} \% \mathrm{H}_{2} \mathrm{O}$ ).

- W2 Model. This window is a three-component plate $0.5 \mathrm{~cm}$ thick. A $0.25-\mathrm{cm}$ thick central plate of water was sandwiched between two $0.125-\mathrm{cm}$-thick plates of Inconel-718.

- W3 Model. This window is a three-component half-cylinder shell $0.5 \mathrm{~cm}$ thick. A $0.25-\mathrm{cm}$-thick central half-cylinder shell of water was sandwiched between two 0.125 -cm-thick half-cylinder shells of Inconel-718.

- W4 Model. This window is a seven-component half-cylinder shell $1 \mathrm{~cm}$ thick. Three $0.2-\mathrm{cm}$-thick half-cylinder shells of water were sandwiched between four $0.1-\mathrm{cm}$-thick half-cylinder shells of Inconel-718.

- W5 Model. This is the same as the W4 model with the exception of the ${ }^{3} \mathrm{He}$ and target material beyond the window.

Table 3-20 shows the results of the calculations. Because the W1 model was homogenous, only results for the total energy deposition were calculated.

In a previous calculation employing a complete mockup of the APT geometry, a homogeneous (W1-type) window was employed. This computation serves as a benchmark for the W1 model in a simplified geometry. The fraction of proton beam energy deposited in the homogenized window in the complete APT calculation was $0.5485 \%$, compared with the $0.5400 \%$ calculated for the simplified W1-model calculation. This is very encouraging and shows that the simple window models of this study provide meaningful results. 


\begin{tabular}{lccccc}
\hline Table 3-20. & Energy Deposited in Various APT Windows & & \\
\hline \multicolumn{1}{c}{ Component } & W1 & W2 & W3 & W4 & W5 \\
\hline 1st Inconel-718 & & 0.2262 & 0.2910 & 0.2482 & 0.2401 \\
2nd Inconel-718 & & 0.2434 & 0.3154 & 0.2683 & 0.2508 \\
3rd Inconel-718 & & & 0.2725 & 0.2662 \\
4th Inconel-718 & & & 0.2775 & 0.2592 \\
Total Inconel-718 & 0.4696 & 0.6064 & 1.0665 & 1.0163 \\
lst Water & 0.0827 & 0.1031 & 0.0850 & 0.0864 \\
2nd Water & & & 0.0968 & 0.0874 \\
3rd Water & & & & 0.0955 & 0.0867 \\
Total Water & & 0.0827 & 0.1031 & 0.2773 & 0.2605 \\
Total & 0.5400 & 0.5523 & 0.7095 & 1.3439 & 1.2768 \\
\hline \hline
\end{tabular}

*Units are percent of beam energy.

The W2 model is a more realistic three-component example of the 0.5 -cm-thick planar window of $\mathrm{W} 1$. There is slightly more energy deposited in a heterogeneous window than in its homogeneous counterpart. About $41 \%$ of the energy is deposited in the first Inconel-718 component, about $44 \%$ in the second Inconel- 718 component, and about $15 \%$ in the water.

The W3 model was constructed to investigate the effects of having to replace a planar window with a cylindrical window for structural strength reasons. Compared with W2, approximately $28 \%$ more energy is deposited in a half-cylinder window. The energy deposition distribution remains the same with $41 \%$ in the first Inconel- 718 component, $44 \%$ in the second Inconel-718 component, and $15 \%$ in the water.

The W4 model represents a thicker (seven-component) half-cylinder window that may be necessary for safety reasons. The energy deposition distribution is $18.5 \%, 20.0 \%, 20.3 \%$, and $20.6 \%$ in the Inconel- 718 components and $6.3 \%, 7.2 \%$, and $7.1 \%$ in the water components.

The W5 model was constructed without the two target planes to investigate how much an even simpler model of the window environment would affect results and to determine the heating caused by beam primary interactions alone (when the heating caused by particles reflected back into the window from the environment is removed). Comparing the W5 and W4 models, only about 5\% of the energy deposited in a window comes from reflected particles. In addition, the energy deposition distribution among the components is about the same except for the last Inconel-718 component that exhibits the largest falloff in energy because it was previously the greatest beneficiary from reflected energy.

In summary, the heat deposited in various windows was investigated. Based on a tradeoff between structural strength and safety versus cooling required, the double-paned, half-cylinder window (W3 Model) is the preferred design.

\subsubsection{Rod Bundles}

It is important to determine the level of modeling detail required in the target bundles to achieve adequate predictions of energy deposition. Therefore, several approximations to rod bundles were tested: homogeneous plates, layered plates, homogeneous hexagons, 
homogeneous cylinders, homogeneous rectangles, and an exact bundle that included every fine detail. An example of the modeling effort is illustrated in Figs. 3-37 and 3-38. For energy deposition (unlike neutronic performance), the results show that:

- Homogeneous or layered plate representations are inadequate (especially for the second row of bundles) - this is not true for neutronic performance.

- Circular or rectangular shapes give nearly the same results as the hexagonal shape, while being much easier to model.

- Energy deposition closely follows mass distribution (as determined by shape).

- Energy deposition in the first-row center bundle can vary $\sim 11 \%$ front-to-back and $\sim 8.4 \%$ center-to-edge.

In all cases of this study, the system was designed for a $50-\mathrm{cm} \times 100-\mathrm{cm}, 1000-\mathrm{MeV}$ proton beam incident on the $55-\mathrm{cm} \times 105-\mathrm{cm}$ double-paned, half-cylinder window model. The window is followed by $50 \mathrm{~cm}$ of helium, which is followed by the first region of target material. This first region is followed by $30 \mathrm{~cm}$ of helium, which is followed by the second region of target material. The rest of the APT T/B regions were neglected and this simplified window/target geometry was used to investigate energy deposition in the first two rows of target bundles.

Modeling the bundles as individual entities rather than as layers appears to have a calculable effect (more than the above mentioned uncertainties) on the energy deposition. The individual modeling of the bundles decreases the first-row energy deposition by $\sim 5.5 \%$, increases the second-row energy deposition by $\sim 29 \%$, and increases the total energy deposition by $\sim 10 \%$.

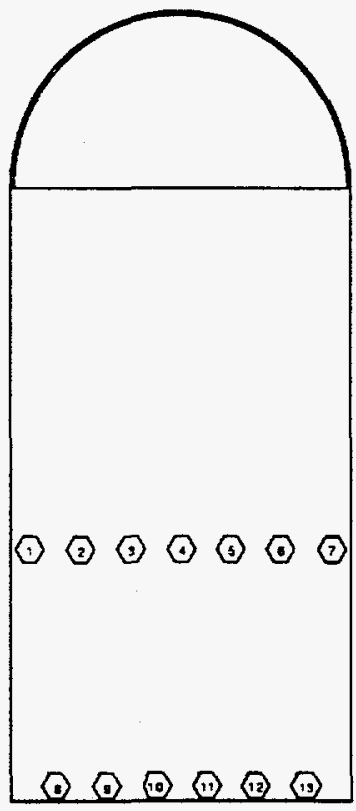

Fig. 3-37. Bundle layout and numbering for case B3, where the target regions are modeled as discrete, homogeneous hexagons. 


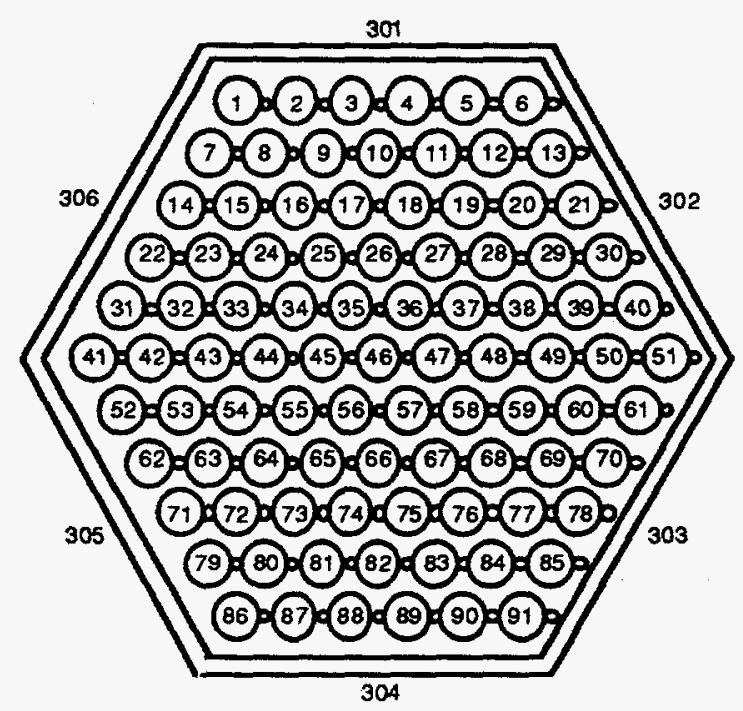

Fig. 3-38. Closeup of the layout and numbering of the detailed bundle modeled in case B4.

In comparing the hexagonal bundle modeling with the detailed bundle modeling, the detailed modeling of the middle bundle in the first row has no significant effect on the energy deposited in that bundle or on the other bundles. This model showed that the energy deposition in the first-row center bundle can vary $\sim 11 \%$ front-to-back and $\sim 8.4 \%$ center-toedge. Except for the latter result and the ability to differentiate energy deposition between coolant and target material, incorporating individual rods in a rod-bundle mockup is not necessary.

Bundles at the ends of the first row have only about $50 \%$ as much energy deposited in them as have the inner bundles because the proton beam only strikes about half of the first-row end bundles.

In summary, plate or layered plate modeling of the target region is inadequate for predicting energy deposition to better than about 30\%. Hexagonal-shaped rod bundles naturally give the best results, although they are hardest to model. Circular-shaped rod bundles and rectangular-shaped rod bundles give nearly the same results, while being easier to prototype. It appears that bundle shape can be chosen for modeling ease and flexibility, which favors the circular or rectangular shape. The results show that a weight percent breakdown of the components serves as a reasonable estimate of the deposited energy distribution in the material components.

\subsection{RADIONUCLIDE PRODUCTION (REFERENCE DESIGN)}

CINDER' 90 calculations were performed for each of 27 materials defined in the LCS Monte Carlo Calculation. Brief descriptions of these materials are given in Table 3-21. Also 
listed in Table 3-21 is the HMCNP-calculated neutron flux ( $<20 \mathrm{MeV})$ used by CINDER' 90 , and the number of nuclides created in each material as calculated by CINDER' 90 .

\begin{tabular}{|c|c|c|}
\hline Material Description & $\begin{array}{c}\text { Flux } \\
<20 \mathrm{MeV} \\
\left(\mathrm{n} \cdot \mathrm{cm}^{-2} \cdot \mathrm{s}^{-1}\right)\end{array}$ & $\begin{array}{c}\text { \# of Nuclide } \\
\text { Species } \\
\text { Produced }\end{array}$ \\
\hline Inconel-718 structure & $3.83 E+14$ & 541 \\
\hline Heavy water & $4.91 E+13$ & 46 \\
\hline SS-304 moderator tank & $1.15 \mathrm{E}+12$ & 210 \\
\hline Al-1100 structure & $1.42 E+14$ & 140 \\
\hline Radial lead target (87 v\% Pb, 10 v\% $\mathrm{D}_{2} \mathrm{O}, 3$ v\% Zirc-4) & $6.60 \mathrm{E}+14$ & 1719 \\
\hline Radial target transition zone $\left(72 \mathrm{v} \% \mathrm{~Pb}, 24 \mathrm{v} \% \mathrm{D}_{2} \mathrm{O}, 4 \mathrm{v} \%\right.$ Inconel) & $8.25 \mathrm{E}+14$ & 1604 \\
\hline 1st Zirc-4 backstop plate $\left(85 \mathrm{v} \%\right.$ Zirc- 4,15 v\% $\left.\mathrm{D}_{2} \mathrm{O}\right)$ & $8.19 \mathrm{E}+14$ & 740 \\
\hline 2nd Zirc-4 backstop plate $\left(87\right.$ v\% Zirc- $\left.4,13 \vee \% \mathrm{D}_{2} \mathrm{O}\right)$ & $7.45 \mathrm{E}+14$ & 693 \\
\hline 3rd Zirc-4 backstop plate $\left(92 \vee \%\right.$ Zirc- $\left.4,8 v \% \mathrm{D}_{2} \mathrm{O}\right)$ & $5.41 \mathrm{E}+14$ & 648 \\
\hline 4th Zirc-4 backstop plate ( 95 v\% Zirc-4, 5 v\% $\left.\mathrm{D}_{2} \mathrm{O}\right)$ & $3.72 E+14$ & 595 \\
\hline Lead backstop plate $\left(97 \mathrm{v} \% \mathrm{~Pb}, 3 \vee \% \mathrm{D}_{2} \mathrm{O}\right)$ & $2.18 \mathrm{E}+14$ & 969 \\
\hline Target chamber ${ }^{3} \mathrm{He}(300$ psia @ $400 \mathrm{~K}$ ) & $9.65 E+14$ & 5 \\
\hline${ }^{3} \mathrm{He}$ heat exchanger zone $\left(75.7 \mathrm{v} \%{ }^{3} \mathrm{He}, 24.3 \mathrm{v} \% \mathrm{Al}-1100\right)$ & $5.25 E+12$ & 100 \\
\hline Inner blanket ${ }^{3} \mathrm{He}\left(55 \mathrm{v} \%{ }^{3} \mathrm{He}, 20 \mathrm{v} \% \mathrm{Al}-1100,25 . v \% \mathrm{D}_{2} \mathrm{O}\right)$ & $1.82 \mathrm{E}+14$ & 134 \\
\hline Middle blanket ${ }^{3} \mathrm{He}$ zone $\left(53\right.$ v\% ${ }^{3} \mathrm{He}, 18$ v\% Al-1100, $\left.29 v \% \mathrm{D}_{2} \mathrm{O}\right)$ & $1.39 \mathrm{E}+13$ & 128 \\
\hline Outer blanket ${ }^{3} \mathrm{He}$ zone $\left(40 \mathrm{v} \%{ }^{3} \mathrm{He}, 23 \vee \%\right.$ Al-1100, $\left.37 \vee \% \mathrm{D}_{2} \mathrm{O}\right)$ & $2.44 \mathrm{E}+12$ & 122 \\
\hline Intrachamber ${ }^{3} \mathrm{He}$ transition zone & $1.01 E+15$ & 501 \\
\hline Inner blanket ${ }^{3} \mathrm{He}$ transition zone & $2.03 \mathrm{E}+14$ & 393 \\
\hline Backstop 3 He (100psia @ 305K) with Aluminum Struts & $5.82 \mathrm{E}+14$ & 136 \\
\hline 1st group W target material $\left(53.66 \mathrm{v} \% \mathrm{D}_{2} \mathrm{O}, 46.34 \mathrm{v} \% \mathrm{~W}\right.$ ) & $1.65 \mathrm{E}+15$ & 1407 \\
\hline 2nd group W target material $\left(49.89 \vee \% \mathrm{D}_{2} \mathrm{O}, 50.11 \mathrm{v} \% \mathrm{~W}\right)$ & $1.91 E+15$ & 1372 \\
\hline 3rd group W target material $\left(45.04 \vee \% \mathrm{D}_{2} \mathrm{O}, 54.96 \mathrm{v} \% \mathrm{~W}\right)$ & $1.91 \mathrm{E}+15$ & 1392 \\
\hline 4th group W target material $\left(40.30 \vee \% \mathrm{D}_{2} \mathrm{O}, 59.70 \mathrm{\vee} \% \mathrm{~W}\right)$ & $1.75 \mathrm{E}+15$ & 1347 \\
\hline 5th group $\mathrm{W}$ target material $\left(34.81 \vee \% \mathrm{D}_{2} \mathrm{O}, 65.19 \mathrm{v} \% \mathrm{~W}\right)$ & $1.48 \mathrm{E}+15$ & 1352 \\
\hline 6th group $\mathrm{W}$ target material $\left(32.55 \mathrm{v} \% \mathrm{D}_{2} \mathrm{O}, 67.45 \mathrm{v} \% \mathrm{~W}\right)$ & $1.17 \mathrm{E}+15$ & 1265 \\
\hline 7th group $\mathrm{W}$ target material $\left(32.55 \mathrm{v} \% \mathrm{D}_{2} \mathrm{O}, 67.45 \mathrm{v} \% \mathrm{~W}\right)$ & $8.92 \mathrm{E}+14$ & 1210 \\
\hline 8th group W target material $\left(32.55 \mathrm{v} \% \mathrm{D}_{2} \mathrm{O}, 67.45 \mathrm{v} \% \mathrm{~W}\right)$ & $6.95 E+14$ & 1163 \\
\hline
\end{tabular}

Input to CINDER'90 consists of regional medium-energy reaction destruction and production rates calculated in LAHET plus the multigroup neutron flux calculated in HMCNP; however, additional input is required to define the irradiation/cooling history.

The irradiation history is assumed to be constant for $600 \mathrm{~d}$, that is, approximating $80 \%$ availability for $2 \mathrm{yr}$, with a beam current of $200 \mathrm{~mA}$ for the irradiation period. Cooling times with increasing lengths were selected to illustrate decay properties over a period of $10^{6} \mathrm{yr}$. and a total of $\mathbf{4 0}$ time steps were used to describe the irradiation/cooling history.

All output capabilities of the CINDER' 90 post-processing code TABCODE were used. This resulted in 21 tables of output, listing properties at all 40 time steps, for each of the 27 materials. These tables were grouped into files by material and stored on the Common File 
System (CFS) at Los Alamos. A print of all files requires about 5800 pages at 64 lines/page. The files are stored in UNIX format, with universal access in the node /apt94/rev1.

It should be clear that the nuclides produced in each material are distributed over the familiar Chart of the Nuclides [3-38], with nuclide masses, A, near and below those of the target nuclides.

Decay products include stable and radioactive nuclides. Radionuclides tend to decay to longer-lived (or stable) nuclides with lower (or zero) decay energies. The total inventory of stable plus radioactive nuclides may be important to some considerations, such as chemical, metallurgical, and neutron absorption properties. The number of different nuclides produced in each of the 27 materials is given in Table 3-21.

Table 3-22 lists the temporal macroscopic neutron absorption cross section-a relatively new product of these calculations. The magnitude of the change in the macroscopic neutron absorption cross section indicates how the performance of the APT system will vary with time after the start of operation. The last column in Table 3-22 shows the percent change in the cross section after 600 days of operation for all 27 materials. The absorption cross section remains constant or increases for all materials except those with an appreciable fraction of ${ }^{3} \mathrm{He}$. The decrease observed in these materials is an artifact of the calculation, and is a result of the decrease in ${ }^{3} \mathrm{He}$ inventory as it is converted to tritium. In reality, the tritium is continuously extracted and the converted ${ }^{3} \mathrm{He}$ is replenished so that the absorption cross section of these zones will remain relatively constant during operation. The largest increase in absorption cross section occurs in the radial lead target, where the increase ranges from 7 to $14 \%$. Because lead has such a low cross section, the addition of even trace amounts of spallation products can have a big impact on the neutronic performance of lead. The radial target transition zone is just above and below the rod bundles. This section of the radial target sees more high-energy particles due to the rectangular shape of the beam footprint; consequently it participates in a greater fraction of spallation reactions per unit volume than does the rest of the radial target. Since the spallation products have, on average, a higher absorption cross section than does lead, this region of the radial target suffers a larger increase in its absorption cross section. The impact that these increases in neutron absorption have on the APT system performance must be determined by an LCS calculation in which the initial material compositions are replaced by the material compositions as calculated by CINDER' 90 at the end of a typical operating cycle. This calculation has yet to be performed.

Figure 3-39 illustrates the calculated distribution of spallation products for the ${ }^{3} \mathrm{He}$ target/blanket system showing that spallation products lie on both sides of the line of stability. Figure 3-40 shows the distribution of spallation product masses by element $\mathrm{Z}$ before and after irradiation by $1-\mathrm{GeV}$ protons.

\subsubsection{Source Term}

The source term associated with irradiated material generally means the inventory of radionuclides present in units of atoms, kilograms, or Ci. Major contributions to decay properties generally vary with time, with short-lived radionuclides dominant during irradiation, and at short cooling times. Long-lived radionuclides typically dominate at longer cooling 
times. An examination of major decay-property contributions in the various regions is beyond the scope of this section.

Table 3-22. Summary of CINDER'90 Calculated Time Dependence of the Macroscopic Neutron Absorption During Operation

\begin{tabular}{|c|c|c|c|c|c|c|}
\hline Material & $\begin{array}{c}\text { At Beam } \\
\text { Turnon }\end{array}$ & 1 day & 10 days & 100 days & 600 days & $\begin{array}{c}\% \\
\text { change }\end{array}$ \\
\hline Inconel-718 structure & $1.01 E-02$ & $1.01 \mathrm{E}-02$ & $1.01 E-02$ & $1.03 \mathrm{E}-02$ & $1.06 \mathrm{E}-02$ & 5.4 \\
\hline Heavy water & $2.19 \mathrm{E}-04$ & $2.19 \mathrm{E}-04$ & $2.20 \mathrm{E}-04$ & $2.25 \mathrm{E}-04$ & 2.33E-04 & 6.3 \\
\hline SS-304 moderator tank & $3.07 \mathrm{E}-02$ & 3.07E-02 & $3.07 \mathrm{E}-02$ & $3.07 \mathrm{E}-02$ & $3.07 \mathrm{E}-02$ & 0.0 \\
\hline Al-1100 structure & $1.42 \mathrm{E}-03$ & $1.42 \mathrm{E}-03$ & $1.42 \mathrm{E}-03$ & $1.42 \mathrm{E}-03$ & $1.43 \mathrm{E}-03$ & 1.1 \\
\hline Radial lead target & 7.31E-04 & 7.31E-04 & 7.33E-04 & $7.43 E-04$ & $7.85 \mathrm{E}-04$ & 7.3 \\
\hline Radial target transition zone & $1.39 \mathrm{E}-03$ & $1.39 \mathrm{E}-03$ & $1.40 \mathrm{E}-03$ & $1.45 \mathrm{E}-03$ & $1.58 \mathrm{E}-03$ & 13.7 \\
\hline 1st Zirc-4 backstop plate & $3.46 \mathrm{E}-03$ & $3.46 \mathrm{E}-03$ & $3.46 \mathrm{E}-03$ & $3.49 \mathrm{E}-03$ & $3.61 \mathrm{E}-03$ & 4.1 \\
\hline 2nd Zirc-4 backstop plate & $3.39 \mathrm{E}-03$ & 3.39E-03 & $3.39 \mathrm{E}-03$ & $3.41 E-03$ & $3.50 \mathrm{E}-03$ & 3.2 \\
\hline 3rd Zirc-4 backstop plate & $3.44 \mathrm{E}-03$ & $3.44 \mathrm{E}-03$ & $3.44 \mathrm{E}-03$ & $3.45 \mathrm{E}-03$ & $3.50 \mathrm{E}-03$ & 1.9 \\
\hline 4th Zirc-4 backstop plate & $3.49 \mathrm{E}-03$ & $3.49 E-03$ & $3.49 \mathrm{E}-03$ & $3.49 \mathrm{E}-03$ & $3.52 \mathrm{E}-03$ & 1.1 \\
\hline Lead backstop plate & $5.11 \mathrm{E}-04$ & $5.11 \mathrm{E}-04$ & $5.11 \mathrm{E}-04$ & $5.14 \mathrm{E}-04$ & $5.30 \mathrm{E}-04$ & 3.7 \\
\hline Target chamber ${ }^{3} \mathrm{He}$ & $2.07 \mathrm{E}-03$ & 2.07E-03 & $2.06 \mathrm{E}-03$ & $1.98 \mathrm{E}-03$ & $1.59 \mathrm{E}-03$ & -23.2 \\
\hline${ }^{3} \mathrm{He}$ heat exchanger zone & $1.98 \mathrm{E}-01$ & $1.98 \mathrm{E}-01$ & $1.97 \mathrm{E}-01$ & $1.92 \mathrm{E}-01$ & $1.65 \mathrm{E}-01$ & -16.5 \\
\hline Inner blanket ${ }^{3} \mathrm{He}$ zone & $1.36 \mathrm{E}-02$ & $1.35 \mathrm{E}-02$ & $1.32 \mathrm{E}-02$ & $1.08 \mathrm{E}-02$ & $3.82 \mathrm{E}-03$ & -71.8 \\
\hline Middle blanket ${ }^{3} \mathrm{He}$ zone & $1.03 \mathrm{E}-01$ & $1.03 \mathrm{E}-01$ & $1.01 E-01$ & 8.95E-02 & $4.67 \mathrm{E}-02$ & -54.6 \\
\hline Outer blanket ${ }^{3} \mathrm{He}$ zone & 7.34E-02 & 7.34E-02 & $7.32 \mathrm{E}-02$ & 7.17E-02 & $6.44 \mathrm{E}-02$ & -12.3 \\
\hline Intrachamber ${ }^{3} \mathrm{He}$ transition zone & $4.00 \mathrm{E}-03$ & $3.99 \mathrm{E}-03$ & $3.96 \mathrm{E}-03$ & $3.64 \mathrm{E}-03$ & $2.34 \mathrm{E}-03$ & -41.5 \\
\hline Inner blanket ${ }^{3} \mathrm{He}$ transition zone & $1.65 \mathrm{E}-02$ & $1.65 \mathrm{E}-02$ & $1.60 \mathrm{E}-02$ & $1.16 \mathrm{E}-02$ & $2.61 \mathrm{E}-03$ & -84.2 \\
\hline Backstop ${ }^{3} \mathrm{He}$ material & $4.81 E-03$ & $4.81 E-03$ & $4.74 \mathrm{E}-03$ & $4.14 E-03$ & $2.05 E-03$ & -57.3 \\
\hline 1st group $W$ target material & $1.48 \mathrm{E}-02$ & $1.48 \mathrm{E}-02$ & $1.48 \mathrm{E}-02$ & $1.50 \mathrm{E}-02$ & $1.59 \mathrm{E}-02$ & 7.0 \\
\hline 2nd group W target material & $1.65 \mathrm{E}-02$ & $1.65 \mathrm{E}-02$ & $1.65 \mathrm{E}-02$ & $1.67 \mathrm{E}-02$ & $1.76 \mathrm{E}-02$ & 6.8 \\
\hline 3rd group $W$ target material & $1.71 \mathrm{E}-02$ & $1.71 \mathrm{E}-02$ & $1.71 \mathrm{E}-02$ & $1.72 \mathrm{E}-02$ & $1.82 \mathrm{E}-02$ & 6.6 \\
\hline 4th group W target material & $1.78 \mathrm{E}-02$ & $1.78 \mathrm{E}-02$ & $1.78 \mathrm{E}-02$ & $1.80 \mathrm{E}-02$ & $1.88 \mathrm{E}-02$ & 5.6 \\
\hline 5th group W target material & $1.87 \mathrm{E}-02$ & $1.87 \mathrm{E}-02$ & $1.87 \mathrm{E}-02$ & $1.88 \mathrm{E}-02$ & $1.95 \mathrm{E}-02$ & 4.6 \\
\hline 6th group W target material & $2.01 \mathrm{E}-02$ & $2.01 \mathrm{E}-02$ & $2.01 \mathrm{E}-02$ & $2.02 \mathrm{E}-02$ & $2.08 \mathrm{E}-02$ & 3.5 \\
\hline 7th group W target material & $2.12 \mathrm{E}-02$ & $2.12 \mathrm{E}-02$ & $2.13 \mathrm{E}-02$ & $2.13 \mathrm{E}-02$ & $2.18 \mathrm{E}-02$ & 2.7 \\
\hline 8th group W target material & $2.53 \mathrm{E}-02$ & $2.53 \mathrm{E}-02$ & $2.53 \mathrm{E}-02$ & $2.54 \mathrm{E}-02$ & $2.59 \mathrm{E}-02$ & 2.3 \\
\hline
\end{tabular}

The calculated reference design total activities, summed over all regions, are slightly lower than total values calculated for the earlier (PEIS) design during the 600-d irradiation history and for cooling times shorter than one day using the same codes, data, and incident beam assumptions. For longer cooling times less than $1000 \mathrm{yr}$., the reference design values are as much as $12 \%$ greater than the earlier values; this difference grows to $170 \%$ greater at one million years cooling when the total activity is negligible.

\subsubsection{Decay Power}

One important property of radionuclides produced in APT is the aggregate decay power. The decay power associated with a nuclide varies with time, and it is calculated simply as the 


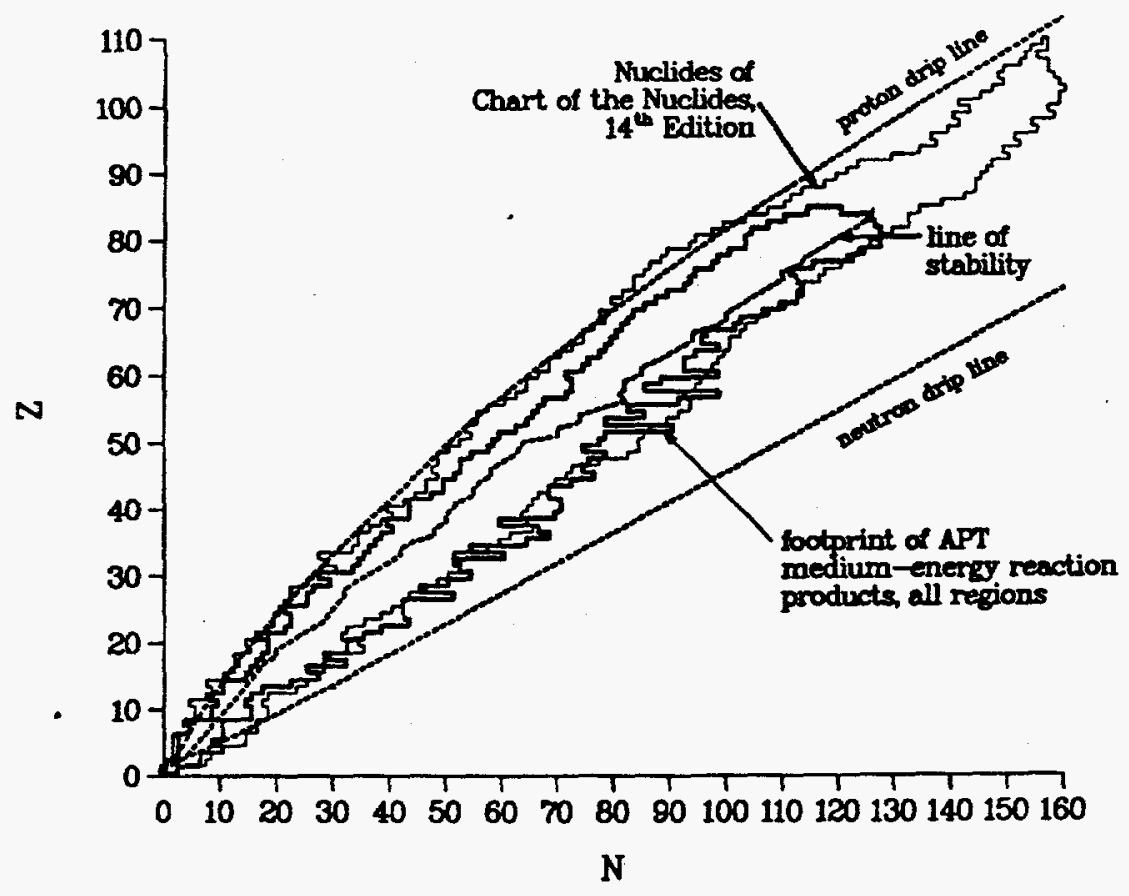

Fig. 3-39. Nuclide chart with APT reference design medium-energy reaction products.

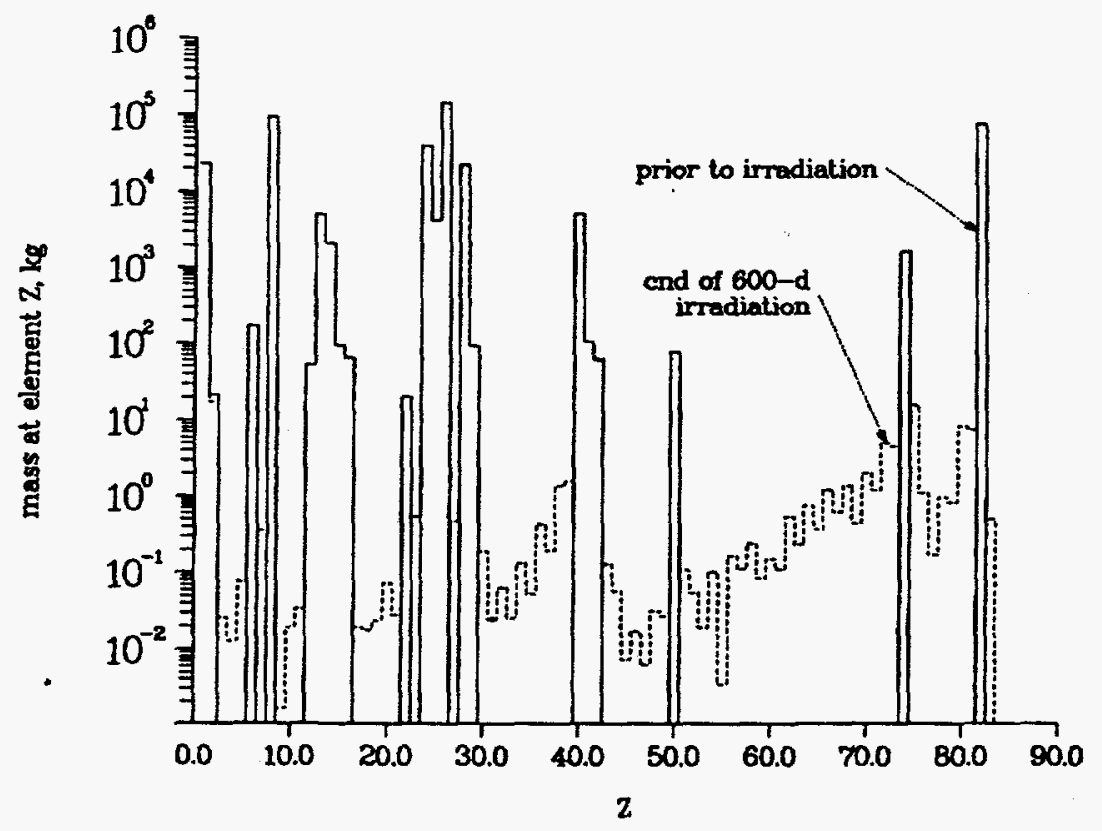

Fig. 3-40. Distribution of APT mass by element $Z$. 
product of the nuclides activity and its average recoverable energy per decay. The total, or aggregate, decay power is the sum of the decay powers associated with each of the nuclides. The use of such summation methods in decay power calculations is widely accepted in the nuclear reactor industry and is an important component of the ANS/ANSI 5.1 Decay Power Standard for LWRs [3-39]. Comparisons of reactor-decay-power experiments and summation calculations have shown agreement within a few percent, reflecting the quality of fission-yield and cross-section data used in the summation calculations [3-40]. The LCS medium-energy reaction-product yields and the cross-section data used in APT decay-power calculations have improved greatly in the past few years. The validity of LCS/CINDER'90 calculations of APT decay power has not been experimentally verified. Therefore some arbitrarily large uncertainty-perhaps $25 \%$-is ascribed to the aggregate APT decay power.

The calculated decay power reveals an important safety feature of the APT systemwithin one second after proton beam shutdown, decay heat in the T/B is less than $1 \%$ of the steady-state power. Figure 3-41 shows a comparison of the time dependence of the relative core power for shutdown of the NPR Heavy Water Reactor versus the ${ }^{3} \mathrm{He}$ APT target/blanket.

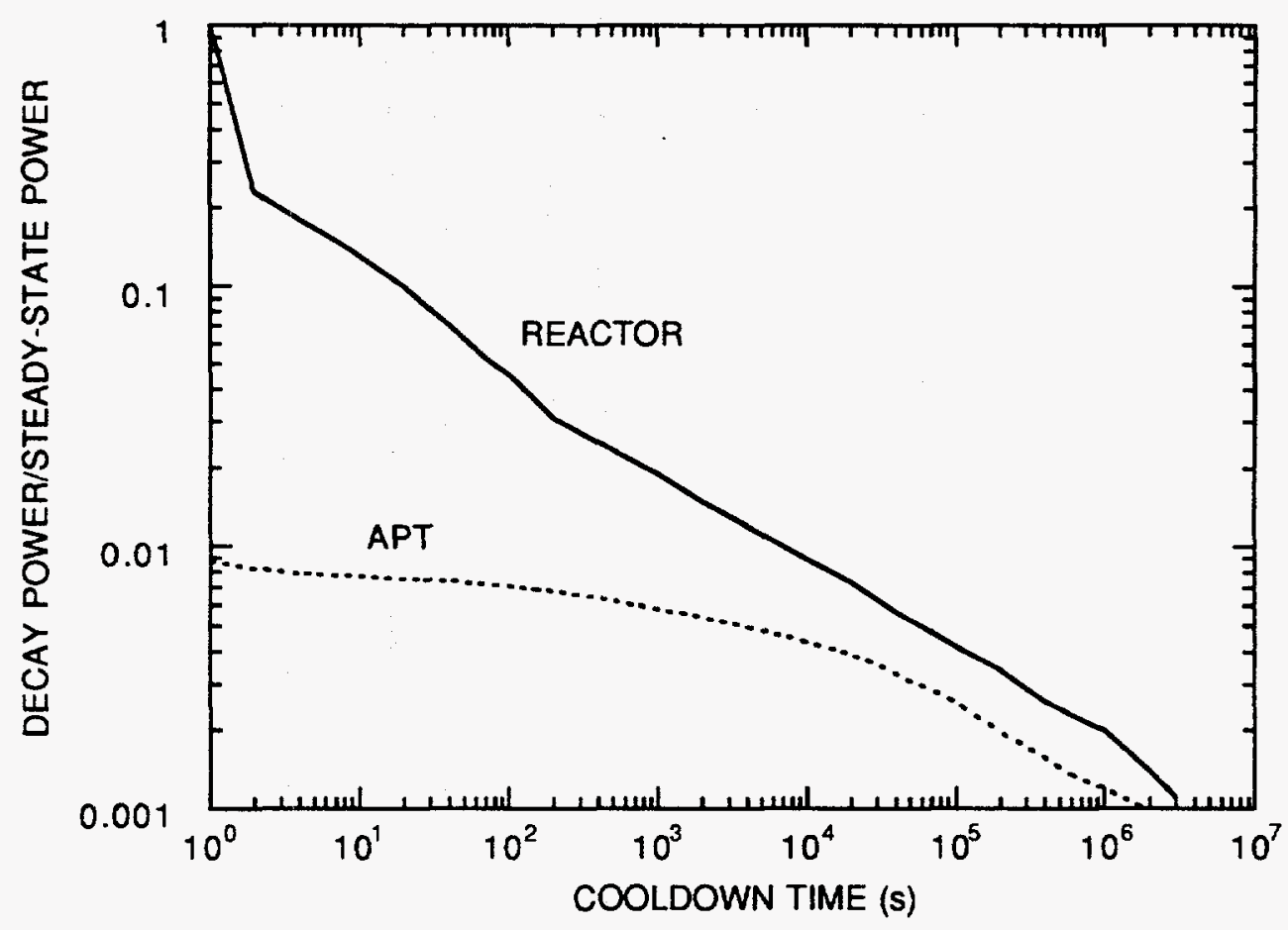

Fig. 3-41. Time dependence of the decay power for the ${ }^{3} \mathrm{He}$ APT target/blanket and the relative core power for shutdown of the NPR Heavy Water Reactor. 


\subsection{SHIELDING}

\subsubsection{Introduction}

Shielding a spallation neutron source is more difficult than shielding a reactor neutron source [3-41] because spallation neutrons have higher energies than fission neutrons. For a spallation neutron source, the highest-energy cascade neutrons approach the energy of the incident proton beam $(e . g .1 \mathrm{GeV})$. Those high-energy neutrons are extremely penetrating and are largely ineffective in producing tritium. Well-designed shielding is needed to prevent highenergy neutrons from causing excessive biological dose rates.

For spallation reactions, we divide energy into two regions: low-energy $(<20 \mathrm{MeV})$ and high-energy ( $>20 \mathrm{MeV}$ ). The $20-\mathrm{MeV}$ energy cutoff is convenient because the continuousenergy cross-section libraries used by HMCNP in our calculations have evaluated cross sections up to $20 \mathrm{MeV}$. As discussed in Section 3.1, low-energy neutron production from a target is the low-energy spallation neutron production plus the net production from low-energy "( $n, x n)$ " reactions. These low-energy neutrons are emitted more or less isotropically and cause shielding problems like those for fission reactors. Figure 3-42 compares a fission neutron spectrum to a $90^{\circ}$ (relative to the proton beam direction) spallation neutron spectrum. The spallation spectrum is characteristic of that calculated for a LANSCE-type target (10-cmdiam $\times 30-\mathrm{cm}$-long cylindrical tungsten target bombarded on axis by $800-\mathrm{MeV}$ protons). Note the presence of the high-energy tail in the spallation neutron spectrum.

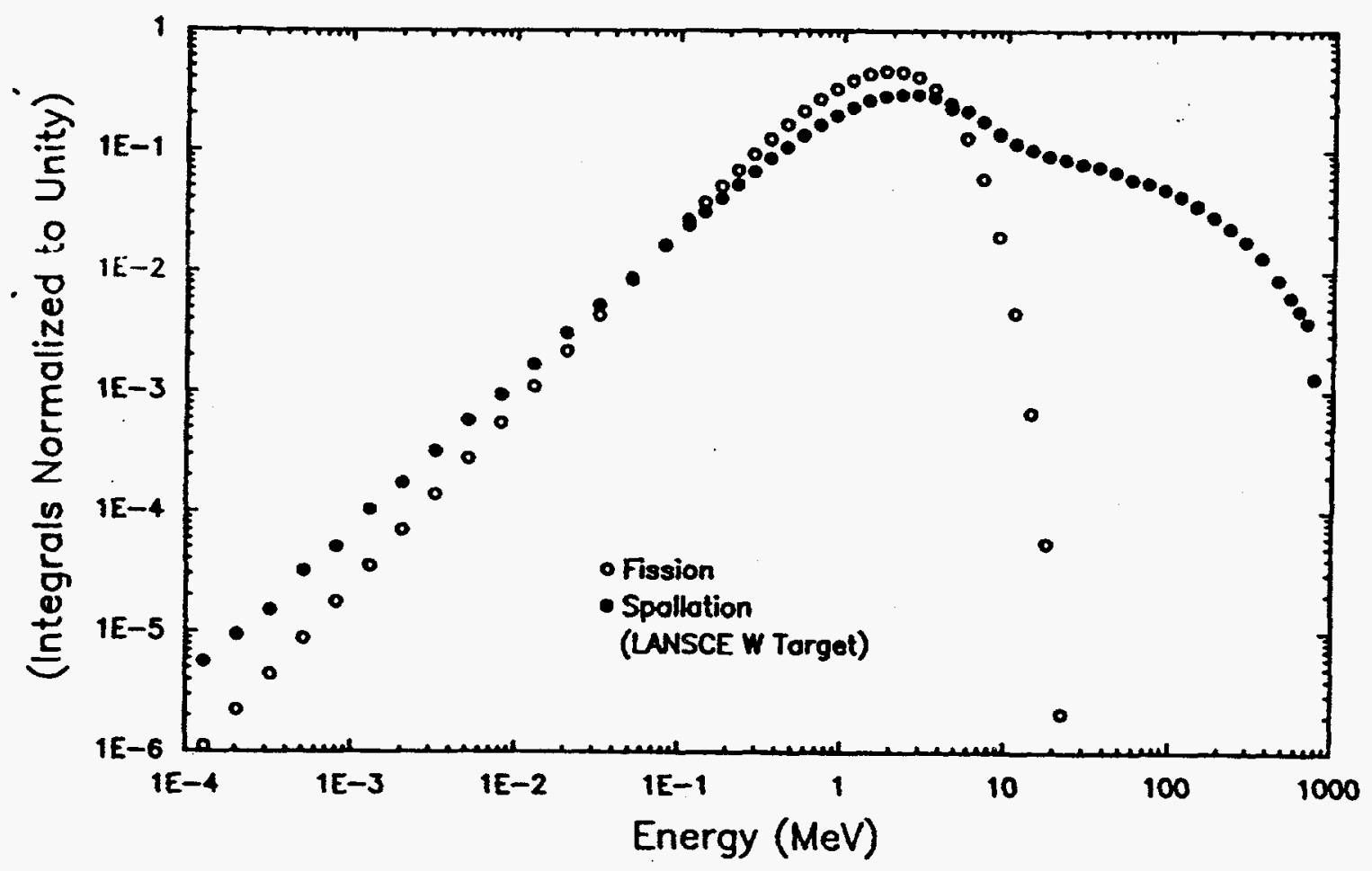

Fig. 3-42. Comparison of neutron production spectra from fission and spallation. 
Spallation source shielding is further complicated by the angular variation of the highenergy neutrons. Iron combined with concrete is required to shield the high-energy neutron component at a spallation neutron source. Concrete or water is an effective neutron shield for a fission neutron source. Consequently, the iron-concrete high-energy neutron shield at a spallation source is also an effective shield for the low-energy spallation neutron component.

High-energy neutrons resulting from nucleon-nucleon reactions have a strong angular dependence. At $0^{\circ}$ to the proton beam direction, high-energy neutrons can have energies up to the incident proton energy. As the angle increases with respect to the proton beam, the highenergy neutron spectrum softens considerably. We illustrate this effect in Fig. 3-43 for 800$\mathrm{MeV}$ protons incident on a thin target of iron (a cross-section calculation). The presence of these high-energy neutrons and their strong angle-dependence are two reasons why shielding a spallation neutron source is quite different than shielding a reactor neutron source.

Shielding a spallation source is also more complex than shielding a reactor source because different leakage neutron spectra are produced depending on whether the proton beam strikes a thin or thick target. In addition, neutron production is also material dependent. Another complexity arises in shielding a spallation source when a shield attenuates high-energy neutrons, because low-energy neutrons are produced (i.e., the shield itself becomes a neutron source). Depending on the application (e.g., thin vs. thick shields), the high-energy neutrons plus their progeny may dominate the dose at a shield surface. Also, spallation reactions in the

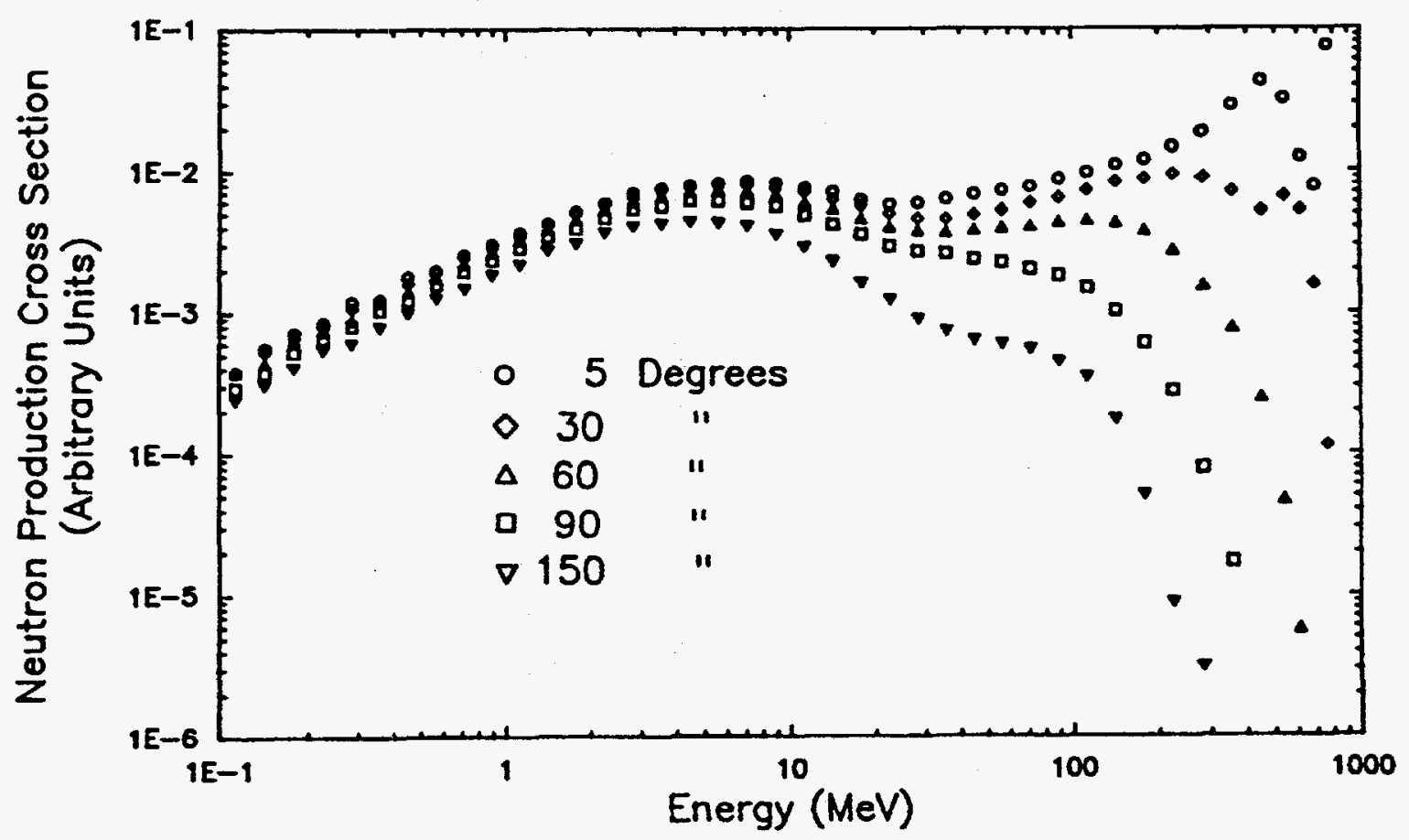

Fig. 3-43. Energy-dependent neutron production cross-sections for 800$\mathrm{MeV}$ protons incident on iron as a function of angle with respect to the proton beam. 
shield create an additional gamma ray source that is not present at reactor source. Shielding a fission neutron source is similar to shielding the primary low-energy spallation neutrons at a spallation neutron source. Typical shielding at a reactor source is light water or concrete; typical shielding at a spallation source is iron followed by concrete.

For a spallation neutron source, the incident neutron spectrum and the shield geometry, composition, and thickness determine whether high-energy or low-energy neutrons dominate the neutron dose at the shield surface and also determine the relative importance of gamma rays. For the first phase of our APT shield design, the criteria for setting the shield thickness was a biological dose rate of $0.1 \mathrm{mR} / \mathrm{h}$ at the shield surface. Other criteria may dominate our APT shield design such as component activation (which affect maintenance and plant availability) or groundwater activation. We plan to look at APT shielding in more detail in FY94, but we first must set the criteria which will drive the shield design.

\subsubsection{Shielding Model}

Preliminary estimates of radiological shielding requirements of the APT target were made using a shielding model developed by Wilkinson and Frankle [3-42]. This model was used to estimate beamspill shielding requirements at LAMPF and is based on the empirical Moyer shielding model [3-43]. For a point source, the dose rate $D$ is given by

$$
D(r, \theta)=\frac{i_{b} H_{0}}{r^{2}} \exp (-\beta \theta) \exp \left(-\sum_{i} \frac{d_{i}}{\lambda_{i}}\right),
$$

where $r$ is the distance between the beamspill point $S$ and a point $P$ at which the dose is measured, $\theta$ is the angle between the beam axis and a line passing through $S$ and $P, i_{b}$ is the proton beam current, $H_{0}$ is the source term, $\beta$ is the angular relaxation, and $d_{i}$ and $\lambda_{i}$ are the path length and dose attenuation length in material $i$. The values of $H_{0}, \beta$, and $\lambda_{i}$, shown in Table 3-23, have been determined empirically by fitting the above equation to results of Monte Carlo simulations of $800-\mathrm{MeV}$ protons impinging on various typical shielding materials.

Table 3-23. Results of Least-Squares Fits of Eq. (3-3) to Monte Carlo Beamspill Simulations*

\begin{tabular}{lll}
\hline & $0 \leq \theta \leq \mathbf{3 0}$ & $\mathbf{6 0}$ \\
$H_{0}\left(\mathrm{mR} \cdot \mathrm{m}^{2} / \mathrm{h} / \mathrm{mA}\right)$ & $8.642 \times 10^{11}$ & $2.955 \times 10^{\circ}$ \\
$\beta$ & 2.06 & 2.3 \\
$\lambda(\mathrm{m})$ & & \\
$\quad$ concrete $\left(2.42 \mathrm{~g} / \mathrm{cm}^{3}\right)$ & 0.57 & 0.50 \\
$\quad$ magnetite concrete $\left(3.63 \mathrm{~g} / \mathrm{cm}^{3}\right)$ & 0.43 & 0.40 \\
$\quad$ tuff $\left(1.6 \mathrm{~g} / \mathrm{cm}^{3}\right)$ & 0.87 & 0.76 \\
$\quad$ natural iron $\left(7.87 \mathrm{~g} / \mathrm{cm}^{3}\right)$ & 0.35 & 0.20 \\
\hline \hline
\end{tabular}

*from Wilkinson and Frankle. 


\subsubsection{Comparisons between Shielding Model, Simulations, and Experiment}

To validate the applicability of this shielding model to a spallation target, we compared results from this model to 3 different targets for which experimental data exist: Case I, a 5-cmdiam $\times 25$-cm-long tungsten target placed in the LANSCE target crypt and surrounded by the LANSCE biological shield; Case II, a 10-cm-diam $\times 25$-cm-long tungsten target placed in the LANSCE target crypt, surrounded by both the reflector-moderator-shield and the LANSCE biological shield; and Case III, a bare 50-cm-diam $\times 200$-cm-long graphite beamstop.

A LAHET simulation of Case I yielded high-energy $(>20 \mathrm{MeV})$ neutron dose rates at the upstream, downstream, and cylindrical surfaces of $7.04 \times 10^{12}, 1.25 \times 10^{12}$, and $5.93 \times 10^{12}$ $\mathrm{mR} / \mathrm{h} / \mathrm{mA}$, respectively; these sum to give a total high-energy neutron source term of $2.49 \times 10^{11} \mathrm{mR} \cdot \mathrm{m}^{2} / \mathrm{h} / \mathrm{mA}$. The difference between this value and the values of $H_{0}$ listed in Table 3-23 may be attributed to self-shielding. For a dose attenuation length in tungsten [3-44] of $0.14 \mathrm{~m}$, the dose at the downstream surface of the target as calculated using the Moyer model and the values listed in Table 3-23 is $2.41 \times 10^{12} \mathrm{mR} / \mathrm{h} / \mathrm{mA}$, within a factor of two of the calculated value listed above. The Moyer model was used to calculate the dose rate at $90^{\circ}$ to the beam axis, assuming a point source with $H_{0}=2.49 \times 10^{11} \mathrm{mR} \cdot \mathrm{m}^{2} / \mathrm{h} / \mathrm{mA}$ and a beam current of $18 \mu \mathrm{A}$. Between the point source and outer shield surface we assume there is $1.02 \mathrm{~m}$ air, $2.85 \mathrm{~m}$ steel, and $0.84 \mathrm{~m}$ magnetite concrete. The dose predicted by the model is $0.50 \mathrm{mR} / \mathrm{h}$, in good agreement with the measured value.

For Case $\Pi$, the total high-energy neutron source term as calculated by LAHET is $1.95 \times$ $10^{11} \mathrm{mR} \cdot \mathrm{m}^{2} / \mathrm{h} / \mathrm{mA}$. Again, self-shielding accounts for the fact that this larger diameter target has a smaller source term than that of Case I. As in Case I, the Moyer model was used to calculate the dose rate at $90^{\circ}$ to the beam axis, assuming a point source with $H_{0}=1.95 \times 10^{11}$ $\mathrm{mR} \cdot \mathrm{m}^{2} / \mathrm{h} / \mathrm{mA}$ and a beam current of $75 \mu \mathrm{A}$. Between the point source and outer shield surface we assume there is $0.39 \mathrm{~m}$ air, $0.30 \mathrm{~m}$ nickel $(\lambda=0.19 \mathrm{~m} \mathrm{[3-46]),} 3.15 \mathrm{~m}$ steel, and $0.84 \mathrm{~m}$ magnetite concrete. The dose predicted by the model is $0.076 \mathrm{mR} / \mathrm{h}$, in good agreement with the measured value of $<1 \mathrm{mR} / \mathrm{h}[3-47]$.

The geometry for Case III is shown in Fig. 3-44. The calculated and measured dose rates for this case have been described in detail elsewhere [3-47]. Unlike Cases I and II, a source term for the carbon target has not been calculated; instead, we use the value listed in Table 3-24. Between the source and detector we assume there is $27 \mathrm{~cm}$ of carbon $(\lambda=0.50 \mathrm{~m}$, [3-44]), $613 \mathrm{~cm}$ of air, and $162 \mathrm{~cm}$ (approx. $5.32 \mathrm{ft}$.) of concrete or magnetite concrete because these two materials should bound the dose resulting from reinforced concrete. The angle between the beam axis and the detector is $70^{\circ}$, and the beam current is $100 \mathrm{nA}$. Results are shown in Table 3-24. The Moyer model does a good job of predicting the expected dose.

Table 3-24. Comparison of Moyer Model Results for Case III with Calculated and Experimental Results

\begin{tabular}{|c|c|c|c|}
\hline Shield material & Calculated [3-47] & $\begin{array}{c}\text { Total dose }(\mathrm{mR} / \mathrm{h}) \\
\text { Moyer model }\end{array}$ & Experimental [3-47] \\
\hline Magnetite concrete & 448 & 373 & \\
\hline Reinforced concrete & & $601^{*}$ & 500 to 700 \\
\hline Regular concrete & 1090 & 631 & \\
\hline
\end{tabular}

*Assumes reinforced concrete is $1 \%$ iron. 


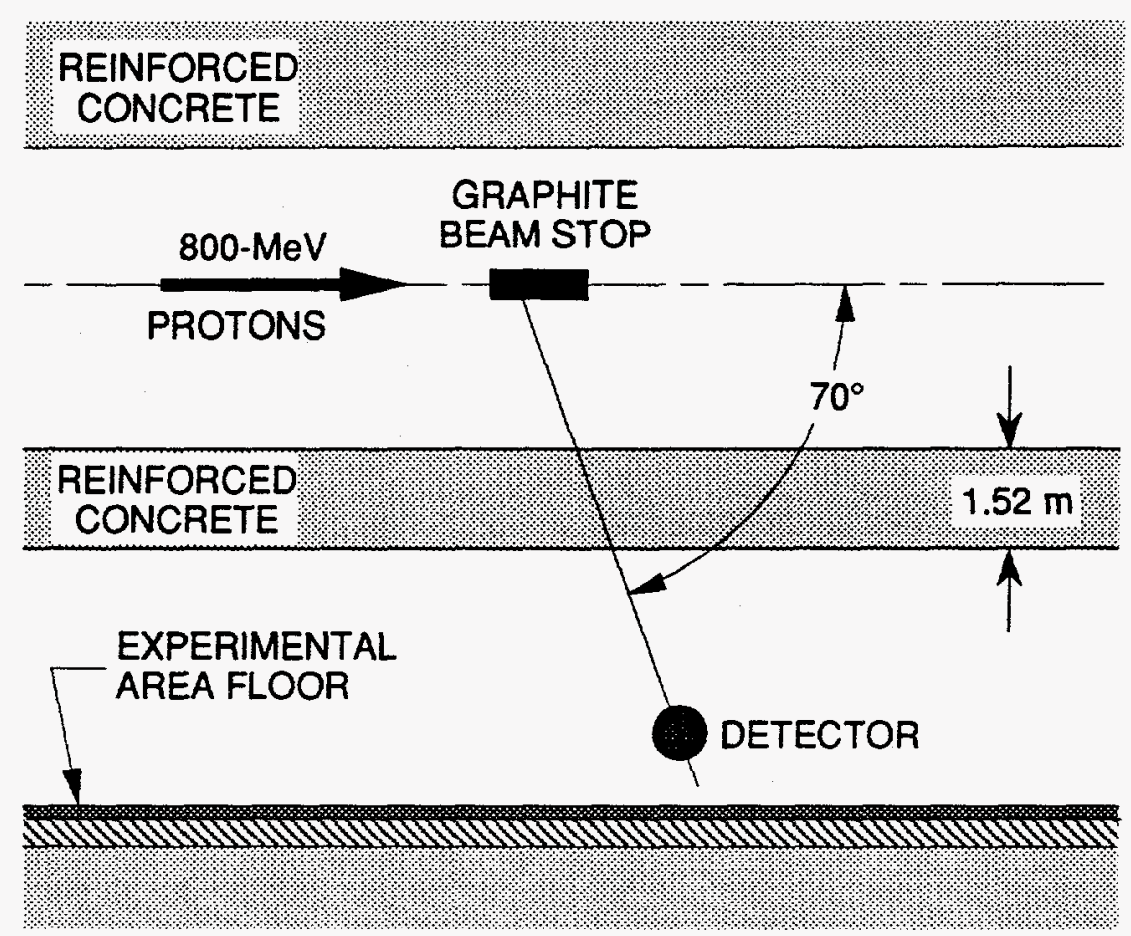

Fig. 3-44. Case III geometry.*

$* 800$-MeV protons strike a 50 -cm-diam $\times 200$-cm-long bare graphite beamstop. A detector is placed in the experimental area, one floor below the beamstop. The angle between the beam axis and a line drawn from the beamstop through the detector is approximately $70^{\circ}$. The vertical distance between the beam axis and the detector is about $7.5 \mathrm{~m}$. The reinforced concrete floor is $1.52 \mathrm{~m}$ thick.

\subsubsection{Application of the Shielding Model to the $3 \mathrm{He}$ T/B System}

The source term $H_{0}$ takes into account that the shield itself is a source of high-energy ( $>20$ $\mathrm{MeV}$ ) neutrons through spallation. Figure 3-45 shows the average high-energy neutron dose $\bar{D}$ and energy $\bar{E}$ for the upstream, downstream, and radial leakage surfaces of the APT target as estimated by LAHET for the APT Physics-III model. To derive a source term for the APT target, we have summed the surface area-integrated high-energy neutron dose over all leakage surfaces. This approach is more conservative than, for example, calculating the dose at $\theta=90^{\circ}$ due solely to the radially emitted high-energy neutron flux. The source term arrived at in this manner is $H_{0}=2.026 \times 10^{10} \mathrm{mR} \cdot \mathrm{m}^{2} / \mathrm{h} / \mathrm{mA}$. This value is somewhat lower than those calculated by Wilkinson and Frankle because very few high-energy protons are passing through the target outer surface, whereas in modeling beamspill, beam protons are presumably the primary contributors to the source term. That is to say, this source term has taken into account the self-shielding of the T/B system, whereas those listed in Table 3-23 are unattenuated source terms. In applying this beamspill model to the APT target, we have attempted to incorporate assumptions in such a way as to overestimate the shield thickness. The target is modeled as a point source with no gap between the source and shield. 


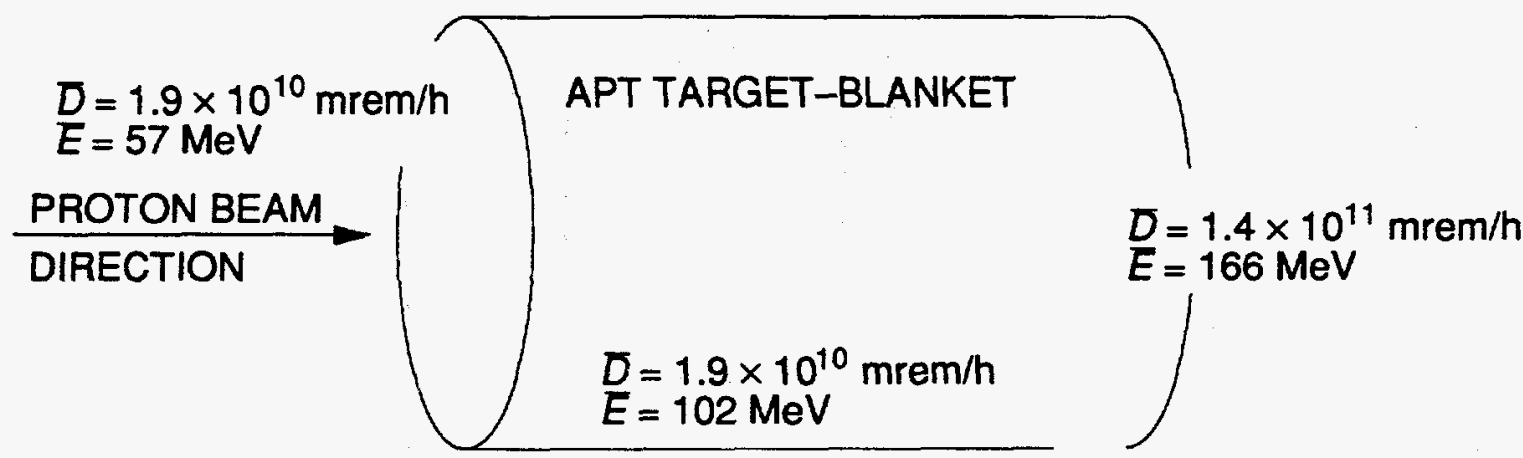

Fig. 3-45. Average high-energy neutron dose and energy from the upstream, downstream, and radial surfaces of the $A P T T / B$, as calculated by the Monte Carlo code LAHET. The proton beam current is assumed to be $200 \mathrm{~mA}$.

For the purpose of estimating shield thickness vs. dose rate at the shield surface, the shield envisioned is iron, interspersed with laminations of magnetite concrete to address the $25-\mathrm{keV}$ window in iron. This would then be followed by a final 1-m layer of magnetite concrete to attenuate low-energy neutrons. Thus, we define here an inner shield (of variable thickness) to consist of $90 \%$ iron and $10 \%$ magnetite concrete and an outer shield (of 1-m thickness) of pure magnetite concrete. As applied to this problem, the Moyer model assumes homogeneity in the shield material. In reality, a composite shield, if properly designed, would be thinner than an equivalent homogeneous shield. In this respect, an estimate of shield thickness based on this model is conservative. Figure $3-46$ is a plot of dose rate vs. inner shield thickness. Based on these results, a radial and upstream shield of $6-\mathrm{m}$ thickness would result in a dose $<0.1 \mathrm{mR} / \mathrm{h}$.

The amount of shielding above the target is fixed by limiting the biological dose rate in the support building (shown in Fig. 3-47) while the accelerator is operating to $0.1 \mathrm{mR} / \mathrm{h}$. A schematic of the APT target region shielding layout is shown in Fig. 3-47. Equipment activation above the target is also a concern, but we expect that if the shield is sufficiently thick to protect personnel, equipment activation will be negligible. On the sides of the target, the shielding is driven by equipment and/or soil and groundwater activation. The downstream end is governed by soil and groundwater activation. Estimates of equipment, soil, and groundwater activation are beyond the scope of this study.

Calculating the biological dose at the support building is straightforward. Based on present estimates of berm thickness and moderator tank (the structure into which the T/B is placed) height, the distance from the top of the moderator tank (where the shielding starts) to the floor of the support building is $13.17 \mathrm{~m}$; this is the value of $r$ in Eq. (1). We then calculate the inner shield thickness required to give the desired dose at the support building. For a dose rate of $0.1 \mathrm{mR} / \mathrm{h}$, the total (inner $+1 \mathrm{~m}$ outer) shield thickness is $5.32 \mathrm{~m}$.

\subsection{TRITIUM IMPLANTATION AND DIFFUSION}

The walls of the Inconel-718 target chamber and hex cans, as well as the aluminum $3 \mathrm{He}$ containers in the blanket of the APT target, are constantly bombarded by high-energy tritons 


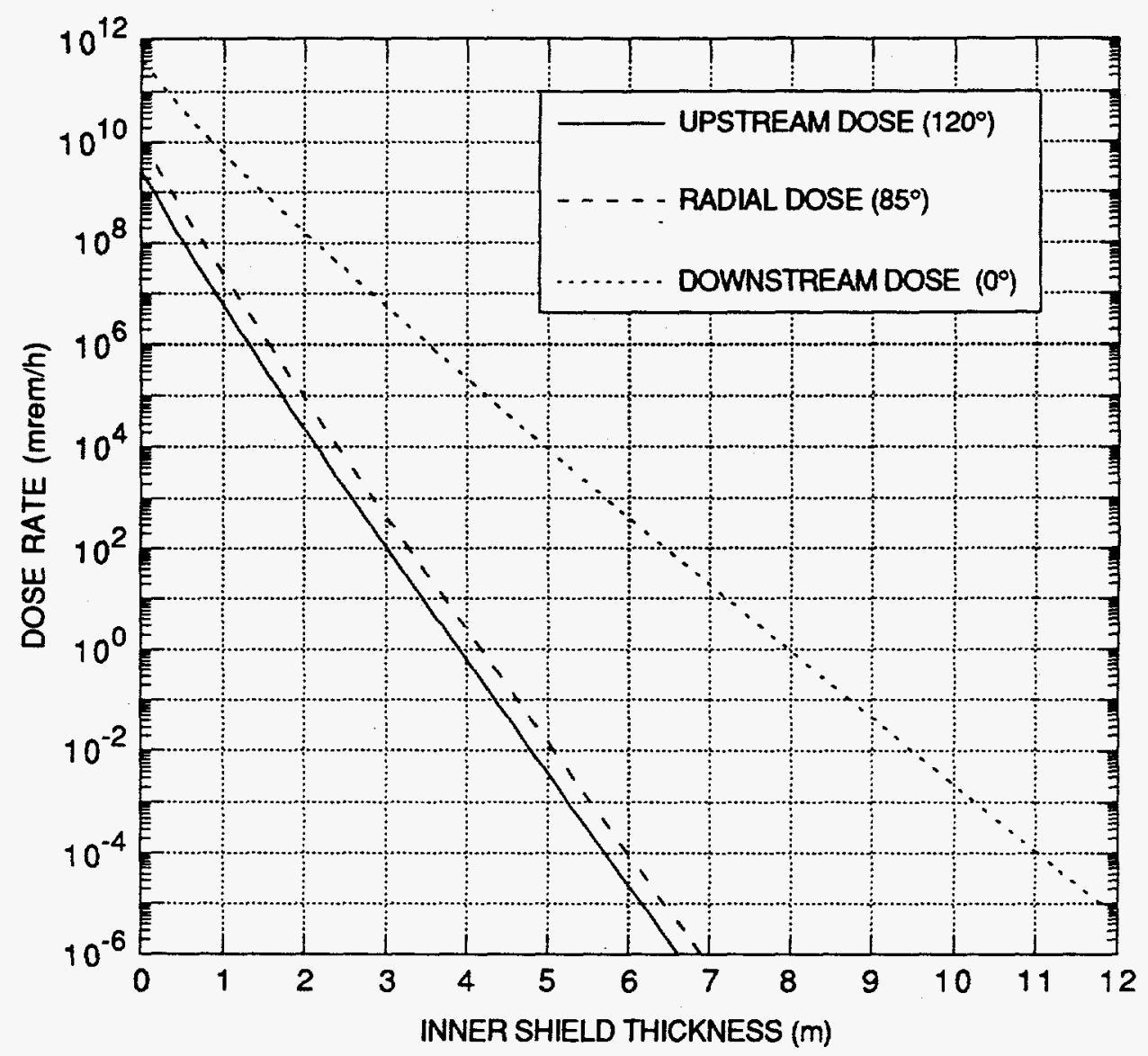

Fig. 3-46. Dose rate at the shield outer surface vs. inner shield thickness.*

*Inner shield composition is $90 \%$ iron / $10 \%$ magnetite concrete, followed by an outer shield of $1 \mathrm{~m}$ of magnetite concrete. Total shield thickness is inner shield thickness plus $1 \mathrm{~m}$.

during operation. Most of the tritons are created when a ${ }^{3} \mathrm{He}$ atom absorbs a low-energy neutron:

$$
n+{ }^{3} \mathrm{He} \rightarrow p+\mathrm{T}
$$

The kinetic energy of the triton emerging from this reaction is $192 \mathrm{keV}$, and, as a first approximation, it can be assumed that the tritons are emitted isotropically in the center-of-mass frame. The probability that an ion would be implanted in a given material is essentially a function of its kinetic energy (as well as other parameters, such as surface topography, presence of adsorbates, and surface condition in general). At $192 \mathrm{keV}$, the kinetic energy of a recoil triton is large enough to ensure that it will be implanted after collision with a wall.

Because the mechanism just described is a tritium-loss mechanism, it was important to determine the fraction of recoil tritons colliding with the walls of the target chamber and blanket ${ }^{3} \mathrm{He}$ chambers. To investigate this, a modified version of LAHET developed at LANL, 


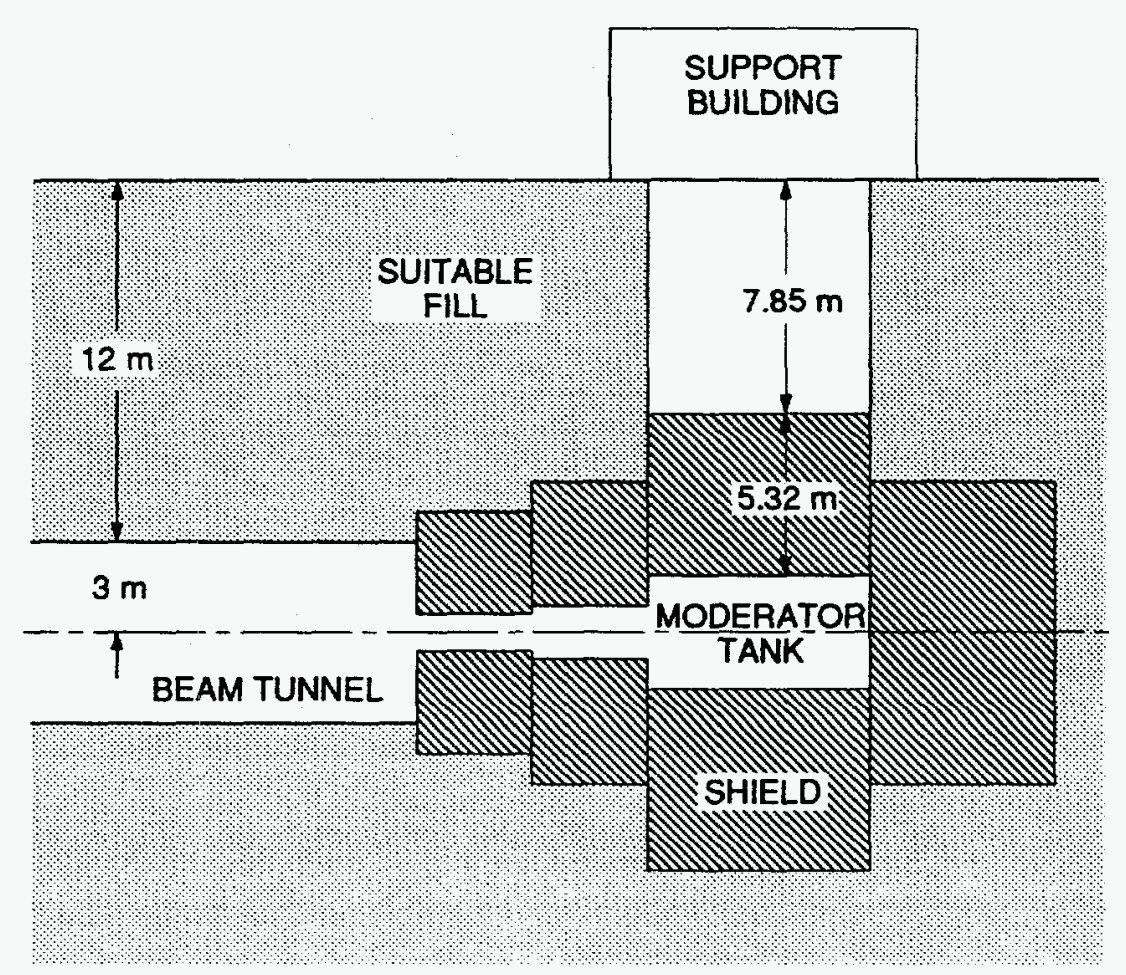

Fig. 3-47. Schematic of the APT target region.*

*This layout shows the assumptions made in estimating the distance between the moderator tank and the support building. The top of the moderator tank is assumed to be $6 \mathrm{ft}$ above beam centerline. The top of the beam tunnel is $3 \mathrm{~m}$ above beam centerline, followed by $12 \mathrm{~m}$ of berm. Assuming the support building is located at the berm surface, the distance from the top of the moderator tank to the support building is $13.17 \mathrm{~m}$. A total shield thickness of $5.32 \mathrm{~m}$ on top of the moderator tank (as shown) would result in a biological dose at the berm surface of $0.1 \mathrm{mR} / \mathrm{h}$.

SUPERHET [3-48] was used, that can handle charged-particle transport in matter more accurately than LAHET at low energies. It was found that, of the total triton production in target chamber, $0.4 \%$ is lost to the walls; in the blanket, $1.9 \%$ is implanted in the walls. These percentages refer to tritium production in the target chamber or in the blanket; they are not fractions of the total production (target chamber + blanket). Because approximately half the tritium production takes place in the blanket and the other half in the target chamber, about $1.2 \%$ of the total production is lost to the walls, a modest fraction of the total production.

\subsection{BEAMSTOP}

The APT facility will require a straight-through beamstop, which will be used for accelerator tuneup and as a place to stop the beam in the event it is necessary to divert it from the tritium-producing area without shutting down the accelerator. Although the beamstop will be infrequently used, it is preferable to have one that produces minimal prompt and residual radioactivity and can accept large excursions in beam tune without compromising its integrity. 
The design presented here uses a graphite stopping-length target with radiative heat transfer to a water-cooled vacuum chamber wall. Although both the beamstop region and the beam transport system are in vacuum, an Inconel-718 window similar in design to that used in the $T / B$ design was included to provide complete isolation of the accelerator and beamstop regions. The design premises are summarized in a memorandum [3-49] by $M$. Cappiello, describing the engineering design.

Calculations were performed using the LAHET Code System (LCS) [3-2] and CINDER'90 [3-50] for an idealized cylindrical system. Further iterations between the engineering and the physics design presented here are necessary, but this calculation is believed sufficient to provide information that can scale for PEIS input. At this time, the Inconel-718 window has not been modeled—using, instead, data from the T/B design.

Two physics design calculations were performed. In the first, a 175-cm-long graphite cylinder divided into $1-\mathrm{cm}$-long segments was used to determine the energy deposition as a function of depth in the graphite. This was done to size the beamstop layers because the intranuclear cascade process gives an energy deposition that is different from proton-only Coulomb processes.

In the engineering design, the graphite section is $23.5 \mathrm{~m}$ long and consists of a series of plates of varying thickness with appropriate separations to allow radiative heat transfer to the tank walls. That feature was represented in the second physics design by a uniform reduceddensity graphite cylinder. The graphite is housed in a water-cooled, cylindrical, aluminum vacuum tank. The aluminum tank has two $2-\mathrm{cm}$-thick double walls with a 5 -cm cooling water layer in between. The beamspot was assumed to have a $150-\mathrm{cm} \times 300-\mathrm{cm}$ footprint. The graphite varies from $300 \mathrm{~cm} \times 150 \mathrm{~cm}$ to $450 \mathrm{~cm} \times 300 \mathrm{~cm}$. The beam and graphite radii used in the physics calculation were chosen to reproduce the cross-sectional areas of the rectangular design and were $119.7 \mathrm{~cm}$ and $207.3 \mathrm{~cm}$, respectively.

\subsection{FUTURE WORK}

We must continue to investigate ways of improving the tritium production efficiency $(\mathrm{T} / \mathrm{p})$ of the $3 \mathrm{He} \mathrm{T} / \mathrm{B}$ system and further enhance the margin to meet the tritium production goal. We will apply new constraints to this endeavor, such as minimizing ${ }^{3} \mathrm{He}$ and $\mathrm{D}_{2} \mathrm{O}$ inventories, mixed waste and radionuclide production, and costs, as well as simplifying the mechanical design. The neutronic optimization studies are important to direct the parallel effort on the mechanical design of the ${ }^{3} \mathrm{He} \mathrm{T} / \mathrm{B}$ system. Depending on the amount of financial support available for this work and priorities for the calculations, we will perform Monte Carlo neutronic studies in the following areas:

- central target zone: size of flux-trap gaps; variable flux-trap gaps; $W / \mathrm{D}_{2} \mathrm{O}$ coolant fraction; rod-bundle thickness; lateral extent of rod bundles; practicality and advantages/disadvantages of using $184 \mathrm{~W}$;

- backstop region: materials; thickness; coolant fraction; minimization of lead; 
- radial target zone: minimize amount of lead; incorporate engineering details into the physics model as they evolve, and evaluate their neutronic impact;

- thickness of Inconel-718 target chamber: investigate tritium production efficiency when both the ${ }^{3} \mathrm{He}$ pressure and thickness of the Inconel target chamber are varied;

- $\mathrm{D}_{2} \mathrm{O}$ gap: further size the $\mathrm{D}_{2} \mathrm{O}$ gap between target chamber and radial target zone using latest physics model;

- energy deposition spatial detail: proton profile; proton beam window; rod bundle; backstop; radial target; moderator tank;

- shield design: dose attenuation; energy deposition;

- soil and water activation calculations and benchmark experiments;

- further benchmarking of the LAHET Code System against existing data: integral (water bath) experiments; bare target integral and differential data; and other data;

- further improvements in physics treatment in LAHET;

- further evaluations of nuclear data library for transport and neutron multiplication to $100 \mathrm{MeV}$;

- calculational support for the ${ }^{3} \mathrm{He} \mathrm{T} / \mathrm{B}(\mathrm{T} / \mathrm{p})$ engineering mockup experiment; and

- further investigations of tritium implantation issues.

The above list of tasks represents an ambitious calculational effort. As mentioned above, the effort will be further focused by budgetary constraints, schedules, and priorities for input to the mechanical design effort. 


\subsection{REFERENCES}

3-1. Radiation Shielding Information Center, "HETC Monte Carlo High-Energy NucleonMeson Transport Code," Oak Ridge National Laboratory report CCC-178 (August 1977).

3-2. R. E. Prael and H. Lichtenstein, "User Guide to LCS: The LAHET Code System," Los Alamos National Laboratory report LA-UR-89-3014 (September 1989).

3-3. T. R. England, "CINDER-A One-Point Depletion and Fission Product Program," Bettis Atomic Power Laboratory report WAPD-TM-334 (August 1962, Rev. June 1964).

3-4. T. R. England, R. Wilczynski, and N. L. Whittemore, "CINDER-7: An Interim Users Report," Los Alamos Scientific Laboratory report LA-5885-MS (April 1975).

3-5. T. R. England, W. B. Wilson, and M. G. Stamatelatos, "Fission Product Data for Thermal Reactors, Part 1: A Data Set for EPRI-CINDER Using ENDF/B-IV," Los Alamos Scientific Laboratory report LA-6745-MS (December 1976).

3-6. T. R. England, W. B. Wilson, and M. G. Stamatelatos, "Fission Product Data for Thermal Reactors, Part 2: User Manual for EPRI-CINDER Code and Data," Los Alamos Scientific Laboratory report LA-6746-MS (December 1976).

3-7. W. B. Wilson, T. R. England, R. J. LaBauve, M. E. Battat, D. E. Wessol, and R. T. Perry, "Status of CINDER and ENDF/B-V Based Libraries for Transmutation Calculations," Proc. Int. Conf. Nuclear Waste Transmutation, Austin, Texas, July 2224, 1980, p. 673 (March 1981).

3-8. W. B. Wilson, T. R. England, R. J. LaBauve, and D. C. George, "CINDER-3: Depletion Code for Class VI Computer," Transactions of the American Nuclear Society 46, 724 (1984).

3-9. F. M. Mann, "Transmutation of Alloys in MFE Facilities as Calculated by REAC," Hanford Engineering Development Laboratory report HEDL-TME 81-37 (August 1982).

3-10. F. M. Mann, "REAC-2: A Users Manual and Code Description," Westinghouse Hanford Company report WHC-EP-0282 (December 1989).

3-11. F. M. Mann, D. E. Lessor, and J. S. Pintler, "REAC Nuclear Data Libraries," Radiation Effects 92, 207 (1986).

3-12. F. M. Mann, "Status of Dosimetry and Activation Data," Proceedings of the International Conference on Nuclear Data for Science and Technology, Mito, Japan, May 30-June 3, 1988, p. 1013 (1988). 
3-13. W. B. Wilson and T. R. England, "Nuclear Data Needs for Studies of Accelerator Induced Neutron Transmutation of Nuclear Waste," Proceedings of the Specialists' Meeting on Fission Products and Nuclear Data, May 25-27, 1992, JAERI, Tokai, Japan; organization for Economic Cooperation and Development Nuclear Energy Agency report NEA/NSC/DOC(92)9, pp. 475-481.

3-14. P. G. Young and W. B. Wilson, "Nuclear Data Requirements for Transmutation," presentation, American Chemical Society, Division of Nuclear Chemistry and Technology Meeting, Denver, Col., March 29, 1993. Los Alamos National Laboratory reports LAUR-93-994 and -1132 .

3-15. Vassilkov et al., in Proceeding of the Eleventh Meeting of the International Collaboration on Advanced Neutron Sources, (ICANS-XI), M. Misawa, Ed. (National Laboratory for High-Energy Physics, Tsukuba, 1991), Vol. 1, pp. 340, 612.

3-16. P. E. Koehler, "Measurement of the LANSCE Neutron Flux from $0.025 \mathrm{eV}$ to 100 keV," Los Alamos National Laboratory report LA-UR-90-290 (1990).

3-17. J. S. Gilmore, G. J. Russell, H. Robinson, and R. E. Prael, "Fertile-to-Fissile and Fission Measurements for Depleted Uranium and Thorium Bombarded by $800-\mathrm{MeV}$ Protons," Nuclear Science and Engineering 99 41-52 (1988).

3-18. V. I. Belyakov-Bodin et al., "Calorimetric Measurements and Monte Carlo Analyses of Medium-Energy Protons Bombarding Lead and Bismuth Targets," Nuclear Instruments and Methods in Physics Research A295, 140-146 (1990).

3-19. V. I. Belyakov-Bodin et al., "Calorimetric Measurements and Monte Carlo Analyses of Medium-Energy Protons Bombarding Uranium Targets," Atomic Energy 70, 339345 (1991).

3-20. V. I. Belyakov-Bodin et al., "Calorimetric Measurements of Medium-Energy Protons Bombarding Beryllium, Carbon, and Aluminum Targets," Nuclear Instruments and Methods in Physics Research A314, 508-513 (1992).

3-21. G. J. Russell, P. A. Seeger, R. G. Fluharty, "Parametric Studies of Target/Moderator Configurations for the Weapons Neutron Research (WNR) Facility," Los Alamos Scientific Laboratory report LA-6020 (March 1977).

3-22. JASON Group Report, January 1992.

3-23. G. J. Russell, E. J. Pitcher, and L. L. Daemen, "Split-Composite Spallation Neutron Source Targets and Accelerator Production of Tritium," Los Alamos National Laboratory report LA-UR-93-2975 (1993).

3-24. H. Lichtenstein, "APT Target Design Study," Los Alamos National Laboratory memorandum X-6:HL-93-76 (January 28, 1993).

3-25. H. Lichtenstein, "APT Target Design Study-II," Los Alamos National Laboratory memorandum X-6:HL-93-125 (February 16, 1993). 
3-26. H. Lichtenstein, "APT Target Design Study-III," Los Alamos National Laboratory memorandum X-6:HL-93-132 (February 18, 1993).

3-27. H. Lichtenstein, "APT Target Design Study-IV," Los Alamos National Laboratory memorandum X-6:HL-93-207 (March 19, 1993).

3-28. H. Lichtenstein, "APT Target Design Study-V," Los Alamos National Laboratory memorandum X-6:HL-93-241 (April 7, 1993).

3-29. D. Weinacht, Los Alamos National Laboratory memorandum MEE-13:92:367, June 30, 1993.

3-30. Unused Reference

3-31. L. D. Smith, "Thermal Analysis of Target Lead Side for APT-LANL," Babcock \& Wilcox report 12-3002060-00 (May, 1993).

3-32. “APT SILC Target Draft Preconceptual Design," ATD/APT93-0007, APT Design Review Meeting, March 1-4, 1993.

3-33. Unused Reference

3-34. J. L. Sapir, "Preliminary APT Survey Calculations," Los Alamos National Laboratory memorandum N-12-91-791, to J. R. Ireland (November 4, 1991).

3-35. R. Streetman, "APT Progress Report for July 14 Through July 20, 1992," Los Alamos National Laboratory memorandum N-12-92, to Gary Russell (July 21, 1992).

3-36. Bob Streetman, "APT Progress Report for July 21 Through July 27, 1992," Los Alamos National Laboratory memorandum N-12-92, to Gary Russell (July 27, 1992).

3-37. R. E. MacFarlane, "TRANSX-CTR: A Code for Interfacing MATXS Cross section Libraries to Nuclear Transport Codes for Fusion Systems Analysis," Los Alamos National Laboratory report LA-9863-MS (February 1984).

3-38. F. W. Walker, J. P. Parrington, and F. Feiner, "Chart of the Nuclides, Fourteenth Edition," General Electric Company (1988).

3-39. ANS/ANSI-5.1-1979. American National Standard for Decay Heat Power in Light Water Reactors.

3-40. W. B. Wilson and T. R. England, "Development and Status of Fission-Product Yield Data and Applications to Calculations of Decay Properties," Transactions of the American Nuclear Society 66, 152 (1992).

3-42. C. A. Wilkinson, D.H. Fitzgerald, S. C. Frankle, R. L. Hutson, and R. J. Macek, "Monte Carlo-Based Formula for Radiation Shielding Assessment in the Forward Direction," in Proceedings of IEEE 1993 Particle Accelerator Conference (May, 1993).

3-43. B. J. Moyer, Lawrence Berkeley Laboratory report UCRL-9769, 1961. 
3-44. "Shielding Against High Energy Radiation," ed. by H. Schopper, Vol. 11 in Numerical Data and Functional Relationships in Science and Technology, Springer-Verlag, 1990, p. 129.

3-45. Unused Reference

3-46. "Shielding Against High-Energy Radiation," in Numerical Data and Functional Relationships in Science and Technology, H. Schopper, Ed. (Springer-Verlag, New York, 1990), Vol 11.

3-47. G. J. Russell, "Equivalent Spherical-Shield-Neutron-Dose Calculations," Proceedings of the 10th Meeting of the International Collaboration on Advanced Neutron Sources, ed. by D. K. Hyer, Institute of Physics, 1989, pp. 803-7.

3-48. A. Gavron, "SUPERHET, Version II," Los Alamos National Laboratory, unpublished.

3-49. M. Cappiello, "Beam Stop Design for APT," Los Alamos National Laboratory memorandum N-12-93-058.

3-50. W. Wilson, E. D. Arthur, C. D. Bowman, L. N. Engle, T. R. England, H. G. Hughes, P. W. Lisowski, and R. T. Perry, "Transmutation Calculations for the Accelerator Transmutation of Waste," Journal of the American Nuclear Society 63, 90 (1991). 


\section{ENGINEERING DESIGN \\ CHAPTER 4}

Page

4.1 INTRODUCTION AND ENGINEERING CONSIDERATIONS ...........................4

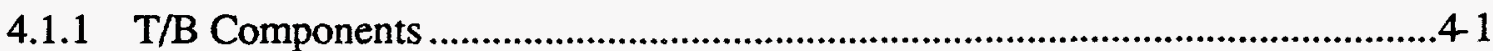

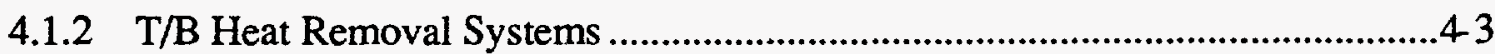

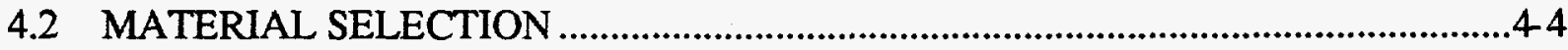

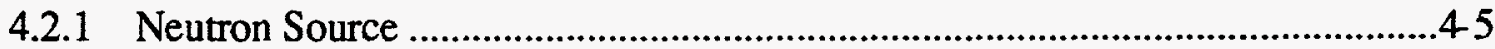

4.2.2 Beam Entrance Window, ${ }^{3} \mathrm{He}$ Chamber, and Rod Bundle Ducts ....................46

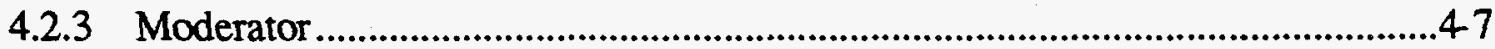

4.2.4 3He Heat Exchanger and Blanket Tubes .....................................................48

4.2.5 Proton Beam Backstop Region ..................................................................48

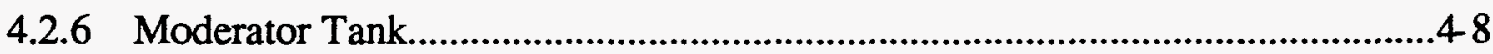

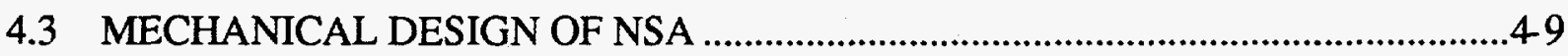

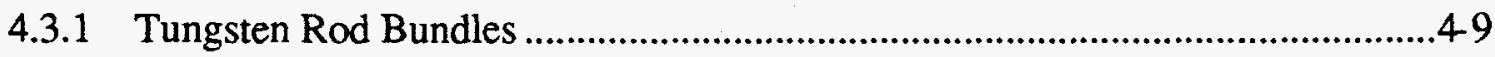

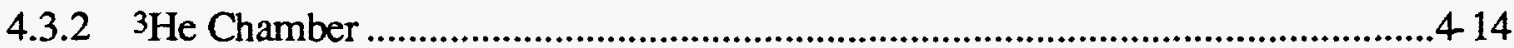

4.3.3 Proton Beam Backstop Region .................................................................415

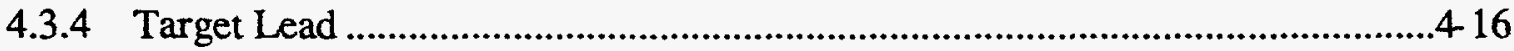

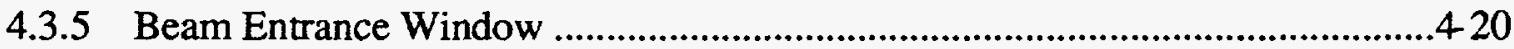

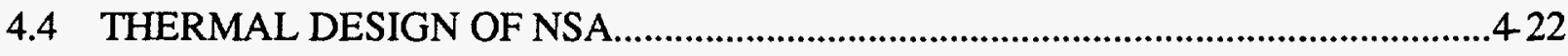

4.4.1 Tungsten Rod Bundles ..................................................................422

4.4.2 3He Chamber ....................................................................................426

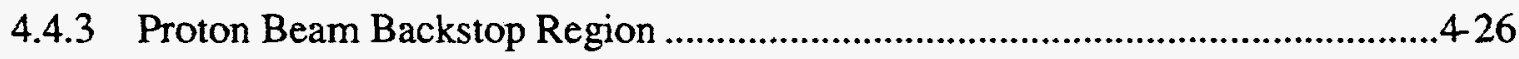

4.4.4 Target Lead Cooling .............................................................................428

4.4.5 Beam Entrance Window ..........................................................................4.33

4.5 MECHANICAL DESIGN OF TARGET MODULE …..........................................4 34

4.6 TARGET/BLANKET HEAT TRANSPORT SYSTEMS ......................................450

4.6.1 Tungsten Heat Transport System (THTS) ................................................450

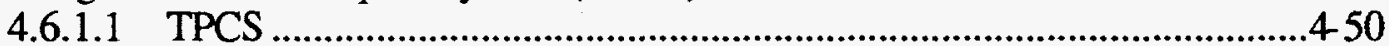

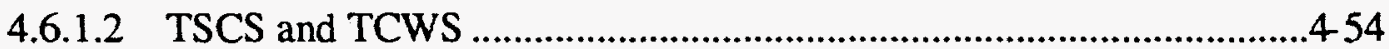

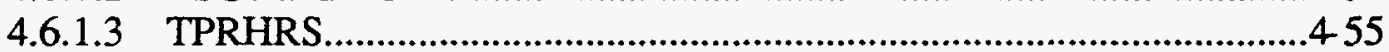

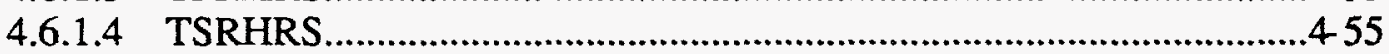

4.6.2 Moderator Heat Transport Systems (MTHTS) ...........................................455

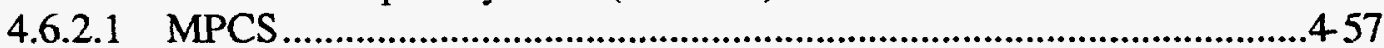

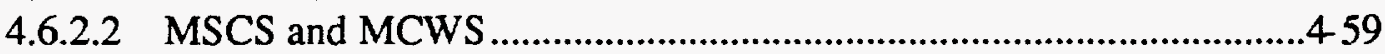

4.6.2.3 MPRHRS ............................................................................6 


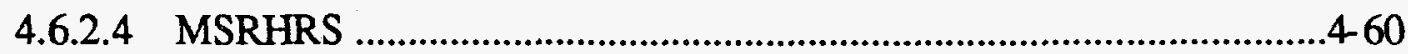

4.6.3 3He Heat Transport Systems (HHTS) .........................................................62

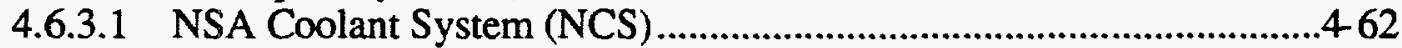

4.6.3.2 Blanket Coolant System (BCS) .......................................................65

4.6.4 Purification Systems ….................................................................................66

4.7 INSTRUMENTATION CONTROL AND PROTECTION SYSTEMS .......................467

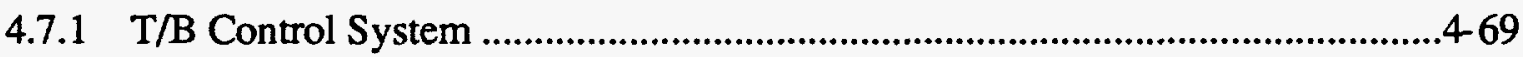

4.7.2 T/B Control Concept ........................................................................................469

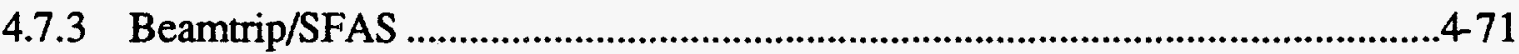

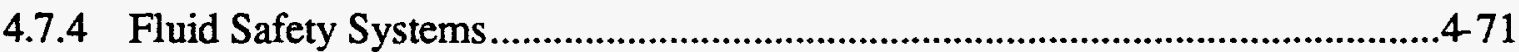

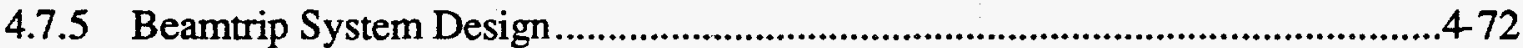

4.7.6 Safety System Sensors …………..............................................................

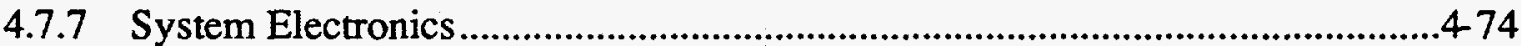

4.7.8 Accelerator Beamtrip Mechanism .............................................................76

4.8 REMOTE-HANDLING SYSTEMS AND OPERATION ….................................4-76

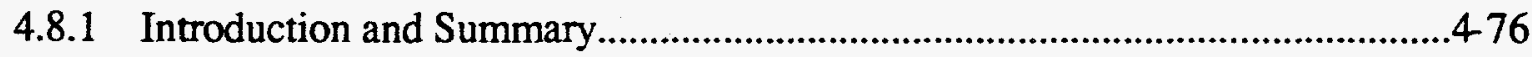

4.8.2 Target Module Refurbishment ....................................................................4. 77

4.8.3 Target Module Retargeting Operation ……...............................................4-79

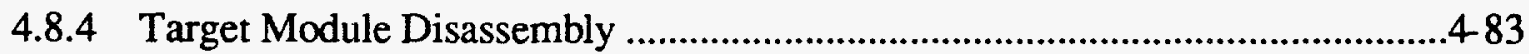

4.8.5 NSA Target Disassembly Sequence ………...............................................484

4.8.6 Target Module Waste Stream .................................................................489

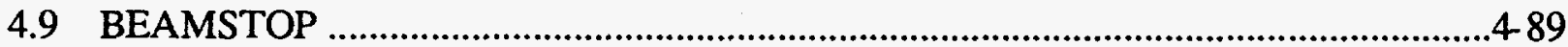

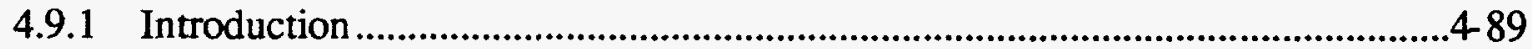

4.9.2 Beamstop Mechanical Design ....................................................................491

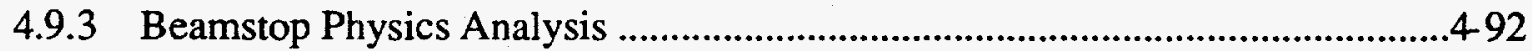

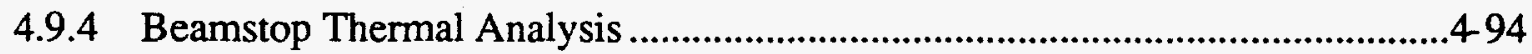

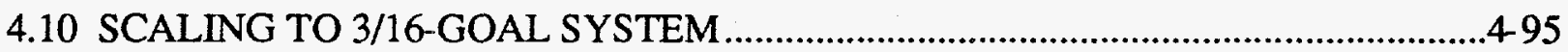

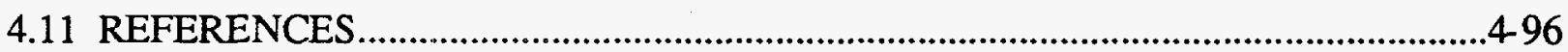

\section{LIST OF FIGURES}

Fig. 4-1. Neutron Source Assembly (NSA)................................................................4-10

Fig. 4-2. Tungsten Rod Bundle Layout Pattern (Rev. 1.5) ...............................................411

Fig. 4-3. Tungsten Rod Bundle End Assembly ..............................................................411

Fig. 4-4. Hexagonal Duct Deformation Due To Internal Pressure Loading.......................413

Fig. 4-5. Target Lead Configuration..................................................................................418 
Fig. 4-6. Finite Element Model Of Beam Entrance Window. ...........................................421

Fig. 4-7. Target Lead $\mathrm{D}_{2} \mathrm{O}$ Fraction...........................................................................429

Fig. 4-8. Moderator $\mathrm{D}_{2} \mathrm{O}$ Coolant Flow Arrangement....................................................430

Fig. 4-9. Target Lead Cooling. .................................................................................431

Fig. 4-10. Target Lead Temperature..........................................................................433

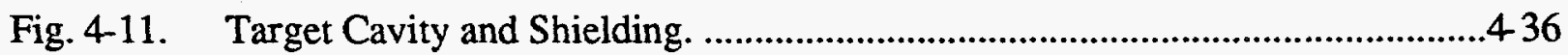

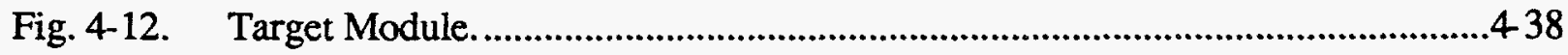

Fig. 4-13. General Arrangement - Cross-Section Elevation. ..............................................439

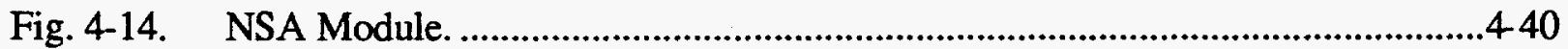

Fig. 4-15. Target Module Assembly.......................................................................441

Fig. 4-16. Target Module General Arrangement - Side Section Elevation........................443

Fig. 4-17. Target Module Tungsten $\mathrm{D}_{2} \mathrm{O}$ Arrangement - Cross-Section Elevation..........4-44

Fig. 4-18. Tungsten $\mathrm{D}_{2} \mathrm{O}$ Coolant Flow Arrangement ......................................................445

Fig. 4-19. NSA Helium Flow Arrangement. .................................................................44

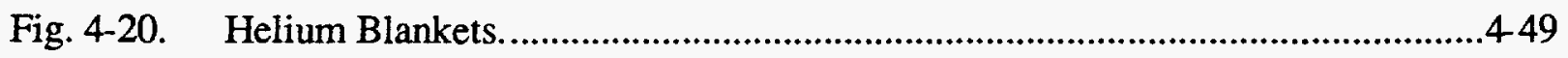

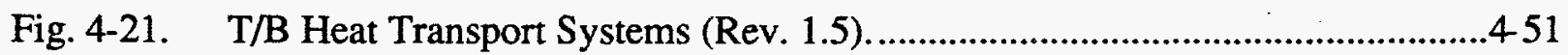

Fig. 4-22. Tungsten Primary Coolant System Flow Schematic........................................452

Fig. 4-23. Tungsten Primary Residual Heat Removal System Flow Schematic................456

Fig. 4-24. Moderator Primary Coolant System Flow Schematic........................................458

Fig. 4-25. Moderator Primary Residual Heat Removal System Flow Schematic. ..............461

Fig. 4-26. NSA Coolant System Flow Schematic. ……..............................................463

Fig. 4-27. Blanket Coolant System Flow Schematic.....................................................464

Fig. 4-28. Purification System Schematic. ...................................................................46

Fig. 4-29. LANL APT Control Concept.....................................................................470

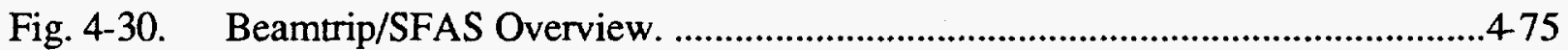

Fig. 4-31. Target Module Assembly..........................................................................4

Fig. 4-32. Decay Heat vs. Time .................................................................................4

Fig. 4-33. Retargeting-Target Disassembly Sequence.....................................................481

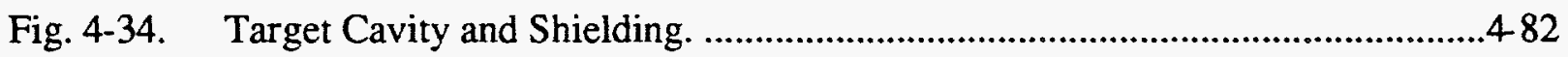

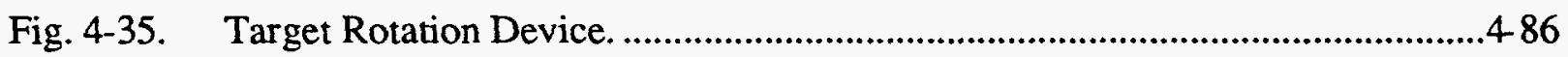

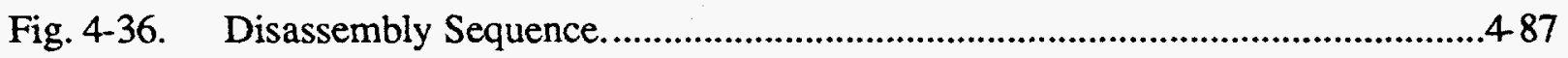

Fig. 4-37. NSA Target Ready for Tungsten Removal. ......................................................48

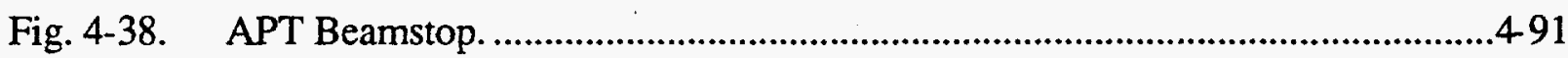

Fig. 4-39. Beamstop Neutron Leakage Flux From Cylindrical Surface.............................493 


\section{LIST OF TABLES}

Page

Table 4-1. Neutron Source Material Comparison …........................................................4-5

Table 4-2. Structural Material Comparisons …….........................................................

Table 4-3. Target Lead Plates Preconceptual Design.......................................................4-19

Table 4-4. Target Lead Parameters (Preconceptual Approximation).................................419

Table 4-5. Tungsten Power Densities in Each Rod Bundle Row......................................423

Table 4-6. Bundle Characteristics for Each Group ......................................................4-24

Table 4-7. High- and Mid-Range-Power Rod Bundles and Ducts...................................425

Table 4-8. Backstop Power Density Summary............................................................427

Table 4-9. Target Lead Cooling Preconceptual Design Parameters..................................4-32

Table 4-10. Estimated Target Module Weight Breakdown...............................................4-37

Table 4-11. LANL APT T/B Startup Procedures........................................................469

Table 4-12. LANL APT T/B Event vs. Trip Matrix......................................................473

Table 4-13. LANL APT T/B Trip Function List ..............................................................473

Table 4-14. Estimated Target Module Waste Stream Breakdown Per Target Module Replacement 490

Table 4-15. LAHET-MCNP Energy Deposition Results................................................4-92

Table 4-16. LAHET-MCNP Proton and Neutron Flux Summary ...................................4.493

Table 4-17. Plate Temperatures and Dimensions.........................................................4.494

Table 4-18. Summary of Masses for a Single Target/Blanket Module ...............................4-95 


\subsection{ENGINEERING DESIGN}

\subsection{INTRODUCTION AND ENGINEERING CONSIDERATIONS}

The proton beam from the APT accelerator is directed toward one of two target/blanket (T/B) modules. One module will be in operation while the other will be in maintenance or standby. A beamstop is physically located between the two T/B modules. Each T/B module has separate cooling loops for its respective neutron source and moderator. Except for sharing the $3 \mathrm{He} /$ tritium separation equipment, the modules operate independently. Note that ${ }^{3} \mathrm{He}$ is removed from one module and put in the other module so that only one ${ }^{3} \mathrm{He}$ charge is needed. The beamstop is designed to take the full beam power, and will be used for beam tuneup and diagnostics. It also has a separate cooling loop.

Each T/B module consists of a centrally located tungsten neutron source, a $3 \mathrm{He}$ chamber enclosing the tungsten neutron source, a surrounding lead neutron multiplier called the target lead, a proton beam backstop region, a heavy-water moderator, a $3 \mathrm{He}$ heat exchanger, and ${ }^{3} \mathrm{He}$ blanket tubes. The neutron source, ${ }^{3} \mathrm{He}$ chamber, target lead, moderator, ${ }^{3} \mathrm{He}$ heat exchanger, ${ }^{3} \mathrm{He}$ blanket tubes, and other structures are contained in a cylindrically-shaped moderator tank. The $3 \mathrm{He}$ gas is circulated through the neutron source and the moderator where the transmutation of tritium takes place through neutron capture. As the ${ }^{3} \mathrm{He}$ is circulated, tritium and other hydrogen isotopes are continually removed for separation by the tritium extraction system, keeping the tritium content in the T/B system at a low level.

The ${ }^{3} \mathrm{He}$ target/blanket (T/B) system includes the major T/B components and the associated heat removal systems. Heat is generated in the $\mathrm{T} / \mathrm{B}$ components due to proton, neutron, and other high-energy particle interactions. Separate heat removal systems have been designed for the tungsten neutron source and the moderator because the heat removal requirements are different in each system. In addition, the moderator acts as the heat sink for the beam entrance window, the ${ }^{3} \mathrm{He}$ chamber, the beam tunnel extension, the target lead, the proton beam backstop region, the ${ }^{3} \mathrm{He}$ in the chamber and in the blanket tubes, and the moderator tank structural components.

The preconceptual design of the ${ }^{3} \mathrm{He}$ T/B system has taken into account several important considerations. These include tritium production, safety and health, and waste minimization. Other important considerations are extensive use of existing technology, material compatibility, design simplicity, reliability, fabricability, operations, remote handling, lifetime, availability, inspectability, and maintainability.

The details of the preconceptual engineering design are given in this chapter. Information on the material selection, the mechanical design, the thermal design, and the heat transport systems for all the major components is provided. In addition, the instrumentation and control, the remote handling systems, and the beamstop are discussed.

\subsubsection{T/B Components}

The neutron source assembly (NSA) includes the tungsten rod bundles, the tungsten inlet and outlet plena, the ${ }^{3} \mathrm{He}$ chamber, the beam entrance window, the beam tunnel extension, the beam entrance nozzle, and the beam tunnel remote connector (See Fig. 4.1). Currently, it 
is planned that the NSA will be replaced every 1 year or longer of full power operation at $75 \%$ capacity. The tungsten rod bundles are the primary source of neutrons. The design features materials and geometries that minimize parasitic neutron capture and maximize production, with the overall goal of a safe design that meets or exceeds the tritium production goal. In addition, the design has made use of the excellent experience at existing spallation sources with tungsten and Inconel and has incorporated the wire-wrapped rod bundle design typical of fast reactors.

Engineering considerations impacted every major design decision. For example, to reduce the heat flux in the neutron source to acceptable levels, the beam spot was expanded to an area of $3822 \mathrm{~cm}^{2}(592 \mathrm{in.}$ ). To increase neutron leakage from the tungsten, the beam spot was made approximately rectangular, $42 \mathrm{~cm} \times 91 \mathrm{~cm}(16.5 \times 35.8 \mathrm{in}$.). To maintain an adequate thermal hydraulic margin for cooling ability, the ratio of the critical heat flux to peak heat flux (Departure from Nucleate Boiling Ratio, DNBR) is kept at or above 2.0. To reduce the amount of structural material in the proton beam and therefore maximize neutron production, the tungsten coolant pressure was minimized. To minimize the amount of moderator in near proximity to the tungsten (and therefore minimize parasitic neutron capture), the coolant was brought in from the bottom and exits at the top of the bundles. To improve safety, both active and passive residual heat removal systems were incorporated into the design. To increase reliability, the active and passive residual heat removal systems were made fully redundant.

The beam entrance window and the rod bundle hexagonal ducts will experience the most intense proton and neutron irradiation damage. Because the NSA is a welded, helium-leaktight structure, removal of the window requires the removal of the NSA. The preconceptual design of the beam entrance window and the choice of structural material for the NSA are both based on the extensive experience at the Los Alamos Meson Physics Facility (LAMPF), where the beamstop window is a double-wall Inconel-718 structure with water cooling. The APT window will operate at similar stress levels and proton fluence. The lifetime estimate for the APT NSA is 1 year or longer at $75 \%$ capacity. Materials research in this area with a focus on extending the lifetime of the NSA is planned as part of the risk reduction program.

The preconceptual design activities for the moderator tank have focused primarily on support of the evolving physics configuration and cooling systems implementation. Additionally, a safety strategy has been adopted for utilization of the moderator tank and primary coolant piping outside of the moderator tank as a highly reliable radionuclide retention boundary that will meet the requirements of the ASME Code Section III, Class 1, pressure boundary. This may allow other coolant boundaries such as the NSA and $3 \mathrm{He}$ blankets, to be considered for noncode classification. Although the primary function of the moderator tank is to retain a level of moderator coolant, it must also be capable of withstanding normal operating pressures and design basis events (DBEs), such as an internal tungsten coolant pipe break and a rupture of the $3 \mathrm{He}$ chamber or blanket tubes. Considerations for support and alignment of internals are also implemented in the concept.

A preconceptual design for the target lead has been developed to meet several key objectives. The extent to which the target lead and the ${ }^{3} \mathrm{He}$ blankets encircle the NSA has been maximized to increase neutron production. The amount of $\mathrm{D}_{2} \mathrm{O}$ and Inconel needed to cool and support the target lead has been minimized to improve tritium production. Thermal hydraulic analyses have shown that the design has safe margins against lead melting, with conservative limits on stress and temperature, to preclude loss of geometry and functionality 
due to thermal creep. Coolant velocities and pressure drops have been minimized to avoid lead erosion and unnecessary structural loads. A passive mode of cooling by natural circulation was developed to withstand loss-of-pump accidents in residual heat mode and to allow natural circulation within the moderator tank. An open coolant flow channel design has been provided to substantially reduce the probability of lead melting due to a local flow blockage. Cooling of the moderator tank heated internal components (such as the beam entrance window, target lead, proton beam backstop region, ${ }^{3} \mathrm{He}$ chamber and blankets, beam tunnel extension, and moderator tank) was accomplished with a single heat removal system to preclude the costs and complications of separate additional systems. Capability for cooling the lead during retargeting was also addressed. To complement the already low waste generation characteristics of the APT ${ }^{3} \mathrm{He}$ concept, the majority of the target lead will be used throughout the 40-year minimum life of the facility to minimize the amount of irradiated-lead mixed waste.

The preconceptual design configuration of the $3 \mathrm{He}$ blanket translates the physics representation of three individual blanket regions into hardware with necessary functional features for meeting engineering design objectives. Maximization of the extent to which the blankets encircle the neutron source was sought. Capability for containment of pressurized ${ }^{3} \mathrm{He}$ and tritium and providing an effective thickness of gas blanketing was addressed. Materials and configuration have been set so as to minimize parasitic neutron capture. Other considerations include "manufacturability", accommodation of remote handling and retargeting sequences, structural support, distribution of moderator coolant, compatibility with water chemistry, and minimization of tritium losses due to permeation into the blanket tube walls. The majority of the target module components can be assembled and checked out at the factory before being shipped to the site to improve the overall reliability of the unit. Significant efforts have been directed to reduce the ${ }^{3} \mathrm{He}$ inventory, the $\mathrm{D}_{2} \mathrm{O}$ inventory, and the inventories of materials in the T/B module. These efforts are reflected in multiple changes to the mechanical design of the target/blanket components including the moderator tank.

A retargeting sequence has been studied during the preconceptual design process in order to identify design issues and provide requirements for demonstrating a practical engineering hardware concept. The mechanical design of the target module allows rapid retargeting which can occur shortly after beam shutdown. Retargeting can be rapidly performed by complete changeout of an entire target module structure. More complex operations such as assembly, disassembly, and target lead recovery and re-use can be performed off-line. The design allows readily operable remote retargeting connections with negligible irradiation effects for high integrity and reduced exposure. The design also provides for recovery and re-use of most of the target lead for mixed waste minimization. All handling needs will be met by attention to mechanical design, operating procedures, and closed-circuit TV monitors, as has been demonstrated at the Hanford and Savannah River sites.

\subsubsection{T/B Heat Removal Systems}

The T/B Heat Transport Systems consist of three separate and independent heat transport systems that remove the thermal energy from various components of the $T / B$ system under normal and off-normal conditions. These systems are: (1) Tungsten Heat Transport Systems (THTS), (2) Moderator Tank Heat Transport Systems (MTHTS), and (3) ${ }^{3} \mathrm{He}$ Heat Transport 
Systems (HHTS). Together, these systems remove the thermal loads under normal operations and provide cooling capability for anticipated operational occurrences (AOOs), design basis events (DBEs), and selected beyond design basis events (BDBEs) for the APT T/B system.

In the design of these systems, there were several important considerations. These include safety, reliability, operation, maintenance, and the use of existing technology. The T/B heat transport systems are based on the "defense-in-depth" principle and utilize both active and passive cooling systems with redundancy to provide high assurance that important safety functions are achieved. For example, the tungsten and moderator heat transport systems use two 50\% loops to remove the energy generated in these systems and to mitigate the effects of LBLOCAs. Active residual heat removal in each of these systems is provided by two $100 \%$ forced-flow cooling systems (only one system is needed to remove the decay heat) that allow small piping sizes and the use of existing nuclear reactor technology. Two independent active residual heat removal systems prevent the complete loss of cooling from a single failure.

In addition, the tungsten and moderator coolant systems have been designed for passive decay heat removal by natural circulation in the event that the active systems are unavailable. The design for natural circulation is provided by establishing sufficient thermal center elevation differences between the primary heat sources, the primary heat exchangers, the secondary heat exchangers, and the water-to-air heat exchangers in the secondary loop. Redundancy in passive decay heat removal is provided because only one loop in natural circulation is required to remove the decay heat. No operator action, valve motion, or active system responses are required to accomplish the transition to natural circulation decay heat removal. Detailed system analyses show that during an unprotected LBLOCA, which is a BDBE, the rod bundles are cooled by natural circulation in the unbroken loop and remain below $160^{\circ} \mathrm{C}\left(320^{\circ} \mathrm{F}\right)$. The passive decay heat removal and natural circulation design is based on experience developed in the NPR-HWR program.

The ${ }^{3} \mathrm{He}$ heat transport systems remove and transport the energy generated in the ${ }^{3} \mathrm{He}$ in the neutron source assembly and blankets to the moderator in the moderator tank. The MTHTS carries the energy to the environment. The use of the moderator as a heat sink for the ${ }^{3} \mathrm{He}$ simplifies the overall system design and reduces the costs of separate additional systems.

The T/B heat transport systems also provide intermediate loops or barriers between the primary loops and the third loops that dump the heat to the atmosphere to reduce the probability of radionuclide leakage from the plant to the environment.

\subsection{MATERIAL SELECTION}

The major APT T/B components are the neutron source, the beam entrance window, the ${ }^{3} \mathrm{He}$ chamber, the rod bundle ducts, the moderator, the ${ }^{3} \mathrm{He}$ heat exchanger and blanket tubes, the proton beam backstop region, and the moderator tank. The material selection criteria for these T/B components include neutronics properties, material lifetime, waste stream, hightemperature capability, strength, corrosion resistance, and amount of operational experience. A discussion of the material choices for the major components is given below. 


\subsubsection{Neutron Source}

Spallation neutron sources are in existence at several facilities worldwide. Neutron sources made of lead, tungsten, tantalum, and uranium have been used with varying success. Both solid and liquid neutron sources have been proposed for new and upgraded facilities. The various candidate materials along with their advantages and disadvantages are given in Table 4-1.

For solid neutron sources, lead is an excellent choice from a neutronics standpoint (good neutron production and low neutron absorption). The main disadvantage is the fact that the melting point is very low at $600 \mathrm{~K}\left(621^{\circ} \mathrm{F}\right)$. Because of this, designers are forced to increase the size of the target in order to reduce the power density to acceptable levels. Also, because of the low melting point, there is much less margin to accommodate local beam-intensity fluctuations or accident conditions. A disadvantage for lead is that after irradiation in the proton beam, it beçomes a mixed waste (both radioactive and chemically toxic). Currently there is no mechanism for permanent disposal of mixed wastes. For these reasons, lead is a poor choice for the primary spallation source but an excellent choice for a neutron multiplier where both the heat deposition rate and radiation damage is greatly reduced, making long lifetimes possible.

Table 4-1. Neutron Source Material Comparison

\begin{tabular}{|c|c|c|c|c|}
\hline Material & $\begin{array}{c}\text { Thermal } \\
\text { Neutron } \\
\text { Absorption }\end{array}$ & $\begin{array}{c}\text { Resonance } \\
\text { Integral Cross } \\
\text { Section (b) }\end{array}$ & Advantages & Disadvantages \\
\hline $\begin{array}{l}238 \mathrm{U} \\
\mathrm{Z}=92\end{array}$ & 2.68 & 277 & high atomic number & $\begin{array}{l}\text { long-lived fission } \\
\text { products, high decay } \\
\text { heat, disposal }\end{array}$ \\
\hline $\begin{array}{l}\text { Tungsten } \\
Z=74\end{array}$ & 18.2 & 350 & $\begin{array}{l}\text { high melting point, high } \\
\text { atomic number, } \\
\text { LANSCE experience, no } \\
\text { cladding required, ease } \\
\text { of disposal }\end{array}$ & $\begin{array}{l}\text { high parasitic neutron } \\
\text { capture }\end{array}$ \\
\hline $\begin{array}{l}\text { Tantalum } \\
Z=73\end{array}$ & 20.0 & 660 & $\begin{array}{l}\text { high melting point, high } \\
\text { atomic number, no } \\
\text { cladding required }\end{array}$ & $\begin{array}{l}\text { high parasitic neutron } \\
\text { capture }\end{array}$ \\
\hline $\begin{array}{l}\text { Lead } \\
Z=82\end{array}$ & 0.171 & 0.14 & $\begin{array}{l}\text { high atomic number, low } \\
\text { parasitic capture }\end{array}$ & $\begin{array}{l}\text { low melting point, } \\
\text { requires cladding, } \\
\text { mixed waste }\end{array}$ \\
\hline Bismuth & 0.034 & 0.18 & $\begin{array}{l}\text { high atomic number, low } \\
\text { parasitic capture }\end{array}$ & $\begin{array}{l}\text { production of } 210 \mathrm{Po} \text {, } \\
\text { low melting point }\end{array}$ \\
\hline
\end{tabular}

Both tungsten and tantalum have the advantage of high melting point and ease of disposal, and they do not require cladding. Their main disadvantage is the high neutron absorption. Tungsten has an edge over tantalum because of its slightly higher atomic number and slightly lower neutron absorption. For the APT ${ }^{3} \mathrm{He}$ design, the use of tungsten is practical because the target can be decoupled neutronically with the use of ${ }^{3} \mathrm{He}$. Thus, the parasitic neutron capture in the tungsten can be made relatively small. Tungsten offers a 
safety advantage because of its very high-temperature capability and melting point $3683 \mathrm{~K}$ $\left(6170^{\circ} \mathrm{F}\right)$. Also, waste disposal is simplified enormously because the irradiated tungsten structures (replaced every 1 year or longer) can be disposed of with onsite burial.

Depleted uranium could boost the neutron production significantly over that of lead or tungsten because of neutron multiplication due to fission. Experience at Rutherford Appleton Laboratory, where a depleted uranium clad in Zircaloy was used as a primary spallation source, has shown that the lifetime is short due to radiation damage. The handling and disposal of the neutron source is also complicated by long-lived actinides and fission products. The decay heat for uranium is similar to that of reactor fuel (about $6 \%$ of full power) because of the buildup of fission products. This is a significant disadvantage over the other candidate materials, which have decay heat rates of about $1 \%$ of full power. However, because of the excellent neutron production possibilities with the use of uranium, it could be considered in the future for a sprint production mode in the event of a national emergency.

Another possibility is a flowing liquid target. In this case, lead and bismuth are excellent candidates. A eutectic mixture of these two elements melts at a temperature as low as $398 \mathrm{~K}\left(256^{\circ} \mathrm{F}\right)$. Designers at a spallation neutron source in Switzerland considered a liquid lead-bismuth target for their upgraded facility. Recently, however, they have opted for a more conservative design of water-cooled Zircaloy and lead rods. In the development of the lead-bismuth target, it was found that the container materials that are compatible with the liquid metal have high neutron absorption, which makes the target less effective. Also, safety problems associated with the production of $210 \mathrm{Po}$ in the bismuth, and its potential release in the case of a target failure, were contributing factors.

Based on the comparisons given above and many neutronics optimization calculations, the materials of choice for the neutron source are tungsten and lead for the primary spallation material and the neutron multiplier, respectively. The use of ${ }^{3} \mathrm{He}$ as the tritium production target makes the use of a moderate neutron absorber such as tungsten possible. By careful physics design as discussed in Chapter 3 , the ${ }^{3} \mathrm{He}$ will neutronically decouple the tungsten and prevent excessive parasitic capture. Lead is the material of choice for the neutron multiplier. Because the energy deposition is reduced in this region, the low melting point can be safely avoided. Also, as a neutron multiplier, the majority of lead could potentially last the lifetime of the facility, thereby reducing significantly the amount of mixed waste that is produced.

\subsubsection{Beam Entrance Window, 3He Chamber, and Rod Bundle Ducts}

The beam entrance window, rod bundle ducts, and $3 \mathrm{He}$ chamber form a helium leak-tight structure and are made of the same material (Inconel-718). HT-9 is to be investigated in the materials irradiation program and is a preferred material because of its reduction in parasitic capture. Inconel-718 has been used successfully in proton irradiation environments and is also a serious contender. The window and the rod bundle ducts are in the direct path of the proton beam and must be sufficiently radiation-damage resistant to provide structural integrity throughout their lifetime. The $3 \mathrm{He}$ chamber must withstand lower-intensity scattered proton and high-energy neutron damage. Candidate structural materials for these applications are shown in Table 4-2. Of these materials, Inconel-718 offers the best hightemperature, high-strength capability. The major elements in Inconel-718 are nickel (52.5\%), chromium (19.0\%), iron (18.0\%), niobium (5.2\%), molybdenum (3.0\%), and cobalt 
(1.0\%). The main disadvantage is its moderate neutron absorption cross section. However, with careful use of the ${ }^{3} \mathrm{He}$, parasitic capture in the material can be reduced significantly, making this a less important factor.

\begin{tabular}{lcclll}
\hline \hline Table 4-2. & Structural Material Comparisons & & Thermal \\
\hline Material & $\begin{array}{c}\text { Resonance } \\
\text { Neutron Cross } \\
\text { Section (b) }\end{array}$ & $\begin{array}{c}\text { Integral Cross } \\
\text { Section (b) }\end{array}$ & Advantages & Disadvantages \\
\hline $\begin{array}{l}\text { Zirconium- } \\
\text { based alloys }\end{array}$ & 0.184 & 1.0 & $\begin{array}{l}\text { low parasitic neutron } \\
\text { capture, LWR } \\
\text { experience } \\
\text { low parasitic neutron } \\
\text { capture, thermal } \\
\text { reactor experience } \\
\text { high-temperature }\end{array}$ & $\begin{array}{l}\text { low strength at very } \\
\text { high temperature }\end{array}$ \\
$\begin{array}{l}\text { Aluminum } \\
\text { strength, proton } \\
\text { irradiation experience } \\
\text { high temperature } \\
\text { Nickel-based } \\
\text { alloys }\end{array}$ & 0.233 & 0.17 & $\begin{array}{l}\text { high parasitic neutron } \\
\text { capture }\end{array}$ \\
$\begin{array}{l}\text { Iron-based } \\
\text { stainless steels }\end{array}$ & 2.5 & 2.2 & $\begin{array}{l}\text { high parasitic neutron } \\
\text { capture }\end{array}$ \\
\hline \hline
\end{tabular}

There is extensive experience with Inconel directly in the proton beam at LAMPF. The beamstop window is a double-wall structure with water cooling, made of Inconel-718. When removed after 6 years of intermittent service, the LAMPF window had not failed and had accumulated an average proton fluence of $0.7 \times 1022 \mathrm{p} / \mathrm{cm}^{2}$. It was replaced as a matter of routine maintenance. No other structural material has accumulated this amount of highenergy $(800-\mathrm{MeV})$ proton fluence, although windows made of stainless steel irradiated to much lower fluence show good performance and could possibly be an alternative material.

The preconceptual design of the APT window operates at lower stress levels and similar proton fluence as the LAMPF window. Also, because the LAMPF beam is pulsed at $120 \mathrm{~Hz}$, the peak flux per pulse is 10 times higher than the average flux for APT. Thus, the conditions experienced by the window are more severe than what is expected for APT. On the basis of the LAMPF experience, the lifetime estimate for the APT beam entrance window, and therefore the neutron source assembly, is 1 year or longer at $75 \%$ capacity. At that point in time the proton fluence at the APT window will be similar to that which the LAMPF window had experienced when it was taken out of service. Because of the hightemperature strength and experience with Inconel-718 in the proton beam, it is the material of choice for the beam entrance window, the rod bundle ducts, and the ${ }^{3} \mathrm{He}$ chamber.

\subsubsection{Moderator}

Candidate materials for the moderator include $\mathrm{D}_{2} \mathrm{O}, \mathrm{H}_{2} \mathrm{O}$, graphite, and beryllium. Of these moderators, $\mathrm{D}_{2} \mathrm{O}$ and graphite have the smallest neutron absorption cross sections and the largest moderating ratios. Because of the importance of reducing parasitic capture, $\mathrm{H}_{2} \mathrm{O}$ was not considered in this preconceptual design. Also, because of the difficulty of 
fabrication, material cost, and toxicity, beryllium was eliminated. Graphite has hightemperature capability, good heat capacity, is compatible with helium, and has favorable neutronic performance. The main disadvantages are the production of ${ }^{14} \mathrm{C}$ in the graphite (a long-lived radioactive waste) and the potential buildup of Wigner energy if the irradiation temperature is less than $480 \mathrm{~K}\left(404^{\circ} \mathrm{F}\right)$. Because of this, $\mathrm{D}_{2} \mathrm{O}$ was chosen for the moderator. Heavy water provides the lowest neutron absorption of the candidate materials, is readily available from Savannah River in the quantities necessary, and offers potential safety advantages and design simplicity. The total heavy water requirements for one $\mathrm{T} / \mathrm{B}$ module and the associated cooling systems is 86 metric tons. The main disadvantage of using $\mathrm{D}_{2} \mathrm{O}$ is the buildup of tritium that will take place and the potential for tritium release in an accident.

\subsubsection{He Heat Exchanger and Blanket Tubes}

The $3 \mathrm{He}$ heat exchanger and blanket tubes must be able to contain $3 \mathrm{He}$ and tritium, have low neutron absorption, and have corrosion resistance to the moderator. High-temperature capability is not required because the tubes are immersed in the moderator, which is at low temperature. For this application, the low neutron absorption materials that are potential candidates are aluminum and Zircaloy. Because of the affinity that Zircaloy has for hydrogen (and therefore tritium), aluminum is the obvious best choice. In addition, aluminum has been used in low-temperature reactor applications at Savannah River with great success and is well characterized with respect to tritium containment. Because the moderator will be kept at a low outlet temperature of $343 \mathrm{~K}\left(157^{\circ} \mathrm{F}\right)$, neutral $\mathrm{pH}$ is applicable and should not cause a corrosion problem with the aluminum. With this water chemistry, the other materials immersed in the moderator water are compatible.

\subsubsection{Proton Beam Backstop Region}

The purpose of the backstop is to "range out" the scattered protons from the tungsten rod bundles and provide additional neutron and tritium production. Lead is the material of choice because of its high neutron production and low neutron absorption characteristics. At the front end of the backstop, the energy deposition is sufficiently high that lead would be difficult to cool and would require a large coolant fraction. The coolant degrades the neutron production and is therefore minimized where possible. Therefore, in this region we have chosen a combination of Zircaloy and lead. Zircaloy is used in the front region where the energy deposition is high, and lead is used in the back region where the energy deposition is low. Using Zircaloy, we can minimize the coolant fraction and have adequate strength and low neutron absorption.

\subsubsection{Moderator Tank}

The moderator tank is a very large structure that must hold the moderator, support the internal structures, be a highly reliable radionuclide retention boundary, and provide neutron shielding. Having a low neutron capture cross section is not an issue. For this application stainless steel is the material of choice. There is ample experience with stainless steel in building this type of structure, and at the temperatures expected, strength is adequate. 


\subsection{MECHANICAL DESIGN OF NSA}

Figure 4-1, Neutron Source Assembly, defines the Neutron Source Assembly (NSA). The NSA includes the following components.

* (88) Tungsten Rod Bundles

* (88) Tungsten Rod Bundle Ducts

* (1) Inlet Plenum

* (1) Outlet Plenum

* (1) Helium Chamber

* (TBD) Helium Chamber Stiffening Plates
* (1) Beam Entrance Window

* (1) Beam Tunnel Extension

* (1) Beam Entrance Nozzle

* (TBD) Beam Tunnel Extension Stiffeners

* (1) Beam Entrance Nozzle

* (1) Beam Tunnel Remote Connector

In addition to several other components, all of the components in the NSA will be replaced every 1 year or longer in conjunction with retargeting. The mechanical design description includes details on the preconceptual design of these components, as well as the structural analysis. While not part of the NSA, this section also includes a description of the preconceptual mechanical design of the target lead and support structures.

\subsubsection{Tungsten Rod Bundles}

Bundles of tungsten rods serve as the primary neutron source for the tritium-making process. Sixteen rows of rod bundles (placed in alternating rows of five bundles in one row and six bundles in the next row, for a total of 88 bundles) serve as the "targets" for the highenergy proton beam from the accelerator (Fig. 4-2). The rows are uniformly spaced at approximately $25.1 \mathrm{~cm}$ (9.9 in.), and the overall length of the rod bundle pattern is approximately $3.8 \mathrm{~m}(12.5 \mathrm{ft})$. The length of the helium chamber including the end caps is approximately $4.5 \mathrm{~m}$ (177 in.). Each of the 88 bundles is composed of 91 tungsten rods wrapped with tungsten wire and encased in an Inconel-718 hexagonal duct. Bundle dimensions are based on a nominal rod diameter of $0.3175 \mathrm{~cm}(0.125 \mathrm{in})$ and a nominal wire diameter of $0.1016 \mathrm{~cm}(0.040 \mathrm{in})$. Rod and wire sizes are varied by position in the rows with smaller rods used in the higher power density front end rows where the beam strikes first and larger rods used in the lower power density back end rows. A more detailed description of the rod and wire sizes is presented in Section 4.4.1. Each tungsten rod is "wire wrapped" with a tungsten wire that makes four complete turns in the $96 \mathrm{~cm}(37.8 \mathrm{in}$.) length of the rod. This helical wire wrap maintains a flow channel between adjacent rods for the $\mathrm{D}_{2} \mathrm{O}$ coolant and also enhances the convective heat transfer coefficient. Note that the rods and wire wrap are constructed from pure tungsten $(>99.95 \% \mathrm{~W})$ and have no cladding. A "keyhole" slot at one end of each of the rods "indexes" the rods relative to each other and also retains them axially within the bundle (Fig. 4-3).

Because the tungsten rods are retained by a straight slot on the opposite end, they are free to grow in the axial direction under thermal loadings. The maximum tungsten rod elongation under normal operating conditions is $0.6 \mathrm{~mm}(0.024 \mathrm{in}$.). Although the rods can 


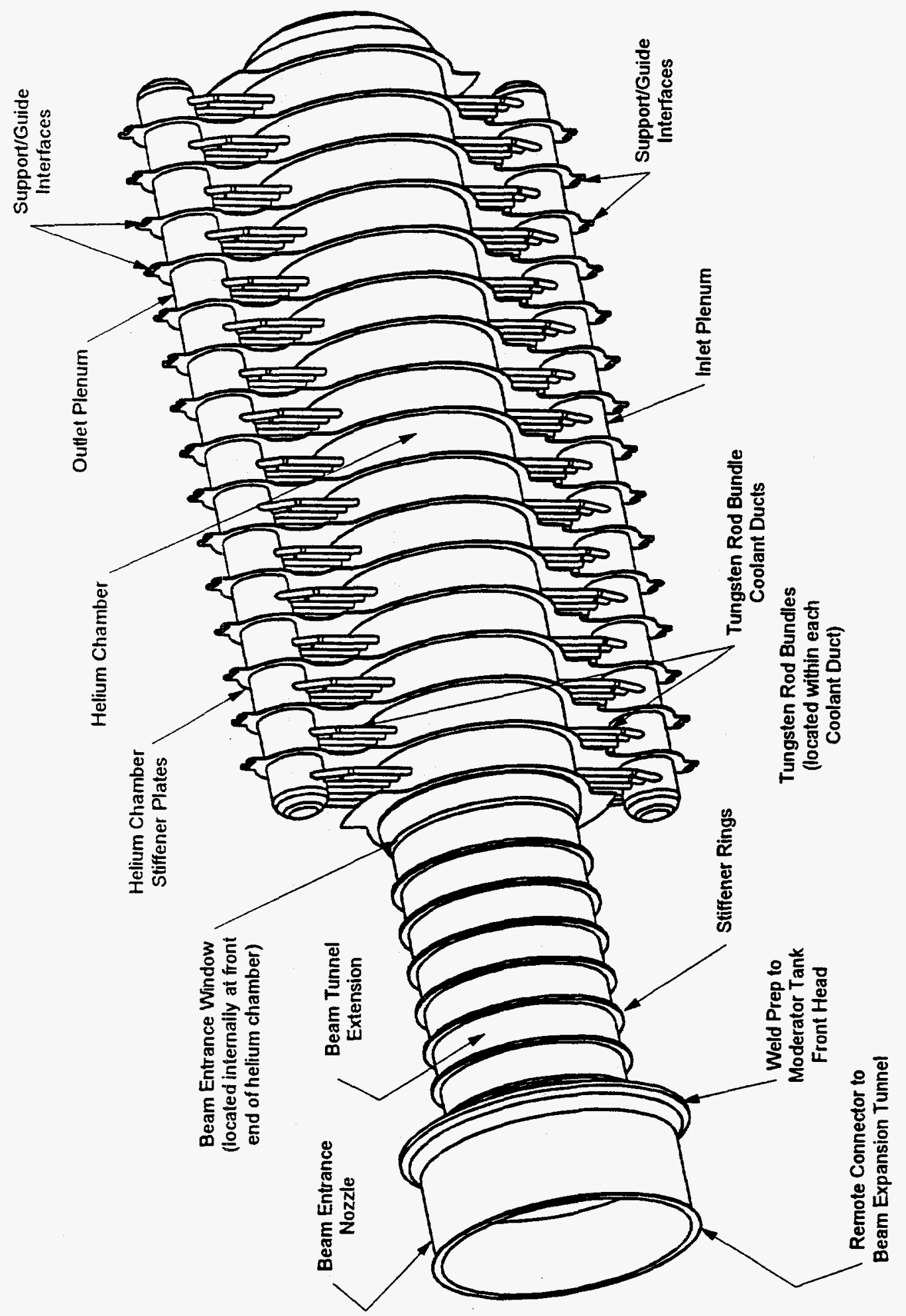

Fig. 4-1. Neutron Source Assembly (NSA). 


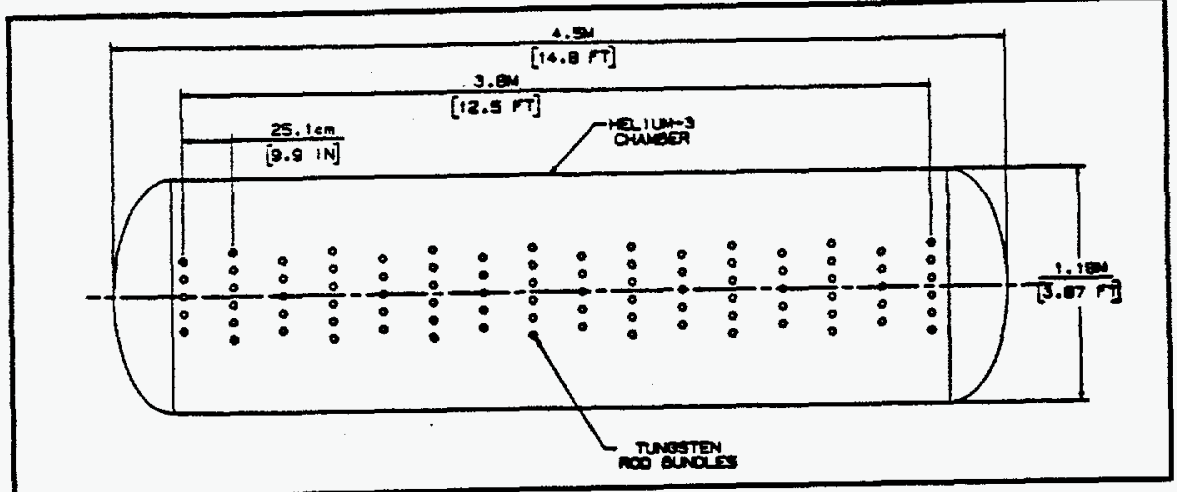

Fig. 4-2. Tungsten Rod Bundle Layout Pattern (Rev.1).

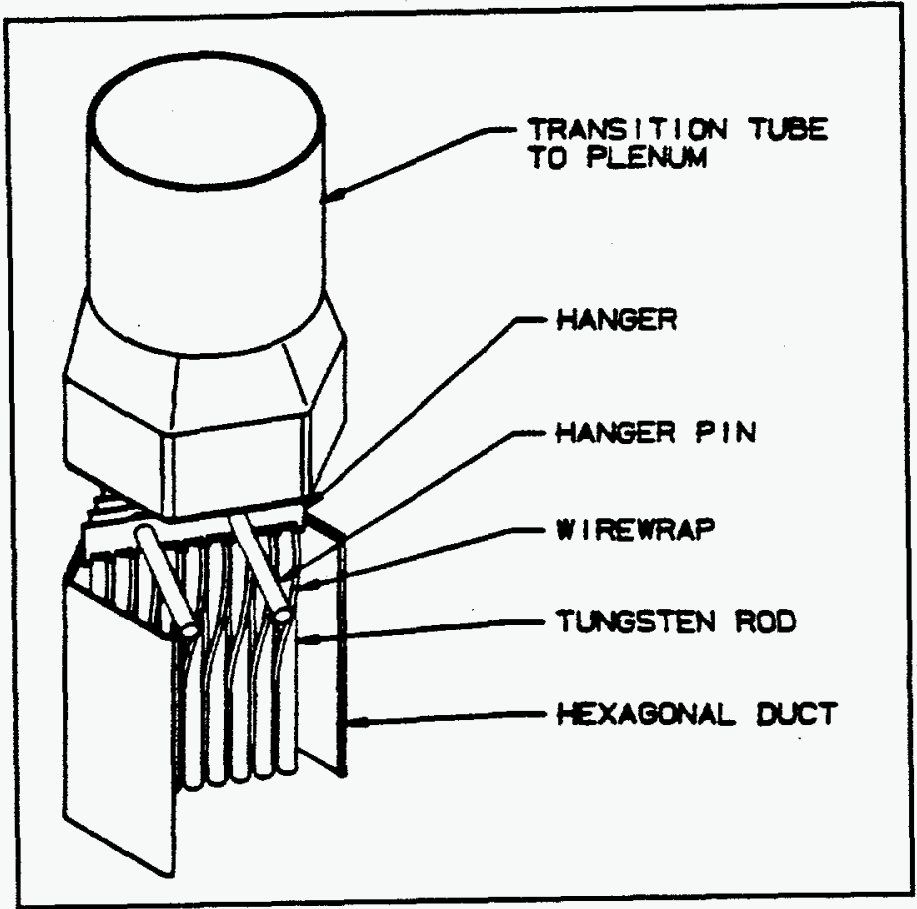

Fig. 4-3. Tungsten Rod Bundle End Assembly

freely expand, they are not entirely free of thermally induced stresses. In fact, the volumetric heating caused by the proton beam results in a nonlinear temperature profile, with the highest temperature at the rod center. The center of the rod tries to grow in both the radial and axial directions. The exterior regions of the rod (near the surface) tend to restrain this growth because they are at a cooler temperature. Thus, the surface of the rod is put into a biaxial tensile stress state. A thermal-mechanical stress analysis using a finite element code (ABAQUS) calculated the effective (von Mises) stress on the surface of the hottest rod to be approximately $6.89 \mathrm{MPa}(1000 \mathrm{psi})$. The von Mises stress is an effective stress measure that is a combination of the principal stresses. Based on a fracture strength of $483 \mathrm{MPa}(70,000$ psi) for tungsten, a significant safety margin exists under normal operational conditions. Even a cyclic stress of this magnitude, which could occur frequently during beam-stop-andstart events, does not present any thermomechanical fatigue problems. A fracture mechanics 
analysis using an alternating stress of $6.89 \mathrm{MPa}(1000 \mathrm{psi})$ and a surface flaw size typical of the processing "scratches" on an available tungsten rod resulted in a stress intensity at the "crack tip" significantly below the fracture toughness for tungsten. Finally, a thermal shock analysis of the tungsten rod was performed. Based on the geometry, material properties, and heat transfer scenario, the calculated temperature change required to cause thermal shock was a factor of 5 above that expected over the time interval of the anticipated beamtrip event. In summary, thermomechanical stress and fatigue analyses, as well as a thermal shock assessment, were made for the tungsten rods, and no thermally induced problems were identified. These calculations were based on the Revision 0 design parameters and will be updated in the next design phase to reflect the current design. While the temperatures in the hottest tungsten rod have increased slightly due to the reduction in size of the beam footprint, the conclusions of this analysis are expected to be similar.

As stated above, the tungsten rods for each bundle are encased in an Inconel-718 hexagonal duct. This duct is also $96 \mathrm{~cm}$ (37.8 in.) long (nominally). Hexagonal-to-circular transition sections are located at each end of the hexagonal duct (Fig. 4-3). These transition sections serve several purposes; namely, they provide a convenient circular cross section for mating to the feed tubes coming from the plena, they provide installation locations for orifice plates that may be needed for flow balancing, and they include a single convolute bellows that will accommodate thermally induced axial growth of the bundle, thereby preventing buildup of any significant axial stresses in the hexagonal duct. The Inconel-718 hexagonal duct has an inside dimension of $4.18 \mathrm{~cm}$ (1.65 in.) across the "flats" and a wall thickness of $0.076 \mathrm{~cm}(0.030 \mathrm{in}$.).

The $0.076-\mathrm{cm}(0.030$-in.) wall thickness used in the hexagonal ducts represents a compromise between physics and mechanical considerations. From a neutronics perspective, the minimization of parasitic capture in Inconel is essential. On the other hand, from a mechanical design point of view, the operational pressure loadings placed on these components must be safely contained without exceeding the available limits of the material. Thus, the high temperature capability coupled with a superior strength were the main reasons for selecting Inconel-718, as opposed to a more neutronically "transparent" material like aluminum, for the hexagonal ducts. Numerous parameter studies were executed using finite element models to determine the minimum wall thickness required to contain the imposed pressure loadings. A worst-case scenario was assumed where the external pressure in the helium chamber was lost while the $1.59 \mathrm{MPa}$ (230 psia) coolant inlet pressure inside the bundle was maintained. This pressure loading is conservative, based on the current design where the coolant inlet pressure has been reduced to $1.44 \mathrm{MPa}$ (209 psia). Note also that the coolant inlet pressure was conservatively applied over the full length of the hexagonal duct. Under this loading situation, the peak von Mises stress in a duct with a wall thickness of $0.076 \mathrm{~cm}(0.030 \mathrm{in}$.$) is on the order of 483 \mathrm{MPa}(70,000 \mathrm{psi})$. Note also that the peak von Mises stresses occur at the vertices of the hexagonal geometry and are due to bending effects because the duct is "trying" to become circular as it deforms. This phenomenon is exhibited in Fig. 4-4, where the deformed mesh is shown superimposed on the undeformed mesh. The flats of the duct are moving approximately $0.015 \mathrm{~cm}(0.006 \mathrm{in}$.) radially outward. To further illustrate that the stresses in the hexagonal geometry are largely due to bending, note that a circular tube of approximately the same "diameter" as the hex with the same wall thickness would experience a circumferential (i.e., "hoop") stress of only $43 \mathrm{MPa}(6300 \mathrm{psi})$ under the same pressure loading. In any case, even with the greatly elevated stress levels attributed to bending effects in the hexagonal duct, a safety factor of approximately 2 (on yield) exists. This stress level and resultant safety factor were deemed to be acceptable. Recall that this 
stress level is for a loading scenario beyond normal operational conditions. During normal operation the $1.59 \mathrm{MPa}$ ( $230 \mathrm{psia}$ ) internal pressure would be counterbalanced by the external pressure of the $3 \mathrm{He}$ in the helium chamber. Originally, this pressure was $0.69 \mathrm{MPa}(100$ psia), resulting in a net outward pressure loading on the hexagonal duct of $0.90 \mathrm{MPa}(130$ psi). The resulting peak von Mises stress under operational conditions would then be 276 $\mathrm{MPa}$ (40,000 psi) (it scales linearly), resulting in an "operational" safety factor of 3.5 . Recent physics design studies have increased the ${ }^{3} \mathrm{He}$ pressure in the chamber to $2.07 \mathrm{MPa}(300$ psia). This external pressure exceeds the $1.59 \mathrm{MPa}$ (230 psia) coolant pressure inside the bundle, resulting in a net operational pressure of $0.48 \mathrm{MPa}$ (70 psi) acting inward. The peak von Mises stress associated with this operational pressure loading would be on the order of $138 \mathrm{MPa}(20,000 \mathrm{psi})$, resulting in an operational safety factor of 7 .

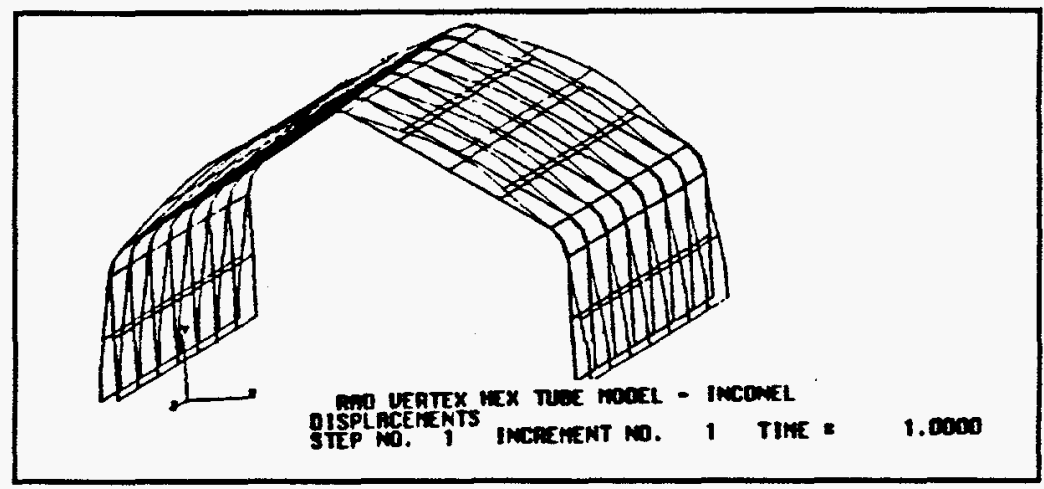

Fig. 4-4. Hexagonal Duct Deformation Due To Internal Pressure Loading.

The inverse abnormal loading case must also be considered. This scenario postulates that the coolant pressure inside the bundle is lost while the external pressure in the helium chamber is maintained at either 0.69 or $2.07 \mathrm{MPa}$ (100 or 300 psia). Under either pressure loading, the hexagonal duct would deflect inward (approximately $0.0076 \mathrm{~cm}$, or $0.003 \mathrm{in}$., at the flats for the $0.69-\mathrm{MPa}$, or 100 -psia, loading and approximately $0.023 \mathrm{~cm}$, or $0.009 \mathrm{in}$., for the 2.07- $\mathrm{MPa}$, or 300-psia, loading). Peak von Mises stresses, again at the vertices of the hexagonal duct, would be 248 and $745 \mathrm{MPa}(36,000$ and 108,000 psi) for the 0.69- and 2.07$\mathrm{MPa}$ (100- and 300-psia) external pressure loading cases, respectively. Neither of these stress levels would cause catastrophic failure because the yield strength of Inconel-718, even at the elevated operational temperature, is approximately 965 to $1034 \mathrm{MPa}(140,000$ to $150,000 \mathrm{psi})$.

As the proton beam passes through the Inconel-718 hexagonal duct, a small portion of its energy is deposited in the duct. A thermal analysis was performed to assess the effect of this parasitic energy deposition. Because the convective heat transfer removal due to the $3 \mathrm{He}$ environment on the exterior surfaces is negligible compared with that on the inside surfaces of the duct, it was neglected. Thus, the exterior surfaces of the duct were assumed to be insulated (a conservative approximation). The $\mathrm{D}_{2} \mathrm{O}$ coolant flowing through the bundle was assumed to provide a convective heat transfer coefficient of $45,300 \mathrm{~W} / \mathrm{m}^{2}{ }^{\circ} \mathrm{K}(7,980 \mathrm{Btu} / \mathrm{h}$ $\left.\mathrm{ft}^{2}-^{\circ} \mathrm{F}\right)$ on the inside surfaces of the duct. A fluid sink temperature of $358 \mathrm{~K}\left(184^{\circ} \mathrm{F}\right)$ was assumed. A thermal analysis of the duct utilizing these parameters resulted in an operational 
temperature for the duct of approximately $427 \mathrm{~K}\left(309^{\circ} \mathrm{F}\right)$. Note that this temperature is well within the operational limits of Inconel-718.

Cyclic loading of the rod bundle duct due to thermal or pressure transients is not considered to be a problem. From a thermal perspective, the duct is free to grow in the axial direction (due to the single convolute bellows located at the transition section). Of course, "radial" growth of the duct due to thermal expansion is essentially unconstrained. Thus, the mechanical design has minimized the potential for the buildup of large thermal stresses. Potential cyclic pressure loadings could result in stresses ranging from 0 to $745 \mathrm{MPa}(0$ to $108,000 \mathrm{psi}$ ). These stresses are only $50 \%$ to $75 \%$ of the yield strength of the material within the operational temperature range. Additionally, very few cycles of this type of loading would be expected. In conclusion, thermomechanical fatigue problems in the rod bundle ducts are not likely to occur due to the conservative mechanical design.

Finally, flow-induced vibration of the tungsten rods within the bundles has been identified as an issue to be addressed. Vibration data were taken on two different occasions using the cold-flow test rod bundle. Although the experimental setup for the cold-flow test does not lend itself to easy vibration data interpretation (i.e., significant pump noise was measured in the first test due to a failure to isolate the pump from the bundle using a bellows), the data reduction is in progress. This task will be addressed in more detail in the next design phase.

\subsubsection{He Chamber}

The $3 \mathrm{He}$ chamber houses the tungsten and is filled with the pressurized $3 \mathrm{He}$ that forms the first layer of the blanket. As shown in Fig. 4-1, the tungsten rod bundle inlet and outlet plena are mounted (bottom and top) in "saddles" that are connected to the $3 \mathrm{He}$ chamber. These plena provide the $\mathrm{D}_{2} \mathrm{O}$ coolant (supply and return) to the tungsten rod bundles. The 3He chamber is a cylindrical tube approximately $4.5 \mathrm{~m}(14.8 \mathrm{ft})$ long including endcaps. It is constructed of Inconel- 718 sheet and has a wall thickness of $0.3175 \mathrm{~cm}(0.125 \mathrm{in}$.). Each end of the main chamber is fitted with an elliptical dome endcap (Standard ASME 2:1 Elliptical Head). Ten stiffening rings with rectangular cross sections of $5.71 \mathrm{~cm} \mathrm{x} 0.7938 \mathrm{~cm}(2.25 \mathrm{in}$. $x 0.3125$ in.) are located on approximately $42 \mathrm{~cm}$ (16.5 in.) centers along the length of the chamber. Note that the stiffening rings and the saddles that support the plena are integral components such that direct load paths for lifting have been achieved through the mechanical design. The plena are cylinders with an inside diameter of $31.59 \mathrm{~cm}(12.44 \mathrm{in}$.). Wall thickness for these components has been specified as $0.40 \mathrm{~cm}(0.16 \mathrm{in}$.).

The first loading scenario involved the normal pressure loadings that the chamber and plena would experience. Heavy water coolant for the rod bundles at a conservative pressure of approximately $1.59 \mathrm{MPa}$ (230 psia) was assumed in the inlet plena. Hand calculations were used to confirm the plenum mechanical design. These calculations were subsequently repeated using a finite element model. ${ }^{3} \mathrm{He}$ pressures ranging from $0.69 \mathrm{MPa}$ to $2.07 \mathrm{MPa}$ (100 psia to 300 psia) have been employed in the evolving physics design calculations. The current mechanical design analysis is based on an internal 3He pressure of $0.69 \mathrm{MPa}$ (100 psia) and reflects the Revision 0 mechanical design. Note that both hand calculations and finite element models were used to assess the ${ }^{3} \mathrm{He}$ chamber design. Peak von Mises stresses in the chamber under a $0.69 \mathrm{MPa}(100 \mathrm{psia})$ internal pressure loading were on the order of $210 \mathrm{MPa}(30,400 \mathrm{psi})$. These stresses are acceptable. 
The second loading to be considered for the ${ }^{3} \mathrm{He}$ chamber was the case where it experiences external pressure loadings. Under normal operating conditions, the NSA is submerged in a $\mathrm{D}_{2} \mathrm{O}$-filled moderator tank and is subjected to the internal pressure loadings outlined above. The inlet to the moderator tank is, however, pressurized to a load approaching $0.38 \mathrm{MPa}$ (55 psia). Two scenarios have been identified where this external pressure in the moderator tank exceeds the internal pressure in the ${ }^{3} \mathrm{He}$ chamber; namely, (1) a normal NSA removal procedure where the submerged helium chamber is "pumped down" before being backfilled with natural helium gas, and (2) an accident scenario where ${ }^{3} \mathrm{He}$ pressure is suddenly lost within the chamber while the external $\mathrm{D}_{2} \mathrm{O}$ pressure is maintained in the moderator tank. Both of these scenarios could potentially buckle the vessel, which has a large (approximately 400:1) diameter-to-wall-thickness ratio. Both hand calculations and finite element analysis using ABAQUS were employed to assess these design conditions. The numerical calculations gave a lowest-energy buckling mode pressure loading of 0.57 $\mathrm{MPa}$ (83 psia), which is well above the expected loads of $0.38 \mathrm{MPa}$ (55 psia). It was these external pressure loading cases that necessitated the external stiffening rings on the helium chamber. The methods and criteria of the ASME Pressure Vessel Code (Sec. III) were used to guide the design of the ${ }^{3} \mathrm{He}$ chamber. Coupled with good engineering judgment, the guidance provided by the code has resulted in a design that will withstand the internal and external pressure loadings described above. However, two aspects of the design remain to be evaluated with regard to the code: (1) the numerous penetrations into the chamber for the rod bundle feed tubes have yet to be analyzed, and (2) Inconel-718 is not a code-rated material (Inconel-800 was used for all code calculations). The full implications of using Inconel-718 (a non-code material) need to be addressed. These issues will be addressed in the next design phase. Also, the current helium chamber design with an internal pressure of $2.07 \mathrm{MPa}(300$ psia) needs to be examined in the next design phase.

\subsubsection{Proton Beam Backstop Region}

The proton beam backstop region is located immediately behind the helium chamber and consists of layers of circular plates of Zircaloy interspersed with 3 He regions (Fig. 4-8). The Zircaloy sections are made of $1.27-\mathrm{cm}(0.5$-in.)-thick plates joined to form a $15-\mathrm{cm}(5.90-$ in.)-thick unit. Coolant channels are machined into one side of each plate to form closed channels after joining.

The $3 \mathrm{He}$ is located in aluminum containers constructed of $0.48-\mathrm{cm}(0.19$-in. $)$-thick sheet, front and back, with $0.16-\mathrm{cm}(0.06$ in.)-thick ribs on a $5.08-\mathrm{cm}$ (2-in.)-pitch welded to the front and back sheets. The ${ }^{3} \mathrm{He}$ in the backstop region is at $0.69 \mathrm{MPa}(100 \mathrm{psia})$. The hoop that makes the circumference part of the container is $0.63-\mathrm{cm}(0.25-\mathrm{in}$.) -thick. This unit is constructed to provide an equivalent thickness of $5 \mathrm{~cm}(1.97 \mathrm{in}$.) of $3 \mathrm{He}$.

Following the Zircaloy and $3 \mathrm{He}$ sections of the backstop is $30 \mathrm{~cm}$ (11.8 in.) of lead that is part of the target lead (described in Sec. 4.3.4). There are four sections of Zircaloy and $3 \mathrm{He}$ units that are a total of $80 \mathrm{~cm}$ (31.5 in.) in length. This plus the $30 \mathrm{~cm}(11.8 \mathrm{in}$.) of lead provides a combined backstop length of $110 \mathrm{~cm}(43.3 \mathrm{in}$.$) .$

Between the first section of Zircaloy plates and the ${ }^{3} \mathrm{He}$ chamber is a transition piece to convert from the elliptical head of the $3 \mathrm{He}$ chamber to a right circular cylinder configuration of the backstop. Because the entire backstop is immersed in the moderator tank fluid, the transition piece displaces the heavy water in this region. This transition piece is essential to 
prevent proton energy loss in water and therefore maximize neutron production. The transition piece is designed similarly to the other $3 \mathrm{He}$ containers, with a flat sheet on one side but with an elliptical part on the side mating to the $3 \mathrm{He}$ chamber. The ribs and the hoop are of the same design as the $3 \mathrm{He}$ containers. All of these units are sized in diameter so they will fit inside the target lead.

Coolant for the backstop is provided by the moderator primary coolant system. Heavy water coolant flows into the bottom of the Zircaloy and lead channels and exits into the moderator tank. The Zircaloy channels are positioned in such a way as to also provide cooling for the ${ }^{3} \mathrm{He}$ containers.

A structural analysis needs to be performed on the proton beam backstop region. This will be performed in the next design phase.

\subsubsection{Target Lead}

Following primary production of high-energy neutrons and other energetic particles from proton collisions in the tungsten rods, additional neutron production is gained from lower energy-spallations and $(n, x n)$ reactions that occur in a lead multiplying region called the target lead.

The preconceptual design development of the target lead has focused on an unclad stacked plate concept. This concept satisfies several key objectives.

- The extent that the target lead and the helium blankets encircle the NSA has been maximized to increase neutron production.

- Thermal hydraulic analyses show that a "coolable" configuration is possible with safe margins against lead melting and low velocities to preclude lead erosion (This is discussed further in Section 4.4.4).

- $\mathrm{D}_{2} \mathrm{O}$ and Inconel needed to cool and support the lead have been minimized for more effective tritium production.

Cursory analyses using conservative limits on stress and temperature have shown that the concept can be engineered to properly address any concerns for loss of geometry and functionality due to thermal creep of the lead material.

The neutral $\mathrm{pH}$ of the moderator is compatible with the unclad lead and corrosion should not be a problem. Unclad lead enhances the fraction of lead within the multiplier zone and thereby improves neutron yield. The basis for selection of lead and it's alternatives is discussed further in Section 4.2.1.

To compliment the already low waste generation characteristics of the APT $3 \mathrm{He}$ concept, over $81 \%$ of the target lead can be configured for lifelong re-use throughout the 40 -year minimum life of the facility for minimization of irradiated lead mixed wastes. About $19 \%$ of the lead will need to be disposed of as a part of normal retargeting. The target lead will also be placed in regions between the plena and helium chamber on the NSA in order to provide a more complete encirclement. Target lead will also be wrapped around the sides of the 
helium chamber to the depth of the NSA stiffening ribs. This enables the reuseable lead sections to clear the NSA stiffening ribs at assembly as it slides along the guide rails.

A logical retargeting sequence (described in Sec. 4.8) has been developed around this configuration in order to demonstrate a practical hardware concept. The majority of the irradiated target lead can be extracted in an in-pool dissassembly sequence and re-installed within a fresh target module for life long re-use throughout the life of the facility. However, new lead could be substituted if needed.

Replacement target modules can be furbished with new or reused target lead as a near final step in the assembly sequence. This capability facilitates a simpler design by minimizing assembly operations in the presence of irradiated lead. Accordingly, the majority of the assembly can be performed at the factory and shipped to site in non-radioactive form. The target lead can be installed in manageable sections for improved handling.

The target lead is configured in (3) principle zones:

- Zone 1 - along the axial length of the NSA helium chamber.

This lead is distinguished by the use of disposable and reuseable lead plates. Some fill plates are placed near the top and bottom of the NSA in between tungsten rod bundle flow ducts and helium chamber stiffeners. Some other disposable lead plates are used to fill the regions between the helium chamber stiffening ribs along the sides of the helium chamber. Some disposable lead is used in order to maximize the degree of lead encirclement while facilitating remote assembly and recovery of the re-useable lead. The re-useable lead in this region provides approximately $70 \%$ encirclement around the helium chamber.

- Zone 2 - along the axial length of the proton beam backstop.

The lead in this region is fully reuseable and can be configured for approximately $95 \%$ encirclement. The working thickness of this lead is greater due to the absence of features which can interfere with axial insertion of the lead sections.

- Zone 3 - a $28-30 \mathrm{~cm}$ thick circular slab located at the rear of the proton beam backstop.

A description of the stacked plate configuration along the axial length of the NSA helium chamber is shown in Fig. 4-5. A key feature of this configuration is that re-useable portions of the target lead can be configured in half section slabs of manageable length (TBD) which partially encircle the flanks of the NSA helium chamber. In back of the helium chamber, along the axial length of the proton beam backstop, the encirclement of reuseable lead can be increased because there are fewer interfering structures.

The lead is presented in thin shaped flat plate segments that are stacked face to face along the axis of the NSA. A summary of the target lead plates is given in Table 4-3. 


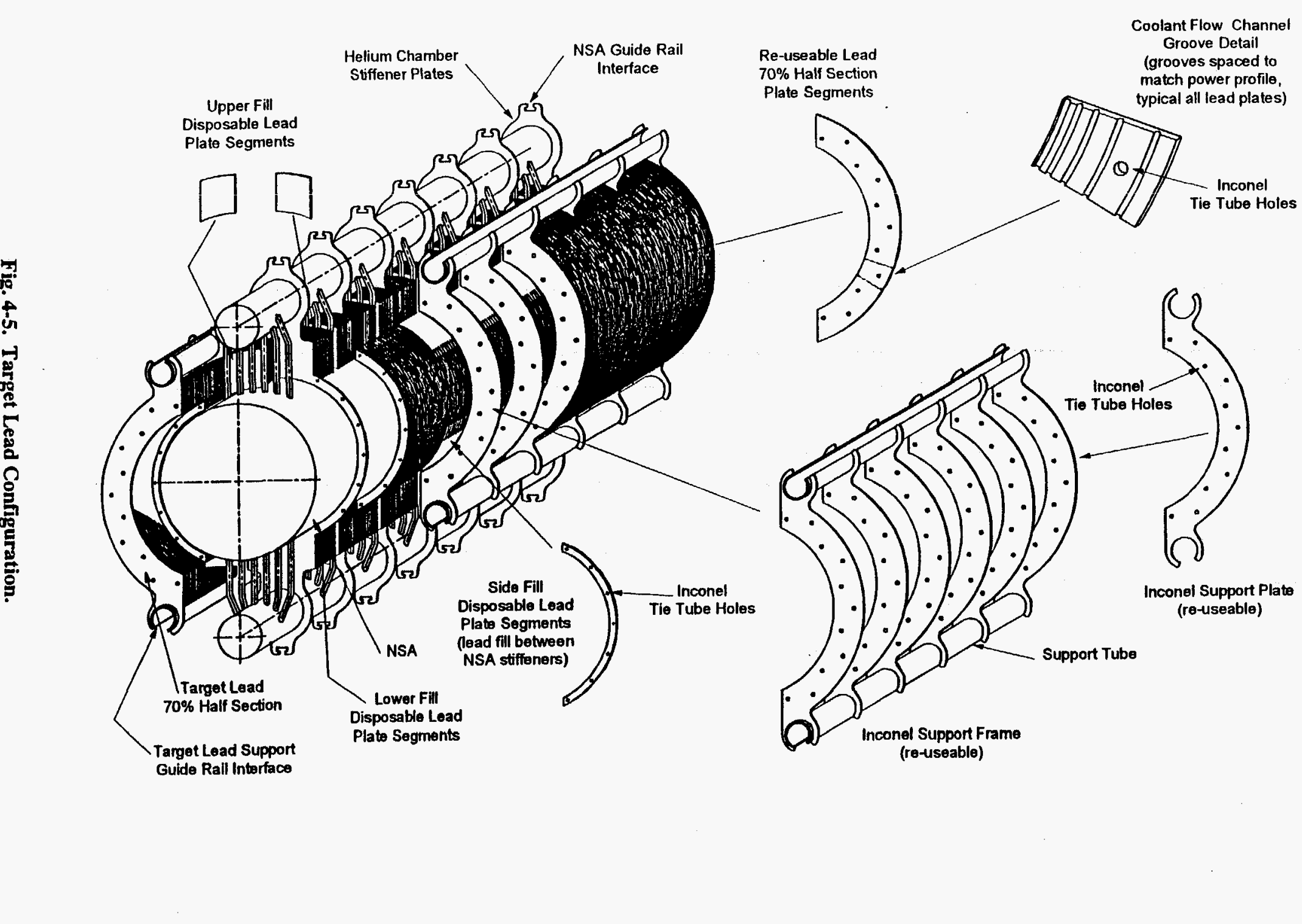

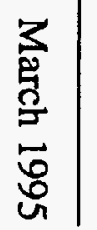


Table 4-3. Target Lead Plates Preconceptual Design

\begin{tabular}{ccc}
\hline Plate Segment Type & No. Pieces & Notes \\
\hline $70 \%$ & 884 & Re-used \\
$95 \%$ & 167 & Re-used Configuration TBD \\
Rear & 36 & Re-used Configuration TBD \\
Upper Fill & 884 & Disposed with NSA \\
Lower Fill & 884 & Disposed with NSA \\
Side Fill & 884 & Disposed with NSA \\
\hline \hline
\end{tabular}

The lead plates within each segment are supported by an Inconel support frame. The frame is constructed of a series of thin Inconel support plates that are spaced at intervals as needed to preclude sagging of the lead plates. Each support plate is captured at two partially looped holes. The loops are captured by heavy wall tubular " $\mathrm{C}$ " shaped members which run the length of each segment. The lead and Inconel support plates are perforated to facilitate axial tie tubes made of Inconel which, with the support tube and inlet header, hold the assembly together. The Inconel plates support the tie tubes, and the Inconel tie tubes support the lead plates. In this fashion, the Inconel volume fraction in the target lead is less than 3\% overall. Preliminary sizing analyses show that the lead can be supported at very low stress levels to preclude creep of the lead plates while maintaining a geometry that can be cooled.

Each lead plate segment contains parallel grooves for channeling coolant. The spacing between the grooves can be varied to match the radial power profile in the lead to minimize the amount of $\mathrm{D}_{2} \mathrm{O}$ in the zone. The grooves are shaped as half circles nearly half the plate thickness in depth.

Adjacent flow channels can be coupled with small diameter bypass holes (not shown) through the lead plates to create an open flow architecture that will reduce the probability of lead melting as a result of a local flow blockage. A summary of the target lead parameters is given in Table 4-4.

Table 4-4. Target Lead Parameters (Preconceptual Approximation)

Radial Thickness
Rear Lead Thickness
Thickness of Lead Plate
Segments
$\%$ Lead (by vol)
zone 1
zone 2
zone 3
\% Inconel (by vol)
zone 1
zone 2
zone 3
$\% \mathrm{D}_{2} \mathrm{O}$ (by vol)

$28 \mathrm{~cm}(11.02 \mathrm{in}$.
$30 \mathrm{~cm}(11.81 \mathrm{in.})$
$0.8 \mathrm{~cm}(0.315 \mathrm{in}$.

$87.4 \%$

$88.2 \%$

$87.5 \%$

$83 \%$

$3 \%$

$2.3 \%$

$3 \%$

$7.4 \%$

$9.5 \%$
Notes

overall

overall

all zones 
Features of the target lead and target module for cooling of the target lead are discussed further in Section 4.4.4. 4.5.

The target lead design for assembly into the target module is discussed further in Section

To accommodate differential thermal expansion of the lead plates during operation, the tie tubes are cold assembled tension free with a slight margin of clearance within the stack of lead plates. During normal operation, the tie tubes are forced cooled from the high pressure side of the flow baffle on the NSA Module through small coolant tubing lines.

\subsubsection{Beam Entrance Window}

The beam entrance window is mounted on the upstream elliptical head of the helium chamber. Its function is to serve as a boundary between the high-pressure $3 \mathrm{He}$ environment inside the helium chamber and the vacuum environment of the proton beam transport tube. The window is constructed of two $0.125-\mathrm{cm}(0.049$-in.)-thick semi-cylindrical shells nominally $47 \mathrm{~cm}(18.5 \mathrm{in}$.) in diameter and $96 \mathrm{~cm}$ (37.8 in.) long that are "nested" with a 0.25 $\mathrm{cm}(0.098 \mathrm{in}$.) coolant gap between them. Partial hemispherical domes cap each end of both the inner and outer shells. An option to be considered is to make the window part of a 2:1 elliptical head. Horizontal spacers between the two 0.125 -cm $(0.049$-in.)-thick shells provide coolant channels and, because they link the two shells together, serve to stiffen the entire assembly. Note that vertical spacers between the shells may offer some safety advantages over the current design; namely, vertical ribs would allow any bubbles that might form during transients to be swept out, and they would also promote cooling via natural convection if pump flow is not available. However, vertical spacers are not as desirable as the horizontal spacers, from a structural perspective. Future design analyses will be employed to study these competing considerations such that an optimal design solution can be achieved.

The beam "footprint" incident on the window covers a rectangular area that is 91 by 42 $\mathrm{cm}(35.8$ by $16.5 \mathrm{in}$.). This "illuminated" area represents approximately $85 \%$ of the available surface area on the window, thus providing a safety margin for slight beam steering errors. $\mathrm{D}_{2} \mathrm{O}$ coolant from the MPCS flows through the coolant channels between the shells to remove the heat deposited by the proton beam as it passes through the material. Inconel-718 was selected as the window material because of its high temperature and strength capabilities. Additionally, considerable experience with Inconel-718 under proton beam irradiation (at fluence levels near those anticipated for the APT system) has been acquired at the LAMPF accelerator at LANL. No radiation-induced damage has been observed in the Inconel-718 components employed in the LAMPF accelerator system.

Finite element analyses were employed to assess the structural behavior of the nested shell structure (Fig. 4-6). Linear elastic stress and buckling analyses were performed with the commercially available finite element code ABAQUS. Parameter studies were executed to determine the optimal shell thicknesses. Again, physics optimizations and mechanical considerations were counter to each other. Minimization of the shell thicknesses was desired from a physics point of view to reduce the proton beam energy losses in the window. Conversely, the pressures to be contained required that the shells be thick enough to maintain the stresses within acceptable levels and prevent buckling. Under normal operational 
conditions, both shells that constitute the window would experience a coolant pressure between the shells of approximately $0.45 \mathrm{MPa}(65$ psia) and the inner shell would also be loaded with a uniform pressure loading due to the $3 \mathrm{He}$ in the chamber. Of course, the "outer" shell has the vacuum environment of the accelerator beam tube on one surface. Physics design studies have recently increased the ${ }^{3} \mathrm{He}$ pressure in the chamber from 0.69 to 2.07 $\mathrm{MPa}$ (100 to 300 psia). To date, the window design described in Revision 0 has been analyzed for several different loading case scenarios. If the only pressure loading is the coolant pressure between the shells (i.e., assume that the ${ }^{3} \mathrm{He}$ pressure has somehow been lost), the maximum von Mises stress in the structure is approximately $152 \mathrm{MPa}(22,000 \mathrm{psi})$. Recall that the von Mises stress is an effective stress measure that is a combination of the principal stresses. It is frequently employed to assess the potential for yielding (i.e., failure) in ductile materials like Inconel-718. A stress level of $152 \mathrm{MPa}(22,000 \mathrm{psi})$ is acceptable for this material. The next loading scenario to be examined with the Revision 0 finite element model applied a pressure of $1.03 \mathrm{MPa}(150 \mathrm{psia})$ to the inside surface of the inner shell and the $0.45-\mathrm{MPa}$ (65-psia) coolant pressure between the shells. Maximum von Mises stresses in this case were on the order of $272 \mathrm{MPa}(39,500 \mathrm{psi})$. Recently, the stress analyses have been updated to reflect the latest window design associated with the reduced beam footprint. A $2.07 \mathrm{MPa}$ (300 psia) $3 \mathrm{He}$ pressure and a $0.45-\mathrm{MPa}(65-\mathrm{psia}) \mathrm{D}_{2} \mathrm{O}$ pressure were simultaneously applied to the finite element model, resulting in a maximum stress level of $399 \mathrm{MPa}(58,000 \mathrm{psi})$. This stress level still results in a safety factor that is greater than 2 (on yield). Further work needs to be done on the window design including exploring different design options to simplify the overall design of the window and the $3 \mathrm{He}$ chamber.

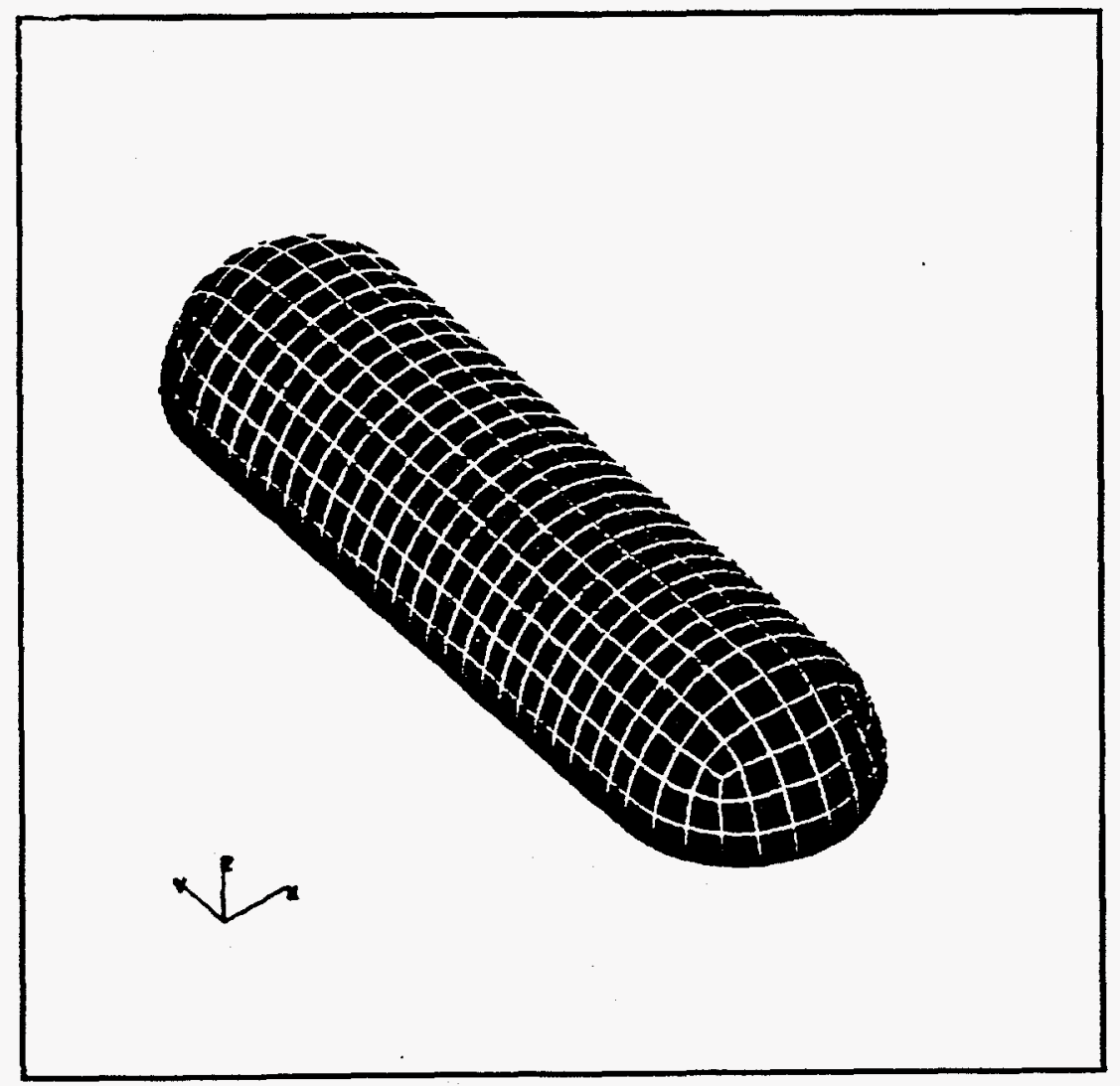

Fig. 4-6. Finite Element Model Of Beam Entrance Window. 
ABAQUS was also employed to evaluate the potential for buckling of the Revision 0 window design. The loading scenario that needed to be checked was the case where the ${ }^{3} \mathrm{He}$ pressure within the chamber has been lost while the $\mathrm{D}_{2} \mathrm{O}$ coolant in the passages between the shells has been maintained. Intuitively, the inner shell would be loaded in a fashion where buckling could occur under this loading scenario. However, the finite element analysis and even a hand-calculation check indicated that the pressure loadings required between the shells to cause buckling were on the order of hundreds of pounds per square inch. The numerical calculation yielded a pressure of approximately $5.17 \mathrm{MPa}$ (750 psi) for the lowestenergy buckling mode, while the hand calculation estimated the pressure to be over $2.07 \mathrm{MPa}$ ( 300 psia). In any case, buckling of the shells that comprise the beam entry window was not deemed to be a problem for a coolant pressure loading of $0.45 \mathrm{MPa}(65 \mathrm{psia})$. These analyses will need to be updated for the current window design in the next design phase.

\subsection{THERMAL DESIGN OF NSA}

The neutron source assembly (NSA) includes the tungsten rod bundles, the tungsten inlet and outlet plena, the ${ }^{3} \mathrm{He}$ chamber, the beam entrance window, the beam tunnel extension, the beam entrance nozzle, and the beam tunnel remote connector. Currently, it is planned that the NSA will be replaced every 1 year or longer of full power operation at $75 \%$ capacity. The following thermal design description includes details on steady-state thermal and hydraulic analyses, as well as a summary of pertinent sensitivity studies. While not part of the NSA, this section also includes a description of the preconceptual thermal design of the target lead.

\subsubsection{Tungsten Rod Bundles}

Inside the ${ }^{3} \mathrm{He}$ chamber, there are 88 tungsten rod bundles arranged in alternating rows of five bundles in one row and six bundles in the next for a total of 16 rows. Figure 4-2 shows the rod bundle layout pattern. Each bundle contains 91 tungsten rods wrapped with tungsten wire with four wraps in the $96 \mathrm{~cm}$ (37.8 in.) rod length. Heat transfer is calculated using the $91-\mathrm{cm}$ ( 35.8 -in.) length directly heated by the proton beam, whereas pressure drop calculations are based on the $96 \mathrm{~cm}$ (37.8 in.) total bundle length. The rod bundle is surrounded by a hexagonal Inconel duct with a $0.0762-\mathrm{cm}(0.030$-in.) wall thickness and a $4.3317-\mathrm{cm}(1.705-\mathrm{in}$.) distance across the outside of the flats. To allow extra room for assembly, inside dimensions of the hexagonal duct are calculated using a factor 1.007 larger than the minimum needed to hold the rods plus wires. Bundle dimensions are based on a nominal rod diameter of $0.3175 \mathrm{~cm}(0.125 \mathrm{in}$.) and a nominal wire diameter of $0.1016 \mathrm{~cm}$ ( $0.040 \mathrm{in}$.). Rod and wire sizes were varied by position in the rows with smaller rods used in the higher power density front end rows where the beam strikes first and larger rods used in the lower power density back end rows. Rod and wire sizes plus any orificing needed are the same for each bundle in a group of two rows of bundles so the flow through each bundle in a group is essentially the same. The rod/wire size and orifice is used to maintain an average outlet temperature of $110^{\circ} \mathrm{C}\left(230^{\circ} \mathrm{F}\right)$ for each group of two rows. Material volume fractions within the bundles ranged from 0.4313 tungsten, 0.0691 Inconel, and $0.4995 \mathrm{D}_{2} \mathrm{O}$ in the first two rows where the rod and wire diameters were $0.2840 \mathrm{~cm}(0.1118 \mathrm{in}$.) and $0.1320 \mathrm{~cm}$ ( $0.0520 \mathrm{in}$.), respectively to 0.6278 tungsten, 0.0691 Inconel, and $0.3030 \mathrm{D}_{2} \mathrm{O}$ in the last six rows, where the rod and wire diameters were $0.3745 \mathrm{~cm}(0.1474 \mathrm{in}$.) and $0.0500 \mathrm{~cm}(0.0197$ 
in.), respectively. The rod/wire size variations were limited in the high power end by keeping the $\mathrm{D}_{2} \mathrm{O}$ fraction less than 0.5 in the highest power bundles and in the low power end by using the minimum wire size commercially available. The total power in all 88 bundles is $76.7 \mathrm{MW}$, and the total mass flow rate required is $409.1 \mathrm{~kg} / \mathrm{s}(901.9 \mathrm{lbm} / \mathrm{s})$ for an average $\mathrm{D}_{2} \mathrm{O}$ temperature increase of $45^{\circ} \mathrm{C}\left(81^{\circ} \mathrm{F}\right)$.

The power deposited is highest in the rows that the beam hits first near the front of the target area and decreases considerably toward the back. Table 4-5 shows the large power variation from the front to back rows. Note that even numbered rows have six bundles, and the outer two bundles are only partially in the beam, so averages for these rows are lower than the corresponding rows of five bundles, where all bundles are completely in the beam. The second row has a higher peak bundle power than the first because it is directly in the proton beam; further, it receives the additional effect of beam scattering from the first row. Tungsten power densities range from $2.56 \mathrm{MW} / \mathrm{in}$ the highest-power bundle to $0.19 \mathrm{MW} / \mathrm{H}$ in the lowest power bundle, with an average power density of $0.99 \mathrm{MW} /$. The power density in the Inconel duct for the highest-power bundle is $1.17 \mathrm{MW} /$, with the average being 0.54 MW/1. All bundles have the same $65^{\circ} \mathrm{C}\left(149^{\circ} \mathrm{F}\right)$ inlet temperature, and the average exit temperature is $110^{\circ} \mathrm{C}\left(230^{\circ} \mathrm{F}\right)$, which is governed by the tungsten primary coolant system heat exchanger design plus keeping a reasonable DNBR margin in the highest-power bundles. Each group of 11 bundles in two rows has the same $110^{\circ} \mathrm{C}\left(230^{\circ} \mathrm{F}\right)$ average exit temperature so that the exit temperature leaving both ends of the exit plenum and entering the cooling loops will be the same.

Table 4-5. Tungsten Power Densities in Each Rod Bundle Row

\begin{tabular}{ccc}
\hline Row & Peak (MW/I) & Average (MW/I) \\
\hline 1 & 2.29 & 2.27 \\
2 & 2.56 & 2.05 \\
3 & 1.86 & 1.78 \\
4 & 2.29 & 1.82 \\
5 & 1.62 & 1.51 \\
6 & 1.68 & 1.36 \\
7 & 1.39 & 1.26 \\
8 & 1.25 & 1.00 \\
9 & 1.04 & 0.93 \\
10 & 0.92 & 0.72 \\
11 & 0.78 & 0.66 \\
12 & 0.66 & 0.52 \\
13 & 0.54 & 0.47 \\
14 & 0.44 & 0.35 \\
15 & 0.37 & 0.32 \\
16 & 0.29 & 0.24 \\
\hline \hline
\end{tabular}

The rod/wire sizes and orificing requirements were calculated as follows for eight groups of bundles with two rows of bundles containing 11 bundles included in each group. The first group is the first two rows that the beam strikes. It uses the minimum rod size to improve the thermal-hydraulic margins and to keep the $\mathrm{D}_{2} \mathrm{O}$ volume fraction below 0.5 in the volume including the tungsten, $\mathrm{D}_{2} \mathrm{O}$, and Inconel duct for improved physics performance. 
Average power in the first group bundles is calculated using the average tungsten and Inconel power densities in the first two rows and the rod and wire sizes in the first two rows. The flow rate is then determined for each bundle by dividing the average bundle power by the product of the specific heat and the $45^{\circ} \mathrm{C}\left(81^{\circ} \mathrm{F}\right)$ average temperature increase from the $65^{\circ} \mathrm{C}$ $\left(149^{\circ} \mathrm{F}\right)$ inlet to the $110^{\circ} \mathrm{C}\left(230^{\circ} \mathrm{F}\right)$ outlet. This flow rate is used with the average bundle power for the group to determine the plenum-to-plenum pressure drop and with the peak bundle power to determine the maximum exit temperature. This pressure drop is then matched in the calculations for the remaining seven groups.

For the second group, an iteration procedure is used to vary the rod/wire size with the average tungsten and Inconel power densities and $a 45^{\circ} \mathrm{C}\left(81^{\circ} \mathrm{F}\right)$ average $\mathrm{D}_{2} \mathrm{O}$ temperature increase to determine the flow rate needed. This flow rate is used to calculate the pressure drop. The rod/wire size is varied until the pressure drop matches that determined for the first (highest power) group. This procedure is continued for succeeding groups until a point is reached where the wire size would need to be smaller than the minimum commercially available. When this occurs, the wire size is limited to the minimum commercially available, and orificing is required to increase the pressure drop to match that of the first group. Table 4-6 shows the rod/wire sizes for each group and the pressure drop that needs to be obtained across orifices for the lower power groups. Note that only the last six rows out of a total of sixteen rows require orificing despite a ratio of 13.5 between the tungsten power densities in the peak and minimum rod bundles. Therefore, varying the rod/wire size is very effective in minimizing required orificing over a very large power range, and it also permits using a lower pressure drop with reduced pumping requirements. Varying the rod/wire size also improves the thermal-hydraulic margins in the high power rod bundles.

Table 4-6. Bundle Characteristics for Each Group

\begin{tabular}{cccccc}
\hline Group & Rows & $\begin{array}{c}\text { Rod Diam. } \\
(\mathbf{c m})\end{array}$ & $\begin{array}{c}\text { Wire Diam. } \\
(\mathbf{c m})\end{array}$ & $\begin{array}{c}\text { Bundle Flow } \\
(\mathbf{k g} / \mathbf{s})\end{array}$ & $\begin{array}{c}\text { Orifice Pressure } \\
\text { Drop (psi) }\end{array}$ \\
\hline 1 & 1,2 & .2840 & .1320 & 7.87 & 0 \\
2 & 3,4 & .3057 & .1123 & 7.14 & 0 \\
3 & 5,6 & .3285 & .0916 & 6.17 & 0 \\
4 & 7,8 & .3476 & .0743 & 5.22 & 0 \\
5 & 9,10 & .3671 & .0566 & 4.14 & 19.9 \\
6 & 11,12 & .3745 & .0500 & 3.06 & 43.3 \\
7 & 13,14 & .3745 & .0500 & 2.12 & 55.4 \\
8 & 15,16 & .3745 & .0500 & 1.47 & \\
\hline \hline
\end{tabular}

Table 4-7 summarizes the thermal-hydraulic results for the highest power bundle (located in second row) and a mid-range bundle. The mid-range bundle is in the ninth row, and it is the highest-power bundle in the last eight rows that the beam strikes. The plenumto-plenum pressure drop is $475 \mathrm{kPa}$ (68.9 psi). An exit plenum pressure of $965 \mathrm{kPa}$ (140 psia) is used, on the basis of net positive suction head requirements for the primary loop pumps. Maximum rod temperatures are 157.0 and $145.4^{\circ} \mathrm{C}\left(314.6\right.$ and $\left.293.7^{\circ} \mathrm{F}\right)$ for the high- and mid-range-power rods, and the corresponding maximum Inconel duct temperatures are $161.8^{\circ} \mathrm{C}$ and $143.6^{\circ} \mathrm{C}\left(323.2^{\circ} \mathrm{F}\right.$ and $\left.290.5^{\circ} \mathrm{F}\right)$. Wall heat fluxes are $1.82 \mathrm{MW} / \mathrm{m}^{2}$ and $0.96 \mathrm{MW} / \mathrm{m}^{2}$ for the high- and mid-range-power rods, respectively. For this stage of the 
design, we are using a minimum DNBR requirement of about 2.0 and a modified Stanton number upper limit of 0.0045 , on the basis of the Savannah River experience. The modified Stanton number measures how close the channel is to onset of significant voiding. For the high-power rods, the DNBR is 1.99 , and the modified Stanton number is only 0.00072 , which is well below the 0.0045 limit based on Savannah River experience. The boiling limit power ratio is 1.59 . Note that the boiling limit power ratio is defined as the ratio of the mass flow rate times specific heat multiplied by the minimum exit subcooling plus the bundle power divided by the bundle power. The minimum exit subcooling is calculated by subtracting the fluid temperature in the film at the exit rod surface from the saturation temperature at the exit pressure. When the boiling limit power ratio decreases to 1.0 , the hottest fluid in the channel would have just reached saturation in the film on the rod at the exit. The minimum bundle exit subcooling is $31.8^{\circ} \mathrm{C}\left(57.2^{\circ} \mathrm{F}\right)$ for the high-power rods and $39.5^{\circ} \mathrm{C}\left(71.1^{\circ} \mathrm{F}\right)$ for the mid-range-power rods.

Table 4-7. High- and Mid-Range-Power Rod Bundles and Ducts

\begin{tabular}{lcc}
\hline \multicolumn{1}{c}{ Quantity } & High & Mid-Range \\
\hline Tungsten Power Density (MW/1) & 2.56 & 1.04 \\
Inconel Power Density (MW/1) & 1.17 & 0.55 \\
Power per Bundle (MW) & 1.755 & 0.994 \\
Inlet Temperature ( $\left.{ }^{\circ} \mathrm{C}\right)$ & 65 & 65 \\
Exit Temperature ( $\left.{ }^{\circ} \mathrm{C}\right)$ & 118.6 & 122.6 \\
Bundle Mass Flow Rate (kg/s) & 7.87 & 4.14 \\
Bundle Average Velocity (M/s) & 9.03 & 7.32 \\
Bundle Reynolds Number & 62255 & 32226 \\
Inlet Plenum Pressure (psia) & 208.88 & 208.88 \\
Bundle Inlet Pressure (psia) & 200.61 & 205.57 \\
Bundle Exit Pressure (psia) & 148.15 & 142.78 \\
Outlet Plenum Pressure (psia) & 140.00 & 140.00 \\
Bundle Pressure Drop (psi) & 52.46 & 62.79 \\
Inlet-to-Outlet Pressure Drop (psi) & 68.88 & 68.88 \\
Maximum Rod Temperature ( $\left.{ }^{\circ} \mathrm{C}\right)$ & 157.0 & 145.4 \\
Maximum Duct Temperature $\left({ }^{\circ} \mathrm{C}\right)$ & 161.8 & 143.6 \\
Heat Flux in Rods (MW/m2) & 1.82 & 0.96 \\
Heat Flux in Duct Wall (Mw/m2) & 0.909 & 0.428 \\
DNBR & 1.99 & 2.73 \\
Modified Stanton Number & 0.000720 & 0.000513 \\
Boiling Limit Power Ratio & 1.59 & 1.69 \\
Bundle Exit Saturation Temperature $\left({ }^{\circ} \mathrm{C}\right)$ & 181.2 & 179.6 \\
Minimum Bundle Exit Subcooling $\left({ }^{\circ} \mathrm{C}\right)$ & 31.8 & 39.5 \\
\hline \hline
\end{tabular}

Because the DNBR for the highest power rods is essentially at the lower limit selected for this stage of the design, a parameter study was performed to determine sensitivity to average exit temperature, inlet temperature, and rod size in the highest-power bundle group. Reducing the average outlet temperature from $110^{\circ} \mathrm{C}\left(230^{\circ} \mathrm{F}\right)$ to $105^{\circ} \mathrm{C}\left(221^{\circ} \mathrm{F}\right)$ and maintaining the $65^{\circ} \mathrm{C}\left(149^{\circ} \mathrm{F}\right)$ inlet temperature increases the DNBR to 2.14 . Reducing the inlet from $65^{\circ} \mathrm{C}\left(149^{\circ} \mathrm{F}\right)$ to $50^{\circ} \mathrm{C}\left(122^{\circ} \mathrm{F}\right)$ and maintaining the same $45^{\circ} \mathrm{C}\left(81^{\circ} \mathrm{F}\right) \mathrm{D}_{2} \mathrm{O}$ temperature change increases the DNBR to 2.13 . Reducing the rod size from $0.284 \mathrm{~cm}$ $(0.1118$ in.) to $0.25 \mathrm{~cm}(0.0984 \mathrm{in}$.) with the original inlet and exit temperatures increases the DNBR to 2.14. Combining all three changes so that the inlet temperature is $50^{\circ} \mathrm{C}\left(122^{\circ} \mathrm{F}\right)$, 
the average exit temperature is $90^{\circ} \mathrm{C}\left(194^{\circ} \mathrm{F}\right)$, and the rod size in the highest-power bundle group is $0.25 \mathrm{~cm}(0.0984 \mathrm{in}$.) gives a DNBR of 2.44 . Therefore, several options can be used to increase the DNBR margin if necessary.

The pressure drop correlations used have been checked against cold flow tests using a full-scale, 91-rod bundle, as described in Ref. 4-2. The calculated bundle pressure drops, including an inlet contraction and exit expansion, differ from the data by no more than $5.9 \%$ over the test range of 3.3 to $7.5 \mathrm{~kg} / \mathrm{s}(7.3$ to $16.5 \mathrm{lbm} / \mathrm{s})$. At the low end of the flow rate range, the calculated pressure drop was $2.9 \%$ above the data; at the high end, it was $5.9 \%$ below the data. The correlation calculates the pressure drop in the bundle very well.

\subsubsection{He Chamber}

The $3 \mathrm{He}$ chamber is subject to a thermal loading due to the neutron fluence. The finite element model developed for the structural analyses described above was also employed in a thermal analysis, using a volumetric heating rate of $88 \mathrm{~kW} /$, a convective coefficient of $10,000 \mathrm{~W} / \mathrm{m}^{2} \mathrm{~K}$, and a sink temperature of $70^{\circ} \mathrm{C}\left(158^{\circ} \mathrm{F}\right)$ on the outside surfaces; assuming the inside surfaces of the vessel to be insulated (due to the poor conductance of $3 \mathrm{He}$ ), a maximum Inconel temperature of approximately $134^{\circ} \mathrm{C}\left(273^{\circ} \mathrm{F}\right)$ was calculated. This operational temperature is acceptable for Inconel-718.

\subsubsection{Proton Beam Backstop Region}

The proton beam backstop region is located immediately behind the ${ }^{3} \mathrm{He}$ chamber and includes layers of $3 \mathrm{He}$, aluminum-1100, and Zircaloy- 4 followed by a lead region at the end of the backstop. Figure 4-8 shows the location of the proton beam backstop region. The $3 \mathrm{He}$ chamber back wall is $0.32-\mathrm{cm}(0.125$-in.) thick. It is followed by an aluminum- 1100 transition piece containing ${ }^{3} \mathrm{He}$ that extends from the elliptical head shape of the back wall to the cylindrical flat plate shape of the rest of the backstop. Between the transition piece and the back wall of the target chamber is a $\mathrm{D}_{2} \mathrm{O}$ flow passage, and there is also a $\mathrm{D}_{2} \mathrm{O}$ flow passage between the transition piece and the first Zircaloy-4 plate. Following the transition piece there are four backstop regions of $15 \mathrm{~cm}$ (5.9 in.) of Zircaloy- 4 cooled by $\mathrm{D}_{2} \mathrm{O}$, followed by aluminum-1000 containing a 5 -cm (2.0-in.) thickness of ${ }^{3} \mathrm{He}$ (flux trap). The final backstop region is $30 \mathrm{~cm}$ (11.8 in.) of lead cooled by $\mathrm{D}_{2} \mathrm{O}$. Each of the aluminum- 1100 regions containing ${ }^{3} \mathrm{He}$ consists of aluminum front and back plates $0.48-\mathrm{cm}(0.19-$ in.). thick, radial walls $0.64-\mathrm{cm}(0.25$-in.) thick, and aluminum ribs to provide strength between the plates that are $0.16-\mathrm{cm}(0.0625$-in.) thick on $5.08-\mathrm{cm}(2.0-\mathrm{in}$.) centers. The Zircaloy-4 plates are assumed to be a maximum of $1.27-\mathrm{cm}(0.50$-in.) thick. The lead thickness was varied to determine how thick it could be for the different power density regions and still stay below a $104^{\circ} \mathrm{C}\left(219^{\circ} \mathrm{F}\right)$ creep limit.

Table 4-8 shows the power densities in the backstop regions. Note the large dropoff in power density in the aluminum and Zircaloy- 4 between the first and last regions. In the physics model for the Table 4-8 power densities, both the Zircaloy-4 and lead regions were assumed to have a $25 \% \mathrm{D}_{2} \mathrm{O}$ volume fraction for cooling. To be conservative, the power densities given for these regions are based on the assumption that all of the power is deposited directly in the Zircaloy-4 or lead. The first lead zone in the physics model was only 1-cm (0.4-in.) thick, and the second included the remaining $29 \mathrm{~cm}$ (11.4-in.). The total 
power in the backstop region is about $17.4 \mathrm{MW}$, with $15.1 \mathrm{MW}$ in the Zircaloy-4 plates. The total $\mathrm{D}_{2} \mathrm{O}$ flow rate needed to cool the backstop region is $225 \mathrm{~kg} / \mathrm{s}(496 \mathrm{lbm} / \mathrm{s})$.

Table 4-8. Backstop Power Density Summary

\begin{tabular}{lc}
\hline \multicolumn{1}{c}{ Region } & Power Density $(\mathbf{k W / 1})$ \\
\hline Inconel Chamber Wall & 81.8 \\
D,O next to Chamber & 17.9 \\
Aluminum & \\
in Canister & 31.4 \\
1st Flux Trap & 23.6 \\
2nd Flux Trap & 13.6 \\
3rd Flux Trap & 3.40 \\
4th Flux Trap & 1.55 \\
3He & \\
in Canister & 0.203 \\
1st Flux Trap & 0.758 \\
2nd Flux Trap & 0.688 \\
3rd Flux Trap & 0.493 \\
4th Flux Trap & 0.414 \\
Zircaloy-4 & \\
1st Zone & 60.4 \\
2nd Zone & 39.6 \\
3rd Zone & 16.3 \\
4th Zone & 5.53 \\
Lead & \\
1st Zone & 4.12 \\
2nd Zone & 1.66 \\
\hline \hline
\end{tabular}

The approximate amount of $\mathrm{D}_{2} \mathrm{O}$ needed to cool each region was determined using the following assumptions. The inlet and exit temperatures were $51.5^{\circ} \mathrm{C}\left(124.7^{\circ} \mathrm{F}\right)$ and $70^{\circ} \mathrm{C}$ $\left(158.0^{\circ} \mathrm{F}\right)$, respectively, and the exit pressure was $241 \mathrm{kPa}(35 \mathrm{psia})$. The pressure drop from inlet to exit was $69 \mathrm{kPa}(10 \mathrm{psi})$ of the total of $138 \mathrm{kPa}(20 \mathrm{psi})$ available from the moderator tank entrance to exit. The remaining $69 \mathrm{kPa}(10 \mathrm{psi})$ was assumed to be available for piping and orificing, as needed. Four types of flow paths were modeled: (1) between the $3 \mathrm{He}$ Inconel chamber wall and the transition piece, (2) between an aluminum plate and Zircaloy-4 plate for either the transition region or a flux trap, (3) between two Zircaloy-4 plates, and (4) between two lead plates. Note that the $3 \mathrm{He}$ chamber wall and aluminum walls are cooled on only one side, while the Zircaloy- 4 and lead plates are cooled on both sides. For each type of flow path the heat flow to the coolant was determined by summing up the power deposited on each side of the coolant path. For example, for Path 3 this would be the power deposited in two half thicknesses of Zircaloy-4 plates. For Path 2 between an aluminum plate and Zircaloy- 4 plate, the total power to the coolant would be the sum of half the power in one Zircaloy- 4 plate, all of the power in one aluminum plate, half the power in the 3 He between the aluminum plates, and half the power in the aluminum ribs between the aluminum plates. The mass flow rate is determined for each path by dividing the heat flowing to the coolant by the product of the specific heat and the difference between exit and inlet temperature. The 
flow passage gap needed was then sized with this flow rate to give the $69 \mathrm{kPa}$ (10 psi) pressure drop. For this stage of the design, the flow passage was modeled as flow between flat plates rather than a more detailed flow through slots in the plates that will probably be used in the next design stage. The heat transfer area on each side of the flow path is the area of the circular plate with the backstop diameter of $118.635 \mathrm{~cm}$ (46.7 in.). The backstop diameter was used as the characteristic length for the flow paths, and the flow path width was determined by dividing the area by the backstop diameter. Temperatures in the metal walls were determined by one-dimensional conduction with uniform heat generation. The peak temperature in the aluminum ribs between the aluminum plates was determined by adding the aluminum plate maximum temperature to the temperature difference through half the rib length-the formula having been based, again, on 1D conduction with uniform heat generation.

Peak metal temperatures are $114.3^{\circ} \mathrm{C}\left(237.7^{\circ} \mathrm{F}\right)$ in the chamber wall, $71.6-79.7^{\circ} \mathrm{C}$ $\left(160.9-175.5^{\circ} \mathrm{F}\right)$ in the aluminum walls, $73.8-123.8^{\circ} \mathrm{C}\left(164.8-254.8^{\circ} \mathrm{F}\right)$ in the aluminum ribs, and $80.2-170.4^{\circ} \mathrm{C}\left(176.4-338.7^{\circ} \mathrm{F}\right)$ in the $1.27-\mathrm{cm}(0.50-$ in.) thick Zircaloy-4 plates. Peak fluid temperatures at the metal surfaces are $80.8^{\circ} \mathrm{C}\left(177.4^{\circ} \mathrm{F}\right)$ for the chamber wall, $71.3-77.4^{\circ} \mathrm{C}\left(160.3-171.3^{\circ} \mathrm{F}\right)$ for the aluminum walls, and $72.2-85.5^{\circ} \mathrm{C}\left(162.0-185.9^{\circ} \mathrm{F}\right)$ for the Zircaloy-4 plates. In the first lead zone, with a power density of $4.1 \mathrm{~kW} /$, lead plates of up to 4-cm (1.6-in.) thickness can be used without exceeding the creep design temperature of $104^{\circ} \mathrm{C}\left(219^{\circ} \mathrm{F}\right)$. In the second lead zone, with a power density of $1.7 \mathrm{~kW} / \mathrm{l}$ the lead can be up to 7 - $\mathrm{cm}\left(2.8\right.$-in.) thick without exceeding the $104^{\circ} \mathrm{C}\left(219^{\circ} \mathrm{F}\right)$ temperature. Peak fluid temperatures at the lead surfaces are less than $75^{\circ} \mathrm{C}\left(167^{\circ} \mathrm{F}\right)$ for all cases. The $\mathrm{D}_{2} \mathrm{O}$ fractions needed in the four Zircaloy-4 regions were $15.3 \%, 12.4 \%, 7.8 \%$, and $4.3 \%$. In the first lead region, the $\mathrm{D}_{2} \mathrm{O}$ fraction needed varies from $4.1 \%$ for $1-\mathrm{cm}(0.4-\mathrm{in}$.) thick plates to $2.3 \%$ for 4-cm (1.6-in.) thick plates. In the second lead region, the $\mathrm{D}_{2} \mathrm{O}$ fraction needed is about $1.1 \%$ for a 7-cm (2.8-in.) thick plate. For all backstop flow paths the DNBR is greater than 4.2.

\subsubsection{Target Lead Cooling}

Preconceptual design activities for cooling the target lead have focused on volumetric minimization of $\mathrm{D}_{2} \mathrm{O}$ (Fig. 4-7) and Inconel used for cooling and support. Coolant velocities and pressure drops have been minimized to avoid lead erosion and unnecessary structural loads. Considerations have been implemented for lifelong use of the lead for minimization of irradiated lead mixed wastes. Cooling of the beam entrance window, the target lead, the proton beam backstop region, the ${ }^{3} \mathrm{He}$ in the chamber and in the blanket tubes, and the moderator tank structural components was accomplished with a single heat removal system to preclude the costs and complications for separate additional systems. The cooling is performed by the Moderator Tank Heat Transport Systems (MTHTS) which are described in Section 4.6. In residual heat removal mode, the indirectly heated internal components are cooled by either the passive natural circulation mode or by the moderator tank residual heat removal system which is the active backup. Capability for cooling the lead during retargeting was also addressed. Thermal-hydraulic analyses have shown safe margins against lead melting and loss of geometry and functionality due to thermal creep. Conservative stress and temperature limits were used. An open coolant flow channel architecture has been provided for the target lead. This architecture will substantially reduce the probability of lead melting as a result of a local flow blockage. 


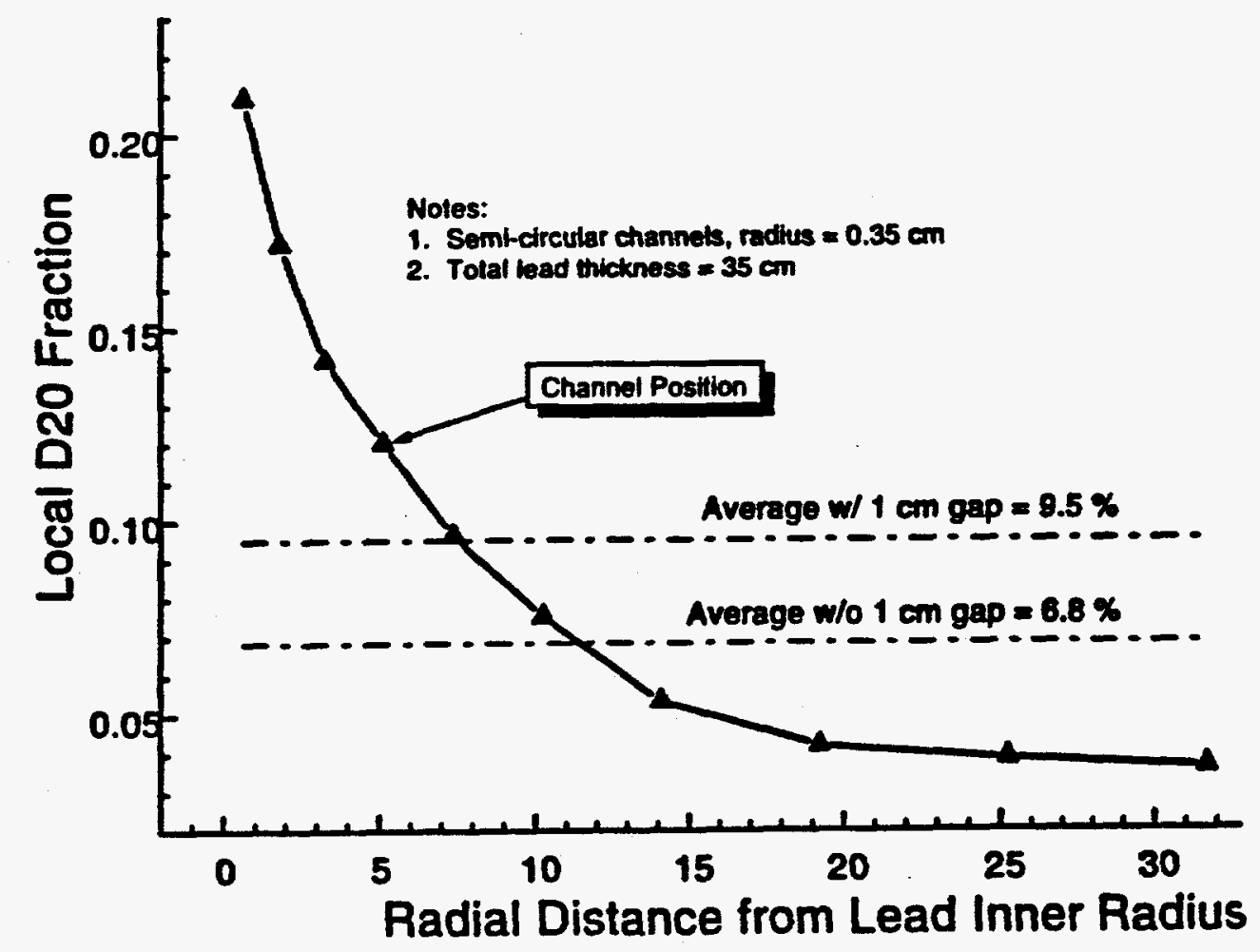

Fig. 4-7. Target Lead $D_{2} O$ Frạction.

Thermal-hydraulic analyses indicate the unclad stacked plate design can be cooled in both the normal operating and residual heat modes with additional margin for continuing design evolution. All analyses were made at a point in the design evolution when the target lead around the helium chamber was of slightly larger ID and thickness. Also the extent of the lead around the helium chamber was approximately $70 \%$. Currently, the design has evolved toward more than $95 \%$ coverage and has incorporated separately cooled disposable and reuseable zones. Additionally, the lead centerline temperature analyses were modeled with grooves on a triangular pitch. Later, a rectangular configuration was selected to incorporate and position the axial tie tube features. Accordingly, the analyses will need to be updated to reflect these changes in the next design phase.

For design basis events such as a moderator coolant LOCA, the analyses found that temperatures near the inner flow channels could increase beyond a $220^{\circ} \mathrm{F}$ normal operating limit. The behavior of the lead under these circumstances will need to be evaluated later along with a clear definition of the design limits associated with these conditions.

The supply of coolant to the target lead is shown in Fig. 4-8, Moderator $\mathrm{D}_{2} \mathrm{O}$ Coolant Flow Arrangement and Fig. 4-9, Target Lead Cooling. In the normal operating mode, most of the moderator coolant is directed by the flow baffle on the NSA Module into a low velocity coolant inlet region underneath the NSA and proton beam backstop (this region is described further in Section 4.5). From there, the coolant can flow upward though the flow 


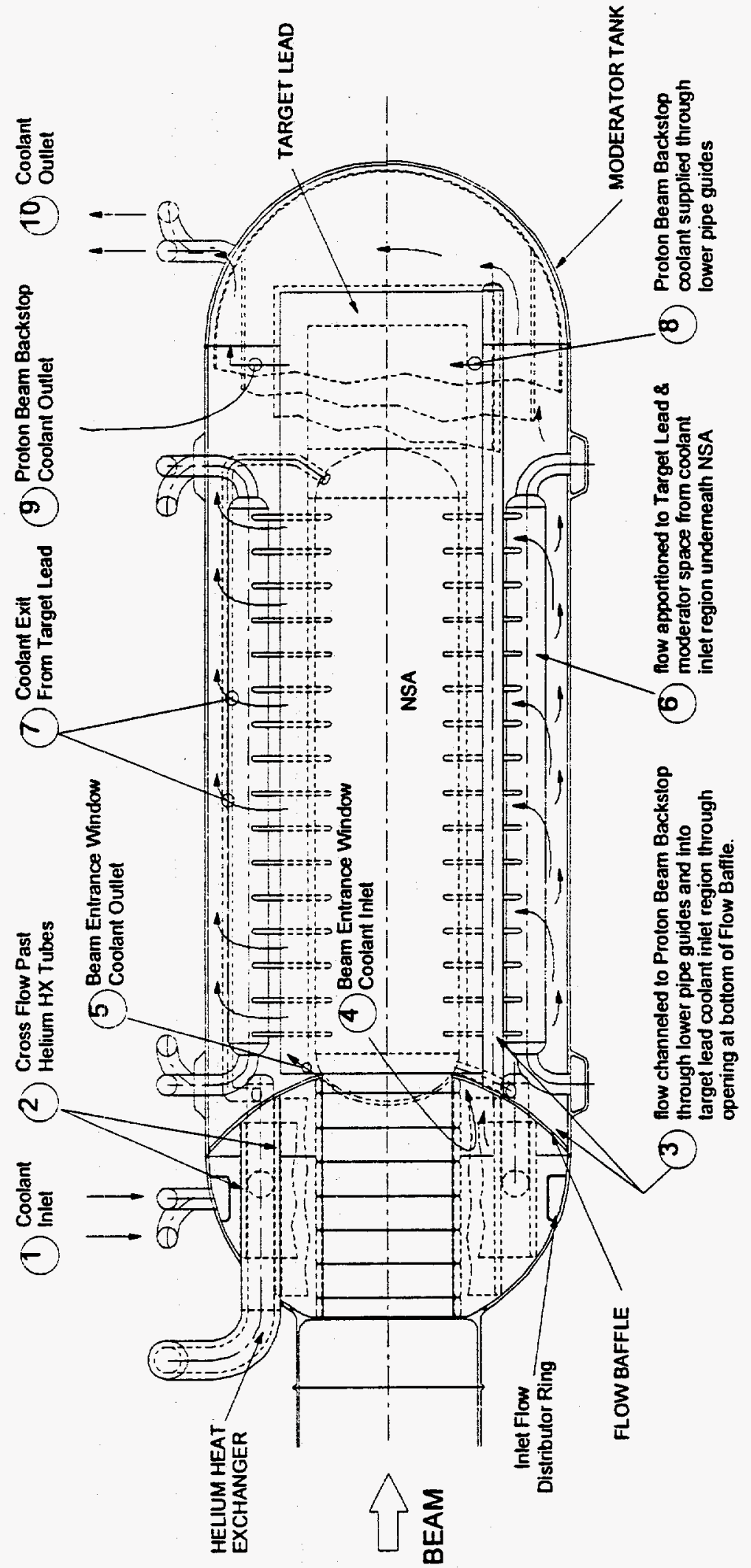

Fig. 4-8. Moderator $\mathrm{D}_{2} \mathrm{O}$ Coolant Flow Arrangement. 


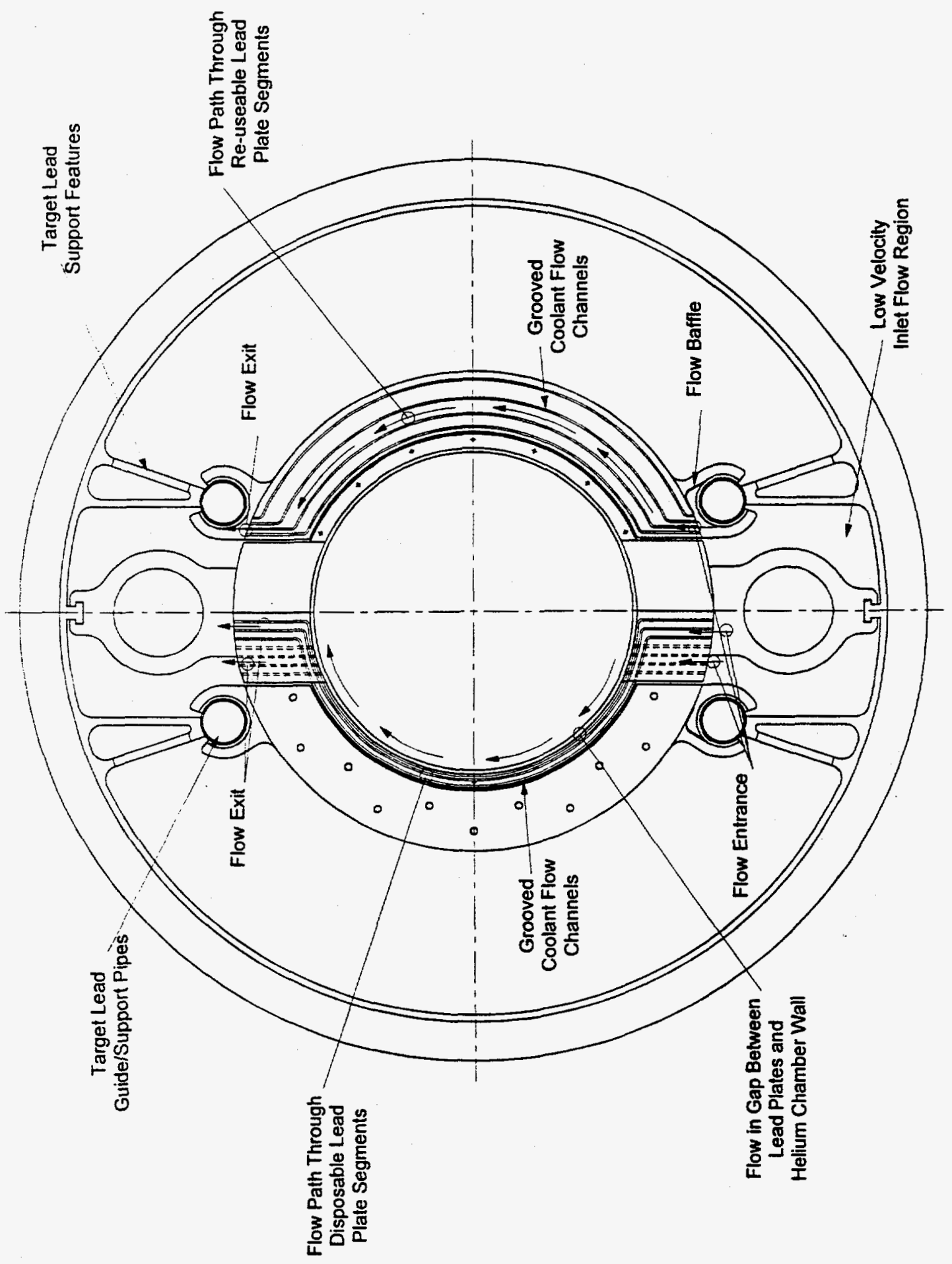

Fig. 4-9. Target Lead Cooling. 
channels in separately cooled disposable and reuseable lead plates. Some of the flow is deliberately apportioned to helium blanket regions in the moderator tank along the sides of the NSA. Upon exiting the target lead below the outlet plenum, the flow re-mixes with coolant in the moderator tank.

In the residual heat removal mode, the target lead is cooled by either the passive natural circulation mode or by the moderator tank residual heat removal system, which is the active backup. The residual heat removal analysis assumed steady state conditions using the peak internal lead residual heating rates one second following beamtrip. A summary of the target lead cooling parameters is given in Table 4-9.

Table 4-9. Target Lead Cooling Preconceptual Design Parameters

\begin{tabular}{cc}
\hline Feature & Preconceptual Value \\
\hline Max. Lead Temperature & $103^{\circ} \mathrm{C}\left(217^{\circ} \mathrm{F}\right)$ \\
Max Coolant Velocity & $2.137 \mathrm{~m} / \mathrm{s}(7 \mathrm{ft} / \mathrm{s})$ \\
(in coolant channels) & $34 \mathrm{kPa}(5 \mathrm{psi})$ \\
Max. unrecoverable pressure drop & $\mathrm{Half}$ Circle \\
Groove Shape & $0.35 \mathrm{~cm}(0.138 \mathrm{in})$. \\
Groove Radius & $\mathrm{TBD}$ \\
No. of grooved Coolant Channels & $\mathrm{TBD}$ \\
Upper and Lower Fill Plates & $\mathrm{TBD}$ \\
Side Fill Plates & $\mathrm{TBD}$ \\
$70 \%$ half section plates & $\mathrm{TBD}$ \\
95\% half section plates & $0.5 \mathrm{~cm}(0.197 \mathrm{in})$. \\
Rear Section plates & Bypass Hole Diameter \\
\hline \hline
\end{tabular}

The maximum temperatures (Fig. 4-10) in the lead occur at the centerlines between flow channels within the metal in regions of highest power density nearest the beam. The maximum lead temperatures were established by first approximating the temperature differences between the surfaces of the flow channel and the lead centerlines using finite element heat transfer models. The fluid and surface temperature variations along the length of the channels were then derived from analytical computer models using standard convection heat transfer correlations. The grooves in the flat plates were sized and spaced to match the operating power profile and to provide low coolant velocities to minimize lead erosion and pressure drop. The peak lead temperatures are below the creep temperature limit of $104^{\circ} \mathrm{C}\left(219^{\circ} \mathrm{F}\right)$.

Additional work will be needed in the future to address target lead cooling during handling. A key issue will be the management of $\mathrm{D}_{2} \mathrm{O}$ inventory in order to maintain high availability. Although there is currently no strategy for accomplishing this, several options may be explored such as:

- Removal of the target module full of heavy water to a cooldown area. This would provide an option to enable the majority of the system $\mathrm{D}_{2} \mathrm{O}$ to be pumped to the other target system. 
- Circulation of a small amount of $\mathrm{D}_{2} \mathrm{O}$ through the lead with all but a small amount of $\mathrm{D}_{2} \mathrm{O}$ drained.

- Forced air or gas cooling.

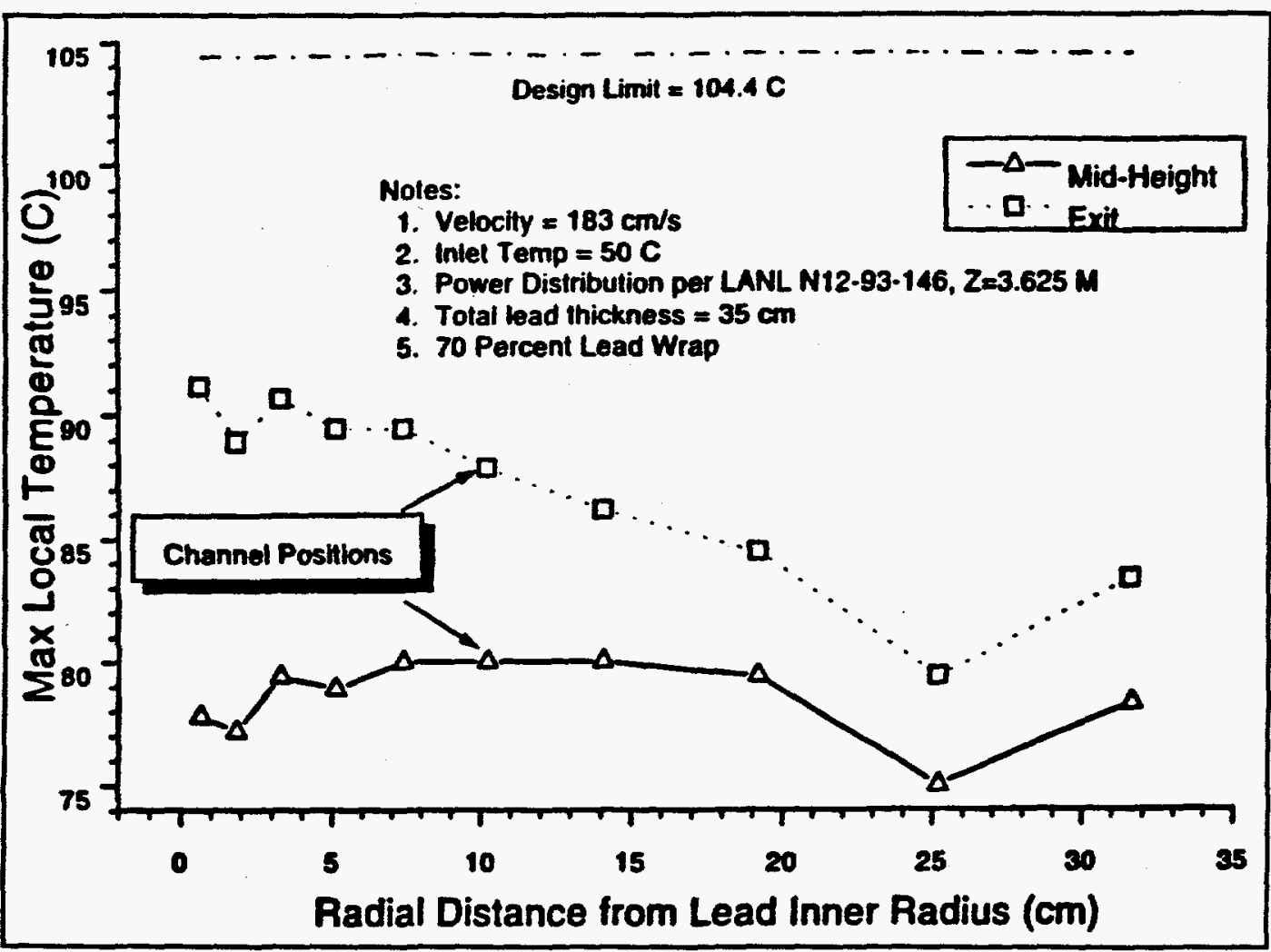

Fig. 4-10. Target Lead Temperature.

\subsubsection{Beam Entrance Window}

The beam entrance window is made up of two half-cylinder Inconel-718 walls with $\mathrm{D}_{2} \mathrm{O}$ coolant flowing between them from the moderator cooling system. The window has nominal dimensions of 47 by $96 \mathrm{~cm}$ (18.5 by 37.8 in.) with the smaller dimension the outer diameter of the half cylinder and the larger dimension the height of the window. The $\mathrm{D}_{2} \mathrm{O}$ flows horizontally through a $0.25-\mathrm{cm}$ gap $(0.098 \mathrm{in}$.) between the two $0.125-\mathrm{cm}(0.049 \mathrm{in}$.) walls. Horizontal spacers between the walls provide flow channels as well as added strength to keep the window from buckling if the target chamber internal $3 \mathrm{He}$ pressure is lost and the $\mathrm{D}_{2} \mathrm{O}$ pressure is maintained in the window. Vertical flow between the two window walls will be considered in the next design phase because it offers the potential safety advantages of sweeping out any bubbles that might form during transients and also lends itself to better natural convection cooling if the pump flow is not available. If vertical flow is used, then the stiffeners will need to be vertical rather than horizontal, so instead of being circular segments they would be rectangular plates. 
The $\mathrm{D}_{2} \mathrm{O}$ inlet and exit temperatures are $51.5^{\circ} \mathrm{C}$ and $70^{\circ} \mathrm{C}\left(124.7^{\circ} \mathrm{F}\right.$ and $\left.158.0^{\circ} \mathrm{F}\right)$, respectively, and the exit pressure is $241 \mathrm{kPa}$ ( $35 \mathrm{psia}$ ). There is $1.429 \mathrm{MW}$ deposited in the window, of which $1.229 \mathrm{MW}$ is deposited directly in the walls and $0.200 \mathrm{MW}$ is deposited in the $\mathrm{D}_{2} \mathrm{O}$. The mass flow rate of $18.5 \mathrm{~kg} / \mathrm{s}(40.7 \mathrm{lbm} / \mathrm{s})$ was determined by dividing the power deposited in the window by the product of the $\mathrm{D}_{2} \mathrm{O}$ specific heat and the $\mathrm{D}_{2} \mathrm{O}$ temperature rise from inlet to exit. This flow rate gives a velocity of $7.24 \mathrm{~m} / \mathrm{s}(23.8 \mathrm{ft} / \mathrm{s})$ and a pressure drop of $87 \mathrm{kPa}(12.6 \mathrm{psi})$ from the window entrance to exit. There is a total of approximately $138 \mathrm{kPa}$ ( $20 \mathrm{psia}$ ) available across the beam entrance window so the remainder can be used for entrance and exit losses plus orificing if needed.

Only part of the window is in the $42-\mathrm{cm}$ by $91-\mathrm{cm}(16.5$-in. by 35.8 -in.) beam. The beam covers 129.2 degrees of the half circle window and only $91 \mathrm{~cm}$ (35.8 in.) of the $96-\mathrm{cm}$ (37.8-in.) height, so $68 \%$ of the window area is in the beam. The power density for this area is $1.03 \mathrm{MW} /$, which gives an average heat flux of $1.288 \mathrm{MW} / \mathrm{m}^{2}\left(408,400 \mathrm{Btu} / \mathrm{hr}-\mathrm{ft}^{2}\right)$. There is a $34.2^{\circ} \mathrm{C}\left(61.6^{\circ} \mathrm{F}\right)$ temperature rise from the bulk fluid to the film at the wall and a $60.6^{\circ} \mathrm{C}$ $\left(109.1^{\circ} \mathrm{F}\right)$ temperature increase through the wall. Therefore, the peak film and wall temperatures at the exit are $104.2^{\circ} \mathrm{C}$ and $164.8^{\circ} \mathrm{C}\left(219.6^{\circ} \mathrm{F}\right.$ and $\left.328.6^{\circ} \mathrm{F}\right)$. The DNBR at the exit is 2.29 , which is above the 2.0 value that is being used as a minimum criteria at this stage of the design. The modified Stanton number is 0.00066 , which is well below the 0.0045 limit based on Savannah River experience. The boiling limit power ratio is 2.34 using the subcooling based on the maximum temperature in the film at the exit. The boiling-limit power ratio is the ratio of the product of flow rate multiplied by the specific heat multiplied by the minimum exit subcooling plus the power in the window divided by the power in the window. When the boiling limit power ratio decreases to 1.0 , the hottest fluid in the channel would have just reached saturation. All three of these parameters indicate there is margin in the critical heat flux, onset of flow instability, and onset of bulk boiling in the beam entrance window thermal-hydraulic design.

\subsection{MECHANICAL DESIGN OF TARGET MODULE}

The mechanical design of the target module provides for:

- A high degree of availability and reduced number of systems.

- Rapid retargeting which can occur shortly after beam shutdown.

- Readily operable remote retargeting connections with negligible irradiation effects for high integrity and reduced exposure.

- Safe cooling of tungsten and other heated components within the target module.

- Practical cooling of heated $3 \mathrm{He}$ gas.

- Minimal 3He inventory.

- Minimal $\mathrm{D}_{2} \mathrm{O}$ inventory.

- Recovery and re-use of most of the target lead for mixed waste minimization. 
- Double containment of $3 \mathrm{He}$ gas throughout the system for helium loss prevention. The moderator tank is considered as a backup containment boundary.

- Valid representation of the physics model for high production efficiency. Near complete encirclement of the $3 \mathrm{He}$ chamber with lead and Helium with a minimum of parasitic materials.

- Manufacturability within the scope of commercial practices and capabilities.

- High integrity radionuclide containment conforming to DOE requirements.

- Shared usage of some heavy water between target modules.

- Shared usage of ${ }^{3} \mathrm{He}$ between target modules.

The general configuration of the target blanket system interface with the balance of plant design is illustrated in Fig. 4-11, Target Cavity and Shielding. This configuration provides for rapid retargeting which can occur shortly after beam shutdown. Retargeting can be rapidly performed by complete changeout of an entire target module structure. More complex operations such as assembly, disassembly and target lead recovery and re-use can be handled off-line. Water, gas, and instrumentation and control (I\&C) connections are made outside of the shielding in a region of reduced radiation. Connector requirements can be satisfied by existing technology for rapid and reliable operation. Retargeting is discussed further in Section 4.8 .

The preconceptual design has focused on a vertical lift configuration. However, provisions for lateral handling such as on rollers or tracks can be investigated in the future for possible improvements in risk and handling ease. With this design, the shield is removed to allow access to the target module. After disconnecting the plumbing, the entire target module can be lifted out as a unit and moved to storage or disassembly pools where additional operations can be performed off-line. In the future, the tradeoffs associated with discarding the entire NSA can also be examined. The target module is sufficiently compact to facilitate handling while submerged in a pool. With this capability, irradiated lead can be transferred and installed into new targets, and old targets can be disassembled with low radiation exposures using well proven pool handling equipment and techniques.

Helium-3 cooling within the NSA is facilitated in part by a helium circulator located remotely from the neutron source. This facilitates a practical cooling system design with minimal concern for irradiation damage to the blower and motor.

The volume envelope of the target module is sufficient to permit a cavity design of minimal additional volume so that pipe breaks can be covered by pressurizer inventory without the need for additional accumulators or $\mathrm{D}_{2} \mathrm{O}$ inventory. A seal interface between the target module and the target cavity will need to be defined in the future to support this capability. In addition Loss-of-Coolant-Accident (LOCA) analyses will also need to be updated to support this design concept. A summary of the estimated target module weight breakdown is given in Table 4-10. 
Table 4-10. Estimated Target Module Weight Breakdown

\begin{tabular}{lr}
\hline Item & Mass \\
\hline Moderator Vessel, Belts \& Lead Supports & 16,618 \\
Pipe \& Strong Back & 10,000 \\
Re-used Target Lead & 57,251 \\
Disposable Target Lead & 13,550 \\
Target Lead Total & 70,801 \\
'3e Blanket Tubes and Supports & 5,052 \\
'He Hx and Piping & 445 \\
NSA & 4,328 \\
$\quad$ Zircaloy - 4 & 4,972 \\
$\quad$ Inconel & 1,476 \\
$\quad$ Tungsten Rods and Wires & 34,583 \\
D2O & 148,275 \\
Total Lift Capacity Required
\end{tabular}

Figure 4-12, Target Module, provides an external view of the target module. The target module consists of the moderator tank, NSA module (including the beam tunnel nozzle and it's remote connector interface with the accelerator beam expansion tube), all components internal to the moderator tank (described further in this section), all piping and I\&C lines outward to the remote connectors, piping strong back, and all integrally attached support features.

All of the pipes enter at the top of the vessel. Pipe runs can be joined to a common "strong back" for connector alignment and support during handling. This enables the piping to be lifted in concert with the moderator tank and internals.

Figure 4-13, Target Module General Arrangement-Cross-Section Elevation, provides a general cross-section view of the principle components of the target module. At assembly the NSA Module is loaded axially into the moderator tank from the front end of the moderator tank shell. With the exception of the target lead half sections, and rear slab (not shown here) all components are disposed of after each retargeting. As the design evolves, further waste minimization work may be performed to develop a configuration in which more of the components can be re-used.

Figure 4-14, NSA Module, shows a principal portion of the target module (not to be confused with the NSA which is described in section 4.3) for the purpose of explaining the assembly sequence for the target module. The NSA Module is loaded axially from the front of the moderator tank at assembly (see Fig. 4-15, Target Module Assembly). The NSA Module is composed of the following components:

* NSA

* Helium Heat Exchanger

* Moderator Coolant Flow Baffle

* Side Fill Disposable Target Lead Plates
* NSA Helium Supply Pipes

* NSA Helium Return Pipes

* NSA Blankets

* Upper and Lower Fill Disposable Target Lead Plates 


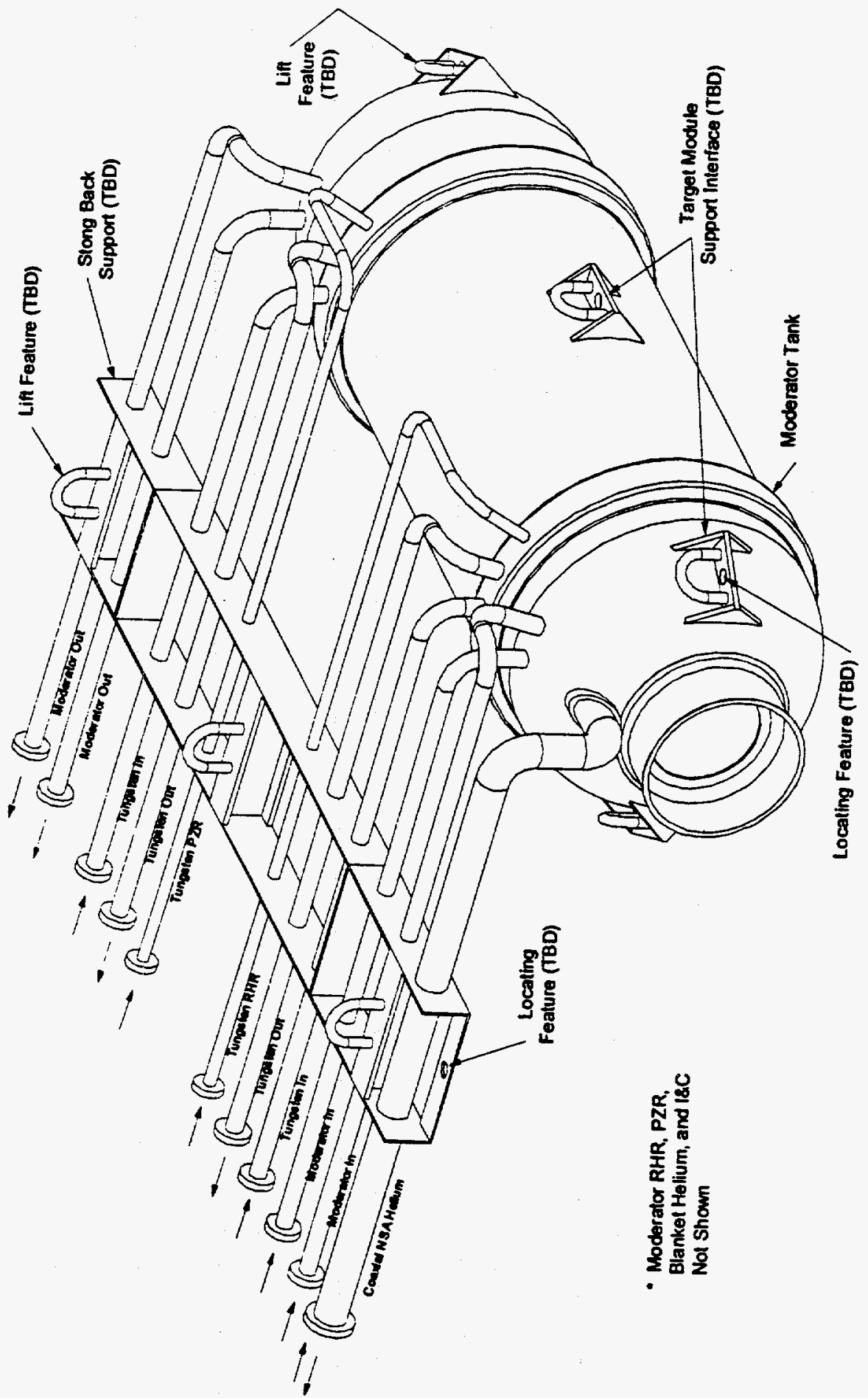

Fig. 4-12. Target Module. 


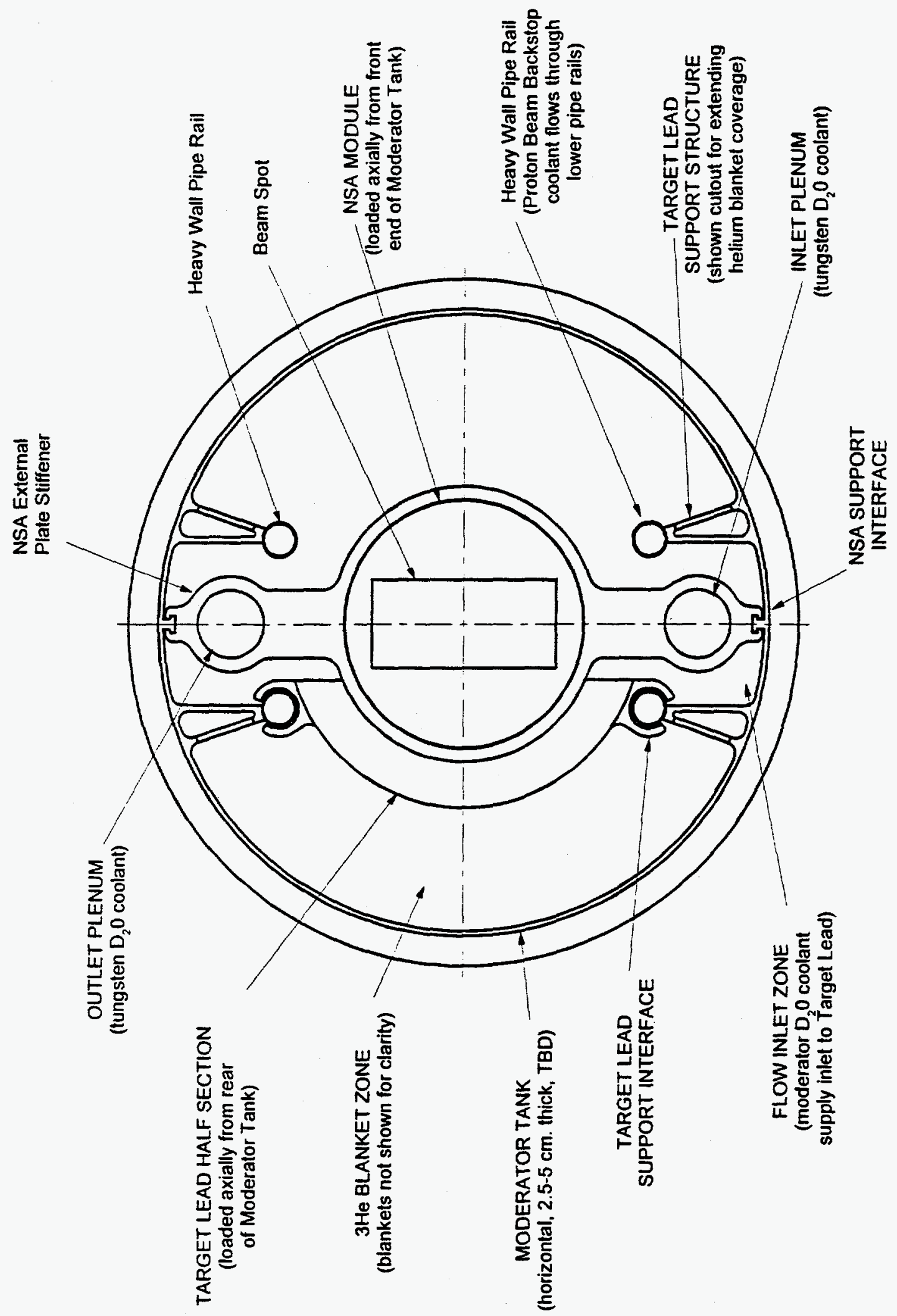

Fig. 4-13. General Arrangement-Cross-Section Elevation. 


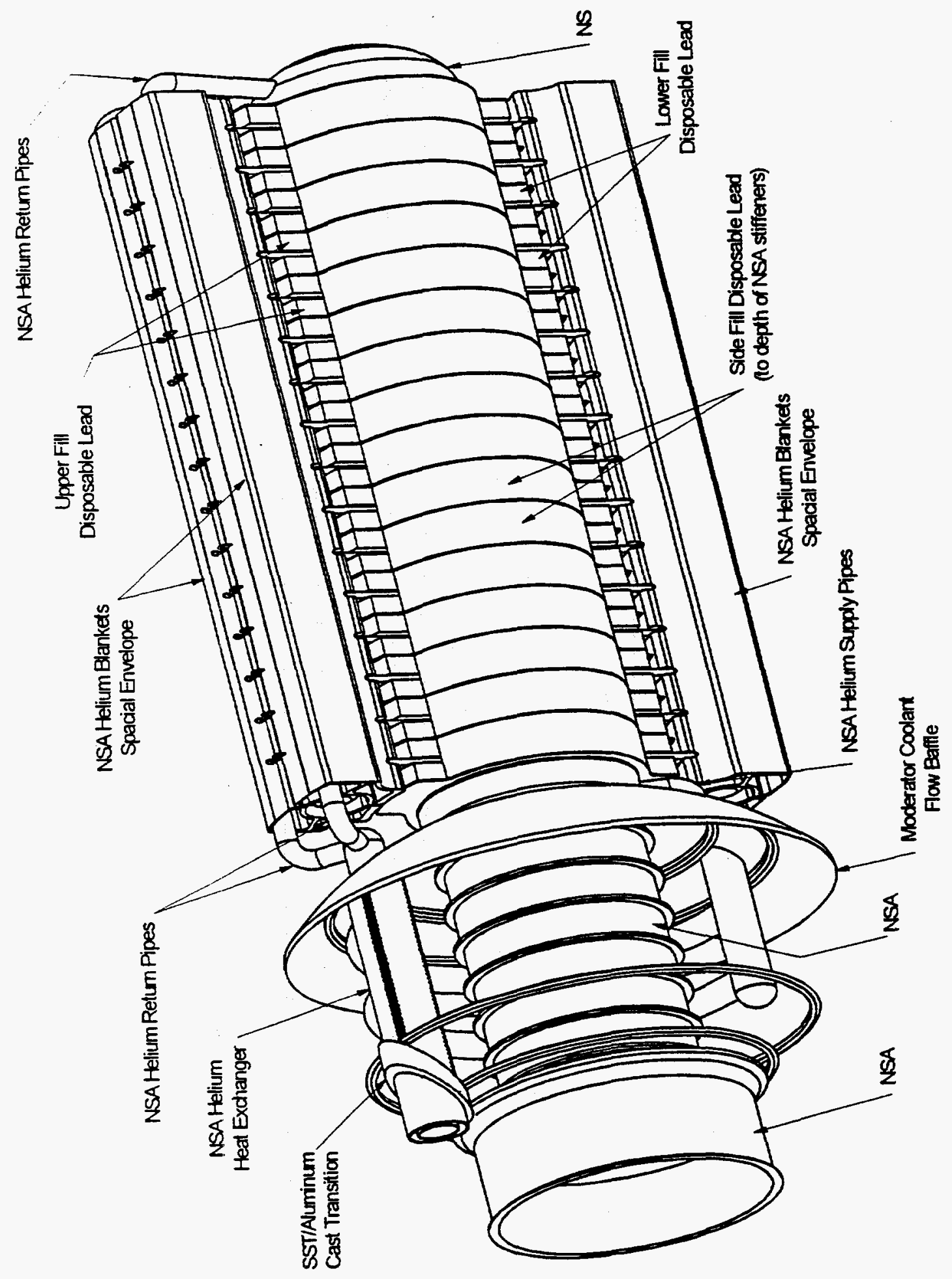

Fig. 4-14. NSA Module. 


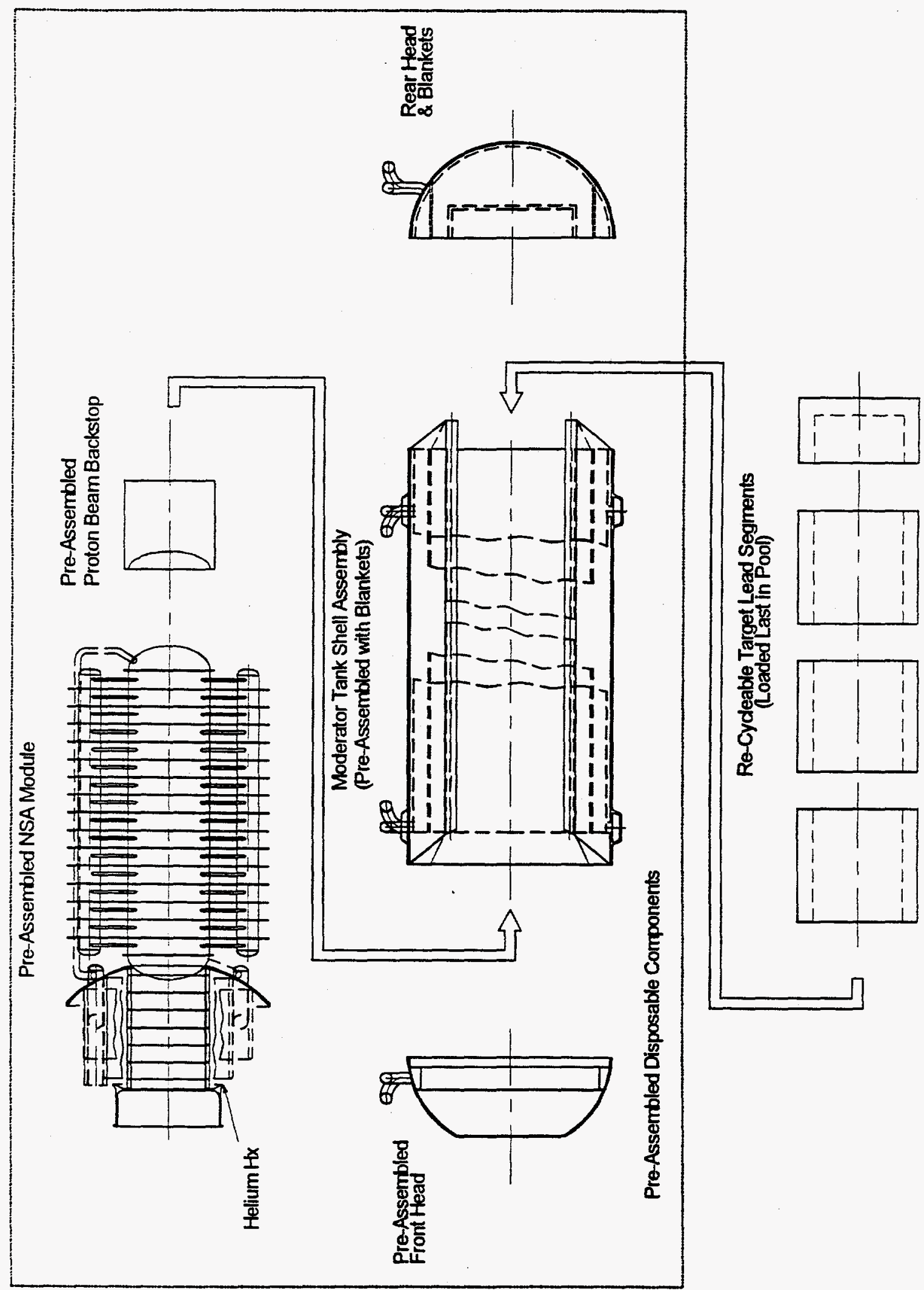

Fig. 4-15. Target Module Assembly. 
Figure 4-13, Target Module General Arrangement-Cross-Section Elevation, and Fig. 4-16, Target Module General Arrangement-Side Section Elevation, show the major features inside the target module. This design provides for an accurate representation of the physics model associated with a $118 \mathrm{~cm}$ ID NSA helium chamber. The NSA external stiffener plates are extended to envelope the inlet and outlet plena, and they incorporate features for NSA support and guidance as well as facilitate axial insertion into a new moderator tank shell during assembly. The helium blankets have been omitted from this view and are described later in this section and are represented as dotted lines in this and all subsequent figures. The target module interfaces with the beam tunnel at the front head with a single remote dry connection.

Near full encirclement of NSA with lead is accommodated. Some disposable lead is configured with the NSA Module to cover the rod bundle duct regions. This lead is noted but not shown. Some additional disposable lead is configured around the NSA to the depth of the NSA stiffeners to facilitate the axial loading of the target lead within the target module as a near final step in the assembly process.

Triangular support plate extensions from the inside of the moderator tank wall provide for load transfer and a support of the target lead. Attached to these are heavy pipe rails to facilitate the axial loading of the target lead in segments. When assembled with the NSA Module, the lower of these two structures defines a low velocity moderator flow inlet zone.

The moderator tank is designed with classical cylindrical and hemispherical shapes for material minimization. The moderator tank should withstand a rupture of the helium chamber with a relatively thin-walled design. The estimated thickness of the tank for this design condition is approximately $4-\mathrm{cm}(1.6 \mathrm{in}$.) thick. Additional sizing will be needed to evaluate the capability of the moderator tank for seismic, lifting, and handling loads. The moderator tank material of construction will be of an appropriate nuclear grade of 304 SST, TBD. In accordance with APT Requirements Document (RD) and General Safety Requirements Document (GSRD) requirements, the moderator tank will be designed and constructed per ASME Code, Section III, Class I. As such it will provide a high degree of safety and assurance against release of spallation products, and protection against loss of ${ }^{3} \mathrm{He}$.

The beam entrance tunnel extending from the NSA helium chamber is shown as circular $\sim 111.9-\mathrm{cm}(44.0-\mathrm{in}$.) ID with external stiffeners attached to a thin wall $\sim 0.8 \mathrm{~cm}(0.3 \mathrm{in}$.). The length of this tunnel is $\sim 1.6 \mathrm{~m}$ (63 in.) and will be optimized in the next design phase to reduce the neutron escape aperture for increased tritium production. Tunnel lengths of up to $2.0 \mathrm{~m}$ have been considered in the physics design. Helium blanket tubes are wrapped about the tunnel between stiffeners. Sizing of these "scavenger blanket tubes" will be performed later.

Figure 4-17, Target Module Tungsten $\mathrm{D}_{2} \mathrm{O}$ Cooling Arrangement-Cross-Section Elevation, and Fig. 4-18, Tungsten $\mathrm{D}_{2} \mathrm{O}$ Coolant Flow Arrangement-Side Section Elevation, show the flow paths within the target module for (2) tungsten coolant loops. The $\mathrm{D}_{2} \mathrm{O}$ coolant enters at the top of the target module through two 170-mm (6-in. nom) pipes into (2) trapezoidal flow belts through which it flows around the outside of the moderator tank to the bottom of the vessel. The flow then proceeds through the inlet plenum, tungsten rod bundle ducts, outlet plenum and finally out the top of the target module through the outlet pipes. The heat generated in the tungsten rod bundles is removed by the Tungsten Heat Transport System (THTS). The use of a flow belt eliminates the need to evaluate pipe breaks occurring 


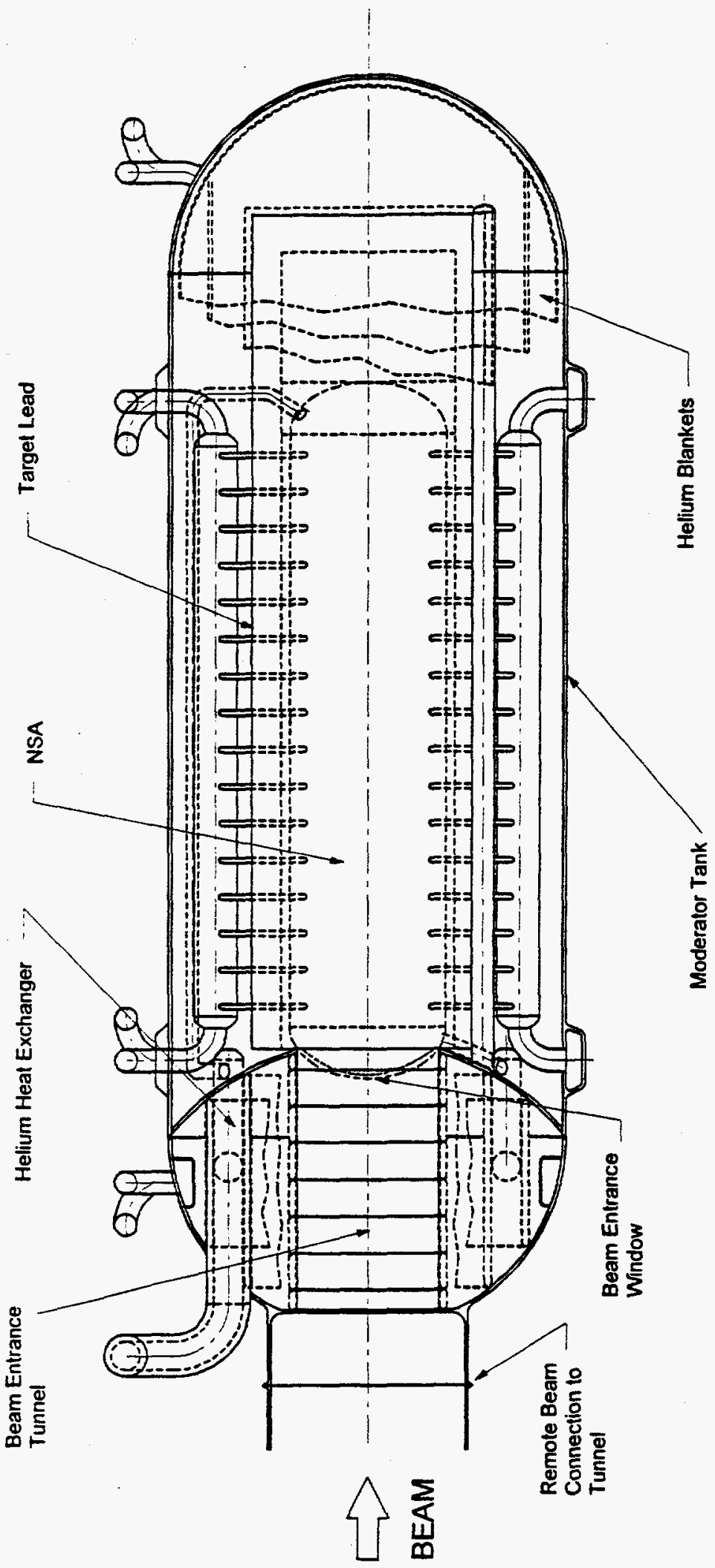

Fig. 4-16. Target Module

General Arrangement - Side Section Elevation. 


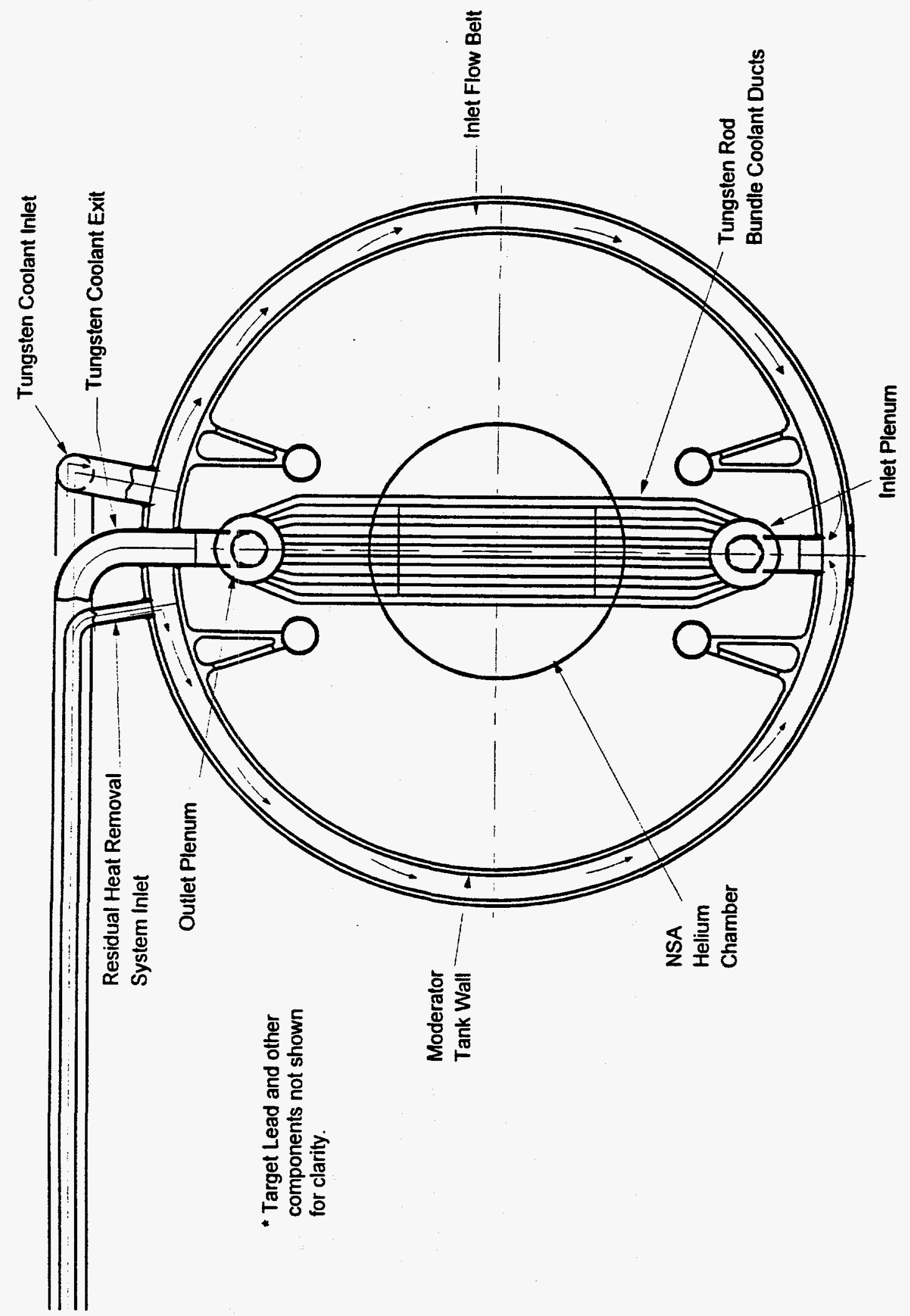

Fig. 4-17. Target Module

Tungsten $\mathrm{D}_{2} \mathrm{O}$ Arrangement - Cross Section Elevation. 


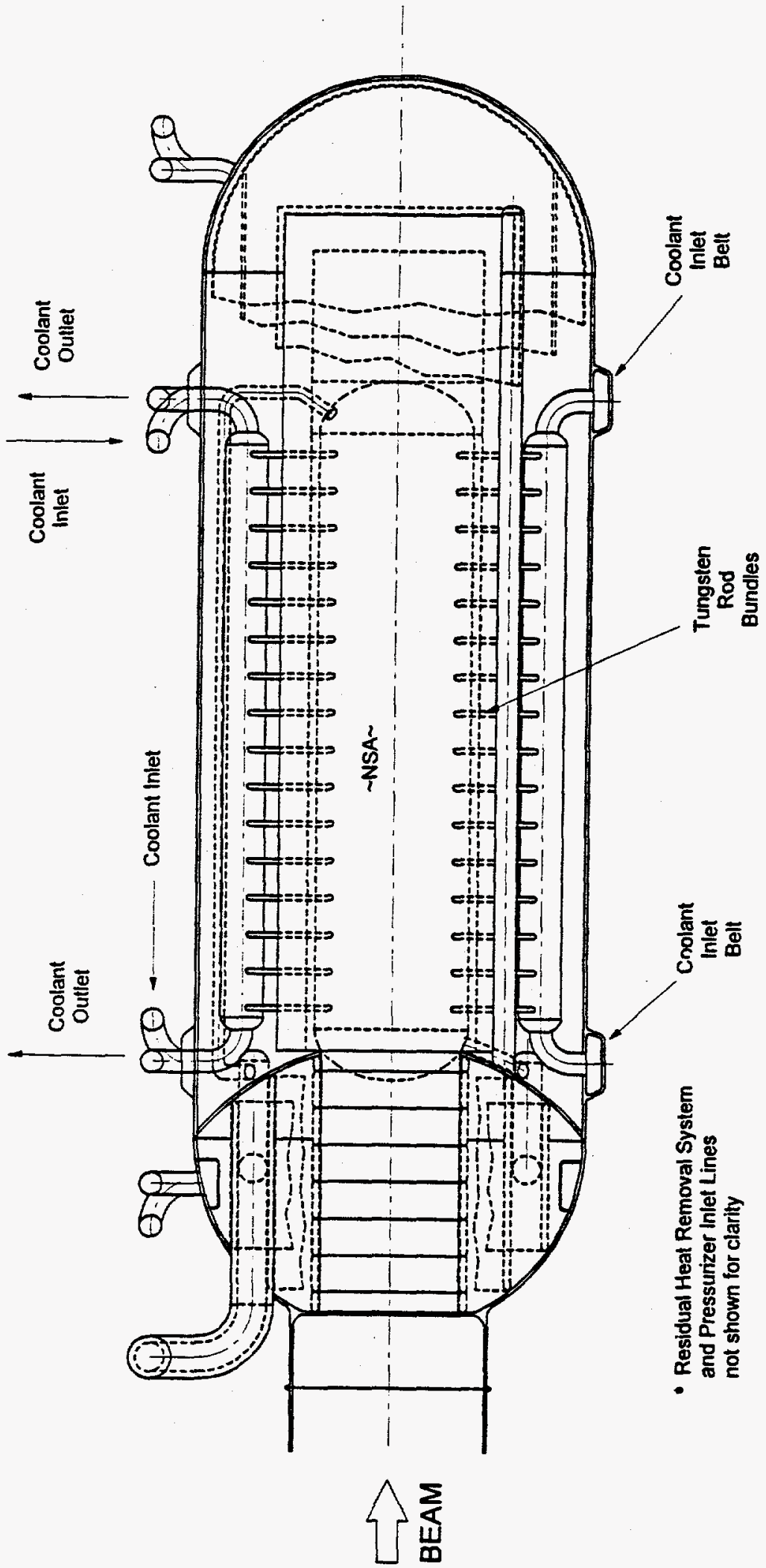

Fig. 4-18. Tungesten $D_{2} O$ Coolant Flow Arrangement. 
below the elevation of the tungsten rods as prescribed by 10CFR50. Tungsten coolant exit legs can be welded from inside of the pipe to facilitate the installation of the NSA module and NSA helium blanket tubing. The Residual Heat Removal System inlet pipe is shown in Figure 4-17. Alternately, the pressurizer line would tie in a similar fashion at the other loop. See Section 4.6 for heat transport systems description.

Figure 4-8, Moderator $\mathrm{D}_{2} \mathrm{O}$ Coolant Flow Arrangement shows the flow of the moderator coolant through the target module. The coolant enters the front end of the moderator tank and is uniformly distributed by an inlet flow distributor ring. This feature precludes high velocity flow impingement on the helium heat exchanger. From there the coolant flows radially inward past two helium heat exchanger tube banks to cool the NSA Helium. Some of the flow enters the target window near the bottom side and exits to a lower pressure side of a large circular flow baffle. Some of the flow is directed through a penetration in the flow baffle into the two lower heavy walled pipe rails through which it flows to the rear of the moderator tank to cool the proton beam backstop. The remainder of the flow is directed through another penetration in the flow baffle into a flow inlet zone directly underneath the NSA. This zone is defined between the lower target lead internal support structures. A slightly greater $(-35 \mathrm{kPa}, 5 \mathrm{psi})$ pressure is maintained within this flow inlet zone for forced cooling of the target lead and flow apportionment to the moderator space. A large portion of moderator coolant is used to cool the target lead as described in Section 4.4.4. The coolant flows up through the target lead and out into the NSA chamber where the coolant exits the target module. The remainder of the coolant in the inlet flow zone is apportioned through flow orifices into the helium blanket zones along the sides of the NSA. This flow is rejoined with the flow exiting the target lead, beam entrance window, and Zircaloy backstop near the top of the moderator tank. The heated moderator coolant then proceeds axially to the rear of the moderator tank where it exits through two coolant outlet pipes.

A future design improvement may be to couple the upper two guide pipes with the lower ones at the rear of the moderator tank to enhance the supply and the distribution of coolant within the moderator tank.

The heat generated in the target lead and the helium heat exchangers is removed by the Moderator Tank Heat Transport System (MTHTS). Flow in the target lead coolant headers is apportioned to the lead and to the moderator tank as described in Section 4.4.4. This flow arrangement maintains the capability to passively cool the lead as described in Section 4.4.4. See Section 4.6 for heat transport systems description.

The heat generated in the helium is removed by the 3 He Heat Transport System (HHTS). Figure 4-19, NSA Helium Flow Arrangement shows the flow of 3He through the target module. From the NSA helium circulator, cold gas enters the moderator tank through a 220 $\mathrm{mm}$ (8-in. nom.) pipe coaxially located inside of a $320 \mathrm{~mm}$ (12-in. nom.) pipe, proceeds through two downcomer pipes, into the lower inlet header, and finally underneath and into the NSA helium chamber through (2) 110 -mm (4-in. nom.) inlet pipes located near the front. As the helium is heated within the helium chamber, it migrates to the rear of the tank and exits through (2) $110 \mathrm{~mm}$ (4-in. nom.) return pipes near the top. From there the gas is channeled to the hot side of a partitioned annulus between a $320-\mathrm{mm}$ (12-in. nom.) and an 220-mm (8-in. nom.) pipe. The gas then flows in parallel through the two banks of helium heat exchanger tubes and returns to the cold side of the partitioned annulus. From there the cold side of the partitioned zone transitions into the outer annulus of the coaxial helium 


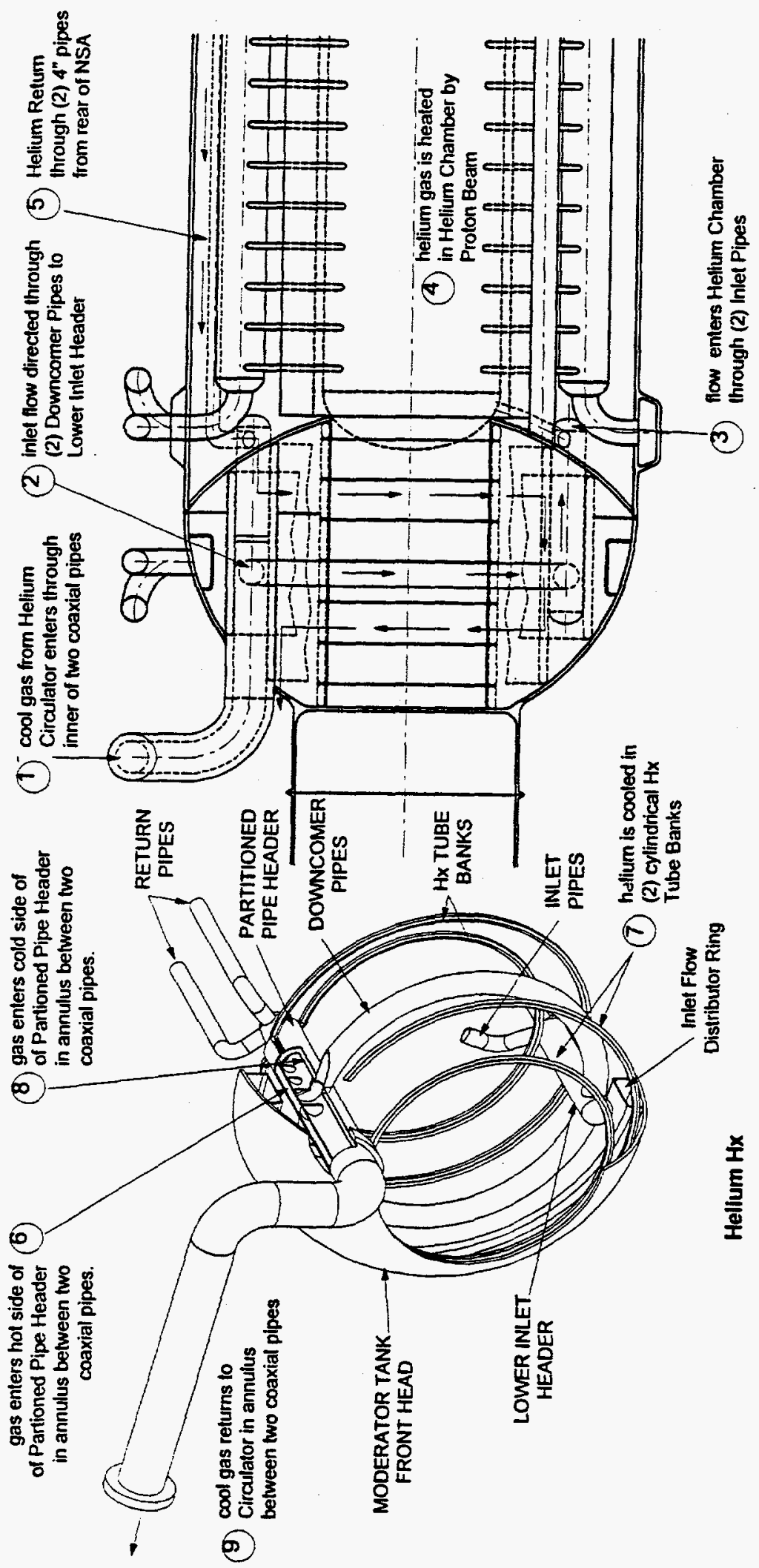

Fig. 4-19. NSA Helium Flow Arrangement. 
conduit from within which the cooled helium flows out of the moderator tank and returns to the NSA helium circulator. The gas circulator is a canned type which is isolated from the target with adequate shielding to preclude concerns for irradiation damage. Selection and sizing of the circulator will need to be performed later with possible need for a special equipment development program. Additional details on the $3 \mathrm{He}$ heat removal system can be found in Section 4.6 of the heat transport systems description.

The production of tritium is accomplished in part by neutron absorption in ${ }^{3} \mathrm{He}$ gas introduced in aluminum-tubed blankets which encapsulate the NSA and target lead. Figure 4-20, Helium Blankets, shows the different types of helium blanket tubes being used. The blankets are operated under $689 \mathrm{kPa}$ (100 psia) helium pressure, substantially less than in the helium chamber. Three layers of helium blankets are defined as the inner, middle, and outer blankets. The inner helium blanket layer is configured with internal heat transfer fins for conductive cooling. All of the other blanket tubes are plain, and can also be cooled by conduction. All blanket tubes are shown horizontal for manufacturability, but alternatives still need to be considered. The blanket tubes are 1100 aluminum for optimum corrosion resistance within the anticipated neutral $\mathrm{pH}$ water chemistry environment.

Some of the blankets are integrally configured with the NSA module to provide for absorption of the neutrons which occur in the regions between tungsten rod bundle ducts. Although they are configured with the NSA Module they will not share the $2067 \mathrm{kPa}(300$ psia) NSA helium within the helium chamber. Instead they will share the $689 \mathrm{kPa}$ (100 psia) helium within the other helium blankets by coupling them together after NSA insertion in the moderator tank. Additional design work will be needed to determine how they can be fit between rows of rod bundle flow channels and the NSA stiffener extensions.

Additional work is needed to improve the manufacturability of the blankets, define blanket interconnections, supports, and bouyancy restraints. One promising approach to blanket construction is to solder or braze the many tube connections in one manufacturing step. The specific braze or solder material needs to be considered in the next design phase along with the impact on production. 


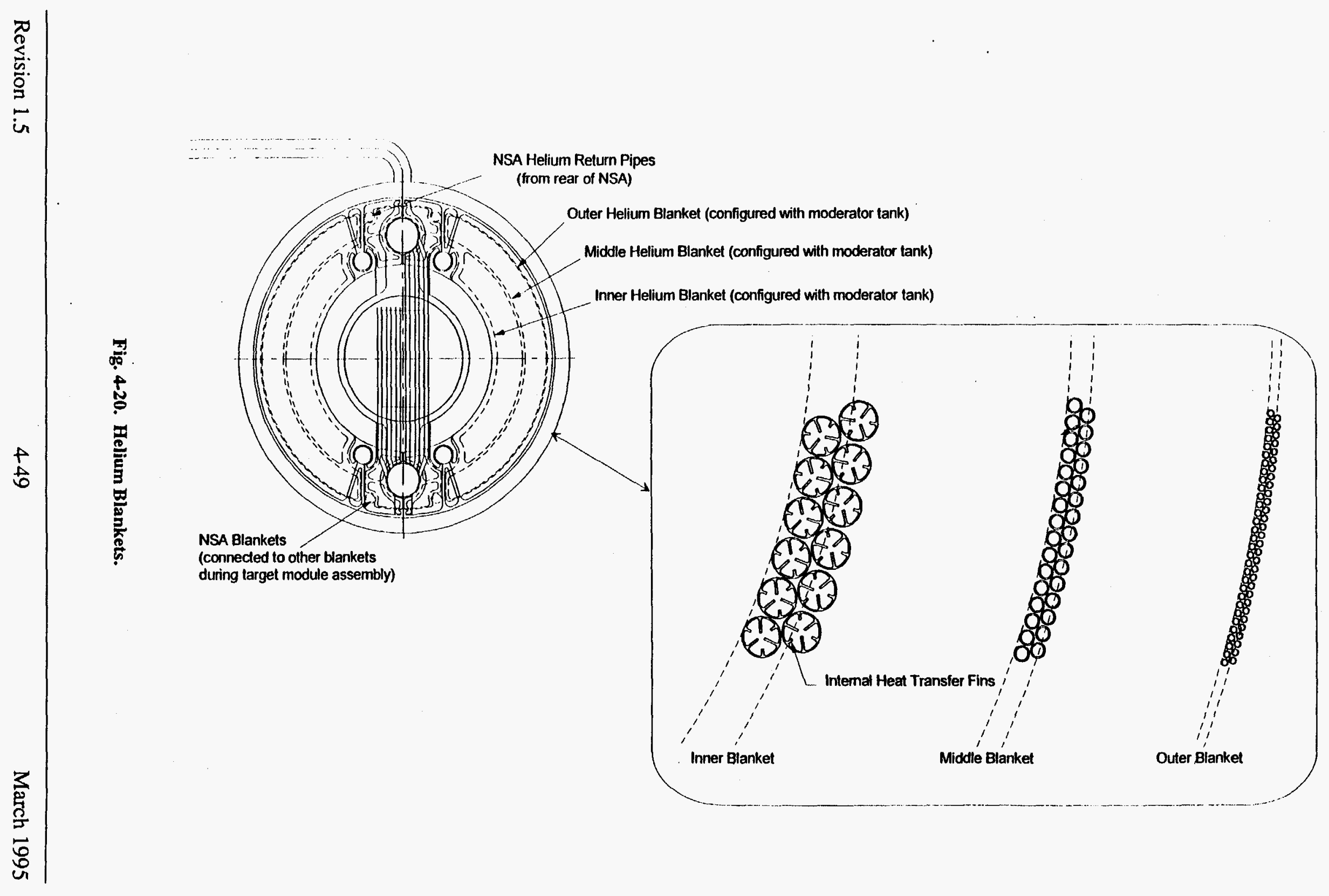




\subsection{TARGET/BLANKET HEAT TRANSPORT SYSTEMS}

The APT 3 He T/B Heat Transport Systems (Fig. 4-21) are broadly grouped into three separate systems corresponding to the components they cool: (1) Tungsten Heat Transport Systems (THTS), (2) Moderator Tank Heat Transport Systems (MTHTS), and (3) 3 He Heat Transport Systems (HHTS). The heat loads associated with the initial design of these systems have changed as the T/B physics design evolves, and the sizing of components will need to be updated in the next design phase. However, the basis system functions, descriptions, and number of components as described below are expected to have minimal changes.

\subsubsection{Tungsten Heat Transport System (THTS)}

The THTS consists of those systems that remove and transport the heat generated in the tungsten rod bundle targets to the environment that will be the ultimate heat sink (Fig. 4-21). The THTS is composed of five systems: the Tungsten Primary Coolant System (TPCS), the Tungsten Secondary Coolant System (TSCS), the Tungsten Circulating Water System (TCWS), the Tungsten Primary Residual Heat Removal System (TPRHRS), and the Tungsten Secondary Residual Heat Removal System (TSRHRS).

The TPCS contains two 50\% capacity primary coolant loops for normal operation where both loops are required to function while the accelerator is operating. The loss of any one loop in the normally operating coolant systems will result in an accelerator trip. The TPRHRS contains two $100 \%$ capacity primary coolant loops for actively removing residual heat from the tungsten target. Each of the TPRHRS coolant loops is capable of removing the total residual heat should the other loop not be available. The TPCS and the TSCS also provide a passive cooling capability for removing tungsten target residual heat through a natural draft water-to-air heat exchanger located in the TSCS. Each primary loop and its associated secondary loop can passively remove the total residual heat load. Thus the active and passive residual heat removal trains are fully redundant.

\subsubsection{TPCS}

The TPCS provides forced circulation of the $\mathrm{D}_{2} \mathrm{O}$ coolant to remove the energy generated within the tungsten target assembly and deliver this energy to the TSCS under all conditions when ac power is available, except for the case when the TPCS is depressurized. When the TPCS is depressurized, the accelerator beam must be shut down and cooling is accomplished by either the passive mode or by the TPRHRS, which is the active backup.

The passive cooling mode is provided through the natural circulation of the TPCS coolant at the residual heat level with the energy being transferred to the TSCS, which is also naturally circulating through to the natural draft water-to-air heat exchanger. Natural circulation in the TPCS and TSCS is accomplished by elevating the heat exchangers so that a thermal driving head exists to cause natural circulation.

All component parts in contact with the tungsten target coolant are fabricated from highly corrosion resistant materials designed to ensure a 40-year minimum life for the TPCS when operated at the temperature, pressure, and water chemistry conditions expected in the TPCS. 
PRE-CONCEPTUAL

LANL-APT TARGET/BLANKET HEAT TRANSPORT SYSTEMS

TUNGSTEN HEAT

TRANSPORT SYSTEMS

(THTS)
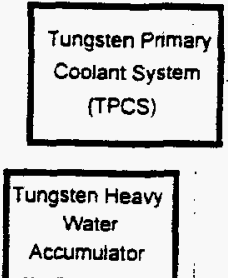

Tungsten Secondary

Coolant System

(TSCS)

Tungsten Passive

Water-to-Air

Heat Exchanger

Envirónment

Tungsten Circulating

Water System

(TCus)

Environment
Tungsten Primary

Resicual Heat

Removal System

(TPRHRS)

Tungsten Secondary

Residual Heat

Removal System

(TSRHRS)

Environment

Envionment

1

3HE HEAT

TRANSPORT SYSTEMS

(HHTS)

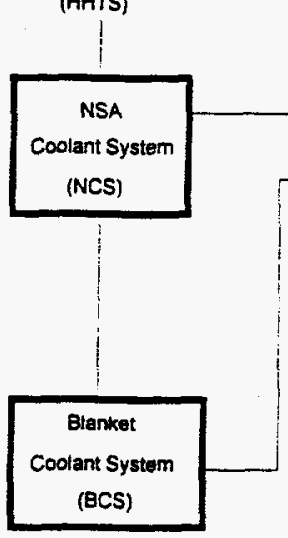

MODERATOR TANK

HEAT TRANSPORT SYSTEMS

(MTHTS)

Moderator Primary

Cootant System

(MPCS)

Moderator Primary

Residual Heat

Removal System

(MPRHRS)

BEW

Bechtel
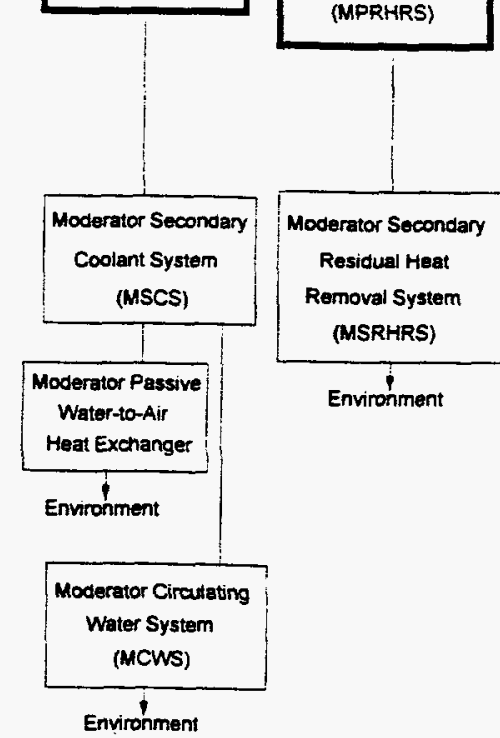

Moderator Secondary

Residual Heat

Removal System (MSRHRS)

Environment

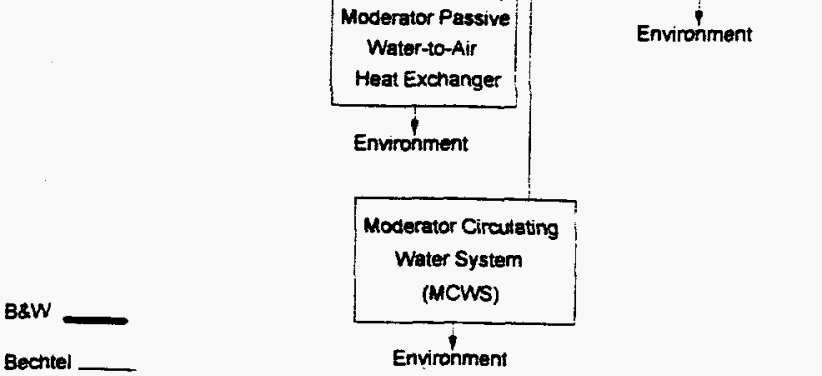

Fig. 4-21. T/B Heat Transport Systems (Rev. 1.5).

The TPCS consists of two loops with each loop connected in parallel to the tungsten target headers. Figure 4-22 is a flow schematic of the TPCS. Each loop removes approximately $50 \%$ of the tungsten target-generated energy and contains one pump, one heat exchanger, connecting piping, and the associated instrumentation to meet the required singlefailure criteria. A pressurizer is connected to one TPCS loop to provide for (1) pressure and $\mathrm{D}_{2} \mathrm{O}$ volume control, and (2) for tungsten target flooding in the event of a LOCA. An accumulator is connected to the other TPCS loop to maintain the tungsten target in a flooded condition in the event of a LOCA or leak. Further studies are needed in the next design phase to determine if an accumulator is still needed in the evolving target module design described in Section 4.5. 


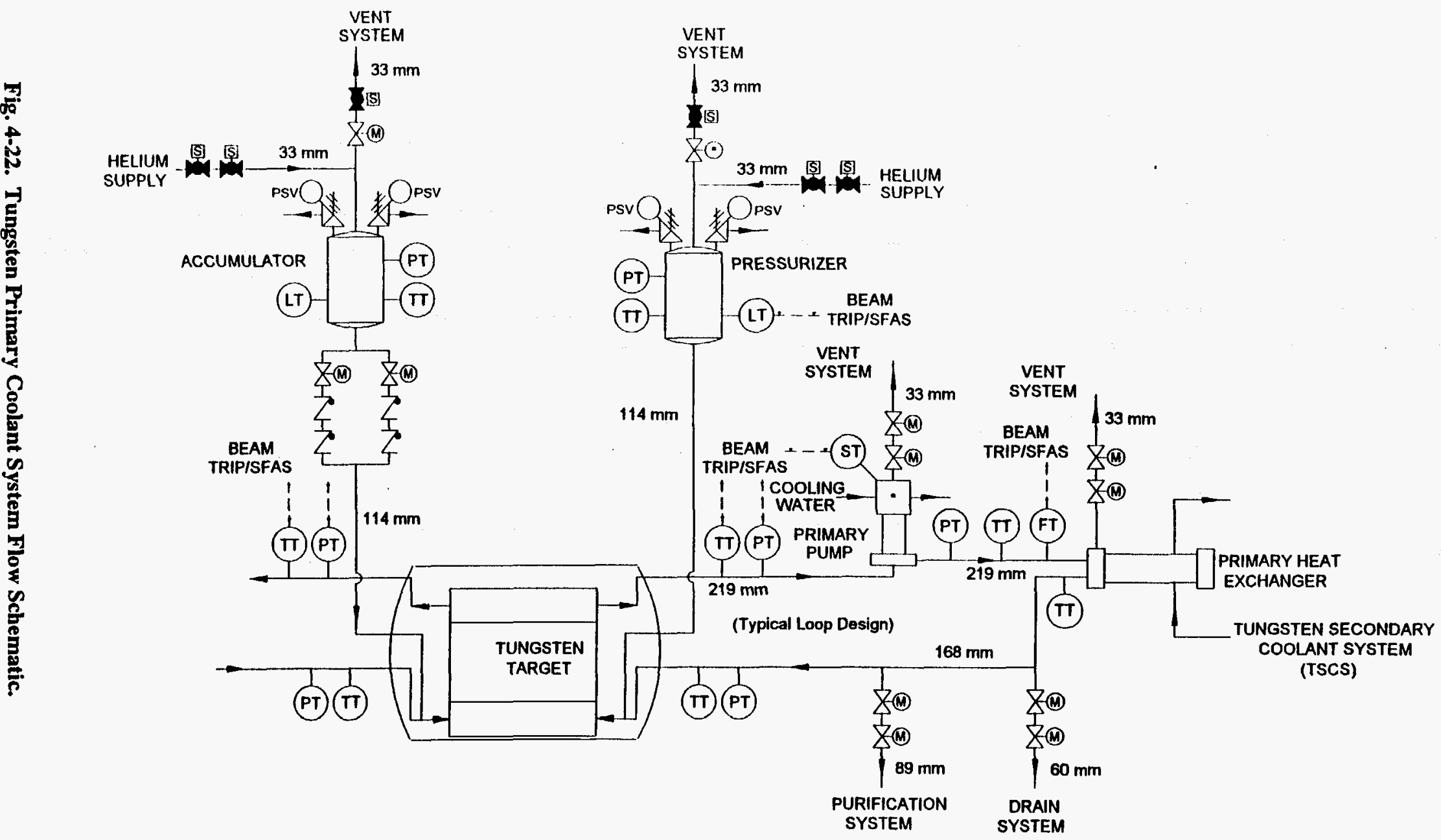


Four features of the TPCS discussed below are pumps, heat exchangers, the pressurizer and accumulator, and piping and valves.

The TPCS primary pumps are vertical canned motor, single-stage, single-suction, mixed-flow units located in the hot-leg piping. Each pump is designed to circulate $50 \%$ of the required flow through the tungsten target. The bottom suction nozzle delivers the $\mathrm{D}_{2} \mathrm{O}$ to the eye of the impeller, and the impeller radially discharges the $\mathrm{D}_{2} \mathrm{O}$ to the diffuser that delivers it to the tangential outlet nozzle. The pump nozzles are butt welded to the TPCS hot-leg piping. The bearings are water lubricated and cooled. An external heat exchanger is provided to remove much of the energy deposited inside the motor and inertial assembly.

The pump motors will have an internally mounted, enhanced inertial unit to extend the flow coastdown of the system to about $45 \mathrm{~s}$, allowing the system to coast down to natural circulation in the event of a loss of offsite power or station blackout. The pumps are located in the hot-leg piping: (1) to provide the necessary pressure in the heat exchanger tubes to ensure that all $\mathrm{D}_{2} \mathrm{O}$ leakage is into the secondary side, and (2) to prevent the formation of a loop seal resulting from the configuration of the pump and the piping. Leakage from the secondary side into the $\mathrm{D}_{2} \mathrm{O}$ would be a source of contamination and a cause for reducing the effectiveness of the tritium production. A loop seal could impede the coolant flow during natural circulation.

The two TPCS heat exchangers are horizontal, two-pass, straight tube, and shell design with the tungsten target $\mathrm{D}_{2} \mathrm{O}$ coolant on the tube side. The TPCS heat exchangers were sized using the Heat Transfer Research, Inc. heat exchanger software. Entrance and exit annuli are provided on the shell side to distribute the coolant flow evenly into the heat exchanger and to eliminate high-velocity fluid impingement cross flow on the outside heat exchanger tubes. A margin was added to the heat transfer surface to allow for surface fouling, tube plugging, and design uncertainties. As the conceptual design evolves, the heat load has changed, and this will require an adjustment in the heat exchanger surface area in the next phase. Optimization of the TCWS and the TSCS also may result in changes in the surface requirements.

The TPCS contains a pressurizer that is charged with natural helium. It maintains the TPCS and the TPRHRS in a subcooled state to promote proper cooling of the tungsten target, to provide the required NPSH for the primary circulating pumps, and to force makeup into the system in the event of a TPCS LOCA. A section of the surge line serves as a return line for one cold leg of the TPRHRS. The pressurizer gas inventory is sized to prevent the charge gas from injecting into the system during a LOCA when the water level is drained to near the bottom of the vessel. The pressurizer contains a power-actuated relief valve, a relief isolation valve, and two code safety valves to protect the TPCS and the TPRHRS from overpressure. Pressurizer controls are provided to maintain a constant gas pressure and a constant water level.

The accumulator is dimensionally identical to the pressurizer but will be operated at a lower natural helium gas pressure. This will ensure that small reductions in TPCS pressure will not cause inadvertent injection. The accumulator is isolated from the system by check valves. The lower pressure causes the connecting line check valves to close, thus preventing the higher-pressure TPCS primary water from backing up into the accumulator. The purpose of the accumulator is to supply additional makeup in the event of a LOCA or leak. The accumulator contains a parallel set of redundant check and stop valves in the connecting line 
to ensure that $\mathrm{D}_{2} \mathrm{O}$ injection will occur in the event of a valve that fails to shut. Like the pressurizer, the accumulator is designed for operation so that the gas will not be injected into the TPCS should a LOCA or leak occur that will drain the accumulator. The accumulator connecting line down stream from the check valves serves as the return line for the second cold leg of the TPRHRS. The accumulator contains valving similar to the pressurizer. The instrumentation for the pressurizer and accumulator includes water level, cover gas pressure, and temperature.

The TPCS piping is seamless 316 restricted chemistry stainless steel piping. All elbows have a long radius to minimize pressure loss. For the preconceptual design phase, all piping and fittings are designed to be butt welded. Consideration will be given in future design phases to bending the piping to eliminate the butt welds. This will reduce the amount of required-inspection and eliminate a potential source of failure.

\subsubsection{TSCS and TCWS}

The TSCS transfers the heat from the TPCS heat exchangers and delivers it to the TCWS under all conditions when ac power is available. A passive cooling function also is provided through the TPCS at the residual heat level. The energy is transported by natural circulation to the atmosphere by a natural draft water-to-air heat exchanger in the TSCS.

Component parts are designed to ensure a 40-yr minimum life for the TSCS when operated at the temperature, pressure, and water chemistry conditions expected in the TSCS.

The TSCS consists of two independent loops, each serving one TPCS heat exchanger and discharging the energy to the TCWS through the TSCS heat exchanger. Each loop removes approximately $50 \%$ of the tungsten target-generated energy and contains the necessary pump, heat exchanger, natural draft water-to-air heat exchanger, connecting piping, surge tank, and associated instrumentation.

A natural draft water-to-air heat exchanger is used in each TSCS loop to remove passively the tungsten target residual heat. Each heat exchanger is sized to remove $100 \%$ of the residual heat load that exists at $200 \mathrm{~s}$ after shutdown and to maintain the tungsten target peak temperature at a safe limit. With both loops operational, the peak temperature will be maintained at an even lower level. The water-to-air heat exchangers are elevated above the primary and secondary heat exchangers to provide natural circulation. The piping to and from the water-to-air heat exchangers is arranged so that it contains no loop seals that could inhibit natural circulation flow. Also, the piping is configured so that flow reversal through the water-to-air heat exchanger does not occur when switching from the forced to natural circulation mode.

Description of the TSCS pumps, heat exchangers, surge tank, piping, and valves is included in the Balance-of-Plant Topical Report [4-4].

The TCWS consists of a natural draft cooling tower, circulating water pumps, piping, and instrumentation. More detailed description of the TCWS is contained in Ref. [4-4]. 


\subsubsection{TPRHRS}

The primary functions of the TPRHRS are to actively remove energy from the tungsten target when the beam is shut down and to cool the tungsten target before replacement when the tungsten TPCS is not available for cooling by either normal operation or natural circulation. The TPRHRS contains two loops each capable of providing $100 \%$ of the cooling of the tungsten target following an accident. Figure 4-23 is a flow schematic of the TPRHRS.

The TPRHRS pumps and heat exchangers are located at a low elevation relative to the TPCS to allow TPRHRS operation when the TPCS is depressurized. Isolation valves are provided to isolate a TPRHRS loop should a break occur in that loop. These isolation valves are required to prevent the draining of the TPCS and the tungsten target by siphoning of the water.

The hot leg of each loop is connected to the tungsten target outlet header. The pipe rises vertically where it exits the moderator tank, then to the primary pump, which is located low to assure adequate NPSH when the system is depressurized and the TPCS is partially drained to allow for maintenance of TPCS components. Each loop uses one canned motor pump, a $\mathrm{D}_{2} \mathrm{O}$ to $\mathrm{H}_{2} \mathrm{O}$ shell and tube heat exchanger, isolation valves, and instrumentation with the redundancy required for a safety related system. The canned motor pump is a vertical, bottom suction, side discharge, single-stage, mixed-flow design.

Outside of the primary isolation valves, the system is safety class 2 . Inside the valves (including the valves) the system is designed to safety class 1 . The pumps and valves can be operated with offsite power or onsite emergency power.

\subsubsection{TSRHRS}

The TSRHRS uses two loops. One loop serves each TPRHRS exchanger. Each train contains an air blast water-to-air heat exchanger, a surge tank, a pump, and the required instrumentation. The pumps, fans, and isolation valves can be operated using either offsite power or onsite emergency power. More detailed description of the TSRHRS is contained in Ref. 4-4.

\subsubsection{Moderator Heat Transport Systems (MTHTS)}

The MTHTS are made up of those systems that remove and transport the heat generated within the moderator tank, including the window, the target lead, the moderator, the proton beam backstop region, the ${ }^{3} \mathrm{He}$ blanket tubes, the ${ }^{3} \mathrm{He}$ in the NSA, and the moderator tank structural components, and transfer the energy to the environment, which is the ultimate heat sink (Fig. 4-21). It consists of the following five systems: the Moderator Primary Coolant System (MPCS), the Moderator Secondary Coolant System (MSCS), the Moderator Circulating Water System (MCWS), the Moderator Primary Residual Heat Removal System (MPRHRS), and the Moderator Secondary Residual Heat Removal System (MSRHRS). All of the systems contain two loops. The main cooling systems contain two $50 \%$ loops for normal operation where both loops are required to function while the accelerator is operating. The loss of any one loop in the MPCS, the MSCS, or the MCWS will result in an accelerator 


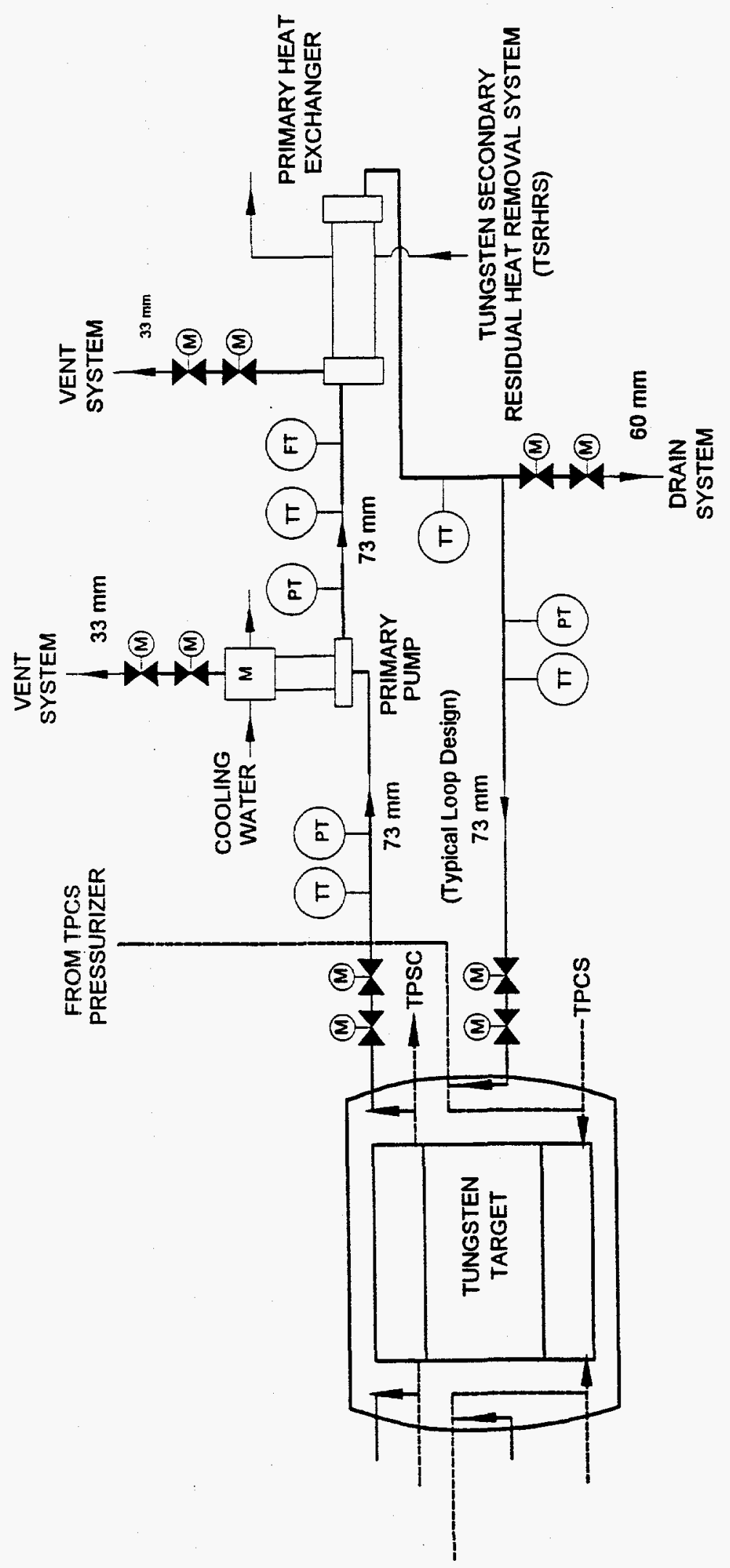

Fig. 4-23. Tungsten Primary Residual Heat Removal System Flow Schematic. 
trip. The MPRHRS contains two $100 \%$ capacity primary coolant loops for actively removing residual heat from the moderator tank. Each of the MPRHRS coolant loops is capable of removing the total residual heat if the other loop is not available. The MPCS and the MSCS also provide a passive cooling capability for removing moderator tank residual heat through a natural draft water-to-air heat exchanger located in the MSCS. Each primary loop and its associated secondary loop can passively remove the total residual heat load. Thus the active and passive residual heat removal trains are fully redundant.

\subsubsection{MPCS}

The MPCS provides forced circulation of the moderator $\mathrm{D}_{2} \mathrm{O}$ coolant to remove the energy generated within the moderator tank, the beam entrance window and $3 \mathrm{He}$, and transfer this energy to the MSCS under all conditions when ac power is available, except for the case when the system is depressurized. When the MPCS is depressurized, the beam must be shut down and cooling is accomplished by either the passive mode or the MPRHRS, which is the active backup.

The passive cooling mode is provided by natural circulation of the MPCS coolant. Residual heat is transported to the MSCS, which is also naturally circulating. The natural draft water-to-air heat exchanger in the MSCS transfers the heat to the atmosphere. Natural circulation in the MPCS and MSCS is accomplished by elevating the heat exchangers so that a thermal driving head exists to cause natural circulation.

All component parts in contact with the moderator coolant are fabricated from corrosion resistant materials designed to ensure a 40-yr minimum life for the MPCS when operated at the temperature, pressure, and water chemistry conditions expected in the MPCS.

The MPCS consists of two independent loops with each loop connected in parallel to the ${ }^{3} \mathrm{He}$ heat exchanger. Figure 4-24 is a flow schematic of the MPCS. Each loop removes approximately $50 \%$ of the energy generated within the moderator tank and contains one pump, one heat exchanger, connecting piping, and the associated instrumentation to meet the required single-failure criteria. The pressurizer is connected to one MPCS loop to provide pressure and $\mathrm{D}_{2} \mathrm{O}$ volume control. It is also a reservoir that provides for moderator tank flooding in the event of a LOCA. The pressurizer is sized based partly on TRAC analysis to accommodate an insurge that would result from a TPCS pipe rupture inside the moderator tank. As such, the pressurizer serves as a relief vessel to prevent overpressurization of the moderator tank. Future TRAC studies are planned in the next design phase to investigate a large break in the helium chamber and the design of the pressurizer and surge line to prevent overpressurization of the moderator tank. The pumps, heat exchangers, pressurizer and accumulator, and piping and valves of the MPCS are discussed below.

The MPCS primary pumps are vertical canned motor, single-stage, single-suction, mixed-flow units located in the hot-leg piping. Each pump is designed to circulate $50 \%$ of the required flow through the moderator tank. The bottom suction nozzle delivers the $\mathrm{D}_{2} \mathrm{O}$ to the eye of the impeller, and the impeller radially discharges the $\mathrm{D}_{2} \mathrm{O}$ to the diffuser that delivers it to the tangential outlet nozzle. The pump nozzles are butt welded to the MPCS hot-leg piping. The bearings are water lubricated and cooled. An external heat exchanger is provided to remove much of the energy deposited inside the motor and inertial assembly. 


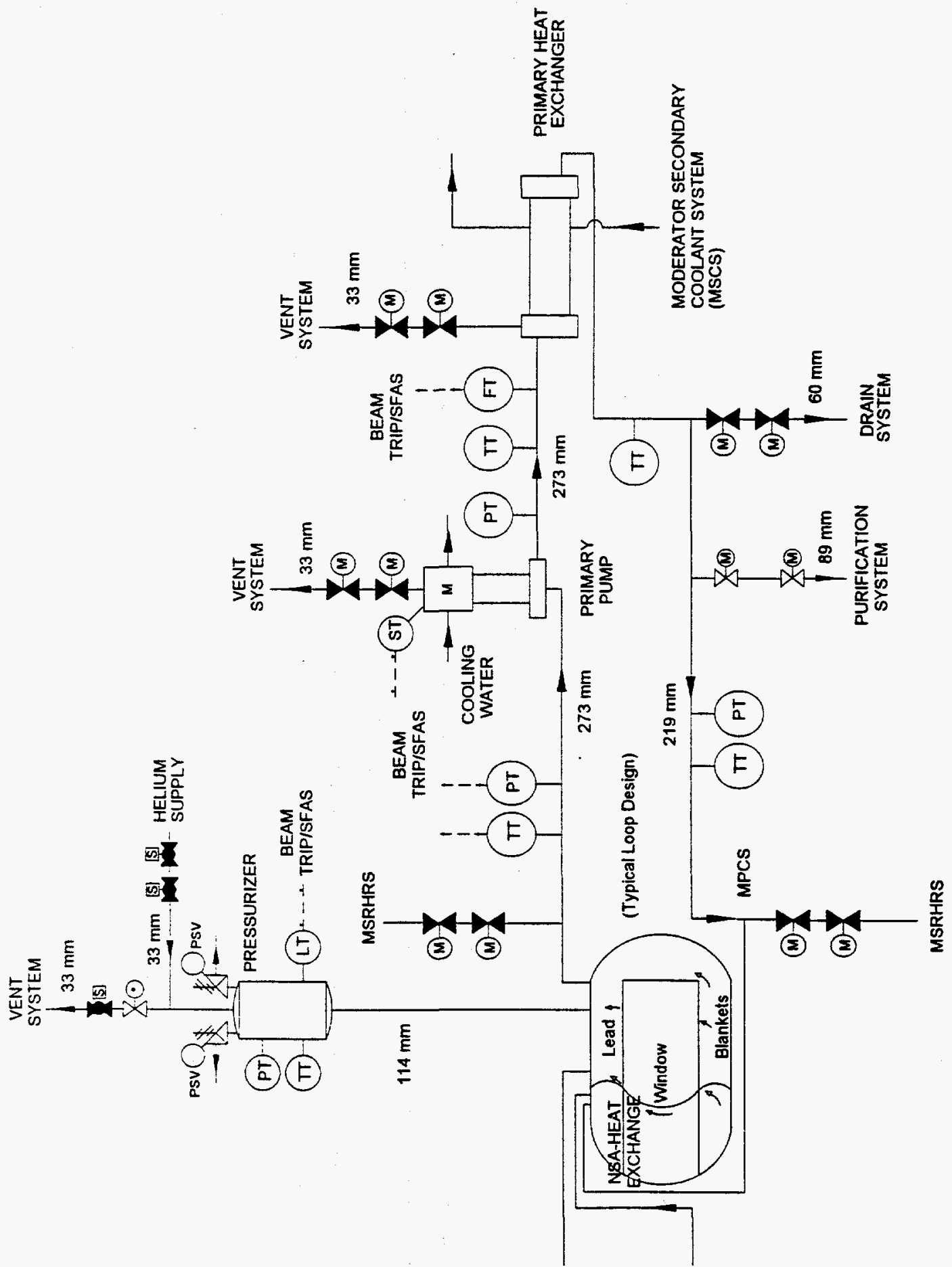

Fig. 4-24. Moderator Primary Coolant System Flow Schematic.

The pump motors will have an internally mounted, enhanced inertial unit to extend the flow coastdown of the system to about $45 \mathrm{~s}$, allowing the system to coast down to natural circulation in the event of a loss of offsite power or station blackout. The pumps are located 
in the hot-leg piping: (1) to provide the necessary pressure in the heat exchanger tubes to ensure that all $\mathrm{D}_{2} \mathrm{O}$ leakage is into the secondary side, and (2) to prevent the formation of a loop seal due to the configuration of the pump and the piping. Leakage from the secondary side into the $\mathrm{D}_{2} \mathrm{O}$ would be a source of contamination and a cause for reducing the effectiveness of the tritium production. A loop seal could impede the coolant flow during natural circulation.

The two MPCS heat exchangers are horizontal, two-pass, straight tube, and shell design with the tungsten target $\mathrm{D}_{2} \mathrm{O}$ coolant on the tube side. The MPCS heat exchangers were sized using the Heat Transfer Research, Inc. heat exchanger software. Entrance and exit annuli are provided on the shell side to distribute the coolant flow evenly into the heat exchanger and to eliminate high-velocity fluid impingement cross flow on the outside heat exchanger tubes. A margin was added to the heat transfer surface to allow for surface fouling, tube plugging, and design uncertainties. As the conceptual design evolves, the heat load has changed and this will require an adjustment in the heat exchanger surface area in the next design phase. Optimization of the MCWS and the MSCS also may result in changes in the surface requirements.

The MPCS contains a pressurizer that is charged with natural helium. It maintains the MPCS and the MPRHRS in a subcooled state to promote proper cooling of the moderator tank internals, to provide the required NPSH for the primary circulating pumps, and to force makeup into the system in the event of a MPCS LOCA. A section of the surge line serves as a return line for one cold leg of the MPRHRS. The pressurizer gas inventory is sized to prevent the charge gas from injecting into the system during a LOCA when the water level is drained to near the bottom of the vessel. The pressurizer contains a power actuated relief valve, a relief isolation valve, and two code safety valves to protect the MPCS and the MPRHRS from overpressure. Pressurizer controls are provided to maintain a constant gas pressure and a constant water level.

The pressurizer will be sized to accommodate an insurge that would result from a TPCS or helium chamber rupture inside the moderator tank. As such, the pressurizer serves as a relief vessel to prevent overpressurization of the moderator tank. The preconceptual MPCS pressurizer is designed to accommodate a rupture of a $2.07 \mathrm{MPa}$ (300 psia) gas pressure in the helium chamber.

The MPCS piping is seamless 316 restricted chemistry stainless steel piping. All elbows have a long radius to minimize pressure loss. For the preconceptual design phase, all piping and fittings are designed to be butt welded. Consideration will be given in future design phases to bending the piping to eliminate the butt welds. This will reduce the amount of required inspection and eliminate a potential source of failure.

\subsubsection{MSCS and MCWS}

The MSCS transfers the heat from the MPCS heat exchangers and delivers it to the MCWS under all conditions when ac power is available. A passive cooling function also is provided through the MPCS at the residual heat level. The energy is transported by natural circulation to the atmosphere by a natural draft water-to-air heat exchanger in the MSCS. Component parts are designed to ensure a 40-yr minimum life for the MSCS when operated at the temperature, pressure, and water chemistry conditions expected in the MSCS. 
The MSCS consists of two independent loops, each serving one MPCS heat exchanger and discharging the energy to the MCWS through the MSCS heat exchanger. Each loop removes approximately $50 \%$ of the moderator tank internals generated energy and contains the necessary pump, heat exchanger, natural draft water-to-air heat exchanger, connecting piping, surge tank, and associated instrumentation.

A natural draft water-to-air heat exchanger is used in each MSCS loop to remove passively the moderator tank internals residual heat. Each heat exchanger is sized to remove $100 \%$ of the residual heat load that exists at $200 \mathrm{~s}$ after shutdown and to maintain the target lead peak temperature at a safe limit. With both loops operational, the peak temperature will be maintained at an even lower level. The water-to-air heat exchangers are elevated above the primary and secondary heat exchangers to provide natural circulation. The piping to and from the water-to-air heat exchangers is arranged so that it contains no loop seals that could inhibit natural circulation flow. Also, the piping is configured so that flow reversal through the water-to-air heat exchanger does not occur when switching from the forced to natural circulation mode.

The MCWS consists of a natural draft cooling tower, circulating water pumps, piping, and instrumentation. Further description of the components in the MCWS is contained in the Balance-of-Plant Topical Report [4-4].

\subsubsection{MPRHRS}

The primary function of the MPRHRS is to remove decay energy from the components within the moderator tank when the beam is shut down and to cool the system down for replacement or repair when the MPCS is not available for cooling either by normal operation or by natural circulation. Figure 4-25 is a flow schematic of the MPRHRS. The MPRHRS contains two loops each capable of providing $100 \%$ of the active cooling of the target lead following an accident. Each loop is designed to be operable when the MPCS is depressurized, as it is capable of cooling the target lead following a LOCA.

The hot leg of each loop is connected to the moderator tank. From the moderator tank the hot leg is routed downward where it connects to the inlet of the MPRHRS primary pump. The pump is located low to assure adequate NPSH when the system is depressurized and the moderator primary cooling system partially drained to allow for maintenance of system components. Each loop uses one single-stage canned motor pump, a $\mathrm{D}_{2} \mathrm{O}$ to $\mathrm{H}_{2} \mathrm{O}$ shell and tube heat exchanger, isolation valves, and instrumentation with the redundancy required for a safety-related system. The canned motor pump is a vertical, bottom suction, side discharge, single-stage, mixed-flow design.

\subsubsection{MSRHRS}

The MSRHRS uses two loops. One loop serves each MPRHRS exchanger. Each train contains an air blast water-to-air heat exchanger, a surge tank, a pump, and the required instrumentation. The pumps, fans, and isolation valves can be operated using either offsite power or onsite emergency power. 


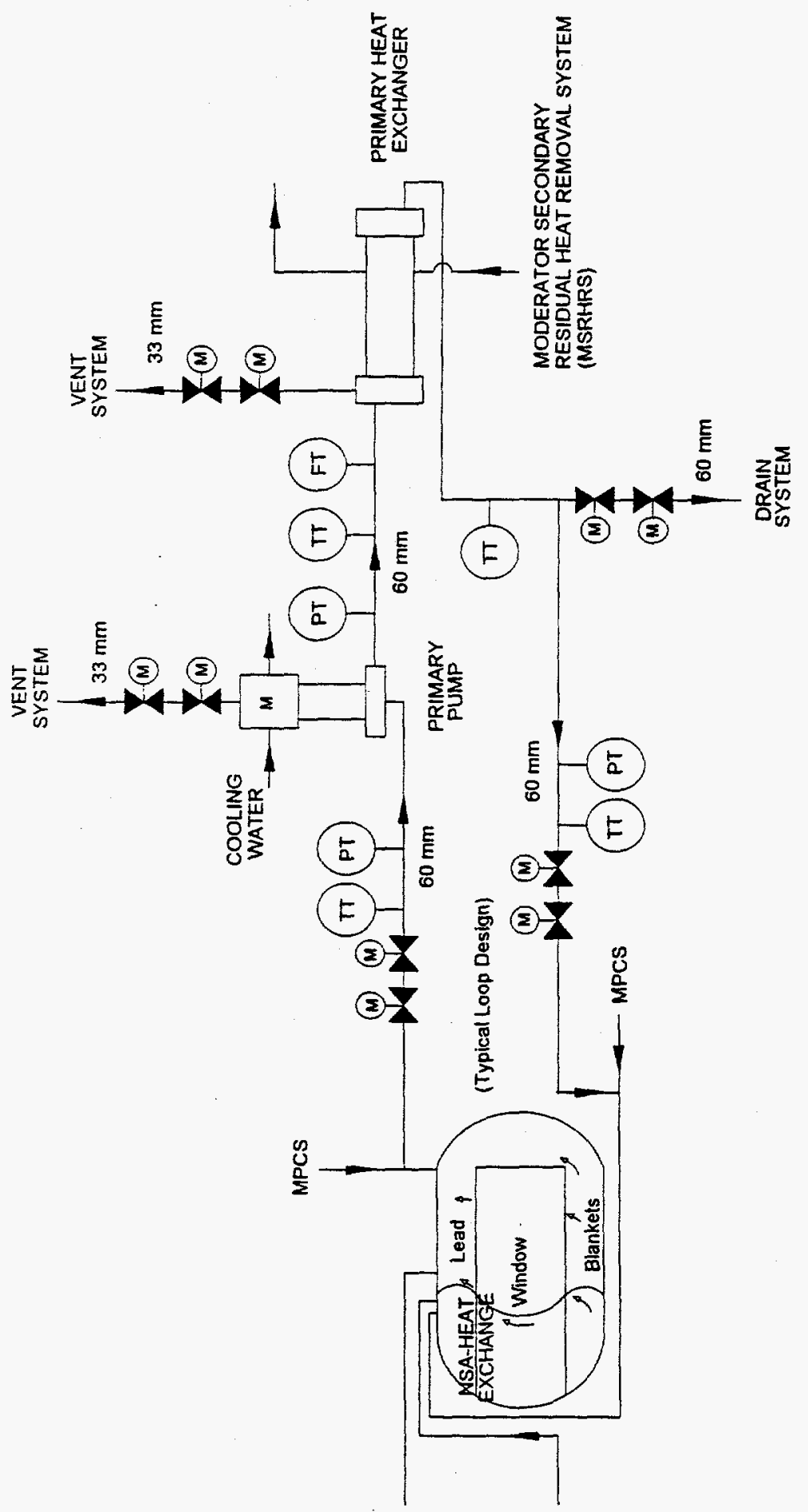

Fig. 4-25. Moderator Primary Residual Heat Removal System Flow Schematic. 


\subsubsection{He Heat Transport Systems (HHTS)}

The HHTS are made up of those components that remove and transport the energy generated in ${ }^{3} \mathrm{He}$ in the neutron source assembly and blankets to the moderator in the moderator tank. It also contains the lines that let down the ${ }^{3} \mathrm{He}$ containing the produced tritium and compress and return $3 \mathrm{He}$ to the neutron source assembly and blankets. It is composed of the following two systems: NSA Coolant System (NCS) and Blanket Coolant system (BCS). All of the systems consist of one loop; refer to Figs. 4-26 and 4-27. The MTHTS carries the energy to the environment, i.e., ultimate heat sink (Fig. 4-21).

\subsubsection{NSA Coollant System (NCS)}

The NCS provides forced circulation of the $3 \mathrm{He}$ in the neutron source assembly to remove the energy generated within the ${ }^{3} \mathrm{He}$ and deliver this energy to the MPCS under all conditions when AC power is available. When the NCS is depressurized or the circulator is not available, the beam must be shut down and the $3 \mathrm{He}$ cooling will be provided by the THTS and the moderator tank heat transport systems..

All component parts in contact with the $3 \mathrm{He}$ are fabricated from materials designed to be compatible with the coolant and interfacial structures.

The NCS consists of one loop connected to the inlet of the moderator tank. Figure 4-19 shows the NSA helium flow arrangement. Figure 4-26 is a flow schematic of the NCS. The system removes $100 \%$ of $3 \mathrm{He}$ generated energy in the NSA and contains one circulator, one heat exchanger, connecting piping, a few high pressure blanket tubes, and the associated instrumentation to meet the required single-failure criteria. The system also contains the $3 \mathrm{He}$ and tritium letdown line for tritium extraction and the $3 \mathrm{He}$ makeup to circulator discharge line.

NCS circulator, heat exchangers, make-up compressor, and piping and valves are discussed below.

Forced flow through the NCS loop is provided by one single-stage, radial circulator. The suction nozzle delivers the $3 \mathrm{He}$ to the eye of the impeller, and the impeller radially discharges it to the diffuser, which in turn delivers it to the return nozzle. The circulator nozzles are butt-welded to the NCS piping.

The NCS heat exchanger, Fig. 4-8, located in the forward section of the moderator tank, was sized to remove the NSA ${ }^{3} \mathrm{He}$ generated energy and maintain the average $3 \mathrm{He}$ temperature in the NSA chamber below $400 \mathrm{~K}\left(260^{\circ} \mathrm{F}\right)$. The NCS heat exchanger is a horizontal, single-pass, helical tube and shell design with the $3 \mathrm{He}$ on the tube side and moderator, $\mathrm{D}_{2} \mathrm{O}$, on the shell side. It was sized based on $2.33 \mathrm{MWt}$ of energy being delivered to the ${ }^{3} \mathrm{He}$. As the conceptual design evolves, the heat load may change slightly. However, it is believed that the heat exchanger is adequately sized for the preconceptual design study.

The NCS coaxial piping and blanket tubes are seamless aluminum that are replaced with each NSA replacement. For the preconceptual design phase, all piping and fittings are considered to be butt-welded. 


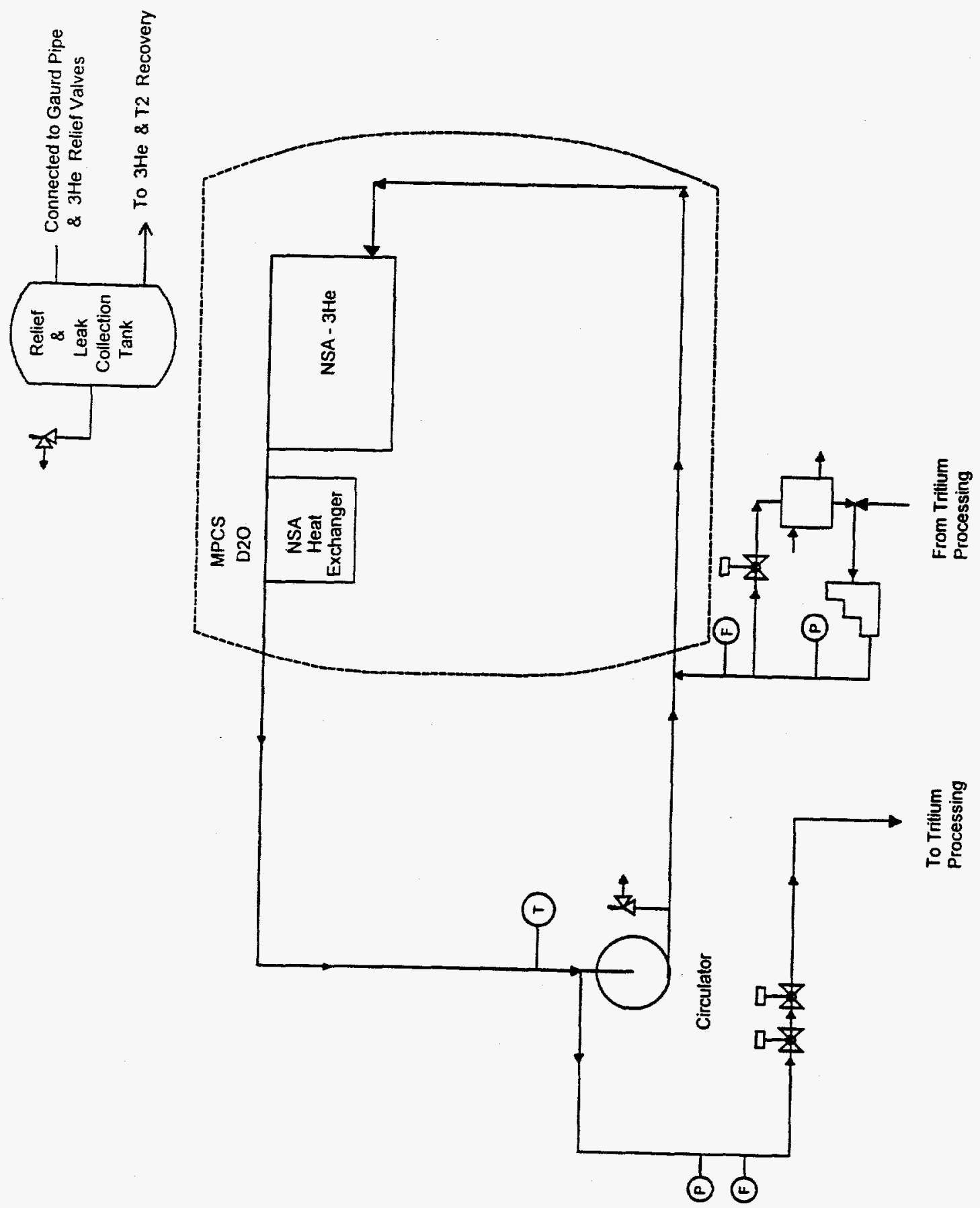

Fig. 4-26. NSA Coolant System Flow Schematic. 


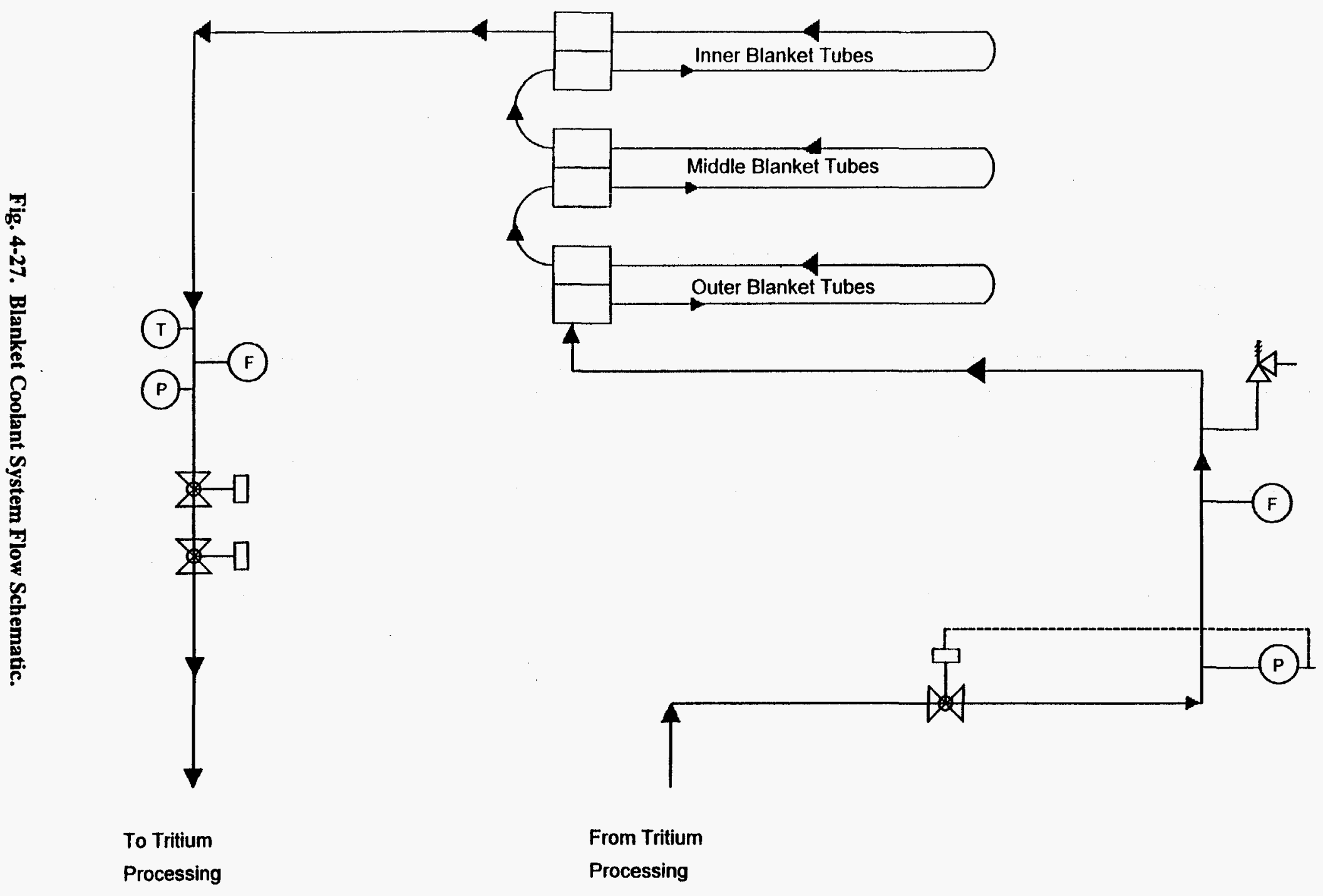


Two supply tubes feed the cool gas from helium circulator into the bottom of the NSA. The helium passes through the NSA where it rises as it is heated and exits the NSA at the top near the backstop end. At the backstop end, some of the helium will pass through the backstop to provide the ${ }^{3} \mathrm{He}$ for tritium production in the backstop. The helium is returned through two tubes from the backstop end of the NSA to the NSA heat exchanger header where it enters the heat exchanger tubes that surround the beam tunnel extension coming into the NSA. Each tube in a bank circles the beam tunnel extension going from a divided header through the tubes to the other section of the header. The return headers are collected into the inner pipe in a coaxial arrangement which penetrates the steel shield. Outside the shield, the center pipe enters a radial circulator which develops the required head for circulation. The $3 \mathrm{He}$ is then returned to the high pressure blanket tubes in the outer section of the coaxial pipe. The coaxial pipe is contained in a third pipe, called the guard pipe, which is held at a slightly negative pressure in order to collect pressure boundary leakage. The leakage is collected in the guard pipe and relief collection tank which is a component of this system.

The $3 \mathrm{He}$ extraction stream is removed from the circulator inlet, the inner coaxial pipe. Its pressure is reduced by flowing the stream through pressure reduction valves/orifices to the tritium extraction line normally held at about one atmosphere pressure, $0.101 \mathrm{MPa}(14.7$ psia). The $3 \mathrm{He}$ flow stream is such that the NSA 3 He volume is processed every day to limit the tritium content in the $3 \mathrm{He}$. The $3 \mathrm{He}$ is returned at charge pressure, $1.034 \mathrm{MPa}$ (150 psia). The makeup compressor compresses it to $2.07 \mathrm{MPa}$ (300 psia) and supplies it to the outer coaxial pipe to supply ${ }^{3} \mathrm{He}$ makeup to the system. Relief valves are provided on the outer coaxial pipe to limit the NSA pressure. These valves discharge to the guard pipe and pressure relief tank.

\subsubsection{Blanket Coolant System (BCS)}

The blanket coolant system contains the low pressure ${ }^{3} \mathrm{He}$ blanket tubes where the ${ }^{3} \mathrm{He}$ is converted to tritium, the letdown lines for the removal of product, and the make-up line. Pressure relief valves are used to limit the pressure in the blanket tubes, and their discharge is to the guard pipe and relief collection tank in the NCS. All of the ${ }^{3} \mathrm{He}$ piping external to the moderator tank is contained inside guard piping. The moderator tank and the MPCS provide the guard pipe function for the piping and tubes inside the moderator tank. The blanket tubes are cooled by conduction from the ${ }^{3} \mathrm{He}$ to the aluminum and then to the surrounding $\mathrm{D}_{2} \mathrm{O}$ moderator, thus the moderator heat transport system transports the energy from the blanket tube surfaces to the environment.

The blanket tubes are grouped into three regions: inner blankets, middle blankets and outer blankets, refer to Figs. 4-20 and 4-27. The volumetric heat generation rates for the blanket tubes decrease dramatically going from the inner to the outer blankets. The volumetric heat generation rates for the different blanket tubes are given in the following list.

$\begin{array}{lll} & \text { Average } & \text { Peak } \\ \text { 3He in Inner Blankets, } \mathrm{kW} / 1 & 0.61 & 1.32 \\ \text { 3He in Middle Blanket, } \mathrm{kW} / \mathrm{l} & 0.095 & 0.21 \\ \text { 3He in Outer Blanket, } \mathrm{kW} / & 0.0018 & 0.0039 \\ \text { Aluminum in Inner Blanket, } \mathrm{kW} / \mathrm{l} & 0.31 & 0.67\end{array}$


The peak to average heat rates in all of the regions is 2.16 . Using only conduction heat transfer in the helium, the temperature rise in the helium will be too large in the inner blankets. Using three internal long fins and three short fins, the temperature increase can be held to $55^{\circ} \mathrm{C}\left(100^{\circ} \mathrm{F}\right)$ or less due to the much higher thermal conductivity in aluminum, about a factor of 1000 . The middle blanket tubes are currently expected to be cooled with no fins. If future analyses result in excessive temperatures, three short fins may be used in the middle blanket tubes. The outer tubes have a much lower heat rate, and they are also smaller in diameter; thus, no enhancement of the thermal conduction is required for them. The average $3 \mathrm{He}$ temperature in each blanket zone will be maintained below $400 \mathrm{~K}\left(260^{\circ} \mathrm{F}\right)$.

All of the blanket tubes use reduced tube diameter U-bends at the backstop end of the moderator tank to form a U-bend that returns ${ }^{3} \mathrm{He}$ to the window end. The two ends of the Utubes are headered (joined) at the window end. One header allows the ${ }^{3} \mathrm{He}$ make-up from the tritium extraction to be distributed between the U-tubes. The $3 \mathrm{He}$ flows into the outer tube bank outer header, through the outer U-tubes to the inner header of the outer tube bank. From the outer blanket it goes to the outer header for the middle tube bank, through the Utubes to the inner header for the middle tube bank. From the inner middle tube header it goes to the outer header serving the inner blanket tubes, through the U-tubes to the inner blanket tube header where it is let down along with the produced tritium. This depressurizes and prepares the ${ }^{3} \mathrm{He}$ for the tritium extraction system.

The makeup line takes suction from the tritium extraction return line that contains a clean $3 \mathrm{He}$ supply, pressure $1.034 \mathrm{MPa}$ (150 psia). The makeup valve is pressure controlled and reduces the pressure to $0.69 \mathrm{MPa}$ (100 psia) for the low pressure blanket tubes. A pair of isolation valves in series is provided in the make-up line to allow for isolation should a problem develop in the blanket region or the makeup supply. The letdown line reduces the pressure from $0.69 \mathrm{MPa}(100 \mathrm{psi})$ to near atmospheric pressure. The letdown line also uses two isolation valves in series to allow isolation and reduction in pressure.

The system flow pattern was chosen to minimize the tritium contained in the blanket low pressure region as it flows from the lower production outer blanket tubes, through the middle blanket tubes, and then through the high production inner blanket tubes. A system relief valve is connected to the inlet piping to protect the blanket tubes from overpressure. Any relief is returned to the guard pipe and relief tank in the NCS.

\subsubsection{Purification Systems}

The functions of the purification systems for the T/B cooling systems are as follows:

- Control coolant chemistry to reduce corrosion and crud deposits on surfaces exposed to coolant.

- Maintain isotopic purity of the moderator/coolant.

- Reduce formation of radiolytically decomposed gas.

- Control the helium content in the coolant to reduce gas release to the coolant on system depressurization. 
- Reduce radioactive contaminants from the water passing through the purification system.

- Provide high-purity makeup water to maintain coolant inventory,

Purification will be required for all normal plant operating modes including startup, production, shutdown, and target changeout. Water chemistry and contamination levels for the TPCS are expected to be different than those for the MPCS. Therefore, separate purification systems were designed for the MPCS and the TPCS. Figure 4-28 shows a flow schematic of a purification system.

Both the MPCS and the TPCS will contain two 100\% capacity purification loops for increased reliability and continuity of operation during replacement of ion exchange beds and filters. Each purification loop will have a degasification holdup tank, a purification pump, a prefilter unit, a mixed bed ion exchange unit, an after filter unit, and a chemical/gas addition system. In addition, the TPCS will have a purification cooler for reducing the coolant temperature before being processed. Also, the TPCS purification systems will include a pair of deuterium absorption units with in-situ regeneration equipment for recovery of $\mathrm{D}_{2} \mathrm{O}$ released in the degasifier tank.

The ion exchange beds will contain mixed deuterated, strong acid and strong base resins. Filter units for both purifications will contain multiple disposable filter assemblies capable of removing $1.26-\mu \mathrm{m}$ particles with $91 \%$ efficiency.

The $\mathrm{D}_{2} \mathrm{O}$ isotopic purification system (Fig. 4-28) includes two steam-heated fractional distillation columns operating under vacuum, in tandem, with the capacity to upgrade approximately $6 \mathrm{gpm}$ of $\mathrm{D}_{2} \mathrm{O}$ from $99.5 \% \mathrm{D}_{2} \mathrm{O}$ enrichment to $99.7 \%$. Each column has a steam-heated reboiler, a water-cooled condenser, and twin condensate pumps. Vacuum will be maintained by a pair of $\mathrm{D}_{2} \mathrm{O}$ sealed mechanical vacuum pumps equipped with exhaust vapor traps to minimize the $\mathrm{D}_{2} \mathrm{O}$ losses. The columns will be operated at a high reflux ratio, and a large number of transfer units will be required to accomplish the required isotopic separation. A moisture separator is installed in tandem with the first reboiler to ensure that steam entering the columns will carry minimum particulates. Upgraded $\mathrm{D}_{2} \mathrm{O}$ is returned as makeup to the TPCS or the MPCS to maintain the required inventory and water quality.

\subsection{INSTRUMENTATION CONTROL AND PROTECTION SYSTEMS}

The T/B instrumentation and control systems consist of both a control system and a beamtrip/safety features actuation system (SFAS). The purpose of the control system is to assure $\mathrm{T} / \mathrm{B}$ parameters are within the design operating range for the system. The purpose of the beamtrip/SFAS is to assure a beamtrip will occur and adequate cooling will be provided if $\mathrm{T} / \mathrm{B}$ parameters deviate from the design operating range. Setpoints for the control systems and beamtrip/SFAS will be based on allowable deviation and instrumentation accuracy as described in Ref. 4-5. The beamtrip/SFAS will be designed to the requirements of the IEEE603 Standard as described in Ref. 4-6. 

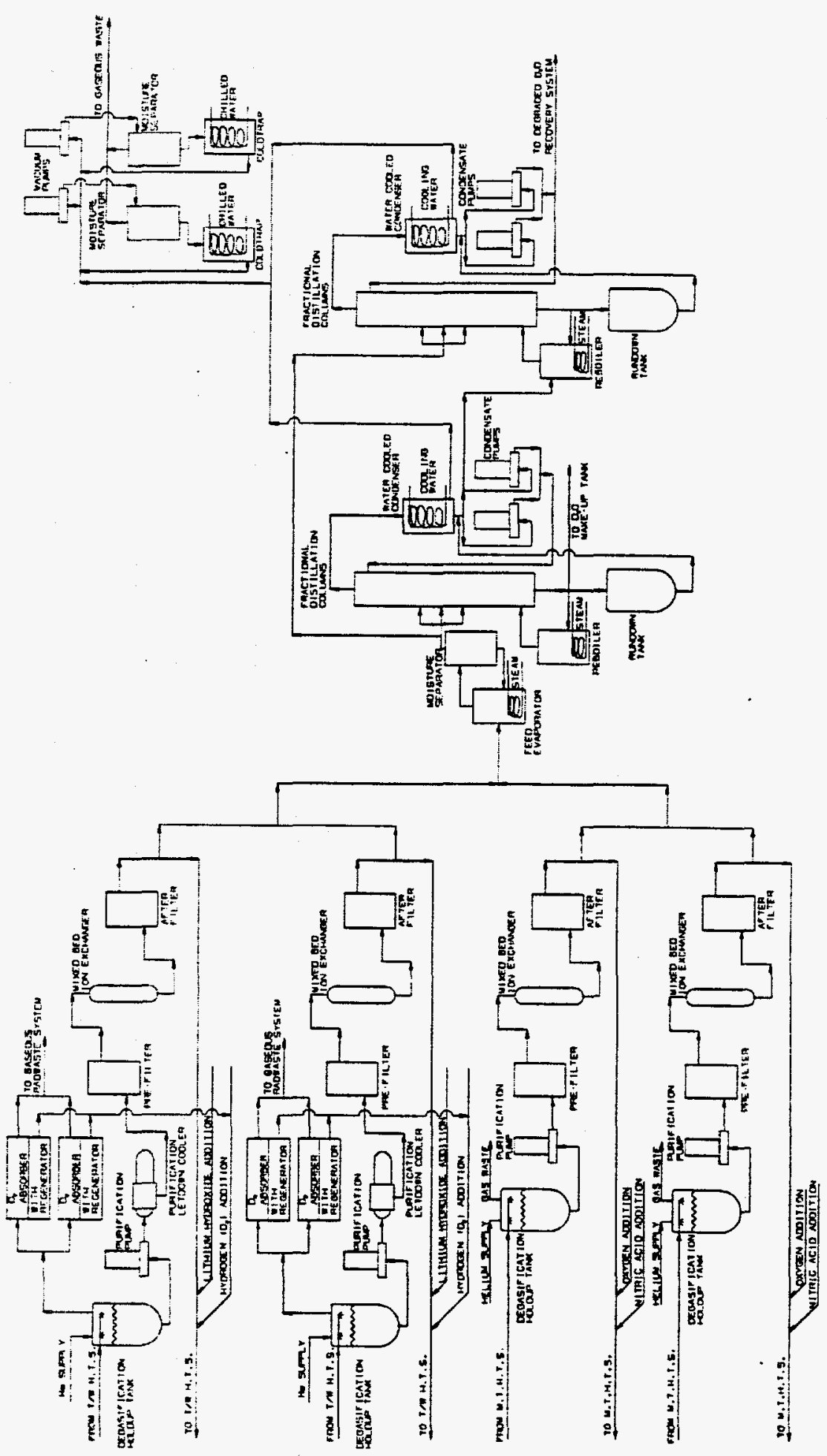

Fig. 4-28. Purification System Schematic. 
The following discussion is for the T/B portion of the APT complex control system. The procedures for handing the $3 \mathrm{He}$ and tritium are a part of the tritium-extraction controls, and the procedures for handling the accelerator are a part of the accelerator controls. The APT complex control system is discussed further in Sec. 6, T/B Operation, and the Balance-ofPlant Topical Report (Ref. 4-4).

\subsubsection{T/B Control System}

A control system is needed for system startup, operation, shutdown, and system interlocks. For all of these operating modes, the design operating parameter nominal values were established, and then the procedures for getting to the operational conditions were established, which lead to the instrumentation, controls, and interlocks that were required. The controls for system startup, operation, and shutdown must maintain parameters within a defined envelope during system normal operation, and the system interlocks must prevent operation or actuation of a system until other conditions are satisfied. The control system is not a safety system; thus the T/B protection will be assured by the beamtrip/SFAS without reliance on the control system.

\subsubsection{T/B Control Concept}

Table 4-11 shows the startup procedure for the T/B control system. The first operation is to fill the Circulating Water Systems (CWS), the Secondary Coolant Systems (SCS), and the Primary Coolant Systems (PCS) for the THTS, and the MTHTS with liquid and then to pressurize the systems. The makeup and purification systems are used for liquid fill to the operating liquid levels in the pressurizers and the gas makeup system is used for gas pressurization to the operating pressure in the pressurizers. After confirming the operating liquid levels and gas operating pressures, the pumps would be started in the CWS, then the SCS, and then the PCS. An interlock would prevent the pumps from operating until NPSH requirements were met. After checking system flow values, the normal makeup and purification flow would be established. After checking the makeup and purification flow, the pressurizer level control would be activated, which would put the control system in automatic mode. A run permit signal would then be sent to the accelerator after all the conditions had been satisfied (standby condition).

Table 4-11. LANL APT T/B Startup Procedures

1. Set pressurizer liquid level and gas pressure to operating values in all systems using liquid makeup and purification system and gas makeup system.

2. Start pumps in each system, starting with tertiary, then secondary, then primary and establish standby steady state.

3. Check and adjust new pressurizer natural helium gas pressure and liquid level as standby.

4. Establish normal purification flow.

5. Turn on liquid makeup and purification system level control.

6. Send run permit signal to accelerator.

7. Turn on accelerator beam, and establish power steady state.

8. Check and adjust new pressurizer gas pressure and liquid level at full power. 
Figure 4-29 graphically shows the control system startup procedure. Starting from the Shutdown conditions of room temperature and pressure, the gas and liquid fill would bring the system to the standby condition. As the beam power is increased, the temperatures would increase, and liquid expansion of the system would be controlled by the pressurizer liquid level control of the letdown and makeup valves in the makeup and purification system. Liquid expansions and contractions due to power level changes would be handled by the pressurizer liquid level control. Figure 4-11 also shows assumptions about other parameters that are involved in the normal operation of the T/B.

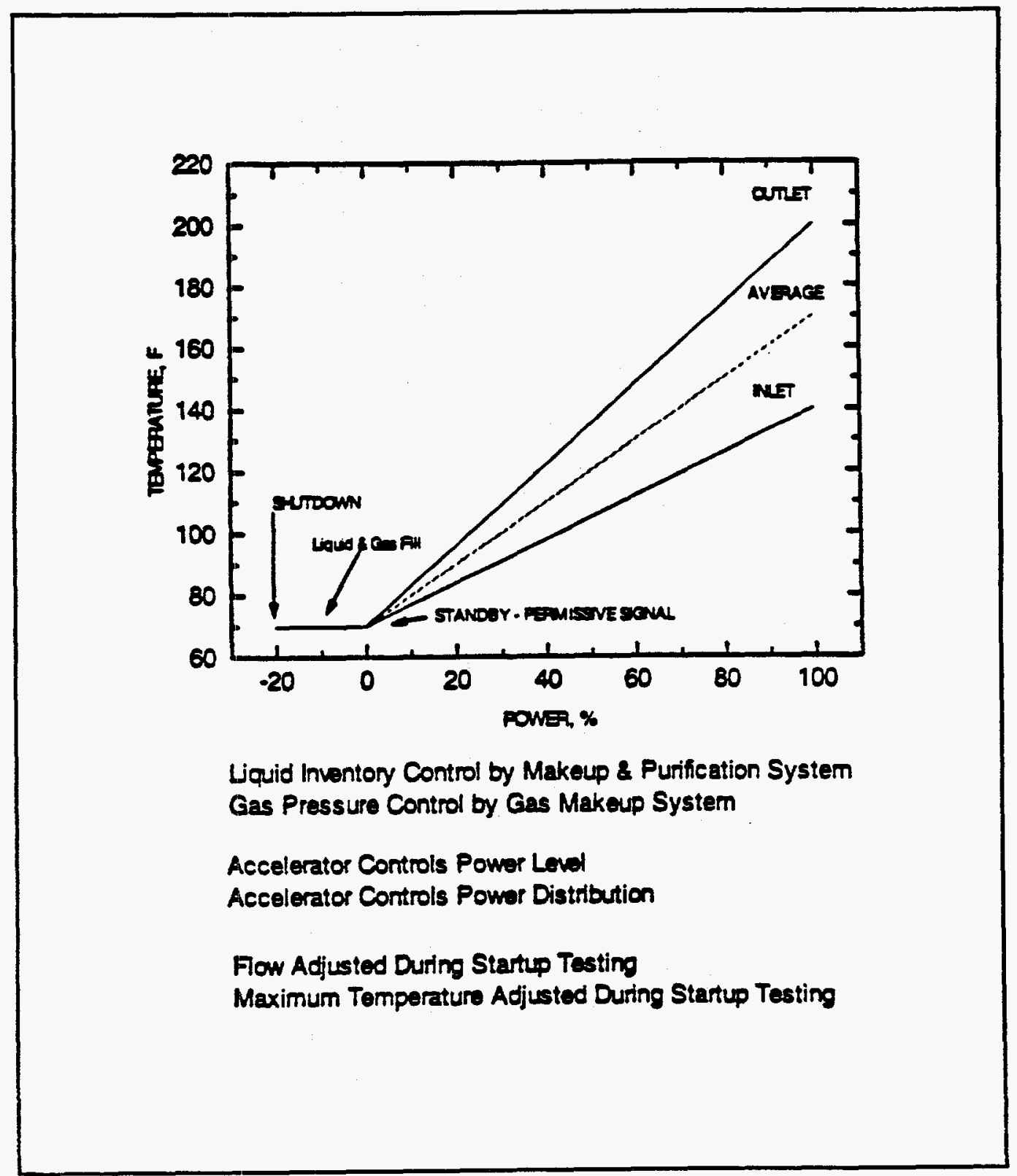

Fig. 4-29. LANL APT Control Concept. 


\subsubsection{Beamtrip/SFAS}

The overall objective of the APT beamtrip/SFAS is to assure highly reliable systems that are designed for single- and multiple-failure effects and still assure that a beamtrip will occur and that adequate cooling is provided. The two key elements of this overall objective are:

- fluid heat removal systems designed to provide active and passive heat transport capability, and

- a protection system designed to assure a beamtrip will occur.

By having both dual active and passive heat removal systems the effects of a single failure can be accommodated. By having both active and passive heat removal systems, the effects of multiple failures can also be accommodated.

The protection system will be designed to IEEE-603 Standards; thus a single failure will neither cause a beamtrip nor prevent a beamtrip because of the two-out-of-four trip logic. For all DBE transients this assurance is provided by having both a primary and secondary trip function for each transient, such that a triple failure (double failure of the primary trip function and a single failure in secondary trip function) could occur and the secondary trip function still operate. The combination of a DBE and a triple failure reduces the transient to a residual risk category (to be confirmed by risk analysis); thus no further trip function protection is required even though it may be available.

\subsubsection{Fluid Safety Systems}

The active fluid safety features are:

- dual loops (THTS and MTHTS) that provide single failure protection, and

- dual RHRS (THTS and MTHTS) that provide single failure protection.

The passive fluid safety features are:

- dual water-to-air heat exchangers (TSCS and MSCS) that provide single-failure protection,

- the pressurizer and accumulator (TPCS and MPCS) that provide rapid fluid inventory control, and

- primary and secondary loop arrangements to assure natural circulation decay heat is removed and that fluid remains in the unbroken loop for LOCA (this allows natural circulation using the water-to-air heat exchangers).

The active and passive fluid systems are fully redundant since either the active residual heat removal (RHRS) or the passive water-to-air heat exchangers can remove the total residual heat load. These passive fluid safety systems are self-contained and do not require ac power to perform their safety function. The passive fluid safety systems rely only on 
natural circulation forces. No operator action or offsite support is required for the passive fluid safety systems, and they will operate indefinitely.

\subsubsection{Beamtrip System Design}

Assurance of a beamtrip is provided by designing the protection system to the IEEE-603 design criteria. These criteria provide single-failure protection because four sensor channels are provided for each trip function, and a single failure will neither cause a beamtrip nor prevent a beamtrip because of the two-out-of-four trip logic. The IEEE-603 criteria will be used for the safety system sensors, the system electronics, and the accelerator beamtrip mechanism.

\subsubsection{Safety System Sensors}

Multiple failure protection is provided by having a primary and secondary beamtrip function for each transient. A triple failure (double failure of the primary trip function and a single failure in secondary trip function) could occur, and the secondary trip function could still operate. The combination of a DBE and a triple failure reduces the transient to a residual risk category; thus no further trip function protection is required even though it may be available.

The process of identifying the primary and secondary trip functions began by considering a large list of transient events that were put into $A O O, D B E$, and $B D B E$ categories. This large list of transients was then reduced to the following list of limiting events:

- LOCA events (hot leg, cold leg, large and small),

- LOFA events (one pump, all pumps, locked rotor),

- flow blockage (partial to complete),

- loss of heat sink (secondary system LOCA, LOFA, etc.), and

- loss of offsite power/station blackout (partial to complete).

Table 4-12 shows the transients and the primary and secondary trip functions associated with each transient. There are other trip functions that would be available for various transients, but the purpose of this list is to assure at least a primary and secondary trip function exists. Table 4-13 shows the event vs. trip matrix of Table 4-12 separated into a list of parameter measurements vs. systems. Figure 4-22 shows the location of the sensors for the TPCS and Fig. 4-24 shows the location of the sensors for the MPCS. Future safety analyses will confirm the final instrumentation list and that the primary and secondary trip functions are adequate.

Actuations are as follows:

(1) RHR start, PCS pump trip, and confinement isolation with LOCA.

(2) RHR start on high PCS temperature.

(3) Diesel start on loss of offsite power (pump power monitors).

(4) Confine ment isolation on high confinement radiation. 
Table 4-12. LANL APT T/B Event vs. Trip Matrix

\begin{tabular}{|c|c|c|c|c|c|c|c|}
\hline . & Event/Trips & $\begin{array}{l}\text { Loop } \\
\text { Press }\end{array}$ & $\begin{array}{l}\text { Pump } \\
\text { Power }\end{array}$ & $\begin{array}{l}\text { Loop } \\
\text { Flow }\end{array}$ & $\begin{array}{l}\text { Loop } \\
\text { Temp }\end{array}$ & $\begin{array}{l}\text { PRZ } \\
\text { Level }\end{array}$ & $\begin{array}{l}\text { Loop } \\
\text { Rad }\end{array}$ \\
\hline 1. & TPCS LOCA outside MT & PRI & & & & & \\
\hline 2. & TPCS LOCA inside MT & PRI & & & & & \\
\hline 3. & TPCS LOCA inside ${ }^{3} \mathrm{He}$ tank & PRI ${ }^{a}$ & & & & & \\
\hline 4. & MPCS LOCA outside MT & PRI & & & & & \\
\hline 5. & MPCS LOCA inside MT & PRI & & & SEC & & \\
\hline 6. & WPCS LOCA outside MT & PRI & & & & & \\
\hline 7. & WPCS LOCA inside MT & PRI & & & & & \\
\hline 8. & ${ }^{3} \mathrm{He}$ Tank LOCA inside MT & b c & & & & & \\
\hline 9. & ${ }^{3}$ He Tubes LOCA outside MT & $\mathrm{b}$ & & & & & d \\
\hline 10. & TPCS LOFA & & PRI & SEC & & & \\
\hline 11. & MPCS LOFA & & PRI & SEC & & & \\
\hline 12. & WPCS LOFA & & PRI & SEC & & & \\
\hline 13. & TPCS Flow Blockage & & & PRI & SEC & & \\
\hline 14. & MPCS Flow Blockage & & & PRI & SEC & & \\
\hline 15. & WPCS Flow Blockage & & & PRI & SEC & & \\
\hline 16. & Loss of Heat Sink-LOCA & PRI & & & & SEC & \\
\hline 17. & Loss of Heat Sink-LOFA & & PRI & SEC & & & \\
\hline 18. & Loss of Offsite Power & & PRI & SEC & & & \\
\hline 19. & Station Blackout & & PRI & SEC & & & \\
\hline 20. & Tube Leak (Rupture) & SEC & & & & & PRI \\
\hline 21. & M\&PS Maloperation & & & & & SEC & PRI \\
\hline $\begin{array}{l}\text { as } \\
b_{\mathrm{P}}\end{array}$ & $\begin{array}{l}\text { tank high pressure. } \\
\text { tank low pressure. }\end{array}$ & $\begin{array}{l}\text { mode } \\
\text { confu }\end{array}$ & $\begin{array}{l}\text { I tank hig } \\
\text { ent radiat }\end{array}$ & ssure. & & & \\
\hline
\end{tabular}

Table 4-13. LANL APT T/B Trip Function List*

\begin{tabular}{llcccc}
\hline & & \multicolumn{4}{c}{ System } \\
\cline { 3 - 6 } & & THTS & MTHTS & WHTS & 3HeT \\
\hline PCS & 1. Loop Low Pressure & $\mathrm{X}$ & $\mathrm{X}$ & $\mathrm{X}$ & $\mathrm{X}$ \\
& 2. Loop High Pressure & & $\mathrm{X}$ & & $\mathrm{X}$ \\
& 3. PRZ Low Level & $\mathrm{X}$ & $\mathrm{X}$ & $\mathrm{X}$ & \\
& 4. PRZ High Level & $\mathrm{X}$ & $\mathrm{X}$ & $\mathrm{X}$ & \\
& 5. Loop Low Flow & $\mathrm{X}$ & $\mathrm{X}$ & $\mathrm{X}$ & \\
& 6. Loop High Temperature & $\mathrm{X}$ & $\mathrm{X}$ & $\mathrm{X}$ & \\
& 7. Loop Pump Power & $\mathrm{X}$ & $\mathrm{X}$ & $\mathrm{X}$ & \\
& 8. Loop Radiation & $\mathrm{X}$ & $\mathrm{X}$ & $\mathrm{X}$ & \\
SCS & $\mathrm{X}$ & $\mathrm{X}$ & $\mathrm{X}$ & \\
& 1. Loop Low Pressure & $\mathrm{X}$ & $\mathrm{X}$ & $\mathrm{X}$ & \\
& 2. Loop High Pressure & $\mathrm{X}$ & $\mathrm{X}$ & $\mathrm{X}$ & \\
& 3. PRZ Low Level & $\mathrm{X}$ & $\mathrm{X}$ & $\mathrm{X}$ & \\
& 4. Loop Low Flow & $\mathrm{X}$ & $\mathrm{X}$ & $\mathrm{X}$ \\
& 5. Loop High Temperature & $\mathrm{X}$ & $\mathrm{X}$ & $\mathrm{X}$ \\
& 6. Loop Pump Power & $\mathrm{X}$ & $\mathrm{X}$ & $\mathrm{X}$ \\
& 7. Loop Radiation & $\mathrm{X}$ & $\mathrm{X}$ & $\mathrm{X}$ \\
CWS & 1. Loop Low Flow & $\mathrm{X}$ & $\mathrm{X}$ & $\mathrm{X}$ \\
& 2. Loop High Temperature & $\mathrm{X}$ & $\mathrm{X}$ & $\mathrm{X}$ \\
\hline
\end{tabular}

*Trip functions unrelated to heat transport systems events include 1) confinement high radiation, and 2) seismic 


\subsubsection{System Electronics}

Figure 4-30 illustrates how the beamtrip/SFAS will work. There are three parts to the system:

- variable sensing,

- input and execute channels, and

- safety actuation channels.

The system will use standard two-out-of-four SFAS logic so that any one channel can be tested and maintained while maintaining redundancy at all times. Each variable to be sensed will have four separate sensors and input/execute channels. There will be two output actuation channels.

There are several means for positively interrupting the accelerator beam in a fail-safe manner. Two technologically independent means for interrupting the beam may be used: one for beamtrip Channel $A$ and the other for Channel B.

The system will be based on proven technology and qualified components. The design of the system will be such that failure of the beam to be interrupted during a AOO, DBE or BDBE falls into the residual risk category.

The following will be the requirements of the system:

- The system is to perform all safety functions required in the presence of single failures.

- Once initiated, manually or automatically, the system will automatically proceed to interrupt the beam. Manual reset will be required after the appropriate steps have followed actuation.

- The various redundant portions of the system will be independent and physically separated from each other.

- The system will be independent of, and physically separated from the effects of the event.

- Credible failures of the other, non-safety-related, control systems will not prevent the beamtrip/SFAS from performing when required.

- The sources of power for the beamtrip/SFAS will meet the requirements of IEEE603. 


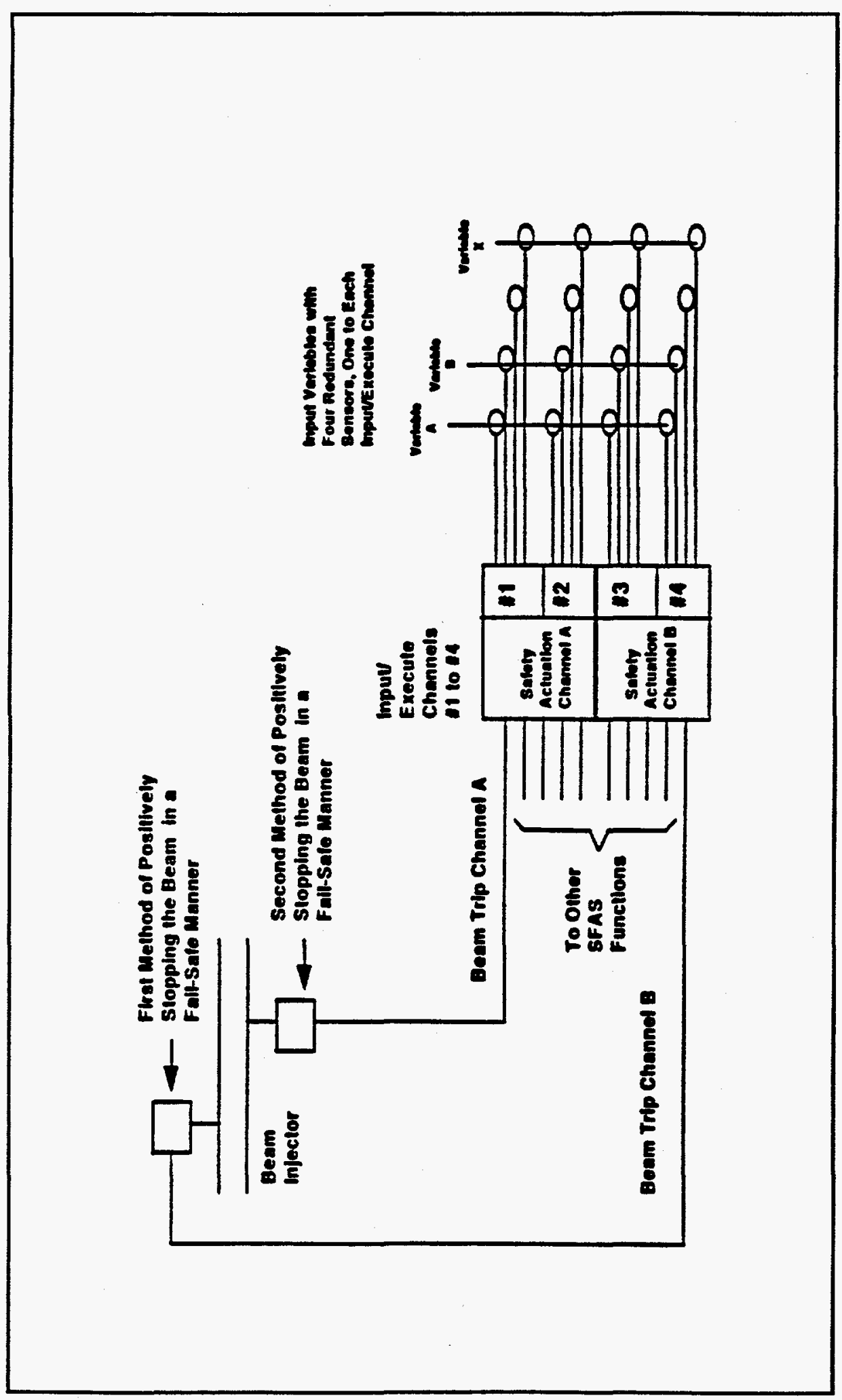

Fig. 4-30. Beamtrip/SFAS Overview. 


\subsubsection{Accelerator Beamtrip Mechanism}

Several means are available for positively interrupting the accelerator beam in a fail-safe manner as follows:

- Turn off the arc drive (actuation time $<10 \mu \mathrm{s}$ ). The arc drive is an RF source (1-2 $\mathrm{kW}$ ).

- Reduce the field in the source solenoid (actuation time $<1 \mu$ s).

- Turn off the extractor voltage (actuation time $<100 \mu \mathrm{s}$ ). This method is used on the GTA injector.

- Activate a deflector magnet in the injector LEBT (actuation time $<1 \mu \mathrm{s}$ ). This method is not as reliable because it requires application of a current to the magnet.

- Kill the RF drive to the RFQ (actuation time $<40 \mu \mathrm{s}$ ). This action eventually would cause damage to the RFQ vanes if the source is left on.

The actuation times assume the given power supply can be turned off without a time delay. For high reliability, the ac power could be turned off to the power supply through a relay; thus the actual time would include the relay opening time and the time for the power supply to discharge.

\subsection{REMOTE-HANDLING SYSTEMS AND OPERATION}

\subsubsection{Introduction and Summary}

To facilitate a practical engineering design, a retargeting sequence for replacing a spent target module with a new target module, and a target module disassembly sequence that would be used to disassemble a spent target module and package the waste for disposal has been integrated into the preconceptual design process as a method for identifying design issues and requirements for demonstrating remote-handling objectives. A step-by-step process was developed for both operations, with input from the design organization. As a result of this activity, practical design issues have been identified and addressed. An evaluation of equipment requirements for cutting, welding, and handling was performed. From this exercise, the disassembly and/or reassembly operations appear to be feasible with reasonable adaptations of commercially available equipment with minimal development. This exercise was a high-leverage design activity that provided early insight into maintenance and operational planning issues.

Where possible, simplicity and utilization of shared equipment was sought in the evaluation of equipment needs for retargeting and disassembly tasks as well as for maintenance and inspection operations. Integration of maintenance and inspection operations with the normal retargeting sequence will be examined in the future using $3 \mathrm{D}$ CAD models. As the target module design evolves, remote-handling issues must continue to be integrated into the design process. Heat removal strategies during handling were needed to address residual heating in tungsten, Inconel, and lead. A cursory check of the residual 
heating in these regions found that cooldown periods to manageable heating rates were consistent with operating schedules.

\subsubsection{Target Module Refurbishment}

Figure 4-31, Target Module Assembly shows the segments associated with the assembly or disassembly of the target module.

The target module preparation sequence for off-line preparation of a target module is listed below. The first five operations involve new non-radioactive components, but the last two operations must be performed under water after the first two target installations because of the radioactive lead reuse.

1. Pre-assembly - New blanket assemblies are installed in a new moderator tank shell and heads, and a new NSA is preassembled and tested with helium blankets, disposable lead portions, NSA helium heat exchanger, flow baffle, NSA helium blankets, Zircaloy backstop and instrumentation.

2. Dry NSA insertion - The new preassembled NSA is inserted axially into the preassembled moderator tank shell from the front (window) end. This can be accomplished most easily with the moderator tank shell turned on end (vertical).

3. NSA welding - Coolant and helium connections are welded and tested. Coolant lines are welded from inside the pipes to avoid possible damage to the NSA helium blankets in the flow channel and extended stiffener regions. Internal helium line connections are welded and tested.

4. Front head attachment - A new moderator tank front head is fitted and welded to the moderator tank shell and to the forward portion of the NSA beam tunnel extension. The penetration of the NSA helium coaxial conduit through the moderator tank wall is sealed. NDE is performed after welding.

5. Pipe welding - New piping runs are fitted to a common strongback (frame) and welded. NDE is performed after welding.

6. Target lead installation - The last components to be installed are the eight target lead segments. These target lead segments are reused and will be radioactive after the first two target installations. Target lead is inserted axially into the moderator tank shell from the rear end. The lead is guided and supported by four heavy pipe rails extending from the inside of the moderator tank wall. This can be accomplished most easily with the moderator tank shell turned on end (vertical). It is assumed that the lead would be installed while the moderator tank is submerged in the disassembly pool.

7. Rear head attachment - A new preassembled moderator tank rear head with associated piping is remotely fitted and welded to the moderator tank shell. Associated piping is joined with the strong back. NDE is performed after welding.

The new target module assembly is now fully checked out and ready for installation. 


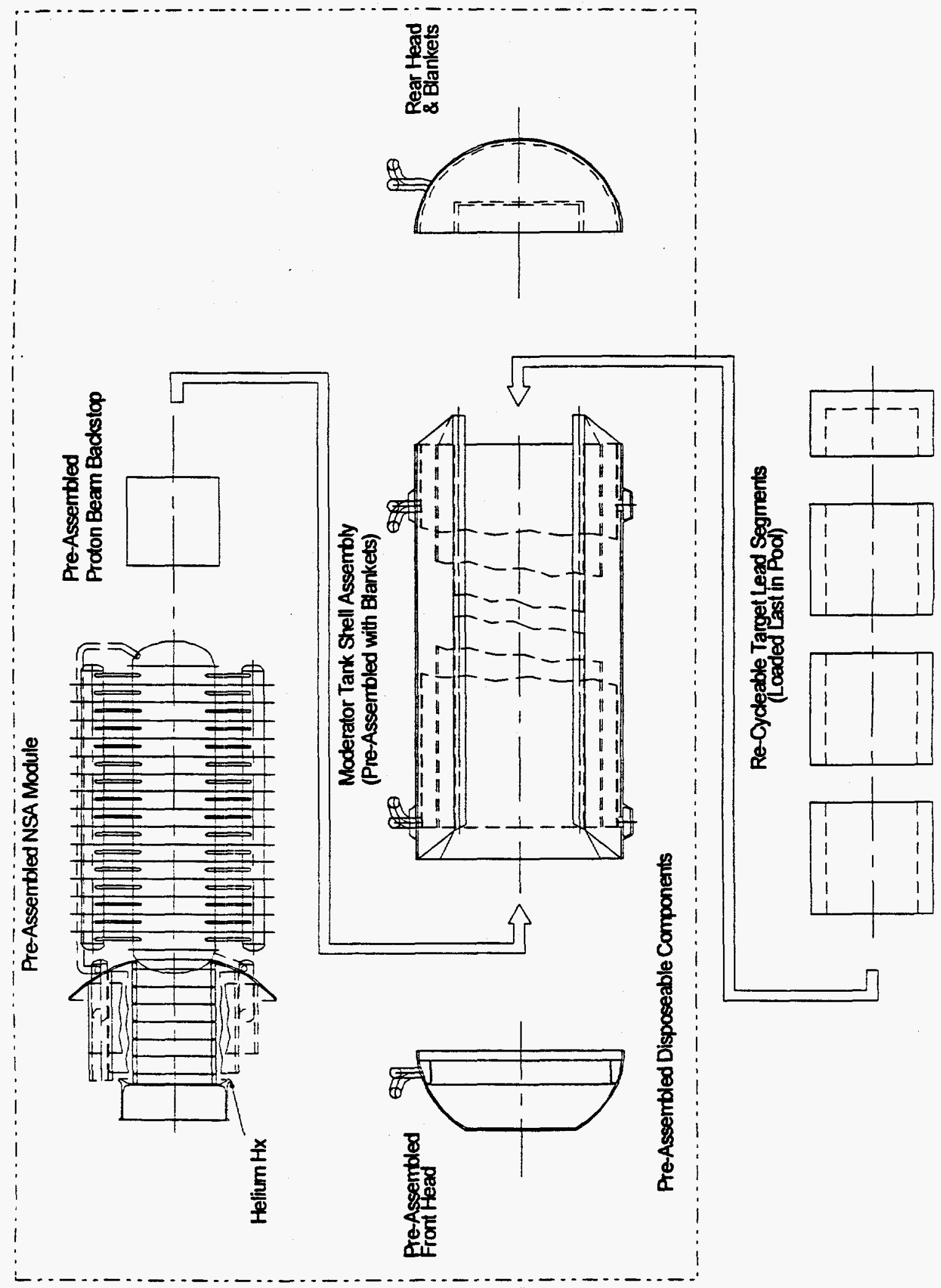

Fig. 4-31. Target Module Assembly. 


\subsubsection{Target Module Retargeting Operation}

This section summarizes the target module retargeting operation. The retargeting sequence begins immediately after shutdown, but the spent target module will not be moved until the peak heat load in the tungsten rod bundles decays to $\leq 40 \mathrm{~kW}$, which corresponds to 90 days after shutdown, see Fig. 4-32. The spent target module will be fully cooled during all handling and storage to insure no target module damage and/or release of activated materials. During removal and transfer, the spent NSA with the 88 tungsten rod bundles will be cooled by two onboard natural convection/fin tube coolers, called the NSA cooling device, which rejects the decay heat to the air. The NSA cooling devices will be sized to keep a $40 \mathrm{~kW}$ spent NSA from overheating if held indefinitely in ambient air. During storage, the NSA cooling device will be submerged in water.

Selection of a 90 day cooldown before the spent target module is moved is based on maximizing availability while minimizing risk, heat load, and exposures. The longer the spent target module cools, the lower the heat load, the radiation levels (exposures), and the risk that the target module could overheat and release radioactive materials. However, the longer the decay period, the longer the period before the new target module will be available

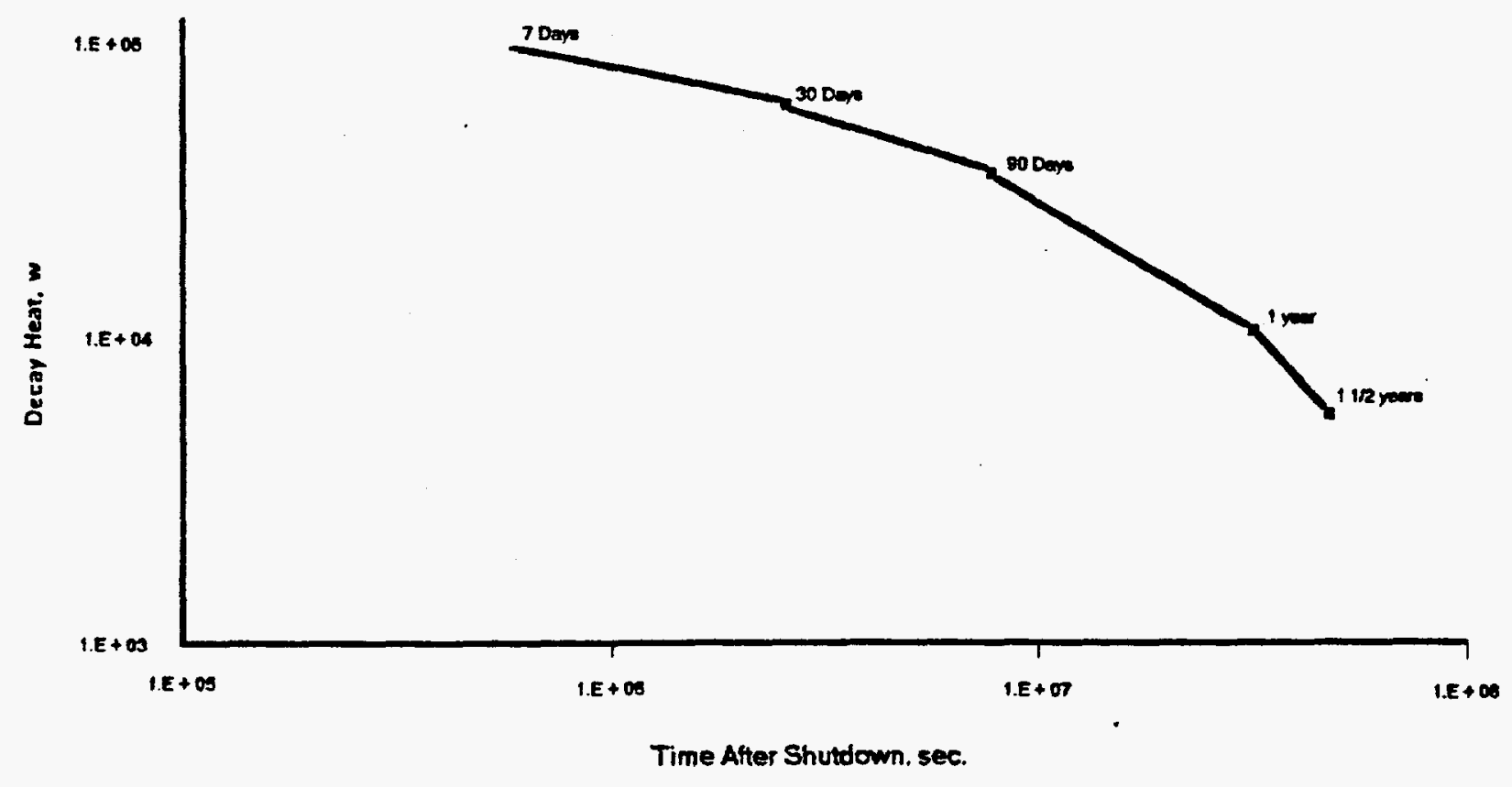

Fig. 4-32. Decay Heat vs Time. 
if needed. This could reduce overall system availability. In the limit, the new target module must be ready before the other in-service target module is spent ( 2.2 years at normal maximum production rate), or system availability will be reduced. The retargeting operations described in Section 4.8 are based on an assumed target lifetime of 2.2 years. The actual target lifetime must await future materials testing and analysis that is planned as part of the risk reduction program. If the new target module is available as a "spare" significantly sooner than the 2.2 years, it could improve availability. From Fig. 4-32, it is evident that the bulk of the decay heat reduction benefit has been achieved after $\sim 90$ days, and the radiation levels should follow the same curve. The 90 day point corresponds to $\sim 40 \mathrm{kWt}$; this heat load can be rejected to ambient air with a relatively simple fin tube cooler with no blower. Moving the spent target module 90 days after shutdown allows the retargeting-target disassembly sequence depicted in Fig. 4-33. In this sequence the new target module is available 0.6 years after shutdown, and the spent target module is disassembled 1.5 years after shutdown. This sequence would mean a spare target module is available $\sim 73 \%$ of the 2.2 years. This appears to be a reasonable goal. Even if the 90 days were reduced to zero, which is impossible due to setup times, there is a minimum target replacement time of 3-4 months, and the spare availability would increase only modestly from 73 to maybe $85 \%$.

The retargeting sequence begins with the spent target module in the sealed and $\mathrm{D}_{2} \mathrm{O}$ filled condition, see Fig. 4-34, and all shield plugs in place. All retargeting equipment will have been serviced and checked, and all administrative approvals will have been received. The retargeting sequence begins with a pre-entry health physics survey, and then any necessary decontamination will be performed to assure the area will be safe to enter. Health Physics personnel will continuously monitor operations during the disassembly sequence, perform periodic surveys, and the area will be decontaminated as necessary to assure doses as low as reasonably achievable (ALARA), but for brevity no further mention will be made of the activities until final closure and cleanup.

The retargeting operations begin by depressurizing the helium tanks, vacuum emptying any ${ }^{3} \mathrm{He}$ lines which are to be broken during retargeting, and then back filling with natural helium. The manipulator and closed circuit TV systems will also be deployed. The exvessel shield cooling lines will be drained and disconnected and the shield blocks over the target module will be removed and moved to storage. The moderator tank $\mathrm{D}_{2} \mathrm{O}$ will then be drained and replaced with $\mathrm{H}_{2} \mathrm{O}$. Moderator tank drain will be deferred until the lead decay heat is sufficiently low ( $\angle \mathrm{TBD} \mathrm{kW}$ ) to assure the lead will not overheat during draining; this is assumed to be a few days to a few weeks. In addition, and in order to assure the lead will not overheat during the moderator tank drain, a small coolant flow will be maintained through the lead during the drain and refill operation. Initially, a small amount of $\mathrm{D}_{2} \mathrm{O}$ will be circulated through the normal lead cooling system. This $\mathrm{D}_{2} \mathrm{O}$ flow will be maintained until the moderator tank is essentially empty; and then, concurrent with the start of the moderator tank refill with normal water $\left(\mathrm{H}_{2} \mathrm{O}\right)$, the lead coolant will also be switched from $\mathrm{D}_{2} \mathrm{O}$ to $\mathrm{H}_{2} \mathrm{O}$. The $\mathrm{H}_{2} \mathrm{O}$ (lead) coolant flow will be from a side stream coming off the moderator tank refill line. This $\mathrm{H}_{2} \mathrm{O}$ (lead) coolant flow will be maintained until the moderator tank refill is complete, and lead system temperatures indicate the lead needs no supplemental cooling beyond that provided by the moderator tank water. This approach assures that minimal mixing of $\mathrm{D}_{2} \mathrm{O}$ and $\mathrm{H}_{2} \mathrm{O}$ will occur and that both the lead and $\mathrm{D}_{2} \mathrm{O}$ can be reused. After the spent moderator tank is drained, the $\mathrm{D}_{2} \mathrm{O}$ will be circulated through the $\mathrm{D}_{2} \mathrm{O}$ purification system and will then be used to fill the newly installed replacement moderator tank. 
Once the $\mathrm{D}_{2} \mathrm{O} / \mathrm{H}_{2} \mathrm{O}$ switch is completed, the target module will be disconnected from the beam tunnel. Any shielding over the piping to the target module will be removed, the helium piping and coolant piping to the target module will be disconnected, and blanks or plugs will be installed on both sides. The NSA cooling devices will then be installed one unit at a time
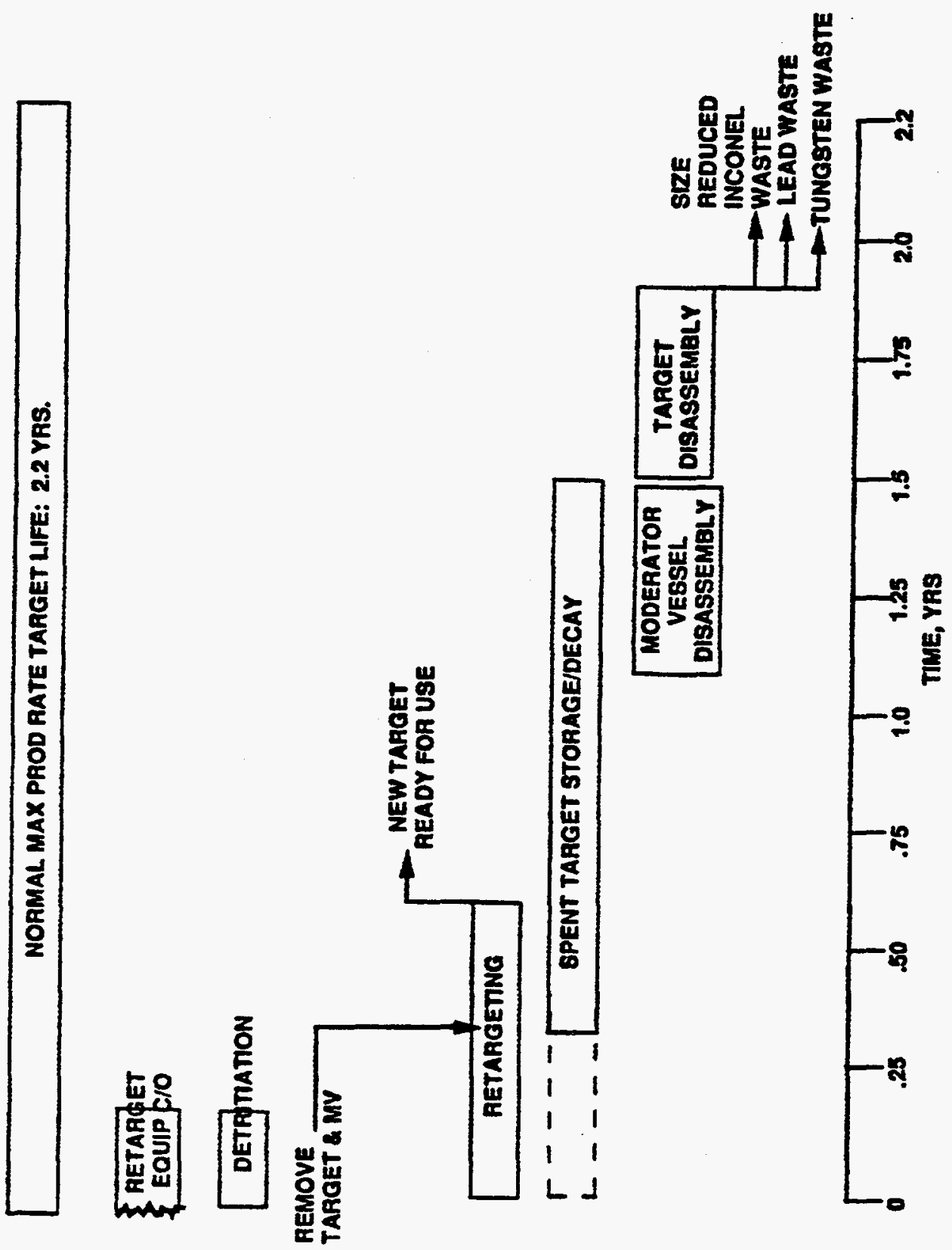

Fig. 4-33. Retargeting - Target Disassembly Sequence. 


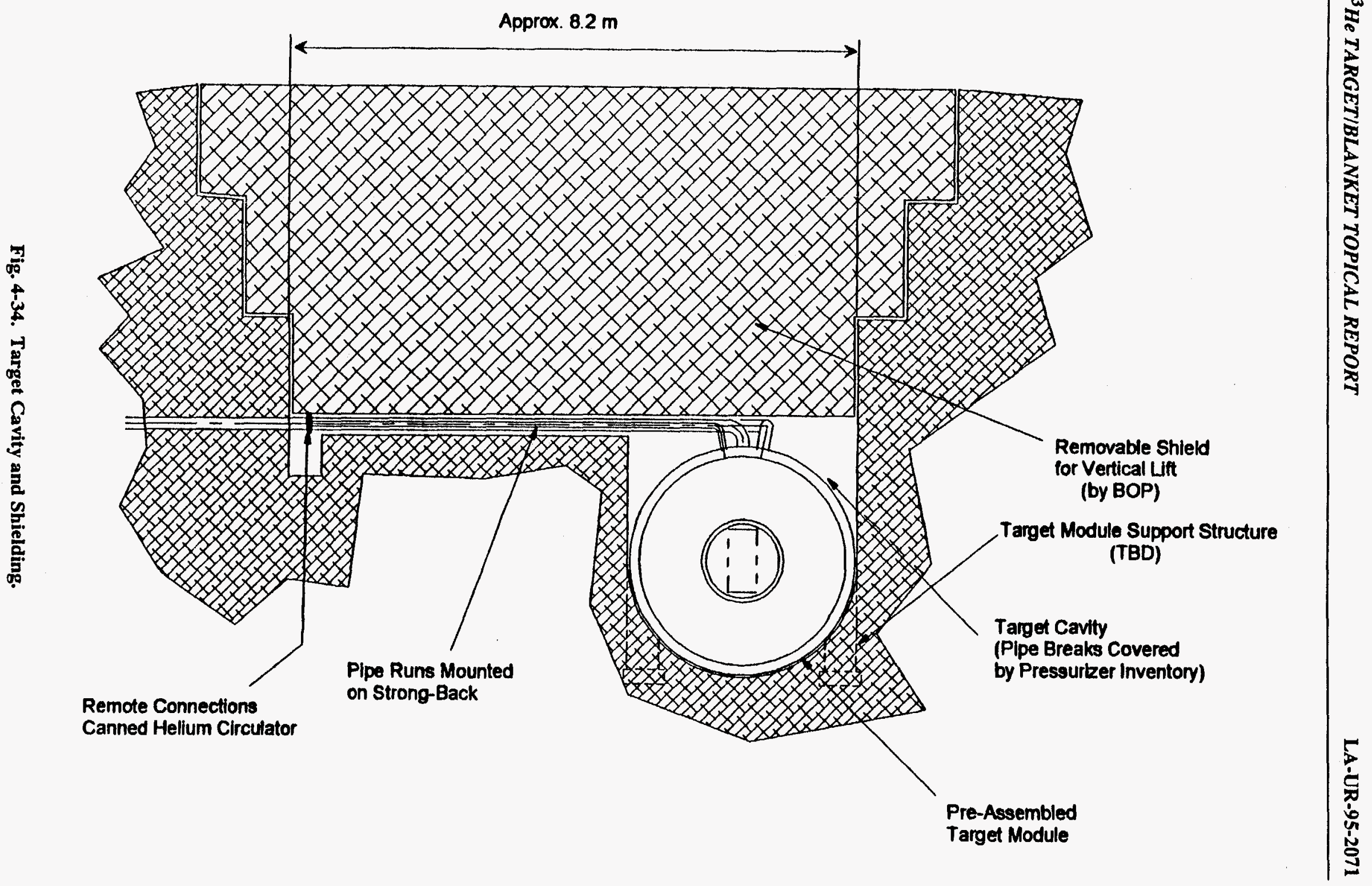


and their operation will be verified. The target module lift fixtures will then be installed preparatory to lift operation. As noted previously, spent target modules will not be moved until the decay heat is $\sim 40 \mathrm{kWt}$, which corresponds to $\sim 90$ days after shutdown. Considering all that has to be done to get ready for target movement, especially if detritiation (which could take several weeks) is required, the 90 days does not represent an impediment to retargeting. The $40 \mathrm{kWt}$ is not a hard number, larger heat exchangers can be used if availability considerations require a quick changeout; from the decay heat curve and the time to get ready, $40 \mathrm{kWt} / 90$ days appears reasonable. Once the target is ready to be moved (or the 90 days ends if it is longer) the target module will be moved to and placed on the horizontal stand in the target storage pool. The lift fixture will be removed and placed around the new target module. The new target will then be lifted out of the storage pool, the $\mathrm{H}_{2} \mathrm{O}$ will be drained, and the new target module will be moved to the beam tunnel and lowered into position. The beam tunnel to target module connection will be made. All instrumentation will be connected, NDE will be performed and the new target module will be checked out. After the new installation is found to be acceptable, the target module will be filled with $\mathrm{D}_{2} \mathrm{O}$. This completes the retargeting.

\subsubsection{Target Module Disassembly}

Target module disassembly will start with the draining of the $\mathrm{D}_{2} \mathrm{O}$ from the tungsten rod bundles. The moderator tank $\mathrm{D}_{2} \mathrm{O}$ will have been drained months earlier, but the tungsten rod bundles will not be drained until the decay heat is $\sim 10 \mathrm{~kW}$ ( $\geq 1$ year after shutdown). Drain starts by connecting the $\mathrm{D}_{2} \mathrm{O}$ drain tank and pressurization lines to the spent NSA cooling device. The spent moderator vessel will then be rotated from horizontal to vertical in the tilt stand; the target module will be oriented with the window down. The $\mathrm{D}_{2} \mathrm{O}$ drain valve will then be opened, and the $\mathrm{D}_{2} \mathrm{O}$ will be drained from the tungsten rod bundles. $\mathrm{A}$ combination of head above the drain tank and high point pressurization will be used to assure a complete $\mathrm{D}_{2} \mathrm{O}$ drain. Once the $\mathrm{D}_{2} \mathrm{O}$ is drained, as indicated by the drain tank level, the drain tank valves will be closed, and the drain valve and pressurization valves will be opened to fill the tungsten rod bundles with storage pool water $\left(\mathrm{H}_{2} \mathrm{O}\right)$. The $\mathrm{D}_{2} \mathrm{O}$ will be purified for reuse after draining.

Once the tungsten rod bundles are filled with $\mathrm{H}_{2} \mathrm{O}$, target module disassembly will be initiated by cutting the seal weld on the dome (rear) end of the target module and then removing the dome, see Fig. 4-33. The dome end is installed last after lead segment installation; and as such, it lifts off with a simple lift sling. The two closed end lead segments are then exposed. They are then lifted out one at a time with their lift sling. The zirconium backstop is then exposed and is lifted out with its lift sling. The 6 semi-circular lead segments are then removed one at a time with their lift sling. The eight lead segments are the only things to be reused at present; further study should allow the reuse of more equipment. The lead segments will be set aside for reuse in the next target module assembly.

With the lead removed from the target module, the weight is reduced by $>70 \%$. The balance of the target module is to be disassembled for disposal. In order to disassemble the balance of the moderator vessel and NSA, the moderator vessel will be returned to the horizontal position. The modulator vessel and the helium piping will be cut away from over the spent NSA, and then the complete NSA will be lifted out with its lift fixture and moved to its disassembly fixture; NSA disassembly will be discussed in Section 4.8.5. In 
disassembling the moderator tank for both NSA removal and for disassembly of the complete moderator tank, the moderator tank shell will be cut into pieces suitable for disposal. A precise cutting plan will be developed during final design to insure the pieces will fit compactly into disposal boxes and to insure the spent NSA can be removed through the resulting opening. Plate grabbers will be used to hold and move the moderator tank pieces to the disposal containers. As the moderator tank shell is cut away, the helium piping will also be cut away in pieces suitable for size reduction in a grater; as before, a precise cutting plan will be developed in final design. In removing the spent NSA, connecting piping and support structure will be cut away per a cutting plan which insures separation while assuring the NSA is supported at all times.

After the spent NSA is removed, the balance of the moderator tank and helium piping will be cut up for disposal. As before, the moderator tank segments will be of an appropriate size for disposal, and the helium piping will be size-reduced in the grater for disposal. As the various segments are removed, any instrumentation (mainly TCs) will be stripped off with a manipulator. Stainless steel, aluminum, Inconel and TCs will be segregated during disposal.

\subsubsection{NSA Target Disassembly Sequence}

This section summarizes the NSA disassembly sequence. The disassembly sequence assumes the following:

1) Two separate storage/disassembly pools, one on each side of the beam stop.

2) One target for disassembly every 2.2 years.

3) Targets will be moved to the storage/disassembly pool $\geq 3$ months after shutdown, and NSA disassembly will start $\sim 1.5$ years after shutdown with a decay heat of $\leq 6 \mathrm{~kW}$. Target disassembly is totally underwater, and decay heat is not significant.

These assumptions are based on the Retargeting - Target Disassembly sequence of Fig. 4-33. With the disassembly timing in Item 2, one set of equipment, movable between the two pools is reasonable. Efficiency is not a requirement. However, a combined pool with one set of equipment will simplify equipment and reduce duplication, e.g., rails. This sequence also assumes that there will be adequate space in the storage/disassembly pools for various fixtures and equipment required to perform these operations. Space requirements will need to be resolved with the BOP design concept in the future.

The NSA disassembly starts with the NSA in a disassembly fixture which can rotate the target $\sim 90^{\circ}$ in each direction about its major axis, see Fig. 4-35. The Inconel chamber has been flooded with water, the $\mathrm{D}_{2} \mathrm{O}$ has been drained from the NSA, and the NSA cooling devices have been removed. Both the NSA cooling devices and $\mathrm{D}_{2} \mathrm{O}$ will be reused. NSA disassembly starts with the removal of the two manifolds and the upward facing cylindrical shell section, see Fig. 4-36. In removing the cylindrical shell section, the cylindrical lead segments, trapped between the shell ribs, must first be moved, see Fig. 4-36. The lead segments will be lifted out after the flange segments trapping the lead against the shell are cut away. In a similar manner, once the manifolds are cut away, the lead segments trapped between the upper and lower manifolds and the shell will have to be removed, see Fig. 4-36. 
The segments are lifted out one at a time after the manifold is cut away. With the Inconel chamber flooded, the order of removal is not significant. Once the manifolds and cylindrical shell section are removed, the Inconel ducts/tungsten rod bundles are exposed, see Fig. 4-37. All cutting will be with a commercial device. Based on previous disassembly pool studies, arc saw cutters were selected originally, but later studies have determined that several other cutting devices are available. Laser, plasma arc, and water jet cutting systems are alternates which will be pursued in later studies. All are feasible, but the water jet appears to be the most attractive at this time. All are commercially available systems. The rotatable handling device will be used to orient the target in support of the cutting device. Although the pieces being cut away are shown as one piece in Fig. 4-36, they will probably be segmented during removal to simplify handling and size reduction. A precise cutting plan will be developed once the NSA design is finalized. Once the various pieces are cut free, they will be moved to a size reduction area where a commercial grater will be used to cut them into small pieces to fit compactly into a waste cask. As part of the cutting plan, pickup points will be established to support each piece as it is being cut off and then carried to the size reduction area. The pickup points will normally be in the stiffening ribs which protrude out from the Inconel shell, manifolds, and tube sheets. Excluding instrumentation (mainly TC's) the only materials are Inconel (shell, manifolds, ducts, and Inconel chips/shavings), lead segments, and tungsten. Waste will be segregated in baskets submerged in the storage pool for shielding. When a waste basket is full, it will be removed from the pool and moved to a shielded drying area (probably a drip pan with air dry). The basket (with waste) will be placed in a cask and shipped to disposal or storage.

After completion of the above, the Inconel duct and tungsten rod bundle array with upper and lower tubesheets is now exposed for disassembly, see Fig. 4-37. The two dome pieces and the lower cylindrical section of the shell remain as a "catch pan" if an Inconel duct and tungsten rod bundle assembly is dropped or disintegrates during handling. For this procedure, the rod bundle is assumed to be intact but relatively fragile. The rod bundle layout pattern is shown in Fig. 4-2. The rod bundle pattern shows the separation between individual assemblies. This will facilitate the disassembly operation. A manipulator, with special adapter to gently grip the hexagonal duct, see Fig. 4-37 - Section B, will be used to support individual tungsten assemblies while the two ends are cut. Cuts will be made in the round portions of the Inconel duct near the tubesheet, see Fig. 4-37 - Section B. After separation, the manipulator will place the tungsten assembly into a tray, Fig. 4-37 - Section $\mathrm{C}$, which holds several assemblies (probably a row), for transport to the size reduction area. The tray will be located as close to the rod bundle as possible to minimize the chance of dropping the assembly. If possible, the tray will be under the assembly during cutting. The tray also cradles the assembly during size reduction. If an assembly breaks up during transport or size reduction, the tray will catch the debris. Depending on how the tungsten rods are supported inside the Inconel duct, the size reduction may be performed in different ways. It may be possible to tip the assembly up at an angle and slide the Inconel out after severing the support connection. This sequence assumes the tungsten will be rigidly tied to the Inconel and that the Inconel duct must be segmented for access to the tungsten. Excluding thermocouples, the only materials present are tungsten and Inconel, which will be separated and size reduced for disposal.

After a row of Inconel duct assemblies are cut free from the upper and lower tubesheets, the tray of assemblies will be moved to the size reduction area. The two round to hex transition ends, see Fig. 4-37 - Section B, will be cut off, and the upper two sides of the hex duct will be cut away (axially) from the other four sides of the hex to expose the tungsten 


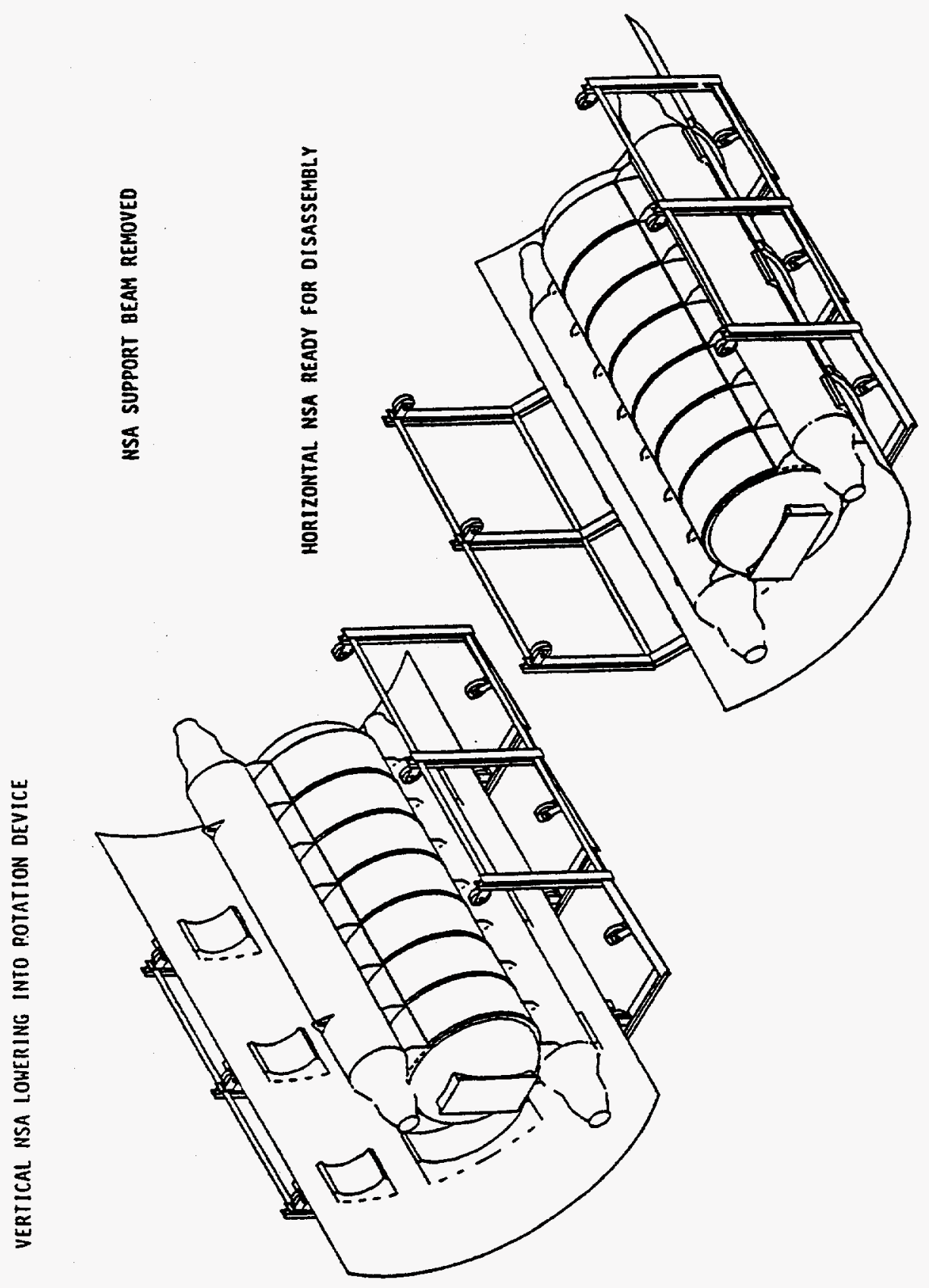

Fig. 4-35. Target Rotation Device. 


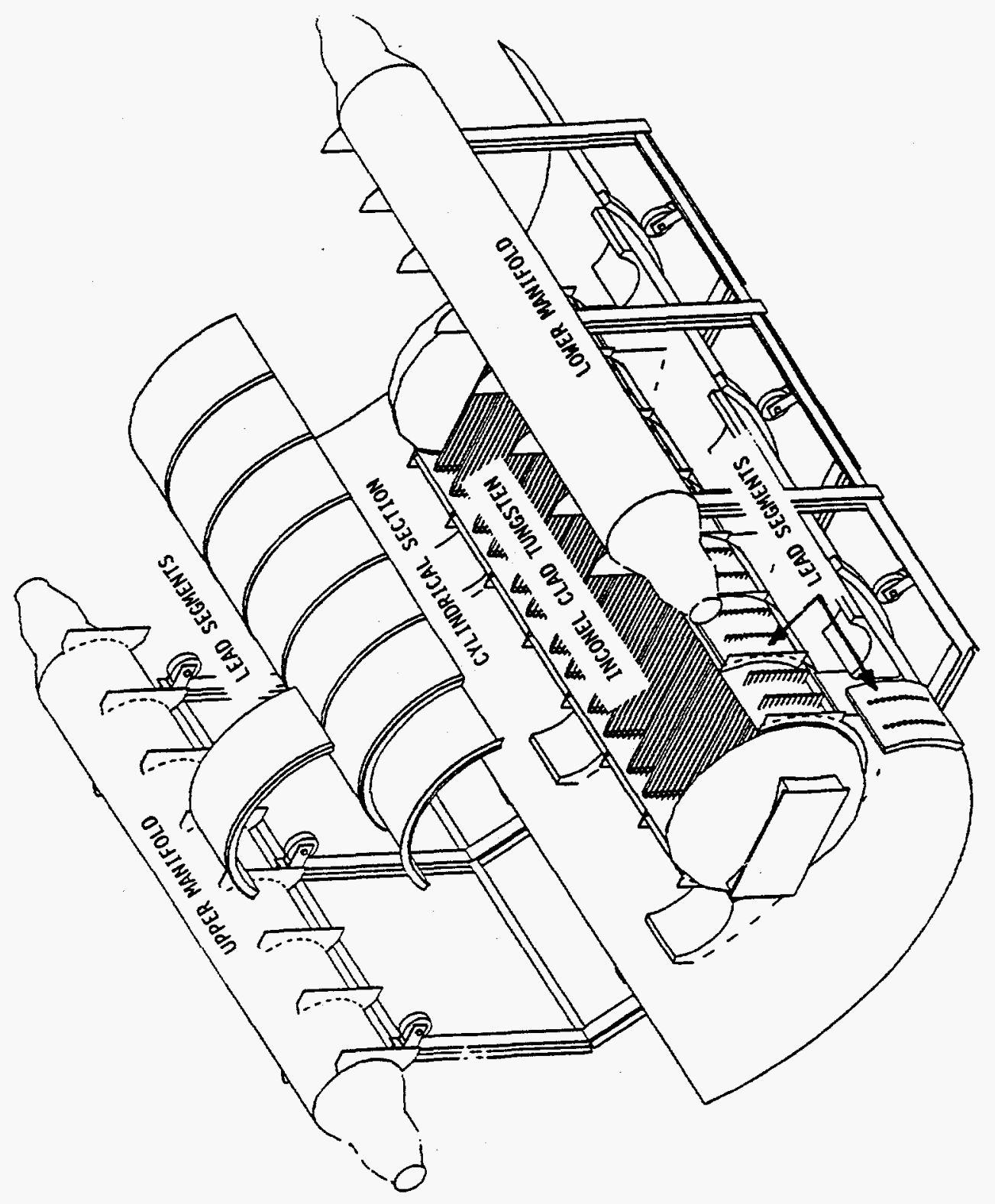

Fig. 4-36. Disassembly Sequence. 


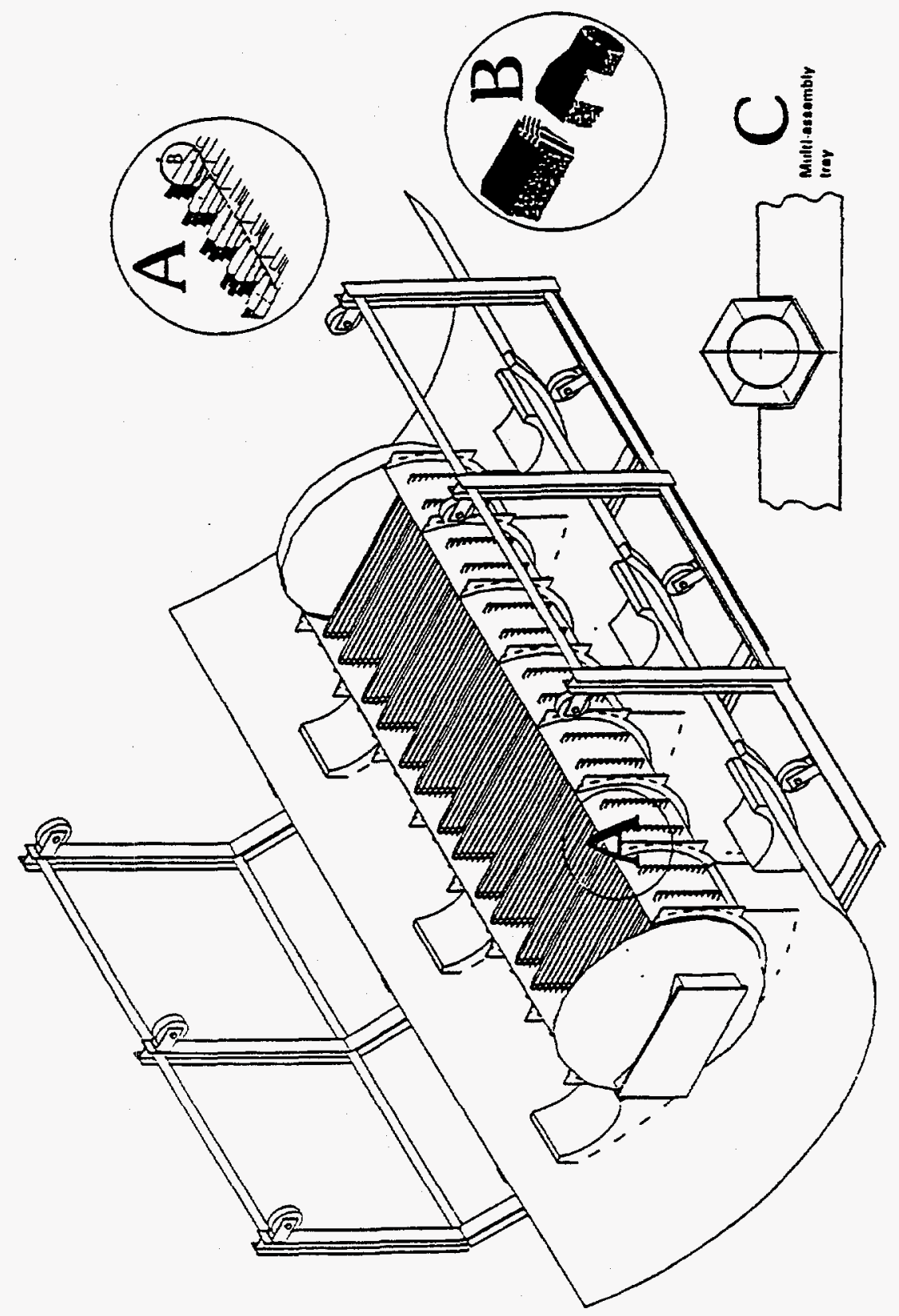

Fig. 4-37. NSA Target Ready for Tungsten Removal. 
(see Fig. 4-37, Section C). The 91-pin tungsten rod bundle will still be held together. The tungsten will be cut in two, which yields two $\sim 2$ foot lengths, and then the tungsten will be placed in the tungsten disposal basket. Any TC's will be placed in the miscellaneous waste basket. After all tungsten is removed, the remaining Inconel duct will be split and placed on the grater cradle. Any TCs or other instruments will be stripped off with the manipulator, and then the Inconel will be size reduced in the grater from where it drops into an Inconel waste basket. The process will be repeated until all of the tungsten assemblies have been separated from the tubesheets, the ducts have been removed, and the Inconel and tungsten have been size reduced and placed in storage baskets. After all of the Inconel duct and tungsten has been removed, all that remains will be the two dome pieces, the two tube sheets and the lower cylindrical section (with lead segments). The cutter will be used to first remove the lead as above and then segment the Inconel into suitable sizes to be shredded, any TCs will be stripped away with the manipulator, and then the Inconel will be shredded in the grater. The Inconel waste baskets may have to limit the volume of Inconel in any basket because of the high concentration of cobalt 60 that will result after irradiating Inconel 718. One possibility is a dual basket with an inner basket for Inconel and an outer basket for tungsten, where the dense tungsten provides some shielding. Any TCs or other instruments will be placed in the miscellaneous waste basket. Any loose parts or debris will be placed into appropriate waste baskets and the area will be cleaned. Waste baskets will be dried, placed in casks and shipped away.

\subsubsection{Target Module Waste Stream}

The current preconceptual target module design and retargeting operation is based on the assumption that the target module is replaced every 2.2 years (600 days of operation). In the current design, the only target module component that would be reused is approximately 81 percent of the target lead. A summary of the estimated target module waste stream weight breakdown is given in Table 4-14. These numbers are based on the data in Table 4-10. All of the waste is low level (solid) except for the disposable target lead which is mixed waste (solid).

In the future, tradeoffs associated with different retargeting operations and component discarding options need to be examined. In particular, the amount of disposable target lead can be reduced to zero if a small reduction in tritium performance is acceptable.

\subsection{BEAMSTOP}

\subsubsection{Introduction}

The APT beamstop will be used sparingly during the life of the facility to perform beam tuneup and diagnostics during commissioning of the accelerator and during startup. Although the beamstop will be used infrequently, it is designed to take the full operating beam energy and current $(1000 \mathrm{MeV}, 200 \mathrm{~mA})$. Also, as one of the safety design goals, the beamstop design should minimize the prompt and residual radioactivity, and accept excursions in beam tune without compromising its integrity. 
Table 4-14. Estimated Target Module Waste Stream Breakdown Per Target Module Replacement

\begin{tabular}{lr}
\multicolumn{1}{c}{ Waste } & Mass \\
\hline Moderator Vessel, Belts \& Lead Supports & $16,618 \mathrm{~kg}(36,636 \mathrm{lb})$ \\
Pipe \& Strong Back & $10,000 \mathrm{~kg} \mathrm{(22,046 \textrm {lb } )}$ \\
Disposable Target Lead & $13,550 \mathrm{~kg}(29,872 \mathrm{lb})$ \\
3He Blanket Tubes and Supports & $5,052 \mathrm{~kg}(11,138 \mathrm{lb})$ \\
3He Hx and Piping & $445 \mathrm{~kg}(981 \mathrm{lb})$ \\
NSA & \\
Zircaloy-4 & $4,328 \mathrm{~kg}(9,542 \mathrm{lb})$ \\
Inconel & $4,972 \mathrm{~kg}(10,961 \mathrm{lb})$ \\
Tungsten Rods and Wires & $1,476 \mathrm{~kg}(3,254 \mathrm{lb})$ \\
\hline \hline
\end{tabular}

Several beamstops are in current use at Los Alamos and other accelerator facilities. Various configurations and materials are used. For example, the Los Alamos Meson Physics Facility beamstop is made of water-cooled copper plates. The GTA, also at Los Alamos, uses a radiatively cooled graphite plate as a beamstop. The plate is positioned within the vacuum chamber and transfers its heat to an air-cooled aluminum tank wall.

For the APT beamstop design, we considered a water-cooled graphite mesh and a radiatively cooled set of graphite plates. Graphite is a preferred material because of its low atomic number and high-temperature capability. The advantage of the radiatively cooled graphite plates is the minimal radionuclide production because of the lack of water coolant directly in the beam. For the water-cooled mesh, the beamstop is smaller, but there is significant tritium production in the water. The drawback of using radiation cooling is the large size of the beamstop required to provide adequate heat transfer area. However, because of the simplicity, safety, and minimal radionuclide production using the radiative cooled graphite, it was chosen as the reference design.

Although both the beamstop region and the beam transport system are in vacuum, it was decided to include a beam entrance window for the beamstop. This provides complete isolation of the accelerator and beamstop regions, and prevents off-gassing of the graphite from contaminating the vacuum system. The window is placed "upstream" of the beamstop at a point where the beam is approximately $42 \mathrm{~cm} \times 91 \mathrm{~cm}(16.5 \mathrm{in} . \times 35.8 \mathrm{in}$.) and will be of similar design to the T/B window (double-wall Inconel with water cooling). Because the window is essentially identical to the window used in the T/B system, it is not included in the beamstop analysis or the technical description provided here.

In this section a beamstop preconceptual design is provided that can operate at full beam power conditions and minimizes radionuclide production. The concept employs radiatively cooled graphite as the basic component. This technique has been successfully used at GTA, but not at this large a scale. The beamstop is physically very large. Future efforts should be directed to reduce the size without compromising the basic design goals. Also, the design should be analyzed for excursions in the beam when these are better defined. 


\subsubsection{Beamstop Mechanical Design}

The beamstop consists of a series of equivalent "plates" of graphite that transfer their heat to a water-cooled tank by thermal radiation. Because large thermal stresses can be built up in flat plate-type geometry, we have chosen to build the plates with close-packed graphite rods. The graphite rods are $2 \mathrm{~cm}(0.8 \mathrm{in}$.) in diameter and are stacked vertically (Fig. 4-38). The rods are held in place with graphite clamps to allow for thermal expansion. For simplicity, the close-packed rods are referred to as plates throughout the remaining technical description.

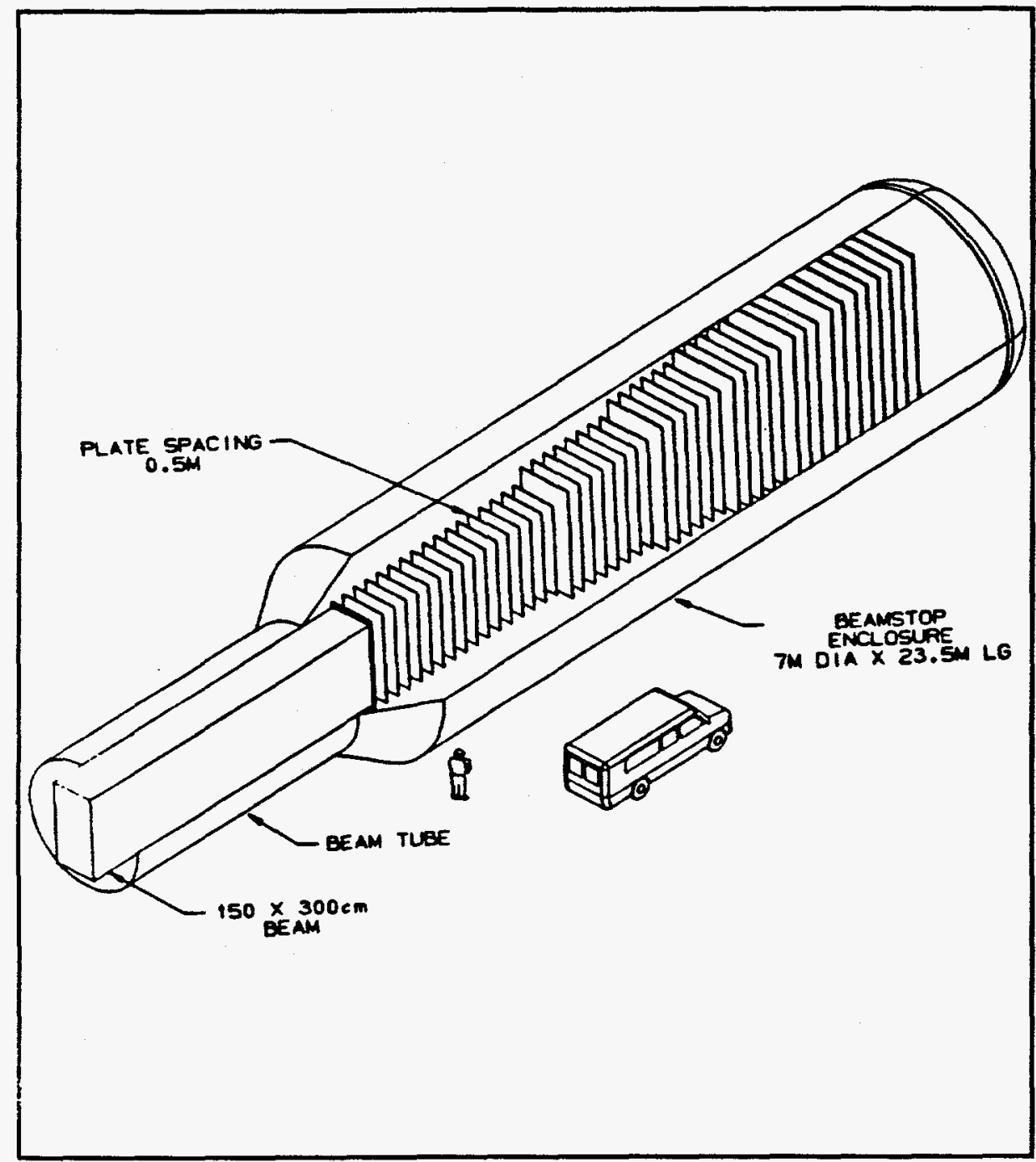

Fig. 4-38. APT Beamstop.

There is a total of 47 graphite plates that vary from $2.0 \mathrm{~cm}$ to $10.6 \mathrm{~cm}(0.8$ in. to $4.2 \mathrm{in}$.) in thickness and vary in size from $150 \mathrm{~cm} \times 300 \mathrm{~cm}(59 \mathrm{in}$. 118 in.) to $300 \mathrm{~cm} \times 450 \mathrm{~cm}$ (118 in. $x 177$ in.). They are placed directly in the path of the beam to stop the high-energy protons. The beamspot striking the first plate is $150 \mathrm{~cm} \times 300 \mathrm{~cm}$ (59 in. $\times 118 \mathrm{in}$.). As the beam interacts with the graphite plates, the protons are scattered. To stop the scattered 
protons, the beamstop plates increase in size as one moves from the front to the back of the beamstop. The distance between the plates varies between $25 \mathrm{~cm}$ and $75 \mathrm{~cm}$ (10 in. and 30 in.) to allow for adequate view factors from the plates to the tank wall. The total length of the beamstop is $23.5 \mathrm{~m}(77 \mathrm{ft})$.

The graphite plates are housed in an aluminum vacuum tank that is $7.0 \mathrm{~m}(23 \mathrm{ft})$ in diameter. The graphite is cooled by thermal radiation to the tank wall, which in turn is cooled with water flowing in a $2.5-\mathrm{cm}$ to $5.0-\mathrm{cm}(1.0 \mathrm{in}$. to $2.0 \mathrm{in}$.)-thick annulus. The use of aluminum as the tank wall material further reduces the amount of radionuclide production.

\subsubsection{Beamstop Physics Analysis}

Two physics design calculations of the beamstop were performed using the LAHET Code System and CINDER90 for an idealized cylindrical system. In the first calculation, a $175-\mathrm{cm}$ (69 in.) -long graphite cylinder divided into $1-\mathrm{cm}(0.4 \mathrm{in}$.$) segments was used to$ determine the energy deposition as a function of depth in the graphite. This was done to size the beamstop layers because the intranuclear cascade process gives an energy deposition that is different from proton-only Coulomb processes.

In the engineering design, the graphite section is $23.5 \mathrm{~m}(77 \mathrm{ft})$ long and consists of a series of plates of varying thickness with appropriate separations to allow radiative heat transfer to the tank walls. This feature was represented in the second physics design by a uniform reduced density graphite cylinder. The beam and graphite radii were chosen to reproduce the cross-sectional areas of the rectangular engineering design and were $119.7 \mathrm{~cm}$ and $207.3 \mathrm{~cm}$ (47.1 in. and $81.6 \mathrm{in}$.) respectively. The aluminum tank was modeled with two $2-\mathrm{cm}(0.8$ in. $)$-thick double walls with a $5-\mathrm{cm}(2$ in.) cooling water annulus in between.

For the $1000-\mathrm{MeV}, 200-\mathrm{mA}$ beam, the calculations show that $110.6 \mathrm{MW}$ is deposited in the graphite, 16.4 MW in the aluminum tank walls and water annulus, and $56.8 \mathrm{MW}$ in the surrounding shield. There is $16.2-\mathrm{MW}$ heat that is converted to mass plus carried away by neutrinos.

Table 4-15 below gives the total energy loss at the average cell center position as measured from the beam entry point.

Table 4-15. LAHET-MCNP Energy Deposition Results

\begin{tabular}{ccc}
\hline Problem Cell Number & Cell Center $(\mathbf{c m})$ & Cell Energy Deposition $(\mathbf{M e V} / \mathbf{p})$ \\
\hline 1 & 117.5 & $1.32 \mathrm{E}+02$ \\
2 & 352.5 & $1.26 \mathrm{E}+02$ \\
3 & 587.5 & $9.79 \mathrm{E}+01$ \\
4 & 822.5 & $7.06 \mathrm{E}+01$ \\
5 & 1057.5 & $4.78 \mathrm{E}+01$ \\
6 & 1292.5 & $3.18 \mathrm{E}+01$ \\
7 & 1527.5 & $2.05 \mathrm{E}+01$ \\
8 & 1762.5 & $1.29 \mathrm{E}+01$ \\
9 & 1997.5 & $8.22 \mathrm{E}+00$ \\
10 & 2232.5 & $5.53 \mathrm{E}+00$ \\
\hline \hline
\end{tabular}


The neutron and proton leakage flux information is summarized below. A typical neutron spectrum (n/MeV/cm $2 / \mathrm{p})$ at the outside of the cylindrical aluminum tank is shown in Fig. 4-39. The proton leakage spectrum, calculated for energies above $20 \mathrm{MeV}$, is quite similar. The surface areas for the graphite, the aluminum tank exit wall, and the cylindrical aluminum tank were $2.70 \times 10^{5} \mathrm{~cm}^{2}, 4.05 \times 105 \mathrm{~cm}^{2}$, and $5.32 \times 10^{6} \mathrm{~cm}^{2}$, respectively. See Table 4-16 for values of LAHET-MCNP proton and neutron flux.

Table 4-16. LAHET-MCNP Proton and Neutron Flux Summary

\begin{tabular}{lcccc}
\hline Surface & $\begin{array}{c}\text { Integrated } \\
\text { Flux (n/cm²/p) }\end{array}$ & $\begin{array}{c}\text { Average } \\
\text { Energy (MeV) }\end{array}$ & $\begin{array}{c}\text { Integrated } \\
\text { Flux }\left(\mathbf{n} / \mathbf{c m}^{2 / \mathbf{p}}\right)\end{array}$ & $\begin{array}{c}\text { Average } \\
\text { Energy (MeV) }\end{array}$ \\
\hline $\begin{array}{l}\text { Entrance Graphite } \\
\text { Material }\end{array}$ & $1.32 \times 10^{-8}$ & 78 & $3.49 \times 10^{-7}$ & 25 \\
$\begin{array}{c}\text { Exit Aluminum } \\
\text { Tank Wall }\end{array}$ & $1.00 \times 10^{-7}$ & 271 & $1.99 \times 10^{-7}$ & 189 \\
$\begin{array}{l}\text { Cylindrical } \\
\text { Aluminum Tank }\end{array}$ & $4.77 \times 10^{-8}$ & 267 & $2.87 \times 10^{-7}$ & 122 \\
\hline
\end{tabular}

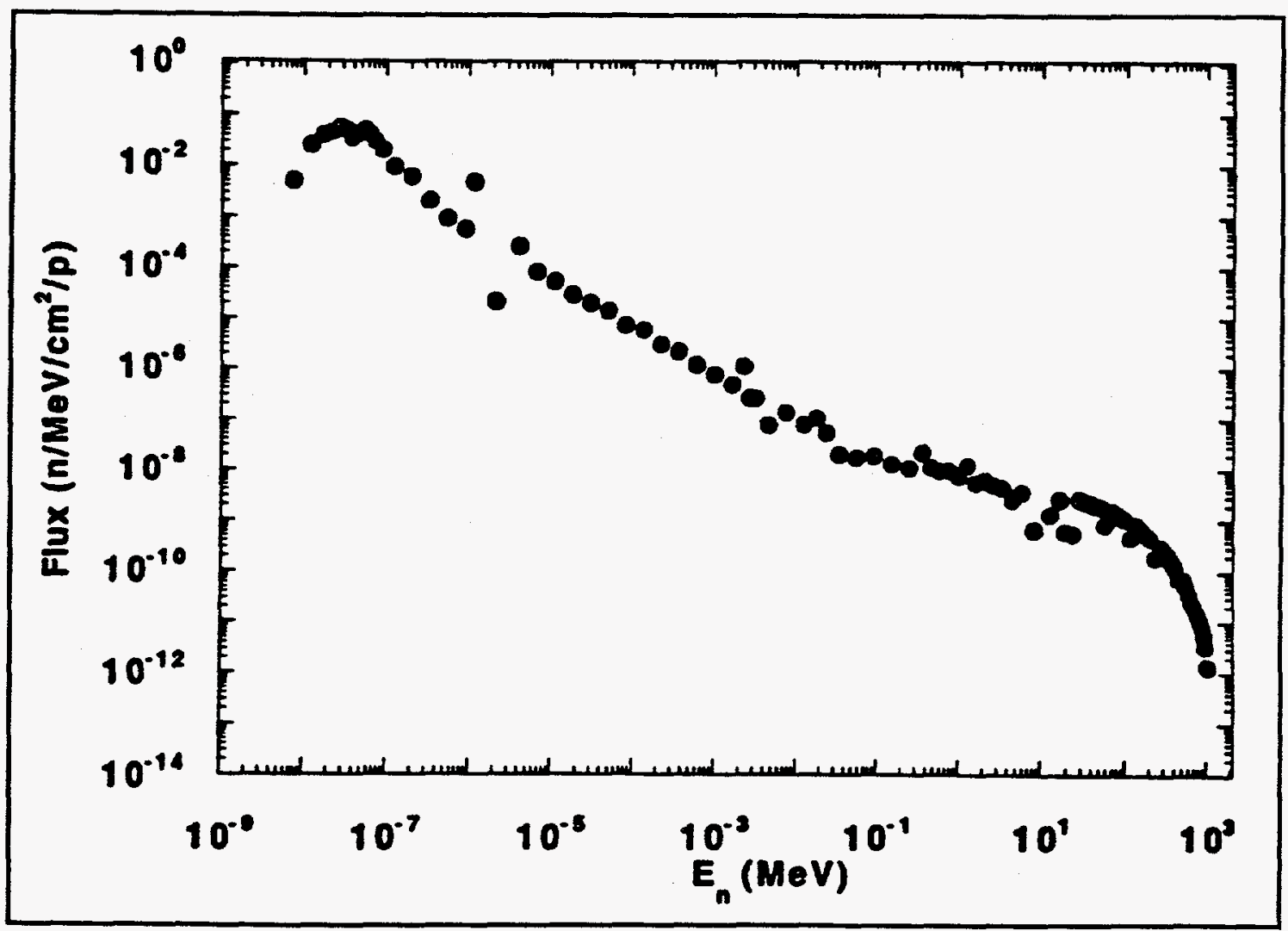

Fig. 4-39. Beamstop Neutron Leakage Flux From Cylindrical Surface. 


\subsubsection{Beamstop Thermal Analysis}

Using the calculated energy deposition as input, a radiation heat transfer analysis was performed on the graphite using closed-form solutions for plate-type geometry. A total of 110.6 MW is deposited in the graphite and radiatively transferred to the tank wall. Plate thicknesses and the distance between the plates were chosen to maintain a relatively constant graphite temperature along the length of the beamstop, therefore minimizing the overall length. The graphite and tank wall emissivities were assumed to both be 0.8 . The tank wall was assumed to be at constant temperature of $373 \mathrm{~K}$.

The results of the analysis are shown in Table 4-17. To dissipate this amount of heat by thermal radiation requires a total of 47 plates that vary from $2.0 \mathrm{~cm}(0.8 \mathrm{in}$.) to $10.6 \mathrm{~cm}(4.2$ in.) in thickness. The plates are in nine separate sections, each with a different thickness. The thin plates are at the front, and the thick plates at the back to accommodate the change in the heat deposition. Most plate temperatures range from $1850 \mathrm{~K}$ to $2050 \mathrm{~K}\left(2870^{\circ} \mathrm{F}\right.$ to $\left.3230^{\circ} \mathrm{F}\right)$. The exception is the last plate, which is calculated to be at $1572 \mathrm{~K}\left(2370^{\circ} \mathrm{F}\right)$. The distance between the plates of $50 \mathrm{~cm}$ ( $20 \mathrm{in}$.) allows for adequate view factors from the plates to the tank wall. The peak plate heat flux is $35.5 \mathrm{~W} / \mathrm{cm}^{2}$. The Ground Test Accelerator (GTA) beamstop heat flux is $47 \mathrm{~W} / \mathrm{cm}^{2}$, Ref. 4-7, for comparison.

The tank that contains the beamstop is $7.0 \mathrm{~m}(23.0 \mathrm{ft})$ in. diam with a $2.5-\mathrm{cm}$ to $5.0-\mathrm{cm}$ (1.0 in. to $2.0 \mathrm{in}$.) annulus containing the water coolant. A total of $127 \mathrm{MW}$ of heat is carried away by the coolant (110.6 MW of radiative heat from the graphite, and $16.4 \mathrm{MW}$ directly deposited in the aluminum tank walls and water annulus). A total of $1012 \mathrm{~kg} / \mathrm{s}$ flows through the annulus to remove the heat. The coolant pressure drop through the annulus is $0.011 \mathrm{MPa}$ $(1.6 \mathrm{psi})$. The inlet temperature is $310.95 \mathrm{~K}\left(100^{\circ} \mathrm{F}\right)$, and the outlet is $340.95 \mathrm{~K}\left(154^{\circ} \mathrm{F}\right)$ with a $30 \mathrm{~K}\left(54^{\circ} \mathrm{F}\right)$ temperature increase. The average heat flux to the wall is $21.4 \mathrm{~W} / \mathrm{cm}^{2}$.

Table 4-17. Plate Temperatures and Dimensions

\begin{tabular}{ccccccc}
\hline Section & $\begin{array}{c}\text { Minimum } \\
\text { Temperature } \\
(\mathbf{K})\end{array}$ & $\begin{array}{c}\text { Maximum } \\
\text { Temperature } \\
(\mathbf{K})\end{array}$ & $\begin{array}{c}\text { Plate } \\
\text { Thickness } \\
(\mathbf{c m})\end{array}$ & $\begin{array}{c}\text { Number } \\
\text { of Plates }\end{array}$ & $\begin{array}{c}\text { Thickness } \\
\text { of Graphite } \\
\text { (cm) }\end{array}$ & $\begin{array}{c}\text { Section } \\
\text { Length } \\
\text { (m) }\end{array}$ \\
\hline 1 & 1897 & 2032 & 2.0 & 17 & 34.0 & 8.5 \\
2 & 1901 & 2059 & 2.5 & 8 & 20.0 & 4.0 \\
3 & 1953 & 2049 & 3.0 & 6 & 18.0 & 3.0 \\
.4 & 1835 & 2022 & 3.5 & 5 & 17.5 & 2.5 \\
5 & 1852 & 2055 & 4.6 & 3 & 13.8 & 1.5 \\
6 & 1930 & 2030 & 6.0 & 2 & 12.0 & 1.0 \\
7 & 1921 & 2036 & 7.0 & 1 & 7.0 & 0.5 \\
8 & 1908 & 2033 & 9.0 & 2 & 18.0 & 1.0 \\
9 & 1572 & 1967 & 10.6 & 3 & 31.8 & 1.5 \\
Total & & & & 47 & 172.1 & 23.5 \\
\hline \hline
\end{tabular}




\subsection{SCALING TO 3/16-GOAL SYSTEM}

The main changes from the $3 / 8$ goal design to the $3 / 16$ goal design are given here for an initial scaled $3 / 16$ design. Both designs use $1000 \mathrm{MeV}$ protons, but the $3 / 16$ design uses half as much current $(100 \mathrm{~mA}$ vs. $200 \mathrm{~mA})$. The distance between the bundles in the beam direction is the same for both designs to give the same total $3 \mathrm{He}$ chamber length. The total beam area for the $3 / 16$ design is half that of the $3 / 8$ design to keep the peak power densities the same. The beam profile is calculated as $33.6 \mathrm{~cm}$ by $56.9 \mathrm{~cm}$ to give a bundle pattern of 4 bundles in the first row, 5 bundles in the second row, and then repeating the 4 by 5 pattern for the remainder of the 16 rows. This gives the same bundle spacing across the beam as for the $3 / 8$ design where a 5 by 6 pattern was used. Both designs used a $2.5-\mathrm{cm}$ envelope of tungsten around the beam profile so the tungsten rod length is $96 \mathrm{~cm}$ for the $3 / 8$ design and only $61.9 \mathrm{~cm}$ for the $3 / 16$ design. A space of approximately $5.6 \mathrm{~cm}$ was used at each end of the diagonal through the tungsten to determine the inner diameter of the $3 \mathrm{He}$ target chamber of $118 \mathrm{~cm}$ for the $3 / 8$ design and only $84 \mathrm{~cm}$ for the $3 / 16$ design. The beam entrance tube used the same $2.5-\mathrm{cm}$ extra length on each end of the tungsten diagonal as for the $3 / 8$ design so it was reduced from a $111.9-\mathrm{cm}$ diameter for the $3 / 8$ design to $77.9-\mathrm{cm}$ diameter for the $3 / 16$ design. The distances of the plena from the center of the target chamber were reduced by the change in target chamber radius from the $3 / 8$ design to the $3 / 16$ design. Plena diameters were not reduced for the $3 / 16$ mass estimates although it is expected that they will be reduced when we complete sizing of the 3/16 design. All of the other thicknesses of $3 \mathrm{He}$ and $\mathrm{D}_{2} \mathrm{O}$ regions in the blanket were kept the same for the $3 / 16$ design as in the $3 / 8$ design. However, the volumes of the blanket regions decreased because the radii at which they occur decrease with the reduction in target chamber radius. The number of tubes in each blanket region was scaled by the ratio of the $3 / 16$ to $3 / 8$ design radii from the center of the target chamber. Volumes of $3 \mathrm{He}$ and aluminum structure in the heat exchanger and blower piping for the cooling system for the high pressure $3 \mathrm{He}$ in the target chamber were reduced by $40 \%$ from the $3 / 8$ to $3 / 16$ design. The reduction in power removal is $50 \%$ so this use of a $40 \%$ reduction is somewhat conservative. The ${ }^{3} \mathrm{He}$ pressure was maintained at $100 \mathrm{psia}$ in the blanket regions as in the $3 / 8$ design, but the ${ }^{3} \mathrm{He}$ pressure was reduced from 300 psia to 260 psia in the target chamber. An average ${ }^{3} \mathrm{He}$ temperature of $400 \mathrm{~K}$ was assumed for all regions for both designs.

A comparison of the materials required for a single target/blanket module for both the 3/16 and 3/8-Goal systems is given in Table 4-18.

Table 4-18. Summary of Masses for a Single Target/Blanket Module

\begin{tabular}{lcc}
\hline Material & 3/16 Goal & 3/8 Goal \\
\hline Aluminum & 4419 & 5497 \\
Heavy Water & 28175 & 34583 \\
Helium-3 & 7.6 & 14.2 \\
Inconel & 3976 & 4972 \\
Lead & 53586 & 70801 \\
Stainless Steel & 14158 & 16618 \\
Tungsten & 778 & 1476 \\
Zircaloy-4 & 2320 & 4328 \\
\hline \hline
\end{tabular}


Scaling of a 3/16-Goal accelerator concept from the 3/8-goal APT point design provides a reasonable starting point for a reduced capacity system, bounding values for ES\&H impacts, and a basis for construction and operating cost estimates. However, such an approach may not generate an optimum design in terms of costs, performance, technical risk, and other factors. Trade studies are planned during the APT conceptual design phase to determine the optimum accelerator configuration to satisfy the Nuclear Posture Review requirements.

\subsection{REFERENCES}

4-1. Unused Reference

4-2. J. Tom Sena and M. A. Merrigan, "APT Thermal Hydraulic Cold Flow Test Report," Los Alamos National Laboratory report LA-UR-93-3582 (August 1993).

4-3. Unused Reference

4-4. “APT Balance of Plant Topical Report," Los Alamos National Laboratory report LA12665-MS (September 1993).

4-5. ISA-RP67.04, Part II-1991, "Methodologies for the Determination of Setpoints for Nuclear Safety-Related Instrumentation," March 1991.

4-6. IEEE Standard 603-1980, "IEEE Standard Criteria for Safety Systems for Nuclear Power Generating Stations" (Revision on IEEE Std 603-1977).

4-7. H. Newman and D. Martinez, "GTA 24-MeV Intermediate Beam Stop Design Report," Los Alamos National Laboratory memorandum MEE-9:G0-92-212 (September 22, 1992). 


\section{SAFETY \\ CHAPTER 5}

Page

5.1 SAFETY APPROACH AND DESIGN CRITERIA ................................................5-1

5.2 SUMMARY OF PEIS ACCIDENT SCENARIOS...................................................

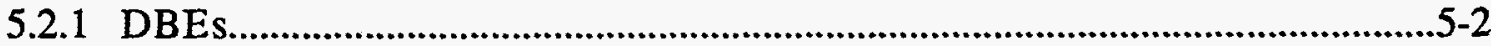

5.2.1.1 Single Helium Tube Break ..............................................................5-3

5.2.1.2 Beam Entrance Window Failure ......................................................5-3

5.2.1.3 Fire in Radwaste System ........................................................................5-4

5.2.1.4 LBLOCA with One Active Residual Heat Removal System ..................5-4

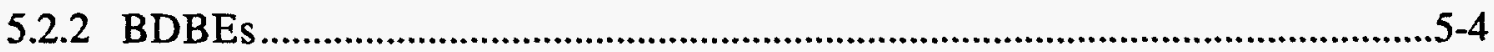

5.2.2.1 Single Assembly Flow Blockage with Delayed Beam Trip ....................5-4

5.2.2.2 Multiple Helium Tube Break or Chamber Failure .....................................5-5

5.2.2.3 LBLOCA with Total Failure of Active Residual-Emergency Cooling System

5.3 SUMMARY OF TRAC MODEL AND LBLOCA ANALYSES …………................5-6

5.3.1 Model Description ................................................................................................

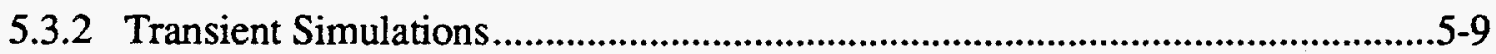

5.4 WINDOW COOLING BY RADIATION HEAT TRANSFER ..................................5-13

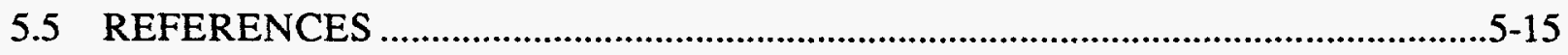

\section{LIST OF FIGURES}

Page

Fig. 5-1. APT primary cooling loop schematic as modeled with TRAC...........................5-7

Fig. 5-2. Pressures in the APT moderator tank during an LBLOCA of a tungsten rod bundle cooling system main coolant pipe, 0 to $10 \mathrm{~s}$.

Fig. 5-3. Maximum structure temperatures for $200 \%$ cold-leg break unprotected LOCA. 


\section{LIST OF TABLES}

Page

Table 5-1. APT Tungsten Rod Bundle Cooling System Design Values..................................5-8

Table 5-2. APT Tungsten Rod Bundle Cooling System Design Parameters..........................5-8

Table 5-3. Comparison of Key Parameters for APT Tungsten Rod Bundle Cooling System LOCA Simulations.

Table 5-4. Break Flow, Integrated Break Flow, and Break Temperature for Hot-Leg LBLOCA, June 1993 Model 


\subsection{SAFETY}

\subsection{SAFETY APPROACH AND DESIGN CRITERIA}

DOE has drafted a General Safety Requirements Document [5-1] for the APT. This document specifies the safety principles and requirements against which the APT will be reviewed and evaluated. The APT safety approach is to develop a design using inherent safety features and active- and passive-engineered safety systems to meet or exceed the safety requirements.

The APT system has a number of inherent safety features. The APT does not use fissile or fertile material, making reactivity-induced transients impossible. The APT has a smaller radioactive inventory and heat source compared with a nuclear reactor. The decay heat in the first seconds after shutdown in the tungsten target is only $0.9 \%$ of total power (compared with $6 \%$ for a nuclear reactor). The accelerator beam can also be shut off very quickly (less than 1 $\mathrm{ms}$, given a trip signal). The use of tungsten is a safety advantage because of its hightemperature capability and melting point $3410^{\circ} \mathrm{C}\left(6170^{\circ} \mathrm{F}\right)$. All of the coolant loops are at low pressure (less than $1.73 \mathrm{MPa}$, or 250 psia) and at low specific energy, so the confinement system will not be subjected to any significant pressure increase in the event of large pipe breaks. The entire target/blanket assembly sits in a tank of heavy-water moderator that in itself is a large heat sink and can be used for cooling under certain accident conditions.

The engineered safety systems include (1) highly reliable instrumentation systems designed to detect accident conditions and rapidly shut down the beam, and (2) active and passive cooling systems to remove the decay heat. The APT beam-trip system for accidents will be designed to IEEE-603 [5-2], with two-out-of-four trip logic and will be designed to have both a primary and secondary trip function for each transient. The result is that a triple protection system failure will still trip the beam (double failure of the primary trip function and a single failure in the secondary trip function could occur, and the secondary trip function will still operate to trip the beam). These protection system features should put initiating events "with failure to trip beam" in the residual risk category. The safety-by-design philosophy utilizes the following steps:

1. Develop a comprehensive list of initiating events and, using engineering judgment and the methodology in Ref. 3, classify the events into the categories of AOOs, DBEs, BDBEs, and residual risk. Residual risk events have a probability of less than $10^{-7} / \mathrm{yr}$, and no action is required to reduce the risk from these events.

2. Establish specific acceptance criteria for AOOs, DBEs, and BDBEs.

3. Perform analyses to identify the limiting event sequence(s) for each event category.

4. Design safety systems and perform analyses to meet the acceptance criteria. Document the functions and design requirements for all safety systems.

5. Utilize redundant and diverse instrumentation to rapidly shut down the beam for all AOOs, DBEs, and BDBEs. 
6. Apply the "defense-in-depth" principle and utilize both active and passive cooling systems with redundancy to provide high assurance that important safety functions are achieved.

7. Design the moderator tank and primary coolant piping outside of the moderator tank as a highly reliable radionuclide retention boundary that will meet the rules and considerations of an ASME Section III, Class I, pressure boundary.

8. Provide an intermediate barrier between radionuclide systems and the environment to reduce the probability of leakage.

The APT safety approach has been developed to assure with high confidence that a beam trip will occur and adequate cooling will be provided if T/B parameters deviate from the design operating range. The safety approach uses redundant and diverse systems to assure high reliability for tripping the beam and removing the decay heat. By having two $100 \%$ active residual heat removal systems (only one system is needed to remove the decay heat), the effects of a single failure can be accommodated. By having both active and passive residual heat removal systems, the effects of multiple failures can also be accommodated.

The proposed safety design criteria are as follows:

1. Ensure a rapid shutdown of the beam for all AOOs, DBEs, and BDBEs.

2. For AOOs, do not exceed critical heat flux limits, allow no significant voiding or flow instabilities, and do not exceed design pressures.

3. For DBEs, maintain the target, blanket, and window component materials below temperature limits; maintain coolable geometry; provide capacity for long term cooling; and ensure that safety systems operate in accident environments.

4. For DBEs and BDBEs, ensure that the confinement system prevents radionuclides from exceeding the offsite release criteria.

\subsection{SUMMARY OF PEIS ACCIDENT SCENARIOS}

At the PEIS information meeting held in Washington, DC, on January 14, 1993, the project participants agreed on the DBEs and the BDBEs that were to be analyzed, documented, and transmitted to SNL as input to the PEIS [5-4]. For the DBE and BDBE LBLOCAs, detailed thermal-hydraulic analyses have been performed and documented (see Section 5.3 for details). For the remainder of the DBEs and BDBEs, formal quantitative analyses have not been performed. Rather, the event scenarios have been delineated, and limiting/maximum radionuclide releases to the confinement building have been identified and documented in the PEIS.

\subsubsection{DBES}

At the January 14,1993 , PEIS meeting, a number of potential DBEs were reviewed and discussed. The result was that four DBEs were identified for documentation in the PEIS. 
These events are: a single helium tube break, a beam entrance window failure, a fire in the most critical radwaste area, and an LBLOCA with active systems responding as designed and a single failure in the most critical active system responding to the event. Descriptions of these events are provided below.

\subsubsection{Single Helium Tube Break}

The radiological release to the confinement for this event is bounded by the BDBE entitled "Multiple Helium Tube Break or Chamber Failure" that is described in Section 5.2.2.2. The source term will be bounded by the bounding BDBE for this DBE (5500 Ci to the environment during the first $24 \mathrm{~h}$ ).

\subsubsection{Beam Entrance Window Failure}

In the APT $3 \mathrm{He}$ target/blanket system, the mechanical interface between the accelerator beam transport tube and the $3 \mathrm{He}$ chamber is a double-walled window made of Inconel-718. One window is attached to the $3 \mathrm{He}$ chamber, and the second window is attached to the accelerator beam transport tube. The MPCS provides for forced circulation of cooling water $\left(\mathrm{D}_{2} \mathrm{O}\right)$ between the two windows. Two scenarios must be considered: failure of the window attached to the $3 \mathrm{He}$ chamber and failure of the window attached to the accelerator beam transport tube. The windows will be designed so that failure of one window does not lead to the subsequent failure of the second window.

The following describes the failure of the window attached to the $3 \mathrm{He}$ chamber. The MPCS average coolant pressure is approximately $45 \mathrm{psia}$ in the window. The $3 \mathrm{He}$ chamber operating pressure is 300 psia. Upon failure of the window, gas will flow from the ${ }^{3} \mathrm{He}$ chamber into the MPCS; the pressure in the MPCS will increase, and the beam will be tripped. A small amount of tritium will accompany the flow of $3 \mathrm{He}$ into the MPCS. However, the integrity of the MPCS pressure boundary will be retained, and there will be no release of radionuclides to the confinement building.

The following describes the failure of the window attached to the accelerator beam tube. The accelerator beam transport tube is evacuated. Upon failure of the window, the MPCS water will rapidly flow into the accelerator beam tube, and all or a significant fraction of the cooling water will vaporize. The introduction of water vapor and possibly some water into the accelerator beam transport tube will be rapidly detected by accelerator beam transport tube protection systems. Upon detection of water vapor in the accelerator beam transport tube, the beam will be tripped. In addition, redundant, fast-acting gate valves will close and isolate the accelerator beam transport tube from the window region. Because the beamline has a slight bend, primarily to stop neutrons backstreaming from the Target/Blanket from reaching beam transport components, any metal debris from a window failure that might pass the fast-closing valves will stop in the shielded neutron backstop region. A small amount of vapor will pass into the accelerator beam transport tube before closure of the gate valves. However, the accelerator beam transport tube is a sealed system having pressure integrity. The pressure integrity of the accelerator beam transport tube will be retained. Therefore, the radionuclides released from the MPCS will remain in the accelerator beam transport tube. They will be allowed to decay in place or removed before cleanup activities are started. No radionuclides will be released to the environment. 


\subsubsection{Fire in Radwaste System}

The complete survey needed to determine the limiting radwaste system fire has not been completed. This is due, in part, to the fact that the design information needed to complete such a survey has not yet evolved. In all cases, applicable release criteria will be satisfied.

\subsubsection{LBLOCA with One Active Residual Heat Removal System}

A double-ended guillotine cold-leg break in the TPCS was simulated with the TRACPF1/MOD3 computer code for $180 \mathrm{~s}$. For this DBE, the plant protection and engineered safety systems performed as designed. The analysis assumed that the most limiting single failure was the loss of power to one residual heat removal system pump.

The short-term ( 0 to $50 \mathrm{~s}$ ) transient results were very similar to the BDBE LOCA. At 60 $\mathrm{s}$, one of the two residual heat removal systems was turned on to provide long-term rod bundle cooling. At $180 \mathrm{~s}$, the rod bundle temperatures had flattened out at approximately $340 \mathrm{~K}$ $\left(152^{\circ} \mathrm{F}\right)$ and would be expected to decrease in time as the power decayed.

The radionuclides released to the confinement building for this DBE are judged to be similar to and bounded by the radionuclides released to the confinement building for the BDBE LBLOCA.

\subsubsection{BDBEs}

At the January 14, 1993, PEIS meeting, a number of potential BDBEs were reviewed and discussed. The result was that three BDBEs were identified for documentation in the PEIS. These events are a single assembly flow blockage with delayed beam trip, a multiple helium tube break or chamber failure, and an LBLOCA with total failure of the active emergency cooling system. Descriptions of these events are provided below.

\subsubsection{Single Assembly Flow Blockage with Delayed Beam Trip}

The event scenario assumes a total flow blockage of a single tungsten rod bundle. It should be noted that the inlets to the tungsten rod bundles will be designed to preclude a total flow blockage. Normally, a large increase in fluid temperature would be detected by the tungsten rod bundle outlet coolant temperature instrumentation and would result in a beam trip. In this scenario, all temperature instrumentation in the blocked bundle is assumed to fail, and thus, the beam is not tripped. The heavy-water coolant in the blocked tungsten rod bundle rapidly heats to saturation and evaporates. In the absence of coolant and with the beam still on, the tungsten rods in the blocked bundle will heat up in a near-adiabatic environment. Some beam energy will also be deposited in the Inconel-718 hexagonal duct of the tungsten rod bundle. Although the temperature of the Inconel duct will begin to increase, it will be at a slower rate than the tungsten rods because the energy deposition is smaller, and a heat sink exists on the outside of the duct where ${ }^{3} \mathrm{He}$ is circulating. As the temperatures of the tungsten rods increase, radiative heat transfer to the Inconel duct will increase, the temperature of the Inconel duct will rise, and the Inconel duct will eventually fail. Once the Inconel duct fails, one or more detectors in the $3 \mathrm{He}$ system will identify that acceptable limits have been exceeded, and the beam will be tripped. Although it is possible to perform analyses to identify and evaluate the Inconel-duct failure mechanism, subsequent trip mechanisms, and conditions of the tungsten rod bundle at the time of duct failure, a bounding release of radionuclides into the 
TPCS and/or the heat transport system for the $3 \mathrm{He}$ was selected. As an upper bound, the total radionuclide inventory of a single tungsten rod bundle is assumed to be released. The radionuclides in a single bundle available for release under this scenario is $1.633 \times 106 \mathrm{Ci}$. The fractions of the total radionuclides that will enter the TPCS and the heat transport system for the $3 \mathrm{He}$, respectively, have not been determined. However, because both the TPCS and the heat transport system for the ${ }^{3} \mathrm{He}$ are primary structural boundaries, there will be no release of radionuclides to the confinement building.

We have also considered whether the heatup of the Inconel duct, either before or shortly after beamtrip, could lead to failure of the penetration at the $3 \mathrm{He}$ chamber/moderator tank fluid interface. A scoping calculation was performed to determine the magnitude of axial conduction in the Inconel duct. This calculation showed that axial conduction is significantly less than radiation heat transfer from the surface of the tungsten rods to the Inconel duct. Therefore, we conclude that failure of the Inconel duct at the penetration will not occur. We note, however, that should this postulated failure occur, the radionuclides would be released to the moderator tank. The radionuclides would still be contained within a primary pressure boundary, and there should be no release to the confinement building.

\subsubsection{Multiple Helium Tube Break or Chamber Failure}

Release of the total $3 \mathrm{He}$ radionuclide inventory to the confinement building is assumed. The total release to the confinement building will be $1.1 \times 10^{5} \mathrm{Ci}$. Confinement building cleanup and processing systems are assumed to be inactive. Therefore, the release to the environment is at the design leak rate (estimated to be $5 \mathrm{vol} . \% / \mathrm{d}$ ) for the confinement building. It is estimated that, for this event, the tritium release to the environment will be less than 5500 $\mathrm{Ci}$ during the first $24 \mathrm{~h}$. Other physical mechanisms that are likely to mitigate the tritium release from the confinement building were not considered in this analysis.

\subsubsection{LBLOCA with Total Failure of Active Residual-Emergency Cooling System}

Double-ended guillotine hot-leg and cold-leg breaks have been simulated to $1000 \mathrm{~s}$ with the TRAC-PF1/MOD3 computer code. For this BDBE, the plant protection systems were assumed to function and trip the beam at $0.2 \mathrm{~s}$, but the two $100 \%$ active residual heat removal systems were assumed to fail. The maximum hot rod temperature, $432.0 \mathrm{~K}\left(318^{\circ} \mathrm{F}\right)$, occurred in the cold-leg break case at the time of beam trip. This temperature is $5.8 \mathrm{~K}\left(10.4^{\circ} \mathrm{F}\right)$ higher than the corresponding steady-state value. For both cases, from the time of beam trip until the pressurizer and accumulator emptied at about $250 \mathrm{~s}$, the temperatures in the system were dominated by the temperature of the pressurizer and accumulator fluid entering the system. Following the emptying of the pressurizer and accumulator, fluid in the TPCS heated up to a condition that would support natural circulation. The simulations verified that the unbroken loop stayed liquid full, as it was designed to do, except for the initial 30 to $40 \mathrm{~s}$ of the transient. After the end of pressurizer and accumulator discharge, natural circulation was established in the unbroken loop, and the maximum hot rod temperature heated up to approximately $365 \mathrm{~K}$ $\left(197^{\circ} \mathrm{F}\right)$ at about $500 \mathrm{~s}$. The tungsten rod temperatures and hexagonal-duct temperatures then slowly decreased for the remainder of the transient as the power decayed. The TRAC simulations verify that in the event of an LBLOCA and the loss of both active residual heat removal systems, the unbroken loop will stay liquid full and support long-term natural circulation and decay heat removal. 
At $1000 \mathrm{~s}$, TRAC calculates a total integrated break flow of approximately $26,000 \mathrm{~kg}$ of liquid. The initial heavy-water mass circulating in the TPCS is approximately $12,300 \mathrm{~kg}$. Therefore, we assume that all of the circulating inventory at the start of the LBLOCA is lost instantaneously out the break to the confinement building. The release to the confinement building is $2.2 \times 10^{5} \mathrm{Ci}$ from the target spallation products and $1.9 \times 10^{4} \mathrm{Ci}$ of tritium, which were resident in the circulating TPCS before the LBLOCA. Release to the environment is expected to be a factor of approximately 40 lower.

\subsection{SUMMARY OF TRAC MODEL AND LBLOCA ANALYSES}

TRAC-PF1/MOD3 has been used to verify selected design concepts for APT cooling systems under accident conditions. TRAC is a reactor safety code developed at Los Alamos for the US Nuclear Regulatory Commission, with the capability to perform best-estimate analyses of LOCAs and other transients in pressurized light-water reactors. It has recently been updated to model heavy-water reactors. TRAC has been used to model APT LOCA DBEs and BDBEs and establish sensitivities to various parameters, such as trip timing. These simulations have demonstrated the validity of the design concepts to mitigate accidents. A LBLOCA, even with the failure of both residual heat removal systems (a BDBE), can be mitigated by natural circulation flow and decay heat removal in the unbroken loop with the tungsten rod bundle temperatures rising only a few degrees above steady-state values. The confinement building loading from the water lost out the break during a LBLOCA is also expected to be small because the majority of the break flow water is subcooled. A more detailed description of the TRAC model and LBLOCA analyses is contained in Ref. 5-5.

\subsubsection{Model Description}

A TRAC-PF1/MOD3 model has been developed to simulate the APT tungsten rod bundle cooling system preconceptual design under LOCA conditions. The TRAC model and analyses described in this report reflect the preconceptual design as of June 1993. Before January 1993, the initial TRAC model was based on design concepts rather than on an actual preconceptual design. Some decisions on the basic configuration of the loops had been made, but actual component sizing and layout had, for the most part, not begun. In January 1993, the TPCS preconceptual design was initiated based on information that reflected a total tungsten rod bundle power of $92 \mathrm{MW}$. As preconceptual design information became available, it was incorporated into the TRAC model. By June 1993, preliminary piping sizes, pressurizer and accumulator sizes, heat exchanger and pump designs, steady-state operating conditions, a primary-to-secondary heat exchanger specification, and piping layouts had been completed. Designers have refined the concepts for the fluid passages from the inlet plenum through the tungsten rod bundles into the outlet plenum. The secondary loops are modeled as sources and sinks to provide the appropriate flow and temperature boundary conditions to the secondary side of the primary heat exchangers. Details of the secondary piping loops are not in the current model. To simulate the passive secondary water-to-air heat exchanger and the secondary loop natural circulation flow rates and temperatures, the secondary flow coasts down over $60 \mathrm{~s}$ from full flow to $2 \%$ of full flow at the initiation of a LBLOCA. The temperature of the secondary loop water entering the primary heat exchanger is held constant during the transient. The secondary loop flow and temperature response is based on previous analyses for a similar secondary loop design with a passive water-to-air heat exchanger for the NPR heavy-water reactor. Figure 5-1 shows the primary cooling loop schematic as modeled 


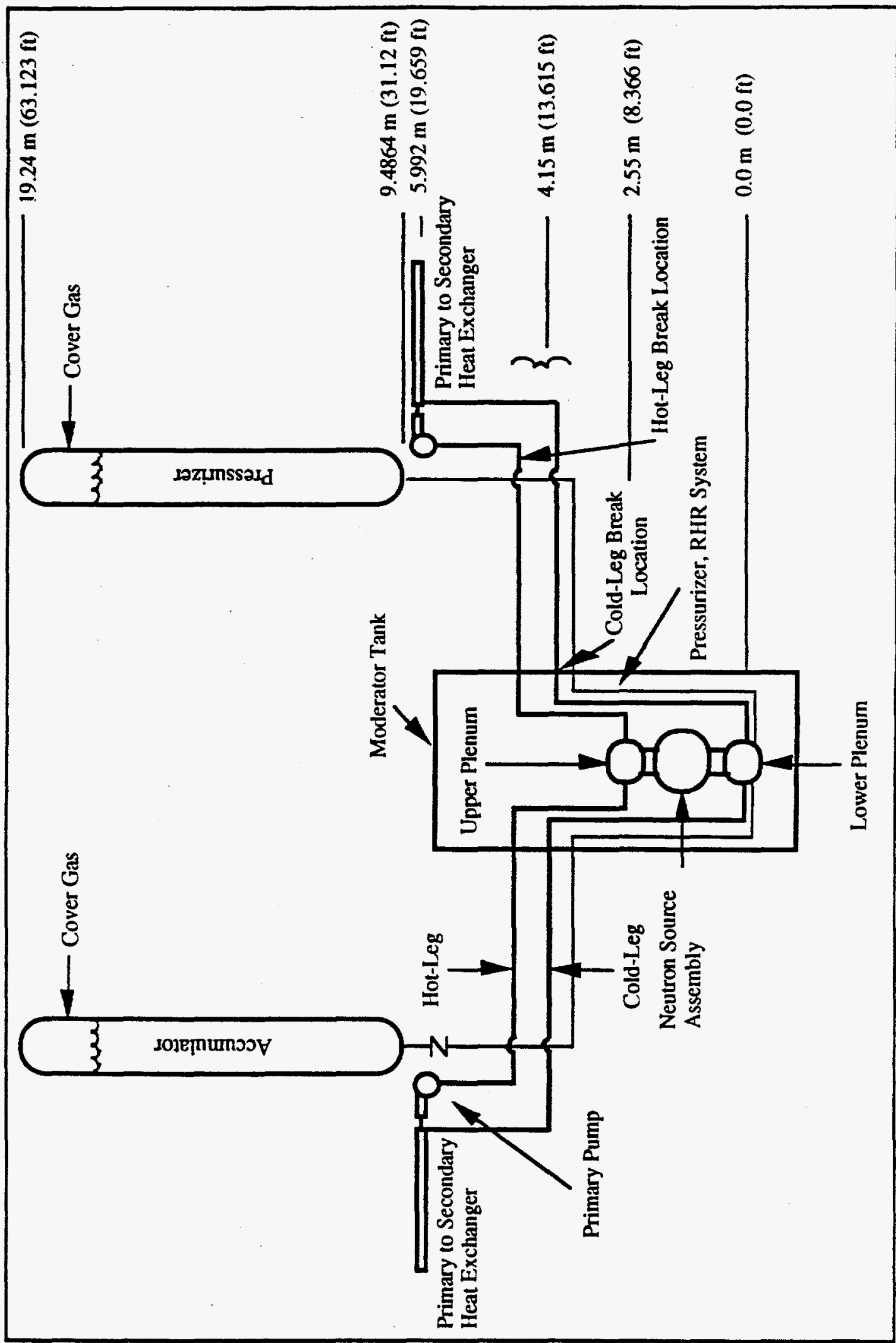

Fig. 5-1. APT primary cooling loop schematic as modeled with TRAC. 
with TRAC. Table 5-1 gives key design information for the tungsten primary coolant system. Table 5-2 gives steady-state thermal-hydraulic information.

Table 5-1. APT Tungsten Rod Bundle Cooling System Design Values

Number of Primary Loops

Primary Piping Hot Leg I.D.

Primary Piping Cold Leg I.D.

Pressurizer and Accumulator Line I.D.

Pressurizer and Accumulator I.D.

Hot Leg Length, Including Pump

Cold Leg Length

Pressurizer and Accumulator Line Length

Pressurizer Liquid Volume

Accumulator Liquid Volume

Pressurizer Gas Volume

Accumulator Gas Volume

Number of Heat Exchangers per Loop

Heat Exchanger Tube Length

Number of Heat Exchanger Tubes

Heat Exchanger Tube Diameter

Pumps per Loop

Pump DP at Nominal Conditions

Pump Torque

Pump Effective Moment of Inertia
2

$0.2027 \mathrm{~m}$ (7.98 in.)

$0.1541 \mathrm{~m}$ (6.07 in.)

$0.1023 \mathrm{~m}$ (4.03 in.)

$1.2192 \mathrm{~m}$ (48 in.)

$33.575 \mathrm{~m}(110.15 \mathrm{ft})$

$35.665 \mathrm{~m}(116.98 \mathrm{ft})$

$18.736 \mathrm{~m}(61.5 \mathrm{ft})$

$10.39 \mathrm{~m}^{3}\left(367 \mathrm{ft}^{3}\right)$

$10.34 \mathrm{~m}^{3}\left(365 \mathrm{ft}^{3}\right)$

$0.7504 \mathrm{~m}^{3}\left(26.5 \mathrm{ft}^{3}\right)$

$0.8070 \mathrm{~m}^{3}\left(28.5 \mathrm{ft}^{3}\right)$

1

$5.395 \mathrm{~m}(17.7 \mathrm{ft})$

2648

$0.0127 \mathrm{~m}(0.5 \mathrm{in}$.)

1 (uses LOFT homologous curves)

$1.034 \times 10^{6} \mathrm{~Pa}$ (150 psi)

$1698 \mathrm{~N} \cdot \mathrm{m}(1252 \mathrm{ft} \cdot \mathrm{lbf})$

$175 \mathrm{~kg} \cdot \mathrm{m}^{2}\left(4153 \mathrm{lbm} \cdot \mathrm{ft}^{2}\right)$

Table 5-2. APT Tungsten Rod Bundle Cooling System Design Parameters

\section{Design Parameter}

Total Bundle Power

Average Bundle Power

Average Bundle Power Density

Average Bundle Inlet Temperature

Average Bundle Outlet Temperature

Average Bundle Flow

Hot Bundle Power

Hot Bundle Power Density

Hot Bundle Inlet Temperature

Hot Bundle Outlet Temperature

Hot Bundle Flow

Total Primary Coolant Flow

Lower (Inlet) Plenum Pressure

Steady-State Coolant Velocity, Hot Leg

Steady-State Coolant Velocity, Cold Leg

\section{Conditions Modeled}

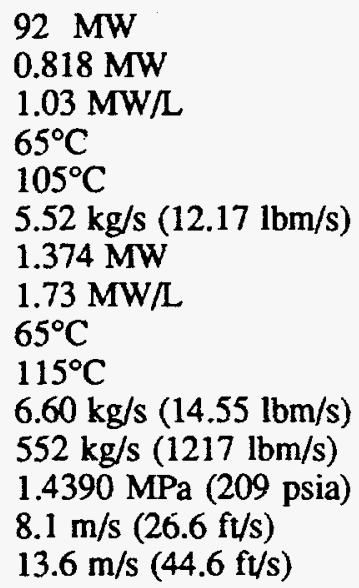

92 MW

$0.818 \mathrm{MW}$

$1.03 \mathrm{MW} / \mathrm{L}$

$65^{\circ} \mathrm{C}$

$105^{\circ} \mathrm{C}$

$5.52 \mathrm{~kg} / \mathrm{s}(12.17 \mathrm{lbm} / \mathrm{s})$

$1.374 \mathrm{MW}$

$1.73 \mathrm{MW} / \mathrm{L}$

$65^{\circ} \mathrm{C}$

$115^{\circ} \mathrm{C}$

$6.60 \mathrm{~kg} / \mathrm{s}(14.55 \mathrm{lbm} / \mathrm{s})$

$552 \mathrm{~kg} / \mathrm{s}(1217 \mathrm{lbm} / \mathrm{s})$

$1.4390 \mathrm{MPa}$ (209 psia)

$8.1 \mathrm{~m} / \mathrm{s}(26.6 \mathrm{ft} / \mathrm{s})$

$13.6 \mathrm{~m} / \mathrm{s}(44.6 \mathrm{ft} / \mathrm{s})$

All components in the TPCS are modeled, but the current TPCS design is based on a total tungsten rod bundle power of $92 \mathrm{MW}$. In the current preconceptual design, the tungsten rod bundle power is approximately $77 \mathrm{MW}$. The TPCS design and the TRAC model will be updated in the future to reflect this change in power level. However, the TRAC results are expected to be similar, and no major changes are expected in the basic design concepts to 
mitigate accident conditions. The current TRAC model also simulates the target region with 99 average-power bundles and 1 high-power bundle. The current preconceptual design has 88 bundles. The TRAC model will be updated to reflect these and any other changes as the APT design evolves.

\subsubsection{Transient Simulations}

Four sets of TRAC transient simulations have been run to support the evolution of the APT design concepts. Most transients have been either protected LBLOCAs, where one RHR system operates to remove decay heat starting $60 \mathrm{~s}$ after the event initiation, or unprotected LBLOCAs, where both RHR systems are assumed to fail, and decay heat removal is through natural circulation in the unbroken loop. The four sets of calculations include

Initial simulations: These simulations were based on preliminary estimates for component and piping size. The configuration was similar to later layouts, except that the primary coolant pumps were located in the cold legs. The time delay between initiation of the accident and trip of the beam was assumed to be $0.6 \mathrm{~s}$. This time delay was based on nuclear reactor experience. The decay heat curve was based on $1 \mathrm{yr}$ of target operation. Results of protected and unprotected cold-leg LBLOCAs were used as input to the March 1993 PEIS report.

Follow on simulations: A second set of simulations used the same sizing estimates but relocated the pumps from the cold legs to the hot legs. The trip delay time was set to $0.2 \mathrm{~s}$ to reflect an estimate from the I\&C group that the time delay between the time a trip condition is reached and the trip of the accelerator would be about $0.1 \mathrm{~s}$. A trip delay of $0.2 \mathrm{~s}$ was chosen as a conservative estimate. The decay power curve was changed to a curve based on 600 days of target operation. Both hot- and cold-leg break cases were run.

Quantifying Pressure Load Simulations: To quantify pressure loading for an LBLOCA of the TPCS inside the moderator tank, several simulations were run. The TPCS remained all liquid during these simulations, so the rod bundle decay heat was easily removed. These simulations showed that the moderator tank pressurizer system proposed by $\mathrm{B} \& \mathrm{~W}$ would limit the pressure response to a short spike with a magnitude of about $0.827 \mathrm{MPa}$ (120 psia).

Latest Simulations: The latest set of simulations was done with the model incorporating all of the component specifications and layout information available by June 1993. These provide a nearly complete preliminary specification for the TPCS. Hot- and cold-leg unprotected LBLOCAs were simulated to verify that the components and systems would perform as intended. The results from these simulations were provided as input to the September 1993 PEIS report.

Table 5-3 summarizes the results of the first, second, and fourth sets of simulations. The highest temperatures were in the initial simulations, with a 0.6-s delay between the time a trip condition was reached and the time the beam tripped. Structure and liquid temperatures rose rapidly between the time of transient initiation and the time of beam trip. Of all the parameters examined, the time delay for the beam trip had the greatest effect on system temperatures. The results were similar for the hot- and cold-leg breaks. 
Table 5-3. Comparison of Key Parameters for APT Tungsten Rod Bundle Cooling System LOCA Simulations

\begin{tabular}{|c|c|c|c|c|c|c|}
\hline & \multicolumn{2}{|c|}{ Initial Model } & \multicolumn{2}{|c|}{$\begin{array}{l}\text { Initial Model with } \\
\text { Modifications }\end{array}$} & \multicolumn{2}{|c|}{ Latest Model } \\
\hline & $\begin{array}{l}\text { Protected } \\
\text { C.L. Break } \\
\end{array}$ & $\begin{array}{c}\text { Unprotected } \\
\text { C.L. Break } \\
\end{array}$ & $\begin{array}{c}\text { Protected } \\
\text { C.L. Break } \\
\end{array}$ & $\begin{array}{l}\text { Unprotected } \\
\text { C.L. Break } \\
\end{array}$ & $\begin{array}{l}\text { Unprotected } \\
\text { C.L. Break }\end{array}$ & $\begin{array}{l}\text { Unprotected } \\
\text { H.L. Break }\end{array}$ \\
\hline $\begin{array}{l}\text { Total Break } \\
\text { Flow }\end{array}$ & $9460 \mathrm{~kg}$ & $10,738 \mathrm{~kg}$ & $8901 \mathrm{~kg}$ & $9640 \mathrm{~kg}$ & $25,858 \mathrm{~kg}$ & $26,749 \mathrm{~kg}$ \\
\hline $\begin{array}{l}\text { Avg. Break } \\
\text { Flow Temp. }\end{array}$ & $326 \mathrm{~K}$ & $320 \mathrm{~K}$ & $328 \mathrm{~K}$ & $325 \mathrm{~K}$ & $313 \mathrm{~K}$ & $316 \mathrm{~K}$ \\
\hline $\begin{array}{l}\text { Max. Hot } \\
\text { Rod Temp. }\end{array}$ & $467.4 \mathrm{~K}$ & $467.4 \mathrm{~K}$ & $425.7 \mathrm{~K}$ & $425.7 \mathrm{~K}$ & $432.0 \mathrm{~K}$ & $426.5 \mathrm{~K}$ \\
\hline $\begin{array}{l}\text { Max. Avg. } \\
\text { Rod Temp. }\end{array}$ & $404.6 \mathrm{~K}$ & $404.6 \mathrm{~K}$ & $392.4 \mathrm{~K}$ & $392.4 \mathrm{~K}$ & $396.4 \mathrm{~K}$ & $392.7 \mathrm{~K}$ \\
\hline $\begin{array}{l}\text { Max. Fluid } \\
\text { Temp., Hot } \\
\text { Bundle }\end{array}$ & $432.9 \mathrm{~K}$ & $432.9 \mathrm{~K}$ & $407.7 \mathrm{~K}$ & $407.7 \mathrm{~K}$ & $413.2 \mathrm{~K}$ & $403.7 \mathrm{~K}$ \\
\hline $\begin{array}{l}\text { Max. Fluid } \\
\text { Temp., Avg. } \\
\text { Bundle }\end{array}$ & $388 \mathrm{~K}$ & $388 \mathrm{~K}$ & $378.8 \mathrm{~K}$ & $378.8 \mathrm{~K}$ & $380.6 \mathrm{~K}$ & $377.9 \mathrm{~K}$ \\
\hline
\end{tabular}

Figure 5-2 shows the pressure response for the in-moderator-tank TPCS LBLOCA from the quantifying pressure load simulations noted above. B\&W specified a relatively large pressurizer in the moderator tank primary coolant system to keep the pressure response of the moderator tank to an acceptable level for this LOCA scenario. These simulations showed that the pressurizer system proposed by $\mathrm{B} \& \mathrm{~W}$ would limit the pressure response to a short spike with a magnitude of about $0.827 \mathrm{MPa}(120 \mathrm{psia})$. The TPCS remained all liquid during these simulations, so decay heat was easily removed. In general, the pressure in the moderator tank will keep the TPCS loops liquid full in case of a break in the TPCS piping within the moderator tank.

Figure 5-3 shows the maximum structure temperatures for the cold-leg break case for the model incorporating the preliminary component specifications (the latest model). Before the beam trip at about $0.2 \mathrm{~s}$, the temperatures increase slightly. The maximum high-powered rod temperature was $432.0 \mathrm{~K}$ at $0.2 \mathrm{~s}$. This was an increase of $5.8 \mathrm{~K}$ over the maximum steadystate temperature. After beam trip, the structure temperatures decreased to slightly above the temperature of the liquid entering the system from the pressurizer and accumulator. Temperatures in the rod bundles rose as the pressurizer and accumulator flows declined before $300 \mathrm{~s}$. Shortly after $300 \mathrm{~s}$ there was a flow reversal, with cooler water above the elevation of the rod bundles falling through the bundles. The water in the bundles again began to heat up before $400 \mathrm{~s}$, eventually reaching a temperature that would initiate and then support natural circulation flow and decay heat removal.

The simulations for the latest model verify that in the event of a LOCA, the intact loop will stay liquid full and support natural circulation. This requires a water-to-air heat exchanger in the TSCS and configuration of the loops so that a break in one primary loop will not cause the other loop to drain. The conditions for doing this are basically the same as the conditions to keep a siphon full and operating. The driving potential for flow is different, but the conditions to keep the piping liquid full are the same. The conditions necessary to keep the unbroken loop all liquid and capable of supporting natural circulation are (1) there can be no path for air 


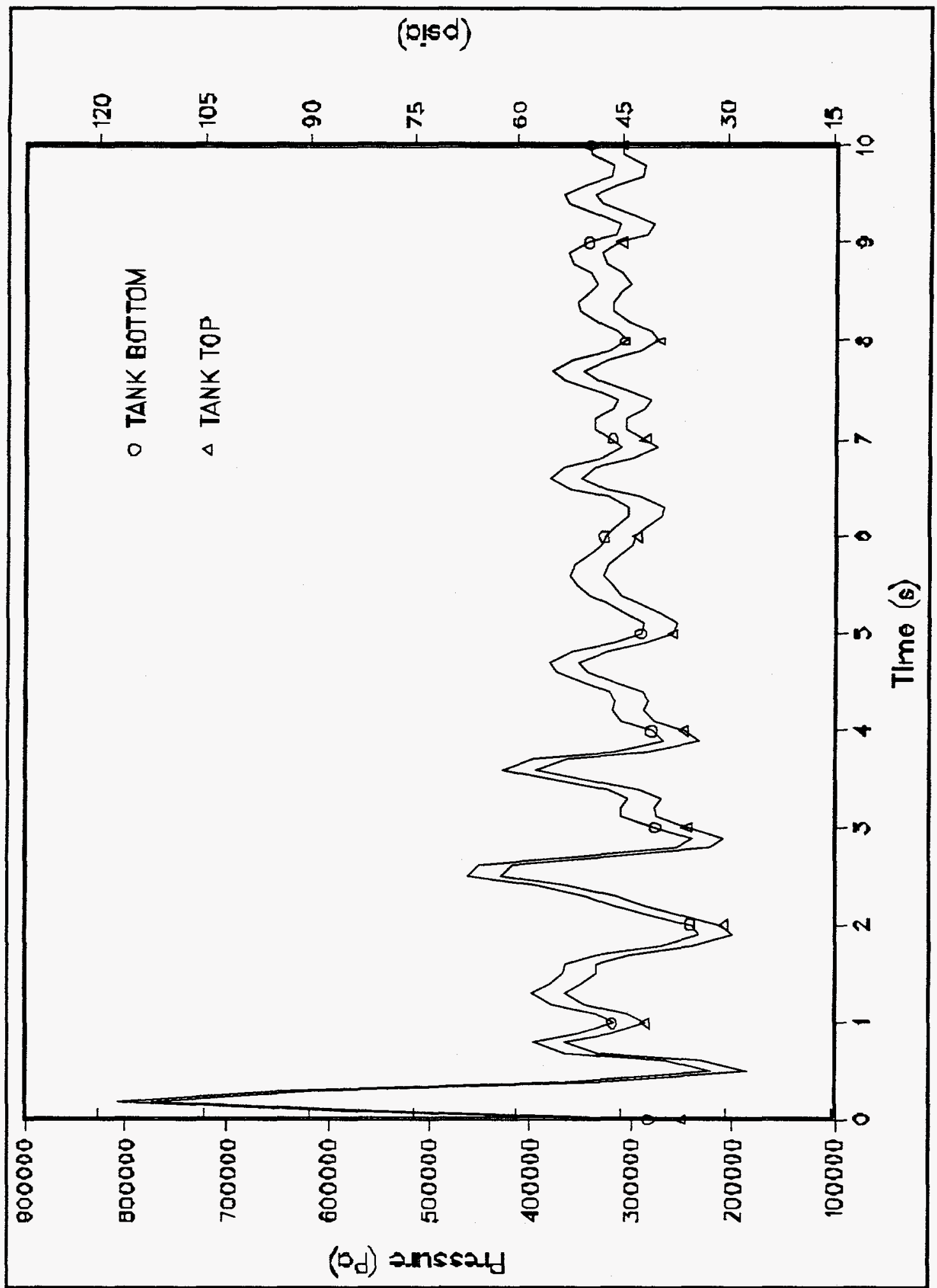

Fig. 5-2. Pressures in the APT moderator tank during an LBLOCA of a tungsten rod bundle cooling system main coolant pipe, 0 to 10s. 


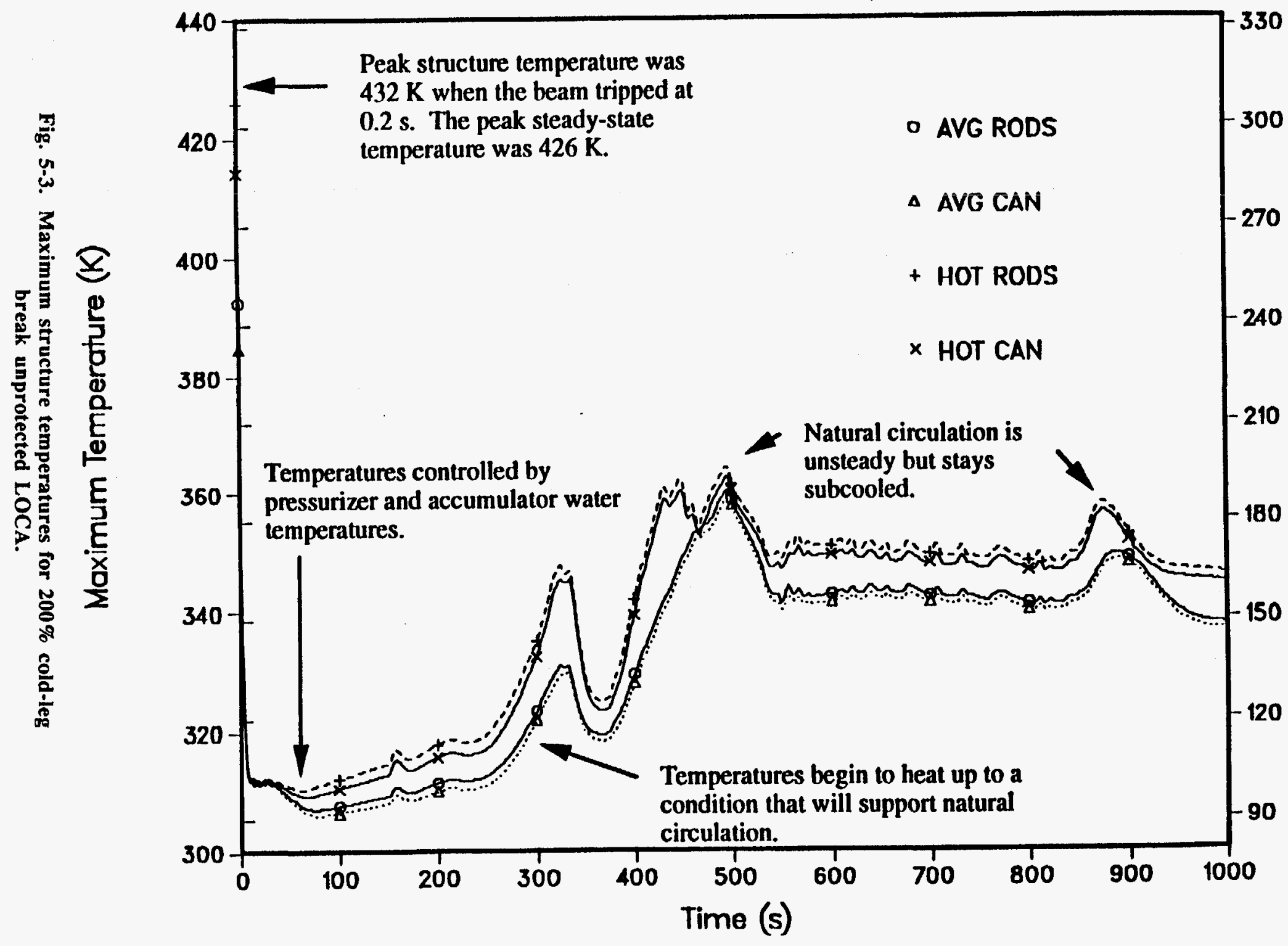


to migrate from the broken to the unbroken loop; (2) the unbroken loop must have space to accommodate dissolved noncondensibles coming out of suspension while still maintaining a liquid flow path; and (3) the pressure at the top of the unbroken loop must be greater than the vapor pressure of the water for all temperature conditions. This last condition requires keeping the elevation difference between the lowest possible point for a main pipe break (outside the moderator tank) and the top of the primary loop small. The piping layout has an elevation difference of $1.8 \mathrm{~m}(6 \mathrm{ft})$ between the elevation at which the piping penetrates the moderator tank and the top of the loops. Keeping these small elevation differences requires a horizontal rather than a vertical heat exchanger. Most of the elevation change in the piping takes place within the moderator tank. As noted previously, a LOCA within the moderator tank will not cause a loop to void because the pressure in the moderator tank is sufficiently high to prevent any voids at the top of the primary loop. The elevation between the thermal centers of the rod bundles and the heat exchanger is $4.72 \mathrm{~m}(15.5 \mathrm{ft})$. The simulations show that this gives adequate driving potential for natural circulation.

Table 5-4 gives break flow and fluid temperature for the hot-leg break case using the latest model incorporating the preliminary component specifications. This case gives the largest total break flow and the highest fluid temperatures. The large increase in total break flow compared to the earlier TRAC models is a result of incorporating the latest Bechtel TPCS piping layout. During the initial $5 \mathrm{~s}$ of the transient, the flow out of the break was saturated. After $5 \mathrm{~s}$, the break flow was subcooled.

Table 5-4. Break Flow, Integrated Break Flow, and Break Temperature for Hot-Leg LBLOCA, June 1993 Model

\begin{tabular}{|c|c|c|c|}
\hline Time (s) & $\begin{array}{c}\text { Break } \\
\text { Flow }(\mathbf{k g} / \mathrm{s})\end{array}$ & $\begin{array}{c}\text { Integrated } \\
\text { Break Flow (kg) }\end{array}$ & $\begin{array}{c}\text { Break } \\
\text { Temperature }(\mathrm{K})\end{array}$ \\
\hline 1 & 269.33 & 277 & 379 \\
\hline 2 & 185.80 & 504 & 379 \\
\hline 3 & 228.07 & 711 & 379 \\
\hline 4 & 207.13 & 928 & 378 \\
\hline 5 & 198.54 & 1131 & 375 \\
\hline 6 & 195.30 & 1328 & 367 \\
\hline 7 & 186.44 & 1519 & 356 \\
\hline 10 & 161.84 & 2036 & 336 \\
\hline 30 & 135.50 & 4967 & 320 \\
\hline 60 & 113.99 & 8714 & 314 \\
\hline 120 & 73.54 & 14,214 & 307 \\
\hline 300 & 31.44 & 24,109 & 313 \\
\hline 600 & 1.95 & 26,436 & 318 \\
\hline 999 & 0.33 & 26,749 & 325 \\
\hline
\end{tabular}

\subsection{WINDOW COOLING BY RADIATION HEAT TRANSFER}

In an accident situation where forced convection cooling to the window is lost (i.e. pump failure), the fast protection system shuts down the beam. The initial decay heat is removed by the flow coastdown and the heatup of coolant in the moderator primary coolant system. This 
section examines the very low probability case where the moderator primary cooling system and the moderator residual heat removal system (both active and passive systems) have both stopped circulating flow through the window. The decay power is then removed by radiation from the front wall of the window to the beam tube walls and from the back wall of the window to the helium chamber walls. A description of the thermal radiation analysis follows.

The APT window design is composed of two walls of Inconel-718 with a gap between the walls for the flow of heavy-water coolant. This double wall structure connects the beam tube to the helium chamber. Therefore, on the accelerator side of the window is a vacuum, and on the helium chamber side is ${ }^{3} \mathrm{He}$ at $2.07 \mathrm{MPa}$ (300 psia). The cooling for the window is provided by the moderator heat transport systems (see Sec. 4.6 .2 for details). At normal operating conditions, a total of $1.43 \mathrm{MW}$ is generated in the two Inconel walls and the heavywater coolant. The total decay heat for both walls and the coolant ranges from $3.55 \mathrm{~kW}$ at $1 \mathrm{~s}$ after beam trip to $2.72 \mathrm{~kW}$ at $1000 \mathrm{~s}$ after beam trip. After $1 \mathrm{yr}$ of cooling time, the decay heat is reduced to $96 \mathrm{~W}$, and after $10 \mathrm{yr}, 3.9 \mathrm{~W}$. The peak decay heat is therefore $1.78 \mathrm{~kW}$ in each wall and occurs immediately after beam trip. For the radiation heat transfer analysis we conservatively assume that the power remains at this peak level. The other pertinent assumptions are

- Heat transfer by thermal radiation, only. The walls are insulated on the coolant side.

- The view factor for the wall facing the beam tube is 1.0 from the window to the beam tube inner surface. The surface of the beam tube is at $373 \mathrm{~K}\left(212^{\circ} \mathrm{F}\right)$ and represents the heat sink.

- The view factor for the wall facing the helium chamber is 0.5 from the window to the helium chamber surface. (The view factor from the full cylindrical window to the chamber would be 0.637.) The surface of the helium chamber is at $373 \mathrm{~K}$ $\left(212^{\circ} \mathrm{F}\right)$. It is in thermal contact with the moderator tank fluid, which is at about $343 \mathrm{~K}\left(158^{\circ} \mathrm{F}\right)$.

- The surface emissivities for all surfaces are 0.5. All surfaces are diffuse gray.

- $1777 \mathrm{~W}$ are generated in each wall. This is assumed to be the steady-state power.

- The heat transfer area is $4770 \mathrm{~cm}^{2}\left(739 \mathrm{in}^{2}\right)$ for each wall, which is the area of the $68 \%$ of the window that is in the beam.

- The conduction heat transfer through the wall is negligible (the walls are about $1 \mathrm{~mm}(0.04 \mathrm{in})$ thick).

- There are no participating media for the radiation (both the vacuum and helium are nonparticipating).

- The heat transfer area of the heat sink is equal to $630,000 \mathrm{~cm}^{2}\left(97,600 \mathrm{in} .^{2}\right)$ for the beam tube wall based on a length of $18 \mathrm{~m}$ (709 in.) and a diameter of $111.9 \mathrm{~cm}$ (44.1 in.). The beam tube is at least $18 \mathrm{~m}$ (709 in.) long. (The results are very insensitive to this length.) 
- The heat transfer area of the heat sink for the helium chamber wall is assumed to be equal to one half the radial helium chamber wall area $\left[72,500 \mathrm{~cm}^{2}\left(11,200 \mathrm{in}^{2}\right)\right]$.

The results show that the accelerator side wall of the window will reach $623.6 \mathrm{~K}\left(663^{\circ} \mathrm{F}\right)$ under these conditions, and the helium chamber side wall will reach $685.5 \mathrm{~K}\left(774^{\circ} \mathrm{F}\right)$. Therefore even in a worst case situation where there is no water coolant, the window will cool itself with radiation heat transfer and remain well below the melting point of Inconel-718 which is $1498 \mathrm{~K}\left(2200^{\circ} \mathrm{F}\right)$.

As a sensitivity calculation, we determined the power level needed for the window helium chamber side wall to reach a point of failure (about $100^{\circ} \mathrm{C}\left(180^{\circ} \mathrm{F}\right)$ below the melting point). In this case the power would need to be increased to $63 \mathrm{~kW}$, which represents about $4.4 \%$ of full steady-state power and is 17.7 times the decay power at $1 \mathrm{~s}$. Thus, this conservative analysis shows that radiation heat transfer will easily remove peak decay heat loads from the window.

\subsection{REFERENCES}

5-1. US Department of Energy, "General Safety Requirements Document for the Accelerator Production of Tritium," Rev. 0, Draft D, US Department of Energy, Office of New Production Reactors document APT-GSR-0001-00 (June 1994).

5-2. IEEE Standard 603-1980, "Standard Criteria for Safety Systems for Nuclear Power Generating Stations," (Rev. on IEEE Standard 603-1977) (November 24, 1980).

5-3. Z. R. Rosztoczy, "General Definitions of AOOs, DBAs, Etc.," US Nuclear Regulatory Commission Advanced Reactors Branch memorandum to J. E. Dyer, US Nuclear Regulatory Commission Advanced Reactors Project Directorate (August 17, 1992).

5-4. "Accelerator Production of Tritium Programmatic Environmental Impact Statement Input Submittal," Sandia National Laboratory report SAND93-2094, (November 1, 1993).

5-5. "APT 3He Target/Blanket Topical Report," Los Alamos National Laboratory report LA-12670-MS, Rev.0 (September 1993). 



\section{T/B OPERATION CHAPTER 6 \\ LIST OF FIGURES}

Page

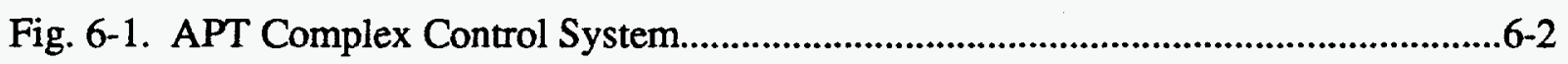





\subsection{T/B OPERATION}

The major systems of the APT complex are the accelerator, the T/B, the TPS, and the BOP. The operation of the T/B system interfaces with the other systems and must conform to the requirements of the other systems to optimize the production of tritium.

The function of the accelerator relative to the T/B system is to create a $200-\mathrm{mA}$ beam of protons, accelerate this beam of protons to $1 \mathrm{GeV}$, and expand the proton beam to a $42-\mathrm{x}-91$ $\mathrm{cm}^{2}$ beam pattern at the $\mathrm{T} / \mathrm{B}$ window. The major accelerator components are the beam injectors, the linac modules, the deflector magnets, the beam expander, and the beamstop. The beamstop is not in the direct path of the T/B but is utilized to focus the proton beam that will be directed to the T/B window.

The function of the T/B systems is to convert the $200-\mathrm{MW}$ proton beam to neutrons and produce tritium from $3 \mathrm{He}$ by neutron interaction. The major $\mathrm{T} / \mathrm{B}$ components are the tungsten neutron source assembly, the lead neutron multiplier or target lead, the $\mathrm{D}_{2} \mathrm{O}$ moderator, the moderator tank, the $3 \mathrm{He}$ blanket tubes and heat exchanger, and the support structures. Other $\mathrm{T} / \mathrm{B}$ systems include the heat removal systems, the purification system, the remote handling system, and the instrumentation and control systems. The $200 \mathrm{MW}$ of energy is distributed between the T/B components, as discussed in Chap. 4, Engineering Design.

The function of the TPS relative to the T/B systems is to remove tritium from the $3 \mathrm{He}$ gas streams and from the $\mathrm{D}_{2} \mathrm{O}$ fluid systems. The $3 \mathrm{He}$ gas streams come from the $3 \mathrm{He}$ chamber and the $3 \mathrm{He}$ blanket tubes. The $\mathrm{D}_{2} \mathrm{O}$ fluid systems are the TPCS, and the MPCS.

The function of the BOP is to provide electric power, heat removal (cooling), HVAC, radiation monitoring, personnel safety, and $T / B$ confinement. The BOP functions are applicable to all other components of the APT complex. The T/B confinement is a physical structure around the T/B that can isolate the $\mathrm{T} / \mathrm{B}$ in the event of a release of radioactive materials or other abnormal situations.

Operation of the T/B is integrated into the overall operation of the APT complex by the APT Complex Control System, as shown in Fig. 6.1. The accelerator and the T/B will be controlled from one common, central control room, and the TPS will be controlled from a separate control room. The control room for the TPS is separate because of special security requirements.

The major interaction between the $\mathrm{T} / \mathrm{B}$ and the accelerator is through the run-permit-andbeamtrip/SFAS feedback. The T/B systems have been designed to accept the 200-MW proton beam from the accelerator at any time after the run permit signal has been supplied to the APT Complex Control System. Thus, no significant feed forward signals from the accelerator to the T/B have been identified during the preconceptual design phase. The beamtrip/SFAS feedback is a safety system designed to IEEE-603 Standards that will assure a termination of the $200-\mathrm{MW}$ proton beam to the $\mathrm{T} / \mathrm{B}$ when either accelerator or $\mathrm{T} / \mathrm{B}$ system parameters are outside of defined limits. The T/B run-permit-and-beamtrip/SFAS are discussed in greater detail in Chap. 4, Sec. 7, Instrumentation Control and Protection Systems.

The only interaction between the T/B and the TPS that has been identified during the preconceptual design is the isolation of the ${ }^{3} \mathrm{He}$ gas streams and the $\mathrm{D}_{2} \mathrm{O}$ fluid streams. The 
normal flow rate of ${ }^{3} \mathrm{He}$ and $\mathrm{D}_{2} \mathrm{O}$ between the T/B and the TPS is a small fraction of the total inventory. If the TPS is shut down, the accelerator and T/B would still be able to operate for some period of time (days or weeks). With the TPS shutdown, the inventory of tritium would build up in the T/B systems. The desire to minimize the tritium inventory in the $T / B$ is the limiting factor on the amount of time the T/B can operate without the TPS.

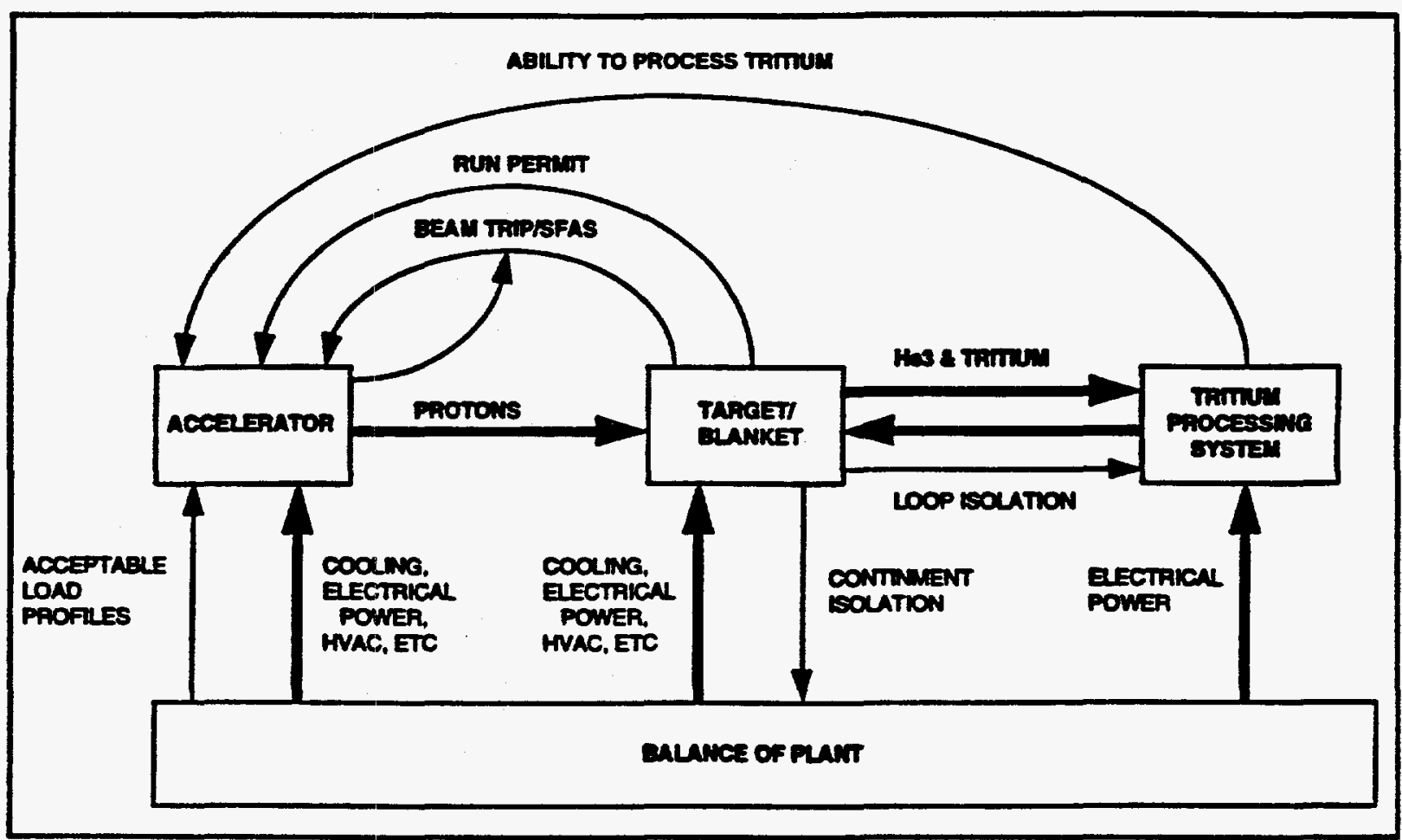

Fig. 6-1. APT Complex Control System. 
T/B INTERFACES WITH BOP

CHAPTER 7 



\subsection{T/B INTERFACES WITH BOP}

The APT $3 \mathrm{He}$ T/B and associated coolant systems interface with the BOP and therefore will require support services from the $B O P$. In the preconceptual design phase, the interfaces were developed sufficiently so that space could be allowed in the confinement building to house the larger equipment, and preliminary costs associated with the interfacing systems/components could be developed.

For interfacing BOP systems that connect directly to the T/B coolant systems, it is assumed that the boundary between the two systems occurs after the two isolation valves that will be required at the interface. In other words, the two isolation valves are considered to be a part of the T/B coolant systems, not a part of the BOP interfacing systems.

The following is a list of the interfacing functions that are provided by the BOP to support the T/B systems and components:

1. The confinement building provides physical support for the T/B primary coolant system components and piping. The supports that are directly connected to the coolant system components are considered part of the T/B component, with the BOP interface occurring at the connection of the support with the building floor, wall, etc.

2. The confinement cooling system removes the heat that is dissipated from the $T / B$ components and coolant systems.

3. The confinement ventilation and air cleanup system prepare and maintain the confinement atmosphere within the environmental conditions required for maintenance of the T/B coolant systems.

4. The confinement penetration system provides routing through the confinement wall for the T/B coolant systems instrumentation and tubing.

5. A leak detection system provides detection of the leakages from the $T / B$ coolant systems. Leak detection is provided for the heat exchanger flanges, valve packing, component drain lines, and relief/safety valves.

6. A heavy-water recovery system collects, processes, and stores radioactive liquid wastes from the $T / B$ coolant systems that have $\mathrm{D}_{2} \mathrm{O}$ concentrations sufficiently high for economical $\mathrm{D}_{2} \mathrm{O}$ recovery. See the summary of typical heavy-water recovery functions, which appears at the end of this chapter.

7. A processing facility removes the light water and/or tritium (if present) from the heavy water that is in the sample tanks and upgrades it for reuse in the T/B coolant systems. The primary purpose of the $\mathrm{D}_{2} \mathrm{O}$ Upgrade System is to upgrade the T/B coolant to maintain the specified $\mathrm{D}_{2} \mathrm{O}$ concentration by removing accumulating $\mathrm{H}_{2} \mathrm{O}$. The $\mathrm{D}_{2} \mathrm{O}$ Upgrade System provides a heavy water "upgrade" to a minimum $\mathrm{D}_{2} \mathrm{O}$ concentration of 99.75 mole \%. The $\mathrm{D}_{2} \mathrm{O}$ Upgrade System upgrades high quality drainage with a $\mathrm{D}_{2} \mathrm{O}$ concentration of 95 mole $\%$ and above. 
8. Provision is made to drain and collect the light water from the secondary side of the primary coolant heat exchangers for maintenance and repair.

9. A cover gas system provides an inert cover gas for the $T / B$ coolant system pressurizers and accumulators during normal operation and for other parts of the $\mathrm{T} / \mathrm{B}$ coolant systems that are drained during shutdown conditions.

10. A liquid radwaste system handles dilute unprocessed or processed liquid waste for release or disposal.

11. A gaseous radwaste system collects and processes the radioactive gases that are generated during plant operations. The system collects gases from the various vents in the T/B coolant systems.

12. A floor drain system collects and handles the nonpiped leakage that may occur from the T/B coolant systems.

13. A process sampling system collects and delivers representative samples of $T / B$ primary coolant to designated sample stations to verify coolant chemistry.

14. Component cooling water cools the canned pump motors in the primary cooling and residual heat removal systems.

15. Secondary cooling water cools the primary and residual heat removal heat exchangers.

16. A postaccident gas vent system that provides for the collection and transfer of $T / B$ coolant system gases after an accident may be required, depending on the final selection of materials for the $\mathrm{T} / \mathrm{B}$.

17. The BOP ac power distribution system provides electrical power for the $\mathrm{T} / \mathrm{B}$ coolant system components such as pumps and valves.

18. A station grounding system provides grounding facilities for the $T / B$ coolant system components.

19. The confinement lighting system provides lighting in areas housing the T/B coolant system equipment to facilitate maintenance and inspection.

20. A plant protection system provides monitoring and trip operations for the $T / B$ coolant systems and interfacing systems.

21. A process component control system provides the monitoring and control of nonsafety cooling system parameters.

Separate systems may be required if the heavy water from the tungsten primary coolant system and the tungsten residual heat removal system cannot be mixed with that of the moderator primary coolant system and moderator residual heat removal system, which was the assumption in the preconceptual design phase. In future phases of work, it will be 
necessary to determine how much of the following equipment will need to be duplicated for the two T/B assembly units and how much can be shared.

The following is a summary of the typical heavy-water recovery functions that will be required for the $T / B$ coolant systems.

- reactor drain tanks located in the confinement building to collect leakage from T/B coolant systems;

- $\mathrm{D}_{2} \mathrm{O}$ drain tanks located in the auxiliary building to receive water from reactor drain tanks;

- high-activity waste tanks located in the auxiliary building to receive water from the $\mathrm{D}_{2} \mathrm{O}$ drain tanks, depending on activity level;

- low-activity waste tanks located in the auxiliary building to receive water from the $\mathrm{D}_{2} \mathrm{O}$ drain tanks, depending on activity level;

- subsystems to clean up $\mathrm{D}_{2} \mathrm{O}$ drained from T/B coolant systems (typical components might include an oil separator, organic trap, ion-to-ion exchangers, two filters, and sample tanks to receive the processed water);

- spent resin tank to collect spent resin from the primary and cleanup demineralizers; and

- inventory holding tanks to receive the total volume of water from the T/B coolant systems. 



\section{TRITIUM PROCESSING SYSTEMS \\ CHAPTER 8}

Page

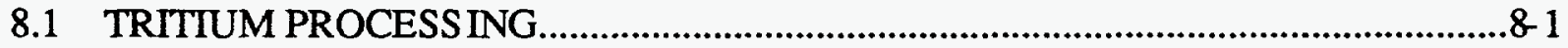

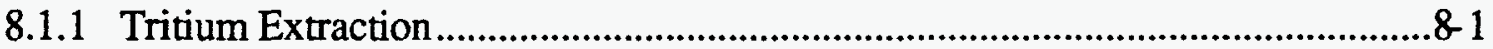

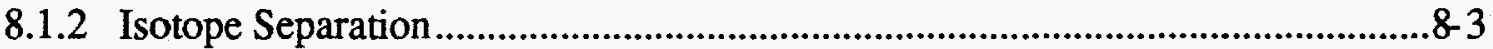

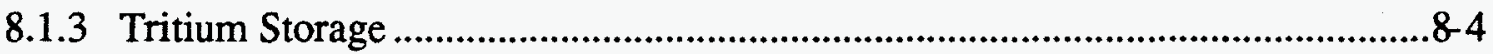

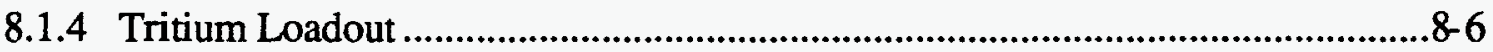

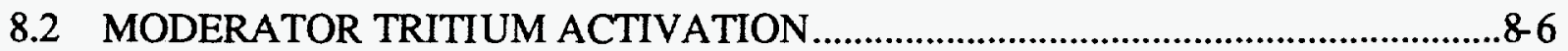

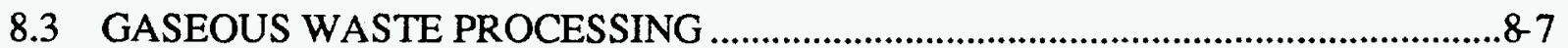

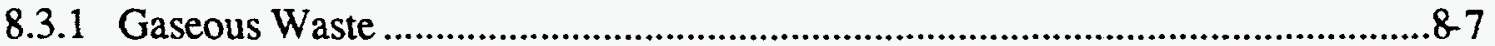

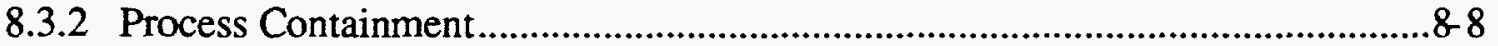

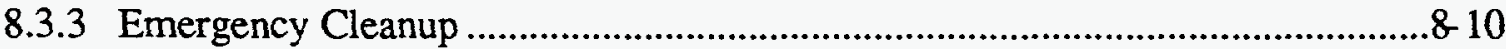

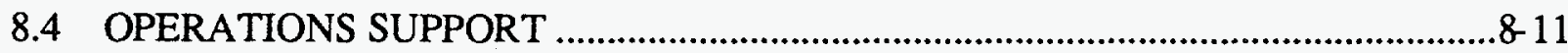

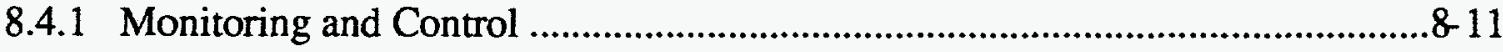

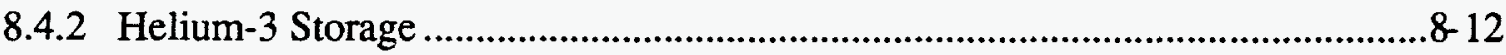

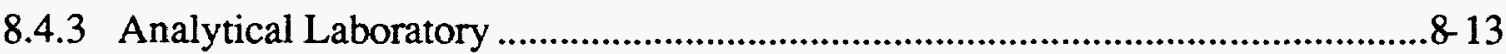

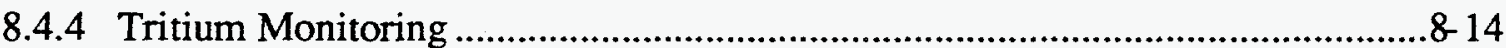

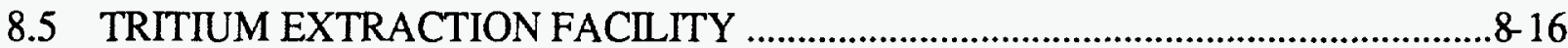

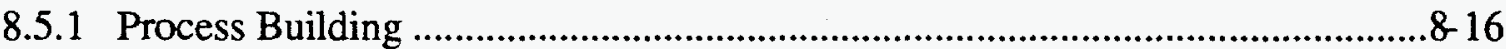

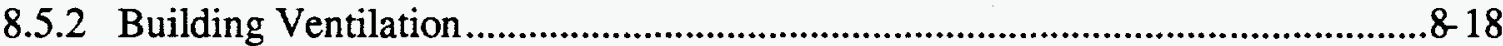

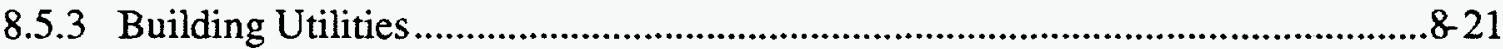

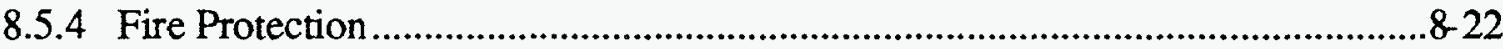

\section{LIST OF FIGURES}

Page

Fig. 8-1. DOE generic components diagram required by tritium supply process ............8-2

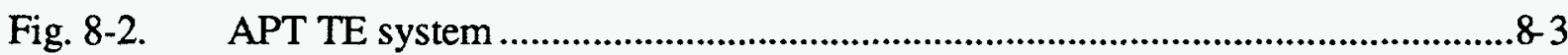

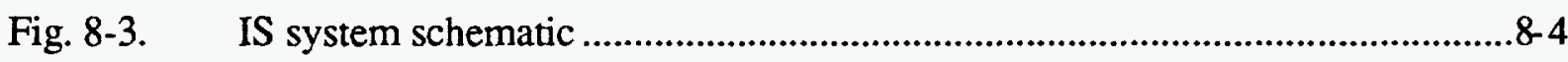




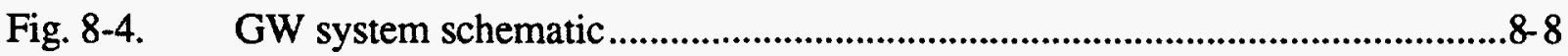

Fig. 8-5. Preconceptual layout-Tritium Extraction Facility ..........................................8-17

Fig. 8-6. HVAC schematic - Tritium Extraction Facility ...............................................8-20

\section{LIST OF TABLES}

Page

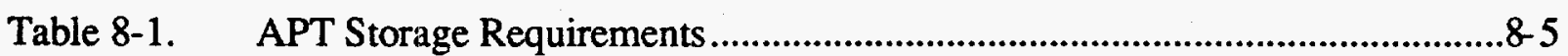

Table 8-2. APT Tritium Monitors ........................................................................... 15

Table 8-3. Tritium Extraction Facility Space Allocation ..............................................8-18 


\subsection{TRITIUM PROCESSI NG SYSTEMS}

\subsection{TRITIUM PROCESSING}

The tritium extraction system used in the $3 \mathrm{He}$ design is part of the overall concept envisioned by the DOE, regardless of the technology selected following the Record of Decision. Figure 8-1 shows the DOE generic diagram of components required by the tritium supply process. The two blocks labeled Tritium Extraction and $3 \mathrm{He}$ Separation represent the systems that remove tritium and hydrogen produced by neutron reactions with ${ }^{3} \mathrm{He}$ in the T/B from the $3 \mathrm{He}$ feed; returning the $3 \mathrm{He}$ to the $\mathrm{T} / \mathrm{B}$ and sending the hydrogen isotopes to an isotope separation process, labeled Purification in the diagram.

\subsubsection{Tritium Extraction}

The tritium extraction system processes $3 \mathrm{He}$ from the $\mathrm{T} / \mathrm{B}$ system to recover hydrogen isotopes $\left(\mathrm{Q}_{2}\right)$ The ${ }^{3} \mathrm{He}$ may contain up to $2 \%$ hydrogen isotopes $\left(\mathrm{Q}_{2}\right)$ and some impurities such as water, methane, ammonia, etc. The $Q_{2}$, an equimolar mixture of tritium and hydrogen isotopes, will be sent to the IS system. The impurities are sent to the GW system and the ${ }^{3} \mathrm{He}$ is returned to the target.

The primary purpose of the TE is to ensure that all impurities have been removed from the $\mathrm{Q}_{2}$ before it is sent to the IS. Otherwise, the IS (which operates at about $25 \mathrm{~K}$ ) would plug because of the formation of impurities ice. The total concentration of condensable impurities in the IS feed stream should be kept below 1 ppm.

It is also important that the TE separate the $\mathrm{Q}_{2}$ from the $3 \mathrm{He}$. Even though the separation could take place in the IS, it can be performed more easily with a permeator in the TE.

Because the impurities encountered by the TE are expected to be minimal, it will not be necessary for the TE to recover the tritium bound up in these impurities (e.g., $\mathrm{CQ}_{4}, \mathrm{Q}_{2} \mathrm{O}$ ). Rather these impurities will be sent to the GW where, if necessary, tritium can be recovered.

Figure 8-2 shows a schematic of the TE system. Tritium $\left(\mathrm{T}_{2}\right)$ that is bred in the APT target is removed from the target by a $3 \mathrm{He}$ carrier gas. This mixture is sent to a PERM. The hydrogen isotopes permeate through the Pd-Ag membrane and are pumped to the IS. The $3 \mathrm{He}$, any impurities, and some $\mathrm{Q}_{2}$ will leave the permeator in the retentate. The impurities (such as water, methane, ammonia, etc.) are expected to be minor but inevitable components that must be removed lest they accumulate to unacceptable levels. Thus, the retentate is sent to an MSB that is normally operated at room temperature. Under these conditions, the MSB will collect such polar molecules as water, ammonia, and carbon dioxide. The MSB effluent is recycled back to the target. If the MSB becomes saturated with polar molecules, the extraction operation can continue by using the MSB bypass line while the MSB is heated and impurities are sent to the GW.

Occasionally, one of two conditions will require cold (liquid nitrogen temperature) operation of the MSB. These conditions are (1) accumulation of nonpolar molecules (such as 


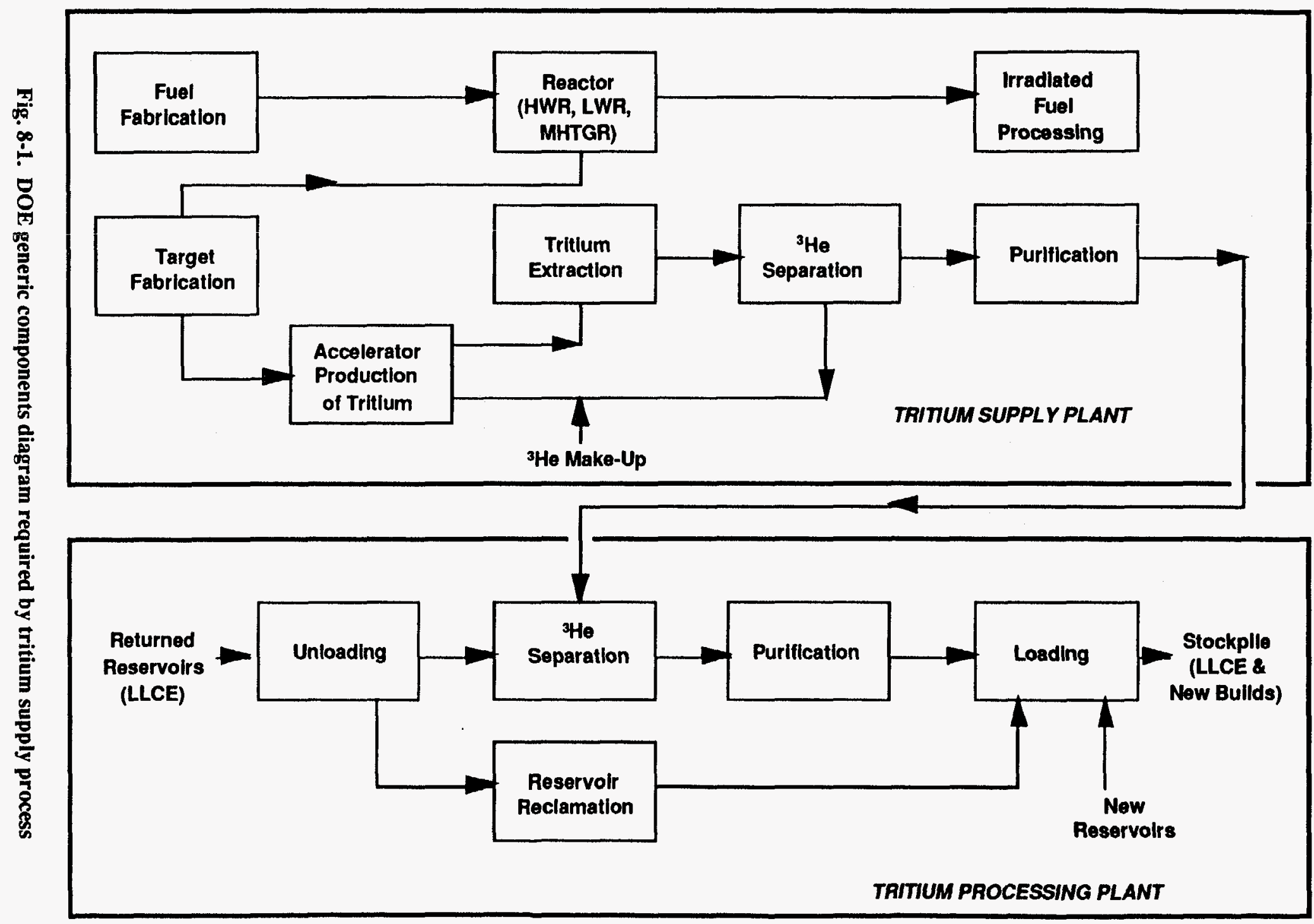


methane) to unacceptable levels in the $3 \mathrm{He}$, and (2) the need to put the $3 \mathrm{He}$ in storage. When either condition occurs, tritium breeding will be interrupted while normal flow continues to extract all elemental tritium from the ${ }^{3} \mathrm{He}$ using the permeator, and then the MSB will be cooled with liquid nitrogen. Under these conditions, the MSB will adsorb everything except 3He. When all impurities have been adsorbed, the MSB will be isolated and evacuated to recover the $3 \mathrm{He}$. Then the MSB will be heated and the impurities sent to the GW. Thereafter, the tritium and impurity-free $3 \mathrm{He}$ may be put in storage or returned to the APT Target System.

\subsubsection{Isotope Separation}

During the normal course of operation of the APT facility, a mixture of tritium and protium $\left(\mathrm{H}_{2}\right)$ will be produced in the T/B system and extracted in the TE. Pretreatment to remove unreacted $3 \mathrm{He}$ is performed by the TE. The IS will provide a cryogenic distillation

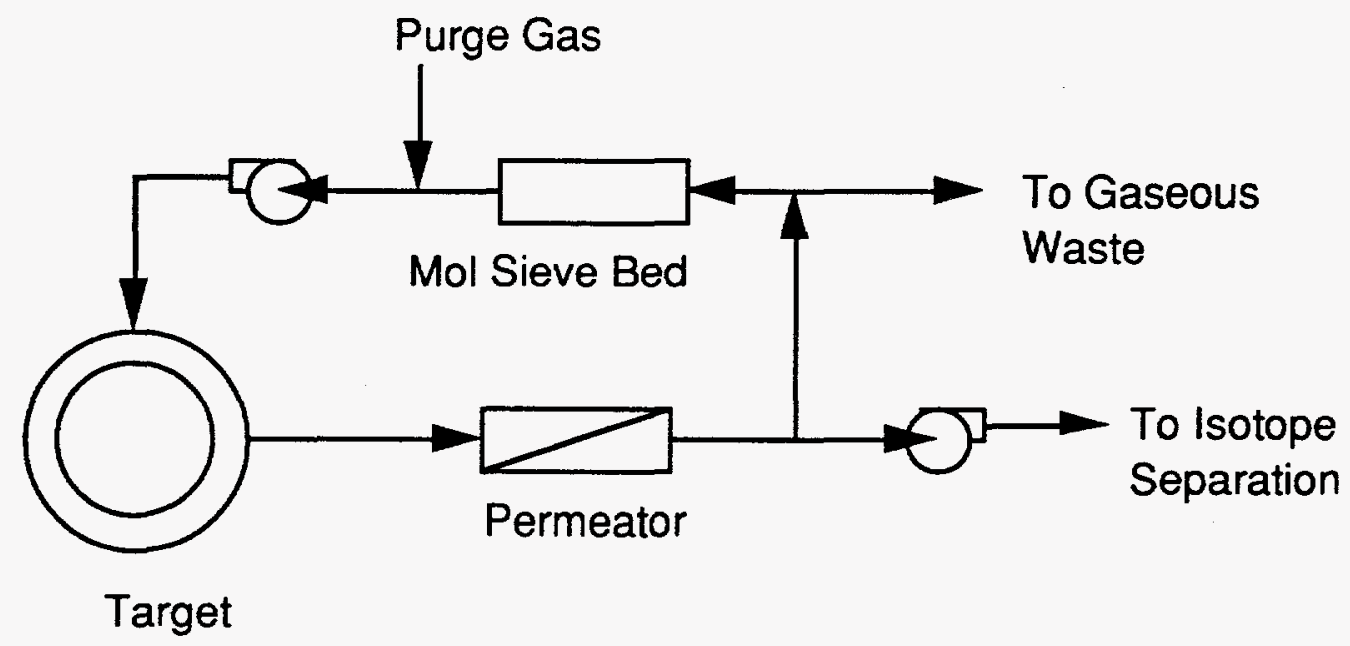

Fig. 8-2. APT TE system

system to concentrate tritium to ca. $99 \%$ and extract hydrogen $\left(\mathrm{H}_{2}\right)$ in a stream that is low enough in $T_{2}$ that it may be discarded. Because $\mathrm{D}_{2}$ is not produced in the target, only the three components $\mathrm{H}_{2}, \mathrm{HT}$, and $\mathrm{T}_{2}$ must normally be processed.

The IS will make the required separations of hydrogen isotopes in a single cryogenic fractional distillation column. The feed will be pretreated by the TE to remove impurities such as $3 \mathrm{He}$ and any condensable materials. A catalytic equilibrator is included in the feed stream to assure complete equilibration. This converts $\mathrm{H}_{2}$ and $\mathrm{T}_{2}$ to $\mathrm{HT}$. This stream is fed to a MTC. A protium-enriched stream is produced from the top of the column. The $T_{2}$ enriched reboiler product is ca. 99 mole\% tritium.

Figure 8-3 shows the flow paths and concentrations of the IS product streams. The column is packed with commercial, stainless steel packing (Helipak). The column is thermally insulated in a vacuum jacket, which also provides secondary containment for the tritium contained in the IS. The MTC jacket contains a $\mathrm{LN}_{2}$-cooled thermal shield at $77 \mathrm{~K}$ 
surrounding the column. All room temperature piping for the MTC is contained in gloveboxes or is otherwise doubly contained.

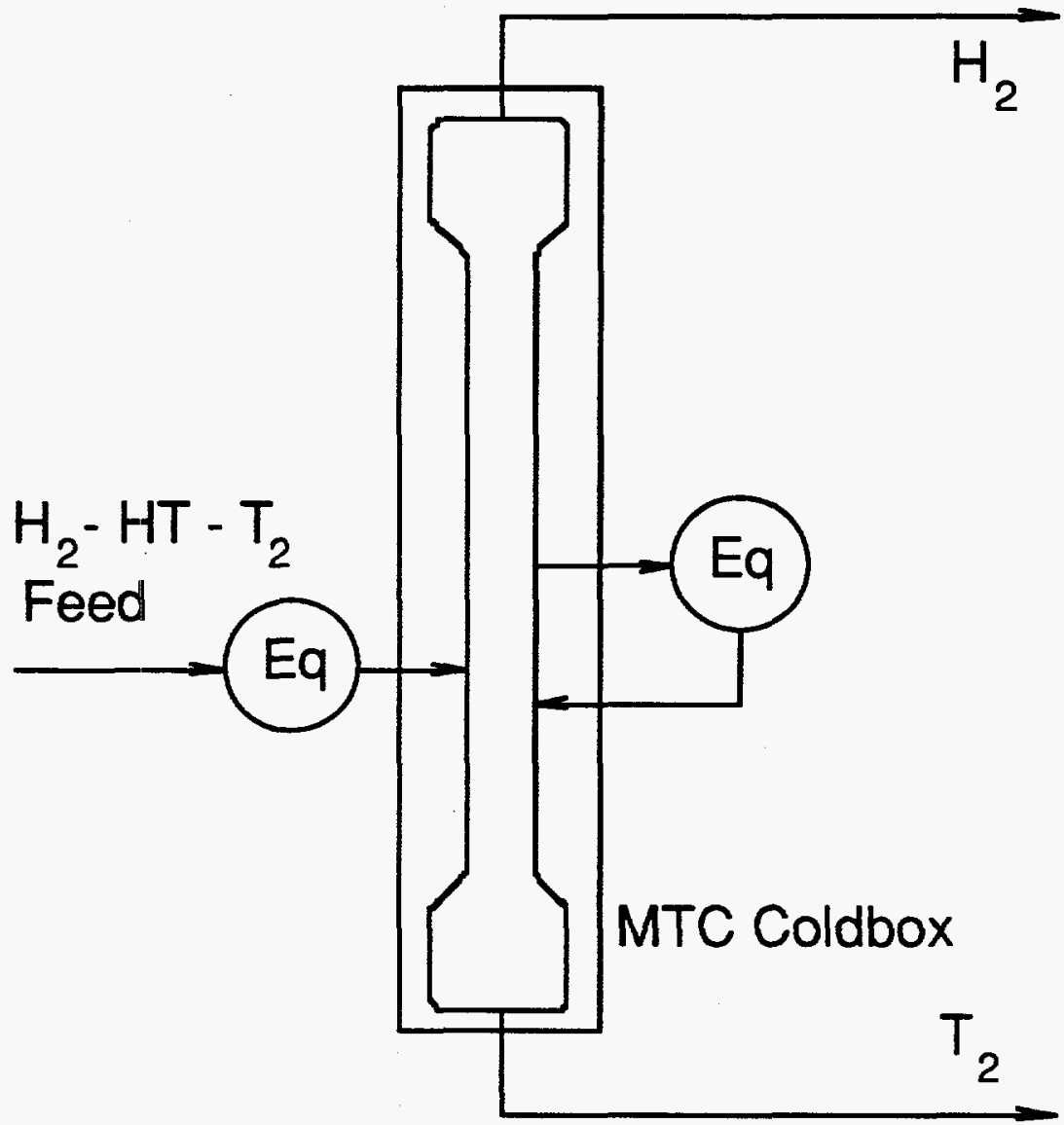

Fig. 8-3. IS system schematic

\subsubsection{Tritium Storage}

Tritium must be stored and assayed in a safe manner at the APT facility. The TS system is provided for this task. This system will provide storage for $\mathrm{H}_{2}, \mathrm{D}_{2}$, and $\mathrm{T}_{2}$ or any hydrogen-isotope mixtures $\left(\mathrm{Q}_{2}\right)$, PVT measurements, transfer pumping, and flow measurements. The proposed system permits tritium and its associated isotopes to be stored as a solid metal-hydride within storage beds or as a gas stored in tanks.

The storage of tritium will satisfy three functional requirements: (1) emergency storage for the $Q_{2}$ inventory following an unscheduled or abnormal shutdown; (2) normal shutdown storage for the $\mathrm{Q}_{2}$ inventory following a normal shutdown; and (3) product storage for high purity product. Storage capacities provided by the TS are summarized in Table 8-1.

The TS facilitates the assay of the tritium for accountability and segregates different isotopic concentrations without adding impurities to the process. The storage must be 
configured in such a way that the system will provide $\mathrm{Q}_{2}$ storage, tritium accountability, and not introduce impurities to the process.

Table 8-1. APT Storage Requirements

\begin{tabular}{lcccc}
\hline Emergency Tank Storage & Mole \% $\mathbf{T}_{\mathbf{2}}$ & $\mathbf{T}$ 2, mole & $\mathbf{D}_{2}$, mole & H2, mole \\
Cryogenic Distillation & 50.0 & 0.5 & 0.0 & 0.5 \\
Moderator Detritiation & $<0.1$ & 0.9 & 1290.0 & 61.0 \\
Tritium Extraction & $<1.0$ & 1.7 & 0.0 & 166.7 \\
Total & 0.2 & 4.1 & 1290.0 & 228.2 \\
& & & & \\
Hydride Bed Storage & Mole \% T2 & $\mathbf{T}$, mole & $\mathbf{D}_{2}$, mole & H2, mole \\
Cryogenic Distillation & 50.0 & 0.5 & 0.0 & 0.5 \\
Moderator Detritiation & $<0.1$ & 0.9 & 1290.0 & 61.0 \\
Tritium Extraction & $<1.0$ & 1.7 & 0.0 & 166.7 \\
Tritium Loadout & $>99.0$ & 100.0 & 1.0 & 0.01 \\
Total & 6.4 & 104.1 & 1291.0 & 228.2 \\
\hline \hline
\end{tabular}

The TS stores tritium and its associated isotopes as a gas in tanks or as a solid metalhydride. Solid-metal-hydride storage is based on $\mathrm{ZrCo}$ (zirconium cobalt) as the getter material. This material is currently being used at the TPL, JAERI, and TSTA. Pressurecomposition isotherms show that hydrogen can be gettered to a residual partial pressure of less than $10^{-4}$ torr at room temperature and regenerated at 500 torr at $400^{\circ} \mathrm{C}$.

An important safety concern for all getter materials is the pyrophoricity of the material. Neither $\mathrm{ZrCo}$ powder nor $\mathrm{ZrCoQx}$ (zirconium cobalt hydrides) powder burns in air at room temperature; however, they are pyrophoric at temperatures above $280^{\circ} \mathrm{C}$. $\mathrm{ZrCo}$ has some attractive advantages over uranium: (1) it is of comparatively low pyrophoricity, and its reactivity toward impurities characteristic of the fusion tritium cycle is low; (2) it is not a nuclear material; (3) it shows a small volume expansion upon hydride formation; and (4) tritium gettered by $\mathrm{ZrCo}$ can be released at relatively low temperatures. The main drawbacks of $\mathrm{ZrCo}$ are the somewhat higher hydrogen dissociation pressure of $\mathrm{ZrCo}$ hydride at room temperatures and the more stringent conditions needed to recover quantitatively the gettered tritium. It is also less fully tested than uranium at this point.

Another advantage of $\mathrm{ZrCo}$ is the dissociation of $\mathrm{ZrCo}$ in hydrogen at pressures above one atmosphere at temperatures above $500^{\circ} \mathrm{C}$. The reaction forms $\mathrm{ZrQ}_{2}$ and $\mathrm{ZrCo}_{2}$. It was observed that this reaction can be reversed if heated to $500^{\circ} \mathrm{C}$ under vacuum. After repeated hydriding, both uranium and $\mathrm{ZrCo}$ become a fine powder, however, it has been observed that $\mathrm{ZrCo}$ does this to a much less of a degree.

The maximum quantity of tritium that can be subject to release following a single process failure is to be less than 20 moles, to minimize the consequences of potential off-site release. This limit is based on the International Thermonuclear Experimental Reactor guidelines. Administrative controls are an acceptable means of limiting this potential release. Redundancy should be provided to assure availability of any storage bed or vessel equal to $99+\%$. 
Availability of $99+\%$ for individual storage units will be assured by providing $100 \%$ redundant metal-hydride storage capacity to compensate for possible bed poisoning. For gases containing $<1$ mole $\% \mathrm{~T}_{2}$, high-capacity, metal-hydride beds and tanks will be used; and for $>1$ mole $\% \mathrm{~T}_{2}$, small metal-hydride beds will be used for storage.

\subsubsection{Tritium Loadout}

The primary functions of the TL system in the TEF are to assay the purified product and to load the assayed product into approved tritium shipping containers. A secondary but necessary function is to recover accountable material from shipping containers returned from the field and to prepare the containers for reuse. In most cases, a returned shipping container can be unloaded, evacuated, and refilled with fresh product, without subsequent physical handling or reconnection.

The shipping container is manually installed into the secondary containment, attached to one of the loading stations, and emptied of its previous contents. A tritium isotopic mixture is pumped into the evacuated shipping container from the premixed and preanalyzed contents of the standard volume, until the proper loading pressure is attained. Total gas assay will be the product of the absolute pressure, the premeasured LP-50 volume, and the inverse absolute temperature, measured at the tank. Solid-state (getter) shipping containers may be filled from gas preloaded into the standard volume, and the assay will be obtained from the pressure difference, volume, temperature, and isotopic analysis measured at the standard volume.

The TEF will have a centralized analytical laboratory, in which gas compositional analysis for the TL system will be accomplished. Though the instruments necessary for assaying the composition are not part of the TL system, sampling lines leading to the analytical laboratory will be included.

When a container leaves the TEF loadout system, it will contain a properly assayed quantity of tritium, the primary container will be sealed and capped, and the secondary container will be fully assembled and sealed so that final finishing, interim storage, and shipment can be accomplished in a normally ventilated room (no fume hoods or glovebox operations).

\subsection{MODERATOR TRITIUM ACTIVATION}

During the normal course of operation of the APT facility, a small quantity of tritium will be produced in the $\mathrm{D}_{2} \mathrm{O}$ moderator through neutron capture in the deuterium. Because the tritium decays with a half-life of 12.6 years, the activation versus decay process is at $89 \%$ of equilibrium at the end of the 40 year plant lifetime (assuming $75 \%$ plant capacity). Thus the maximum concentration of tritium in the moderator will occur at the end of life.

The total amount of tritium in the moderator of both target/blanket modules at the end of the 40 year life of the plant is calculated to be about $572,000 \mathrm{Ci}$. This takes into account the decay of tritium that occurs during the production. The total volume of the moderator $\mathrm{D}_{2} \mathrm{O}$ in both target/blanket modules is about $97,200 \mathrm{~L}$. In about 10 years of operation, the concentration of tritium would reach $2.0 \mathrm{Ci} / \mathrm{L}$, the current goal for maximum activation. At the end of life, it would reach $5.9 \mathrm{Ci} / \mathrm{L}$. To maintain the $2.0 \mathrm{Ci} / \mathrm{L}$ level for the life of the 
plant would require about $287,600 \mathrm{~L}$ of $\mathrm{D}_{2} \mathrm{O}$. The other option is to detritiate the $\mathrm{D}_{2} \mathrm{O}$. To avoid the extra expense and complication of building a detritiation facility, it is a simple matter to increase the size of the moderator volume. Thus, for the current design base it is recommended to provide a total moderator $\mathrm{D}_{2} \mathrm{O}$ volume of $287,580 \mathrm{~L}$ if it is necessary to maintain the activation at or below $2.0 \mathrm{Ci} / \mathrm{L}$.

\subsection{GASEOUS WASTE PROCESSING}

\subsubsection{Gaseous Waste}

The GW processes tritium contaminated effluent from the TEF before it is released to the environment. Tritium is removed by catalytic oxidation of elemental tritium and tritiated compounds to water and oxides of carbon and nitrogen and subsequent removal of tritiated water with molecular sieve beds. This technology has been demonstrated by over 10 years of operation at the TSTA and elsewhere.

The exhaust stream is monitored for tritium before the release to the stack. If tritium levels are higher than the administrative set point, the gas is automatically recycled for further processing. The GW is monitored and controlled by computer. Little operator intervention is necessary for daily operations. All major components are redundant. The complete system is secondarily contained in a separate room that interfaces with the RD. GW design requirements are

- maximum discharge tritium concentration $20 \mathrm{mCi} / \mathrm{m}^{3}$,

- maximum discharge flow rate to stack $180 \mathrm{~m} 3 / \mathrm{h}$, and

- maximum storage capacity $108 \mathrm{~m}^{3}$.

Figure $8-4$ shows the GW system. The main system components, such as the catalytic reactor and molecular sieve beds, are fabricated on separate skids and joined through a double-valved purge system. This design provides component isolation, evacuation, and inert gas back-filling capabilities, and permits the valves to be separated from their exterior flanges, reducing tritium releases and personnel exposure during maintenance operations.

The GW is divided into two major sections, the waste-gas-receiving section (subatmospheric) and the waste-gas-processing section (above atmospheric). The principal components within the waste-gas-receiving section are the inlet RM, CF, LPR, and the ISR. Effluent gas from TEF process systems is monitored for tritium before entering the GW. Tritium streams with $<100 \mathrm{Ci} / \mathrm{m}^{3}$ are sent to the LPR. Streams with $>100 \mathrm{Ci} / \mathrm{m}^{3}$ are directed to the ISR.

The major components of the waste-gas-processing section are the CR, OHX, MSB, MSRS, CB, and the RMEX. The catalytic reactor consists of three reactors operating at room temperature, $500^{\circ} \mathrm{C}$, and $650^{\circ} \mathrm{C}$, respectively; two economizers, ECON1 and ECON2, to conserve energy; and two preheat heat exchangers. Reactors CR2 and CR3 utilize both external and internal heating. The gas exiting from the catalytic reactor is cooled to room temperature by the OHX. Moisture contained in the effluent stream is removed by a series of 
molecular sieve beds, MSB1, MSB2, MSB3, and MSB4, that are internally electrically heated to $300^{\circ} \mathrm{C}$ for regeneration. The MSRS provides low-dew-point regeneration gas for the MSBs. The MSRS also collects tritiated water for tritium assay and disposal to molecular sieve. The GW effluent stream, now devoid of water and with $<20 \mathrm{mCi} / \mathrm{m}^{3}$, is sent to the stack.

If the tritium concentration reaches $20 \mathrm{mCi} / \mathrm{m}^{3}$, the stack valve is closed and the system goes into the recycle mode. In this mode, gas is circulated through the output section until the concentration reaches $15 \mathrm{mCi} / \mathrm{m}^{3}$. The pressure in the output section can vary from 1 to 3 bar depending on the input to the system. Conversion efficiency is improved at the higher pressure.

The major safety concern of this system is a release of tritiated water to the atmosphere or to other TEF areas. This concern is mitigated by secondary containment within a sealed room and interface with RD system. All major components or components that may degrade are redundant.

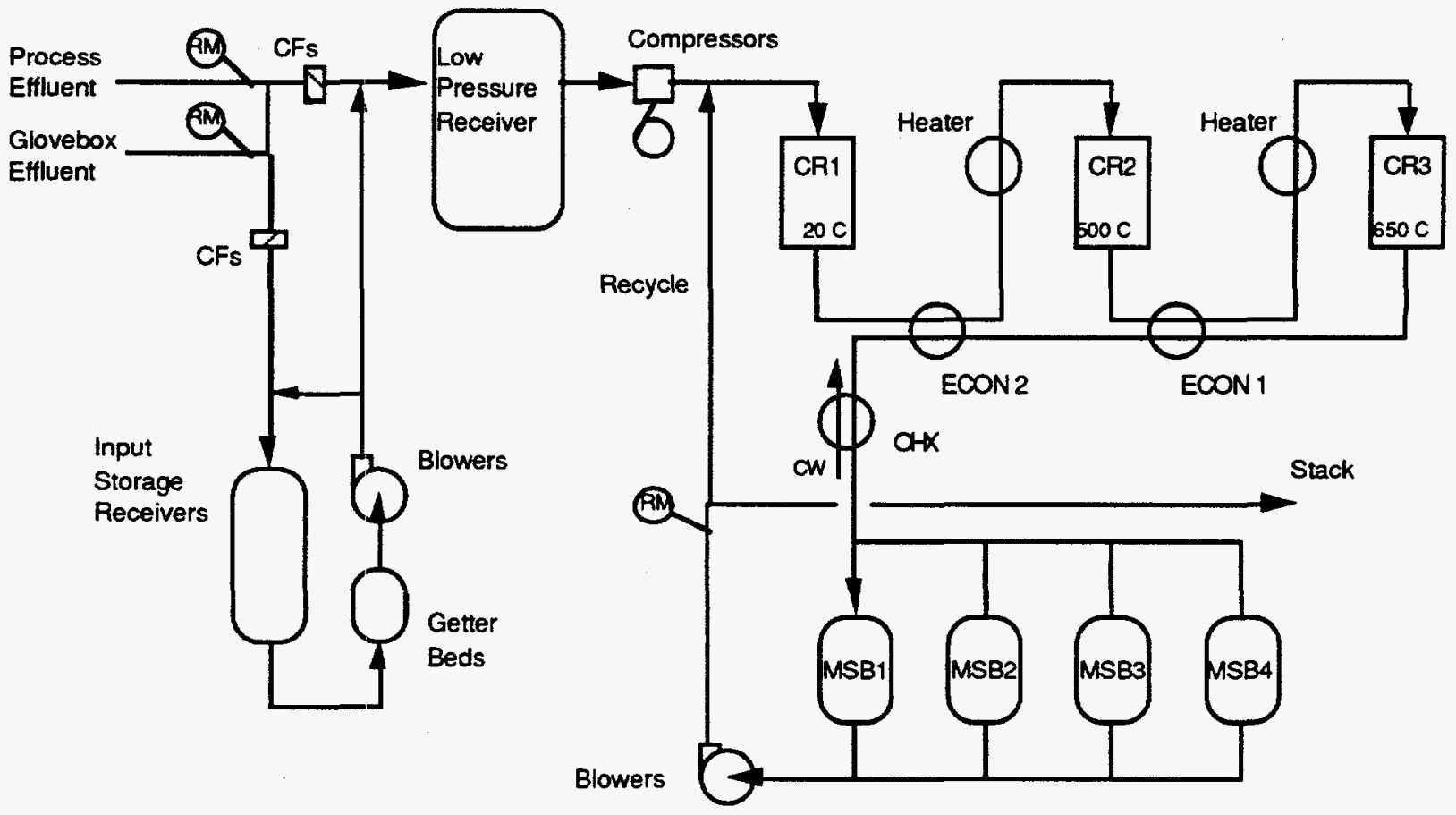

Fig. 8-4. GW system schematic

\subsubsection{Process Containment}

The PC system confines releases of tritium to gas-tight volumes until the affected enclosure can be treated by the GW system. Because the Tritium Extraction Facility is a non-reactor nuclear facility it is not necessary to have a containment system. We have therefore chosen a confinement system as discussed below. By providing enclosures for isolating tritium from areas routinely accessed by operating personnel, the PC system maintains the room atmosphere at a safe concentration for workers for any accident that does 
not breach the secondary containment. By providing interim containment for accidental releases, PC also helps limit environmental releases.

The principal component of PC is a series of interconnected gloveboxes enclosing all main process systems and the tritium piping that interconnects them. In addition, several nonprocess gloveboxes are used for contaminated equipment maintenance and contaminated waste handling. The gloveboxes are maintained at sub-atmospheric pressure by dry nitrogen purge and exhaust. Purge and exhaust components operate automatically when out-of-range pressures, or high oxygen or tritium concentrations are detected. Each glovebox is equipped with an internal tritium monitoring instrument, an oxygen-concentration sensor, a gas purging system, and provision for leak-testing internally installed components from outside the glovebox.

All gloveboxes containing process piping and components are equipped with an airlock antechamber for passing in tools and replacement components. Each antechamber is sized to hold the largest component likely to be replaced in each glovebox during the life of the facility.

Tritium gas-transfer lines between process systems are enclosed in secondary piping. Each secondary containment line is sealed off from its connecting glovebox at one end and open to the glovebox atmosphere at the other. This arrangement ensures that leakage from any inter system transfer line is detected by a glovebox monitor, enables the leakage to be routed to the GW system for cleanup, and prevents cross contamination between interconnected gloveboxes.

Because the size of the RD and GW components may preclude mounting them in a conventional glovebox, they are located inside separate, air-tight rooms (with ambient air atmosphere) ducted directly to the TEF ventilation system. If tritium is detected inside these rooms, the room is isolated from the ventilation system and cleaned up by the EC.

Fume hoods are installed in proximity to the waste handling and product load-out gloveboxes. These hoods provide space for short term storage, for repair of components with low levels of tritium contamination, and for secondary packaging and unpackaging of tritium shipping containers.

All structures supporting gloveboxes and other PC components are designed and secured to the building structure so that they will meet seismic requirements. Interbox PC piping includes flexible sections to permit relative motion between boxes of without breach of the secondary containment, and without transmitting seismic loads via this piping.

Dry nitrogen is chosen as the glovebox atmosphere to avoid combustible mixtures after tritium is released. Even though the boxes are static except when being flushed to reduce oxygen or tritium levels, significant quantities of makeup gas must be supplied for cleanup operations. Dry nitrogen is less expensive and more readily available than helium or argon, which offer only slight advantages over nitrogen. Nitrogen is also compatible with pressurized nitrogen pneumatic valve supplies, which are most conveniently exhausted inside gloveboxes. 
Aluminum is specified for gloveboxes because it is easier to clean up after a release of tritium into the box. Aluminum has lower solubility and permeability for hydrogen and water than does stainless steel, the only other material generally used for gloveboxes. At ambient conditions, tritium reacts with oxygen and exchanges with the water vapor normally present in air to synthesize significant quantities of HTO. Reaction of $\mathrm{H}_{2}$ and $\mathrm{O}_{2}$ to form water on metal surfaces is slower with aluminum than with stainless steel, in which nickel acts as an efficient catalyst. Aluminum boxes are also less expensive, lighter, and easier to modify for penetrations. Stainless steel is nevertheless acceptable for TPS PC applications.

\subsubsection{Emergency Cleanup}

The primary function of the $\mathrm{RD}$ is to remove tritium from the operating area atmosphere in the event of an accidental release. This capability is required to assure significant tritium will not be released to the environment. In the event of a tritium release to an operating area, the tritium content of the air will be reduced to a level that will permit personnel to re-enter the operating area without supplied air. The effluent gases from the RD system will either be discharged to the environment or recycled to the room, depending upon the contamination level.

A collection manifold system, extending from the RD system to the various rooms within the tritium boundary, will route the room air to the RD system. A parallel duct system will recycle the purified air back to the room from which it was exhausted. Both duct systems will be manifolded in the RD room such that all four tritium recovery units in the system can be independently connected to each duct system. A blower in each tritium recovery unit will provide the motive force to transfer the gases through the tritium recovery units and the duct systems.

Each blower will exhaust to a purification train that will include a regenerative heat exchanger, a catalytic reactor, a cooler, two drying towers, and a filter. The exhaust gases from the blower will be routed through a regenerative heat exchanger where the exit gases from the catalytic reactor will be used to preheat them. The preheated gases will then be routed to the catalytic reactor where they will be heated to $400^{\circ} \mathrm{F}$ and passed through the catalyst bed where oxidation will take place. The oxidized gases exit the catalytic reactor and are routed back to the regenerative heat exchanger where they are precooled by heat exchange with the inlet gases. From the regenerative heat exchanger the gases are routed to a water-cooled heat exchanger where they are cooled to $60^{\circ} \mathrm{F}$. The cooled gases exiting this heat exchanger are then rerouted to the drying towers where they pass through two stages of molecular sieve that adsorb the water vapor. The effluent from the drying towers will discharge to the atmosphere by way of the building ventilation exhaust system.

The EC is capable of removing postulated tritium releases from any operating area to a level of $20 \mu \mathrm{Ci} / \mathrm{m}^{3}$ within $24 \mathrm{~h}$. The EC is based on catalytic oxidation of elemental tritium to tritium oxide and subsequent removal of the tritium oxide by molecular sieve. The catalytic reactors are designed to oxidize $99.9999 \%$ of the tritium (excluding tritium in hydrocarbon compounds) to tritium oxide in one pass. Sufficient molecular sieve capacity is provided to contain all of the water vapor associated with cleanup of an operating area without regeneration during the cleanup. 
The EC provides sufficient redundancy to meet $150 \%$ of the capacity requirement for cleanup of any operating area. Ducting is provided to connect the EC to each of the operating areas within the tritium boundary such that gases from any one of these areas can be routed to the EC and then recycled to the same operating area without interrupting the ventilation of the other operating areas within the tritium boundary. The catalytic reactors are to be maintained at operating temperature during periods when the EC is in the standby mode. The capability to regenerate the molecular sieve in place is to be provided. The EC is capable of controlling the distribution of its outlet flow between recycle and exhaust to the stack to compensate for infiltration into the area being cleaned.

\subsection{OPERATIONS SUPPORT}

\subsubsection{Monitoring and Control}

The MC system is an integrated system of hardware, software, and instrumentation through which the control and operation of the TEF and contained TPS are effected. The MC assures safe handling of large amounts of tritium on a routine basis. More than trace releases to the environment are not acceptable. Exposure of operating personnel to hazardous radiation levels will not be allowed. All these functions must be performed with a high degree of reliability.

The primary purpose of the MC system is to monitor and control TPS operation. It provides the after functions:

- automatic control and monitoring for the TEF systems;

- an operator interface to the devices that control the TPS;

- coordination of measurements and control to be consistent with physical restrictions and operator commands;

- provision for alarms and corrective action when parameters exceed safety limits; and

- real time data acquisition, reduction, and display.

The MC consists of the hardware and software necessary to monitor, control, and display data from the TEF unit processes. The TEF system is complex, requiring several thousand measurements and controls. Some systems are monitored and controlled automatically, using complex control algorithms. Automatic control will be provided by a local computer provided for each unit process or by the TEF Master Computer. Operator commands, stored in a macro file, will be executed with a single command.

The MC hardware consists of central, fully redundant computers connected by a LAN to microcomputers that control each TPS unit process.

The TEF operators will monitor and control processing operations through the MMI that uses color terminals for display. These terminals and their associated software allow the 
operator to determine the status of the TEF or any part of the TPS. The displays are color flow diagrams with explanatory text and messages.

\subsubsection{Helium-3 Storage}

The function of the HS is to supply $3 \mathrm{He}$ to the target chamber. To accomplish this function, the system must have the capability to receive $3 \mathrm{He}$ from shipping containers, store the $3 \mathrm{He}$ in a tank, and transfer the $3 \mathrm{He}$ to the T/B system. Because it may be necessary to rapidly remove ${ }^{3} \mathrm{He}$ from the target chamber in the event of abnormal operation or to provide temporary storage of $3 \mathrm{He}$ during target maintenance, the system will maintain an evacuated reservoir for this purpose. The HS will consist of a compressed helium storage tank and distribution header, a low pressure charge vessel, a diaphragm charge pump, a low pressure transfer pump, and a large vacuum vessel.

The HS performs the following operations:

- Loading. In this mode the system transfers $3 \mathrm{He}$ from a shipping container to a storage tank within the system. The storage tank must have provisions for measuring the quantity of helium that was transferred from the shipping container and to sample the ${ }^{3} \mathrm{He}$ to determine the tritium content. The system must provide pumping capability to allow essentially all of the helium to be removed from the shipping container.

- Charging. In this mode the system transfers helium from the collection tank to the target chamber. This filling operation is to be completed within one 8-h shift.

- Makeup. In this mode the HS transfers small, accurately measured quantities of helium from the collection tank to the target area at a rate equal to the rate at which helium is converted to tritium.

- Recovery. In this operating mode the HS must rapidly receive and safely store the target chamber helium in the event that rapid removal from the target chamber is required.

The HS consists of piping, tanks, pumps, and instrumentation configured to accommodate the four operating modes. The helium must not contaminate the helium inventory with oil, particulate, or other gases. Tritium and helium accountability requirements must be satisfied. Because the helium may contain significant amounts of tritium, secondary containment is provided, either by gloveboxes or by walk-in enclosures. The HS must have sufficient storage capacity to store safely the inventory of the target chamber plus the working inventory required to assure an adequate supply of clean helium for normal accelerator operation. The HS provides a mechanism to recover helium contaminated with tritium from the target chamber and to safely contain these gases until they can be returned to the target chamber or transferred to the TE system. The helium loading manifold is to be configured to allow several shipping containers to be connected independently.

The system will maintain a low-pressure reservoir for emergency recovery of target chamber helium. The helium subsystem will consist of a compressed helium station and 
distribution header, a low-pressure charge vessel, a diaphragm charge pump, a low-pressure transfer pump, and a large vacuum flask.

Bulk helium will be supplied in compressed gas bottles. A pressure let-down station will provide a controlled release of the high-pressure gas to the helium charge vessel. A diaphragm compressor will be provided to complete the helium transfer after the bottle pressure has fallen below the charge vessel's working pressure.

Accommodation will be made for sampling of the bulk helium before it is introduced into the charge vessel. The high-pressure manifold will be designed to allow nitrogen purge and evacuation to the gaseous waste system to minimize possible tritium contamination.

In operation, the charge vessel will supply the blanket charge and maintenance system. Vessel pressure will be maintained initially by the compressed gas bottle pressure reduction manifold. When the pressure in the compressed gas bottle falls below the charge vessel operating pressure, a diaphragm pump will start. Subsequently, the charge vessel pressure will ride on the compressor discharge.

\subsubsection{Analytical Laboratory}

The function of the AL is to support analysis requirements for TE, IS, TS, TL, MD, GW, $\mathrm{RD}$, and other general analysis requirements.

The AL will determine the quality, composition, and isotopic ratios of various systems, components, and materials that will be used or generated during the process of producing tritium. Typical determinations will include

- isotopic ratios of hydrogen, deuterium, tritium, and various compounds containing these isotopes;

- analysis of the helium feed stock, helium feed to TE and purified helium from TE;

- assay of the final product;

- assay of residual product in returned shipping containers;

- analysis of moderator water, cooling water and process generated water; and

- analysis of samples taken from GW and EC systems.

The following analytical instruments and materials are to be provided in the laboratory:

- double focusing ultra high resolution gas mass spectrometer;

- custom constructed laser Raman spectrometer,

- liquid scintillation counter with refrigerated sample chamber;

- gas chromatograph with mass spectrometer detector; 
- calorimeter; and

- gloveboxes for containment of radioactive analytical operations.

The laboratory will also serve as a secondary source for calibration of the various online instrumentation used throughout the TEF. Furthermore, the laboratory is to provide final determination of waste stream compositions before disposal.

\subsubsection{Tritium Monitoring}

The TM system monitors and controls the operation of the tritium processing systems to reduce the likelihood of uncontrolled releases of tritium and to minimize personnel and public exposure to airborne tritium.

To accomplish this goal, TM performs the following functions:

- monitoring glovebox atmosphere for tritium;

- monitoring tritium concentrations in room and room exhaust air;

- monitoring the stack effluent for potential tritium releases to the environment;

- initiating alarms when preset concentrations are reached so that appropriate corrective actions may be taken;

- initiating cleanup of the glovebox atmospheres when tritium concentrations reach a preset value; and

- isolating the room when the room air concentration reaches a preset value so that cleanup of the room by the emergency cleanup system may be initiated.

The TM consists essentially of several tritium monitors that are categorized according to their function. Table 8-2 lists these monitors and some of their properties. With the exception of the stack bubbler, the instruments are ion-chamber instruments chosen because of their proven reliability, versatility, and sensitivity.

Each glovebox where tritium is handled is monitored by a single monitor of the flowthrough ionization chamber type.

Two alarm settings are to be incorporated into each instrument-the low setting to trigger cleanup of the glovebox atmosphere by the gaseous waste system and the high setting to alert personnel of a major release of tritium into the glovebox. Depending on use and operating history, the alarm settings may be different for different gloveboxes. The alarm signals are fed to the computer in the control room, which then initiates cleanup operation. Cleanup is terminated by the computer at a lower concentration level, again determined by 
the operating history of the glovebox and (largely) by the background level of the glovebox itself and of the glovebox monitor.

All instruments are equipped with local, control room, and support center readouts and/or alarms.

Table 8-2. APT Tritium Monitors

\begin{tabular}{|c|c|c|c|}
\hline Name & Detector Type & Range, $\left(\mu \mathrm{Ci} / \mathbf{m}^{3}\right)$ & Alarm, $\left(\mu \mathrm{Ci} / \mathrm{m}^{3}\right)$ \\
\hline Glovebox Monitor & $\begin{array}{r}\text { Flow-through } \\
\text { ion chamber }\end{array}$ & $1-106$ & $10^{3}, 10^{5}+$ low flow \\
\hline Room Monitor & $\begin{array}{r}\text { Flow-through } \\
\text { ion chamber }\end{array}$ & $1-10^{6}$ & $\begin{array}{l}20,10^{2}, 10^{4} *+ \\
\text { low flow }\end{array}$ \\
\hline $\begin{array}{l}\text { Room Monitor (high } \\
\text { range) }\end{array}$ & $\begin{array}{l}\text { Open-walled } \\
\text { ion chamber }\end{array}$ & $10^{3}-10^{9}$ & None \\
\hline $\begin{array}{l}\text { Room Monitor (cold } \\
\text { area) }\end{array}$ & $\begin{array}{l}\text { Open-walled } \\
\text { ion chamber }\end{array}$ & $1-10^{3}$ & 20 \\
\hline Duct monitor & $\begin{array}{l}\text { Open-walled } \\
\text { ion chamber }\end{array}$ & $1-10^{6}$ & $20,10^{2}, 10^{4 *}$ \\
\hline \multicolumn{4}{|l|}{ Stack Monitors } \\
\hline 1. Real Time & $\begin{array}{l}\text { Flow-through } \\
\text { ion chamber }\end{array}$ & $1-106$ & $50,104 *+$ low flow \\
\hline $\begin{array}{l}\text { 2. Continuous } \\
\text { Sampler }\end{array}$ & $\begin{array}{l}\text { Glycol/water } \\
\text { bubbler }\end{array}$ & $3 \mathrm{mCi}$ - infinity & Flow + Temp. \\
\hline
\end{tabular}

*The room evacuation alarm is triggered at $10^{4} \mu \mathrm{Ci} / \mathrm{m}^{3}$.

The room monitoring system consists of standard and high-range room monitors strategically located in rooms where tritium is handled, and of room exhaust monitors located at points where room air is exhausted through the exhaust ducts.

Room air is exhausted either through exhaust registers or by way of entry or fume hoods. The exhausted room air will be monitored (preferably) by an open-walled exhaust monitor with the chamber located inside the duct such that, if there is more than one branch exhaust duct from the same room, the chamber will be strategically located to monitor the air past the point where the branch ducts come together.

A total release of the tritium inventory in a particular room may result in the upper range of the standard room monitor(s) in that room to be exceeded. For those rooms, high-range monitors will be installed.

A single low-range monitor will be used to monitor the intake air to Zone IV to warn of any tritium released from the Zone II stack that might re-enter the building through the ventilation system. This monitor will have its readout and (single) alarm in the control room.

DOE and EPA regulations require that facilities handling the quantities of tritium to be found in the TEF monitor the effluent with a real-time monitor and with a passive, continuous sampler. 


\subsection{TRITIUM EXTRACTION FACILITY}

\subsubsection{Process Building}

A preconceptual layout for the Tritium Extraction Facility is shown in Fig. 8-5. Architecture of the operating areas was driven by the following considerations:

- The operations cells receive input at one end and discharge tritium product at the other. Therefore, the receiving and shipping areas are on opposite ends of the building.

- Personnel access into the operations cells logically should not be through the receiving area, shipping area, or the HVAC room. Therefore, only one side of the process cell is available for routine personnel access.

- Personnel monitoring, anti-C clothing change rooms, personnel decontamination showers, and the health physics laboratory need to be collocated near the operations cells entry.

- The operations cells include Area A and Area B. The other process areas include Experimental Rooms 1 and 2, the Gaseous Waste Room, the Emergency Cleaning Room, the Analytical Laboratory, the Tritium Storage Room, the Helium Storage Room, and the Product Vault. These areas are all within the tritium boundary.

- The control room and support center are located on opposite sides of the operations cells to provide complete visual coverage of functions and personnel.

- Emergency egress will comply with the Life Safety Code, NFPA 101.

The architectural design of the APT-TEF building was driven by the function and support requirements of the tritium operations cells and process areas. The result is an architectural form tailored to the processes and functions to be performed. Development of the conceptual design proceeded in the following sequence:

1. Space requirements of the tritium operations cells and process areas.

2. Development of process support and facility functions and their space requirements.

3. Preliminary floor plan and elevation development.

4. HVAC and facility systems design.

5. Structural design.

6. Design integration and finalization. 


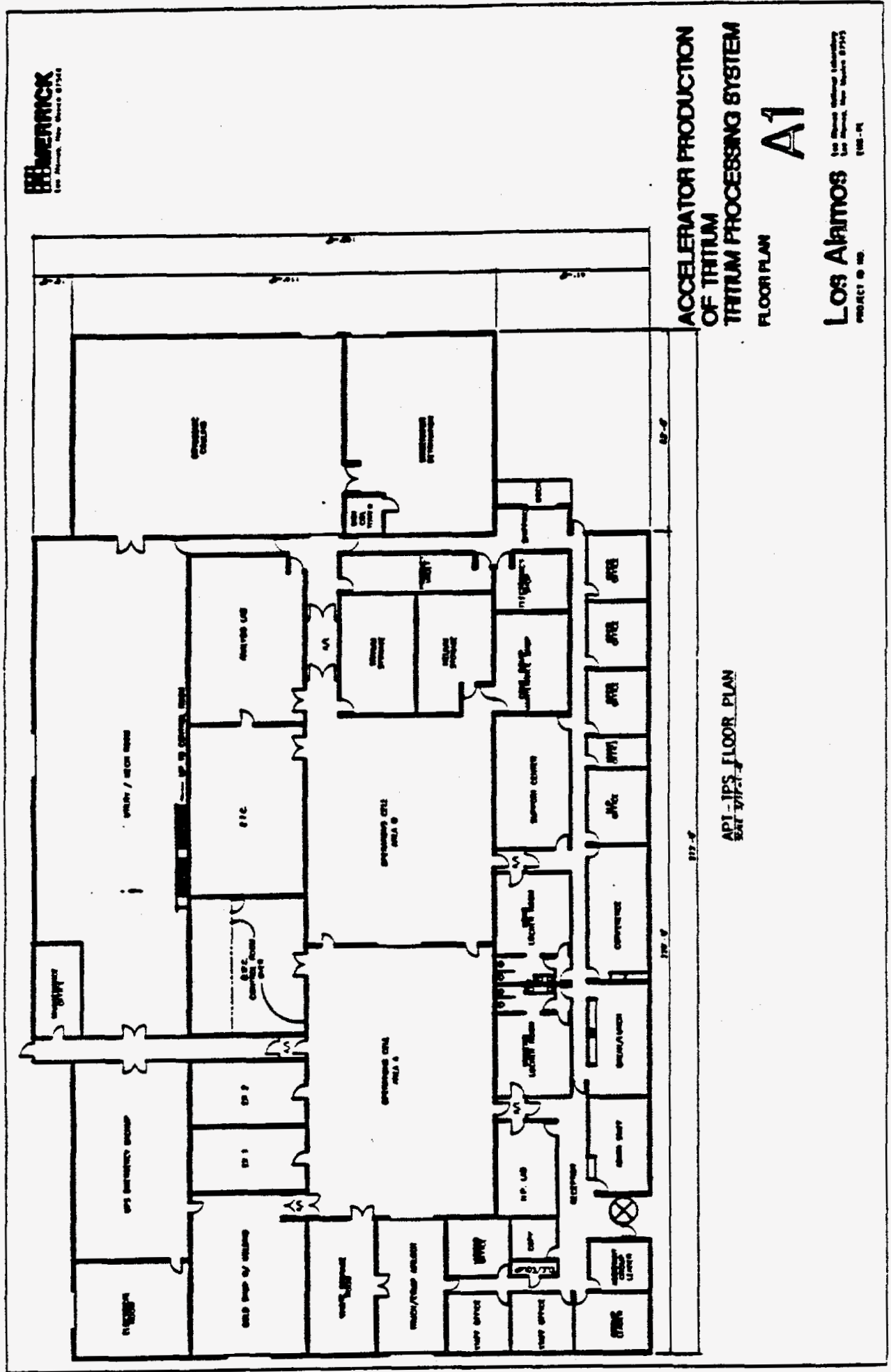

Fig. 8-5. Preconceptual layout-Tritium Extraction Facility. 
The building is structured around the central feature of the operations cells. It must have a secondary confinement structure designed to withstand design basis earthquakes, fires, and other accidents that might release radioactive or hazardous material outside of the site boundary. This tertiary confinement structure is a concrete enclosure that is large enough to contain the operations cells and the other process areas within the tritium boundary.

The elevation of the secondary confinement structure was determined in response to the process-equipment vertical height. The highest point in the cell is approximately $36 \mathrm{ft}$ at the top of the roof trusses. The overall ceiling height has been established by allowing for an overhead bridge crane hook height of $21 \mathrm{ft}$.

The ceiling in the operations cells and other process areas will be designed to facilitate the collection and removal of tritium in the event of an accidental release. This will be accomplished by sloping the ceiling upward to a confining enclosure (capture point) at the high point of the ceiling.

The support functions for the operations include such things as a health physics laboratory, receiving area, shipping area, maintenance rooms, a break room, control room, support center, offices, and others, as detailed in the Space Summary. The support areas do not provide any form of hazard confinement. Therefore, the construction of these areas will be conventional and will include a steel structure, panel walls, interior finishes, and typical commercial ventilation and environmental control. The support building surrounds the tertiary confinement structure.

The total areas in net square feet (NSF) for the four principal categories are summarized in Table 8-3. Circulation is approximately $6 \%$ of the building programmed space (NSF).

Table 8-3. Tritium Extraction Facility Space Allocation

\begin{tabular}{|c|c|}
\hline Area & Square Feet \\
\hline Operations Cells Area & $8,700 \mathrm{NSF}$ \\
\hline Operations Support Area & \\
\hline Equipment Rooms & $8,886 \mathrm{NSF}$ \\
\hline Personnel Areas & $13,884 \mathrm{NSF}$ \\
\hline Administrative Support Area & $5,580 \mathrm{NSF}$ \\
\hline Circulation & 2,566 NSF \\
\hline Subtotal & $\overline{39,616}$ NSF \\
\hline Walls & $2,482 \mathrm{NSF}$ \\
\hline Total (Gross Square Feet) & $\overline{42,098}$ GSF \\
\hline
\end{tabular}

\subsubsection{Building Ventilation}

The HVAC system for the Tritium Production Facility consists of two independent HVAC air-handling systems, one for the tritium production areas of the facility and one for the office and other cold support areas. Two HVAC air-handling systems and the zones that they serve 
were chosen because of the radiological release and contamination potential of individual rooms per DOE Order 6430.1A and ERDA 76-21. The contamination control plan showing the individual room operating pressures and their associated HVAC system is shown in Fig. 8-6. The first system is classified as a Zone II HVAC system because of the potential for tritium release and contamination potential within certain rooms within the TEF. This system will operate in a constant volume, once-through mode of operation to eliminate the potential for recycling tritium in the event of release. All areas that are served by the Zone II HVAC system are maintained at a negative pressure relative to the atmosphere. This ensures that there is no exfiltration from areas within the TEF that have the possibility of becoming tritiated.

The remaining areas of the building are classified as Zone IV because of their low contamination potential and will be served by the Zone IV HVAC system. This system will operate in a constant volume mode with a minimum amount of return air exhausted.

The building's Zone I areas are those in which tritium is confined. Most Zone I areas are the gloveboxes inside the process areas. Because these gloveboxes are purged by the nitrogen supply system in concert with Gaseous Waste system and not the air-handling system, there is no Zone I HVAC system. There is also no Zone III HVAC system because of the contamination classification of a Zone III area per DOE Order 6430.1A.

The Zone II HVAC system for the TEF ventilates, conditions, and maintains required space-design pressures. The HVAC system maintains the integrity of the confinement zone by maintaining a pressure differential between Zone $I I$ and any surrounding zone. This ensures that all airflow is into areas of highest probable contamination and that all exhaust is through the TEF stack. The following are the major functions of the Zone II HVAC system:

- The HVAC system maintains a negative pressure differential between the secondary confinement and tertiary confinement. This will ensure that all airflow is from an area of lower probability of contamination (Zone IV) to an area of higher probability (Zone II).

- The HVAC system must be integrated with the RD system to isolate a room from the HVAC system during a tritium release and to allow the $\mathrm{RD}$ system to remove tritium from the room. This will prevent release of tritium to other rooms or to the atmosphere. During an RD operation on a contaminated room, the remaining rooms will remain on the HVAC system, and the contaminated room will be kept at a negative pressure relative to the atmosphere by the RD system.

At the room level, the supply and exhaust air ducts are equipped with fast acting butterfly isolation dampers. In the event that tritium release is detected in a room, these dampers will close and isolate the room from the Zone II HVAC system. This action not only prevents the exhausting of tritiated air directly to the atmosphere but also prevents the spreading of tritium throughout the facility. At the same time the HVAC room isolation dampers are closing, the EC fast-acting butterfly isolation dampers are opening and the EC becomes operational, which allows the $\mathrm{EC}$ to begin the cleaning process for the contaminated room. 


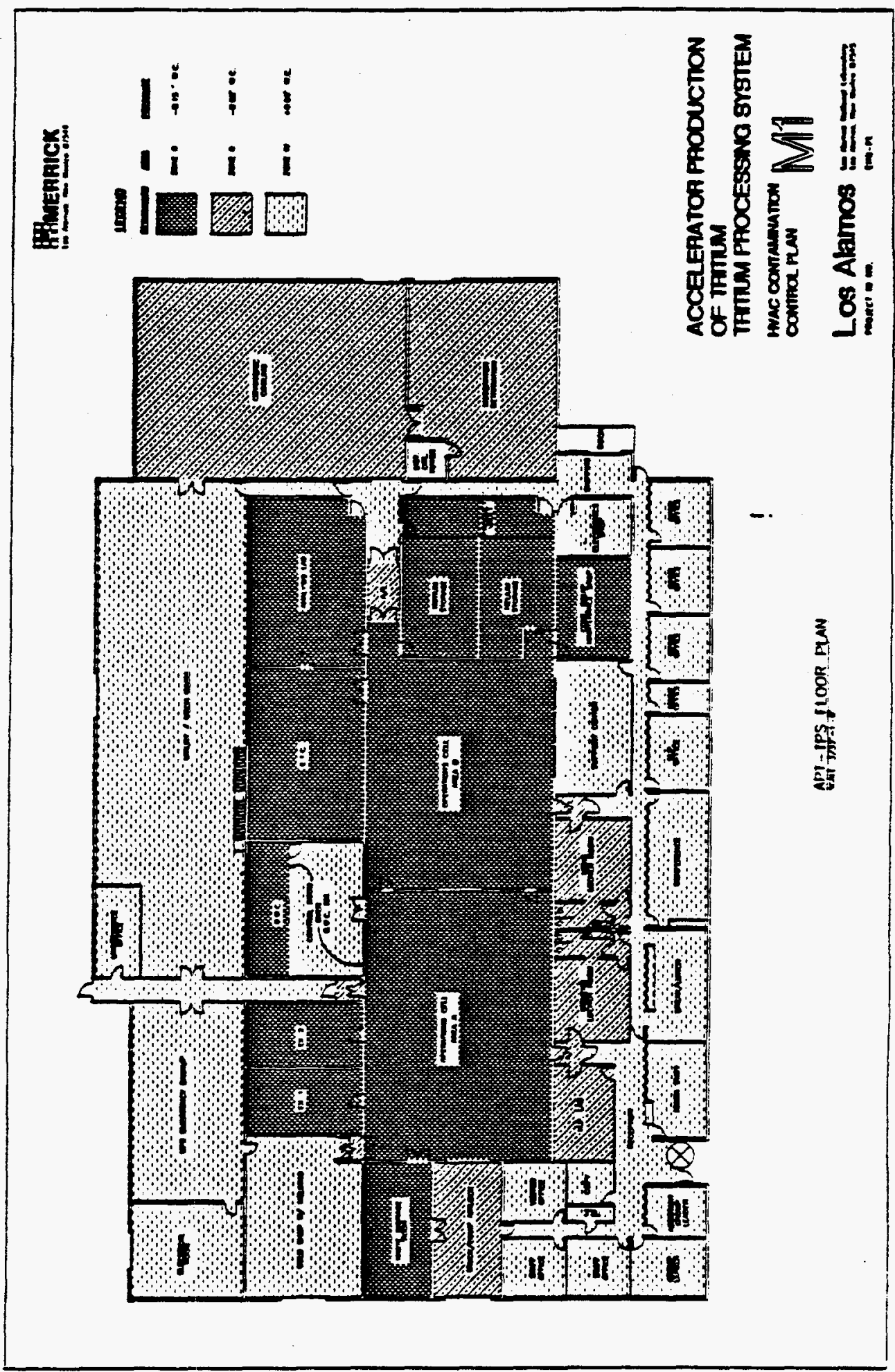

Fig. 8-6. HVAC schematic-Tritium Extraction Facility. 
The preconceptual system design for the Zone IV HVAC system consists of an outside air intake louver, supply air-handling unit, duct mounted air flow measuring stations, atomizing humidifier, terminal heating coils, return/exhaust air fan, and an exhaust air louver. The supply air-handling unit comprises a hot water preheat coil with an integral face and bypass damper, $30 \%$ and $85 \%$ ASHRAE rated filters, chilled water cooling coil, and a centrifugal supply air fan.

The HVAC system is designed to operate in a recirculating mode, where approximately $15 \%$ to $20 \%$ of the return air is exhausted, and outside makeup air is drawn into the system to satisfy outside air requirements. The HVAC system also incorporates an outside air economizer to use outside air for free cooling. The Zone IV air is drawn through an outside air intake louver, conditioned through a hot water-face and bypass preheat coil, through two stages of filtration ( $30 \%$ and $85 \%$ ), cooled by a chilled water cooling coil, and supplied by a backward-inclined centrifugal air fan. The air is distributed by the supply-air ductwork after having been humidified by a duct-mounted atomizing humidifier. The air is then heated by a terminal heating coil before it is delivered to the space. The air is then returned by a return/exhaust air fan. Space pressures are established by initially balancing the system to design space pressures.

\subsubsection{Building Utilities}

Mechanical and electrical systems are provided to supply facility and process utility requirements for communications, sanitary sewer, breathing air, nitrogen systems, potable water, instrument air, fire protection, and component cooling water. Electrical power, cooling water, and natural gas will be supplied to the TEF from the balance of plant systems.

Emergency electrical power will be provided with associated switch gear and controls for safety class systems, should any be identified through safety analysis. Standby power will be provided with associated switch gear and controls for systems with severe production or economic impacts, should any be identified.

UPS systems will be provided for selected portions of the process control systems, accountability systems, safety monitoring, and alarms. The UPS will be compatible with the electrical system and shall include sufficient battery charging capability to maintain the system operation during normal conditions and sufficient battery capacity to provide an orderly shutdown on loss of normal, emergency, or standby power.

A voice-paging and intercom system shall be provided throughout the facility, minimizing personnel access and egress to controlled areas. The telephone system will provide standard telephone service to outlets throughout the facility. The design is to provide capability to incorporate secure telephone service. A public-address system will include the capability of paging or alert from the computer control room or from other balance-of-plantsite central-paging locations to the APT. A LAN is to be provided to facilitate computer communication throughout the TEF.

The sanitary sewer system will provide liquid-waste collection from all noncontaminated drains, including cold maintenance floor drains, restrooms, sinks, and custodial drains. No drains that originate within in the tritium boundary shall connect to the sanitary sewer. 
A breathing air system will provide clean, dry air for consumption by technicians wearing supplied air respirators and suits while conducting decontamination, maintenance, and waste management tasks. Self-Contained Breathing Apparatus (SCBA) bottles meeting breathing air specifications will be supplied.

Nitrogen systems provide a continuous supply of LPN and HPN gas, which is used for general pneumatic, utility, and purge-gas requirements in the TEF. HPN is used to operate the pneumatic instrumentation and equipment in the process gloveboxes. LPN is used to purge the glovebox enclosures. A liquid nitrogen supply is also available for process operations.

Potable Water is supplied to the facility from the balance of plant water main. Potable cold water is used to supply the mechanical systems, safety showers, sinks, restrooms, custodial service sinks, and drinking fountains. Hot water is provided by local instantaneous electric water heaters.

An instrument air system will supply clean, dry, oil-free air for facility control instruments, HVAC control systems, and general utility use.

A separate cooling water system will be used in the TEF as a coolant for the TEF processes. The MD system will have the largest cooling requirements.

\subsubsection{Fire Protection}

The function of the fire-protection water system is to provide an adequate water supply for the facility's sprinkler system in the event of a fire within the facility. These systems will be designed as a pre-action system within the tritium confinement and the control room and a wet-pipe sprinkler system in the remaining areas of the building.

The fire-detection and fire-alarm system will provide an electrically operated, totally solid-state, single supervised, closet-circuit fire alarm and sprinkler supervisory system. The system shall include the capability of providing a zone-enunciated indication of the fire alarm, water-flow alarms, and sprinkler-supervisory signaling service. 


\section{TECHNOLOGY BASE AND FUTURE DEVELOPMENT \\ CHAPTER 9}





\subsection{TECHNOLOGY BASE AND FUTURE DEVELOPMENT}

Throughout the preconceptual design work described in this report, we have identified items that require additional study to place the technical feasibility on a firmer basis and to quantify experimentally the design, safety, and environmental characteristics of APT.

The participants in this study have proposed a 4-yr technology development program that, if approved and funded by the DOE, would carry APT through the conceptual design, preliminary design and into the start of final design. That program is presented in the $A P T$ Technology Development Program Four-Year Plan and contains considerably more detail than would be appropriate here. We therefore present, in bullet form, specific items identified in the physics, engineering, and nuclear methodology benchmarking areas. The list is not intended to be all-inclusive but to indicate areas of priority for near-term future technology development.

- Physics design of the neutron source and blanket tritium production regions

- Optimize design of central neutron source region-include optimization of the size and geometry of flux-trap gaps; $\mathrm{W} / \mathrm{D}_{2} \mathrm{O}$ coolant fraction; rod-bundle thickness; adjustment of the lateral extent of rod bundles; and the potential use of tungsten enriched in $184 \mathrm{~W}$.

- Optimize design of backstop region-optimization of materials; thickness; coolant fraction; and minimization of lead are needed.

- Optimize design of radial target zone-minimize amount of lead; incorporate engineering details into the physics model as they evolve and evaluate their neutronic impact.

- Optimize Inconel-718 helium chamber design-adjust thickness as a function of pressure, examine alternate materials.

- Update design to reduce $\mathrm{D}_{2} \mathrm{O}$ and ${ }^{3} \mathrm{He}$ requirements.

- Improve energy deposition spatial detail-proton profile; proton beam window; rod-bundle backstop; radial target; moderator tank.

- Determine moderator tank wall cooling needs.

- Determine shield cooling needs.

- Complete preconceptual physics design of neutron backstop, 20-MeV beamstop, and $100-\mathrm{MeV}$ beamstop.

- Investigate the use of a light water moderator.

- Continued engineering design of the T/B and beamstop

- Investigate moderization of the design to allow for component changeout.

- Continue to develop a preconceptual design for the $3 \mathrm{He}$ Heat Transport Systems, and analyze the system for normal operation and limiting accident conditions.

- Develop a preconceptual orifice design for the tungsten rod bundles needing orificing to distribute the flow and achieve a uniform power to flow ratio.

- Develop a preconceptual design to preclude a total flow blockage to a rod bundle assembly. 
- Update the preconceptual design of the TPCS, MPCS, NCS, and BCS to reflect the latest thermal loads associated with each system (the present design of the TPCS and MPCS are based on heat loads from January 1993 that are outdated).

- Perform TRAC analyzes and investigate major ruptures of the $3 \mathrm{He}$ chamber and blanket tube piping into the MPCS to support item \#1 and the design of the moderator tank and pressure relief system.

- Update TRAC LOCA analyses in the TPCS to reflect the latest thermal/mechanical design and to establish if an accumulator is needed with the current target cavity arrangement.

- Continue to develop a preconceptual purification system design to control the circulation, precipation and plating of radioactive spallation and corrosion products in the TPCS.

- Continue to develop the preconceptual thermal-mechanical design for the moderator tank and internal components (beam extension tunnel, beam entrance window, target lead, proton beam backstop region, $3 \mathrm{He}$ heat exchanger and blanket tubes, and support structures) that satisfies tritium production and manufacturing requirements and will minimize the volume of required helium3 and heavy water.

- Continue to develop the remote-handling systems and operations to improve availability and reliability.

- Continue the work initiated in FY 1993 to support the development of an accident delineation document with safety analyses that presents a firm understanding and quantification of the T/B system safety characteristics.

- Review the T/B preconceptual design to ensure that it meets all the requirements in the updated (TBD) GSRD and RD.

- Perform design studies using lithium as the tritium production target.

- Continue to develop I\&C requirements for all T/B systems.

- Complete preconceptual engineering design of neutron backstop, 20-MeV beamstop, and 100-MeV beamstop.

- Design the shield-cooling system.

- Design the support structures to handle seismic requirements.

- Develop a seal interface design between the target module and the target cavity.

- Update tungsten rod bundle decay heat analyses to support different retargeting options.

- Benchmarking and validation of nuclear design methodology and improvement of materials information

- Benchmark the LAHET Code System against existing data: integral (water bath) experiments; bare target integral and differential data; and other data, including the recently performed physics design $\mathrm{T} / \mathrm{p}$ test.

- Benchmark the CINDER'90 Code against existing and new data: radionuclide production experiments; induced radioactivity, decay heat.

- Improve the existing nuclear database by performing key measurements of neutron production and neutron multiplication reactions.

- Extend the evaluated nuclear data library to $100 \mathrm{MeV}$ to bridge the gap between conventional neutron transport codes and LAHET.

- Complete physics design T/p measurements at $800 \mathrm{MeV}$ and continue support for prototypic $T / p$ measurement. 
- Design experiment to provide data on radiolysis, corrosion, and radiological contamination of the tungsten rod bundles and their coolant.

- Perform further tests of a number of materials including irradiated tungsten and Inconel samples to determine their properties and confirm their suitability for the APT application. 
